\title{
SELECTED STUDIES OF THE PRINCIPLE OF RELATIVE FREQUENCY IN LANGUAGE
}


LONDON : HUMPHREY MILFORD

OXFORD UNIVERSITY PRESS 


\section{SELECTED STUDIES OF THE PRINCIPLE OF RELATIVE FREQUENCY IN LANGUAGE}

BY

GEORGE KINGSLEY ZIPF

INSTRUCTOR IN GERMAN AT HARVARD UNIVERSITY

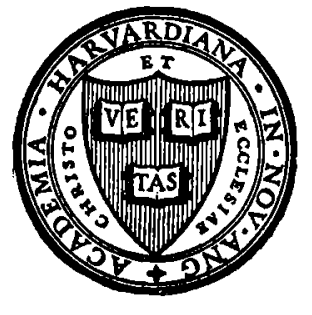

HARVARD UNIVERSITY PRESS

CAMBRIDGE, MASSACHUSETTS

I $93^{2}$ 
COPYRIGHT, I932

BY THE PRESIDENT AND FELLOWS OF HARVARD COLLEGE

PRINTED AT THE HARVARD UNIVERSITY PRESS

CAMBRIDGE, MASS., U. S.A.

Brought to you by | MPI fuer Psycholinguistik 


\section{INTRODUCTION}

THE present paper, consisting of the results of several investigations subvened by liberal grants from the General Education Board's Appropriation for Studies in the Humanities, deals with the Principle of Relative Frequency, a general theory of language first advanced publicly by me in 1929. The investigation of the theory, which began some years before publication in I929, had even by that time reached such proportions that in the original article I could include only such material as I deemed especially salient for the demonstration of the new hypothesis. Since then, the material has again increased considerably, not only from my own independent research, but likewise from the comments and suggestions kindly tendered by critics both here and abroad. Hence again, my space compels only selected studies, a selection which is designed in part to increase the illustrative material, in part to penetrate further into the analysis of that material, and in part to extend the limits of the very theory itself. The carefully deliberated arrangement is, however, such that $I$ hope the reader casually perusing the following pages will sense the fundamental organic unity of the underlying principle. The unity of the Principle of Relative Frequency, its ramifications and scope, its bearing on the theory of Gestalt Psychology on the one hand and the theory of Psychoanalysis on the other, and its general implications both linguistic and otherwise, I am at present combining into a modest volume to appear in the immediate future.

It is because of this volume that I now include as appendices all the essential source material upon which the argument of the following pages is based. Only after the most careful discussion of the matter with colleagues and with the Chairman of the General Education Board's Appropriation was it decided to print this unwieldly evidence. If, as they say, the Chinese phonetic transcriptions in the appendix and the lists of the Chinese words and syllables arranged according to frequency of occurrence prove themselves of interest and value to those working on grammars, readers, or stenographic systems in Chinese, and if the list of all words occurring in four of Plautus' plays, arranged according to frequency of occurrence, together with the cards with line references for each word (deposited in the Harvard College Library) should prove of value for one working on Plautus, all of that is incidental to the purpose of their inclusion here. Perhaps that is more than a sufficient justification. I however include them solely that there should be no doubt as to the method used for the results and as to the veracity of the results obtained, for these results offer overwhelming proofs of the Principle of Relative Frequency. Though the evidence they give represents but a small portion of the total evidence available, yet it is of such a nature as to establish at least certain aspects of the theory beyond a shadow of a doubt.

It is needless to say that it would have been impossible for me to gather the ensuing material without the help of loyal assistants. The tediousness and need of accuracy of the work called for almost superhuman patience. One who has never attempted such an investigation will never understand the enormous debt of thanks I owe to Mr. Kan Yu Wang, Mr. H. Y. Chang, Miss Dorothy Priest, Miss Mary Campbell, and my wife for their assistance, assistance which would have been mainly impossible without the generous subvention of the General Education Board's Appropriation for Studies in the Humanities.

Cambridge, Massachusetts

January 6, 1932

1 G. K. Zipf, "Relative Frequency as a Determinant of Phonetic Change," Harvard Studies in Classical Philology, Vol. XI (1929), pp. 1-95. 


\section{CONTENTS}

Part I. Relative Frequency in Respect to Chinese Phonology . . . . . . . . . I

PART II. Relative Frequency, Abbreviation, and Semantic Change . . . . . . . 8

Appendix A. Frequency of Occurrence of Words in Plautus' Aulularia, Mostellaria, Pseudolus, and Trinummus . . . . . . . . . . . . . . . . . . $3^{\mathbf{I}}$

Appendix B. Phonetic Transcriptions of 20,000 Syllables of the Dialect of Modern Peking. . . . . . . . . . . . Plates I-50

APPENDIX C. Frequency of Occurrence of Words in the 20,000 Syllables of Appendix B . . . . . . . . . . . . Plates $1 a-5 a$

Appendix D. Frequency of Occurrence of Syllables in the 20,000 Syllables of Appendix B . . . . . . . . . . . . . Plates $x b-7 b$ 


\section{PART I \\ The Principle of Relative Frequency in Respect to Chinese Phonology}

THE present paper sets forth the results of an investigation undertaken chiefly to determine whether or not the modern vernacular of Peking, China, agrees with Indo-European tongues in substantiating the Principle of Relative Frequency. That an oriental language would offer valuable and final evidence was apparent likewise to others. ${ }^{1}$ And that of the many oriental languages, Chinese, or, more particularly, the dialect of modern Peking, would be the most ideal from almost every angle, I hope to make clear in the ensuing paragraphs.

But before proceeding to the evidence, it would be wise to review in brief the main points of the theory which the evidence seems to support. For the theory of Relative Frequency, unlike every other linguistic theory, does not look for the causes of linguistic changes in climate, geography, vocal organs, childhood mispronunciations, and so on, but simply maintains that any change in a given language is brought about by the inherent structure of that language itself. The gist of the theory is that any element of speech which occurs in spoken language more frequently than some other similar element demands less emphasis or conspicuousness than that other element which occurs more rarely. In other words, what is expected does not need or receive the emphasis or conspicuousness of the relatively less expected. A few examples will perhaps make this clearer.

The ever recurring endings of a word do not generally need as much emphasis as the root; the verbal auxiliaries, being more frequent, usually do not demand or receive as much emphasis as the main verb; so too the article less than its noun. The general tendency toward abbreviation, syncope, toward the fusion of endings, suffixes, prefixes, auxiliaries, articles, and particles offers further possibilities of proof. In fact this seems to me the very crux of Satzphonetik, as I hope will be apparent to the reader in Part Two of this paper. If one goes deeper than the word, however, and takes a paradigm which has some endings rigidly accented and others regularly without accent, one will find that accent is coupled with comparative rarity of occurrence, while lack of accent is invariably linked with high frequency of usage. ${ }^{2}$

In the light of all this, I formulated the Principle of Relative Frequency: The accent or degree of conspicuousness of any word, syllable, or sound is inversely proportionate to the relative frequency of that word, syllable, or sound, among its fellow words, syllables, or sounds in the stream of spoken language. As any element's usage becomes more frequent, its form tends to become less accented, or more easily pronounceable, and vice versa.

1 Cf. Ed. Hermann, Philologische Wochenschrift, Vol. 5I (1931), p. 602.

2 For a detailed discussion of supporting evidence, cf. Zipf, op. cit., pp. $5 \mathrm{ff}$. In support of these views, cf. Elise Richter Archiv filr das Studium der neweren Sprachen, Vol. 157 (1930), pp. 29 I ff. Against these views cf. Ed. Herman, $0 p$. cit., pp. $599 \mathrm{ff}$. As I expect soon to be able to point out, the e-o gradation in the o-declension and thematic verbs is the answer to Professor Hermann's shrewd question. 
This principle becomes extremely important in respect to sounds (phonemes). ${ }^{1}$ For strangely enough sounds, as we shall see, differ decidedly in degree of conspicuousness. The voiced aspirate $d h$ for example is more conspicuous, both phonetically and acoustically, than the media $d$, since it has everything the $d$ possesses, plus the additional increment of aspiration. Because of this additional increment of aspiration, the $d h$ is more difficult to pronounce than a $d$ and, likewise, more audible to the hearer: in other words, it is both phonetically and acoustically more conspicuous. So too a lenis or a fortis $d$ is more conspicuous than the correspondingly lenis or fortis tenuis $t$, since the $d$ possesses everything the $t$ has, plus the increment of voice. In a like manner, the afficate $t s$ is phonetically and acoustically more conspicuous than a $t$, because in addition to the $t$ it has a following spirant. But we can say nothing in respect to the relative conspicuousness of a spirant, for example the $b$. For though the $p$ lacks the increment of explosiveness of the tenuis, it can more than compensate for this lack by a prolonged duration (e. g. $b p b$ ). Obviously this arrangement of the dentals can be correspondingly extended to labials, palatals, velars, or indeed to any other or every other sound which will similarly admit of a comparative analysis.

That the conspicuousness of a sound is only determinable relative to another sound has seemed to some critics of this theory unfortunate. But in spite of the brilliant work of modern experimental phonetics, phoneticians have not yet discovered any method which can give for any sound, let alone for every sound, either the actual absolute amount of energy required for its pronunciation, or an absolute measure either of its audibility or its psychic conspicuousness. And it is doubtful that such a measure will ever be found. For this conspicuousness depends to a large degree upon the amplitude or loudness, a thing which varies greatly in words, and therefore in sounds, according to the mood or emotional state of the speaker. But in regard to the relative conspicuousness of a sound, the arrangement of $d h$ to $d, d$ to $t$, and $t s$ to $t$ is satisfactory. For regardless of how loudly or softly an individual speaks, a lenis or fortis $d$, let us say, will be both more audible and more difficult to pronounce than the correspondingly lenis or fortis $t$.

And now, if we can say that a lenis or fortis media is more conspicuous than a correspondingly lenis or fortis tenuis, analysis of language should show, if the Principle of Relative Frequency be correct, that the less conspicuous tenuis should occur in cursive language more frequently than the

\begin{tabular}{|c|c|c|c|c|c|c|}
\hline & $t$ & $d$ & $\boldsymbol{k}$ & $g$ & $p$ & $b$ \\
\hline Bulgarian . . . . . . . . & $7.54 \%$ & $3.55 \%$ & $2.98 \%$ & $1.46 \%$ & $2.82 \%$ & I. $32 \%$ \\
\hline English . . . . . . . . & 7.13 & $4 \cdot 31$ & 2.71 & .74 & 2.04 & $\mathrm{r} .8 \mathrm{I}$ \\
\hline Russian $\ldots \ldots \ldots \ldots$ & 7.49 & 3.42 & 3.49 & 1.10 & 2.19 & 1.76 \\
\hline Italian $\ldots . . . \ldots \ldots$ & 7.02 & 4.74 & 3.63 & $.4 I$ & 2.78 & .89 \\
\hline Swedish $\ldots \ldots \ldots \ldots$ & 7.64 & $5 \cdot 4^{8}$ & $3 \cdot 52$ & 2.50 & 1.20 & $I .32$ \\
\hline Hungarian . . . . . . & 7.18 & $3 \cdot 30$ & $5 \cdot 72$ & 2.45 & $I .04$ & $r .7 T$ \\
\hline German ......... & 6.42 & 3.75 & 2.24 & 1.84 & $I .30$ & I.34 \\
\hline French $\ldots \ldots \ldots \ldots$ & 6.28 & 3.55 & $4.8 \mathrm{I}$ & .76 & 3.54 & 1.39 \\
\hline Czech $\ldots \ldots \ldots \ldots$ & 5.60 & 3.73 & 3.93 & .15 & $3 \cdot 52$ & 1.86 \\
\hline Spanish $\ldots \ldots \ldots \ldots$ & 4.27 & 5.20 & 3.82 & .07 & 2.64 & 2.05 \\
\hline Sanskrit . . . . . . . & 6.65 & 2.85 & 1.99 & .82 & 2.46 & .46 \\
\hline Greek . . . . . . . . & 7.58 & 2.87 & 4.07 & I.74 & $3 \cdot 3^{8}$ & .49 \\
\hline Latin $\ldots \ldots \ldots \ldots$ & $7 \cdot 72$ & 3.41 & $3.7 I$ & .96 & 2.01 & 1.40 \\
\hline
\end{tabular}

1 For the sake of those who prefer the word phoneme, an extremely useful philological term which does not yet enjoy the frequency of usage it deserves, let me say that the older and more generally used word sound which I shall adhere to in this paper may be replaced throughout this paper by the word phoneme without modifying my meaning in the slightest degree. By sound changes I mean, as practically every investigator of this field now means, phoneme changes. For the meaning of phoneme see Leonard Bloomfield, "A Set of Postulates for the Science of Language," Language, Vol. II (1926), I57. In addition one may profitably consult Edward Sapir, "Sound Patterns in Language," Language, Vol. I (1925), 37. 
more conspicuous media. In other words, we should expect in counting the sounds of a reasonably long piece of colloquial prose to find by actual count the tenues more numerous than their corresponding mediae - more $t$ 's than $d$ 's, more $k$ 's than g's, more $p$ 's than $b$ 's.

The table on page 2 , reprinted from my original article, illustrates that this is strikingly true. ${ }^{1}$ And the conclusions to be drawn from the table are clear.

When, either because of change of the frequency of usage of words in the folk vocabulary, or because of the addition of new or the deletion of hitherto familiar words, the relative frequency of a sound increases, the sound tends to become less conspicuous, either by diminishing the conspicuousness of one or more of its increments, or by abandoning one or more of its increments entirely. So a fortis $d$ may become a lenis $d$, or even a $t$. And conversely, if the sound becomes relatively rarer, it may add to its increments. In this fashion a $t$ may become a $d$, a $t h$, or a $t s$; or a lenis $d$ may become a fortis $d$ or a $d h$. In this fashion sound shifts take place.

The German and Scandinavian $b$ are examples of the former. Having become more frequent than $p$, as our table shows, the German and Scandinavian $b$ have weakened in both their increment of voice and explosiveness, and have become a sound not very different from a softly voiced English $p$. Spanish likewise exhibits a similar change with its $d$, which, because of its excessive frequency, has in most positions lost its increment of explosion and become an $\circlearrowright$. Hence it happens that the same fundamental sounds in various languages may vary in degree of explosiveness, amount of aspiration, or voice. Some critics believe this variation vitiates the Principle of Relative Frequency. On the contrary, it would be highly odd, as will appear below, if this variation were not there. For a more complete discussion of the table I must however refer the reader to the original article. Let us turn now to the objections that have been made to the table, inasmuch as the present Chinese data are intended to answer most, if not all, of them.

The first objection is by far the most frequent, namely that the statistics do not represent phonetic transcriptions. To this may I say again that the figures in the table for some of the languages do represent actual phonetic transcriptions; for some of them only approximate phonetic transcriptions; and for some, notably those for the dead languages, in considering letters as sounds, come as close to a phonetic transcription as we shall ever obtain. It is commonplace to say that our alphabets are not phonetically exact. But, in so saying, let us not forget that our alphabets in general, especially in regard to consonants, do not always utterly and completely disregard phonetic equivalents, representing, as it were, no earthly connection with the actual pronunciation. Sanskrit, as a matter of fact, is so exact in its alphabet that one might safely say that it is truly a phonetic writing. But be that as it may. If the reader is not impressed by the incontestable correlation of the percentages in the table, he may disregard the table on whatever grounds he wishes. But he may not discard the Chinese statistics on the ground of phonetic transcription, for the Chinese statistics represent careful and exact phonetic values; the transcriptions themselves are included in the appendix.

The second objection deals with conspicuousness. ${ }^{2}$ It has been objected that a sound which is easy for one person to pronounce may be difficult for another, meaning by this that a German, for example, finds the English th more difficult than does an Englishman. But this again has nothing to do with the Principle of Relative Frequency. Just as the relative frequency of a sound means the frequency of the sound relative to the other sounds of the same dialect and ob-

1 Zipf, op. cit., p. 61. The various sources of these statistics and control figures, together with a criticism of the method by which the statistics were obtained, are given on pp. $42 \mathrm{ff}$.

2 Cf. E. Hermann, op. cit., p. 602 . 
viously not to sounds in general, so too the conspicuousness of any sound is relative only to the conspicuousness of other sounds in the same dialect. It would be absurd to think that this law should work for $t$ in general and $d$ in general; a fortis $t$, as said before, may be more conspicuous than a lenis $d$. If so, it should be less frequent than the $d$. Moreover it is not likely that any sound or group of sounds will be as easy for a speaker to utter or a hearer to perceive as a sound or sounds of the native speech pattern. If the reader wishes, he may discard the previous table on the somewhat doubtful grounds that a French $t$, for example, is pronounced in many ways, and add that the striking correlation is nothing but random chance. But he may not discard the Chinese figures for that reason; for the analysis has been restricted to the modern vernacular dialect of Peking.

The third, and I think the last, serious objection, is that the same sound varies in intensity, and hence conspicuousness, according to the nature of the surrounding sounds. This is undoubtedly true. As Professor Elise Richter points out, ${ }^{1}$ among other excellent examples, the Ger$\operatorname{man} p$ at the beginning of a word, between syllables, and at the end of a word represents three different and phonetically distinct sounds; she indicates that the percentages for German $p$ are, therefore, not valid until these $p$ 's are each segregated. She suggests recourse to experimental phonetics. It has also been suggested by another that I avoid the issue entirely by using the word phoneme instead of sound, implying thereby that sounds whose difference is detectable only by the skillful reading of a recording apparatus may be regarded for linguistic purposes as identical sounds. I myself had hoped to avoid this troublesome issue on page $4 \mathrm{I}$ of the original article by stating that when a sound became more frequent, and therefore tended to diminish in conspicuousness, its weakening would first commence in those positions where surrounding sounds were propitious for a weakening. For example, if a $b$, becoming too frequent, tended to lose, let us say, its increment of voice, it would in all probability lose it sooner and easier if surrounded by voiceless sounds, than it would between vowels; it would be more apt to lose its voice in gebst or hebst than in geben or heben. And if one considers language as something dynamic, something living, and one happened to record the language phonetically as a gradual weakening were taking place, one could not reasonably say that the Principle failed to function simply because a difference of articulation was observable in different positions.

However, there is an easier way to refute the phoneticist than by attempting to demonstrate to him that the very phenomena he points to as disproof are in reality an essential part of the working of the Principle. Let us take rather a language where this problem does not arise and test its consonants. Let us examine the dialect of Peking, which is monosyllabic in structure. In the dialect of Peking, a consonant is used only at the beginning of a syllable and is always followed by a vowel. There is no such thing as a final consonant. The language, being essentially monosyllabic, offers no serious problem of intersyllabic consonants. In respect to the position of the consonant, everything is absolutely constant. There is nothing to consider but the actual conspicuousness of the sound.

Nor is there any difficulty in determining which consonant in each pair is more conspicuous. Even the earliest romanizations show it; all phoneticists agree about it; it is at once evident to anyone listening to a native of Peking speak. The fortis tenuis aspirata is decidedly more conspicuous than the faint lenis media. ${ }^{2}$ For so weak is the voice in the lenis media, that there is doubt as to whether it should best be transcribed with a Roman media or tenuis (e. g. by a $b$ or $p$ ). And so strong is the aspiration and force of the tenuis, that several of the transcriptions indicate it

1 Op. cit., pp. 293 ff.

2 For a careful and thorough discussion of Chinese phonetics, see Bernhard Karlgren: Enudes sur la phonologie chinoise, Leyden and Stockholm, I915, Pp. 223 fi.

$$
\text { [4] }
$$


with a following ${ }^{c},\left(e \cdot g \cdot p^{c}, k^{0}, t^{0}\right.$, etc., as in the Wade System). For my own transcriptions $I$ have used the phonetic system recommended by Chao, purely for its greater simplicity and brevity: the media is used to represent the lenis, the tenuis transcribes the fortis tenuis aspirata. But that the reader may not be confused, the percentages for all sounds in the tables on pages 6 and 7 indicate the four different ways in current use for transcribing Chinese sounds.

And so the Chinese figures will be an answer to every criticism made of this portion of the theory. If Professor Hermann on page $602{ }^{2}$ does not find it strange that the sounds of most of the languages agree roughly in percentages, inasmuch as they go back to the same dialect four thousand years ago, such an argument, as strained as it seems to me,drops when one considers Chinese. If Professor Hermann on page 603 , in concluding his criticism, expresses his surprise, after all, that the tenues should be generally more frequent than their corresponding mediae, and wonders at the reason, let him look at these figures and remember that on page 599 he did, after all, concede that the theoretical side of the argument appeared incontestable.

In the light of the Principle of Relative Frequency, the faint lenes mediae should be decidedly more frequent than their corresponding fortes mediae aspiratae, and they are:

\begin{tabular}{|c|c|c|c|c|c|c|}
\hline & $d / t$ & $b / p$ & $g / k$ & $c j / c \varepsilon$ & $t \xi / t f$ & $t z / t s$ \\
\hline $\begin{array}{l}\text { lenis media ..... } \\
\text { fortis tenuis aspi- }\end{array}$ & $6.18 \%$ & $2.37 \%$ & $2.58 \%$ & $2.69 \%$ & $2.44 \%$ & $2.63 \%$ \\
\hline rata .......... & 2.56 & .56 & I.02 & 1.04 & I.23 & 1.40 \\
\hline
\end{tabular}

May it be observed that likewise in Chinese the dentals (cerebrals) are decidedly more frequent than the labials or gutturals. ${ }^{3}$ In the event that one be interested in the number of occurrences of all sounds, he will find in the tables, on pages 6 and 7 , the actual number of occurrences together with the individual percentages according to the various systems of transcription. The column with an $\mathrm{X}$ at the top is Chao's recommended phonetic system which is used in the transcriptions given in full in Appendix B.

The figures are based on a transcription of twenty selections of colloquial Chinese, each selection one thousand syllables or ideographs long. The transcription reads from left to right; each square contains a Chinese ideograph representing a syllable. In the upper left hand corner of each square is the number of that syllable in the selection of a thousand syllables; the number at the lower right is the tone of the word $\left({ }^{*}, \mathrm{r}, 2,3,4\right)$; and underneath is the phonetic transcription for each syllable. The tones and transcriptions are according to Dr. Yuen Ren Chao's A Phonetic Dictionary of the Chinese National Language, Shanghai (r926), the Commercial Press. Though Chao gives all the transcriptions for each word, my transcriptions in the appendix use only the "Broader System of International Phonetic Symbols" based upon "L'écriture phonétique internationale exposé populaire," published by the International Phonetic Association.

1 See infra.

2 Op. cit.

3 See Fu Lu: Etude Experimentale sur les tons du Chinois, Paris and Peking, 1925, p. 105, for a transcription, according to a different system, of 255 Peking words. The frequency of the consonants in Lu's transcription indicates the same relationship as my own and can be considered a control. 
In the 20,000 syllables, there are 20,000 vowels, and 17,338 consonants. The number of tones are as follows:

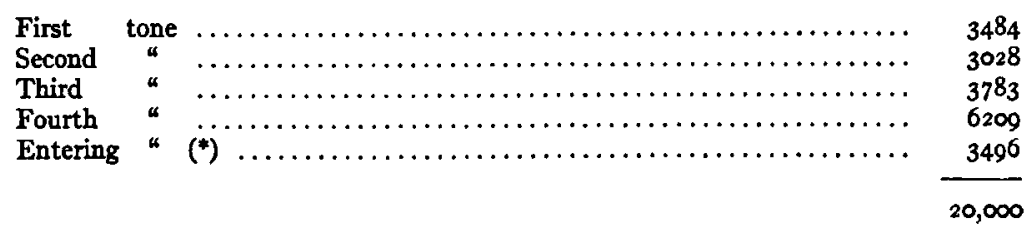

The names of the twenty different writings from each of which a selection of one thousand connected syllables was taken are given in Chinese on the last plate $\left({ }_{7} B\right)$ of Appendix D.

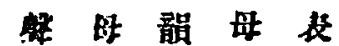

\begin{tabular}{|c|c|c|c|c|c|c|}
\hline 注 & 急 & $\begin{array}{l}\mathbf{4} \\
x \\
x\end{array}$ & $\begin{array}{l}1 \\
\\
\end{array}$ & $\begin{array}{c}\text { Wiado } \\
\text { 委 } \\
\text { 䊁 }\end{array}$ & Count & $\%$ \\
\hline 今 & $\mathbf{b}$ & $\mathbf{b}$ & $\mathbf{b}$ & $\mathbf{p}$ & 887 & 2.37 \\
\hline$x$ & $\mathbf{p}^{c}$ & $\mathbf{p}$ & $\mathbf{p}$ & $p^{c}$ & 210 & .56 \\
\hline$\infty$ & $\mathbf{m}$ & $\mathbf{m}$ & $\mathbf{m}$ & $\mathbf{m}$ & 815 & 2.18 \\
\hline E & $\mathbf{f}$ & $f$ & $f$ & i & 395 & 1.06 \\
\hline$\pi$ & $\mathbf{v}$ & $\nabla$ & $\nabla$ & $\mathbf{w}$ & 0 &.$\infty$ \\
\hline 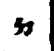 & व̊ & $\mathbf{a}$ & d & $t$ & 2306 & 6.18 \\
\hline \pm & $\overline{t^{c}}$ & $t$ & $t$ & $t^{c}$ & $95^{8}$ & 2.56 \\
\hline 3 & $\mathbf{n}$ & $\mathbf{n}$ & $\mathbf{n}$ & $\mathbf{n}$ & 371 & .99 \\
\hline s & 1 & 1 & 1 & 1 & 1096 & 2.93 \\
\hline$\ll$ & $\bar{g}$ & $g$ & 8 & $\mathbf{k}$ & 965 & 2.58 \\
\hline 7 & $\mathbf{k}^{e}$ & $\mathbf{k}$ & $k$ & $\mathbf{k}^{2}$ & 382 & 1.02 \\
\hline$\pi$ & n & y & $\mathbf{q}$ & (粎) & 220 & .59 \\
\hline$r$ & $x$ & $x$ & h & $\mathbf{h}$ & $78 \mathrm{I}$ & 2.09 \\
\hline 4 & $\overparen{619}$ & cj & $y$ & ch & 1005 & 2.69 \\
\hline$<$ & $\widehat{\cos ^{c}}$ & cs & ch & $\mathbf{c l i}^{\mathrm{C}}$ & 389 & 1.04 \\
\hline$\infty$ & $\mathrm{n}$ & n & $\boldsymbol{n}$ & $\mathbf{n}$ & 117 & $.3 \mathrm{I}$ \\
\hline$T$ & 9 & s & $\mathbf{s h}$ & hs & 423 & I.I3 \\
\hline in & $t_{1}$ & $t_{3}$ & $\mathbf{j}$ & ch & 912 & 2.44 \\
\hline 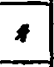 & $\widehat{t}]^{c}$ & tf & ch & $\operatorname{ch}^{4}$ & $45^{8}$ & I.23 \\
\hline $\boldsymbol{P}$ & 1 & 1 & th & $\mathbf{B b}$ & 1714 & 4.59 \\
\hline 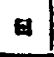 & 3 & 3 & $\mathbf{r}$ & j & 552 & I. 48 \\
\hline
\end{tabular}

\begin{tabular}{|c|c|c|c|c|c|c|}
\hline 洒 & 承 & $\begin{array}{l}\text { 葛 } \\
\mathbf{x} \\
\mathrm{X}\end{array}$ & 臂 & Wade & Count & $\%$ \\
\hline$\pi$ & $\hat{t_{1}}$ & $t z$ & $t z$ & ts, $1 t z$ & 983 & 2.63 \\
\hline 5 & $\widehat{\mathrm{tg}^{e}}$ & to & ts & $t^{c} s_{1} I_{t}^{c} z_{z}$ & 522 & 1.40 \\
\hline 2 & 8 & a & 8 & B, $1_{\text {SB }}$ & 877 & 2.35 \\
\hline 日 & 3 & 3 & $r$ & in & 1302 & 3.49 \\
\hline 20 & $z^{+}$ & 2 & $z$ & $\mathfrak{n}$ & $32 \mathrm{I}$ & .86 \\
\hline-1 & 1 & $\bar{i}$ & 1 & 1 & $27 \times 7$ & .28 \\
\hline$x$ & $\mathbf{u}$ & $\mathbf{u}$ & $\mathbf{u}$ & wu, - $u^{\wp}$ & $\mathrm{r} 38 \mathrm{I}$ & 2 \\
\hline 4 & $\mathbf{y}$ & $y$ & $\dot{u}$ & $\mathbf{j u},-\mathbf{i}$ & 363 & .97 \\
\hline $\mathbf{r}$ & $\alpha$ & $a$ & 8 & $\mathbf{a}$ & 794 & 2.13 \\
\hline 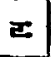 & d & 0 & 0 & $0,(e)^{26}$ & II99 & 3.21 \\
\hline 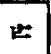 & $\theta$ & $\theta$ & e & $\theta$ & 279 & .75 \\
\hline 世 & $\theta$ & 6 & $\theta$ & $\hat{\theta}$ & 264 & $.7 \mathrm{I}$ \\
\hline 7 & at & ai & ai & $\mathbf{a i}$ & 673 & 1.80 \\
\hline$\checkmark$ & èt & ei & el & el & 115 & .31 \\
\hline 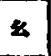 & gove & do & aO & 80 & 580 & 1.55 \\
\hline$x$ & öy & ou & ou & ou & $34 \mathrm{I}$ & $.9 I$ \\
\hline 4 & an & an & an & an & 615 & r.65 \\
\hline 4 & en & en & en & on & 879 & 2.35 \\
\hline$\star$ & an & $a y$ & $\mathrm{aq}$ & ang & 492 & 1.32 \\
\hline$<$ & $\Delta y$ & on & eq & eng & 420 & I.3I \\
\hline$\Omega$ & 2 & ex & er & er & 106 & .28 \\
\hline
\end{tabular}

[6] 


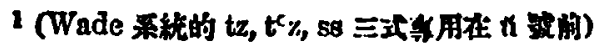

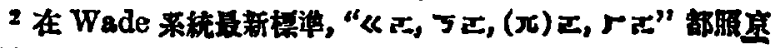 音相作“-

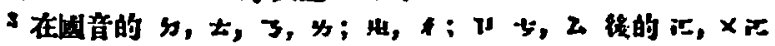

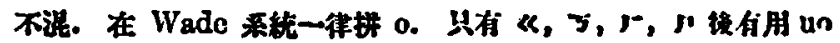
的证。

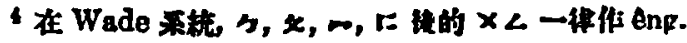

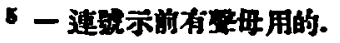

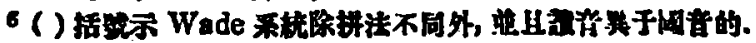

\begin{tabular}{|c|c|c|c|c|}
\hline 注 & 营 & $\begin{array}{l}\text { 萬 } \\
\text { 䒪 } \\
\text { X }\end{array}$ & $\begin{array}{l}\text { 羅 } \\
\text { 促 }\end{array}$ & Wade \\
\hline$-\gamma$ & $i \alpha$ & id & is & ya, -ia \\
\hline - & id & io & io & $\begin{array}{l}\text { (yüeh, } \\
\text {-üeh) }\end{array}$ \\
\hline-4 & jè & io & ie & $\begin{array}{l}\text { yeh, } \\
\text {-ieh, }\end{array}$ \\
\hline -5 & iar & iai & iai & $\begin{array}{l}\text { yai, } \\
\text { (ieli) }\end{array}$ \\
\hline$-x$ & inov & iuo & iao & $\begin{array}{l}\text { Jao, } \\
\text {-ino }\end{array}$ \\
\hline$-x$ & iu & iu & iu & Ju, -iu \\
\hline-5 & ien & $i \varepsilon n$ & ien & $\begin{array}{l}\text { yen, } \\
\text {-ien }\end{array}$ \\
\hline-4 & in & in & in & yin, -in \\
\hline 一兀 & $i \propto y$ & iun & ian & $\begin{array}{l}\text { Jang, } \\
\text {-iang }\end{array}$ \\
\hline-2 & in & in & iq & $\begin{array}{l}\text { fing, } \\
\text {-ing }\end{array}$ \\
\hline$x y$ & $u \propto$ & ud & ua & wa, - $11 \mathrm{a}$ \\
\hline$x z$ & go & 110 & no & $\begin{array}{l}\text { Wo, - uo, } \\
(0)^{3}\end{array}$ \\
\hline
\end{tabular}

Count \%

$260 \quad .70$

$174 \quad .47$

$300 \quad .80$

$79.2 \mathrm{I}$

$694 \quad \mathrm{I} .86$

$593 \quad 1.59$

$865 \quad 2.32$

$442 \quad 1.18$

$406 \quad 1.09$

$49 \mathrm{I} \quad 1.32$

62.17

$583 \quad 1.56$

\begin{tabular}{|c|c|c|c|c|c|c|}
\hline 法 & 焉 & $\begin{array}{l}\text { 营 } \\
\boldsymbol{x}\end{array}$ & 䉓 & Wade & Count & $\%$ \\
\hline$\times 5$ & nal & uai & uai & wai, -nai & 78 & .21 \\
\hline$x$ & në드. & nei & nei & wei, -ui & 523 & 1.40 \\
\hline$x=$ & tan & uan & uan & wan, -uan & 326 & .87 \\
\hline$\times 4$ & uən & nən & uen & wen, -un & 169 & .45 \\
\hline$x \pi$ & tran & uan & $\operatorname{tag}$ & wang, -uang & 95 & .25 \\
\hline$x<$ & זע & on & oq & $\begin{array}{l}\text { (wêng), ung, } \\
\text { (eng)t }\end{array}$ & 718 & 1.92 \\
\hline 山民 & yेd & yo & üo & -ỉeh & 0 &.$\infty$ \\
\hline 山上 & ye & je & to & Jïeh, -iith & 49 &. $\mathrm{I} 3$ \\
\hline$\omega$ & $\bar{y} \in n$ & $y \in n$ & ilen & yüan, -ïian & 93 & .25 \\
\hline Wh & gn & $\mathbf{y n}$ & th & yün, -ün & 45 & .1 \\
\hline$\mu<$ & ion & ion & iog & yung, -iung & II 4 & .30 \\
\hline
\end{tabular}




\section{PART II}

\section{Relative Frequency, Abbreviation, and Semantic Change}

THE present study deals with the problem of semantic change and is intended to show the relationship between semantic change and abbreviation. Much has been written on the subject of semasiology by linguists, psychologists, and philosophers, yet, as far as I know, little or nothing has been done from the angle of abbreviation. And believing that abbreviation is the very crux of the matter, I prefer to disregard, for the present, all previous investigations of the subject and to build up my present theory by a consideration of the obvious examples of semantic change such as they occur in the everyday language of an individual - of any individual, be he old or young, wise or foolish, sick or well, mad or sane. In the ensuing pages much will be said about the "meaning" of words and a very great deal about the "emotional quality and intensity" of words, domains hitherto and for no good reason considered primarily proper for the philosopher and psychologist. But since these properties of words are, to my mind, not divorced from abbreviation, as incomprehensible as that may seem to the reader at this time, I shall keep this paper within the limits of linguistics, and try to avoid first, an intricate portrait of what is considered the nature of human mind, and secondly, the eternal question of the relationship of the "symbolic" to the "real," hoping, thereby, to escape two knotty problems which invariably spell the downfall of semasiologists who begin with a contemplation of mind or reality.

For frankly, we know nothing about the nature of human mind and very little about reality. There are theories to be sure, but these theories conflict so gravely that a frank examination of the facts of language will, in my belief, be more apt to throw light on the process of mind than theories of mind on the process of language. For the facts of language are the facts of language, just as the facts of gravitation are the facts of gravitation. And just as we should smile at a physicist who devoted his time to explaining how much more picturesque nature would be if gravitation followed the inverse cube instead of the inverse square, so we should smile at the person who spends his time trying to persuade us that "correct" language should be of this nature and not of that. Hence, how one should express oneself is of no concern in this paper, but rather how one does express oneself. To be sure, the reader will occasionally glimpse behind my statements the thoughts of others. The reason I do not interlard the present paper with references to the investigations of others is not that $I$ do not willingly concede that the works of others have often been either an inspiration or a re-assurance, and that without them this theory of abbreviation would have prospered more slowly, but rather that this theory for better or for worse should stand on its own feet without resting in any way upon authority.

Before proceeding further, there is another point to be considered inasmuch as this paper is addressed primarily to linguists. The theory of Relative Frequency is a statistical law and, as such, falls within the laws of probability. Let us review, briefly, what is meant in part by probability. When the odds are even, it means that either eventuality covered by the odds may 
happen; when the odds are ten to one, it means that either eventuality may happen; when the odds are a trillion to one, either eventuality still may happen, and it is no disproof of a law, based on probability, if that remote chance should take place. The entire Principle of Relative Frequency is based on probability. Sound changes are too general and consistent to allow that sound laws are anything but basic, dynamic, pervasive laws; but the exceptions are numerically too abundant and conflicting that they may all be charged without further evidence to the account of prehistoric linguistic substrata or prehistoric dialectal divergences. One cannot deny the possible influence of linguistic substrata and remote dialectal divergences; but that pure chance plays some rôle in sound change likewise cannot be disregarded. For, after all, how else can one explain the enormous diversity following upon any major sound shift?

I allude to all this now because some have taken exception to the Principle of Relative Frequency simply because it is statistical. For statistics are hateful to the human mind; they are painfully definite for the group without being particularly definite for the individual. Undoubtedly, a primary law which knows no fluctuation within itself is pleasanter. If nature had consulted man in the matter, we should all have suggested primary laws, from the instinctive fear that some day Whirl might otherwise become king, forgetting first of all that even in a statistical law it is difficult for Whirl to become complete king, forgetting, likewise, that in many personalities Whirl does seem in a way to be king. But nature did not consult us in the matter and has seen fit to let the laws of chance govern vast portions of the basic order of the physical universe, as well as no small amount of the biological. You may disregard the Principle of Relative Frequency because you do not consider the statistics significant; you cannot discard it, however, on the grounds that a statistical law is not respectable. And so with the critics who have resented the mathematical nature of this theory, and whose names I here omit, I can only agree that it is perhaps not pleasant to know that all colors go back to three primary colors, that all matter is reducible to protons and electrons in motion, yet nature really seems to have been so bold as to decide these difficulties without asking for the philologist's exquisite opinion. And now to the matter at hand.

There are four important characteristics which are easily recognizable in words, perhaps not all four in every word, but certainly there are words which have at least one and even more, nor are the characteristics so unusual that the reader will not recognize them as important. The first is meaning, that elusive attribute of a word, so important and yet often so hard to describe. Secondly, there is quality, by which I mean that a word may represent something desirable for the ego (positive quality) or something undesirable for the ego (negative quality). "Honor" is generally positive; "death" generally negative. Thirdly, words have emotional intensity, which is nothing more than value: comparatively speaking, the intensity indicates how much we should like to obtain the positive or how much we should like to avoid the negative. The fourth characteristic I shall call order; and for the present, let us consider order nothing more than the word's part of speech, - adverb, noun, etc. I shall not try to define these four characteristics further here, but by examining the processes of semantic change try to make clearer my understanding both of their nature and of their importance in semantic change.

By semantic change I shall mean a change whereby one symbol is temporarily or permanently substituted for another. Examples are without number. Calling a "man" a "dog," a "woman" a "peach," the "moon" a "candle," may be considered temporary semantic changes, until their use in this sense becomes so frequent and general that the original primary meaning of "dog," "peach," and "candle" becomes secondary to the new primary meaning of a particular kind or aspect of "man," "woman," and "moon." When a given substitution occurs but rarely, we call the change a metaphor. However, as the given substitution becomes ever more frequent and gen-

[9] 
eral, the originally rare meaning of the word finally becomes a sub-meaning, a co-integral part of the word, a connotation. In this fashion, "kid," at one time "a young goat," came to have the connotation "child" or "boy." Why "kid" was ever substituted for "child" or "boy" need not concern us now; it is simply self-evident that once substituted the permanency of the substituted meaning depends largely, if not entirely, upon the frequency of occurrence of the word with the substituted meaning.

This one thing we must remember, since it is important for any study of semantic change, that there is no fixed boundary between metaphor or connotation. It is disputable whether "nut" is merely a metaphor, or whether by now "queer person" is not a connotation of "nut." Likewise, there is no definite law establishing for all time what is the primary meaning or denotation of a word and what its secondary meaning or meanings, the connotations. If we take the word "bughouse" as an example, it is questionable whether the primary meaning is "insane asylum," or "house for the preservation or propagation of bugs."

In determining the primary meanings of words, one is apt to feel that either the original meaning or the oldest meaning or the aptest meaning is decisive. But that none of these are final is evident from the fact that often at a given moment the original or oldest meaning is either obsolescent, obsolete, or indeed even entirely unknown. Whatever "lord" means today, it certainly does not mean as in Old English "guardian of the loaf." And as for aptness of designation, one can bring forth examples without limit to illustrate the frequent inaptness of designation. If one takes "cocktail," "high-ball," "marshmallow," "bootleg," without examining the special history of each word and without knowing what the words refer to, one would simply be at utter loss to guess their meaning. Age, derivation, and appropriateness must be all discarded if one seeks the absolute in the meaning of a word. Sometimes they explain the primary meaning; sometimes not. To my mind, it is highly doubtful that there is an absolute primary meaning over any period of time, or within a group of individuals. One gains best insight into meaning by examining shifts of meaning, - semantic changes.

The temporary, semi-permanent, and permanent semantic changes, for so I shall henceforth designate the sliding scale from metaphor through connotation to complete and irrevocable substitution, differ among themselves only in respect to degree of probability. The greater the probability that any given word when used will have the derived or secondary meaning instead of the primary meaning, the greater the degree of permanency of the semantic change. Every vocabulary at any time reflects these various stages of semantic development in its words so clearly that the semasiologist may rightly say that meaning is shifting and determinable in respect to primarity only by the relative frequency of usage. And this frequency of use likewise explains our feelings about the importance of the original meanings or the fitting meanings of words. For often these meanings remain primary. Yet that original meanings or apt meanings so often continue to remain primary meanings is simply because of a favoring probability. And it is this favoring probability as we shall see that prevents every temporary semantic change from automatically becoming permanent, thereby replacing the older meanings.

"Ham" means to us a kind of meat, to a theatrical circle a "poor actor"; "lamb" means to us a kind of animal, to the racketeer a "guillible person." And so we might proceed with many words whose primary meanings, or the permanency of whose semantic changes, varies between different social, occupational, regional, cultural circles. A permanent semantic change in one circle may be only temporary or unheard of in another. Hence, permanency is really a relative affair, primarily relative to a given circle in which it enjoys a preponderant relative frequency of usage. A person is a member of many circles, - his family, his office, his club, the circle covered by his journals, his church, - and this free interlocking is the conservative influence in language. For within a given

$$
\text { [10] }
$$


circle a certain meaning, arrived at by semantic change, may, because of the rights of relative frequency, be undisputed king, and yet in other circles be unheard of. Indeed, a word may have a different, decided primary meaning in every different circle and yet, when one measures the relative frequency of occurrence of meanings in the large group which includes all the other circles, find that all these local primary meanings are insignificant. Anything that tends to hinder the free interlocking of these social and occupational circles, whether the impediment be caused by nature in the form of rivers, oceans, mountains, deserts, and the like, or by man himself through custom barriers, hostile feelings, different and separated market centers, travel, differences in culture and religion, and the like, will tend to remove the conservative influence of the large group and cause the language to drift apart slowly into dialects. Each small circle broken from the whole pursues its semantic changes unhindered until its vocabulary as used becomes more and more unintelligible to a separated circle. If the changed frequency of the changed vocabulary upsets the inner balance of vowels and consonants, as explained in Part One, phonetic changes may add to the cleavage. One circle cannot expand at the expense of others, or one circle cannot isolate itself from all others without upsetting the stability of meaning, which is based on the relative frequency or relative probability of occurrence.

The Norman invasion of England or the Middle Ages in Germany exemplify the consequences of an expanding circle and of contracting circles. Conservative change in language is found where the status remains fairly fixed through time, and is not at all connected with the amount of slang in the language. For all languages have slang; if the slang of today does not in toto become the good usage of tomorrow, it is not because slang is not older or not more appropriate than good usage, for it is often very old and its usage reflects its suitability. Language in good usage has the laws of chance to thank for its longevity, and not, contrary to the belief of schoolmasters, any inherent venerability or suitability. This is, to my mind, an explanation of the phenomena covered by Johannis Schmidt's Wellentheorie, and is the basis of anything important in the current theories on linguistic substrata. However a word may receive its meaning, and whether the meaning is original or appropriate or not, the permanency of the meaning and of the word itself is ultimately a matter of Relative Frequency. The primary meaning simply is the customary or usual meaning. A word may change its meaning over night. Yet only those semantic changes are likely to remain which are of service to a decided majority. The meaning of a word is far from stable. But let us turn to a consideration of just what a word is.

A word, whether it be written, spoken, felt, or thought is at root, regardless of the degree of attendant consciousness, a mental event. To be sure, sometimes it may be perceptibly accompanied by gestures of the vocal cords or other members of the body, or, as in the case of pure thinking, it may not. In itself, a word is nothing but an event in time. The meaning is supplied by the mind of the speaker and the mind of the auditor, if there be an auditor. The meanings do not necessarily need to agree between speaker and auditor, nor is there necessarily a consistency of meaning for the speaker or hearer over a period of time. Now "cat" means "feline animal," now it means "woman." If the mental event is in the mind of the speaker, the word is an expression; if in the mind of the auditor, a perception, regardless of the age, wisdom, experience, training, health, or sanity of either. The definition of a word as a mental event applies to every living organism that has language. "Correct" meaning or "proper" meaning of a word signifies nothing more than that the circle or circles in which that meaning enjoys high relative frequency seem to you, because you are or are not in them, "proper" and "correct."

But though a word exists both as a perception and as an expression, I shall henceforth, as far as possible, consider it only as an expression, - not because it is more interesting or important as an expression, but because a word is always an expression, even in an hallucination, though not

$$
\text { [ I I ] }
$$


always a perception. Furthermore, let us continue our examination for convenience by first examining only the spoken word. For the spoken word is a mental event which is always accompanied by perceptible gestures. By gestures, I do not mean primarily gestures of the hands, legs, arms, but rather certain small gestures of the membranes and tissues of the throat, mouth, lips, lungs, - phonetic gestures. Yet that is not all. The circulation of the blood, breathing, and other basic physiological processes, though not restricted to the spoken word, are also an extremely essential part of a spoken word and I shall call them substrata gestures. ${ }^{1}$

We have these substrata gestures, which are an essential part of the spoken word and which, as far as we can see, are a constant escort of mental events through life, varying to be sure in their activity according to the nature of the mental event. But of the mental event we know nothing more than it is connected with meaning and that the same meaning is not always linked with the same phonetic gestures or the same substrata gestures. Taking the spoken word as standard, that is, that part of the spoken word which we shall call phonetic gestures, we find that the meaning may vary from metaphor through connotation; likewise, the substrata gestures may vary denoting, thereby, an emotion differing both in quality and intensity. The mental event contains something more than meaning; it contains both quality and intensity in addition; it contains the momentary appraisal of the meaning.

By quality, I mean the nature of the mental event, whether the mental event expressing the meaning at a given time is positive or negative. If the mental event seems ultimately to be on the one hand a reaching out into environment to grasp in any fashion for some support for the organism from the environment, the mental event has a positive quality. If, on the other hand, the mental event is a shrinking back from environment to escape and to defend the organism from the environment, the mental event has a negative quality. That these qualities exist in language is indisputable. It is likewise indisputable that they exist as part and parcel of the mental event and substrata gestures. Though the quality of a word may differ from speaker to speaker and from time to time with the same speaker, yet it is, none the less, exclusively a part of the mental event. Quality like meaning is subjective; yet quality is not meaning. The quality of a word is part of what we shall call the inner vividness of a word. The remaining part of inner vividness is what we loosely call intensity.

To make inner vividness clearer, let us take the word "cat" for an example, as any given person may use it at any given time. Disregarding the meaning of the word at that time, the quality will be either negative or positive. That is, "cat" will to that person at that time be either desirable for the ego, or undesirable, or neutral, or more positive than negative, or more negative than positive. In other words, the mental event "cat" may be colored by desire or fear, or by neither, or a mixture of the two where one or neither may prevail. In addition to this quality is the intensity. If the quality is anxiety, the intensity is the amount of anxiety at that time with that person. The more the anxiety, the greater I shall call the intensity. The same holds true of desire, or a mixture of anxiety and desire, or of fear, remorse, or hatred. How low the intensity of a mental event may be is problematical. But that it may go so high as to derange or destroy the

1 These basic physiological processes - the functioning of nerves, lungs, liver, heart, and the like - which I call substrata gestures, are concomitant with the mental event, and intimately linked with it. If the mental event varies suddenly in emotional intensity, the activity of the substrata gestures is apt to vary: sudden wrath or anxiety, and a sudden change in heart-beat and rapidity of breathing. Whether the wrath or anxiety causes the change in the substrata gestures, or whether the change in the substrata gestures causes the wrath or anxiety, it is hard to say. Much has been argued on each side. It is sufficient for the purposes of this paper to assume that the mental processes and the physiological processes are concomitant, without an attempted inquiry into which comes first in time or importance. If the reader finds this procedure arbitrary, let him miss several meals and notice the emotional condition of his mind; and having done so, let him wait until he is beset with worry and anxiety, and notice the condition of his appetite. 
organism is not unheard of. All this is contained in the inner vividness of the mental event "cat." As to the meaning, it may have meant "feline animal," or "woman," or any number of things.

As meaning may fluctuate, so too may quality and intensity fluctuate. Each one may vary among persons, or within the same person from time to time. Even if there is no instrument to measure intensity, or record quality, our experience tells us that they are not necessarily constant. The word "whiskey" may be uttered with desire in one individual and with aversion in another. Within twelve hours the same person may shift from high desire to complete aversion. In fact, if it were possible for a person to compile the words he uses and the occasions at which he uses them in expressing himself, he would be surprised to see the differences in meaning, quality, and intensity of many of the words over a period of time. Yet I believe he would be equally surprised to find that these fluctuations in meaning and inner vividness were not absolutely at random, as he might expect from a day to day observation. Though it is conceivable that a word has a different meaning every time it occurs, yet it is probable that many words will have but a few meanings and will show among those meanings decided preferences; the meanings will arrange themselves as primary, secondary, etc., even though there be competing meanings for the first, second, third place.

So, too, the positive or negative quality of a word, though it may be randomly shifting for some words, certainly with other words will show a decided preference for either the positive or negative or a certain mixture of the two. Like meaning, quality may not be invariably constant, but like meaning, it may have a marked tendency or preference.

In the like manner, the intensity, although it too may fluctuate greatly for some words, should for others show a tendency toward a more marked stability, high or low. To be sure, a man while starving might use "bread" with a considerably higher intensity than he would while walking by a bakery shop after dinner. Yet though these fluctuations may be great, depending upon the varying situations, still you would hardly be willing to say that from day to day the meanings, qualities, and intensities of your words vary absolutely at random. So, too, you cannot say that they do not vary at all, estimating meanings, qualities, intensities as they are at the beginning and at the end of a period of time - a day, a month, a year, a decade, a score of years, a life-time. If the inner vividness for many words does not fluctuate at all over a given period, or if it fluctuates a great deal over a period of time, this may be symptomatic of a serious mental condition. Yet no matter how serious the mental condition of the individual, the events of his mind would constitute language.

In light of the above, we may say then of inner vividness that for a considerable group of individuals at one time, or for a single individual over a considerable period of time, words in spite of all their fluctuations show a tendency toward a preference of meaning, toward a stability of quality, whatever the quality may be, and toward a constancy of intensity. To be sure, we have no positive way of knowing whether or not this applies to all events of the mind, since we have no way of examining mind except through its expressions, and we can only appreciate expressions of the mind when they have something approaching a preference for meaning, a stability of quality, and a constancy of intensity. But that it is in general true for the mental events of spoken language is crystal clear, since all communication depends upon a tacit "acceptation" of primary meanings, qualities, and intensities. The "accepted" meanings need only be approximate, likewise the qualities and intensities, varying more or less as they do from individual to individual. But that there is a general agreement somewhere is evidenced by the very existence of dictionaries. In other words, the usual meaning is the usual meaning, the usual quality the usual quality, the usual intensity the usual intensity. We are again in the field of probability. 
And even in cases where the spoken language is not what we normally consider communication, that is, in the case of the language of a person raving with madness or delirium, still this language over a period of time reveals to the skillful observer a certain consistency of meaning, quality, and intensity - perhaps at utter variance with the "accepted" inner vividness - yet none the less, fairly consistent, relative to the patient.

And even where speech is not accessory to mental expression, that is, in our dreams and phantasies, though the meanings of the symbols are illusive even to the dreamer, and the qualities and intensities far from obvious, yet the great specialists in that field have made it clear that some of the symbols tend to recur with a consistency of inner vividness, not only frequently consistent to the individual, but sometimes consistent over great groups of people.

As a matter of fact, there must be a rough consistency of inner vividness throughout life, even though the mental event is in no way perceptible. At least, every living mortal assumes a day to day consistency both in himself and in others. We assume that we shall like and dislike tomorrow what we like and dislike today; that we shall think and feel and "reason" tomorrow as we think and feel and "reason" today. We expect the same of others; and yet our surprises, disappointments, and sudden fears show that in this we are often mistaken. It is not only that external conditions alter, but we, likewise, change, change where to all intents and purposes externals are identical. Inner vividness is only something approximate, something that varies within a range; something constant enough so that life and society can generally function with an amazing degree of orderliness, yet something fluctuating enough so that life and society generally function also with an amazing amount of conflict and friction.

What has all this to do with semantic change, the reader may ask. It is the propelling cause behind semantic change. We have seen how meaning may shift; this illustrates why meanings do shift, why one word is substituted for another at all. When a mental event, from whatever cause, deviates decidedly from the normal in quality or intensity, another word may be substituted which more nearly approaches the quality and intensity dictated by the exigencies of the moment. This is not the whole story of semantic change, but it is by far the most significant part, for the similarity in quality and intensity is to my mind far more important in the substitution than similarity in meaning. In calling a "woman" a "peach," or a "cat," or a "rose," or a "bitch," or a "vampire," or a "mouse," are we in the last analysis doing much more than simply substituting the quality and perhaps intensity (I say advisedly "perhaps") of "peach," "cat," "rose," "bitch" and so forth? Surely there can be little doubt about the substitution of quality. One would rarely refer endearingly to a person as "pest-house."

How great a rôle the accepted intensity of the substituted word plays in the substituting is problematical. I am of the opinion that it is in many cases negligible. For in many instances of substitution, the substituted word has normally only a mild or indifferent intensity, and the intensity of the substitution arises much more from the fact that an unusual epithet has been bestowed than that it is an epithet of unusual intensity. I am far from convinced that a word of normally high emotional intensity, such as "bitch," is selected for substitution because of its high emotional intensity; I believe rather that its present high emotional intensity has arisen from former sporadic substitution of unpleasant nature, - a seemingly paradoxical proposition which we shall presently develop.

When asked or desiring to give the clear meaning of an event, we do not normally "substitute"; we "explain." Definition, exposition, synthesis, analysis, or whatever else you are inclined to put under the heading of logical explanation, seem to me primarily a question of order or sequence of words. In "explanation" it is a matter of using the "accepted" meaning and "accepted" inner vividness as far as possible. Occasionally in "explanation" there is metaphor and

$$
\left[\mathrm{I}_{4}\right]
$$


connotation. But are not metaphor and connotation, that is, semantic change, rather used to convey vividness than clarity? Is it not true that the more an explanation is in the world of metaphor, which is the world of feeling, the less likely it is that the auditor will follow, or, if he follows, the less likely that he will lend to the words the meaning with which the speaker intends to endow them? For with metaphor, by its very nature, the primary meaning is not to prevail. But with explanation, clarity depends upon a fairly strict adherence to "accepted" meaning and "accepted" inner vividness, and if I may here say so, upon a comprehensible order - coherence.

This is then really the very problem of semantic change, the relationship between emotional intensity and clarity of meaning. Though we cannot define emotional intensity or clarity of meaning, we are all conscious of what they are; and I believe the solution to the riddle is to be found in what I term "order." Order, as I shall later explain and define it, is the essential difference between high intensity and clear meaning. Such a statement is in itself utterly meaningless. I mean that clarity tends to collect around "normal" order, and intensity around abnormal or "broken" or unusual order. I do not say there is any causal relationship, I simply state what I hope to show is a fact. The more primary the meanings, the more fixed the qualities, the more normal the order, the lower will be the concomitant intensity. In other words, the usual is not emotionally intense, a proposition which is certainly not unheard of. Hence, intensity, like meaning, must somehow, even though only remotely, be connected with relative frequency. Though absolutely nothing is known definitely about order other than that it is everywhere extremely important, let us proceed to examine the facts of order as far as language reveals them.

Every language has a pattern, so rigid that certain small violations of the pattern may lead instantly to obfuscation or incomprehensibility. And yet the pattern is so loose and supple that an individual within his pattern may describe his entire consciousness, regardless of what it be. But there is no divine decree behind a linguistic pattern, though the schoolmaster is rare who does not believe so. If we compare pattern with pattern, putting one language beside another, we find nearly every conceivable order represented in the various patterns - adjectives generally precede in one, follow in another; verbs precede in one, follow in another; inflectional suffixes are added in one, prefixes in another, neither in a third. If one looks at linguistic patterns, one is forced to conclude that here, if anywhere, the usual order is the usual order. We say "the black horse" instead of "horse black the," because it makes better sense; yet the reason it makes better sense is not because of divine decree, but because the order is more usual.

Again, exactly what normal order is, I, for one, do not know. When one says normal order, one means usual order. When one speaks of the normal course of events, one means the usual course of events. When the order of certain events becomes so fixed that one does not even think of the possibility of changing, we refer to those events in that crystallized order as habit. Again, there is obviously no divine decree behind order, although the temptation is always great to assume that our habits, or our arrangement of events in the flow of time, is "proper" and "correct." The more often in the past a certain event preceded or succeeded another certain event or events at a relatively approximate interval in the flux of time, the more we are inclined to consider those events as being in a fixed order and expect they will continue to occur in the future in the like arrangement. When $A$ is always followed by $B$, or $B$ always preceded by $A$, we say that $A$ and $B$ relative to each other are in firm sequential order; and judging from our past experience, we postulate the same for the future. The less fixed the order in the past, the less positively do we prognosticate the arrangement in the future. Let us express this feeling in different terms: the more often that two or more events occur in a certain sequential order in the stream of time, the more fixed we consider those events in order. In other words, briefly, the greater the relative frequency 
of an event in a certain sequence or sequences rather than in no sequence, the more fixed the order of the event.

This is first of all true regardless of causal relationship, whatever causal relationship may mean. If great portions of life processes are found in a causal relationship in a sense other than that outlined by the theory of Relative Frequency, the above statement of order will still hold true, in spite of the fact that "causation" should be found the basic factor of arrangement. Secondly, the definition of order does not necessitate the organism's "comprehension" of the inner arrangement or of the possible appropriateness of the order. "I planted a seed, a tree grew, and here is an apple from the tree." Seed, tree, apple, are in fixed normal sequence, even though we cannot explain the connecting steps. The reason we say that planting a seed and after a certain interval plucking an apple is reasonable and understandable is not that it is in the least understandable or reasonable according to our present knowledge, but that it is usual. Let a man marry his grandmother and it is incomprehensible to us; let a man beget a child and most of us do not wonder an instant about the processes involved. And yet, one is as much or as little reasonable and understandable as the other. We make a greater stir about the one than the other because it is less usual. Not a small amount of human experience may be lined up behind the acid statement that the greater the relative frequency the less the ado. And can we not say that one of the reasons that a pleasant thing becomes unpleasant to us is because it suddenly becomes too frequent or too rare in our life, or in the wrong order? "There is a time for all things." And does not the comprehensibility of the event become clouded, and isn't the meaning of the event unsettled by sudden shifts in frequency? Just as every event has some kind of quality and some "meaning," so, too, every event of mind must have a degree of determinacy of order. Every event must be preceded and succeeded by another event, from the first event to the last event, whenever that may be. There is then no harm in estimating future probabilities of sequence from past occurrences. And yet we also have breaches in normal order.

Every word has syntactical possibilities and limitations; a word is unthinkable except as somehow ordered in the stream of time. Any breach against usual order tends to unsettle the stability of both quality and meaning, and to increase the emotional intensity. A breach in order does not cause the unsettling, for one may just as reasonably commence with an unexpected emotional intensity and find order deranged. One may argue the causal relationship until the end of time; broken order is simply emotional intensity; emotional intensity is simply broken order they are concomitant. One is definable only in terms of the other. You may consult all the specialized works on emotion you will; you may read investigations intended to measure, investigations intended to analyze, investigations intended to cure. We talk daily about emotions - yet what are they? There is no part of the brain reserved for them; what we consider the "causes" of them can arise in the lungs, liver, or in the mind itself. They can have a source anywhere. All definitions of the emotions seem to gravitate about the proposition that emotion is inalienably connected with conflict. Is there any harm in saying that conflict is broken order, a deviation from whatever cause, from the normal course of events? Do not your greatest sorrows, greatest fears, greatest joys come from an unexpected change in the habitual course of events? Is not the intensity of the emotion greater, the greater and more unexpected the deviation? Yet as the unexpected event recurs more and more, - in fact becomes less unexpected and more usual, in fact more and more a matter of course, - does not the intensity correspondingly diminish? Of course there are flash-backs, but we expect that in probability. But the greater the relative frequency, the less the likelihood of flash-backs. And if to our above factors of meaning, quality, intensity, and order, we add likewise gradients of attention, fatigue, volition, as we shall when all of this is 
brought together in one volume, a certain sense of every-day reality will begin to collect around the Principle of Relative Frequency.

But what is order, you may ask? Order, as used here, is but the sequence of events. In the sequence of the events A, B, C, D, E, the greater the determinacy, in the light of probability, with which one event and not another appears immediately or at a certain interval instead of less immediately or at an uncertain interval, or not at all, before or after another event, the more fixed the order of those events. After the degree of probability of a certain sequence becomes so great that the likelihood of non-occurrence in that sequence becomes negligible, we begin to speak phenomenologically of causation.

The reader may rebel at this definition, partly because he feels that Whirl may become king, and partly because order may with passing time become very determined. As to Whirl, let me say that with order so defined Whirl's chances of partial or complete regency in human personalities are, of course, definitely existent, and numerous human beings behind or not behind locked doors give pitiful evidence to the veracity of this statement. The objection of constantly greater determinacy of certain sequences at the expense of others is just as valid. When, out of all possible events arranged according to probability of occurrence, one event instead of any of the others occurs, that event fractionally increases, thereby, its probability of re-occurrence by correspondingly diminishing the probability of the others. So it is that with passing years an individual, regardless of his species, becomes more fixed and set in his ways, and a new order or a new intensity or a new quality suffices to annihilate him, even though it be only of a slight degree of deviation from the normal, so slight that in the individual's youth he would have adjusted himself to it comparatively easily.

And why does not language become so crystallized, for language represents this order par excellence? It does. But the great thing that saves language from becoming entirely crystallized is abbreviation. For when the sequence A, B, C, D, E becomes so fixed that whenever A occurs the odds are enormous that $B, C, D, E$ will follow, the economy of language abbreviates $B, C, D$, $\mathrm{E}$, using only $\mathrm{A}$. So, too, when the odds are in favor of $\mathrm{B}$, or $\mathrm{C}$, or $\mathrm{D}$, or $\mathrm{E}$, so that when any one or more of them occur, it or they will occur in this sequence, that symbol or those symbols may stand for the sequence. There is nothing in the nature of meaning that it cannot be contained in one symbol rather than in another or others, provided that it once becomes established either by abbreviation or semantic change, which are to all intents and purposes the same thing. When for the sequence A, B, C, D, E one of the symbols therein contained is substituted, we call it abbreviation, when an outside symbol $X$ is substituted, we call it semantic change. I speak of symbols advisedly; a symbol is something that stands for something else. But I shall show that a symbol is also a mental event. The conservative tendency which causes abbreviation is the same tendency which maintains the abbreviation and tends to prevent regression to the original sequence which, wherever it may exist after it has been abbreviated, contains the meaning attached to the new symbol. And so the symbol arising from abbreviation may combine with other symbols arising from abbreviation until a fixed sequence arises, and a new abbreviation takes place - abbreviation upon abbreviation ad infinitum. If we delve into the roots of a symbol, we may hit strata after strata of symbols in ever more fixed and crystallized order. When does one finally reach reality, do you ask? I likewise ask. The order may become so firm as to be subconscious, instinctive, to be reflexive, to be structural - these are the substrata gestures, of which the permutations and combinations in time make up consciousness. Where is reality? Go even into cell structure and you are still in the field of symbolism.

For as we review the development of life, as far as it is known to us, we see that these abbreviations of sequences arising by chance have led to the greatest variety of species. And as a member 
of a species begins to develop, if it has an embryonic stage at all, it rehearses, as we all know, the entire previous essential history of its species in abbreviated order. There is no essential difference between an amoeba distending its wall for a food particle, a dog digging for a bone, a child reaching for a cookie, and an old man crying for bread. They are all reacting to their environment in a different pattern with different relative frequencies. As the relative frequency becomes greater, the likelihood of definite inheritability becomes greater. You do not need to think your hand is less a symbol than your words simply because you can perceive the material substance in the one. The essential development, movement, gesture, ordering in your hand are really important; the molecules are replaced remarkably often throughout life. Look at your hand in the light of evolution and consider it a slowly developing abbreviation of manifold gestures of defense and aggression therein centered, becoming ever more crystallized in the order of its development and functions, all intimately connected, like a word, with the whole ego. Indeed, we might go on indefinitely with this theory, regarding physical disease as interrupted or broken essential physiological order, regarding mental disease as broken or interrupted instincts or habits, showing that our intelligence and reason, of which we are so proud, have been obtained at the expense of the stability of instinctive process, a bargain of the past which, as our propensities for drugs and narcotics indicate, is not now always unanimously considered wise. And it is fitting that such a theory of life should come from the philologist, even though his expression of it would be somewhat facilitated by using terms of the Gestalt and Freudian systems of psychology, those two powerful systems which have so greatly aided our understanding of ourselves.

The modern theoretical linguist has a position of natural advantage. He finds his evidence all sorted and classified for him, evidence going back thousands of years and extending over millions of square miles, the record of billions of mortals, studied and orderly arranged by some of the most brilliant minds of the last and present centuries. And though he works alone he has at his hand the fruits of investigation and speculation of the modern psychologists, physicists, chemists, philosophers to encourage and point the way. Yet the greatest and most unique advantage he has in the very nature of his material. He can observe both by looking into himself and at others. The problem of change and mutation is the same for him as for the biologist; but the linguist can observe mutations taking place in his very lifetime without subjecting his object of experiment to a strain which, in being great enough to break established order, may be more than sufficient to annihilate the object. However, all of this is easy to say. Let us now turn to the proof. For the great problem is how we can demonstrate that this theory of abbreviation and the functional relationship of order and emotion is true. And the great impediment to proof is the question of what in all language shall we take as a basic unit for measurement.

For there has never been agreement as to what the unit of language is. One may argue equally plausibly for sounds, syllables, words, phrases, clauses, sentences, and so forth, and each one of these units is serviceable, depending upon the nature of the problem. Yet there is no common basic unit which unites phonology with morphology, with syntax, with semantic change. We have no measuring rods, scales, or watches to shift pints into pounds, or hours into meters. Taking sounds as a unit, we find evidence to support the contention that the greater the relative frequency, the less the conspicuousness, as defined and illustrated in Part One of this paper. Taking syllables as a unit, every philologist is willing to concede that increased frequency of usage is apt to wear away the syllable. And elsewhere ${ }^{1} \mathrm{I}$ have shown that syllabic accent - that great support of form - is apt to diminish with increasing frequency. But when we come to words, let alone phrases, clauses, and sentences, we are frankly at our wits' ends. What is the common unit that will include all these?

3 Zipf, op. cit., pp. 5 ff. 
The common unit is a time triangle. When I say that A is an abbreviation for A B C D E, a sequence which happened to be preceded by event $Y$ and followed by event $Z, I$ have really said that the abbreviation $A$ is a short cut through time from $Y$ to $Z$, and have given thereby an example of a time triangle:

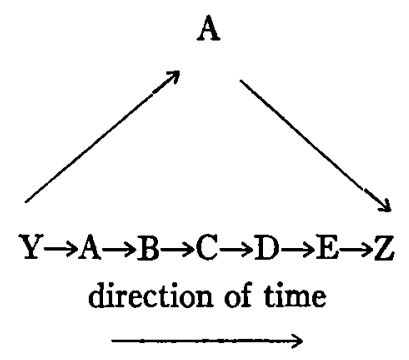

$\mathrm{Y} \mathrm{A} \mathrm{Z}$ is shorter than Y A B C D E Z. If one substitutes an outside symbol, $\mathrm{X}$ (semantic change) for the sequence, $\mathrm{Y} \mathrm{X} \mathrm{Z}$ is still shorter in time than Y A B C D E Z. It is shorter because one event intervenes instead of five. The sum of $\mathrm{Y} \mathrm{A}+\mathrm{A} \mathrm{Z}$ is shorter than Y A B C D E Z. ${ }^{1}$ If the reader wonders at this, let me assure him that $I$ am not measuring by solar time, even though solar time ultimately belongs most decidedly in the picture. I can make the time of this triangle clearer to you by a little experiment.

Let me lock you in an enclosure from which sun and starlight are excluded. I shall take your watch, but in return I shall give you everything you ask for, except any object by which you can deduce solar time. Is the experiment clear? Now as long as you are entertained there, you will find time short; while you are bored, you will find time long. Is that not true? But, according to what measure is it long or short? I think you will admit that your feelings about slowness or swiftness of passing time have little or nothing to do with a clock, but everything to do with the nature and intensity of your emotions at the time.

Now, that subjective stream of time according to which you say that a given portion of your life seemed long or short, seemed to pass especially slowly or rapidly, is the same stream of time in which I shall say that the unit of language forms a time triangle. I shall call it psychic time. I shall say the unit of language is a mental event which in turn is a time triangle in psychic time. "But this psychic time is not absolute like clock time. How can you use it as a measure?" It is just exactly as little absolute as clock time; that is precisely the point of the matter. In going from $\mathrm{Y}$ to $\mathrm{Z}$ by means of $\mathrm{A}$, it takes less time than from $\mathrm{Y}$ to $\mathrm{Z}$ by means of $\mathrm{A} B \mathrm{CD}$ E. And since time is merely that by means of which we measure velocity, we may, therefore, say that the mental event $\mathrm{A}$, being the interval between $\mathrm{Y}$ and $\mathrm{Z}$, is relatively faster than A B C D E, being also the interval between $\mathrm{X}$ and $\mathrm{Z}$.

Without the abbreviation, the events of your mind run, let us say, $\rightarrow$ V W Y A B C D E Z $\rightarrow$; with the abbreviation the events run $\rightarrow \mathrm{V} \mathrm{W} \mathrm{Y} \mathrm{A} \mathrm{Z} \rightarrow$ and the only way you can tell from the stream of consciousness that abbreviation has taken place (or semantic change, if one uses $\mathrm{X}$ instead of $\mathrm{A}$ ) is that there is a sudden acceleration between $\mathrm{Y}$ and $\mathrm{Z}$. If $\mathrm{Y}$ and $\mathrm{Z}$ in turn are likewise abbreviations or substitutions and thereby enjoy acceleration, there is then no way of telling

1 Cf. A. S. Eddington: The Nature of the Physical World, New York, Macmillan (I930), pp. II I ff., for a clear and popular description of the time triangle in physics. This book, together with A. N. Whitehead's Symbolism, Its Meaning and Effect, New York, Macmillan (1927), and Kurt Koffka's The Growth of the Mind, New York, Harcourt Brace (1928), together with many happy discussions of Freudian principles with Professor H. A. Shaw at Harvard, have been helpful to me in revamping and expressing the Principle of Relative Frequency, though it has often been impossible to reconcile some of the more speculative theories of the Gestalt with the facts of language.

[I9] 
that $A$ is an abbreviation except from the fact that it does not slow down. I need not point out that V W Y A Z are loosely ordered mental events, and should they through relative frequency of occurrence become sufficiently crystallized to justify abbreviation, and should some other event than $A$ be used for the abbreviation, and should $A$ likewise disappear from other sequences by the same process, A may utterly disappear from consciousness, and simply be carried on in essence by the abbreviating symbols. This is the structure of language to my mind. And "meaning" is really what lies behind, or if you will, below the symbols. But before we proceed to quality and intensity of mental events, let us sum up what we have said on the basis of our assumptions: the greater the relative determinacy of order, the less the relative speed, and vice versa. Though this is an astonishing statement, I shall, none the less, prove that it is true, as an analysis of language will show.

Now, it follows from what we have said before that the greater the relative acceleration, the greater the proportionate emotion, for emotion is but a lack of determinacy of order. V W Y A Z are less determined in order than V W Y A B C D E Z, even though A B C D E are the only events in determined sequence. It seems almost as if the persons who coined "abbreviation" and "emotion" were somehow conscious of the Principle of Relative Frequency. What happens to A B C D E while abbreviated, I do not know (nor do you). But that they are not annihilated forever is evidenced by their chance occurrence again in that sequence in the stream of consciousness. Surely such an exposition explains the significance of order, intensity, and meaning, but it leaves quality out of the picture. And yet quality is most important, for it furnishes the motive.

One may proceed from $A$ to $B$ for one of several reasons. It may be an escape from $A$ because $A$ is unpleasant (in which case $A$ has a negative quality); or one may proceed from $A$ to $B$ because $B$ is more pleasant (in which case B is more positive or is just as positive with a greater degree of intensity). We may speak of negative as fear and positive as desire. And these two well recognized qualities, as roughly as they are here stated, are really the propelling motives behind the sequence of events. With a proper understanding of quality, we can ultimately gain great light on this theory from previous studies of the neurosis, and, if this theory of emotion be correct, throw a great deal of light on the study of the neurosis. The reader may not understand the important rôle of quality in life. He may say that he can understand why an individual should want to put a negative event behind him, or flee from a low positive intensity to a higher positive intensity, but why should he wish to leave a very high positive intensity for something else?

In simple words, you have said "I can see why a person wants to escape an unpleasant event, or leave a pleasant event for a more pleasant event, but I cannot see why he should want to leave a very pleasant event for one which is less pleasant." In short, why don't we always do just what we like most to do? A very frequent question in this world! Let us try to answer it according to Relative Frequency. Let us assume that the event has a high positive intensity, + A. Now let us assume that there are, likewise, $+\mathrm{B}$ and $+\mathrm{C}$, different, but approximate in intensity. Let us now restrict our activity to them: $+\mathbf{A}+\mathbf{B}+\mathbf{C}+\mathbf{C}+\mathbf{B}+\mathbf{A}+\mathbf{B}+\mathbf{C}+\mathbf{B}$, etc. Being limited to three events, they can have only a limited number of permutations. No matter how hard you try, with passing time they will develop sequences, and with the high relative frequency of the sequences, order will become more crystallized. With increased crystallization or determinacy of order, emotional intensity begins to decrease. And after the intensity diminishes far enough it will be less than the emotional intensity of events other than A, B, C, and to these other events you then proceed. For as you conceded a moment ago, you can easily understand passing from pleasant event or events to more pleasant events.

If you would like to experiment in the matter, pick out the most delicious article of diet, or the most exquisite piece of music, or the most refreshing form of exercise, mental or physical, and con- 
fine yourself to it day on end, week on end. You will become tired of it? I agree. Fatigue is a function of relative frequency and serves to keep you from increasing the relative frequency too fast, as I hope to explain when I have more available space. But in spite of the fact that you are becoming tired of your favorite dish of lobster that you have eaten daily for ten days, continue eating lobster for another ten days. And now how do you feel about lobster? So you are sick of it and don't want another! After a further ten days of forceful feeding of lobster you would most definitely not want any more at all. The erstwhile high positive intensity has become a high negative intensity. This is surely an every-day phenomenon, familiar to all. Over-indulgence brings satiety. And yet, to my mind, it is nothing but too rapid acceleration of the relative frequency. The attendant derangement of the substrata gestures which may be broadly termed sickness, whether it be an upset stomach or cancer of the skin, is in my opinion in the last analysis due to nothing more than a breach in crystallized order, from whatever source or trauma that you wish. In increasing the relative frequency of lobster you have broken the strict order of the everyday; it is both the increased frequency of the one and the broken order of the other that leads to the resultant trouble. Certainly the fault is not with the lobster; for customary activities are appallingly different among individuals and everywhere we notice that what is food for one may be poison for another. It is regrettable to me that right at this point where the physiological, mental, and evolutionary aspects of the Principle of Relative Frequency are becoming so pertinent we must return to linguistic considerations. But before returning to linguistic considerations, let me say here for the sake of any mathematician who may plan to formulate the ensuing data more exactly, the ability of the highly intense positive to become the highly intense negative, in my opinion, introduces the devil into the formula in the form of $\sqrt{-I}$. And now to linguistics.

Highly emotional mental events are rare. If they are of negative quality, they tend to be rare because of a natural desire to avoid them. If they are positive, they tend to occur rarely because of their tendency to shrink in emotional intensity when their frequency increases. And they by the same token tend to be manifold in variety. For nothing can intervene between mental events except mental events; and these intervening mental events will be positive or negative or neutral, and subject to the same general laws of abbreviation. This tendency of mental events to be rare and manifold in variety I shall call the life tendency. In simple terms it is the tendency to increase experience by a hazardous seeking for the new. But, opposing the life tendency, which increases variety, is abbreviation which reduces number when frequency increases. I shall call this tendency to decrease number and variety by abbreviation when relative frequency increases, the death tendency, being willing to agree if the reader insists that this is roughly identical with Freud's death instinct, though I have no space to argue the point. Life tendency and death tendency are constantly counterbalancing each other, - emotional intensity on the one hand versus the stability of meaning and order on the other. As I look at life, I think one can say that the one tendency takes us from our mothers' womb and with passing time hands us over more and more to the other tendency which gives us surcease in the grave. In many ways we seem to be like the physical universe, which we are told expands only to contract. But be that as it may. If it be true that there is this balance in mind between the rare occurrence of many dissimilar events and the frequent occurrence of a small number of events, this balance is the basic law of life as well as mind. Let us translate the law into terms of language.

Let us take the word "automobile," and remember that we shall use as our measure the time triangle. Shall we call the sounds the events? If you like; there are eight of them and the sequence is A B C D E F G H. These sounds occur in other sequences, but their order in this sequence, as in other sequences, is very fixed, and the emotional intensity of any one is practically nil. Sounds are meaningless if not fixed in sequences. And each sound represents particular ges- 
tures of various organs of the mouth, plus the substrata gestures. The phonetic gestures are crystallized in arrangement and sequence in time. As a sound increases in relative frequency, these gestures are abbreviated more and more, losing, thereby, what I call conspicuousness. Shall we take syllables as the events? There are four syllables in "automobile" and the sequence may be referred to as $\mathrm{L} \mathrm{M} \mathrm{NO}$ which is really an abbreviation of A B C D E F G H which are substrata when the measurement is of syllables. $\mathrm{L}, \mathrm{M}, \mathrm{N}$, and $\mathrm{O}$ each occur elsewhere presumably. Though fairly crystallized, accent tends in the long run to hover about the relatively rare as I have shown elsewhere.' Let us assume that the sequence $\mathrm{L} M \mathrm{M} \mathrm{O}$, for one reason or another, becomes relatively more frequent. Any one, two, or three of the mental events may be abbreviated, those going first which are less essential, that is, which occur in more other sequences. So we may take "auto," or "bil" (as in Icelandic) for example. If "au," or "aut," or "to," or "mo" do not occur as abbreviations, it may be because they are already abbreviations or symbols of something else and would lead to confusion, or it may be simply chance. Can we shorten "auto" further? Yes, by semantic change, we can substitute "car." This tendency toward abbreviation and substitution is evidenced by the tendency of short words to be frequent and long words rare.2

But now let us take the word "automobile" as a single symbol, $\mathrm{X}$, and consider, as substrata, syllables as well as sounds. Very good, but then we must have a stream of words in which the symbol $\mathrm{X}$ occurs among its fellows so that we may estimate its frequency. If all we have said about Relative Frequency, abbreviation, and semantic change be true, then in examining a suffcient number of words in the stream of language, we should expect to find many different words occurring relatively rarely, and only a few occurring relatively frequently. That would be the proof of the pudding; and it happens to be true, regardless of how inflected or uninflected the language may be.

I have investigated the words of 20,000 syllables of modern colloquial Chinese of Peking (the same selections of which the sounds were examined in Part One). The actual words with their frequency of occurrence are to be found in Appendix $C$. I have investigated the frequency of occurrence of all words in Plautus' Aulularia, Mostellaria, Trinummus, and Pseudolus, ${ }^{3}$ and the words together with the number of their occurrences may be found in Appendix A. In addition, I have taken R. C. Eldridge's analysis ${ }^{4}$ of the frequency of occurrence in American newspapers of selections totalling 43,990 words (exclusive of numerals and proper names). I have not used F.W. Kaeding's ${ }^{5}$ elaborate investigations of the German language because unfortunately his results are not in a form serviceable to statistics. But Kaeding agrees (page $54 \mathrm{ff}$.) with my own results and those of Eldridge, that many words occur rarely, and only a few frequently. Below are tabulated the number of words and the times of their occurrence, in Chinese, English, and Latin that is, in a negligibly inflected, in a slightly inflected, and in a fairly highly inflected language, so selected for their importance for future work. Under each language the left-hand column gives the number of times the word occurs, the right-hand column gives the number of words, each one of which occurs that many times. In parentheses after the number of words in Chinese and Latin are given the number of syllables. For Chinese the actual number of words in each frequency which have one, two, three, etc., syllables is given with a small number at the upper right to desig-

\footnotetext{
1 See supra, p. I.

2 See infra, p. 23.

${ }^{3}$ According to the Oxford edition. The elision of $e s t$ with a preceding word is indicated by italics (e. g. inopiast); the elision of $e s$ is indicated by apostrophe (e.g. pessumu's). Differences in vowel quantity were not recorded, nor would their consideration seriously alter the statistics, as the reader will see for himself by examining the list of words in the Appendix.

- R. C. Eldridge: Six Thousand Common English Words, copyright IgII, privately printed at Niagara Falls, New York.

s F. W. Kaeding: Bäufigkeitswörterbuch der deutschen Sprache, Steglitz, bei Berlin, 1898.
} 


\begin{tabular}{|c|c|c|c|c|c|c|c|c|c|}
\hline \multicolumn{5}{|c|}{ Chinese } & \multicolumn{2}{|c|}{ ENGLISH } & \multicolumn{3}{|c|}{ Laten of Plautus } \\
\hline $\begin{array}{l}\text { Number of } \\
\text { Occur- } \\
\text { rences }\end{array}$ & $\begin{array}{l}\text { Nunber of } \\
\text { Words }\end{array}$ & Numbe & r of Word & Is with their Syllables & $\begin{array}{l}\text { Number of } \\
\text { Occurrences }\end{array}$ & $\begin{array}{l}\text { Number of } \\
\text { Words }\end{array}$ & $\begin{array}{l}\text { Number of } \\
\text { Occurrences }\end{array}$ & $\begin{array}{l}\text { Number of } \\
\text { Words }\end{array}$ & $\begin{array}{c}\text { Average } \\
\text { number of } \\
\text { Syllables }\end{array}$ \\
\hline$I$ & 2046 & $\left(3 \div 5^{1}\right.$ & $1571^{2}$ & $144^{3} \quad I 4^{4} \quad I^{6} \quad I^{6}$ & I & 2976 & $\mathbf{I}$ & 5429 & $(3.23)$ \\
\hline 2 & 494 & ( $110^{1}$ & $35^{82}$ & $\begin{array}{ll}23^{3} & \left.3^{4}\right)\end{array}$ & 2 & 1079 & 2 & I 198 & (2.92) \\
\hline 3 & 216 & $\left(59^{1}\right.$ & $147^{2}$ & & 3 & 516 & 3 & 492 & $(2.77)$ \\
\hline 4 & 100 & $\left(24^{1}\right.$ & $73^{2}$ & $\left.3^{3}\right)$ & 4 & 294 & 4 & 299 & $(2.05)$ \\
\hline 5 & 99 & $\left(39^{1}\right.$ & $5^{8^{2}}$ & $\left.2^{3}\right)$ & 5 & 212 & 5 & 161 & (2.60) \\
\hline 6 & 66 & $\left(24^{1}\right.$ & $41^{2}$ & $\left.\mathrm{I}^{3}\right)$ & 6 & I5I & 6 & I 26 & $(2.53)$ \\
\hline 7 & $4^{I}$ & $\left(16^{1}\right.$ & $\left.25^{2}\right)$ & & 7 & 105 & 7 & 87 & $(2.39)$ \\
\hline 8 & 25 & $\left(10^{1}\right.$ & $14^{2}$ & $\left.I^{3}\right)$ & 8 & 84 & 8 & 69 & (2.44) \\
\hline 9 & 30 & $\left(13^{1}\right.$ & $15^{2}$ & $\begin{array}{ll}I^{8} & \left.I^{4}\right)\end{array}$ & 9 & 86 & 9 & 54 & $(2.35)$ \\
\hline 10 & 20 & $\left(13^{1}\right.$ & $\left.7^{2}\right)$ & & 10 & 45 & ro & 43 & $(2.32)$ \\
\hline I I & 25 & $\left(14^{1}\right.$ & $\left.\mathrm{II}^{2}\right)$ & & II & 40 & II & 44 & (2.29) \\
\hline I2 & 22 & $\left(15^{1}\right.$ & $\left.7^{2}\right)$ & & I2 & 37 & I2 & 36 & $(2.30)$ \\
\hline I3 & 10 & $\left(6^{1}\right.$ & $\left.4^{2}\right)$ & & 13 & 25 & I3 & 33 & $(2.30)$ \\
\hline 14 & 14 & $\left(7^{1}\right.$ & $\left.7^{2}\right)$ & & 14 & 28 & $r_{4}$ & 31 & $(2.09)$ \\
\hline 15 & 13 & $\left(5^{1}\right.$ & $\left.8^{2}\right)$ & & 15 & 26 & 15 & 13 & $(2.07)$ \\
\hline 16 & ro & $4^{1}$ & $5^{2}$ & I) & I6 & I 7 & 16 & 25 & $(2,40)$ \\
\hline 17 & 10 & $6^{1}$ & $\left.4^{2}\right)$ & & 17 & 18 & 17 & $2 \mathrm{I}$ & $(2.09)$ \\
\hline 18 & 6 & $\left(2^{I}\right.$ & $\left.4^{2}\right)$ & & 18 & ro & 18 & $2 \mathrm{I}$ & $(2.04)$ \\
\hline 19 & 5 & $\left(4^{1}\right.$ & $\left.\mathrm{I}^{2}\right)$ & & Ig & 15 & I9 & II & (2.18) \\
\hline 20 & 5 & $\left(5^{2}\right)$ & & & 20 & 16 & 20 & I5 ) & \\
\hline 21 & 4 & $\left(3^{1}\right.$ & $\left.\mathbf{I}^{2}\right)$ & & $2 I$ & 13 & $2 I$ & Io & \\
\hline 22 & 2 & $\left(2^{1}\right)$ & & & 22 & II & 22 & 8 & \\
\hline 23 & 5 & $\left(4^{1}\right.$ & $\left.I^{3}\right)$ & & 23 & 6 & 23 & 8 & $(2.08)$ \\
\hline 26 & 3 & $\left(2^{1}\right.$ & $\left.\mathrm{I}^{2}\right)$ & & 24 & 8 & 24 & 9 & \\
\hline 28 & 4 & $\left(3^{1}\right.$ & $\left.\mathrm{I}^{2}\right)$ & & 25 & 6 & 25 & I I & \\
\hline 29 & 4 & $\left(I^{1}\right.$ & $\left.3^{2}\right)$ & & 26 & Io & 26 & 7 & \\
\hline 30 & 6 & $\left(4^{1}\right.$ & $\left.2^{2}\right)$ & & 27 & 9 & 27 & 9 & $(a m)$ \\
\hline 32 & 6 & $\left(4^{1}\right.$ & $\left.2^{2}\right)$ & & 28 & 6 & 28 & I 2 & $(2, \infty)$ \\
\hline 33 & 2 & $\left(I^{1}\right.$ & $\left.I^{2}\right)$ & & 29 & 5 & 29 & 4 & \\
\hline 34 & I & & & & 30 & 4 & 30 & 4. & \\
\hline 35 & I & & & & $3 I$ & 6 & $3 \mathrm{I}$ & 8 & \\
\hline 36 & I & & & & 32 & 4 & 32 & 3 & \\
\hline 37 & I & $\left(I^{2}\right)$ & & & 33 & 6 & 33 & 4 & \\
\hline $3^{8}$ & $\mathbf{I}$ & $\left(\mathrm{I}^{2}\right)$ & & & 34 & 2 & 34 & 6 & $(2.05)$ \\
\hline $4 I$ & 4 & & & & 35 & 5 & 35 & 3 & \\
\hline 43 & 2 & $\left(2^{2}\right)$ & & & 36 & 3 & 36 & 5 & \\
\hline 44 & 2 & $\left(x^{1}\right.$ & $\left.\mathbf{I}^{2}\right)$ & & 37 & 2 & 37 & 7 & \\
\hline 45 & 3 & $\left(I^{1}\right.$ & $\left.2^{2}\right)$ & & 39 & 2 & 38 & 2 & \\
\hline 46 & I & & & & 40 & 4 & 39 & 4 & \\
\hline 47 & 2 & & & & $4 I$ & I & 40 & 3 & \\
\hline 50. & $\mathbf{I}$ & $\left(I^{2}\right)$ & & & 42 & 7 & 4I & 3 & \\
\hline 52 & I & & & & 43 & I & 43 & 4 & \\
\hline 55 & 2 & & & & 44 & 4 & 44 & I & \\
\hline 57 & I & & & & 45 & $\mathbf{I}$ & 45 & $\mathbf{I}$ & \\
\hline 58 & $I$ & & & & 46 & 2 & 46 & I & \\
\hline 60 & I & & & & 47 & 5 & 47 & 3 & \\
\hline 66 & 2 & $\left(I^{1}\right.$ & $\left.\mathrm{I}^{2}\right)$ & & 48 & $I$ & 48 & I & $(1.70)$ \\
\hline 68. & I & & & & 49 & 3 & 49 & I & \\
\hline 72 & I & & & & 50 & 3 & 50 & 2 & \\
\hline 73 & $\mathbf{I}$ & & & & $5 I$ & I & 51 & 2 & \\
\hline 75 & I & & & & 52 & 3 & 53 & 4 & \\
\hline 78 & I & & & & 54 & I & 54 & $\mathbf{I}$ & \\
\hline 81 & $\mathbf{I}$ & & & & 55 & I & 55 & I & \\
\hline 83 & $\mathbf{I}$ & & & & 56 & $\mathbf{I}$ & 56 & 2 & \\
\hline IOI & 2 & & & & 58 & 2 & 58 & I & \\
\hline $102-905$ & I 2 & $\left(I 2^{1}\right)$ & & & 60 & $\mathbf{I}$ & $6 r$ & 3 & \\
\hline 13248 & 3332 er & $.8 \%$ & & & $6 I-4290$ & 71 & $62-514$ & $7 \mathrm{I}$ & $(1.40)$ \\
\hline
\end{tabular}


nate the number of syllables, thus permitting us to see the tendency of long words to occur rarely, which we should expect from the Principle of Relative Frequency and Abbreviation. Where the number of syllables is not marked, the words are monosyllables. In Latin the parentheses embrace the average number of syllables of all the words having the given occurrence.

The astonishing thing about this marked tendency is not that it is new - for it is well-known among linguists and as self-evident as gravitation. The astonishing thing is that it should occur to anyone to build up a law of language and mind upon it. But even more astonishing it is that, like gravitation, there is not only a pull toward abbreviation, but the pull toward abbreviation varies in an orderly fashion as the frequency increases. That is, regardless of whether the language is highly inflected or not (and this is very important to show the functional interrelationship of morphology and syntax), the number of words decreases with a marked similarity upon increase of usage. But the overwhelming disclosure is this, that the formula for abbreviation is $a b^{2}=k$, a formula exactly identical to that of gravitation.

For we can plot these figures on double logarithmic charts, marking off on the horizontal axis the actual number of words which occur at a given frequency, and marking off on the vertical axis the frequency with which the words occur. The charts for the words in Plautus and English are given opposite on page 25. The upper chart on page 26 is for Chinese words. The lower chart on page 26 , for Chinese syllables, should be disregarded. The reasons for its inclusion are given below. The diagonal line represents the formula $\mathrm{ab}^{2}=\mathrm{k}$, and the reader can judge for himself if this does not pass through the indicated points with reasonable closeness.

The deviations from the line $\mathrm{ab}^{2}$ are in all three cases remarkably slight. If it deviates in English at the very low frequencies, that is because Eldridge omitted proper names and numerals which are as much words as any of the others. But the slight deviation is due to two other causes: (I) the natural probability of error; (2) the fact that they are based on written language and not spoken or thought, and hence the authors of the selections, in making any erasure for re-writing, have artificially distorted what was the normal course of events.

You may ask, "why did you not also plot the words with high frequencies which, according to the lists on page 23 , would make the diagonal line suddenly jump, and not follow the straight line of the formula $a b^{2}=k$ in the way you indicate by the dotted line on all three charts? For Plautus, for example, you did not include words which occurred more of ten than fifty times. And so too, correspondingly, for English and Chinese."

You will find that the line would jump at exactly that point where, according to the formula, fractional words are called for. I shall call this the scattering point and it is where the problems of syntax, morphology, and semantic change meet. For when we say that the square of the frequency of occurrence times the number of words possessing that frequency remains constant, we must perforce have, with high frequencies, fractional words which do not exist. This demand of the formula for fractional words is represented by the sudden spurt of frequency with ever greater intervals in between.

The chart for Chinese syllables I include here simply because the present publication seemed ideal for the inclusion (in Appendix D) of the frequency lists of Chinese syllables and tones, of which I shall make future use in attempting to show that the degree of inflection of a language is possible of rough computation.

It is with true regret that my space now necessitates conclusion, without my even broaching the question of language as a perception. If one chooses to regard what I call a sequence as a Gestalt, or to compare my definition of emotion with that of the Freudian school, well and good. That I have not done so myself is partly because I wished the Principle of Relative Frequency to 

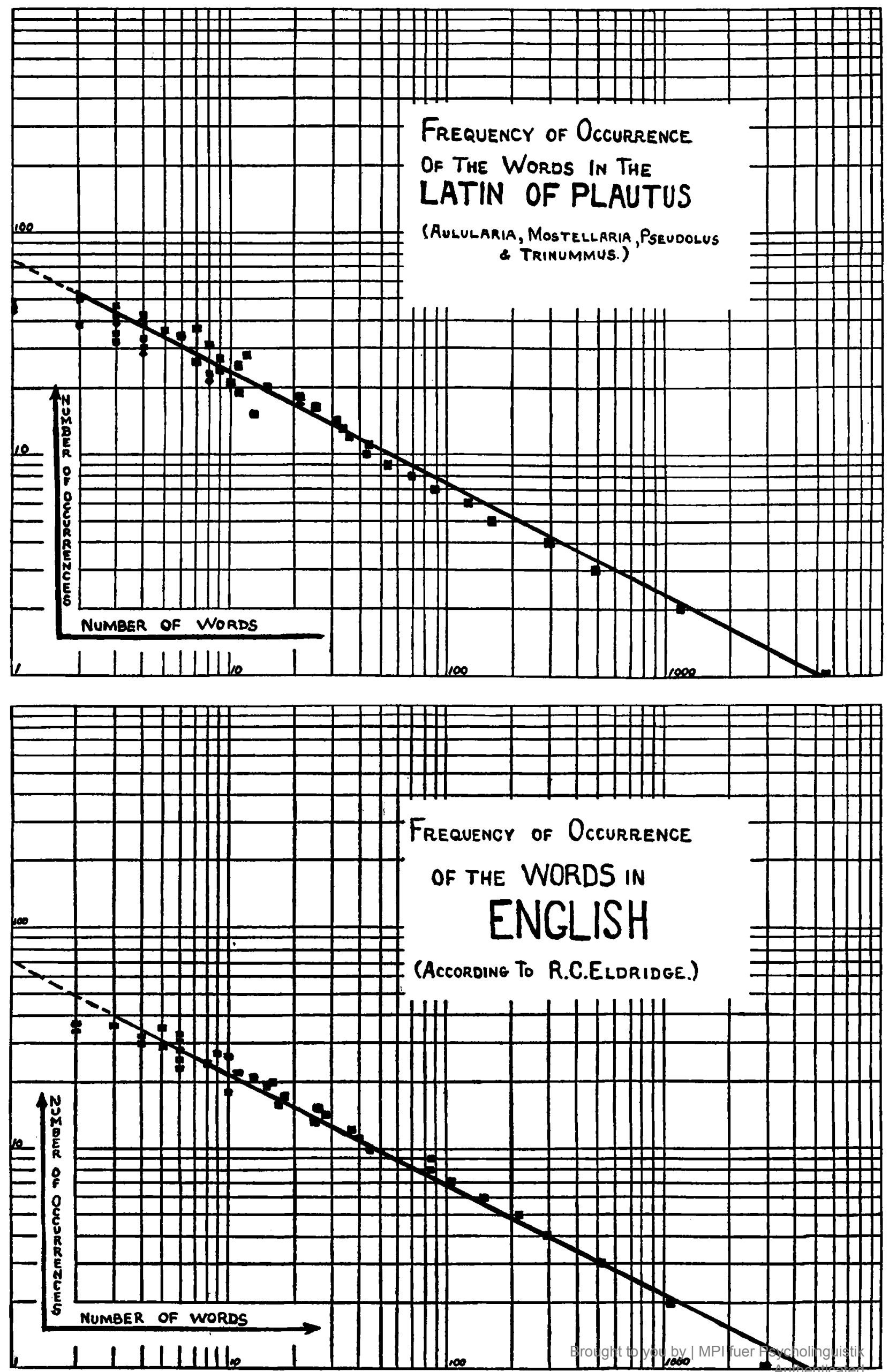

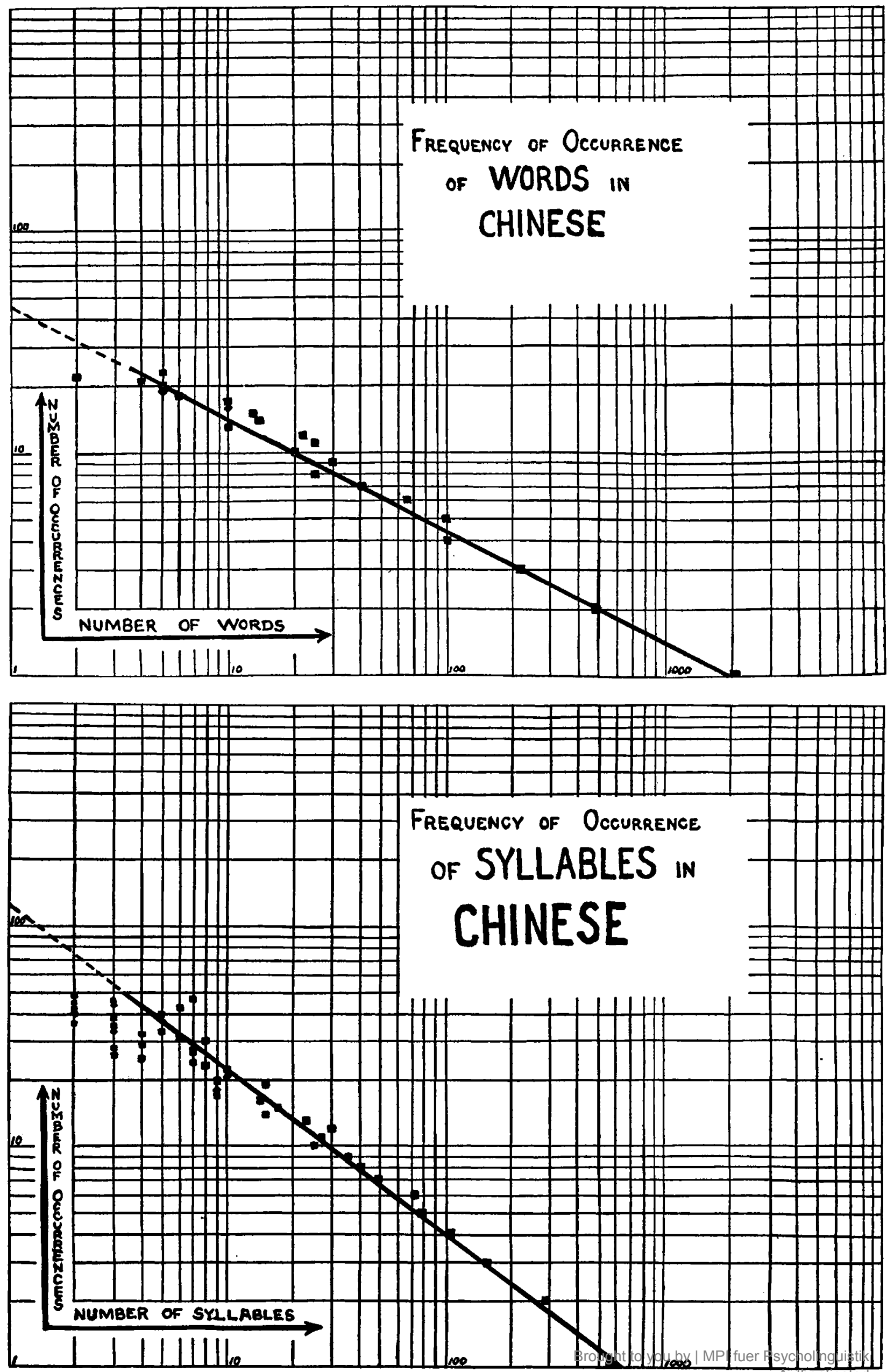
stand on its own feet, and partly because some of the theories of the Gestalt, as I understand them, definitely do not agree with the phenomena of language as I observe them.

And as we have argued of words, so we may argue of phrases, clauses, sentences, regarding in turn the phrase, the clause, the sentence, as the symbol, and considering the words as substrata, even though in doing this, order and abbreviation play a less perceptible rôle. The word seems roughly to be that unit of consciousness in which the degree of determinacy of order and the intensity of emotion are within such a range as to be both perceptible. And where does the Principle of Relative Frequency stop? It stops where neither order nor value are existent. In other words it is all pervasive in life. I believe that from the single cell to the most highly organized society of individuals the law prevails that Frequency times Conspicuousness is Constant. 
Brought to you by | MPI fuer Psycholinguistik Authenticated Download Date | 7/4/17 11:30 AM 


\section{APPENDIX A}


Brought to you by | MPI fuer Psycholinguistik Authenticated Download Date | 7/4/17 11:30 AM 


\title{
FREQUENCY OF OCCURRENCE OF WORDS
}

\author{
In Plautus' Aulularia, Mostellaria, Pseudolus, and Trinummus
}

$\begin{array}{ll}\text { quid } & 514 \\ \text { ego } & 506 \\ \text { me } & 482 \\ \text { est } & 473 \\ \text { ut } & 445 \\ \text { te } & 408 \\ \text { et } & 373 \\ \text { si } & 349 \\ \text { in } & 338 \\ \text { non } & 308 \\ \text { mihi } & 305 \\ \text { tu } & 300 \\ \text { tibi } & 296 \\ \text { qui } & 295 \\ \text { atque } & 275 \\ \text { nunc } & 250 \\ \text { quod } & 245 \\ \text { hic } & 212 \\ \text { ad } & 199\end{array}$

abi

$\begin{array}{lr}\text { iam } & \text { I9I } \\ \text { hoc } & \text { I88 } \\ \text { quam } & \text { I70 } \\ \text { sed } & \text { I70 } \\ \text { esse } & \text { I69 } \\ \text { nam } & \text { I69 } \\ \text { id } & \text { I59 } \\ \text { neque } & \text { I45 } \\ \text { ita } & \text { I40 } \\ \text { hercle } & \text { I34 } \\ \text { ne } & \text { I29 } \\ \text { ubi } & \text { I23 } \\ \text { mi } & \text { I22 } \\ \text { modo } & \text { I14 } \\ \text { quae } & \text { I13 } \\ \text { rem } & \text { I08 } \\ \text { quom } & \text { 102 } \\ \text { haec } & \text { Ior } \\ \text { sunt } & 98\end{array}$

eam

$\begin{array}{ll}\text { uolo } & 74 \\ \text { di } & 72 \\ \text { bene } & 70 \\ \text { aedis } & 68 \\ \text { homo } & 67 \\ \text { quo } & 66 \\ \text { uis } & 66 \\ \text { an } & 64 \\ \text { hominem } & 64 \\ \text { quia } & 64 \\ \text { haud (hau) } & 63 \\ \text { argentum } & 62 \\ \text { istuc } & 62 \\ \text { res } & 62 \\ \text { at } & 61 \\ \text { quis } & 61 \\ \text { scio } & 61 \\ \text { sis } & 58 \\ \text { illum } & 56\end{array}$

nos dum ea facere sine aurum pro uos credo huic magis dare 39 igitur $\quad 39$ malum 39 nomen 39 dicam 38 numquam 38

7 Words Occur 37 Times

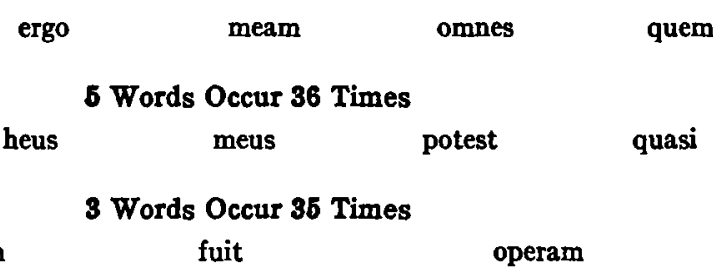

animum

6 Words Occur 34 Times

aedibus

domum

illic

lubet

opsecro

quoi

4 Words Occur 33 Times

caussa

enim

ero

tuom

3 Words Occur 32 Times

apud

ipsus

quaeso

senex

8 Words Occur 31 Times

sic simul

4 Words Occur 30 Times

domi

hac

per

potius

4 Words Occur 29 Times

erit

nimis

re

satis

12 Words Occur 28 Times

autem

dabo

em

equidem

habeo

foras ibi illud tecum 40

sibi

44
43
43
43
43
$4 I$
$4 I$
$4 I$
40
40
40
39
39
39
39
38
38

\section{[3I ]}




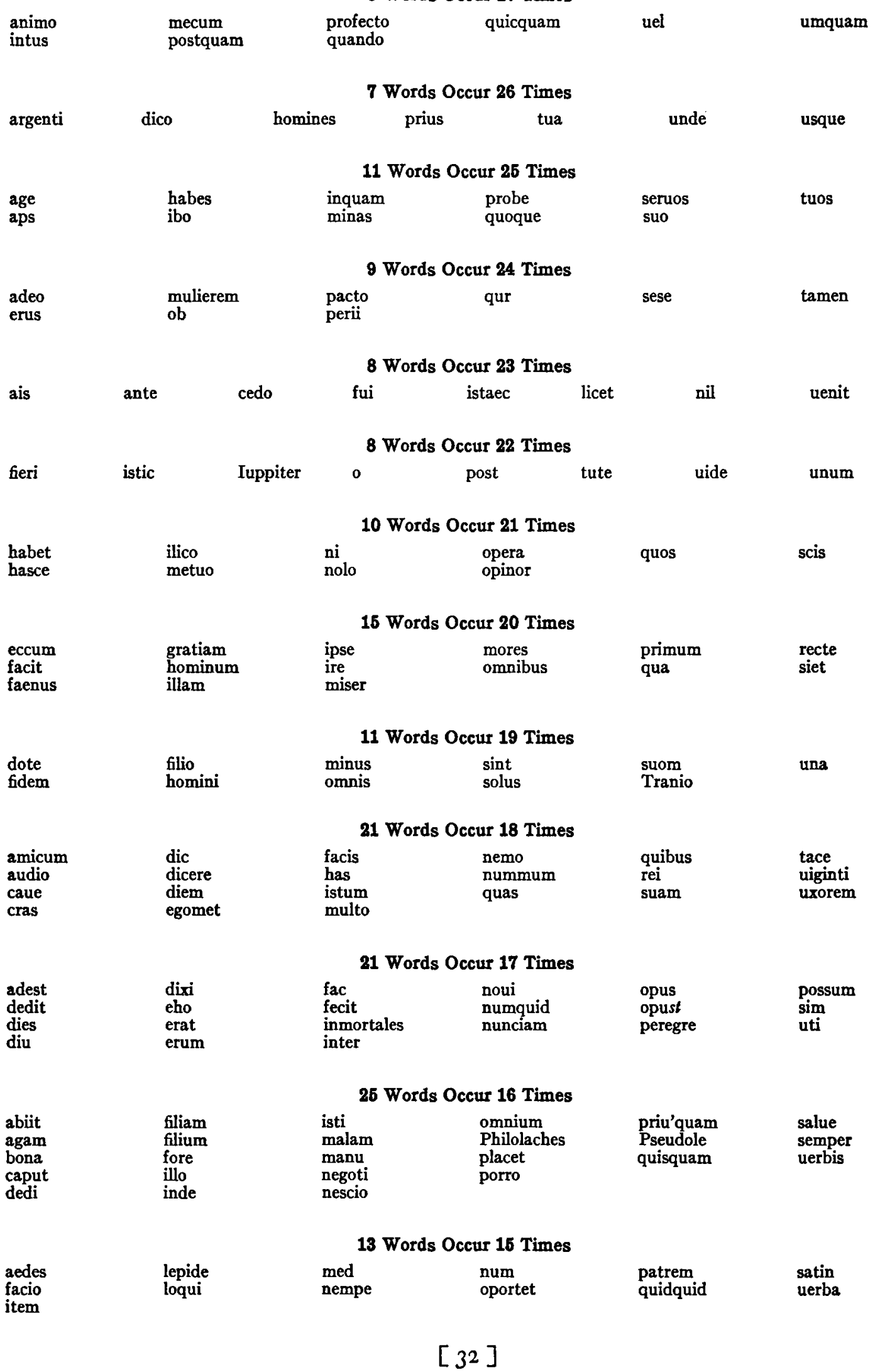


31 Words Occur 14 Times

$\begin{array}{ll}\text { adulescens } & \text { dudum } \\ \text { aequom } & \text { eius } \\ \text { aetatem } & \text { epistulam } \\ \text { arbitror } & \text { faxo } \\ \text { cenam } & \text { fores }\end{array}$

dicis

$\begin{array}{ll}\begin{array}{l}\text { actutum } \\ \text { agis }\end{array} & \begin{array}{l}\text { certum } \\ \text { animus } \\ \text { ecquid } \\ \text { argento } \\ \text { auri } \\ \text { egoni } \\ \text { eilus } \\ \text { estis } \\ \text { etsi }\end{array} \\ & \\ \text { alii } & \text { cor } \\ \text { aliud } & \text { corde } \\ \text { amicam } & \text { det } \\ \text { apage } & \text { dicas } \\ \text { Charmides } & \text { dicta } \\ \text { consilium } & \text { die }\end{array}$

consilium die

36 Words Occur 12 Times

$\begin{array}{ll}\text { dotem } & \text { iussit } \\ \text { eloquar } & \text { leno } \\ \text { facias } & \text { maxume } \\ \text { faciunt } & \text { meae } \\ \text { genus } & \text { molestus } \\ \text { illuc } & \text { nimium }\end{array}$

\section{Words Occur 11 Times}

$\begin{array}{ll}\text { aduorsum } & \text { capite } \\ \text { aetate } & \text { certe } \\ \text { ago } & \text { cupio } \\ \text { alium } & \text { dem } \\ \text { ament } & \text { dixit } \\ \text { amor } & \text { esset } \\ \text { Ballio } & \text { fateor } \\ \text { bonum } & \text { fit }\end{array}$

amico

ausculta

bono

censeo

coquom

coquos

da

datur

$\begin{array}{ll}\text { abeo } & \text { dedisse } \\ \text { ac } & \text { eas } \\ \text { aduenit } & \text { ere } \\ \text { ait } & \text { exemplum } \\ \text { aliam } & \text { extemplo } \\ \text { continuo } & \text { faciant } \\ \text { crucem } & \text { feci } \\ \text { dari } & \text { fiet } \\ \text { dat } & \text { fuge }\end{array}$

\begin{tabular}{|c|c|}
\hline $\begin{array}{l}\text { accipe } \\
\text { admodum } \\
\text { adulescentem } \\
\text { aedium } \\
\text { agas } \\
\text { agat } \\
\text { ain } \\
\text { alia } \\
\text { aliquem } \\
\text { amicis } \\
\text { attinet } \\
\text { audire }\end{array}$ & $\begin{array}{l}\text { aulam } \\
\text { cape } \\
\text { censes } \\
\text { cito } \\
\text { consilia } \\
\text { conuenit } \\
\text { credam } \\
\text { debet } \\
\text { ducere } \\
\text { ecquis } \\
\text { eheu } \\
\text { eorum }\end{array}$ \\
\hline
\end{tabular}

$\begin{array}{ll}\text { Harpax } & \text { minae } \\ \text { hicquidem } & \text { modis } \\ \text { illis } & \text { multum } \\ \text { ingenio } & \text { mutuom } \\ \text { istas } & \text { neu } \\ \text { loquor } & \text { ostium } \\ \text { mala } & \text { postea }\end{array}$

\section{Words Occur 10 Times}

$\begin{array}{ll}\text { foro } & \text { malo } \\ \text { forum } & \text { mane } \\ \text { his } & \text { manus } \\ \text { idem } & \text { meis } \\ \text { mali } & \text { pectore }\end{array}$

33 Words Occur 13 Times

$\begin{array}{llll}\text { eugae } & \text { illa } & \text { minis } & \text { oculis } \\ \text { facinus } & \text { istis } & \text { minus } & \text { rebus } \\ \text { facta } & \text { istoc } & \text { multa } & \text { salutem } \\ \text { fiat } & \text { loquere } & \text { nobis } & \text { Stasime } \\ \text { fide } & \text { manum } & \text { nusquam } & \text { uides } \\ \text { filius } & & & \end{array}$

$\begin{array}{llll}\text { habent } & \text { meas } & \text { probus } & \text { sororem } \\ \text { habere } & \text { miles } & \text { Pseudolus } & \text { sumus } \\ \text { habitat } & \text { misero } & \text { quidum } & \text { tene } \\ \text { hisce } & \text { miserum } & \text { quoniam } & \text { tuae } \\ \text { istam } & \text { nostrum } & \text { saluos } & \text { uiam } \\ \text { jube } & \text { omnem } & \text { sane } & \text { uidetur } \\ \text { lenoni } & \text { palam } & \text { soror } & \text { ultro }\end{array}$

54 Words Occur 9 Times

$\begin{array}{ll}\text { gratia } & \text { morem } \\ \text { habeas } & \text { moribus } \\ \text { istac } & \text { moror } \\ \text { istos } & \text { natus } \\ \text { melius } & \text { nihili } \\ \text { mentem } & \text { nimio } \\ \text { merito } & \text { nugas } \\ \text { mille } & \text { obuiam } \\ \text { modum } & \text { optumum }\end{array}$

69 Words Occur 8 Times

$\begin{array}{ll}\text { eos } & \text { magna } \\ \text { facile } & \text { magnum } \\ \text { feceris } & \text { malus } \\ \text { fecisti } & \text { mei } \\ \text { gaudeo } & \text { mirum } \\ \text { gnatus } & \text { negotium } \\ \text { hos } & \text { nequam } \\ \text { huius } & \text { occidi } \\ \text { ibidem } & \text { omni } \\ \text { iste } & \text { oppido } \\ \text { it } & \text { os } \\ \text { Lesbonice } & \end{array}$

$\begin{array}{ll}\text { plus } & \text { Simo } \\ \text { quantum } & \text { tui } \\ \text { sat } & \text { tuis } \\ \text { sciam } & \text { uelim } \\ \text { scire } & \text { uolt }\end{array}$

$\begin{array}{ll}\text { officium } & \text { sequere } \\ \text { pudet } & \text { sumbolum } \\ \text { quamquam } & \text { tantum } \\ \text { quemquam } & \text { ted } \\ \text { saluom } & \text { uelle } \\ \text { senem } & \text { uobis }\end{array}$

$\begin{array}{ll}\text { praedicas } & \text { seruom } \\ \text { puere } & \text { solet } \\ \text { quadraginta } & \text { tandem } \\ \text { reddam } & \text { ueniat } \\ \text { rogitas } & \text { uera } \\ \text { rogo } & \text { uerbum } \\ \text { scin } & \text { uidi }\end{array}$

$\begin{array}{ll}\text { perdant } & \text { Scapha } \\ \text { Phoenicium } & \text { sequor } \\ \text { posse } & \text { sicut } \\ \text { propera } & \text { sub } \\ \text { Pseudolum } & \text { terra } \\ \text { quoiquam } & \text { tun } \\ \text { redde } & \text { uirtute } \\ \text { redi } & \text { uolui } \\ \text { roga } & \text { uolunt }\end{array}$

$\begin{array}{ll}\text { pectus } & \text { rus } \\ \text { pedibus } & \text { scias } \\ \text { perdidit } & \text { sermonem } \\ \text { Philto } & \text { spondeo } \\ \text { postulas } & \text { stulte } \\ \text { potin } & \text { thensaurum } \\ \text { pridem } & \text { uah } \\ \text { propere } & \text { uideor } \\ \text { quaero } & \text { uitam } \\ \text { rationem } & \text { uostra } \\ \text { ruri } & \text { utrum }\end{array}$


87 Words Occur 7 Times

$\begin{array}{ll}\text { accipere } & \text { date } \\ \text { actumst } & \text { deaeque } \\ \text { aequomst } & \text { deos } \\ \text { amica } & \text { dici } \\ \text { amicus } & \text { dicito } \\ \text { aquam } & \text { dictis } \\ \text { arbitratu } & \text { e } \\ \text { audacter } & \text { ecastor } \\ \text { Callicles } & \text { eloquere } \\ \text { certo } & \text { epistula } \\ \text { certumst } & \text { erga } \\ \text { contra } & \text { eri } \\ \text { coqui } & \text { eris } \\ \text { credere } & \text { faciat } \\ \text { dabit } & \text { facito }\end{array}$

$\begin{array}{ll}\text { factis } & \text { mater } \\ \text { fecisse } & \text { maxumam } \\ \text { ferre } & \text { misere } \\ \text { filiae } & \text { misit } \\ \text { foris } & \text { more } \\ \text { frater } & \text { nequiquam } \\ \text { genere } & \text { nulla } \\ \text { illius } & \text { nummo } \\ \text { intellego } & \text { nuptias } \\ \text { Iouis } & \text { nuptiis } \\ \text { ius } & \text { omne } \\ \text { lege } & \text { optume } \\ \text { Lesbonicum } & \text { partem } \\ \text { locum } & \text { patre } \\ \text { manibus } & \end{array}$

patris
peto
plane
potes
propter
quanti
queo
quinque
quisque
repperi
responde
sciunt
seni
seruo

manibus

126 Words Occur 6 Times

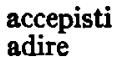

adire

aduenis

adulescenti

aeque

agere

agit

agrum

alio

alios

alius

alteram

amabo

amare

amat

amicos

amoris

amplius

aperite

audi

audin

ausculto
Callidamates
censeas
cuncta
cura
dabis
dabitur
dederis
des
do
domo
dos
ducam
duco
duo
egestatem
emi
etiamne
faceres
facies
facite

$\begin{array}{ll}\text { Fides } & \text { manufesto } \\ \text { foret } & \text { Megadore } \\ \text { foribus } & \text { meos } \\ \text { fuerit } & \text { mortuom } \\ \text { gnatam } & \text { mulier } \\ \text { gnato } & \text { multis } \\ \text { hem } & \text { namque } \\ \text { hominis } & \text { negat } \\ \text { homost } & \text { neminem } \\ \text { horum } & \text { nequeo } \\ \text { ingenium } & \text { nescis } \\ \text { interim } & \text { nostras } \\ \text { Ioni } & \text { nouom } \\ \text { ipsi } & \text { oculos } \\ \text { ipsum } & \text { olim } \\ \text { itidem } & \text { pax } \\ \text { iubeo } & \text { petit } \\ \text { loquitur } & \text { placide } \\ \text { lubens } & \text { possis } \\ \text { ludos } & \text { postremo } \\ \text { maiorum } & \text { praedico }\end{array}$

praeter
prope
proxumo
Pseudolo
quapropter
quippiam
quisquis
quoiusmodi
ratio
rogas
rusum
salua
sapis
scelestus
scies
scit
secum
sedulo
sentio
sin
soleo

sortem

speculo

st!

subito

sui

summo

sumptu

taces

teneo

tibicinam

tribus

uendidit

uenio

uiden

uidere

uiri

uita

uocant

uoles

usus

utinam

161 Words Occur 5 Times

$\begin{array}{ll}\text { abis } & \text { canes } \\ \text { accepisse } & \text { cena } \\ \text { adfinitatem } & \text { cesso } \\ \text { aduenio } & \text { coctum } \\ \text { aduenisse } & \text { condicionem } \\ \text { culpam } \\ \text { aduorte } & \text { dedisti } \\ \text { aes } & \text { dicant } \\ \text { aetati } & \text { dignum } \\ \text { ager } & \text { dimidium } \\ \text { agitur } & \text { disperii } \\ \text { aibat } & \text { dixeris } \\ \text { aiio } & \text { docte } \\ \text { aliis } & \text { doctum } \\ \text { aliquis } & \text { ecce } \\ \text { amantem } & \text { eras } \\ \text { amanti } & \text { estne } \\ \text { amet } & \text { exi } \\ \text { amicitiam } & \text { fabulam } \\ \text { amorem } & \text { faxint } \\ \text { anum } & \text { fero } \\ \text { apscede } & \text { fides } \\ \text { apsente } & \text { flocci } \\ \text { Athenis } & \text { fuat } \\ \text { auferam } & \text { fur } \\ \text { boues } & \text { furem } \\ \text { Calliclem } & \text { futurum } \\ \text { Callipho } & \\ & \end{array}$

$\begin{array}{ll}\text { gentium } & \text { mentionem } \\ \text { geram } & \text { metuam } \\ \text { geras } & \text { metum } \\ \text { gnate } & \text { militi } \\ \text { habe } & \text { minime } \\ \text { habuit } & \text { multos } \\ \text { hi } & \text { neuis } \\ \text { huiius } & \text { nihilo } \\ \text { iamne } & \text { noctu } \\ \text { incedit } & \text { noster } \\ \text { inimicos } & \text { nostra } \\ \text { inprobis } & \text { nostrae } \\ \text { inspicere } & \text { nullam } \\ \text { iratus } & \text { nuptum } \\ \text { itast } & \text { occasio } \\ \text { lenonem } & \text { opere } \\ \text { lepida } & \text { oportuit } \\ \text { loco } & \text { optuma } \\ \text { loquar } & \text { optumo } \\ \text { Lysiteles } & \text { ordine } \\ \text { Macedonio } & \text { paene } \\ \text { magister } & \text { pariter } \\ \text { magnam } & \text { patri } \\ \text { magnis } & \text { patriam } \\ \text { malis } & \text { pessume } \\ \text { maxumo } & \text { petam } \\ \text { Megaronides } & \text { petere } \\ & \end{array}$

$\begin{array}{ll}\text { petunt } & \text { sinam } \\ \text { Philippum } & \text { sino } \\ \text { Philolachem } & \text { siquidem } \\ \text { pistrinum } & \text { soli } \\ \text { possim } & \text { strenue } \\ \text { possunt } & \text { stultus } \\ \text { postulo } & \text { suos } \\ \text { potissumum } & \text { tamquam } \\ \text { praedam } & \text { tenes } \\ \text { pretio } & \text { Theopropides } \\ \text { principio } & \text { totum } \\ \text { probo } & \text { triginta } \\ \text { proinde } & \text { uae } \\ \text { properas } & \text { uideas } \\ \text { quaerit } & \text { uidisse } \\ \text { quidque } & \text { uin } \\ \text { quique } & \text { uirginem } \\ \text { quorum } & \text { uitae } \\ \text { redeo } & \text { ullam } \\ \text { rerum } & \text { unam } \\ \text { reuortar } & \text { uoluit } \\ \text { saepe } & \text { uortant } \\ \text { sciant } & \text { uortat } \\ \text { secus } & \text { uox } \\ \text { sententia } & \text { urbe } \\ \text { serui } & \text { uror } \\ \text { seruitutem } & \\ & \end{array}$

[34] 
299 Words Occur 4 Times

\begin{tabular}{|c|c|c|c|c|c|}
\hline \\
\hline $\begin{array}{l}\text { abeam } \\
\text { abeat }\end{array}$ & $\begin{array}{l}\text { clam } \\
\text { clanculum }\end{array}$ & $\begin{array}{l}\text { facto } \\
\text { factumst }\end{array}$ & $\begin{array}{l}\text { magno } \\
\text { maiorem }\end{array}$ & $\begin{array}{l}\text { parui } \\
\text { pati }\end{array}$ & $\begin{array}{l}\text { scelesta } \\
\text { sceleste }\end{array}$ \\
\hline abin & cogito & factus & malae & patiar & $\begin{array}{l}\text { sceleste } \\
\text { scilicet }\end{array}$ \\
\hline addecet & commodum & famam & maxumum & pauci & scimus \\
\hline aderit & conduxit & feram & Megadorus & pauper & sermone \\
\hline adhuc & consili & ferat & meliust & pedes & sies \\
\hline adueni & consilio & ferunt & memini & penes & signum \\
\hline adueniens & corruptum & filia & menses & perdidi & simile \\
\hline aduenientem & credidi & fliai & meque & perge & similis \\
\hline adulescente & credis & frustra & meridie & periisse & simus \\
\hline aetas & culpa & furcifer & metuont & periit & solent \\
\hline agedum & cures & fures & meumst & perit & solum \\
\hline agite & currere & gloriam & minoris & Philematium & specta \\
\hline agitis & damno & gnatae & miseret & piget & speculum \\
\hline aiebat & danunt & gnati & miseris & placeat & Strobile \\
\hline alias & dapsilis & gnatum & mittam & Polymachaero- & sua \\
\hline alicunde & dato & gradum & mitte & plagides & suamque \\
\hline aliquantum & datum & habeam & mittere & populi & Surus \\
\hline aliquid & daturum & habui & mitti & populo & tabellas \\
\hline aliquo & deceat & hae & mones & possint & taceo \\
\hline alter & dedero & heia & moram & possit & talentum \\
\hline alteri & Delphium & heri & mortalem & potare & temperi \\
\hline alterum & demum & heu & mortalis & pote & tempestas \\
\hline amant & denuo & hosce & mortuost & potero & tempus \\
\hline amator & deum & illae & muliere & potui & tergum \\
\hline ambo & dicat & illaec & munus & potuit & totus \\
\hline ambula & dictum & indicium & nemini & prae & tris \\
\hline amo & diues & inimicum & nomine & praeda & tune \\
\hline animi & divitias & iniuriam & noscere & praesens & ualide \\
\hline aperit & dolos & insidias & nostro & praesenti & uellem \\
\hline apstulisse & domino & interii & nouisti & preti & uelles \\
\hline argumenta & dormitum & inuitus & nouo & procul & ueneris \\
\hline astant & duas & ipsa & noxiam & proloqui & ueniam \\
\hline astat & duce & istanc & nullo & promisi & uenisse \\
\hline attulit & ducit & istinc & oblitus & pueri & ueri \\
\hline audes & eadem & itaque & occepi & pultabo & uesperum \\
\hline audis & ecfodiam & iubes & occlude & quaeras & uicissim \\
\hline aula & egon & laborem & oculi & quamuis & uideam \\
\hline auro & emisse & leges & oh & quidnam & uidisti \\
\hline bis & erilem & legibus & onustam & quiduis & uinum \\
\hline bonae & erili & lenone & oppidum & quindecim & uir \\
\hline bonam & esses & Lesbonico & oras & quippe & uirgini \\
\hline bonis & Euclionis & liber & orat & quispiam & uiros \\
\hline caelum & euenit & liberauit & ornamentis & quoîus & uiuis \\
\hline cantharum & experiar & liceat & ornata & quoius & unguentis \\
\hline capere & exsequi & licebit & ornatus & redeam & uolturios \\
\hline caueas & extra & litteris & ostende & refert & uoluptas \\
\hline Charmidem & facilius & longe & pactum & reliqui & uostram \\
\hline circumducere & facinora & ludo. & par & saltem & uostrum \\
\hline ciues & factiones & magni & pars & sapio & uterque \\
\hline & & 492 Word & cur 3 Times & & \\
\hline abduxit & aio & argentario & carnufex & conloqui & danista \\
\hline abest & alibi & argenteam & centum & conspicio & darem \\
\hline abii & aliquam & arraboni & certa & consulere & data \\
\hline abite & aliter & attat & cetera & contempla & debentur \\
\hline accedam & allatum & attuli & ceteris & copiast & decem \\
\hline Accheruntem & altera & audiui & ceterum & coquere & deinde \\
\hline accipiam & amabit & aufer & cibum & cordi & deliciae \\
\hline adesse & amabunt & auribus & circumspice & cornicem & demissis \\
\hline adfinis & amatorem & ausim & cluet & credat & dentes \\
\hline aduecti & amicae & auxilio & coepit & crederet & detur \\
\hline aduenienti & amice & Ballionem & colas & credideris & dices \\
\hline aduentu & amittat & beluam & comissatum & crepuit & dicit \\
\hline aduorsa & angiportum & beneficium & comoediis & crux & dicto \\
\hline aduortite & antehac & beneuolentem & compellabo & cupit & dignu's \\
\hline aedificare & antidhac & bibam & concedam & curare & dique \\
\hline agito & antiquom & bibere & concessero & curem & dis \\
\hline agnum & anus & Calidore & condimentis & curo & diuinis \\
\hline agro & apstrudam & Callicli & condiunt & curram & diuites \\
\hline ah & apstulerit & canem & conductus & cursuram & dixeram \\
\hline aha & apstuli & capio & congrediar & custodes & dixisti \\
\hline aiebant & aram & capitis & conloquar & damni & dixti \\
\hline
\end{tabular}




\begin{tabular}{|c|c|c|c|c|c|}
\hline & & & & & \\
\hline doleo & gratias & lucri & optumumst & propemodum & speciem \\
\hline dolis & gregem & macellum & optumus & propero & spero \\
\hline dolo & gynaeceum & machaera & orationem & prosus & sponden \\
\hline dolum & habeat & magisque & orator & proxuma & stas \\
\hline dominum & haben & magnufice & oro & proxumum & stulta \\
\hline donicum & habeto. & malas & otiose & publico & sumam \\
\hline dormit & habitatio & malos & otium & puer & sumes \\
\hline dotis & habitet & maneam & paenissume & puero & summis \\
\hline drachumam & haeret & matrem & paenitet & pugnam & sumptus \\
\hline dubitas & herbas & matrimonium & pallium & pulchre & super \\
\hline duint & homine & maxuma & parata & quaeris & surge \\
\hline dulce & hominibus & mel & parentes & quaeritas & suspicer \\
\hline eat & honorem & mendaciis & parietes & quaerunt & tabellis \\
\hline ebrium & hospes & mendacium & parum & quaestioni & tanta \\
\hline ecfecta & hostis & mentiar & paucis & quattuor & temere \\
\hline ecferri & ianua & messis & paullo & queat & terram \\
\hline edim & ianuam & metu & pauperem & quicquid & tetigi \\
\hline edunt & ibit & mihique & peccaui & quicum & tibist \\
\hline egere & ignoscas & milite & pecudes & quidam & totae \\
\hline eget & illinc & militis & peculio & quoquam & tria \\
\hline ehem & illorum & miseram & pecunia & quoquo & trium \\
\hline elocutus & illunc & morae. & peius & ratem & tuas \\
\hline emoriar & impera & mortuis & pendere & recipere & tuorum \\
\hline empta & imperium & mortuos & perdas & recipe & tutelam \\
\hline eram & inanis & mos & perdidisti & recipit & uale \\
\hline erus & innoxium & $\operatorname{mox}$ & perdit & recta & uelint \\
\hline eu & inops & mulieres & perductet & reddere & uelit \\
\hline eueniat & insanum & mulierum & pergam & regem & uelut \\
\hline eueniunt & insidiis & nate & perpetuom & reliquit & uenalem \\
\hline exemplis & insipientior & nato & pessum. & reperire & uendidisse \\
\hline existumat & interdius & nauem & pessumis & repudium & uenerit \\
\hline exit & interea & necauit & petas & roges & ueni \\
\hline expeto & inuenire & neget & Philolachetem & sacruficem & uenimus \\
\hline expetunt & inuentum & Neptune & pietatem & saluere & ueniunt \\
\hline exturbauit & inuito & nequior & pigeat & sapere & uerberas \\
\hline fabri & ipsam & neuolt & piscis & sauia & uerbero \\
\hline fabula & istae & noctesque & placent & scelerum & uestis \\
\hline fabulae & istunc & nonne & plenam & scelus & uetera \\
\hline fabulare & ite & nosce & pone & sciat & ueteres \\
\hline fabularier & ito & nosmet & poplo & scibam & uetus \\
\hline facie & iubet & nostram & poscam & scibo & uicino \\
\hline facturus & iudicem & nostris & poscere & scite & uictu \\
\hline factust & Iuno & nouisse & posco & sei & uidentur \\
\hline fallaciis & iure & nouos & posset & Seleucia & uidet \\
\hline familia & iusserat & nullum & possies & sempiternum & uillam \\
\hline familiam & iussero & nullus & posthac & senecta & uirgo \\
\hline fassu's & iussi & numerum & postulat & sententiam & uivom \\
\hline faxis & lanios & nummos & postules & serio & uiuos \\
\hline fecero & laudant & occasionem & postulet & sero & uitium \\
\hline feres & lenonis & occepisti & poteris & seruat & ullum \\
\hline ferme & lepidis & occulto & potior & seruatum & unguenta \\
\hline flagitium & lepidum & occupatus & praeceptis & seruem & ungulis \\
\hline fori & Lesbonici & oculum & praesente & seruet & uno \\
\hline formido & lex & odio & praetorem & seruis & unus \\
\hline Fortuna & liberos & olet & primumdum & simitu & uoltis \\
\hline fugiam & liberum & opino & prior & sinas & uolumus \\
\hline fustibus & liceret & oportere & probum & sola & uostrorum \\
\hline futurumst & ligna & opperiar & profectus & soles & uter \\
\hline gerere & liquet & opseruare & promisit & sollicitat & utor \\
\hline gestio & lubeant & opsignatas & promitte & somnis & utut \\
\hline graphicum & lubenter & opsonium & promittere & somno & uxores \\
\hline & & $1198 \mathrm{Wo}$ & ccur Twice & & \\
\hline abducere & accepti & actum & adgrediar & adulescentia & agitat \\
\hline abduceres & acceptum & adde & adiit & aduorsus & agninis \\
\hline abduceret & accersam & addideris & adlegauit & aduortas & agundae \\
\hline abeamus & accerse & adducam & admísit & aegre & agunt \\
\hline abeas & accessit & adduxi & admoenire & aegrotant & aiunt \\
\hline abibam & accipias & adeam & adsiet & aequius & aliae \\
\hline abierit & accipiat & adeas & adsiste & aequo & alieno \\
\hline abire & accubas & adferre & adsum & agedum & alienum \\
\hline accepi & accubat & adferret & adueniat & agerem & aliorum \\
\hline accepit & acerrume & adfinem & aduenire & agimus & aliquanto \\
\hline
\end{tabular}




\section{Words Occur Twice - Continued}

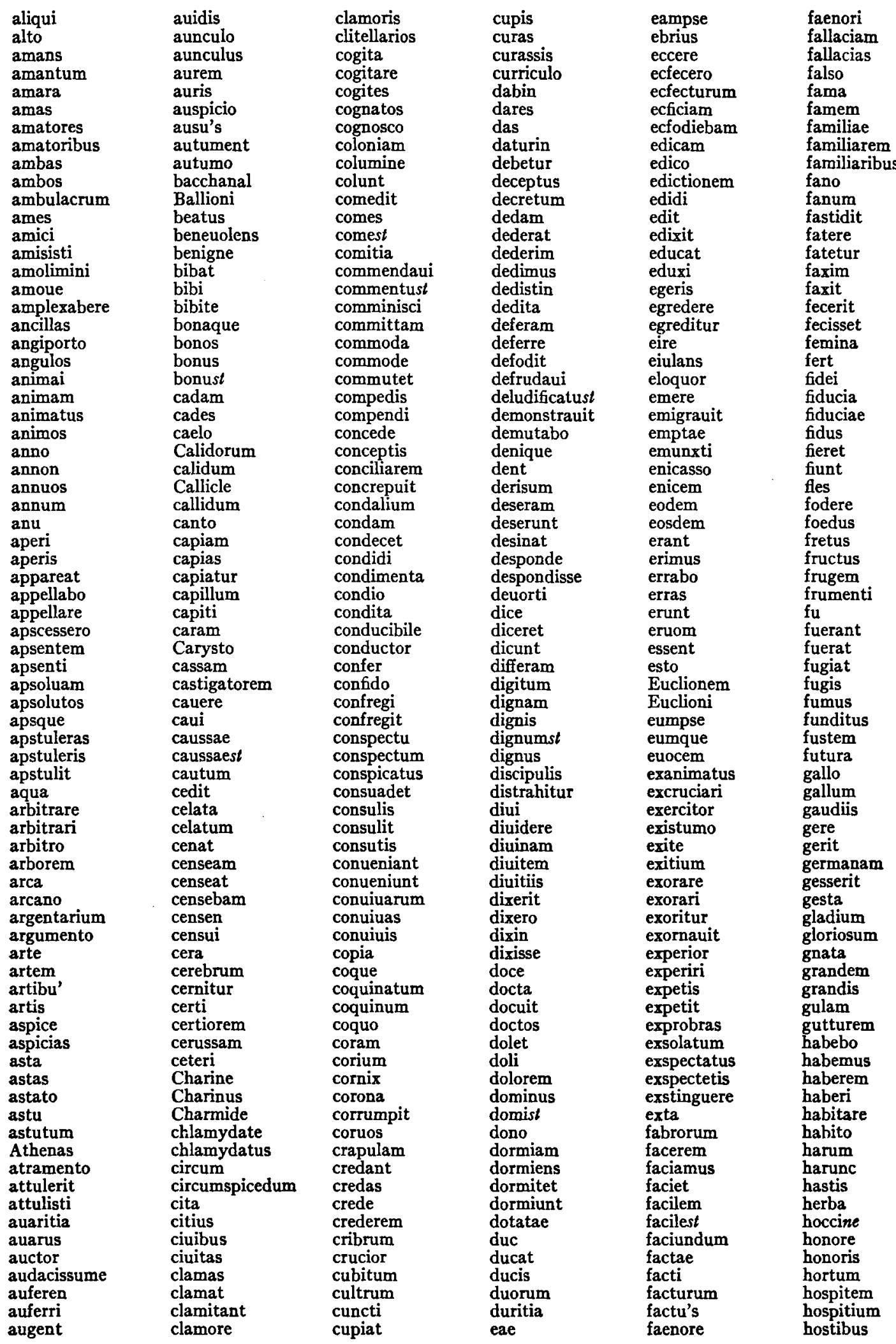

[37 ] 


\begin{tabular}{|c|c|c|c|c|c|}
\hline iamdudum & iura & memorem & nominat & paullum & potis \\
\hline ibis & iusseram & mendacia & noua & pauperi & praecipio \\
\hline ignem & iuuentute & mensa & nouistin & pauperibus & praedices \\
\hline ignis & iuues & meopte & nouit & pauperiem & praedones \\
\hline ignobilis & iuxta & mercatus & nudius & peccatis & praemium \\
\hline illac & labore & mercedem & nugari & pecauisse & praequam \\
\hline illas & labra & mercedis & nugator & peculi & praesensit \\
\hline illast & labris & mercimoni & nullust & pecuniam & praestituta \\
\hline illequidem & lacrumas & merenti & nummus & pedem & praeterea \\
\hline illisce & lacrumis & meres & numquis & peiiurum & praeterire \\
\hline illos & laetus & meriti & nutricem & peiorem & prandium \\
\hline imagine & lapidem & merui & obiurigem & pellegam & primo \\
\hline imaginem & latum & meruisti & obnoxius & pellegere & principium \\
\hline imperas & legionem & metuas & occasiost & peni & priorem \\
\hline imperatumst & legiones & metuere & occidam & penus & probant \\
\hline imperauit & lepidam & metuit & occidis & pepulit & probare \\
\hline imperia & Lesbonicus & mihimet & occidisti & percontabor & probiorem \\
\hline impero & libera & mihist & occipias & percontare & probis \\
\hline impetratum & liberare & militarem & occludam & perconteris & probos \\
\hline impetraui & libere & mina & occupabo & perdere & probrum \\
\hline impleuisti & liberi & minimi & occupes & perdiderit & prohibet \\
\hline improbi & liberis & minimo & ocius & perdis & proin \\
\hline impulsu & linguam & minore & octoginta & perditum & promam \\
\hline incedere & liquido & mira & odi & perditus & promus \\
\hline incenatus & litterae & miror & odos & perduci & propius \\
\hline incipit & litterarum & misereat & offendet & perductorem & proprium \\
\hline indeque & loca & miseria & olant & perduint & Pseudolust \\
\hline indicem & locare & misericordia & olere & pergin & publice \\
\hline indignum & locaui & missa & oleum & pergulam & publicum \\
\hline indiligens & loces & mitto & omitte & periclis & pudeat \\
\hline indoctus & loci & mittunt & omnino & periclum & pudere \\
\hline industria & locis & modi & onera & periere & pudico \\
\hline inest & locus & molestos & onerabo & perierint & pudicum \\
\hline ineunte & longior & molestum & onustos & perierit & pugna \\
\hline infamiam & longius & molestumst & operae & periimus & pulchra \\
\hline infectum & longum & moneo & opes .. & periisti & pulchrum \\
\hline infelix & lubeat & monere & opperiri & perlubens & pultando \\
\hline infitias & lubido & monitum & opportune & permities & pultare \\
\hline ingeni & lubidost & montis & opsignatum & permitto & pultas \\
\hline ingere & luco & mora & optes & perpetuo & purpura \\
\hline inhiat & lucro & morata & optestor & persequi & purpuram \\
\hline inicere & ludis & moriuntur & optigere & peruorse & purus \\
\hline inimici & Lysiteli & mortui & optigit & pessumi & puteo \\
\hline inimicis & maiore & mulieri & optuere & pessumo & putes \\
\hline inopia & maledictis & mulieris & optulit & petat & puteum \\
\hline Inopiam & malest & muliones & optumi & petimus & putus \\
\hline inprobi & malitia & mulos & opulento & petito & quadrilibrem \\
\hline inprobior & mamma-madere & multas & opulentus & Philolachi & quaerere \\
\hline inprobum & mammia & munditiis & orare & Philtonis & quaeritat \\
\hline inprudens & mancupio & mundo & oratio & pignori & quaesti \\
\hline inpune & mandatum & murenam & oratum & pinguior & quaestione \\
\hline inpure & mandauit & narrauero & Orcus & Piraeum & quaestum \\
\hline inquito & $\operatorname{mani}$ & natali & ores & placere & qualis \\
\hline inridere & manta & natalis & ornamenta & places & quandoquidem \\
\hline inrides & manufestarium & natum & ornatu & plagis & quanta \\
\hline insciens & mastigia & nauci & otio & planissume & quanto \\
\hline institui & mauellem & necesse & palmam & plateam & quantumst \\
\hline intemperiae & maxumast & necne & para & Plautus & querere \\
\hline interest & mearum & negas & parant & pluris & quicquamst \\
\hline interuisam & meast & neges & paratus & plurumum & quidlubet \\
\hline intra. & mecastor & nego & parce & poeta & quiesce \\
\hline inueniam & media & nemost & parcum & pollicitust & quietus \\
\hline inueniat & medio & nequeam & parcus & popli & quippini \\
\hline inuenio & Megadoro & nequeas & parsimoniam & porta & quit \\
\hline io & melior & nequis & participem & portam & quoia \\
\hline Iouem & meliora & nequiter & participes & porticum & quoiuis \\
\hline ipsusne & meliores & nescias & partitudo & possem & quonam \\
\hline irem & memineris & nescioquem & parumper & postid & quondam \\
\hline ista & meminisse & nescit & parumst & postilla & quot \\
\hline istius & memor & neue & patinae & postis & radicitus \\
\hline isto & memora & nilne & patinas & postremum & rape \\
\hline itane & memorant & nimisque & patrissat & potat & rapere \\
\hline itur & memorare & noli & patronus & potesse & ratione \\
\hline iubeam & memoras & noluit & paullisper & potestate & ratu' \\
\hline
\end{tabular}

[ $\left.3^{8}\right]$ 
1198 Words Occur Twice - Continued

\begin{tabular}{ll} 
rectam & scitum \\
rectius & scorta \\
red & scortis \\
reddet & scortum \\
reddite & scripsit \\
redditur & scriptam \\
redeat & scriptum \\
redii & semel \\
rediisse & semul \\
redit & senatu \\
refer & senatum \\
referre & sene \\
regiones & senes \\
regionibus & sentit \\
renuntiant & seque \\
reperio & sequimini \\
repperisse & sermo \\
repperisti & sermonibus \\
repudies & seruant \\
respexis & seruas \\
respice & seruassint \\
respicias & seruiam \\
restas & seruio \\
rex & seruire \\
rogitem & seruit \\
sacra & sescenta \\
sacrum & seu \\
saeuiter & sextus \\
saginam & sicine \\
saluam & siem \\
salus & Siluani \\
salutat & Simia \\
salutis & similem \\
sanus & Simonem \\
sapiens & sitis \\
sapit & sitne \\
sarcinam & sodalem \\
satine & solere \\
scelestiorem & solide \\
scibas & solio \\
sciet & solitum \\
scires & solitun \\
& \\
\hline &
\end{tabular}

soluta
somnias
somnum
sonitu
sorori
spectatores
spernit
stabulum
Staphyla
Stasimus
statum
stimulis
sto
stultitia
stultitiam
suae
subdole
subolet
sumbolust
sume
sumet
summa
summas
summos
sumne
sumpsit
sumptum
superfit
suppetat
Surorum
surrupias
surrupui
surrupuisti
suscenseo
suscensui
suscita
suspendere
suspicarier
suspicio
suspicionem
suspicor
sutor

\begin{tabular}{|c|c|c|}
\hline sycophanta & tuto & uisne \\
\hline sycophantam & tutum & uisum \\
\hline sycophantari & ualere & uiues \\
\hline sycophantast & uales & uiuet \\
\hline sycophantiae & ualet & uiuit \\
\hline sycophantiam & uapulabis & uiuitur \\
\hline sycophantias & ubinam & uiuont \\
\hline tacere & uectus & ulciscar \\
\hline tactiost & uehicla & ulcisci \\
\hline talentis & uehit & ullo \\
\hline tali & uellet & ullus \\
\hline tangere & uenalis & umbra \\
\hline tangerem & uendas & umbram \\
\hline tantis & uendidi & uni \\
\hline tantisper & uenis & uocare \\
\hline tanto & uentrem & uocas \\
\hline tantumdem & uentri & uocat \\
\hline teneam & Venu' & uoce \\
\hline tenebras & uenusta & uocem \\
\hline tenent & uerberibus & uociuas \\
\hline teneor & uerborum & uoco \\
\hline tenet & uereor & uocor \\
\hline tergi & uestem & uolat \\
\hline tergo & uias & uolentibus \\
\hline tertiam & uiatico & uoltu \\
\hline tertius & uicini & uoluisse \\
\hline test & uicinia & uoluntate \\
\hline tetigistin & uicinum & uorte \\
\hline Thensauro & uicinus & uortit \\
\hline thensauros & uicisti & uosmet \\
\hline tigna & uictor & uoster \\
\hline time & uictum & uostras \\
\hline tis & uictumas & uotat \\
\hline tolerare & uigila & uotet \\
\hline tot & uile & urbem \\
\hline tot & uincam & usui \\
\hline totidem & uincunt & utamur \\
\hline traho & uini & utendam \\
\hline triduom & uiris & utere \\
\hline Trinummo & uirtutem & utimur \\
\hline & virum & utram \\
\hline
\end{tabular}

\section{Words Occur Once}

$\begin{array}{ll}\text { abalienare } & \text { abituru's } \\ \text { abalienarier } & \text { abitus } \\ \text { abalienatur } & \text { abripite } \\ \text { abdiderunt } & \text { abrogant } \\ \text { abducant } & \text { abusus } \\ \text { abducas } & \text { accederes } \\ \text { abducat } & \text { accedito } \\ \text { abducet } & \text { acceperam } \\ \text { abducito } & \text { acceperim } \\ \text { abducitur } & \text { acceperis } \\ \text { abducturum } & \text { accepto } \\ \text { abducturus } & \text { acceptor } \\ \text { abduxisse } & \text { accersat } \\ \text { aberat } & \text { accersunt } \\ \text { aberis } & \text { accesseris } \\ \text { abes } & \text { accessi } \\ \text { abeundum } & \text { accessio } \\ \text { abeunt } & \text { accheruntis } \\ \text { abierunt } & \text { accidisse } \\ \text { abhinc } & \text { accidunt } \\ \text { abibo } & \text { acciperem } \\ \text { abiectas } & \text { accipi' } \\ \text { abiero } & \text { accipient } \\ \text { abiisse } & \text { accipies } \\ \text { abiisses } & \text { accipite } \\ \text { abiisti } & \text { accipito } \\ \text { abimus } & \text { accolae } \\ \text { abiret } & \text { accommodem } \\ \text { abit } & \text { accuba } \\ \end{array}$

accubans
accubantis
accubes
accubitum
accubo
accumbas
accumbe
accumbere
accurarier
accurassis
accusas
accusem
accusites
acerui
aceti
acidissumi
acie
acrem
acriorem
acriter
acrius
acta
actam
actast
acturu's
acturus
aculeus
acutis
adaeque

adaxint
adcurare
adderes
addidero
addidit
addito
additum
additur
adduc
adducerem
adducite
adduint
addunt
adduxit
adegero
ademit
aderat
adero
adesses
adessuriuit
adeste
adeundi
adeunt
adfatim
adfectat
adfer
adferat
adferet
adferetur

adfero
adferrier
adfert
adfertur
adferto
adfigi
adfines
adfini
adfinitate
adfinium
adflictas
adflicter
adfligi
adfuerunt
adglutinant
adgrediundust
adhibendust
adhibet
adhibete
adibit
adigit
adii
adiisse
adiisti
adimerem
adimit
adit
adiungit
adiunxeris

adiurasti

adiutorem

adiutrice

adiutum

adiuua

adiuuabere

adiuuare

adiuuas

adlegatu

adlicere

adloquar

adloqui

admemordit

adminiclum

admittam

admonitus

admordere

adorna

adpetit

adplicare

adplaudere

adportas

adposita

adprime

adprobare

adpropera

adsedero

adsentabor

adsentari 
5429 Words Occur Once - Continued

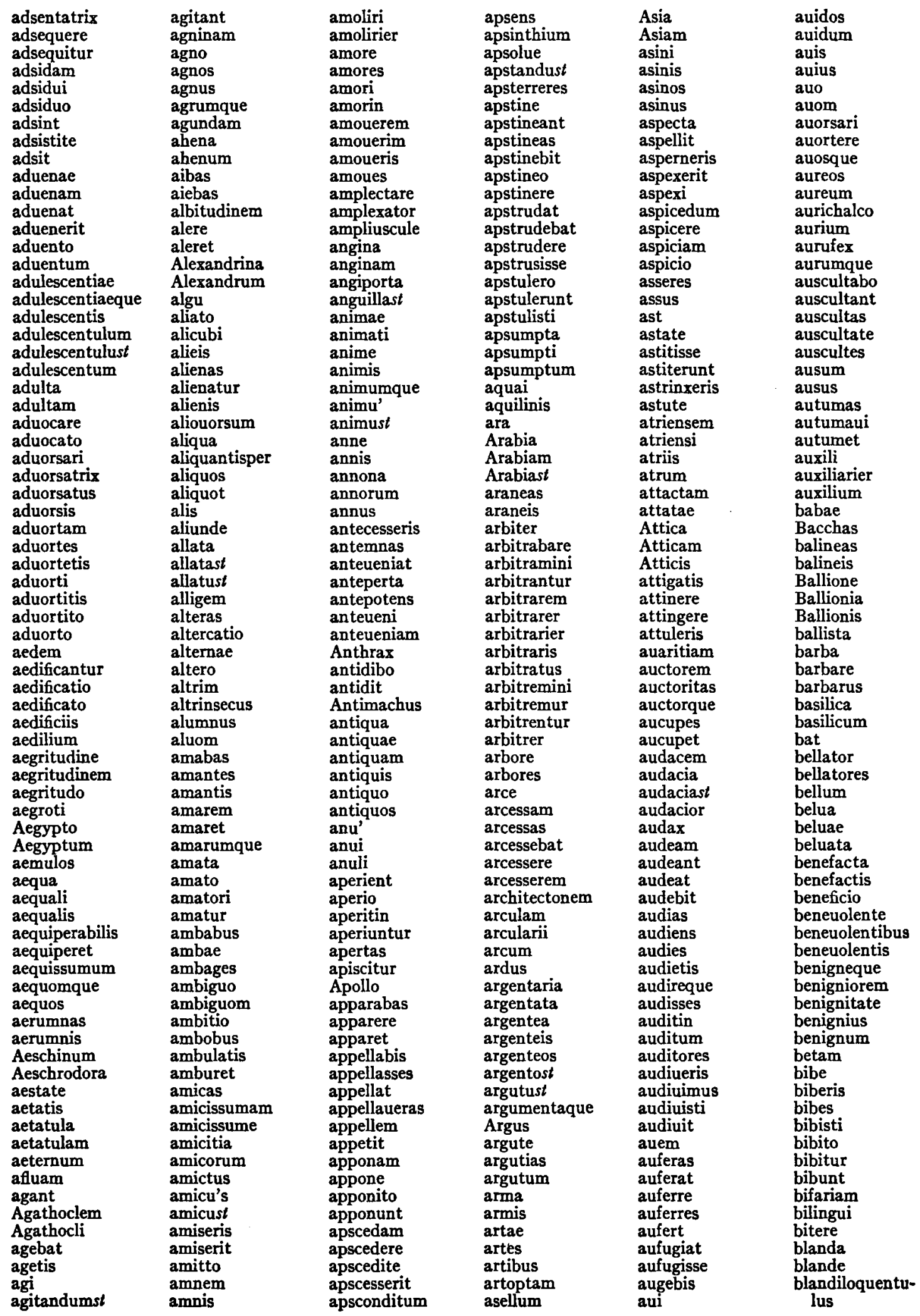

[40] 


blandis
blanditerne
blanditur
blandius
blandus
blandust
blitum
bombax
bonan
bonasque
bone
bonisne
bonist
bonumst
bos
bouem
bracchia
bracchium
brassicam
breuis
breuem
bubula
bubulam
bubulcitarier
bubuli
bubulis
bubus
bucaedae
bustirape
c'
caculam
cadas
cadere
cado
cadum
caeca
caecus
caedere
caedite
caedito
caedundum
caeno
caenum
caeruleos
calamitatis
calamo
calator
calceolarii
calet
Calidori
Calidoro
Calidorus
callent
callet
Callias
Callidamatem
Callidamati
Callidemides
callidiorem
Callimarchus
Callinicus
Calliphone
Callippus
callum
Campanica
Campans
campos
cancer
candefacere
candida
candido
candorem
cantantem
cantari

\begin{tabular}{|c|c|}
\hline $\begin{array}{l}\text { cantas } \\
\text { cantat } \\
\text { cantharus } \\
\text { cantheriis } \\
\text { cantores } \\
\text { cantrices } \\
\text { caperratam } \\
\text { capessere } \\
\text { capesses } \\
\text { capitale } \\
\text { capitali } \\
\text { Capitolio } \\
\text { capitur } \\
\text { capiundas } \\
\text { capram } \\
\text { capsit } \\
\text { capta } \\
\text { captandumst } \\
\text { captas } \\
\text { captat } \\
\text { capti } \\
\text { captio } \\
\text { captioni } \\
\text { captum } \\
\text { capturum } \\
\text { captus } \\
\text { cara } \\
\text { carbunculus } \\
\text { carcerem } \\
\text { carent } \\
\text { careo } \\
\text { carinarii } \\
\text { cariora } \\
\text { carissumum } \\
\text { carissumust } \\
\text { carnaria } \\
\text { carnarium } \\
\text { carnuficium } \\
\text { caros } \\
\text { carum } \\
\text { caruisse } \\
\text { cassidem } \\
\text { caste } \\
\text { castigabilem } \\
\text { castigabit } \\
\text { castigare } \\
\text { castigatum } \\
\text { castrandum } \\
\text { casum } \\
\text { catam } \\
\text { catapirateria } \\
\text { cataractria } \\
\text { cati } \\
\text { catum } \\
\text { catus } \\
\text { caudicali } \\
\text { caueam } \\
\text { caueant } \\
\text { cauebo } \\
\text { cauendum } \\
\text { cauendumst } \\
\text { caueo } \\
\text { cauerem } \\
\text { caueres } \\
\text { cauero } \\
\text { cauet } \\
\text { cauillam } \\
\text { caupones } \\
\text { causificor } \\
\text { caussam } \\
\text { caussast } \\
\text { caute } \\
\text { cautiost } \\
\text { cautiores } \\
\text { cal }\end{array}$ & $\begin{array}{l}\text { cauto } \\
\text { cautos } \\
\text { cecidit } \\
\text { cecidero } \\
\text { Cecropio } \\
\text { cedis } \\
\text { cedodum } \\
\text { cedunt } \\
\text { celebra } \\
\text { celem } \\
\text { celere } \\
\text { celerem } \\
\text { celeri } \\
\text { celerior } \\
\text { celeriter } \\
\text { celerius } \\
\text { celes } \\
\text { celo } \\
\text { celocem } \\
\text { cenaene } \\
\text { cenandum } \\
\text { cenas } \\
\text { cenati } \\
\text { cenaverat } \\
\text { cenavero } \\
\text { cenes } \\
\text { censebit } \\
\text { censebo } \\
\text { censet } \\
\text { censetur } \\
\text { censione } \\
\text { censu } \\
\text { census } \\
\text { centuriata } \\
\text { cepero } \\
\text { cepisse } \\
\text { cepit } \\
\text { cepolendrum } \\
\text { ceram } \\
\text { Cercobulus } \\
\text { Cerconicus } \\
\text { Cererin } \\
\text { Cereris } \\
\text { certast } \\
\text { certior } \\
\text { certis } \\
\text { certissumus } \\
\text { certius } \\
\text { certos } \\
\text { certust } \\
\text { cerussa } \\
\text { ceterae } \\
\text { ceteros } \\
\text { cetum } \\
\text { Chares } \\
\text { Charmidatus } \\
\text { Charmidei } \\
\text { Charmidi } \\
\text { chlamyde } \\
\text { chlamydem } \\
\text { chorago } \\
\text { Chrysidem } \\
\text { cibo } \\
\text { cibust } \\
\text { cicimandro } \\
\text { Ciliciam } \\
\text { cinaedus } \\
\text { circiter } \\
\text { circumcirca } \\
\text { circumdari } \\
\text { circumducam } \\
\text { circumducat } \\
\text { circumduce } \\
\text { circumspectabam }\end{array}$ \\
\hline
\end{tabular}

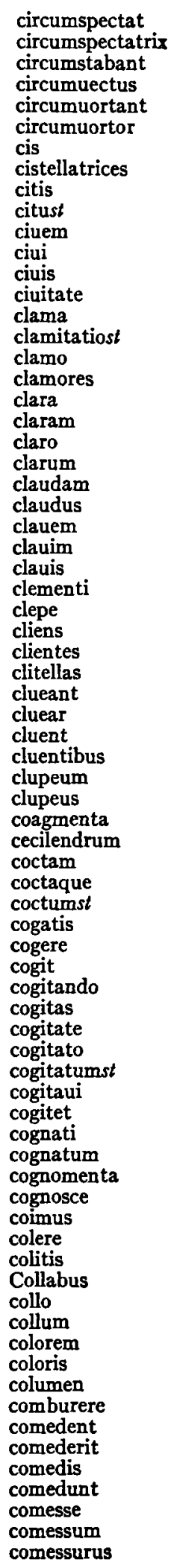

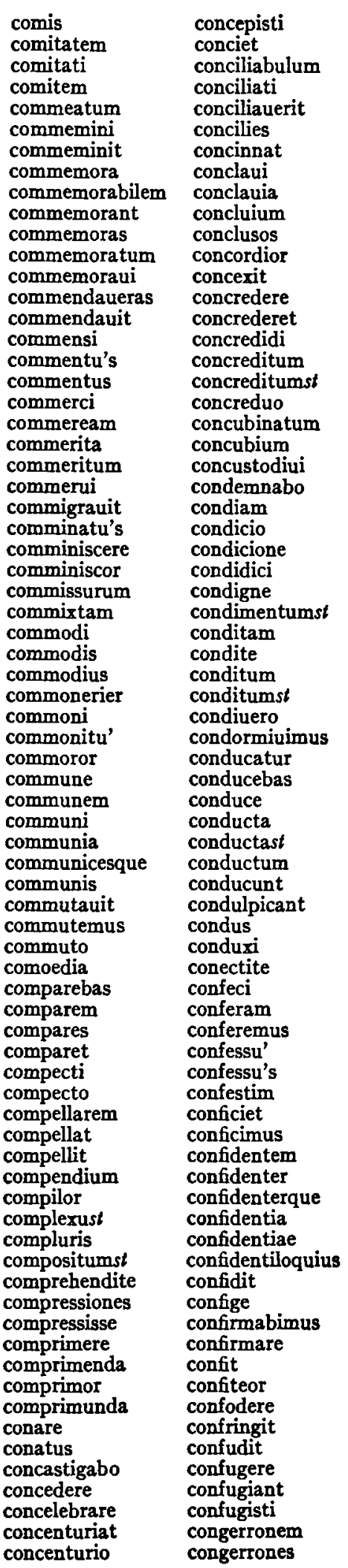

[4I ] 


\begin{tabular}{|c|c|c|c|c|c|}
\hline & & 6429 Words Occ & Once - Contin & & \\
\hline congerunt & contemplat & costae & cupidum & decorari & deridebo \\
\hline congestae & contemplo & cottabi & cupiebam & dedecorant & derideo \\
\hline congestum & conteram & cottidianumst & cupienter & decorum & derideri \\
\hline congialem & conterere & cottidie & cupies & decretumst & deridiculum \\
\hline congliscat & contiens & coxerint & cuppes & decumus & deripiamus \\
\hline congrediri & contigit & crassam & curabit & decumo & desedisti \\
\hline congrio & continens & crassas & curabitur & decus & deserat \\
\hline congrione & continere & crassis & curabo & dedas & deserere \\
\hline congrum & contione & crassitudine & curant & dederam & deseres \\
\hline $\begin{array}{l}\text { cohiberent } \\
\text { conicere }\end{array}$ & $\begin{array}{l}\text { controllam } \\
\text { controuorsia }\end{array}$ & $\begin{array}{l}\text { crastini } \\
\text { crastinum }\end{array}$ & $\begin{array}{l}\text { curantur } \\
\text { curata }\end{array}$ & $\begin{array}{l}\text { dederamque } \\
\text { deducam }\end{array}$ & $\begin{array}{l}\text { deseret } \\
\text { deseruerunt }\end{array}$ \\
\hline coniecit & contuderunt & creas & curatam & defaecato & deseruit \\
\hline coniectura & contui & crebri & curate & defaecatumst & deseruitque \\
\hline coniuent & contumeliam & crebro & curatiost & defendat & desideres \\
\hline coniunctus & conturbabunt & credatis & curatum & defetigo & desidia \\
\hline conlaudant & contutu & credibile & curaui & deficatam & desidiam \\
\hline conlegit & $\begin{array}{l}\text { condenerai } \\
\text { conuenero }\end{array}$ & $\begin{array}{l}\text { crealaerim } \\
\text { credidero }\end{array}$ & $\begin{array}{l}\text { curer } \\
\text { curiae }\end{array}$ & $\begin{array}{l}\text { derormata } \\
\text { defossa }\end{array}$ & desiste \\
\hline conlibitum & conueni & credier & curialium & defrudes & desistum \\
\hline conligatis & conueniam & credito & curio & defrustratur & despoliator \\
\hline conligi & conueniat & creditum & curiosam & defrutum & despondeat \\
\hline conlinunt & $\begin{array}{l}\text { conueniebatne } \\
\text { conuenimus }\end{array}$ & crediturus & curis & degam & desponderi \\
\hline conlocaui & $\begin{array}{l}\text { conuenumus } \\
\text { conuenire }\end{array}$ & $\begin{array}{l}\text { creauas } \\
\text { crepent }\end{array}$ & currendumst & $\begin{array}{l}\text { aego } \\
\text { dehibuisti }\end{array}$ & $\begin{array}{l}\text { aesponaes } \\
\text { despondi }\end{array}$ \\
\hline conlocem & conuenisse & creta & currenti & dehinc & despondisti \\
\hline conlocet & conuenistin & crimen & curriculum & deices & despondit \\
\hline conlocutu's & conueniundust & crimina & curro & deiuuare & desponsatam \\
\hline conlutulentet & conuentum & criminaret & cursim & deis & desquama \\
\hline $\begin{array}{l}\text { conqueritur } \\
\text { conqueror }\end{array}$ & $\begin{array}{l}\text { conuicium } \\
\text { conuiuae }\end{array}$ & $\begin{array}{l}\text { crinis } \\
\text { Crinnus }\end{array}$ & $\begin{array}{l}\text { cursori } \\
\text { cursu }\end{array}$ & $\begin{array}{l}\text { delatum } \\
\text { delectauerit }\end{array}$ & $\begin{array}{l}\text { destinant } \\
\text { destinat }\end{array}$ \\
\hline conquiescere & conuiui & croccibat & curuo & delicatu's & destinatam \\
\hline conquiescito & conuocaui & cruci & custodem & deliciis & destiteris \\
\hline conquiniscet & conuocem & cruciabiliter & custodelam & deliciisque & destitisti \\
\hline conquiniscito & conuoco & cruciatu & custos & deliquimus & desubito \\
\hline conrepserit & copi & cruciatum & cyathum & deliquit & deterior \\
\hline conscius & copiaest & cruciatur & dabam & Delphio & deteriorem \\
\hline $\begin{array}{l}\text { conscribito } \\
\text { conscriptam }\end{array}$ & $\begin{array}{l}\text { copiam } \\
\text { copias }\end{array}$ & & dabimus & Delphis & $\begin{array}{l}\text { deterrui } \\
\text { deterrums }\end{array}$ \\
\hline & $\begin{array}{l}\text { copias } \\
\text { copulantur }\end{array}$ & $\begin{array}{l}\text { crudum } \\
\text { cruminam }\end{array}$ & $\begin{array}{l}\text { dabisne } \\
\text { dabunt }\end{array}$ & $\begin{array}{l}\text { deluctaui } \\
\text { deludam }\end{array}$ & $\begin{array}{l}\text { deterruma } \\
\text { detexit }\end{array}$ \\
\hline consectarier & coquas & crura & dabuntur & deludis & deteritque \\
\hline consenserunt & coquant & cruricrepidae & damnare & demas & detexundam \\
\hline consequere & coquat & cubandumst & damnoseis & demeret & detraxe \\
\hline conseruauit & coquinare & cubat & damnum & demeritas & detrudi \\
\hline conseruorum & coquite & cubem & danistam & demerui & detrusti \\
\hline consident & coquo' & cubiculum & danisticum & deminuam & detuli \\
\hline $\begin{array}{l}\text { considero } \\
\text { consignabo }\end{array}$ & $\begin{array}{l}\text { coquont } \\
\text { corbulis }\end{array}$ & $\begin{array}{l}\text { cubitare } \\
\text { cucule }\end{array}$ & $\begin{array}{l}\text { dant } \\
\text { daret }\end{array}$ & $\begin{array}{l}\text { demonstratae } \\
\text { demonstretis }\end{array}$ & $\begin{array}{l}\text { deturbault } \\
\text { deu' }\end{array}$ \\
\hline consignemus & corcotarii & $\begin{array}{l}\text { cucure } \\
\text { cuculus }\end{array}$ & daretur & $\begin{array}{l}\text { demonstretis } \\
\text { dempserat }\end{array}$ & $\begin{array}{l}\text { deu } \\
\text { deueneris }\end{array}$ \\
\hline consiliis & corcotis & cudere & Dareum & demumst & deuenires \\
\hline consilist & corculum & culcitulam & datam & demutant & deuincis \\
\hline consistam & cordique & culina & datare & demutauit & deuincit \\
\hline consistunt & coriandrum & culleis & dataria & denegem & deuinxere \\
\hline consociauit & Corintbiensem & culleo & datas & denis & deuorari \\
\hline $\begin{array}{l}\text { consolatur } \\
\text { conspexeris }\end{array}$ & $\begin{array}{l}\text { corio } \\
\text { cornibus }\end{array}$ & $\begin{array}{l}\text { culpari } \\
\text { culpent }\end{array}$ & datat & & deuoravi \\
\hline $\begin{array}{l}\text { conspexeris } \\
\text { conspexi }\end{array}$ & $\begin{array}{l}\text { cornibus } \\
\text { comu }\end{array}$ & $\begin{array}{l}\text { culpent } \\
\text { culpes }\end{array}$ & $\begin{array}{l}\text { datis } \\
\text { datu }\end{array}$ & $\begin{array}{l}\text { dentatum } \\
\text { denumerato }\end{array}$ & $\begin{array}{l}\text { deuorec } \\
\text { deuorsum }\end{array}$ \\
\hline conspicari & cornua & cummam & daturu's & denumerauero & deuorteris \\
\hline conspicere & corolla & cumque & datus & deorsum & deuortor \\
\hline conspicor & corollas & cumulatissume & dea & deorum & dexteram \\
\hline constituatur & coronam & cunctae & debebas & deosque & dexteras \\
\hline constitueram & coronas & cunctam & debent & depellar & diabathrarii \\
\hline constrictis & corpora & cunctis & debeo & dependi & Diapontius \\
\hline constringam & corporis & cunctos & debere & deperit & dicebat \\
\hline consudaueris & corporum & cunila & deberet & deplorabundus & dicendumst \\
\hline consuetudine & corpus & cunis & debes & deprome & dicet \\
\hline consuetudines & corrigere & cupi' & deblaterauisti & deponat & dicetis \\
\hline consuetum & corrigis & cupiam & debuit & deportatum & dicimus \\
\hline consulas & corrumpat & cupias & decedam & deportere & dicisne \\
\hline consului & corrumpe & cupidae & decent & deposiuit & dicitur \\
\hline $\begin{array}{l}\text { contabefacit } \\
\text { contechnatus }\end{array}$ & $\begin{array}{l}\text { corrumpi } \\
\text { corrumpiter }\end{array}$ & $\begin{array}{l}\text { cupide } \\
\text { Cupidinem }\end{array}$ & $\begin{array}{l}\text { deceret } \\
\text { decidunt }\end{array}$ & $\begin{array}{l}\text { depromi } \\
\text { depromptum }\end{array}$ & $\begin{array}{l}\text { dictator } \\
\text { dictatorem }\end{array}$ \\
\hline contemnam & corrupisse & cupido & deciens & depugnat & dictitatur \\
\hline $\begin{array}{l}\text { contemnit } \\
\text { contemnor }\end{array}$ & $\begin{array}{l}\text { corruptor } \\
\text { coruom }\end{array}$ & $\begin{array}{l}\text { Cupido } \\
\text { cupidos }\end{array}$ & $\begin{array}{l}\text { declaramus } \\
\text { declinaui }\end{array}$ & $\begin{array}{l}\text { deputas } \\
\text { derepente }\end{array}$ & $\begin{array}{l}\text { dictu } \\
\text { dictumst }\end{array}$ \\
\hline & & & & & \\
\hline
\end{tabular}

[42] 


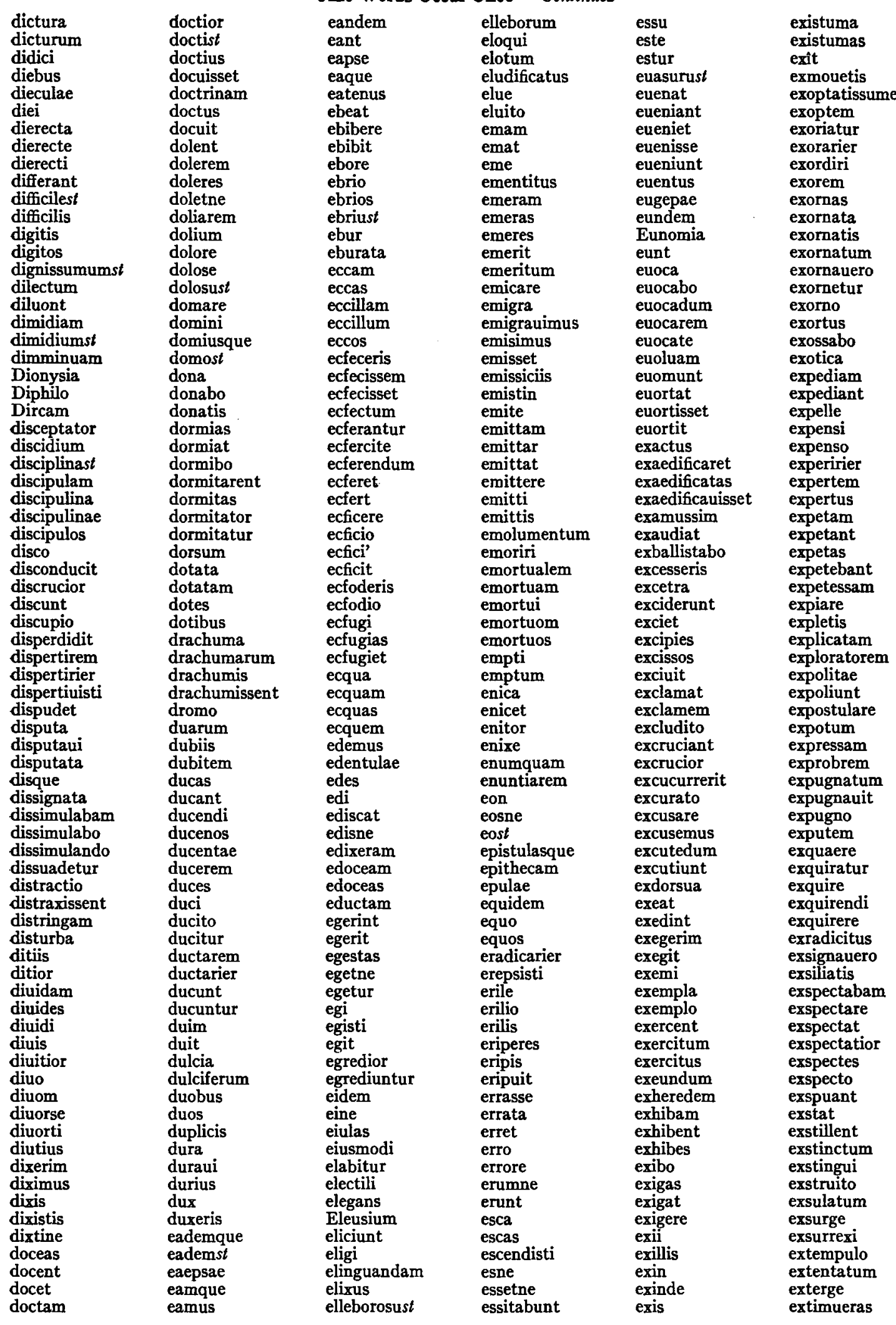

[43] 


\begin{tabular}{|c|c|c|c|c|c|}
\hline extis & feras & fluont & fuitne & glandium & hereditas \\
\hline extolli & feratur & foco & fulguritae & gloriae & heres \\
\hline extollunt & ferebat & focum & fullo & gnaruris & hesternum \\
\hline extrudit & ferentarium & fodi & fullones & graderere & hiberna \\
\hline extrusisti & feret & foedarim & fullonius & gradibus & hicin \\
\hline extudi & feriam & foede & fulmentas & gradietur & hicine \\
\hline exturba & ferias & follem & fundamento & gradu & hiemem \\
\hline exturbasti & ferio & fontem & fundamentum & Graece & hilari \\
\hline exuuiis & ferit & forabunt & fundes & grandinem & hilarus \\
\hline exussum & ferocem & forent & fundis & grandibo & Hilurica \\
\hline faba & ferratile & formai & fundus & grandior & hircum \\
\hline faber & ferreis & formicis & fungaris & graphice & hircus \\
\hline fabris & ferret & formidas & fungatur & graphicus & hirquina \\
\hline fabrum. & ferriteri & formidolosas & fungi & gratiae & hiscunt \\
\hline fabulabimur & ferriterium & fors & fungino & gratiasque & historiam \\
\hline fabulatast & ferritribaces & fortasse & fungit & gratiis & hiulca \\
\hline fabulor & ferrum & fortem & furandum & gratis & hocine \\
\hline facete & fertur & fortis & furatum & gratulator & hodiest \\
\hline facetis & feruefaciunt & fortiter & furcilles & gratum & holera \\
\hline faciebat & feruont & fortuito & furibus & grauabor & holitores \\
\hline $\begin{array}{l}\text { faciem } \\
\text { facient }\end{array}$ & festinate & fortunabunt & furinum & grauaris & holus \\
\hline $\begin{array}{l}\text { tacient } \\
\text { facillumum }\end{array}$ & $\begin{array}{l}\text { festine } \\
\text { festinet }\end{array}$ & unam & im & iem & minist \\
\hline facimus & festino & fortunatam & fusti & grauida & homunculi \\
\hline facin & festo & fortunataque & galea & grauior & honeste \\
\hline facinorum & feta & fortunatas & Gallicis & grauiter & honestet \\
\hline facitis & fiam & fortunate & gallina & grauius & horno \\
\hline factam & framus & fortunatorum & gallinacea & greges & Honor \\
\hline factio & fictor & fortunatus & gallinacio & Grumio & honori \\
\hline factiosum & fictorem & fortunatu's & gallinacius & gurguliost & honos \\
\hline factius & ficturae & frangat & gallinae & gustare & hora. \\
\hline factost & fideli & frangere & gallus & gustato & horriola \\
\hline factu & fideliam & frangitis & garrire & gustauerit & horridularum \\
\hline facturi & fidelis & frate & gaudeant & gutta & horti \\
\hline facunde & fidelitasque & fratrem & gaudeas & guttam & hospite \\
\hline faeceos & fidelitatem & fratri & gaudent & gymnasium & hospiti \\
\hline & fideliter & fraude & gaudere & gymnastica & hostias \\
\hline faenusculum & Fidi & fraudem & gaudia & habeant & hosticas \\
\hline falas & fidicinas & traudulenta & gaudjusque & habebam & hostilibus \\
\hline $\begin{array}{l}\text { rallacrae } \\
\text { fallere }\end{array}$ & & Irauaulente & gaudium & EDIS & hostisne \\
\hline fallis & fierent & fricari & gemitu & habeon & huiusmodi \\
\hline fallunt & fieres & frigida & genera & habitas & humani \\
\hline falsa & filias & frit & generest & habitatum & humanis \\
\hline falsidicum & finem & frons & generibus & habitauit & huncine \\
\hline falsis & firma & frontem & generum & habitent & aceam \\
\hline falson & firmiora & frugalior & geniumque & habiti & iaceant \\
\hline famae & firmiorem & frumentariis & gens & habiturum & iacebis \\
\hline famas & firmitate & frumento & gentiumst & habiturust & iacentem \\
\hline fame & firmitatem & frumentum & genua & habitus & iacentis \\
\hline fameque & firmum & frustramini & genuerunt & habuerim & iacere \\
\hline fames & frmus & frustrationes & gerat & habuisti & iacitur \\
\hline famigeratio. & fissile & frutex & geratur & habuitne & iactari \\
\hline famigeratori & fissorum & fuant & gerebat & haecine & iamque \\
\hline famigeratorum & fixae & fuas & geretur & haecst & ibam \\
\hline familiariter & flabelliferae & fuco & germana & hahae & ibas \\
\hline fartim & flagitabere & fue & gerrae & hahahae & ibat \\
\hline fas & flagitare & fueram & gerundum & hallec & ibist \\
\hline fatear & flagitator & fueris & Geryonaceo & hamum & ibitur \\
\hline es & flagiti & fuerunt & gessi & hapalopside & ictus \\
\hline acis & flagitio & fugam & gessisse & har & idcirco \\
\hline fa & flagris & fugat & gessit & hariolus & identidem \\
\hline faxem & flagritribae & fugatis & gestam & harpaga & ideo \\
\hline febrim & flammarii & fugere & gestant & harpagatum & idne \\
\hline fecerim & flare & fugereque & gestandust & harpagauit & idoneum \\
\hline fecerunt & fleam & fugin & gestas & Harpage & idoneus \\
\hline fecimus & flens & fugit & gestast & harpago & Iasonem \\
\hline felat & flentem & fugitat & gestire & Hedylium & ignarissumis \\
\hline feliciterque & fleueris & fugitiuam & gestis & herbasque & ignaui \\
\hline & floccum & fugitiue & gestores & herbis & ignauia \\
\hline as & floreas & fugitiuost & gignitur & herclest & ignauiam \\
\hline feniculum & fluctibu' & fugitorem & glabrae & Herculem & ignauissumus \\
\hline ferant & fluctus & fugiunt & glabriorem & Hercules & ignauom \\
\hline ferare & fluctusque & fuist & gladio & Herculi & ignauos \\
\hline
\end{tabular}

[44] 
5429 Words Occur Once - Continued

\begin{tabular}{|c|c|c|c|c|c|}
\hline ignorandust & incommodi & iniurium & integumentum & iocus & laetitia \\
\hline ignosco & incommoditates & iniuste & intellegam & Ionica & laetitias \\
\hline ignota & incommodum & inlexit & intellegas & Ioue & laeto \\
\hline gnotam & inconciliare & inlocabilem & intellegere & ipsissumus & laeua \\
\hline iisti & inconciliastin & inluuies & intellegis & ipso & laeuam \\
\hline ilicet & inconsultu & inmanem & intellegit & ipson & lamberas \\
\hline illanc & increpitauit & inmemor & intempestiuos & ira & lamentare \\
\hline illest & incumbat & inmemorem & intende & iram & lanarius \\
\hline illicinest & incursent & inmoene & interbibere & irasci & lanii \\
\hline illic'st & incus & inmortale & interbitat & irati & languorem \\
\hline illine & incuses & inmortalia & intercedat & irato & lanterna \\
\hline illist & incuties & inmortalis & intercepi & iratum & lapides \\
\hline illoc & indagator & inmundus & interduim & ires & lapidi \\
\hline imago & indaget & inmuni & interdum & iret & laqueo \\
\hline imber & indaudisse & inmunis & interfecero & isque & Larem \\
\hline imbre & indaudiuerit & innocentem & interficiant & isse & large \\
\hline imbremque & indicabis & inopem & interfieri & ist & largiri \\
\hline mbres & indicant & inopiast & interibi & istacin & largiter \\
\hline imbricesque & indicare & inpia & interiit & istacine & largitu's \\
\hline imbrim & indicassis & inpium & interimam & istest & Lari \\
\hline imbuas & indice & inportunam & interminatust & istiusmodi & laruae \\
\hline immigrat & indidem & inpoti & intermissum & istocine & lasciuiae \\
\hline immigraui & indidi & inpransus & intermortui & istorum & laserpici \\
\hline mmutare & indidit & inproba & interpellatio & itan & lassus \\
\hline mmutassis & indignis & inprobae & interpolare & iterant & late \\
\hline impellere & indipisces & inprobe & interpoles & iterari & latebis \\
\hline imperabo & indipiscor & inprobiores & interpretari & iterum & latebricolarum \\
\hline imperata & inducti & inprobissumum & interpretes & itidemque & latebris \\
\hline imperator & indoles & inprudentem & interrogare & itinera & latebrose \\
\hline imperatum & indomito & inprudenti & interrumpere & iturust & latent \\
\hline mperauerim & indotata & inpudens & interstringam & iubeat & latera \\
\hline imperes & indotatam & inpudice & interuias & iubebis & lates \\
\hline imperiis & indotatas & inpurae & interuorteret & iubebo & latitabat \\
\hline imperio & induitur & inpurate & intestina & iuben & latiu' \\
\hline imperiosus & indunt & inpuris & intestinis & iubent & latrocinatum \\
\hline imperitas & indusiarii & inque & instituta & iuberes & latu \\
\hline impertio & industriae & inquinaui & intolerabiles & iubeto & latuit \\
\hline mpertit & industriis & inquit & intolerandum & iucundumst & lauabo \\
\hline mpetrabilis & industrior & inrideas & introierit & iudex & lauat \\
\hline impetrabit & induxeris & inrideat & intuli & iudicare & lauatio \\
\hline impetrare & induxerunt & inridet & intuor & iudicium & lauationem \\
\hline impetrari & in' & inriqua & inuasit & iudico & lauatum \\
\hline impetrassere & inepte & inructas & inueneris & iugis & laudari \\
\hline impluat & ineptus & insaniam & inuenerit & iuisse & laudas \\
\hline npluit & ineuscheme & insaniaeque & nero & iunculae & laudasse \\
\hline mponam & infamis & insanin & inueni & iurando & laudauisse \\
\hline mponas & infecta & insanis & inuenias & iurandum & laudem \\
\hline imponentur & infectores & insatietatibus & inuenies & iurauistin & laudent \\
\hline imposisse & infensae & inscendam & inuenietis & iuratori & laudis \\
\hline impulsor & inferi & insciente & inuenimus & iuratus & laudo \\
\hline impulsore & inferiorem & inscita & inuenires & iussisse & Lauerna \\
\hline impune & infert & inscite & inuenisse & iusta & laui \\
\hline lanem & infidelis & inscitissumus & inuenisti & iustus & lauis \\
\hline aniis & infidum & inscribit & inuento & juuabo & lauit \\
\hline nanilogistae & infit & insepultum & inuentu & iuuas & lautaque \\
\hline cedo & infortunio & inseruibis & inuenturum & iuuerit & lectae \\
\hline cedunt & infortunium & inseruire & inuentu's & iuuentis & lectisterniator \\
\hline cenati & infumo & insidiantur & inuestigare & iuuentutem & lecto \\
\hline cendes & ingenia & insimulas & inuideat & iuxtaque & lectus \\
\hline cendor & ingenuom & insipiens & inuident & labella & lectust \\
\hline ceptat & ingerimus & insipientiast & inuidere & labellis & legam \\
\hline certa & ingredere & inspectas & inuides & labitur & legerit \\
\hline certi & ingrediri & inspectasque & inuidia & laborent & legerupa \\
\hline certo & ingreditur & insperata & inuidus & labores & legerupam \\
\hline certumst & inhiauit & inspice & inuisam & labori & legioni \\
\hline essi & inhonestis & inspiciat & inuises & laboris & legis \\
\hline idi & inibis & instipulatus & inuitet & labos & lemniscos \\
\hline ipissis & inicit & instrenuos & inuoca & Lacina & lenam \\
\hline itas & iniecisti & instruere & inuoco & Laconicam & lenoniam \\
\hline clementer & iniimus & insula & inuolem & lacrumam & lenonium \\
\hline clutae & inimico & insulas & inutilem & lacrumans & lenoniumst \\
\hline cohatam & inimicus & insulsum & iocaris & lactibus & lenonius \\
\hline lae & quiore & insuper & ioculo & laetantem & lenonum \\
\hline mmode & uria & integra & iocum & laetificantur & lepidast \\
\hline
\end{tabular}

[45] 


\begin{tabular}{|c|c|c|c|c|c|}
\hline lepidi & luci & malificus & meministin & minitare & mordicibus \\
\hline lepidissume & lucrifugas & malim & meminit & minor & morer \\
\hline lepidissumum & luctator & malique & memorarier & minusculum & mori \\
\hline lepido & luculentum & malitiam & memorat & mirabilis & morigerae \\
\hline lepidus & lucus & malitiast & memorato & mirare & morigeru's \\
\hline leto & ludam & malorum & memoret & mirari & morior \\
\hline leuem & ludere & malumque & memoriam & miremini & morologis \\
\hline leues & ludibundus & malumst & men & mirimodis & moros \\
\hline leuiorem & ludicram & malust & mendacem & mirumst & morosos \\
\hline libella & ludificabo & mammicula & mendaciloquius & mis & mors \\
\hline libellai & ludificat & mandasse & mendaciloquom & Misargyrides & morsiunculae \\
\hline libellam & ludificatorem & mandatast & mendax & miscent & mortales \\
\hline libello & ludificatu' & mandatus & mendicabula & misceres & mortarium \\
\hline liberam & ludificatust & mandatust & mendicare & misces & mortem \\
\hline liberant & ludificauerit & maneat & mendice & misera & mortua \\
\hline liberasse & ludificetur & manebo & mendicis & miserae & mortuam \\
\hline liberasso & ludimus & manere & mendico & miseras & mortuo \\
\hline liberast & ludiust & manesne & mendicus & misereret & mostra \\
\hline liberat & ludus & manicas & mens & misereri & mostrare \\
\hline liberate & lumbrice & maniplatim & mensam & miserescat & moueant \\
\hline liberaui & lupos & mansi & mense & miseri & moues \\
\hline liberet & lupus & mantant & mensulam & miseriae & mouet \\
\hline liberorum & luscus & manubrium & mensum & miseriis & mucci \\
\hline liberosque & lustrantur & manufesta. & mentire & miseritumst & mula \\
\hline libertam & lutitant & manulearii & mentis & miserius & mulcari \\
\hline libertas & luto & manuleatam & mentitust & miseros & mulcet \\
\hline libram & lutost & manumst & meosque & miserrume & muli \\
\hline libro & lutum & manuplares & mequidem & miserrumeis & mulierculam \\
\hline licentiam & luxantur & manuplaris & mera & miserrumus & mulieremque \\
\hline licetne & Luxuriae & mare & merast & miserumst & mulsi \\
\hline licetque & Lyconides & mari & mercatum & misi & multabo \\
\hline lictumst & macchaera & maria & mercatura & misisse & multae \\
\hline lignean & maccidem & maris & mercaturan & misissem & multam \\
\hline limbularii & Macedoni & maritumis & mercedest & misisset & multasque \\
\hline limen & Macedonia & Massici & mercimonio & missus & multauit \\
\hline lineam & Macedoniensem & mastigiae & mercist & misti & multi \\
\hline linguis & Macedonius & materiae & merear & mittatur & multiloquom \\
\hline linteones & macerant & matronae & merendam & mitteres & multipotenti \\
\hline linteum & macero & matronarum & merens & mitteret & multoque \\
\hline linum & macet & matronis & merere & mittimus & munera \\
\hline linquere & Machaerio & matula & meret & mittis & munerigeruli \\
\hline liqueant & machinas & matulam & meretricis & mittit & murmurat .. \\
\hline liquida & mactant & matura & meretricium & mixtum & murobatharii \\
\hline liquidust & mactari & maturam & meretur & modesti & murrinam \\
\hline liquitur & macto & maturate & merita & modestia & murum \\
\hline litationem & madeam & mauelim & meritam & modestiam & musice \\
\hline litem & madefactatis & mauelis & meritus & modicis & mussari \\
\hline litteram & madent & mauelit & meritust & moenia & mussitabas \\
\hline litteras & madeo & mauis & meruerunt & Molas & mutabit \\
\hline litterasque & madet & mauolo & meruisse & molestam & mutam \\
\hline locassem & madide & maxumas & merum & molestiaeque & muttire \\
\hline Iocassim & madidum & maxumi & messem & molestu's & mutuam \\
\hline locata & madulsam & maxumist & messim & molestust & mutuis \\
\hline locos & maerens & meai & metere & molles & mutumst \\
\hline locost & maerore & mean & metimur & mollior & myropolae \\
\hline locuples & maeroris & meaque & metit & molliter & nac \\
\hline locutus & maestitiaeque & Medea & metuant & mollitiis & nactus \\
\hline longae & maestitudoque & mediam & metuculosa & molocinarii & naenia \\
\hline longi & maestust & medicabo & metuebam & mone & nanctu's \\
\hline longissuma & mage & medicamento & metuenda & moneas & nare \\
\hline longo & magisterio & medico & metuis & monendu's & narrabit \\
\hline loquaces & magnas & meditabar & metus & monet & narrabo \\
\hline loquendi & magnisque & meditabor & meu's & monstra & narras \\
\hline loquentis & magnuficus & meditati & migrare & monstrare & narrat \\
\hline loquerer & magnus & meditatum & migrat & monstres & narrato \\
\hline loqueris & maitiores & meditatus & mihin & montem & narraui \\
\hline lora & malaque & medullitus & milia & montes & narret \\
\hline loris & maledicis & mehercle & miliens & moramur & narro \\
\hline lubeat & malefacta & Melinum & militariis & morare & natalem \\
\hline lubentissumo & malefice & mellam & militaris & moratur & nati \\
\hline lubitum & maleficos & mellillae & militem & moratus & natu \\
\hline lubuit & maleficum & memento & miluinis & morbo & natura \\
\hline lucem & maleque & meminerunt & miluom & morbum & naui \\
\hline lucernam & malesuada & meministi & miluos & morbus & nauis \\
\hline
\end{tabular}

[46] 


\begin{tabular}{|c|c|c|c|c|c|}
\hline nebulam & nouam & obrepat & onerem & optumis & paritas \\
\hline necdum & nouarum & obrepseris & oneris & opturat & parricida \\
\hline necessitas & nouas & obrepsisti & onus & opulentiae & pansi \\
\hline necullum & nouercam & obsonas & operaest & opulentiores & parsimonia \\
\hline negabas & noueris & obuenerit & operamque & opulentissumus & parsimoniae \\
\hline negabo & nouerisne & obuoluto & operast & orabo & parsis \\
\hline negauit & nouerit & obuortat & operi & orant & parsissem \\
\hline neglegens & nouicium & occasiuncula & operiri & orasque & parta \\
\hline neglegentes & novorum & occedamus & operito & oratione & partas \\
\hline neglegentiam & nox. & occepistis & opibus & orationis & parte \\
\hline neglegere & noxia & occepit & opifex & oratiost & particeps \\
\hline negotiis & nu & occeptumst & opinione & orauit & partum \\
\hline negotio & nubant & occessit & oporteat & ordinem & parturit \\
\hline negotiosust & nubent & occideret & opperiam & orditur & parua \\
\hline negotist & nucem & occiderit & opperiare & ordo & paruis \\
\hline negotiumst & nuda & occidimus & opperibor & ore & paruisti \\
\hline nent. & nugacissume & occidit & opperire & orgiorum & paruolus \\
\hline Neptuni & nugae & occidor & opperitur & oritur & paruom \\
\hline Neptuno & nugamini & occipiam & opperitus & oriundi & paruomst \\
\hline nequamst & nugare & occipiat & opplebo & ornatum & pascite \\
\hline nequaquam & nugatorem & occipitio & oppletae & ossa & passim \\
\hline nequeat & nugatori & occisa & oppletus & ostendam & passum \\
\hline nequeunt & nugatorias & occisus & opponam & ostentarier & pastum \\
\hline Neri & nugatorium & occlusa & Opportunitas & ostendi & patagiarii \\
\hline neruo & nugatur & occlusae & opportunius & ostentat & pateant \\
\hline nesciam & nugigerulis & occlusioremque & opportunum & ostio & patefeci \\
\hline nesciat & nugis & occlusti & opportunus & otiosse & patere \\
\hline nescioquid & nulli & occulant & oppressiunculae & otiosus & paterer \\
\hline nescioquo & nulliu' & occultabo & opprimat & oues & paternam \\
\hline nesciunt & nullumst & occultandaest & opprimitur & ouis & paternj \\
\hline neutrubi & numeras & occultatum & opprobra & pacem & paterque \\
\hline nidoricupi & numeratae & occultassis & opprobrarier & pacisci & patibulatum \\
\hline nihilist & numeratis & occultum & oppugnando & pacta & patientia \\
\hline nimiae & numeratum & occulunt & oppugnas & pactam & patientissumumst \\
\hline nimias & nummis & occupassis & opsaeptast & pactast & patinis \\
\hline nimioque & nummorum & occupato & opscurasse & pactionem & patior \\
\hline nimirum & numnam & occupatu's & opsecraueram & paedagogus & patiunda \\
\hline niteant & numqui & occupauit & opsecrauisset & paenulast & patiundumst \\
\hline nitidior & nundinalest & occupias & opsecrauit & palamst & patres \\
\hline nitidiuscule & nuntiaret & occurrit & opsequentem & pallam & patria \\
\hline nitidiusculum & nuntias & occurro & opsequentis & pallas & patrium \\
\hline nitorem & nuntiate & ocelle & opsequi & pallio & patrone \\
\hline nituntur & nunties & ocello & opserendo & palliolatim & patrono \\
\hline niue & nuntiet & ocellu's & opseri & palliost & patronum \\
\hline nobilest & nuntietur & oculata & opseruabo & palpum & pauca \\
\hline nobilis & nuntii & oculeus & opseruauit & panem & paucas \\
\hline nobiscum & nuntium & oculicrepidae & opseruemus & panis & pauciores \\
\hline noceas & nupsisti & oculisque & opsessor & panticesque & pauciorum \\
\hline noceat & nupta & oculus & opseueris & pantopolium & paucorum \\
\hline nocet & nuptiae & oderit & opsidione & papauerem & paue \\
\hline noctem & nusquamst & odiosas & opsignatam & papillarum & paullulum \\
\hline noctes & nutrice & odiossaeque & opsignato & parabit & pauperant \\
\hline noctis & nutrix & odiosus & opsisti & parabo & pauperculum \\
\hline noenum & obambulatum & odorem & opsit & parare & pauperioris \\
\hline nolebam & obdormiui & odores & opsonate & parasite & pauperiorum \\
\hline nolint & obducam & offendam & opsanari & parasitos & pauperis \\
\hline nolit & obest & officio & opsonauit & parasitus & pauperrumum \\
\hline nolui & obicere & offigantur & opsoni & parat & paupertate \\
\hline nominabo & obicias & offuciam & opsoniumque & paratae & paupertatem \\
\hline nomini & obicitur & offusam & opstant & paratast & pauperum \\
\hline nonum & obiciunt & oggerunt & opstinauit & paratis & pauxillo \\
\hline noram & obiectare & ohe & opstringit & paratragoedat & pauxillulo \\
\hline noscitat & obiectas & olent & opstrinxero & paratum & pauxillum \\
\hline nosco & obiectast & oleo & optegere & paraui & peccarim \\
\hline nosque & obiectent & oles & optemperem & parcere & peccata \\
\hline nosse & obiectes & oliui & optemperas & parcior & peccatum \\
\hline nossem & obiurgandus & oliuom & optines & parcissumum & peccauisti \\
\hline nostramne & obiurgans & Olympico & optio & parcunt & pectori \\
\hline nostri & oblectem & omine & optrudere & parent & peculiaris \\
\hline nostronum & oblinam & omittis & optrunco & parerem & pedisequa \\
\hline nostrost & oblitum & omnibust & optuerier & pari & pedisequos \\
\hline nota & obnoxios & onerabij' & optumam & pariant & peiiurare \\
\hline notis & obnoxiosae & onerat & optumas & parietem & peiiurauisti \\
\hline notisque & oboluisti & onere & optumast & parietum & peiiure \\
\hline
\end{tabular}

[47] 


\begin{tabular}{|c|c|c|c|c|c|}
\hline $\begin{array}{l}\text { peiiuri } \\
\text { peiiurissume } \\
\text { peiiurius } \\
\text { peiius } \\
\text { peior } \\
\text { Peliam } \\
\text { pellego } \\
\text { pellerem } \\
\text { pellis } \\
\text { pellucet } \\
\text { pendam } \\
\text { pendeant } \\
\text { pendens } \\
\text { pendentem } \\
\text { pendes } \\
\text { pendet } \\
\text { pendis } \\
\text { pendo } \\
\text { penetrarem } \\
\text { penetraui } \\
\text { penetrauit } \\
\text { penitus } \\
\text { pensilem } \\
\text { peperceris } \\
\text { peperisse } \\
\text { peperit } \\
\text { perambula } \\
\text { peracuit } \\
\text { perbene } \\
\text { perbitere } \\
\text { perbonam } \\
\text { perbono } \\
\text { perbonum } \\
\text { percepi } \\
\text { percoles } \\
\text { percontabere } \\
\text { percontari } \\
\text { percontarier } \\
\text { perculsust } \\
\text { percussi } \\
\text { percussisses } \\
\text { percussit } \\
\text { percutiam } \\
\text { perdat } \\
\text { perde } \\
\text { perderes } \\
\text { perdidero } \\
\text { perdidici } \\
\text { perdidisse } \\
\text { perdita } \\
\text { perdoces } \\
\text { perdocte } \\
\text { perduellibus } \\
\text { perduellis } \\
\text { perduim } \\
\text { perduxerunt } \\
\text { pereat } \\
\text { peregri } \\
\text { peregrina } \\
\text { peregrinos } \\
\text { peregrinum } \\
\text { peregrinus } \\
\text { perendie } \\
\text { perendinum } \\
\text { pereo } \\
\text { perfacile } \\
\text { perfeceris } \\
\text { perferam } \\
\text { perfidiam } \\
\text { perfiass } \\
\text { perfidis } \\
\text { perfossor } \\
\text { perfrigefacit } \\
\text { pergere } \\
\end{array}$ & $\begin{array}{l}\text { pergite } \\
\text { pergitin } \\
\text { pergraecaminei } \\
\text { pergraecamini } \\
\text { pergraecari } \\
\text { pergraphicus } \\
\text { pergunt } \\
\text { perhibeatur } \\
\text { pericli } \\
\text { periclumst } \\
\text { periclo } \\
\text { periculo } \\
\text { periissem } \\
\text { periisses } \\
\text { perinde } \\
\text { perire } \\
\text { peris } \\
\text { peristromata } \\
\text { peritissumus } \\
\text { perlongumst } \\
\text { permadefecit } \\
\text { permanascere } \\
\text { permanauit } \\
\text { pernam } \\
\text { pernegare } \\
\text { pernegat } \\
\text { pernego } \\
\text { pernoui } \\
\text { pernouit } \\
\text { pernix } \\
\text { perperam } \\
\text { perpessu's } \\
\text { perpeti } \\
\text { perpetiar } \\
\text { perpetior } \\
\text { perpetraui } \\
\text { perpetuae } \\
\text { perpetuat } \\
\text { perplacent } \\
\text { perplexarier } \\
\text { perpluant } \\
\text { perpluit } \\
\text { perpluont } \\
\text { perpotasse } \\
\text { perpotaui } \\
\text { perscrutabor } \\
\text { perscrutatus } \\
\text { perscrutaui } \\
\text { persentiscat } \\
\text { persequor } \\
\text { perspecta } \\
\text { perspexi } \\
\text { perspicue } \\
\text { pertegito } \\
\text { perterruit } \\
\text { pertimescit } \\
\text { pertundat } \\
\text { perturbat } \\
\text { pertussum } \\
\text { peruenant } \\
\text { perueneris } \\
\text { peruenerit } \\
\text { peruenisti } \\
\text { peruiamst } \\
\text { peruigilat } \\
\text { peruium } \\
\text { peruolgatumst } \\
\text { pessulis } \\
\text { pessumaa } \\
\text { pessumu's } \\
\text { peste } \\
\text { petaso } \\
\text { petasus } \\
\text { pete } \\
\text { a }\end{array}$ & 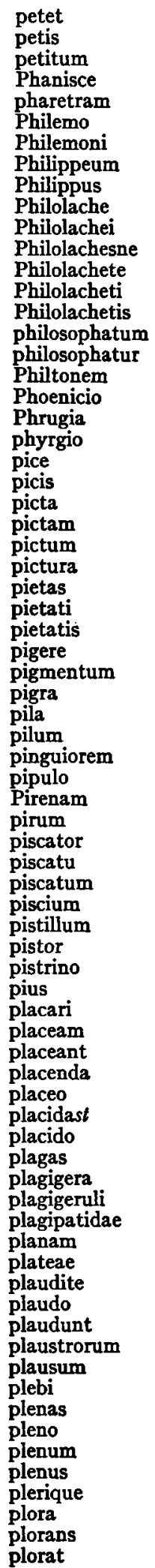 & $\begin{array}{l}\text { pluma } \\
\text { plumbeos } \\
\text { plumbeum } \\
\text { plura } \\
\text { pluribus } \\
\text { plurimis } \\
\text { pluris } \\
\text { plurumae } \\
\text { plurumi } \\
\text { plurumis } \\
\text { plurumos } \\
\text { plusque } \\
\text { pocula } \\
\text { poeniceo } \\
\text { polliceor } \\
\text { pollicetur } \\
\text { pollicitatio } \\
\text { pollicitos } \\
\text { pollucibiliter } \\
\text { Polymachaero- } \\
\text { plagidem } \\
\text { Polymachaero- } \\
\text { plagidi } \\
\text { polypos } \\
\text { ponam } \\
\text { ponat } \\
\text { pondo } \\
\text { ponere } \\
\text { ponitur } \\
\text { Ponto } \\
\text { Pontum } \\
\text { ponunt } \\
\text { popularem } \\
\text { populares } \\
\text { populum } \\
\text { porcinam } \\
\text { porge } \\
\text { poriciam } \\
\text { porrectam } \\
\text { porrexi } \\
\text { portast } \\
\text { portenditur } \\
\text { porticus } \\
\text { portitorem } \\
\text { portitores } \\
\text { portitori } \\
\text { portorium } \\
\text { portu } \\
\text { portum } \\
\text { poscas } \\
\text { posce } \\
\text { poscerem } \\
\text { posceret } \\
\text { poscis } \\
\text { posiueris } \\
\text { posiui } \\
\text { posses } \\
\text { possidere } \\
\text { possidet } \\
\text { possiet } \\
\text { possimus } \\
\text { possumus } \\
\text { poste } \\
\text { posterorum } \\
\text { posticulo } \\
\text { posticulum } \\
\text { posticum } \\
\text { postidea } \\
\text { postulare } \\
\text { Postumus } \\
\text { pota } \\
\text { potandus } \\
\text { potantis } \\
\text { potarier } \\
\end{array}$ & $\begin{array}{l}\text { potate } \\
\text { potatum } \\
\text { potem } \\
\text { poterat } \\
\text { poteriis } \\
\text { poterit } \\
\text { poterunt } \\
\text { potestas } \\
\text { potestatem } \\
\text { poti } \\
\text { potiatur } \\
\text { potiorem } \\
\text { potitus } \\
\text { potuisti } \\
\text { praebeo } \\
\text { praeberi } \\
\text { praecellet } \\
\text { praecepi } \\
\text { praecepta } \\
\text { praeceptor } \\
\text { praecidam } \\
\text { praecipere } \\
\text { praecipitauit } \\
\text { praecipitem } \\
\text { praecipue } \\
\text { praecipuos } \\
\text { praecurram } \\
\text { praedae } \\
\text { praedatoribus } \\
\text { praedicem } \\
\text { praedicet } \\
\text { praedicitur } \\
\text { praediti } \\
\text { praedo } \\
\text { praefectum } \\
\text { praefectus } \\
\text { praeficio } \\
\text { praefulcior } \\
\text { praegnatem } \\
\text { praehiberem } \\
\text { praemandatum } \\
\text { praemature } \\
\text { praemostrauit } \\
\text { praemostro } \\
\text { Praenestinus } \\
\text { praeoccupabo } \\
\text { praeoptauisti } \\
\text { praeponeres } \\
\text { praeripiam } \\
\text { praerodat } \\
\text { praerodatis } \\
\text { praesagibat } \\
\text { praesegmina } \\
\text { praesensisset } \\
\text { praesentaria } \\
\text { praesentarïs } \\
\text { praesentarium } \\
\text { praesentem } \\
\text { praesentes } \\
\text { praesertim } \\
\text { praesidebo } \\
\text { praestat } \\
\text { praestinem } \\
\text { praestitutast } \\
\text { praesto } \\
\text { praestolabor } \\
\text { praestrigiator } \\
\text { praeterhac } \\
\text { praeuortar } \\
\text { praeuortaris } \\
\text { praeuortere } \\
\text { prandero } \\
\text { prandio } \\
\text { prata } \\
\text { ala }\end{array}$ & $\begin{array}{l}\text { precator } \\
\text { prehendam } \\
\text { prehenderit } \\
\text { prehendunt } \\
\text { prehensi } \\
\text { premam } \\
\text { pretium } \\
\text { pretiumst } \\
\text { primaria } \\
\text { primoribus } \\
\text { primus } \\
\text { principi } \\
\text { priuatae } \\
\text { priuatum } \\
\text { priuos } \\
\text { proauo } \\
\text { probasse } \\
\text { probest } \\
\text { probior } \\
\text { probiores } \\
\text { procedere } \\
\text { procellae } \\
\text { processerunt } \\
\text { processi } \\
\text { processit } \\
\text { procreandis } \\
\text { procudam } \\
\text { procurator } \\
\text { procures } \\
\text { prodegeris } \\
\text { prodi } \\
\text { prodigi } \\
\text { prodire } \\
\text { prodit } \\
\text { profanum } \\
\text { profecturus } \\
\text { profero } \\
\text { proferto } \\
\text { proferunt } \\
\text { profesto } \\
\text { proficisci } \\
\text { profugiens } \\
\text { profundere } \\
\text { progigni } \\
\text { prognatum } \\
\text { progredimino } \\
\text { progreditur } \\
\text { prohibeas } \\
\text { prohibere } \\
\text { prohibes } \\
\text { prohibessis } \\
\text { prohibessit } \\
\text { promat } \\
\text { promere } \\
\text { promeres } \\
\text { promerita } \\
\text { promi } \\
\text { promiscam } \\
\text { promiseram } \\
\text { promisisse } \\
\text { promissi } \\
\text { promittitis } \\
\text { promptas } \\
\text { pronuper } \\
\text { propemodo } \\
\text { properant } \\
\text { properare } \\
\text { properate } \\
\text { properatost } \\
\text { properes } \\
\text { properet } \\
\text { propinare } \\
\text { propinqua } \\
\text { propinquitate }\end{array}$ \\
\hline
\end{tabular}

[48] 


\begin{tabular}{|c|c|c|c|c|c|}
\hline propior & putidum & quotumo & relinqui & reuocamus & sapies \\
\hline propitia & puto & quouis & relinquo & reuocas & sarcina \\
\hline propitii & $\mathbf{q}$ & radebat & reliquiae & reu'que & sarcinatores \\
\hline propolae & quadrigis & radicibus & remisit & Rhadamantem & sarcire \\
\hline proprio & quaedamst & rapacidarum & remittam & rides & sarciri \\
\hline propterea & quaen. & rapax & remittat & ridetis & sarmenta \\
\hline propromptu & quaepiam & rapiam & remitte & ridicularia & Sarsinatis \\
\hline $\begin{array}{l}\text { prorogare } \\
\text { prorogatur }\end{array}$ & $\begin{array}{l}\text { quaepiamst } \\
\text { quaeq' }\end{array}$ & $\begin{array}{l}\text { rapi' } \\
\text { rapitur }\end{array}$ & $\begin{array}{l}\text { remittere } \\
\text { remoramque }\end{array}$ & $\begin{array}{l}\text { ridicule } \\
\text { riuabo }\end{array}$ & $\begin{array}{l}\text { sarta } \\
\text { satellites }\end{array}$ \\
\hline proscinditur & quaequomque & raptas & remorantur & & satias \\
\hline prosilui & quaeram & raptores & remoratur & rogandi & satietate \\
\hline prosint & quaerat & rationesque & renuntiabo & rogant & satius \\
\hline prospere & quaere & ratis & renuntiantur & rogare & satiust \\
\hline propereque & quaerebas & ravim & renuntiare & rogat & saucaptidem \\
\hline $\begin{array}{l}\text { prospicere } \\
\text { prostibulum }\end{array}$ & $\begin{array}{l}\text { quaererem } \\
\text { quaerite }\end{array}$ & reantur & renuntiatum & rogaturu's & $\begin{array}{l}\text { sauiis } \\
\text { sauium }\end{array}$ \\
\hline prostituam & & $\begin{array}{l}\text { rear } \\
\text { rebitur }\end{array}$ & $\begin{array}{l}\text { renunta } \\
\text { reor }\end{array}$ & $\begin{array}{l}\text { roget } \\
\text { rogitant }\end{array}$ & $\begin{array}{l}\text { Sauium } \\
\text { saxo }\end{array}$ \\
\hline prostituat & quaeritemus & recens & reparcunt & rogito & saxum \\
\hline prosum & quaeritet & recenti & repente & ruant & scabrae \\
\hline proterritum & quaerito & recepit & repentino & rubicundo & scaenam \\
\hline prothyme & $\begin{array}{l}\text { quaeritur } \\
\text { quaerundum }\end{array}$ & recipiam & reperias & ructare & scaeuast \\
\hline protollet & quaesso & recita & $\begin{array}{l}\text { reperiatur } \\
\text { reperiri }\end{array}$ & $\begin{array}{l}\text { ructus } \\
\text { ruere }\end{array}$ & $\begin{array}{l}\text { scalpurrire } \\
\text { scandit }\end{array}$ \\
\hline protractum & quaestio & recludat & reperit & rufus & Scaphae \\
\hline proueniant & quaestus & reclude & repertam & rumicem & Scapham \\
\hline prouenit & quaeuis & recommentatu's & repetas & rumori & scapulis \\
\hline prouidero & quali & recomminiscar & repetundi & ruont & scatat \\
\hline prouinciae & qualine & Recordatu' & repetunt & rustici & scelera \\
\hline prouinciam & qualubet & recten & reposcam & rusticus & scelestae \\
\hline prouincias & quamobrem & recurre & reposco & rusus & scelestam \\
\hline prouocas & quamue & recursem & repperero & sacerrumus & scelestior \\
\hline prouorsus & quantas & redactust & repperissem & sacrilege & scelestum \\
\hline prox & quantillo & reddat & reprehendam & sacris & scelestu's \\
\hline proxumam & quantillum & reddatur & reprehende & sacruficassem & scibis \\
\hline proxumas & quantis & redderem & reprehendit & saeclo & sciens \\
\hline proxume & quaquam & redderes & reprehensi & saeculum & scindant \\
\hline proxumumst & quaque & reddes & reputes & saepissume & scindere \\
\hline proxumus & quartus & reddeturne & reputo & saeuiendum & scintillam \\
\hline pube & quasique & reddi & requiritas & saeuomque & scirpea \\
\hline publicae & quassas & reddidi & rere & sagina & scita \\
\hline publicisne & quater & reddit & resciscat & sagittas & scitis \\
\hline pueros & quempiam & redditae & resciuerit & sagittatis & scitius \\
\hline puerum & quemque & reddituru's & resciui & sagittis & scitne \\
\hline pugnabo & querimonia & redditurus & resciuisse & saliat & scito \\
\hline pugnis & queunt & reddundum & resciuisti & salicto & scitust \\
\hline pulchram & quian & reddunt & resecroque & salillum & sciui \\
\hline pulchrior & quibam & redduxit & resignatas & salit & sciuin \\
\hline pulchriores & quiddam & redemi & resonat & Salsipotenti & scortantur \\
\hline pulchrius & quiddamst & redhibere & respicere & salsis & scribam \\
\hline pulmento & quietae & redierit & respiciam & saluae & scribuntur \\
\hline pulmentum & quieto & rediero & respicis & saluete & scrobes \\
\hline pulpamentis & quinam & rediget & respondeam & saluomque & scrutari \\
\hline pultabit & quinauicenaria & redigunt & respondeat & saluomst & scurra \\
\hline pultadum & quincto & rediit & respondendi & Salus & scurras \\
\hline pultandi & quingentos & redipisci & respondeo & salutabant & scuta \\
\hline pultaui & quinis & reditio & respondi & salutant & secat \\
\hline pultem & quintibi & redito & respondisset & salutare & secreto \\
\hline pultiphagus & quintus & rediturum & responset & salutas & sectatur \\
\hline pumex & qui's & reducem & responsiones & salute & sector \\
\hline pumiceos & quisquamst & referam & responsum & saluti & secunda \\
\hline pungit & quoad & referas & restat & salutigerulos & securi \\
\hline Punica & quocum & referret & restim & saluto & securim \\
\hline puplicis & quodam & refers & restinguas & sanae & sedebas \\
\hline pure & quoiam & refertur & Iestio & sanctast & sedem \\
\hline purget & quoidam & reges & restiteras & sanctumst & sedens \\
\hline purigant & quoii & regi & restitit & sandaligerulae & sedent \\
\hline purpurata & quoipiam & reginae & restituis & sanein & sedentarii \\
\hline purpurissum & quoiuismodi & regione & retinere & Sangarionem & sedet \\
\hline puta & quoium & regno & retineri & sanguinis & seduxi \\
\hline putas & quoiumst & regredere & retullit & sapiat & seges \\
\hline putat & quomodo & relictae & retunsast & sapienter & segnities \\
\hline putatur & quopiam & relicui & retunsumst & sapienti & segregant \\
\hline putefacit & quotiens & relicuos & reueniat & sapientia & segregent \\
\hline puten & quotumas & relinques & reuenit & sapientiam & segreges \\
\hline
\end{tabular}

[49] 


\begin{tabular}{|c|c|c|c|c|c|}
\hline Seleuciam & sinito & speres & suauisauiatio & supsequar & tecta \\
\hline semita & sintne & spernere & suauitate & supsequere & tectum \\
\hline senectus & siquid & spernis & Subballio & supsequitur & tectus \\
\hline senectuti & siris & Sphaerio & subblandiebar & supsequor & tegam \\
\hline senis & sistendae & splendorem & subditiuom & supsiet & tegit \\
\hline senserim & sistere & spolia & subducemus & supstruont & tegoribus \\
\hline senserit & sisti & spoliem & subducta & suram & tegulas \\
\hline sensi & sita & sponsione & subegero & surdu' & telam \\
\hline sensim & sitast & sponte & subegi & Sure & temerarium \\
\hline sensit & siti & spopondi & subest & surgam & temeti \\
\hline sententiasque & situm & spurcificum & subeunt & surgedum & temperabo \\
\hline sentiat & situmst & sta & subigere & surgunt & temperei \\
\hline sepeliui & situst & stabile & subigis & suris & tempestatem \\
\hline septem & siue & stabit & subigit & Suro & tempestates \\
\hline septumas & siueris & stacta & subigor & surrupere & tempta \\
\hline sepulcro & sobrie & stamus & subitost & surruperem & temptat \\
\hline sequitur & sobriu' & $\operatorname{stan} t$ & subitum & sumuperent & temptaui \\
\hline sera & socco & Stasimum & sublegam & surruperes & tempust \\
\hline serere & socerum & stat & subleui & surruperet & ten \\
\hline sermonatus & socienno & statin & subleuit & surrupio & tendant \\
\hline sermones & sociofraude & statis & sublinat & surrupuisse & teneant \\
\hline sermoni & socordiamque & statuam & sublingulo & surrupuit & teneas \\
\hline sermonisque & Socratem & statuisti & subniger & suscenseas & teneat \\
\hline serua & sodalis & statur & subolebat & suscenses & tenebris \\
\hline seruanda & sodalitate & sternuas & subolem & suspendito & teneris \\
\hline seruando & sodes & stilis & subueni & suspendium & teneto \\
\hline seruari & sol & stimulatricem & subuenisset & suspicamini & tenetur \\
\hline seruasso & solam & stimulatrici & subuenisti & suspicentur & tenuisti \\
\hline seruator & solas & stimulorum & subuenit & suspicetur & teque \\
\hline seruauero & sole & stimulum & succreuere & suspicione & tequidem \\
\hline seruaui & soleam & stipendium & sucophantis & sustinent & ter \\
\hline seruauisti & soleant & stipularier & sudor & sustinet & terere \\
\hline serue & solearii & stirpe & sues & susum & terginum \\
\hline seruent & soleas & stolide & sulco & sutores & teritur \\
\hline seruiant & soleatis & stolidius & sumas & sycophantae & terminos \\
\hline seruitum & solitus & strata & sumen & sycophantiose & terras \\
\hline seruitus & solitu's & stratioticum & sumere & symbolo & terrestris \\
\hline seruitutis & solstitiali & stratioticus & sumeret & Syrus & territas \\
\hline seruiui & solstitialis & stratu' & summam & taberna & terta \\
\hline seruiunt & soluam & strenui & summatum & tabernaculo & tertio \\
\hline seruorumque & soluere & strenuom & summus & tabernam & tertium \\
\hline seruosne & soluite & strenuosos & sumpsero & tabulas & terunt \\
\hline sescentae & solutum & strepitust & sumpsi & tacebo & testem \\
\hline sesquipede & solutumst & strigibus & sumpsimus & tacente & testest \\
\hline seuerumque & somniatis & Strobilum & sumpsisse & tacet & testudineum \\
\hline seuocas & somniauit & Strobilus & sumpti & taciturnitati & tetigerunt \\
\hline seraginta & somnu' & Strolum & sumptibus & tactu' & tetigisti \\
\hline sextum & sonant & strophiarii & sumptui & taesumst & tetigit \\
\hline sibique & sonat & strophiis & sumptumque & taeterrume & tetulit \\
\hline Sibulla & sonitum & structa & sumptumst & taetriorem & texier \\
\hline siccoculum & sonitus & studeat & sumptusque & taetrius & textores \\
\hline siccus & sopor & studeo & supellex & tale & theatri \\
\hline Sicyone & sorbereque & studes & superant & talenta & thensaurarios \\
\hline Sicyonem & sorbitione & studet & superare & talento & thensauti \\
\hline Sicyoni & sororis & stultam & superauit & tametsi & thensaurus \\
\hline signis & sors & stulti & superbe & tangam & Theopropidem \\
\hline signo & sospitalis & stultiloquentiam & superbum & tangeres & thermopolio \\
\hline silentio & sospitent & stultis & supercilium & tangite & thermopotasti \\
\hline Siluano & sparsa & stultitiae & superi & tantam & thylacistae \\
\hline simia & spatium & stultitiast & superiores & tantas & tibicem \\
\hline Simiae & specieque & stultitiis & supero & tantillo & tibicina \\
\hline Simiam & specimen & stultius & superstes & tantillum & tibicinae \\
\hline Simoni & spectabit & stultum & suppeditat & tanton & tibicinamque \\
\hline simulacrumque & spectant & stultu's & suppetant & tantu & tibicinas \\
\hline simulant & spectare & suadeam & suppingi & tantumst & tibicinasque \\
\hline simulas & spectato & suadeas & supplex & tappetia & tibin \\
\hline simulauit & spectatorum & suades & supplicabo & tarde & tibique \\
\hline simules & specto & suadetur & supplicat & tardus & tigillo \\
\hline simultatem & speculabor & suarum & supplicatum & tarpezitae & timeam \\
\hline simulter & speculatum & suas & supplici & tarpezitam & timeas \\
\hline sinapis & speculatur & suasore & suppositicium & tarmes & timeo \\
\hline sincerum & speculoclaras & suaui & supremi & taurus & times \\
\hline singillatim & Spemne & suauior & supremum & taurum & timida \\
\hline sinistera & speras & suauis & supremus & techinae & timido \\
\hline
\end{tabular}

[50] 


\begin{tabular}{|c|c|c|c|c|c|}
\hline & & 5429 Words Occu & Once - Con & & \\
\hline timidus & tuaique & uein & uicerit & uisitatus & uoluptates \\
\hline timorem & tuamst & ueis & uici & Visso & uoluptati \\
\hline timui & tuapte & uela & uicine & uisu' & uoluptati' \\
\hline tinnis & tuast & uelintne & uicinos & uiuam & uoluptatibu' \\
\hline & $\begin{array}{l}\text { tuisque } \\
\text { tuli }\end{array}$ & uellent & uicit & $\begin{array}{l}\text { uiuamus } \\
\text { viuat }\end{array}$ & uoluptatum \\
\hline $\begin{array}{l}\text { tintinnabuulis } \\
\text { tintinnabulum }\end{array}$ & tulissent & $\begin{array}{l}\text { uellicat } \\
\text { ueluti }\end{array}$ & $\begin{array}{l}\text { uictibus } \\
\text { uictis }\end{array}$ & $\begin{array}{l}\text { uiuat } \\
\text { uiue }\end{array}$ & $\begin{array}{l}\text { uolutaui } \\
\text { uomitum }\end{array}$ \\
\hline titubanti & tumultuoso & uenales & uictitabam & uiuito & vorem \\
\hline titubes & tunc & uenalium & uictitare & uite & uorsa \\
\hline titubet & tunica & uenderes. & uictoria & uitia & uorsabatur \\
\hline $\begin{array}{l}\text { tolerabis } \\
\text { toleres }\end{array}$ & $\begin{array}{l}\text { tunicam } \\
\text { tunicas }\end{array}$ & $\begin{array}{l}\text { uendidisti } \\
\text { uendito }\end{array}$ & $\begin{array}{l}\text { uictorum } \\
\text { victuraque }\end{array}$ & $\begin{array}{l}\text { uitilena } \\
\text { uitio }\end{array}$ & $\begin{array}{l}\text { uorsari } \\
\text { uorsatur }\end{array}$ \\
\hline toleret & tuomst & uenditurum & $\begin{array}{l}\text { uicturaque } \\
\text { uictust }\end{array}$ & uitulinam & $\begin{array}{l}\text { uorsatur } \\
\text { uorso }\end{array}$ \\
\hline tollat & tuost & uendundust & uideare & uituperarier & uorsoriam \\
\hline tollet. & turanne & ueneficae & uideat & uituperas & uorsus \\
\hline tonsilia & turbae & ueneficu's & uideatur & uiuam & uorsute \\
\hline $\begin{array}{l}\text { tonsor } \\
\text { totas }\end{array}$ & turbant & uenenis & uidebis & uiuere & uorsutus \\
\hline totust & $\begin{array}{l}\text { turpat } \\
\text { turbauimus }\end{array}$ & $\begin{array}{l}\text { ueneram } \\
\text { uenerare }\end{array}$ & $\begin{array}{l}\text { uydebo } \\
\text { uidebunt }\end{array}$ & $\begin{array}{l}\text { uixerit } \\
\text { uiximus }\end{array}$ & $\begin{array}{l}\text { uortere } \\
\text { uorterit }\end{array}$ \\
\hline totos & turbauit & uenerim & uidelicet & uixisse & uorti \\
\hline tractare & turbellas & uenias & uidendum & uixit & uortitur \\
\hline & $\begin{array}{l}\text { turbidast } \\
\text { turbidos }\end{array}$ & uenient & uidendumst & $\begin{array}{l}\text { ulciscare } \\
\text { Vlizem }\end{array}$ & uostrae \\
\hline $\begin{array}{l}\text { tractaut } \\
\text { traderem }\end{array}$ & $\begin{array}{l}\text { turbbidos } \\
\text { turbines }\end{array}$ & $\begin{array}{l}\text { uenlet } \\
\text { uenibit }\end{array}$ & $\begin{array}{l}\text { uldeon } \\
\text { uideri }\end{array}$ & & $\begin{array}{l}\text { uostrarum } \\
\text { uostro }\end{array}$ \\
\hline tradiderunt & turbo & ueniisse & uideto & ulmearum & uostros \\
\hline tradier & turget & ueniret & uidetque & ulmeis & uotem \\
\hline $\begin{array}{l}\text { trado } \\
\text { tragulam }\end{array}$ & turpes & ueniri. & uideturne & ultumas & uotes. \\
\hline $\begin{array}{l}\text { tragulam } \\
\text { trahe }\end{array}$ & & uenisti & uidit & & urbani \\
\hline $\begin{array}{l}\text { trane } \\
\text { trahere }\end{array}$ & $\begin{array}{l}\text { turpilucricup1- } \\
\text { dum }\end{array}$ & $\begin{array}{l}\text { uenitne } \\
\text { uenti }\end{array}$ & $\begin{array}{l}\text { ungians } \\
\text { uigilanti }\end{array}$ & $\begin{array}{l}\text { umiditate } \\
\text { unae }\end{array}$ & $\begin{array}{l}\text { urbanus } \\
\text { urbis }\end{array}$ \\
\hline trahi & turpitudinem & uentriosus & uigilas & uncti & urit \\
\hline Tranione & tuquidem & uenturum & uigiliam & unctitant & urnam \\
\hline Tranium & turtures & uentus & uigilias & unctiusculo & uspiam \\
\hline tranquilla & tu's & uenustates & uigiliis & unctor & usquamst \\
\hline $\begin{array}{l}\text { tranquille } \\
\text { transcendere }\end{array}$ & $\begin{array}{l}\text { tus } \\
\text { tusculum }\end{array}$ & $\begin{array}{l}\text { uerberabilissume } \\
\text { uerberare }\end{array}$ & $\begin{array}{l}\text { uigilo } \\
\text { uilest }\end{array}$ & $\begin{array}{l}\text { undam } \\
\text { undique }\end{array}$ & $\begin{array}{l}\text { usquequaque } \\
\text { usquin }\end{array}$ \\
\hline transeat & tutin & uerberari & uiliores & unguem & ussurae \\
\hline transfigi & tutor & uerberarier & uilissumust & unguendam & ussust \\
\hline $\begin{array}{l}\text { transigam } \\
\text { transiges }\end{array}$ & $\begin{array}{l}\text { uaginam } \\
\text { ualeat }\end{array}$ & $\begin{array}{l}\text { uerberate } \\
\text { uerberauisti }\end{array}$ & $\begin{array}{l}\text { uim } \\
\text { uinarium }\end{array}$ & $\begin{array}{l}\text { unguis } \\
\text { ungulas }\end{array}$ & $\begin{array}{l}\text { usu } \\
\text { usum }\end{array}$ \\
\hline transmarinus & ualen & uerbeream & uincere & uniuorsis & usura \\
\hline transuorsum & ualent & uerberonem & uinces & uniuorsum & usurpari \\
\hline $\begin{array}{l}\text { transuorsus } \\
\text { treceni }\end{array}$ & $\begin{array}{l}\text { ualeo } \\
\text { ualete }\end{array}$ & $\begin{array}{l}\text { uerbist } \\
\text { uerbo }\end{array}$ & $\begin{array}{l}\text { uincite } \\
\text { uinciti' }\end{array}$ & unos & $\begin{array}{l}\text { usurpaui } \\
\text { usust }\end{array}$ \\
\hline trecentis & ualui & uerbumst & uinctam & uocabo & $\begin{array}{l}\text { usust } \\
\text { utar }\end{array}$ \\
\hline tribu'ne & ualuisti & uereare & uinctus & uocari & utare \\
\hline tricas & ualuistin & uerecundari & uindex & uocauerit & utenda \\
\hline triennio & $\begin{array}{l}\text { uana } \\
\text { uanidicis }\end{array}$ & $\begin{array}{l}\text { uerecundiam } \\
\text { uerumst }\end{array}$ & $\begin{array}{l}\text { unnolentum } \\
\text { uiolarii }\end{array}$ & $\begin{array}{l}\text { uoces } \\
\text { uocis }\end{array}$ & $\begin{array}{l}\text { utendumst } \\
\text { uterum }\end{array}$ \\
\hline triennium & uapulem & uerus & uirgarum & uocitantur & utibile \\
\hline trifur & uapulo & uesanum & uirginis & Volcano & utibiles \\
\hline $\begin{array}{l}\text { trifurcifer } \\
\text { triplici }\end{array}$ & $\begin{array}{l}\text { uaria } \\
\text { uasa }\end{array}$ & uestibulum & uirgis & uolebam & utile \\
\hline $\begin{array}{l}\text { trpnlic } \\
\text { triplicis }\end{array}$ & $\begin{array}{l}\text { uasa } \\
\text { uasis }\end{array}$ & $\begin{array}{l}\text { uestrpıca } \\
\text { uestitu }\end{array}$ & $\begin{array}{l}\text { uiris } \\
\text { uiritim }\end{array}$ & $\begin{array}{l}\text { uolebas } \\
\text { uolgo }\end{array}$ & $\begin{array}{l}\text { utitur } \\
\text { utra }\end{array}$ \\
\hline tristi & uastities & uestitu's & uirium & uolpes & utramque \\
\hline trisuiros & uaticinamini & uetere & uiro & uolsus & utrique \\
\hline triuenefica & uberi & ueterem & uirtus & uolturium & utrisque \\
\hline triuerunt & uberrume & ueteris & uirtuti & uolueram & utrumuis \\
\hline mphe & $\begin{array}{l}\text { uberrumos } \\
\text { ubiauomaue }\end{array}$ & $\begin{array}{l}\text { uetulae } \\
\text { vexillum }\end{array}$ & $\begin{array}{l}\text { uirtutibus } \\
\text { uirtutis }\end{array}$ & $\begin{array}{l}\text { uoluere } \\
\text { voluimus }\end{array}$ & $\begin{array}{l}\text { uxorio } \\
\text { Xytilis }\end{array}$ \\
\hline $\begin{array}{l}\text { trina } \\
\text { triplicia }\end{array}$ & uecturam & ui & $\begin{array}{l}\text { uurtutis } \\
\text { uisam }\end{array}$ & $\begin{array}{l}\text { uoluimus } \\
\text { uoluisses }\end{array}$ & $\begin{array}{l}\text { Xytulis } \\
\text { zamiam }\end{array}$ \\
\hline Troianum & uehar & uiaticum & uise & uoluisti & zonarii \\
\hline Truthus & eenter & uicem & uisitata & volup & zonarius \\
\hline tuaeque & uehunt & & & & \\
\hline
\end{tabular}

[5I] 
Brought to you by | MPI fuer Psycholinguistik Authenticated Download Date | 7/4/17 11:30 AM 
APPENDIX B 
Brought to you by | MPI fuer Psycholinguistik Authenticated Download Date | 7/4/17 11:30 AM 


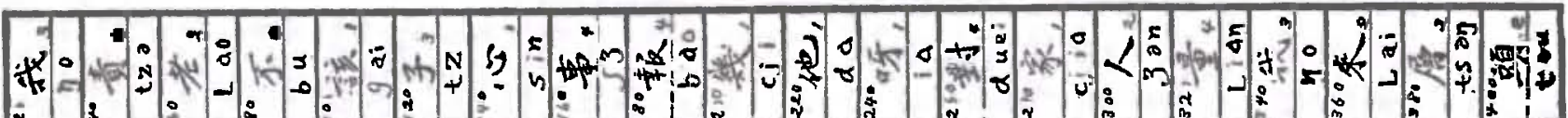

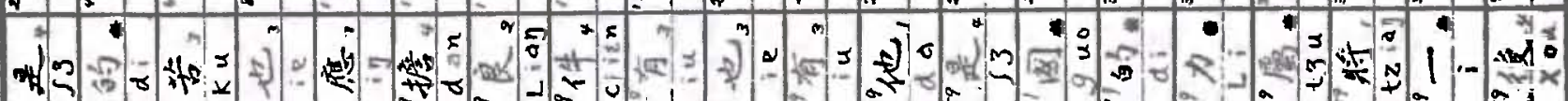

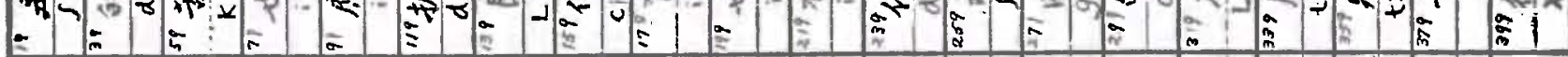

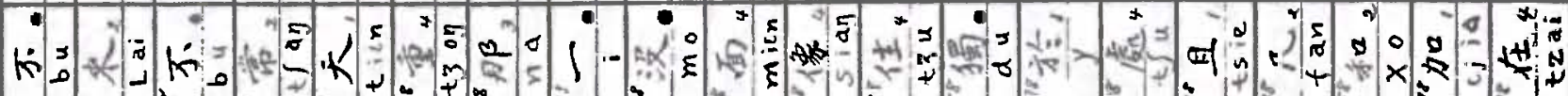

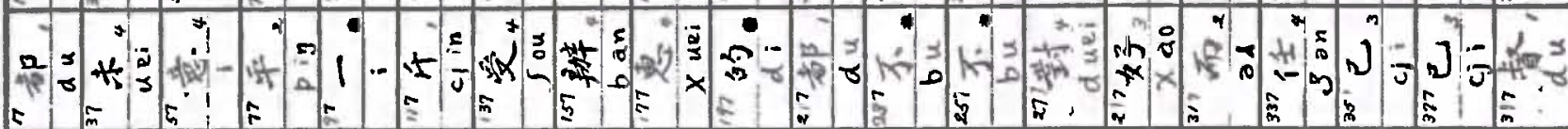

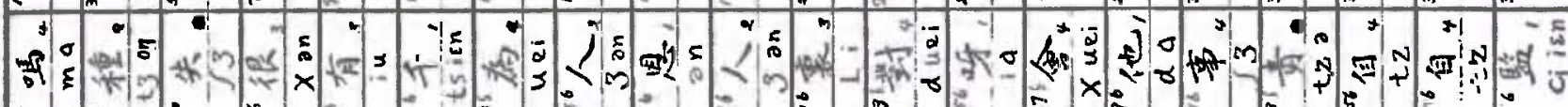

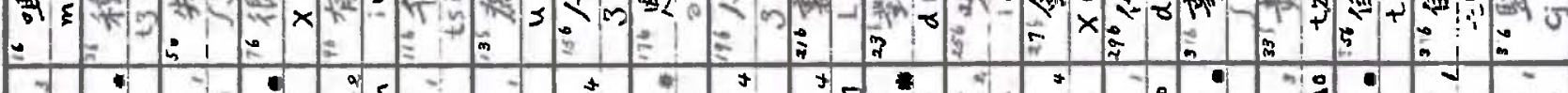

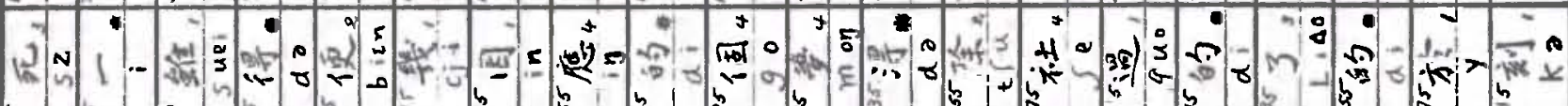

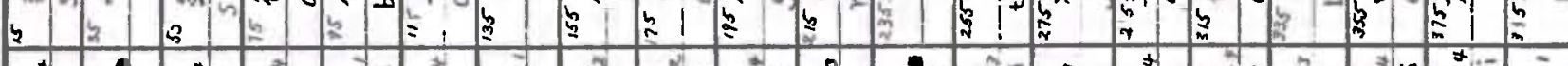

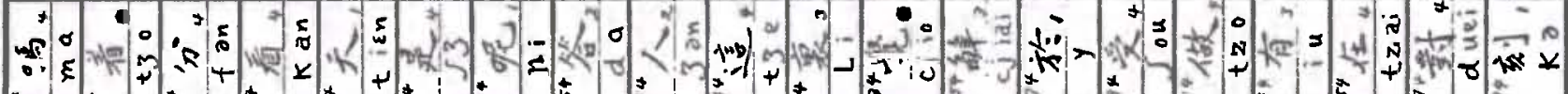

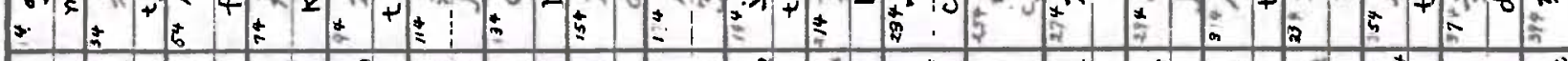

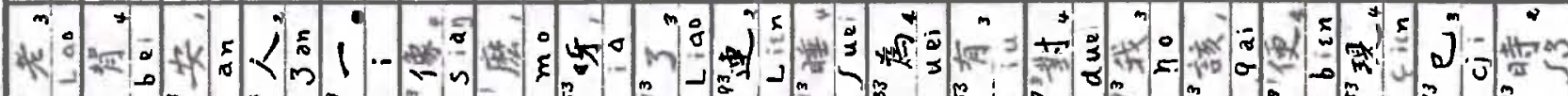
C m m m m m m m $m$

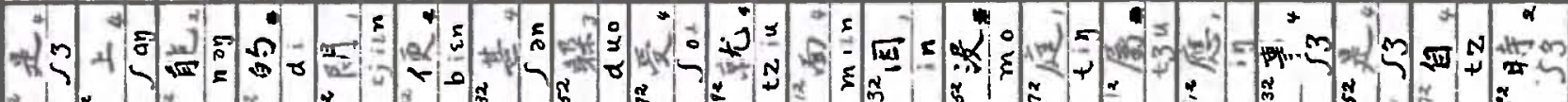

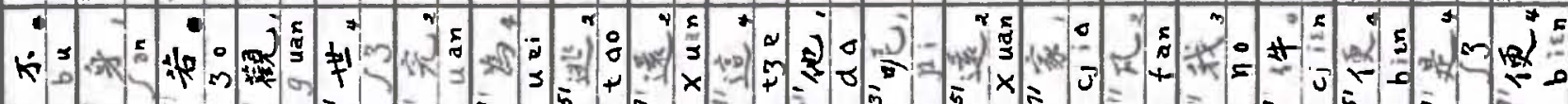

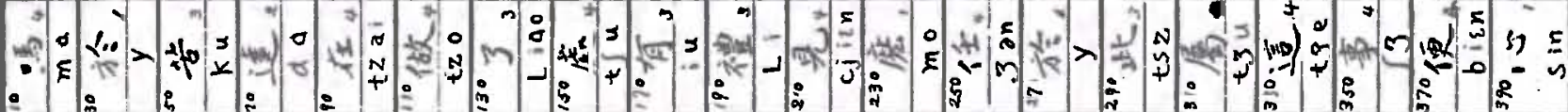

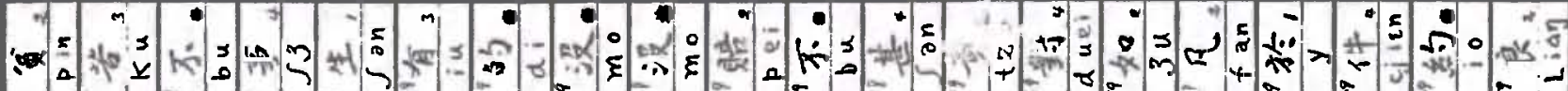

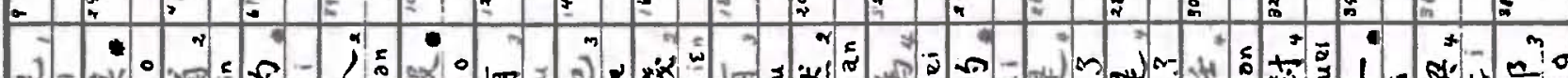

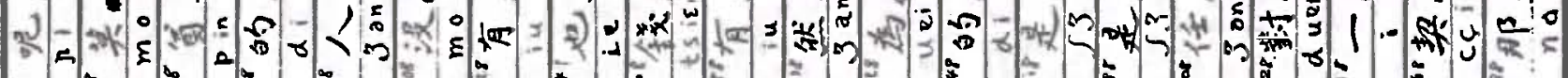

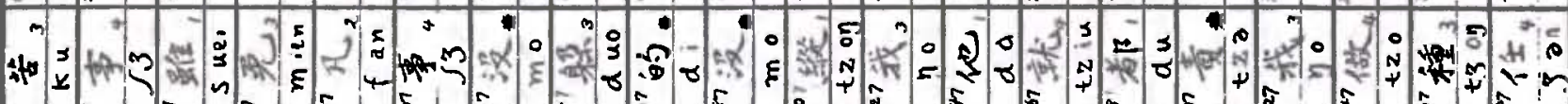

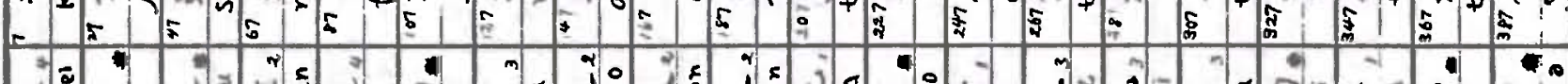

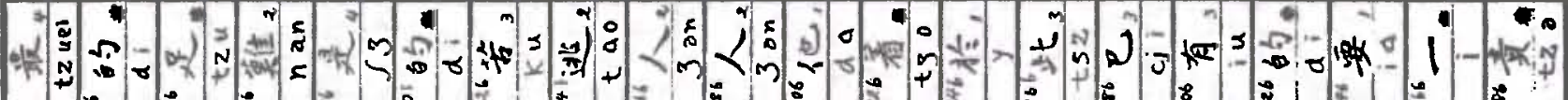
-

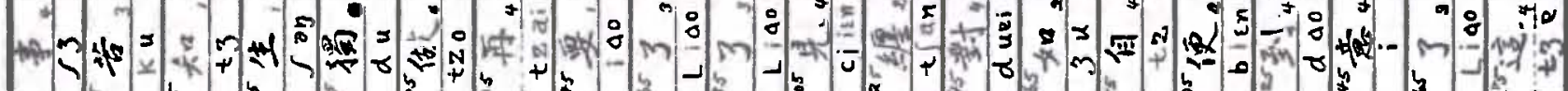

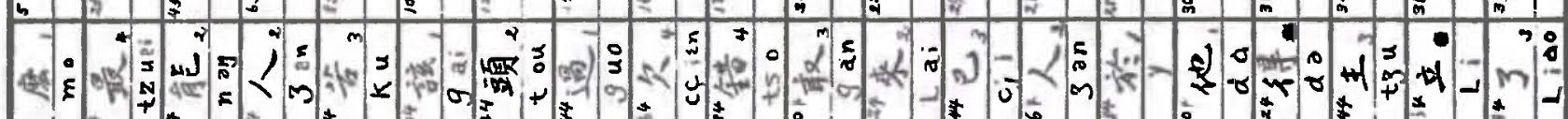

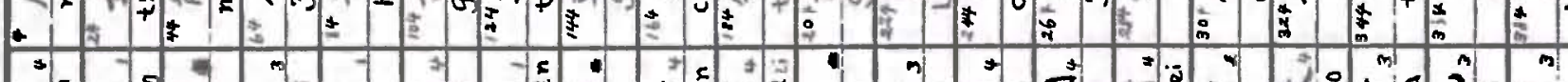

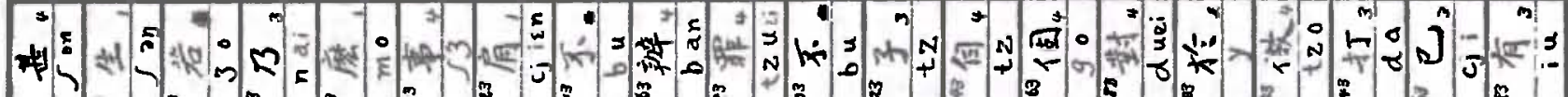

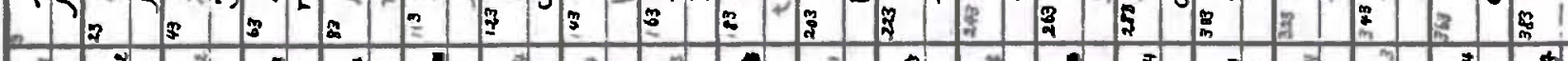

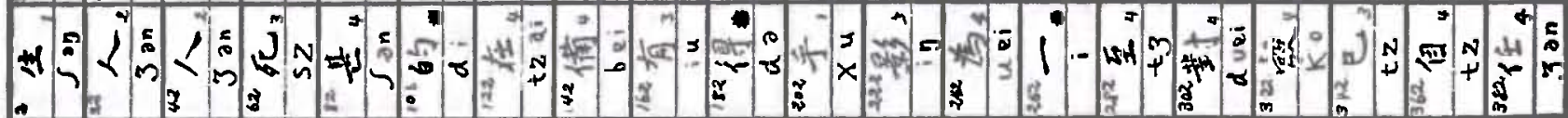

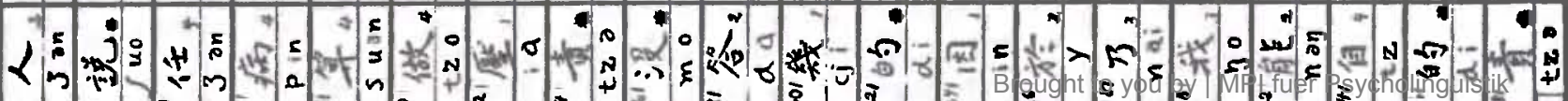


15.5

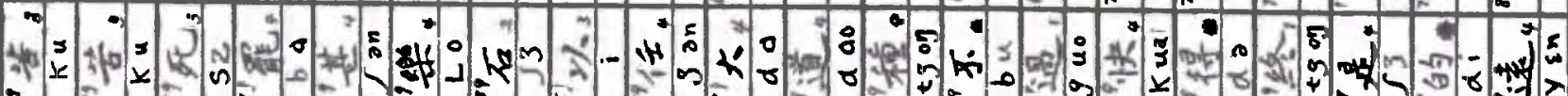

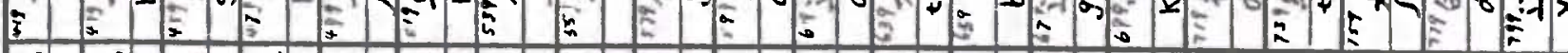

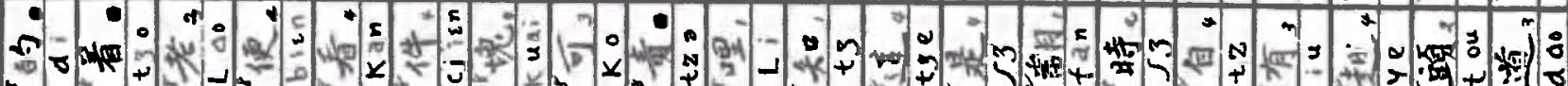
c $=2$

5) 5

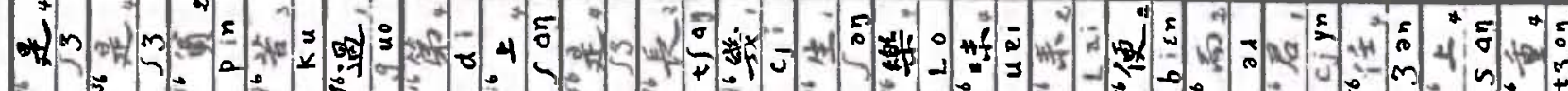

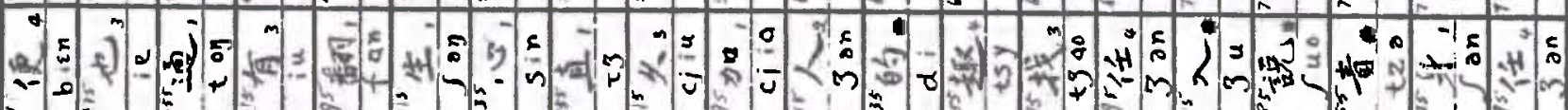

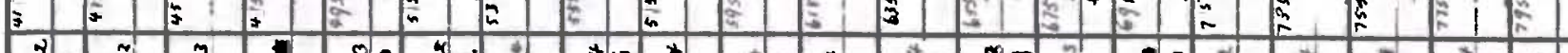

(20.

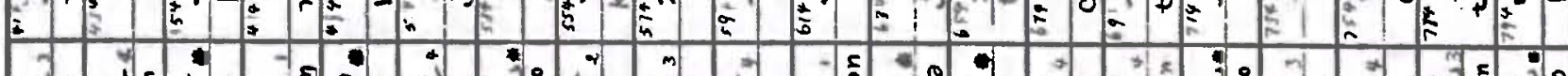

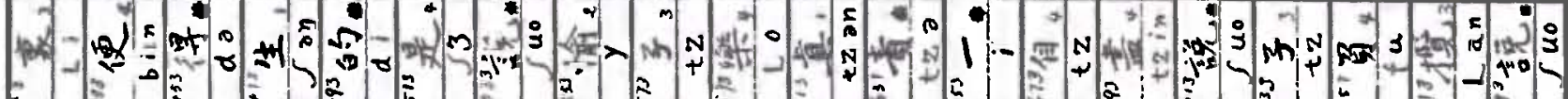

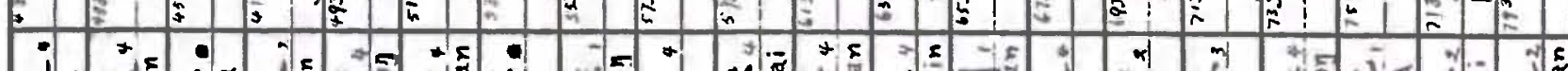

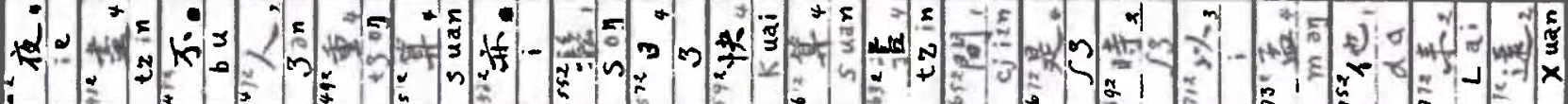

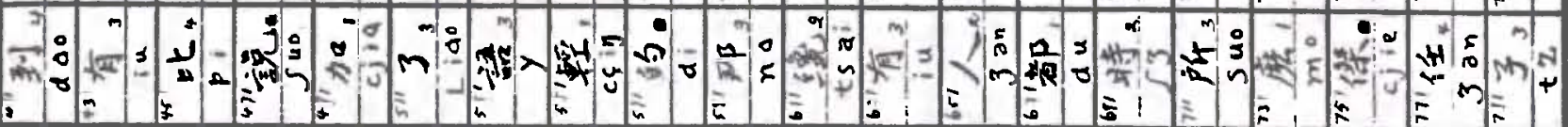

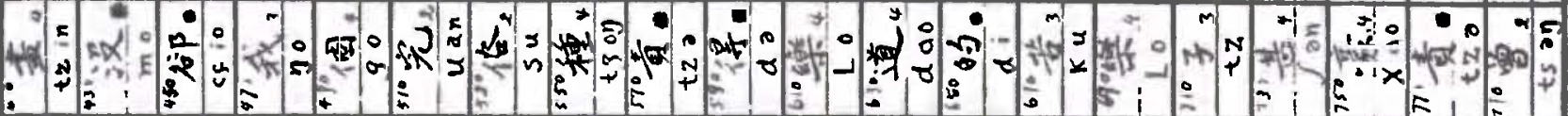

for 3 (

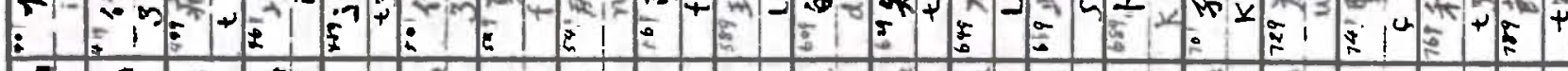

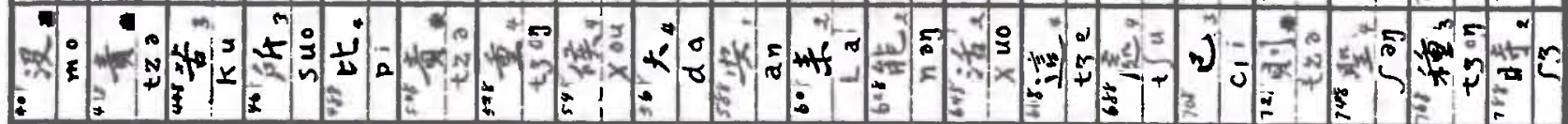

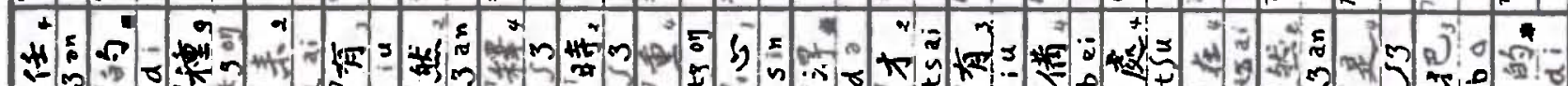

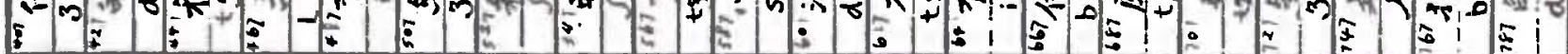

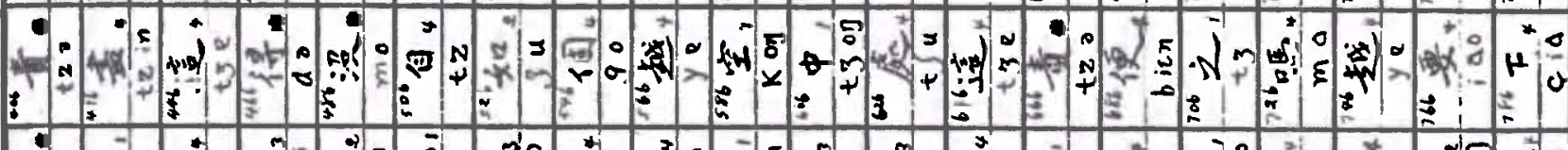

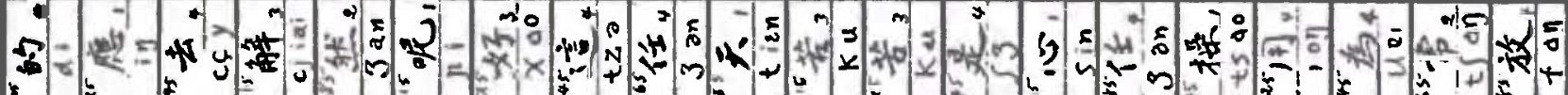

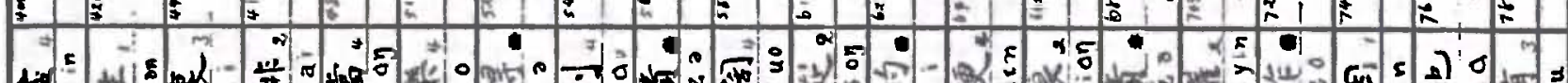

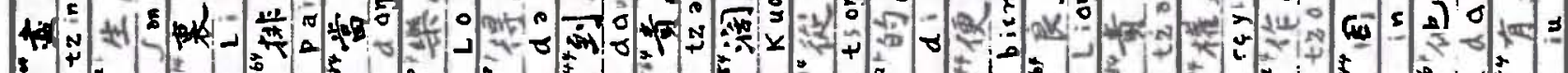

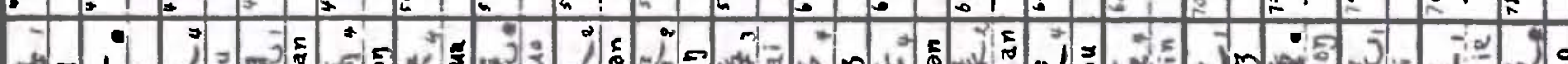

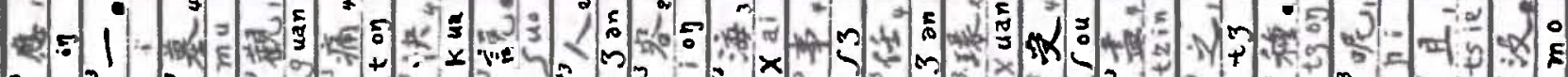

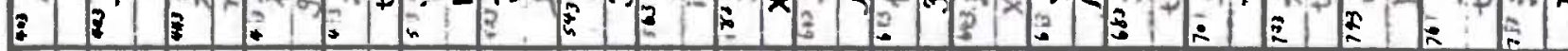

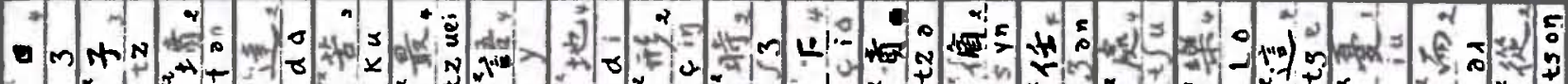

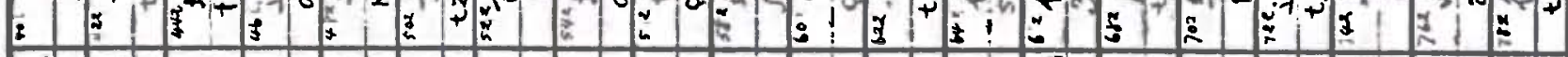

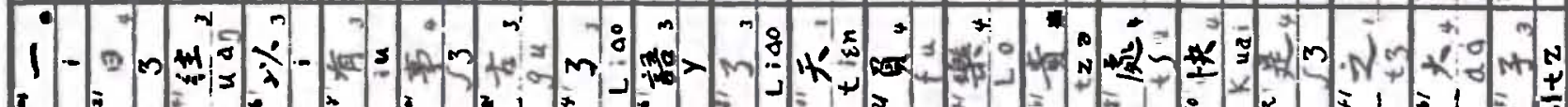

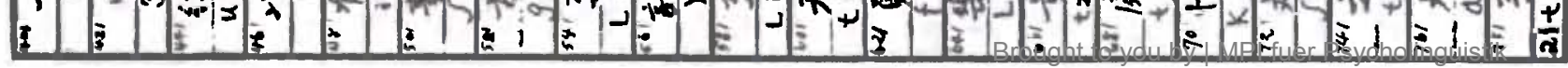




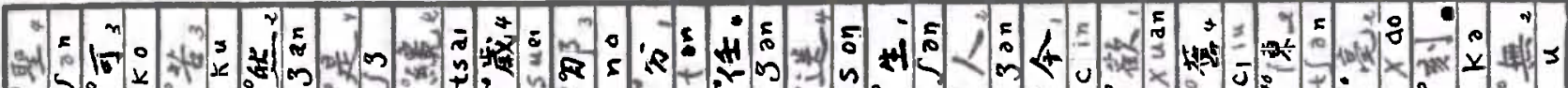
A

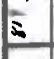

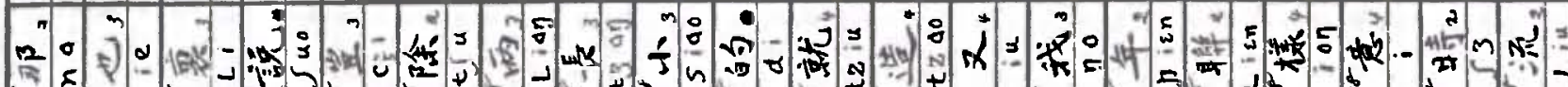

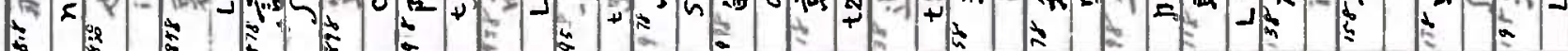

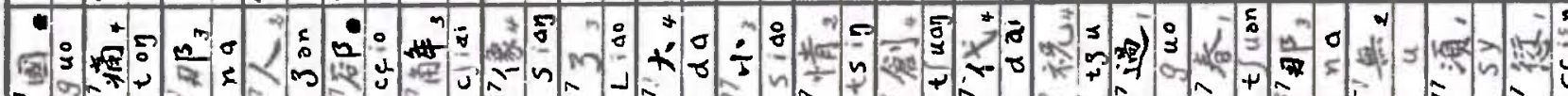
$E$ E

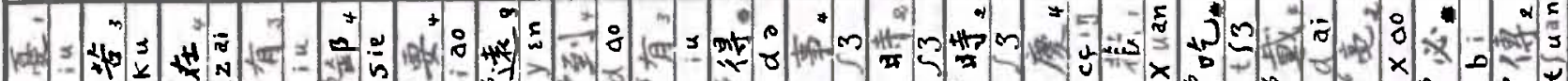
(2)

Y 5 . 7 -

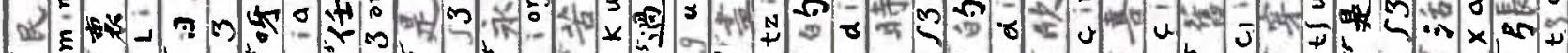

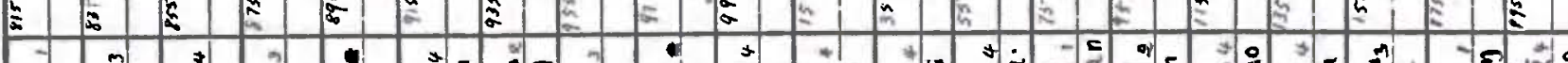

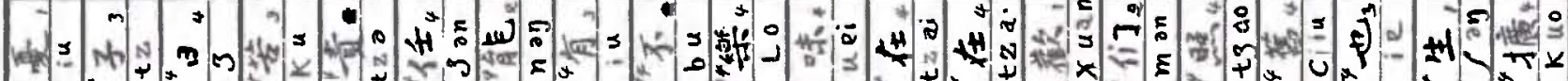

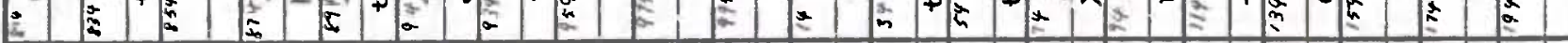
G.

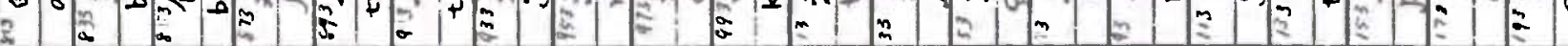

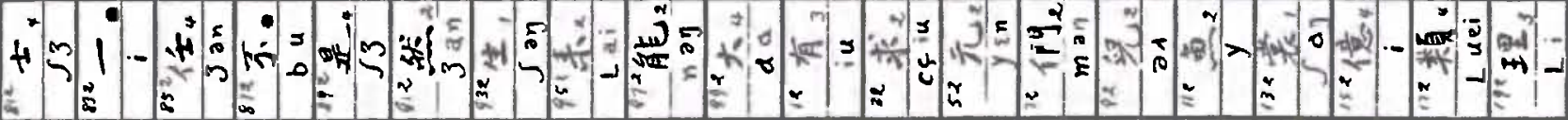

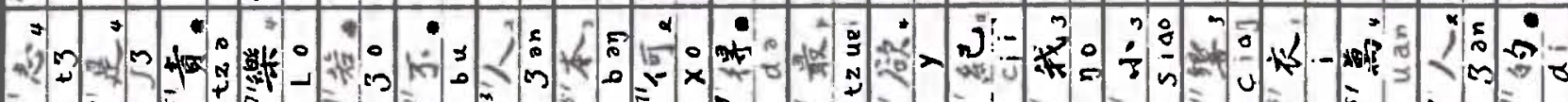

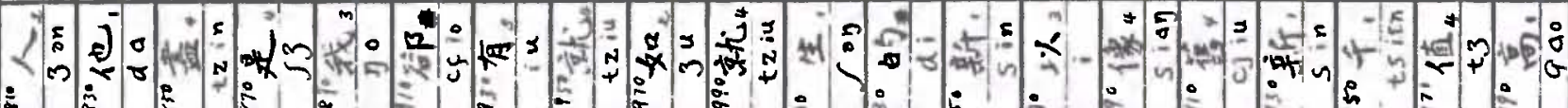

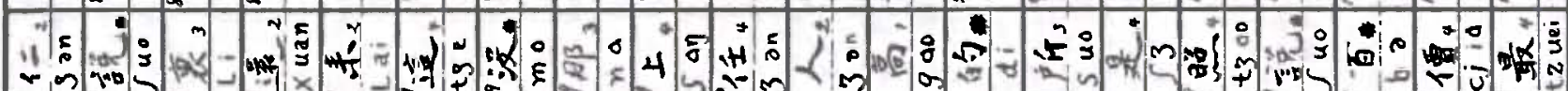

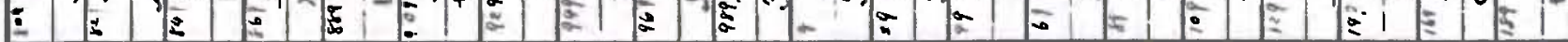

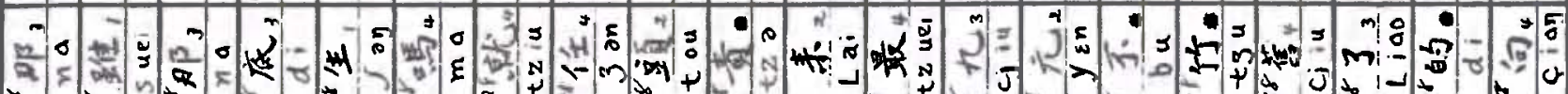

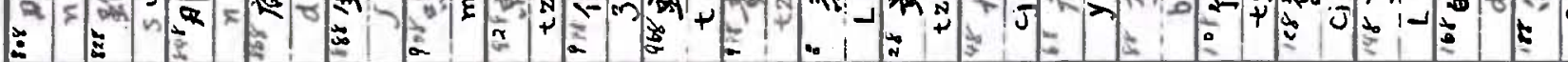

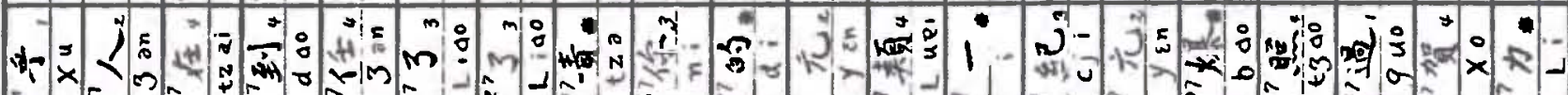

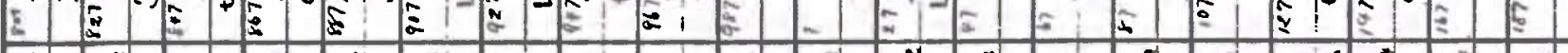

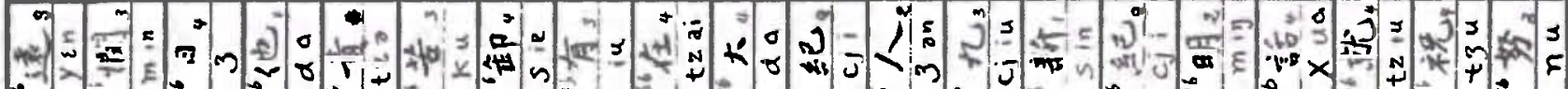

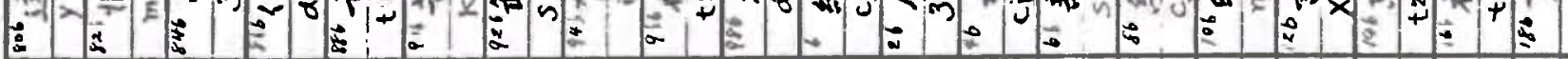

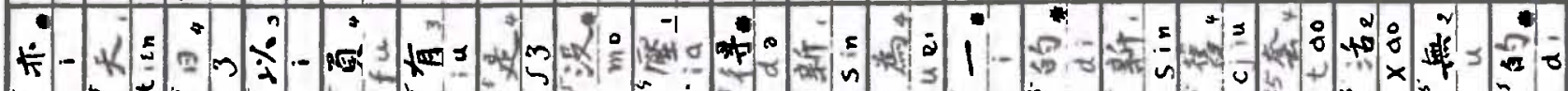

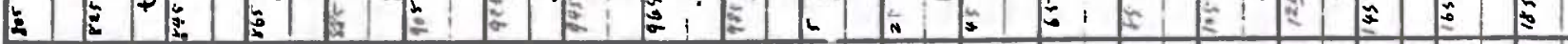

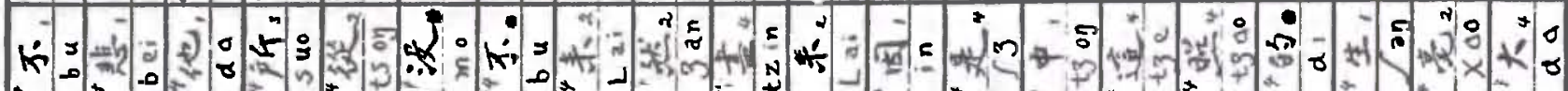

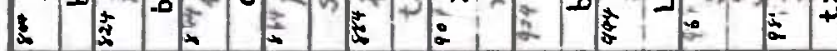

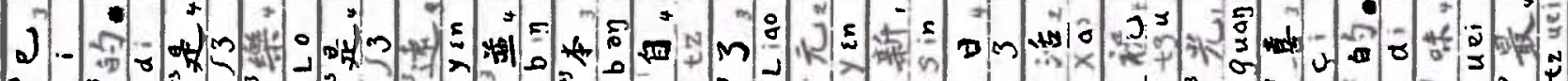
:

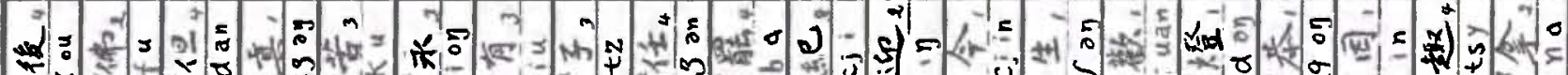

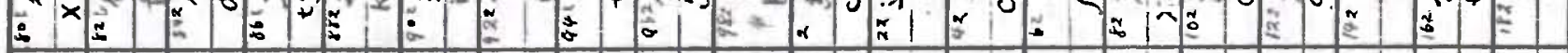

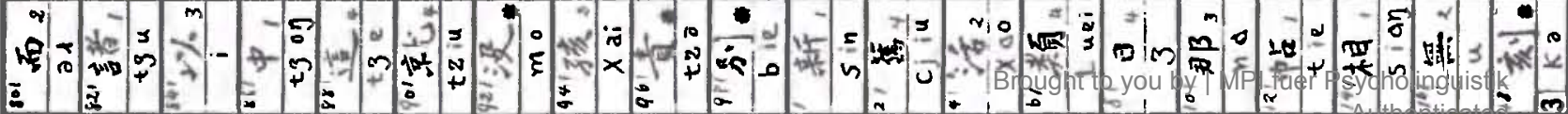




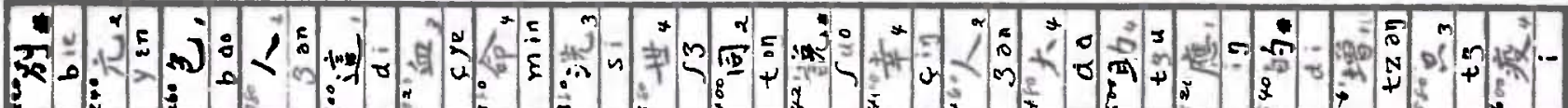
2y.

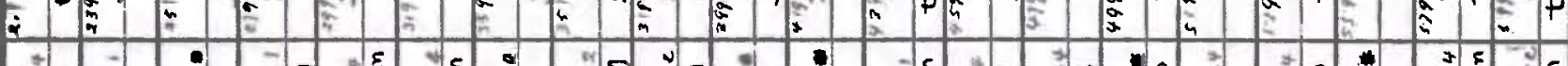

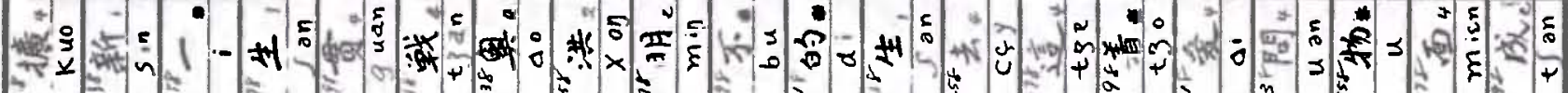

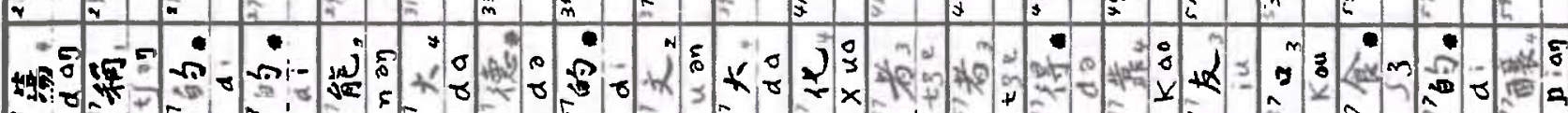
a

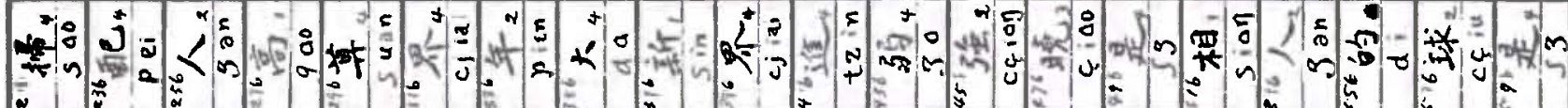

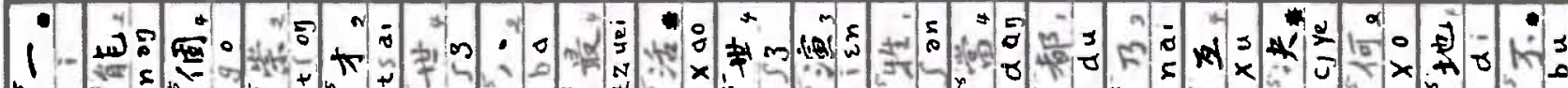

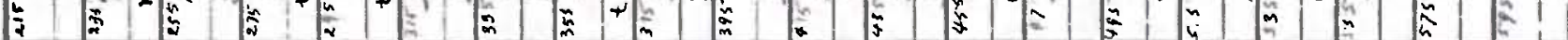

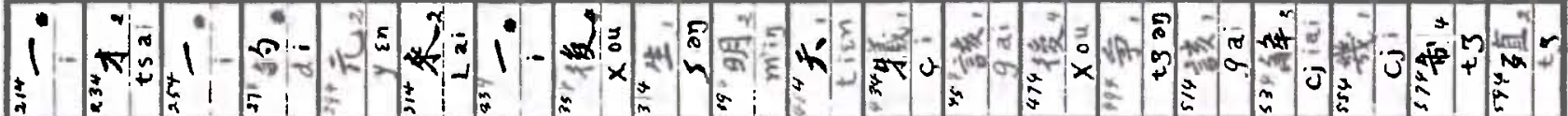

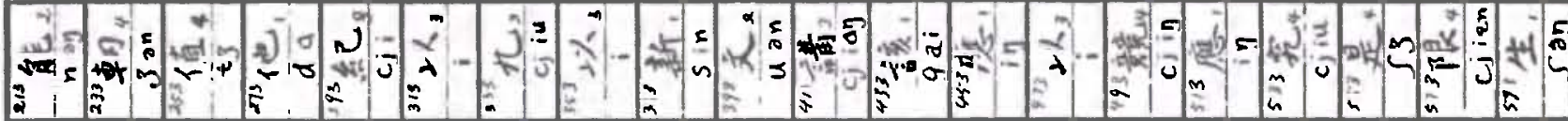
स

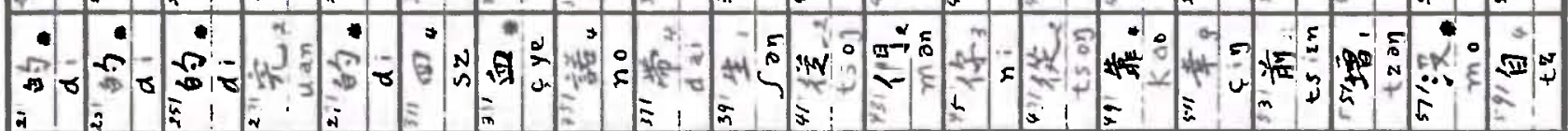

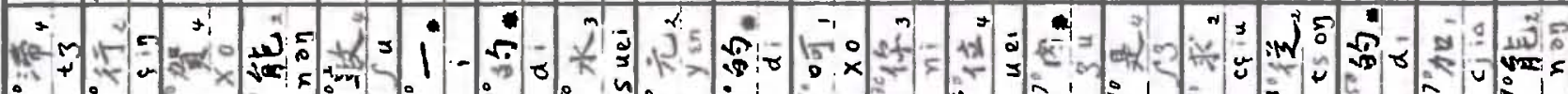

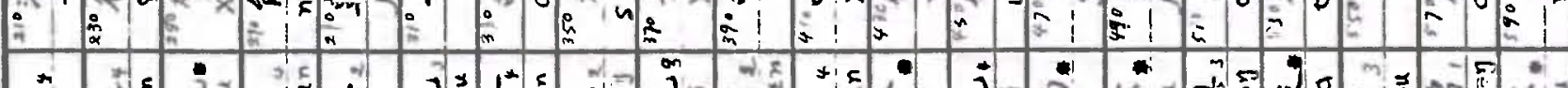

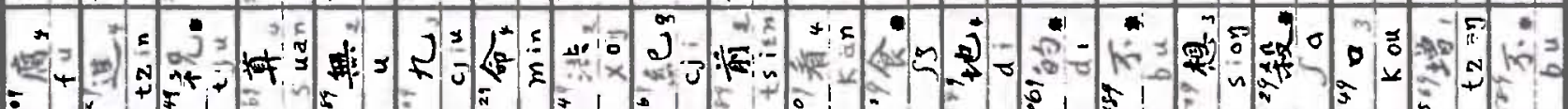

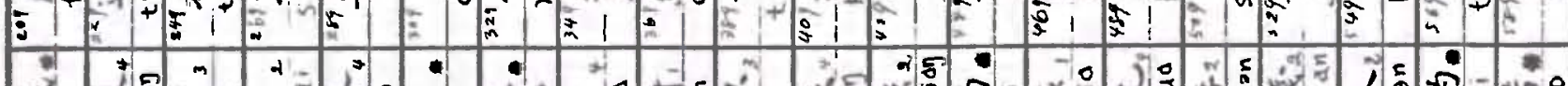

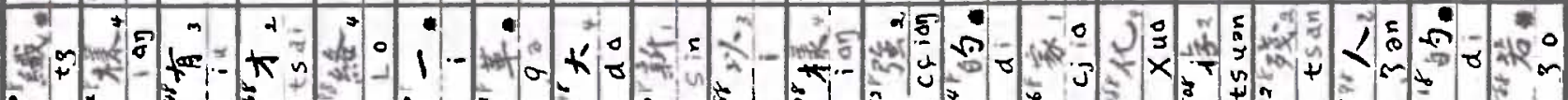

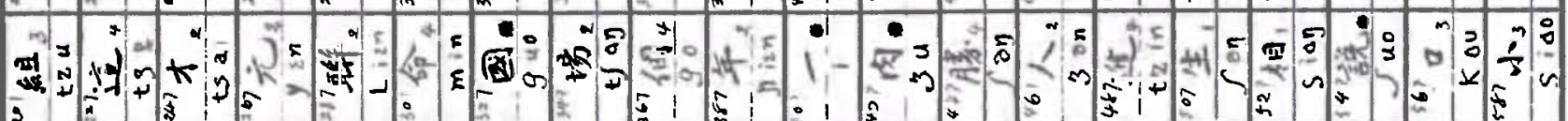

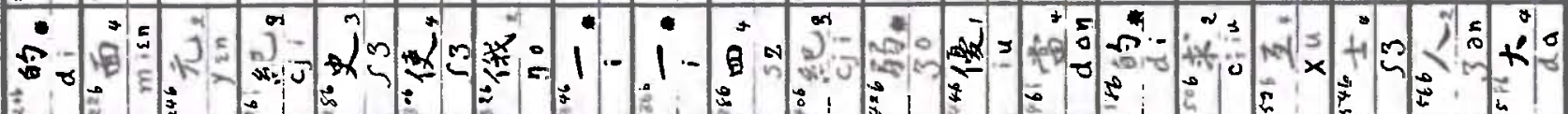

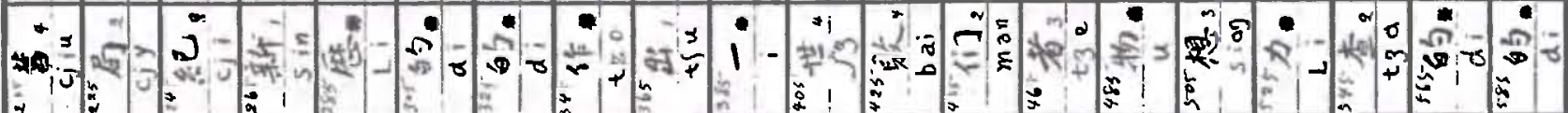

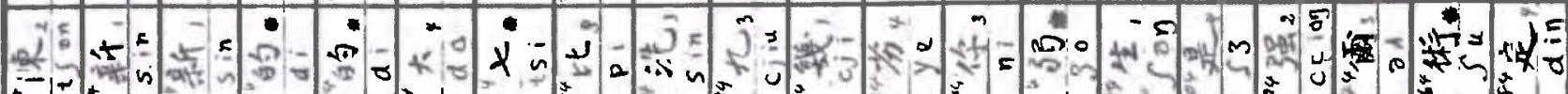

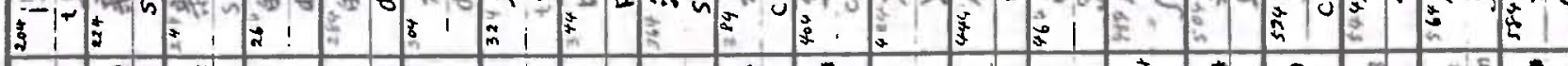

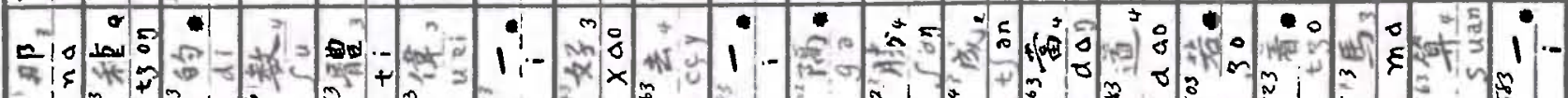

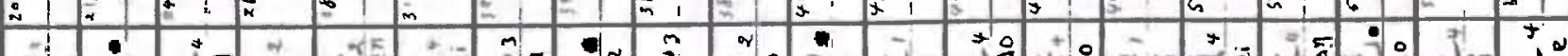

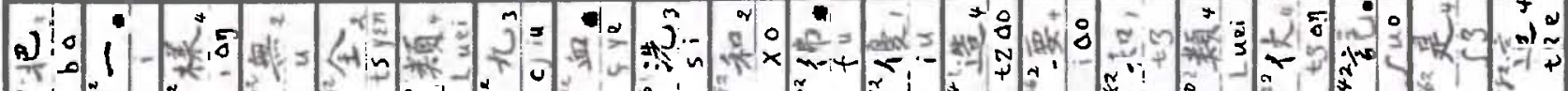

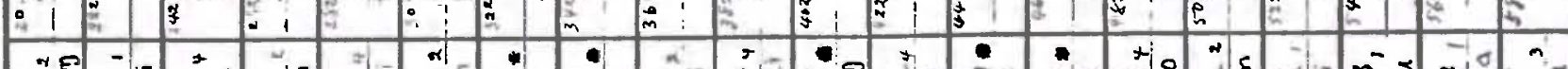
(a) इ U. 


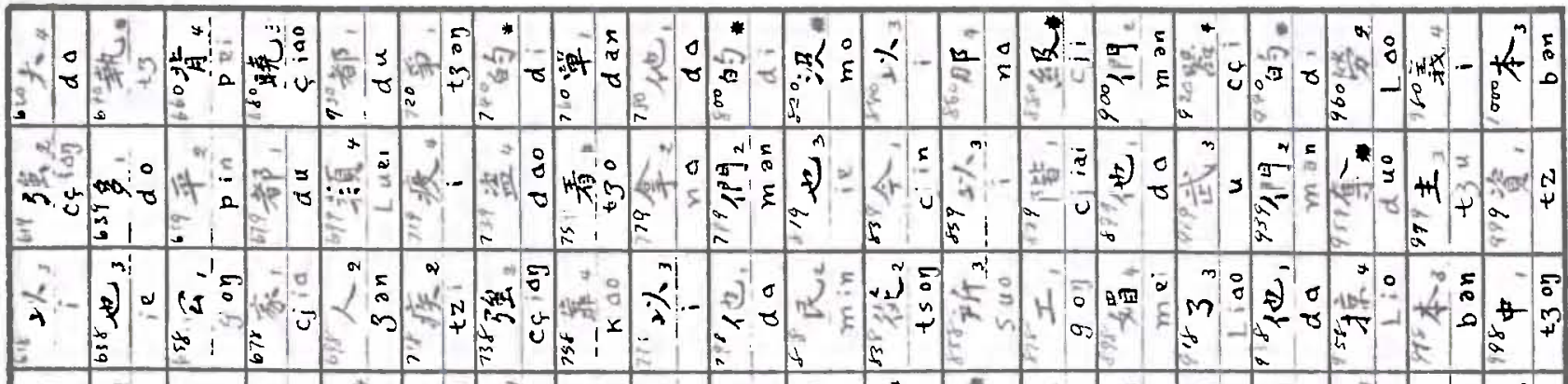

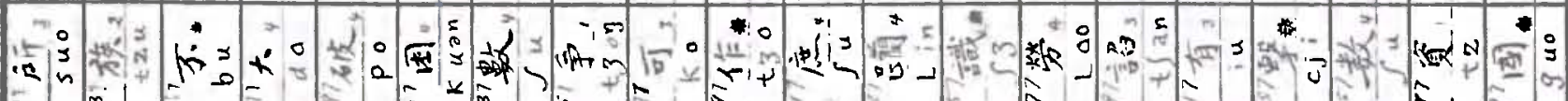

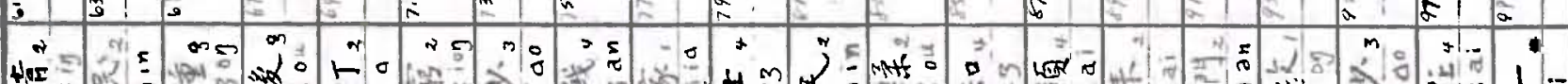

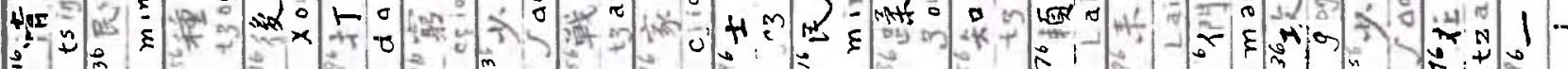

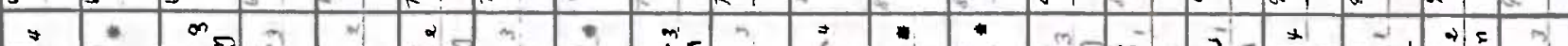

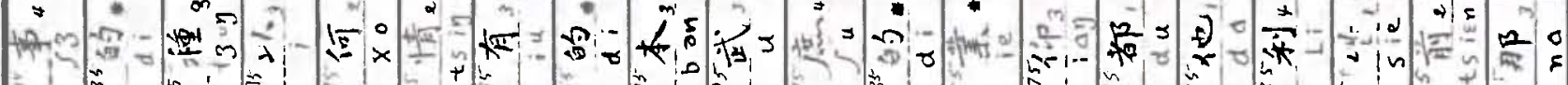

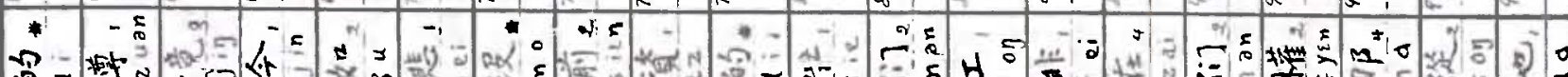
$\checkmark$ o ot

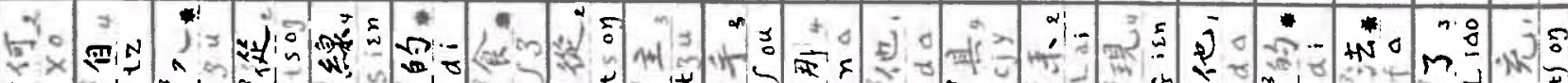

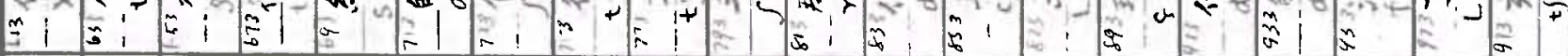

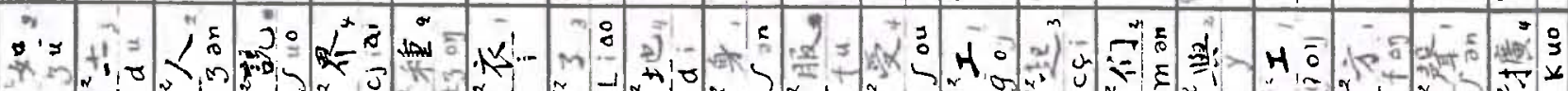
3.

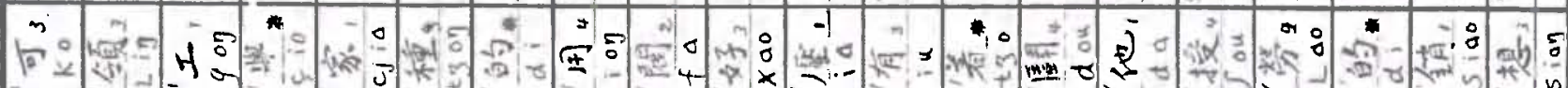

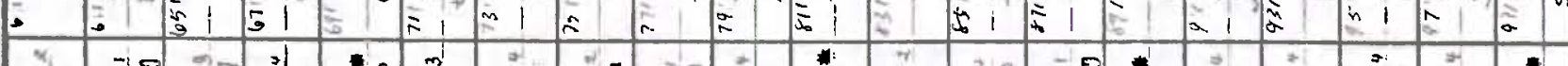

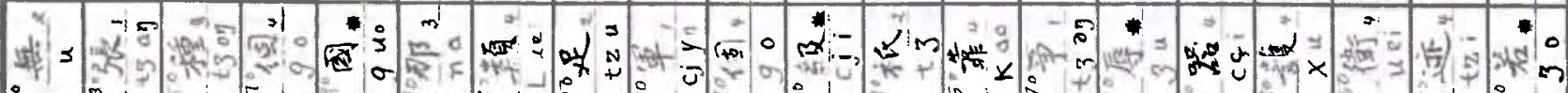

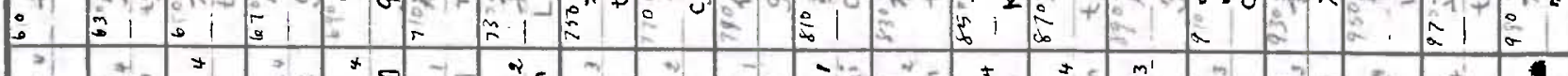

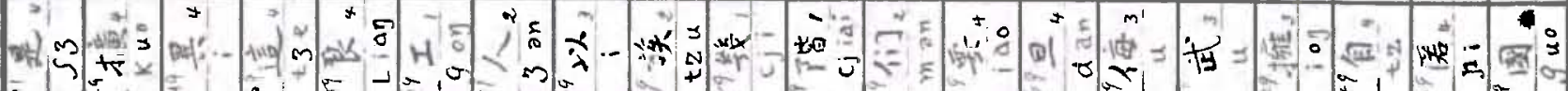

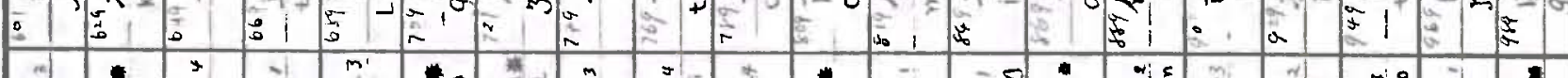

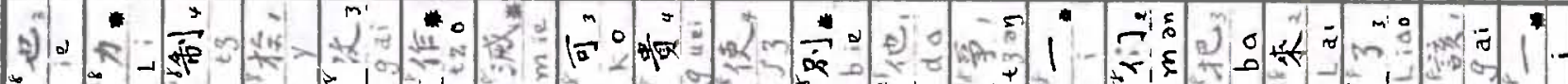

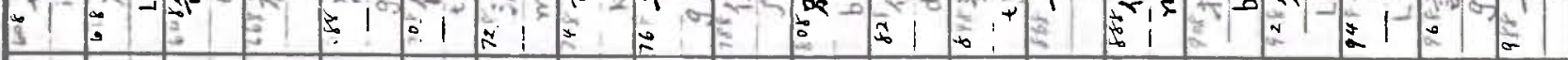

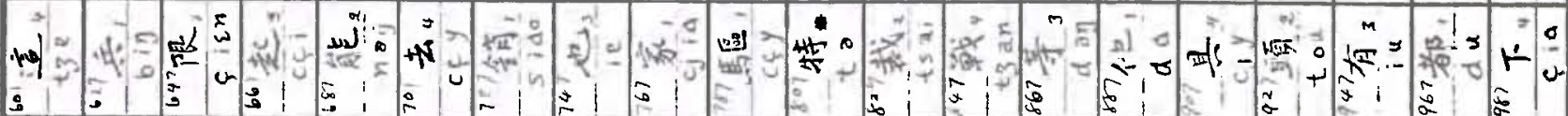

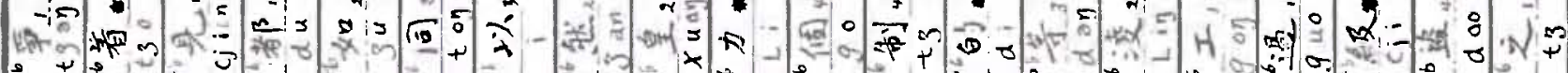

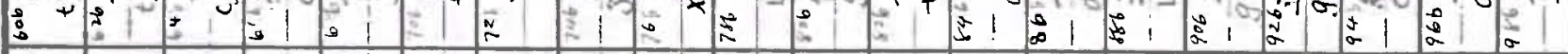

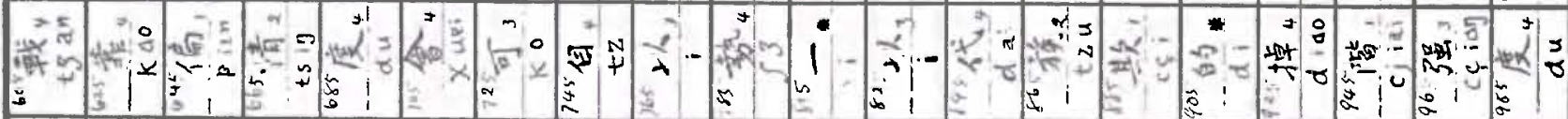

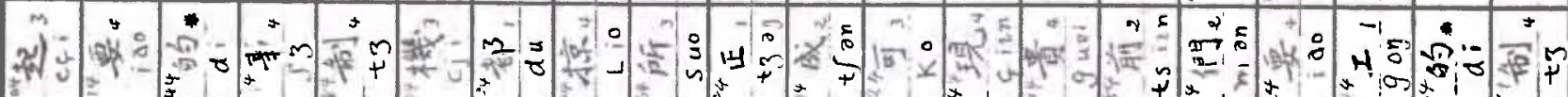

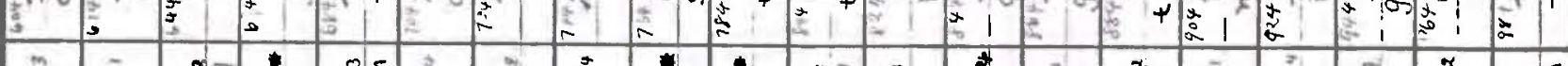

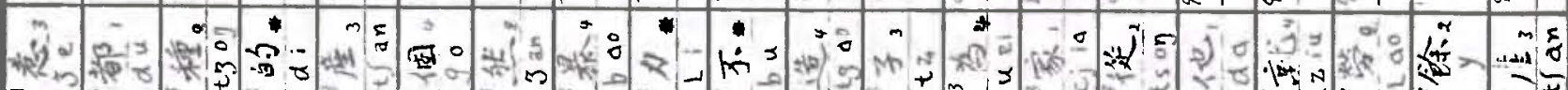

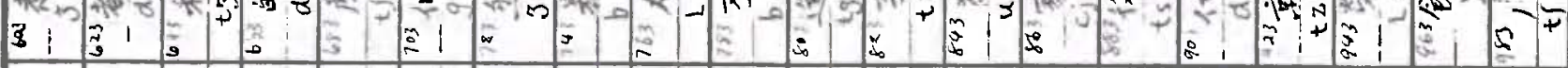

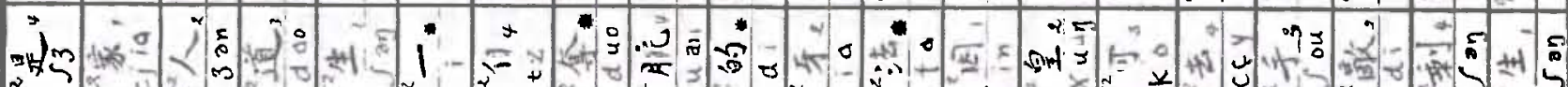
药

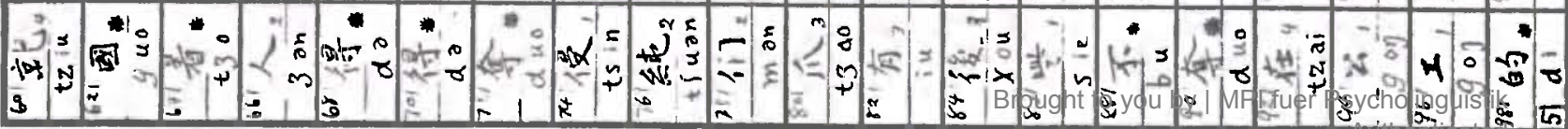




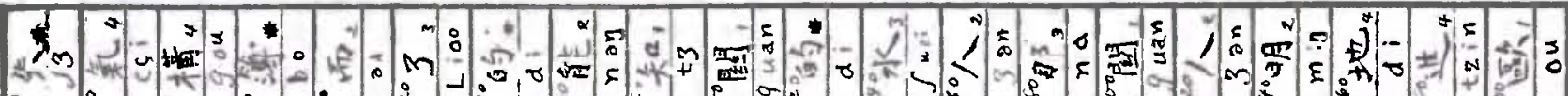

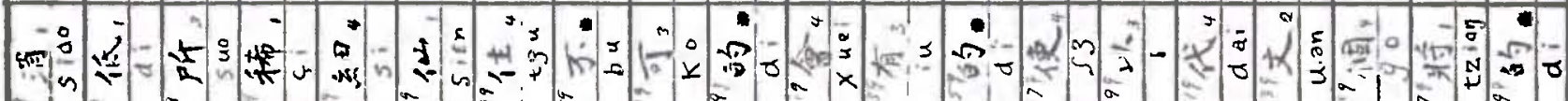
KAl

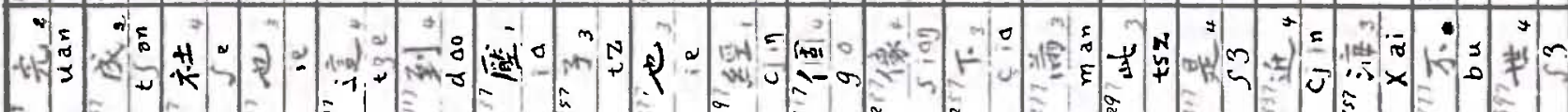

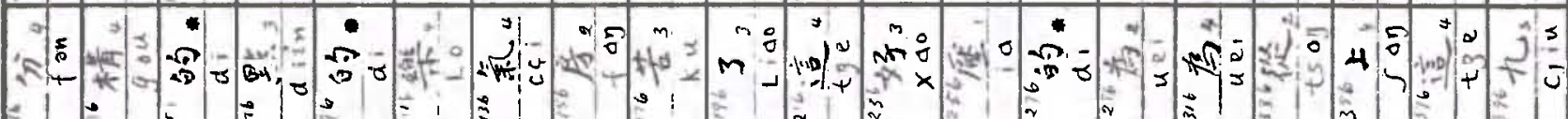

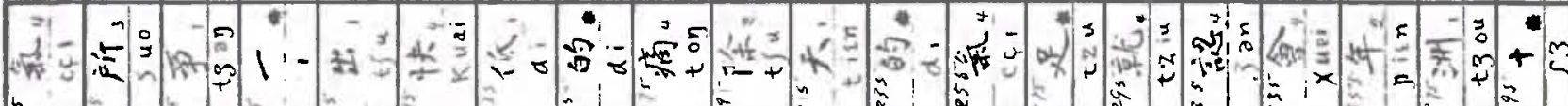

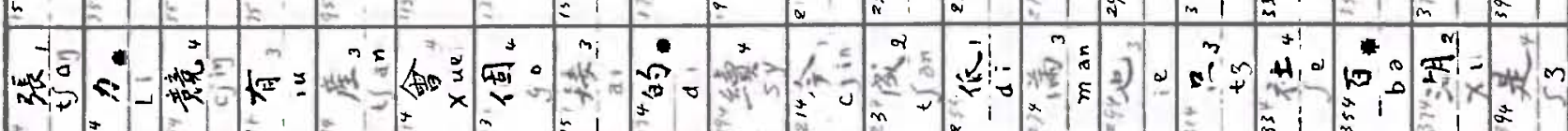

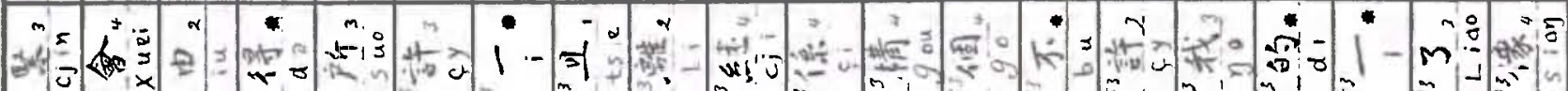

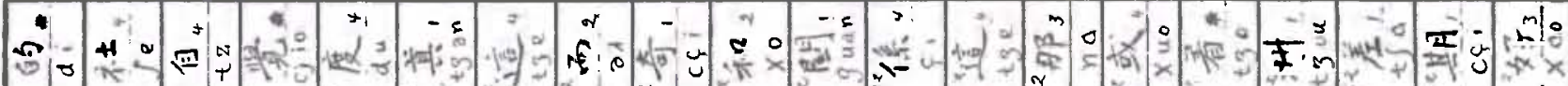

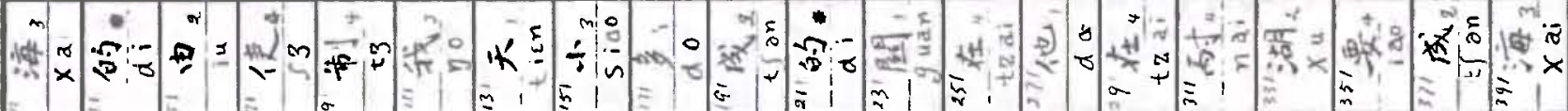

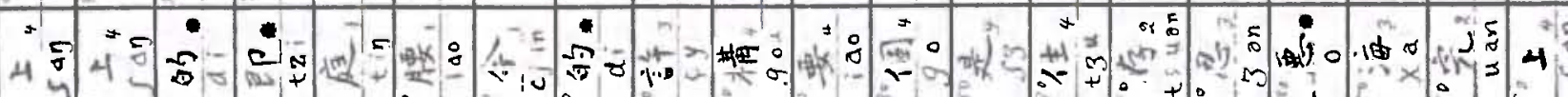
H

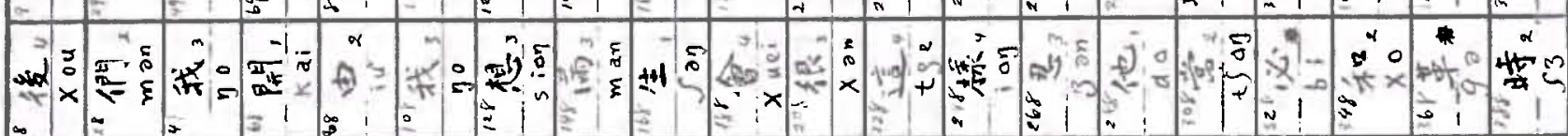

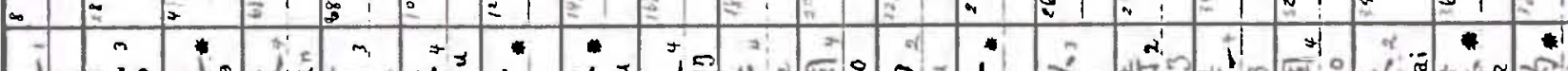

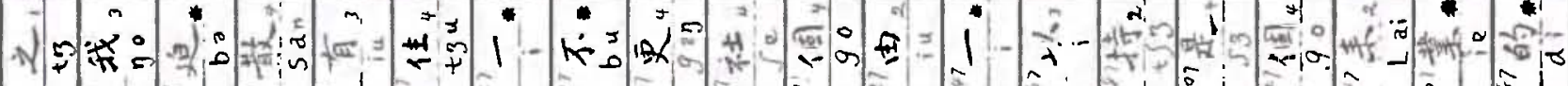

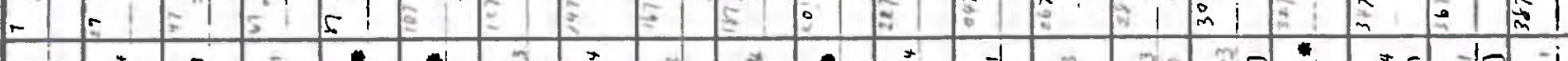

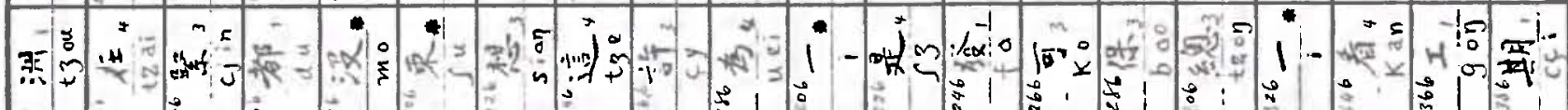

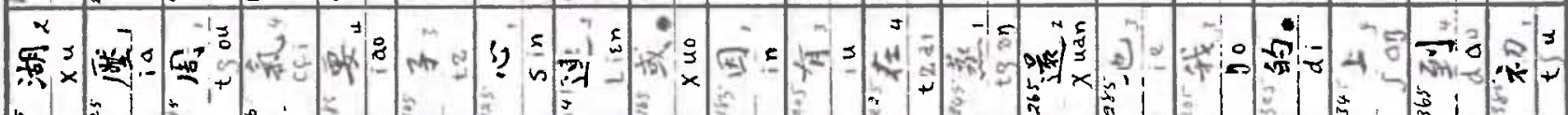

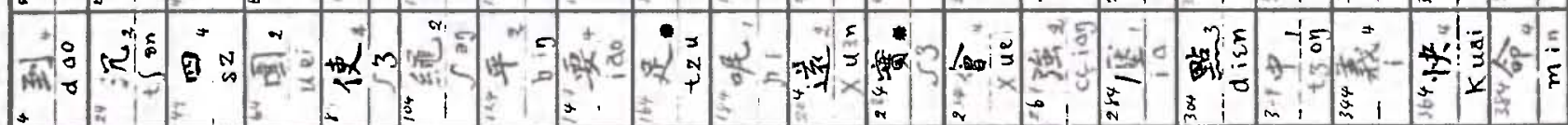

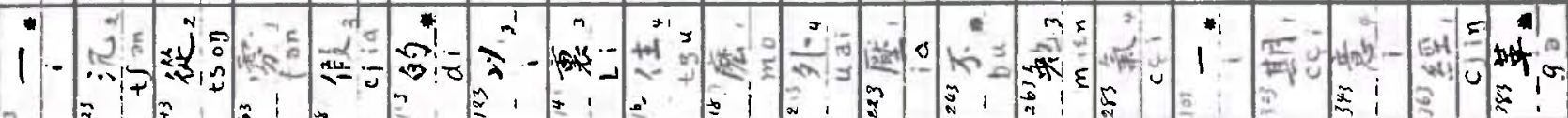

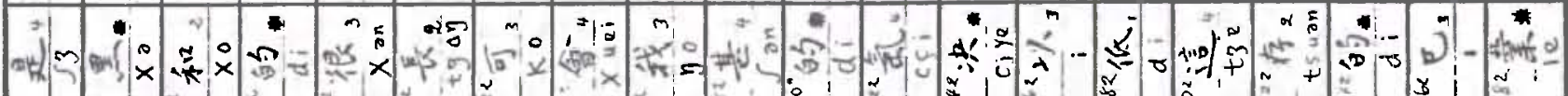

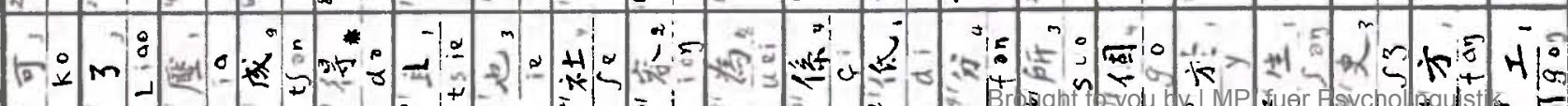




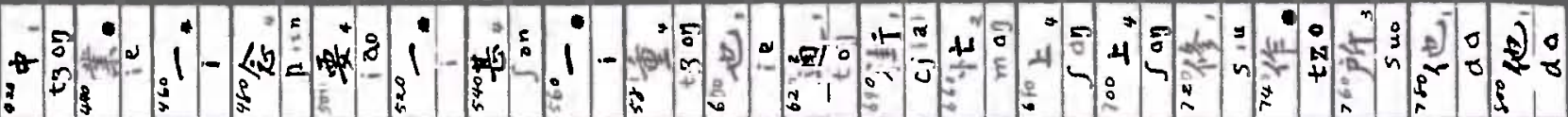

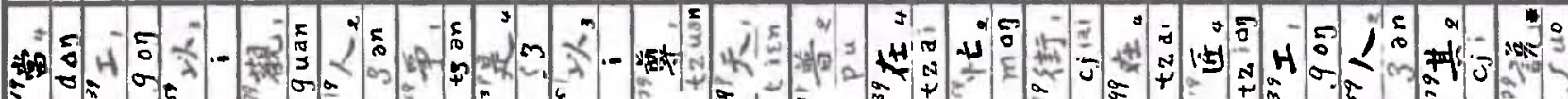

(1)

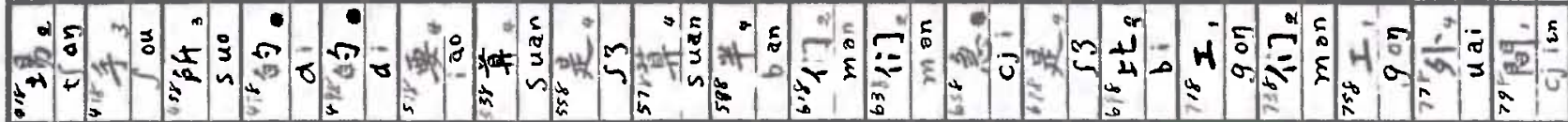

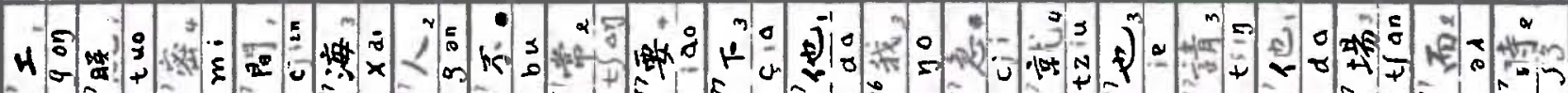

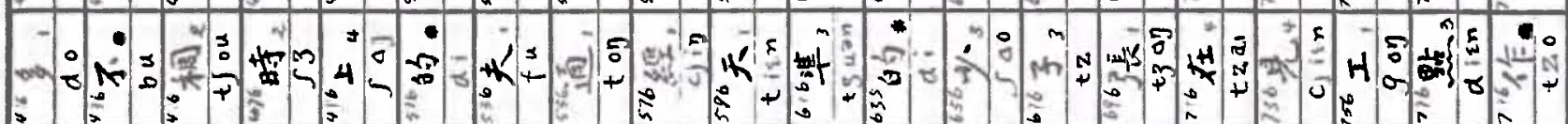

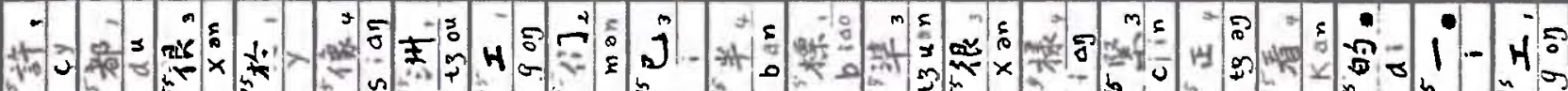

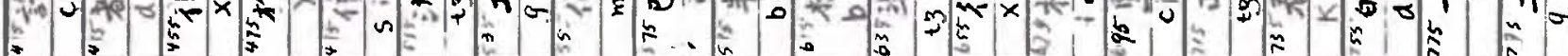

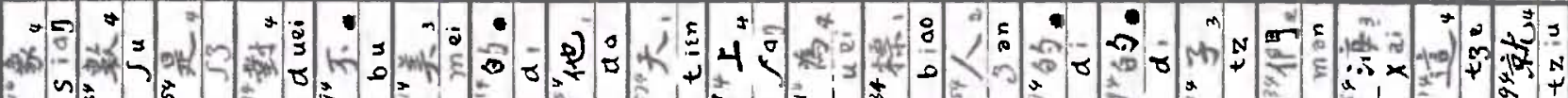

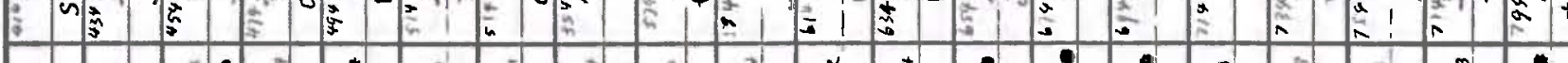

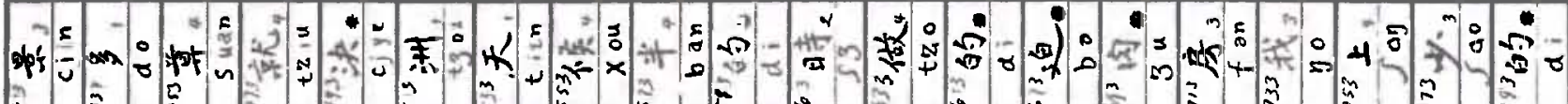

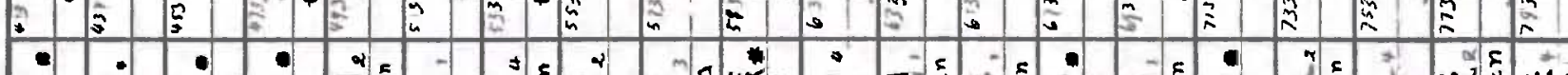

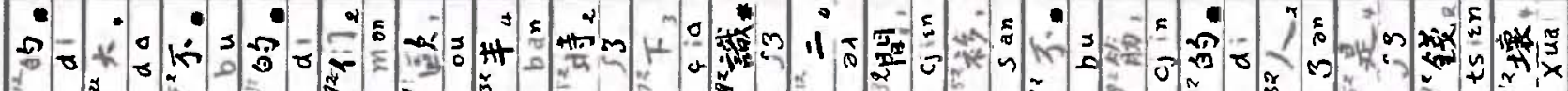

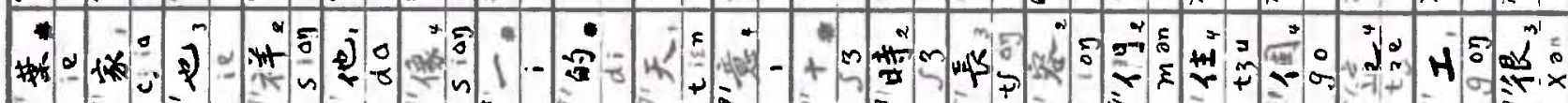
-

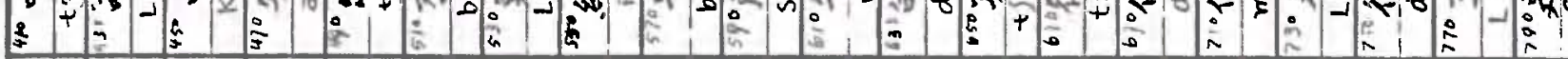

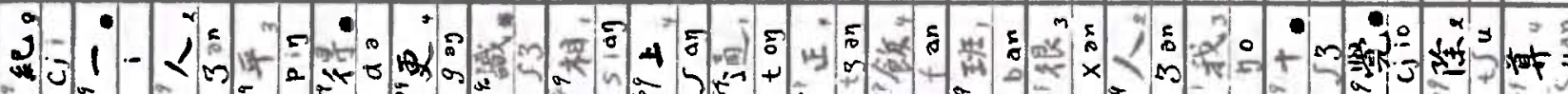

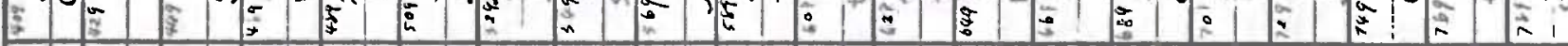

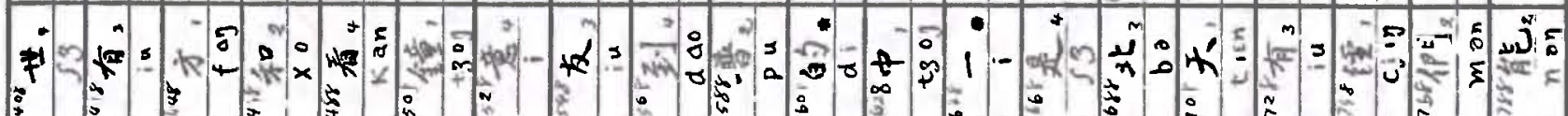

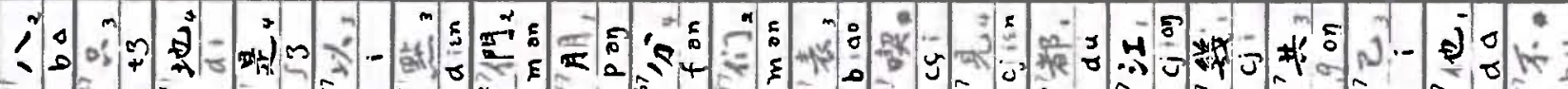

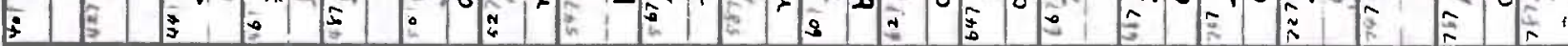
$+2=0$ is

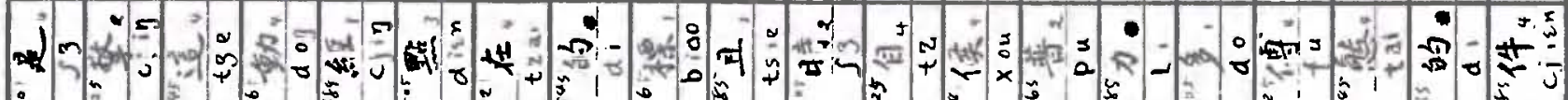

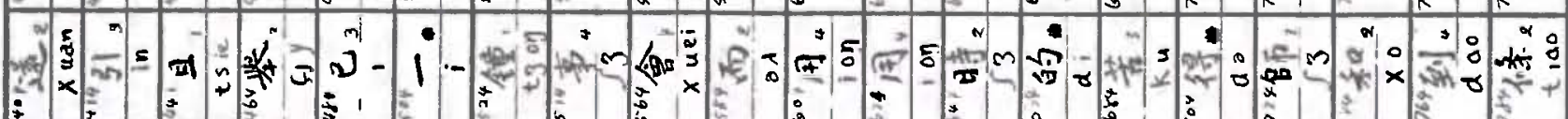

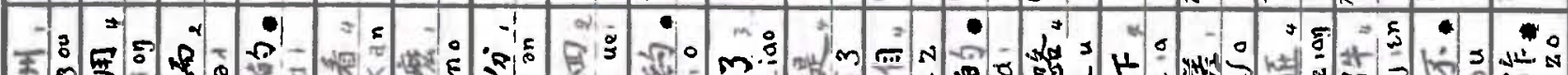

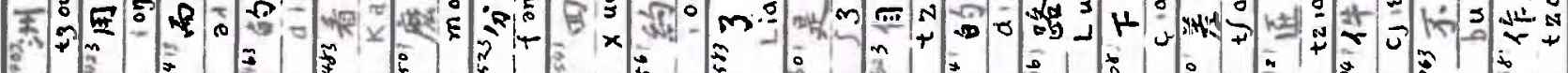

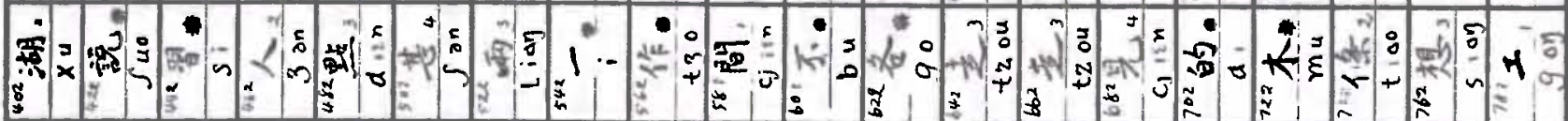

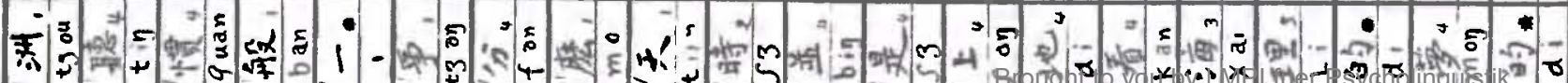

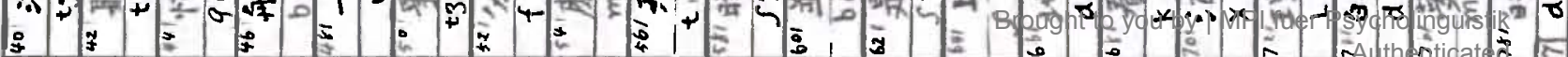




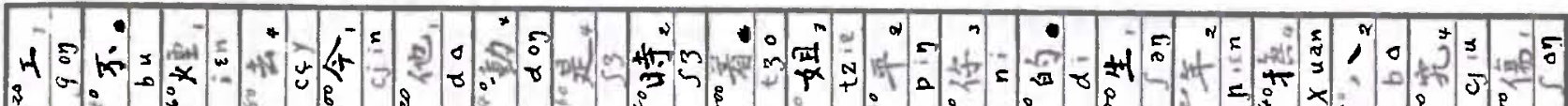

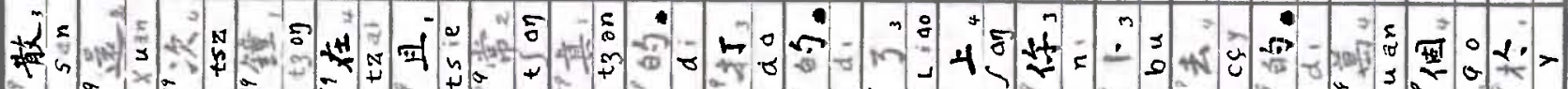

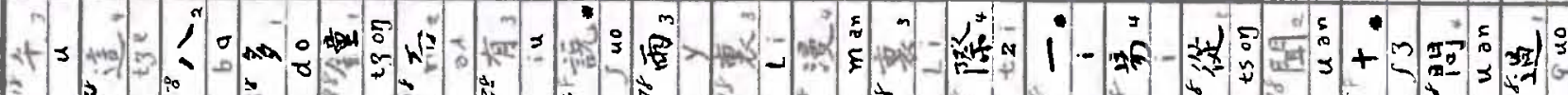

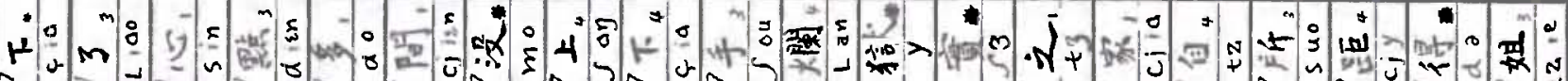

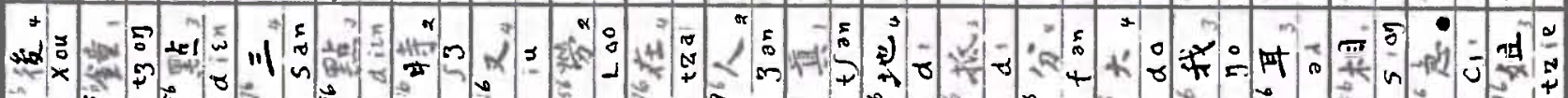

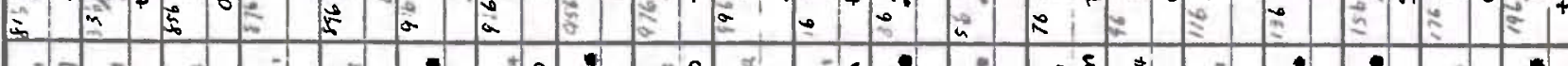

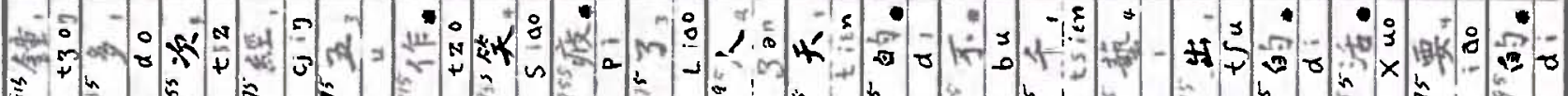

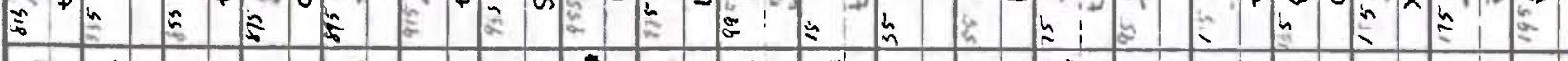

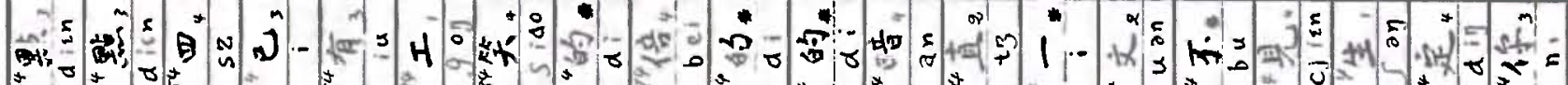

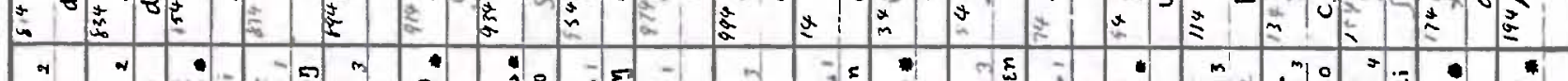

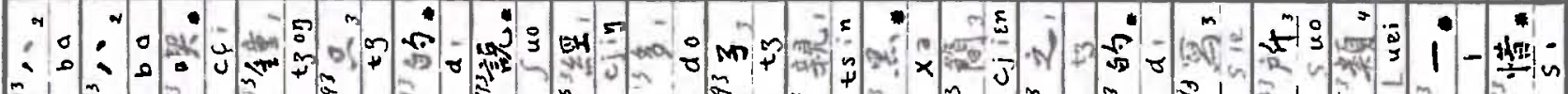

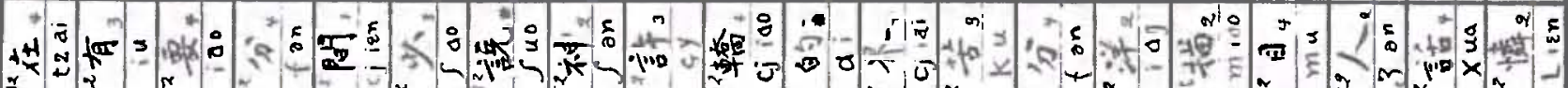

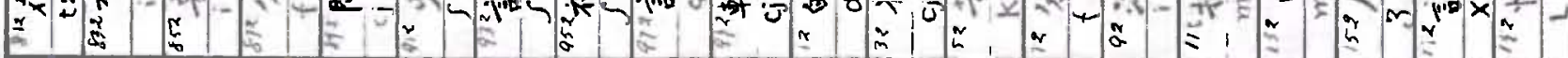

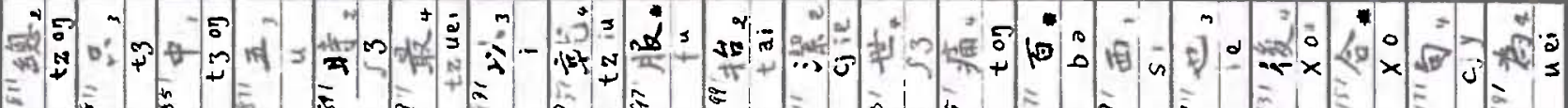

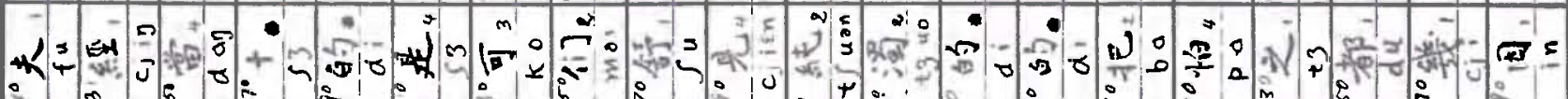

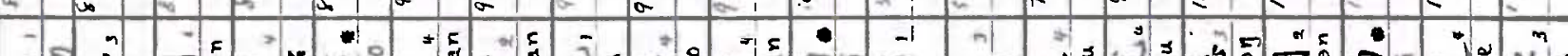

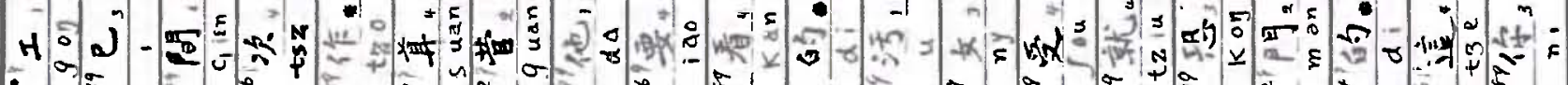
$\therefore$ E E E E a n 势

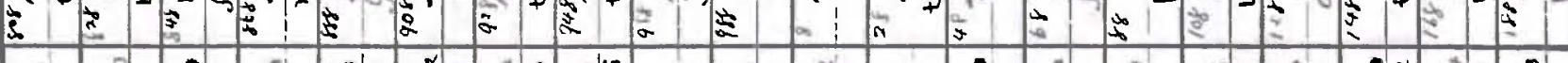

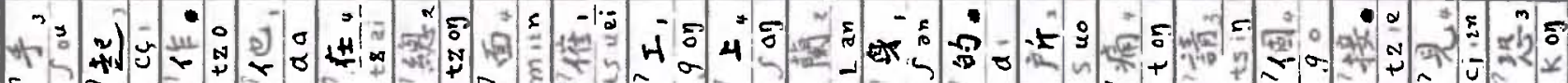

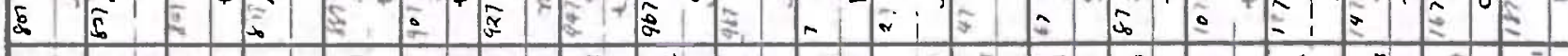
W०

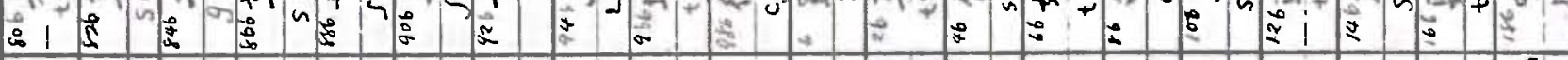

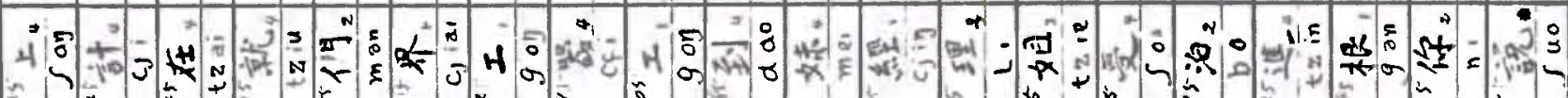

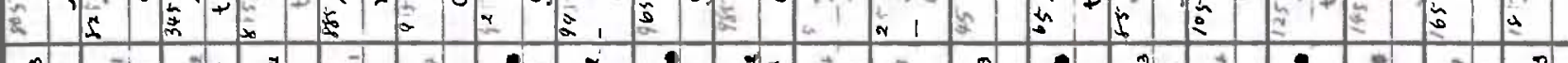
of $\begin{aligned} & 7 \\ & 0\end{aligned}$ z F.

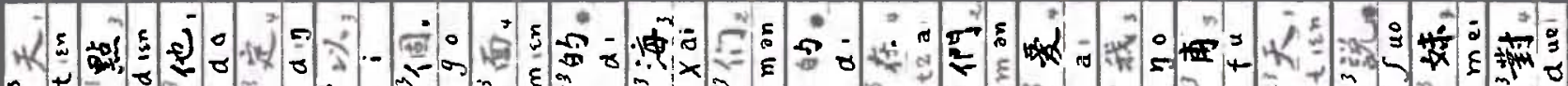

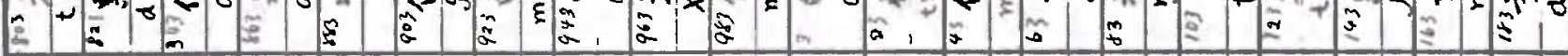

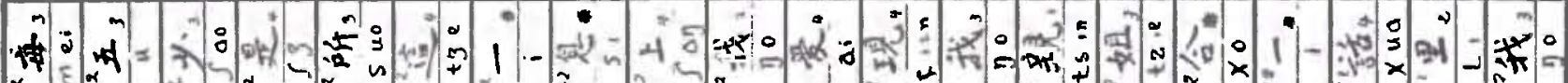

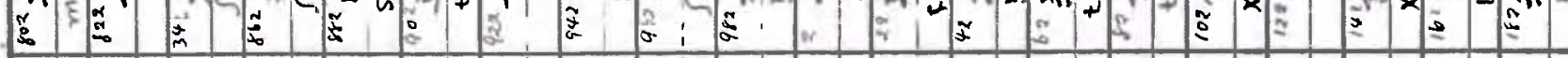

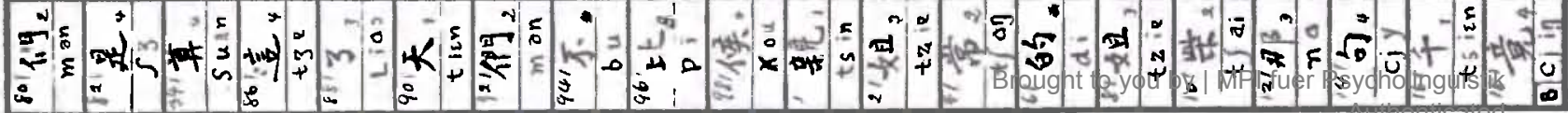




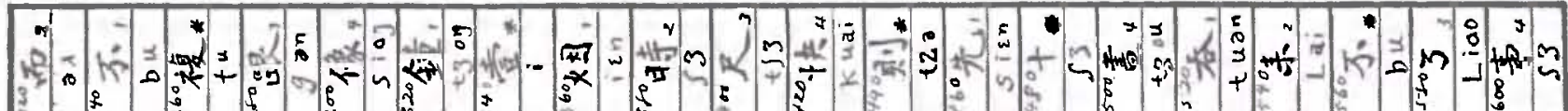

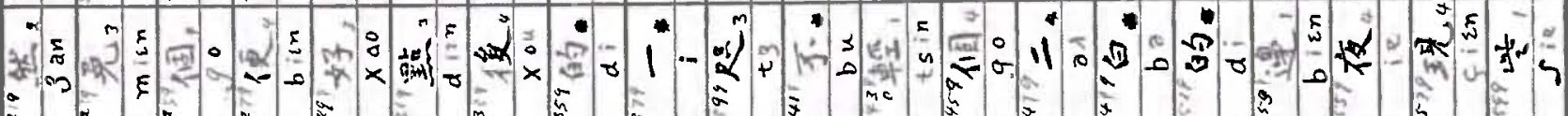

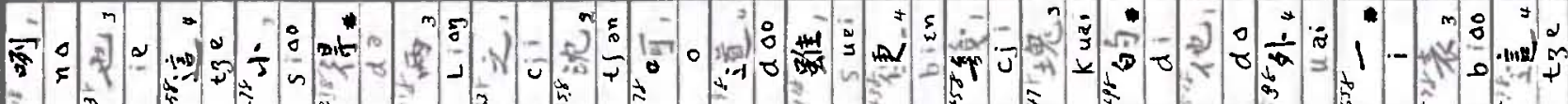

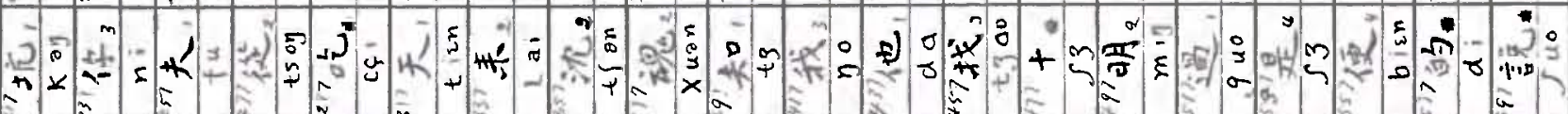

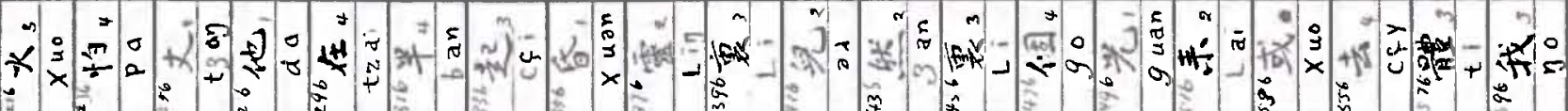

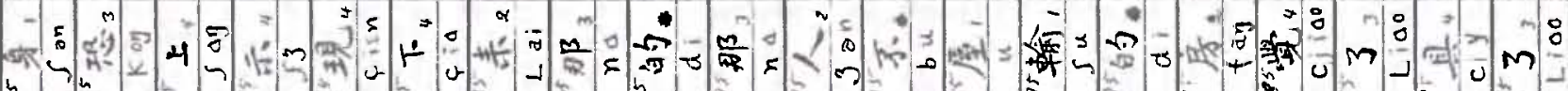
\&

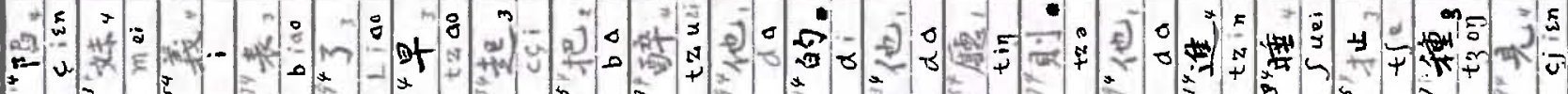

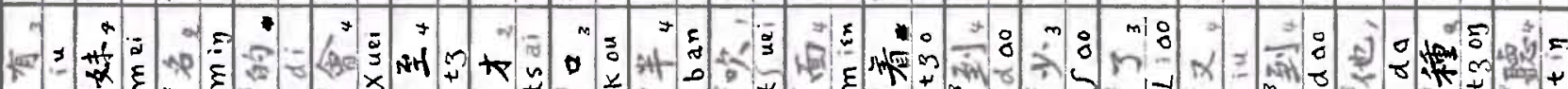

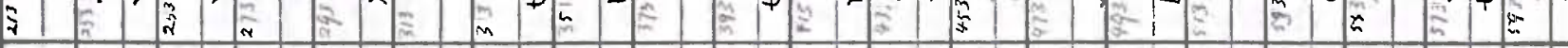
s.

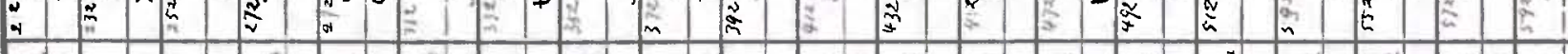

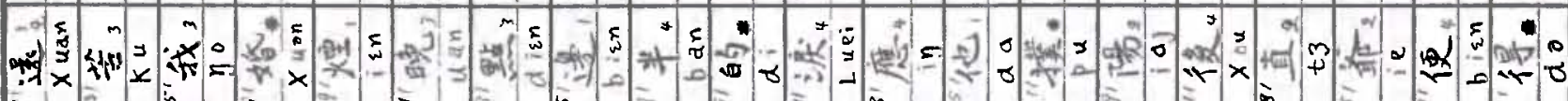

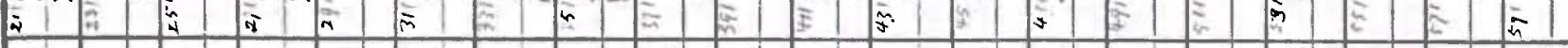

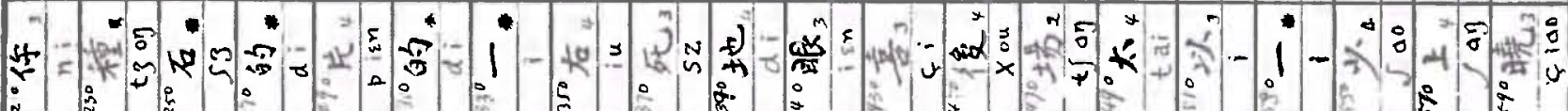
*

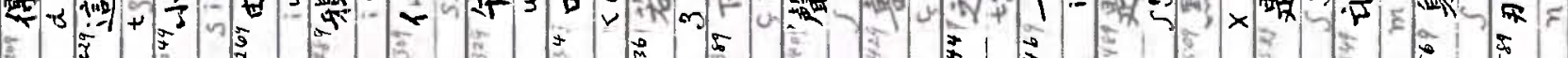

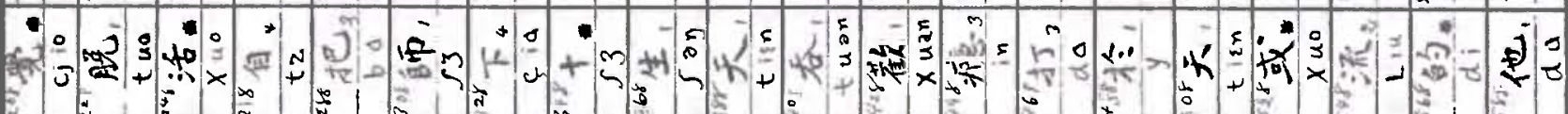

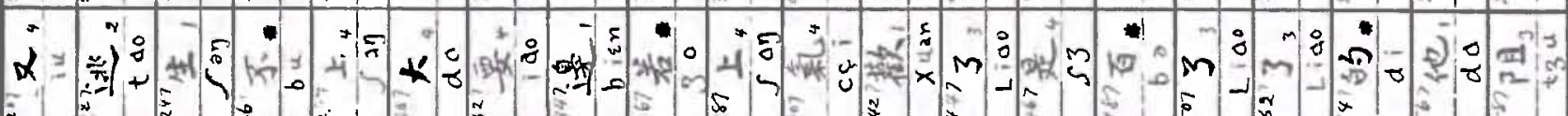

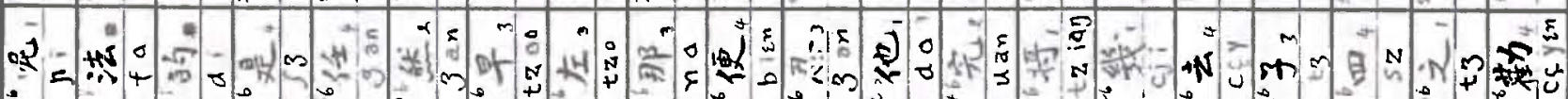

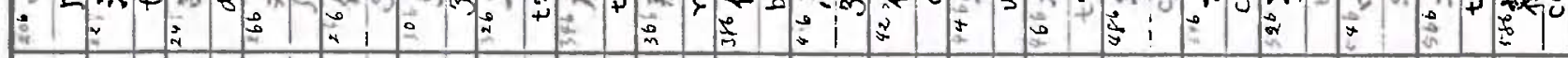

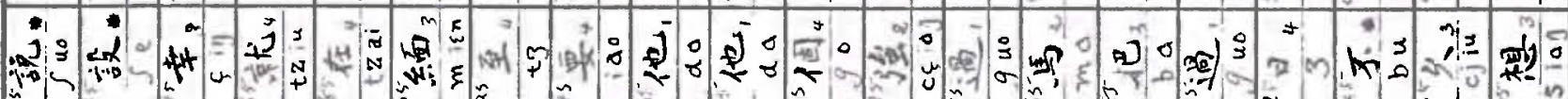

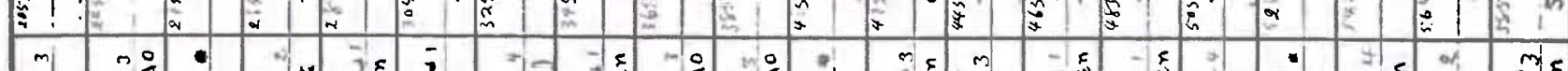

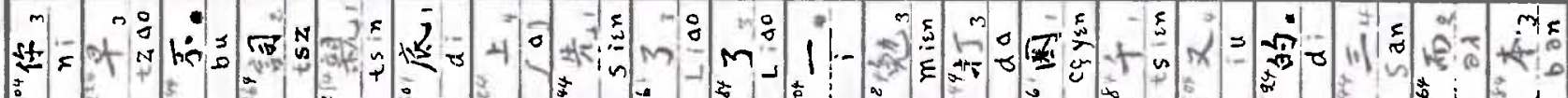

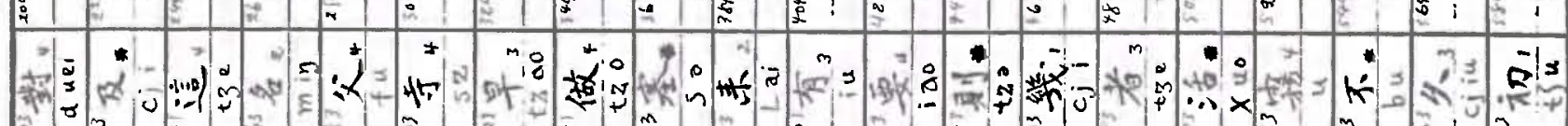

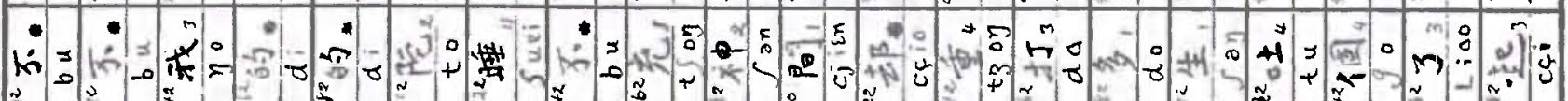

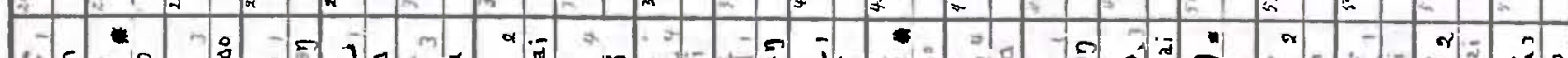

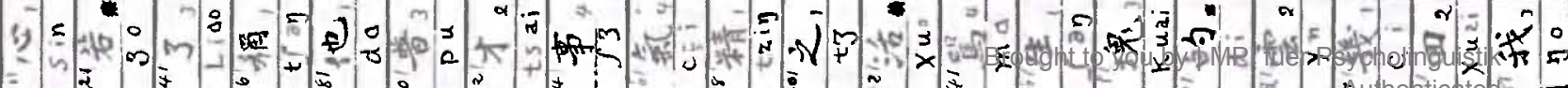

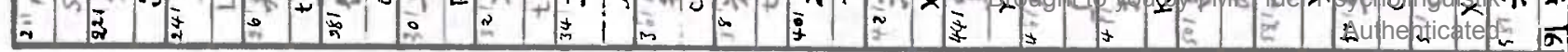




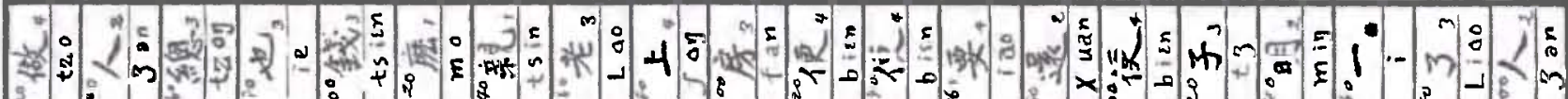

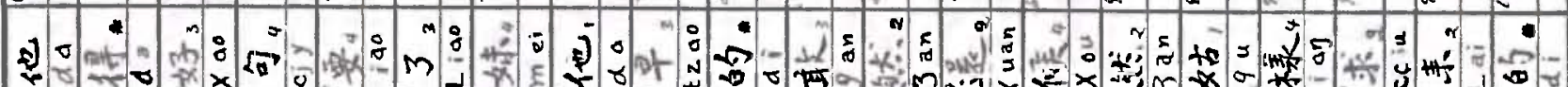

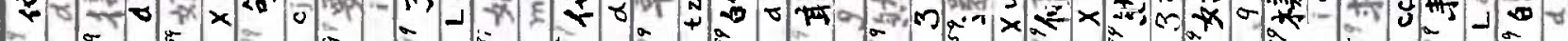

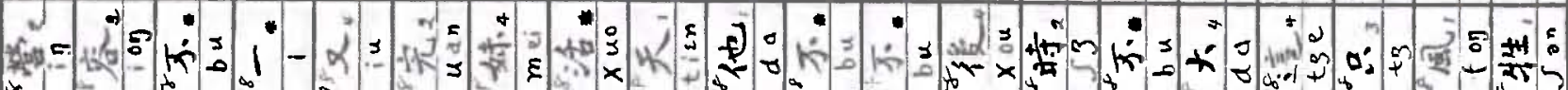

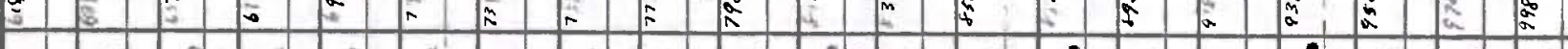

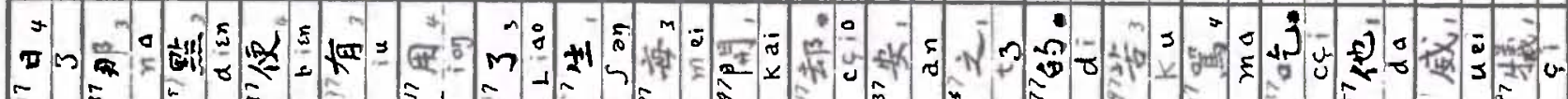

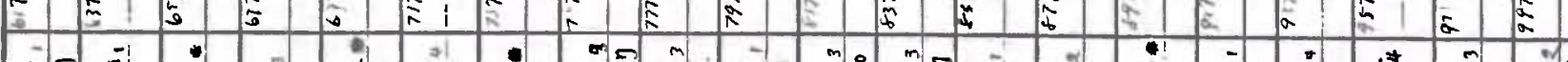
अ:

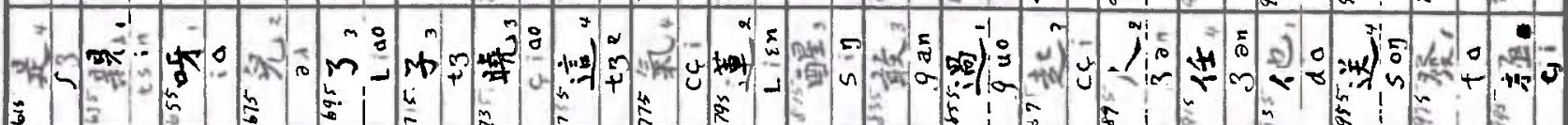

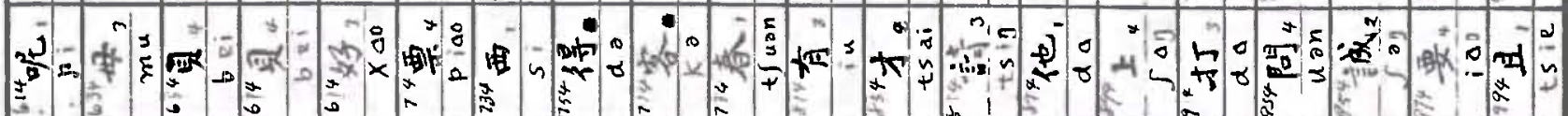

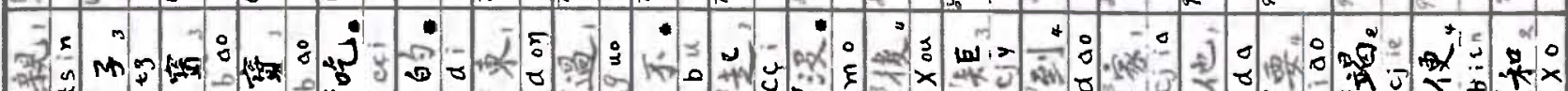

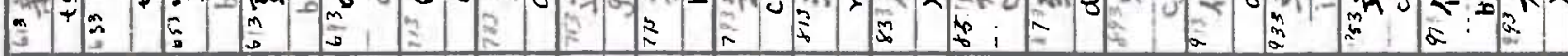

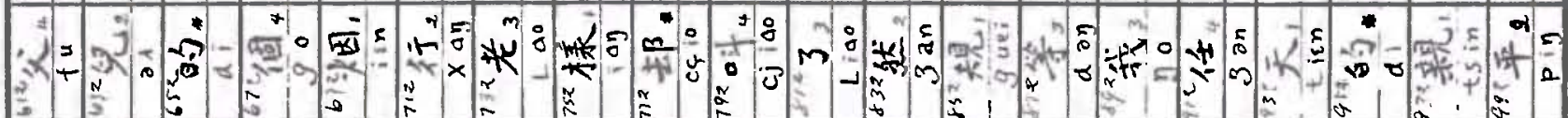

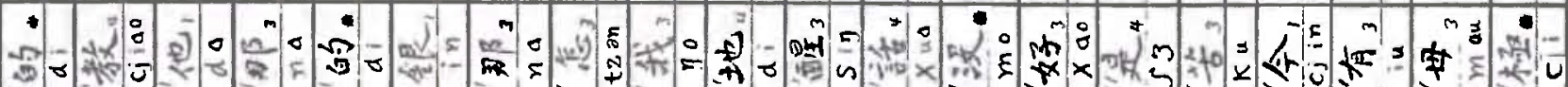

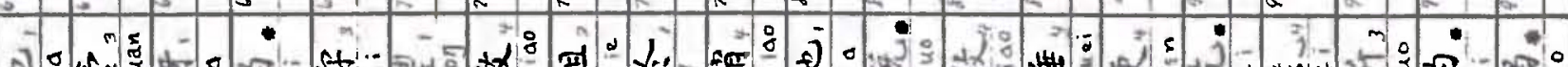

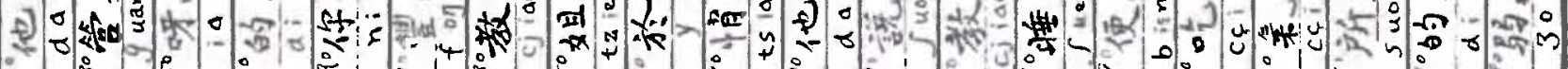

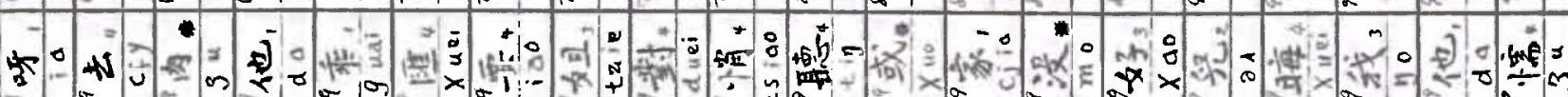

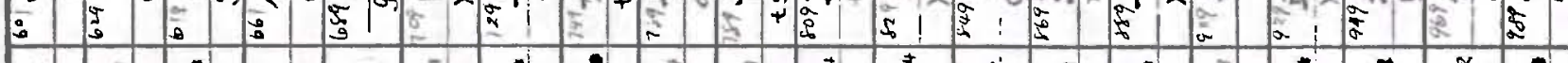

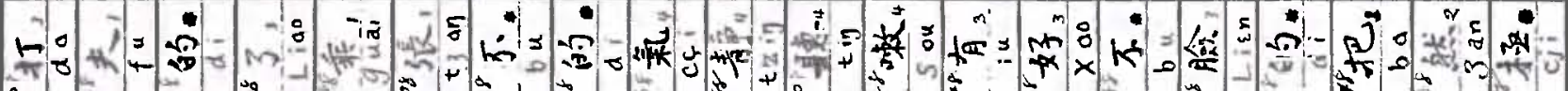

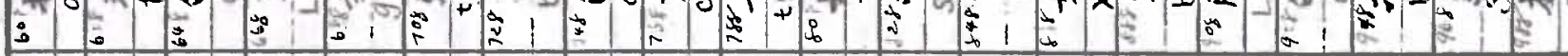

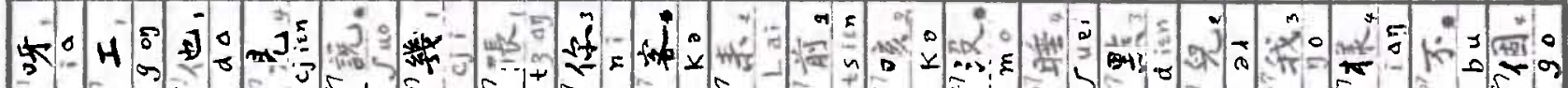

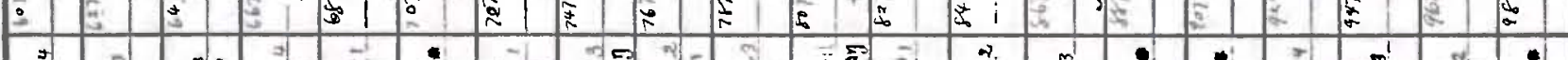

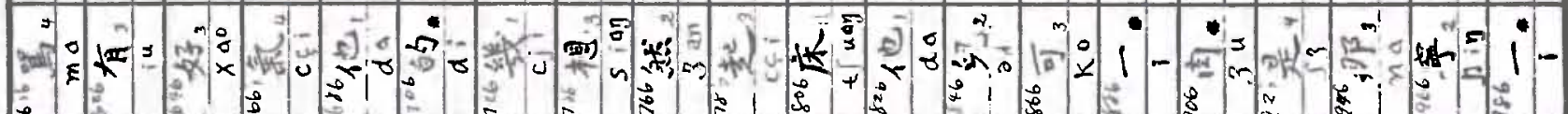

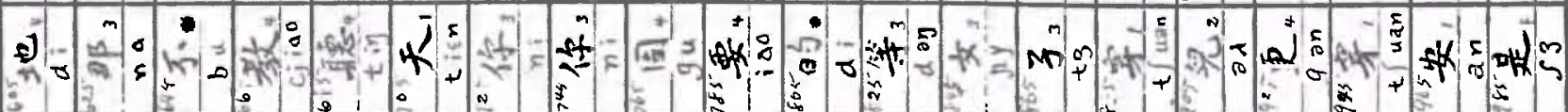

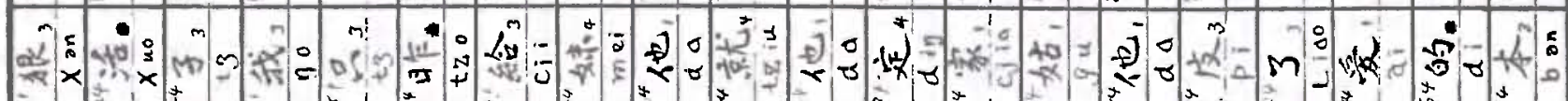

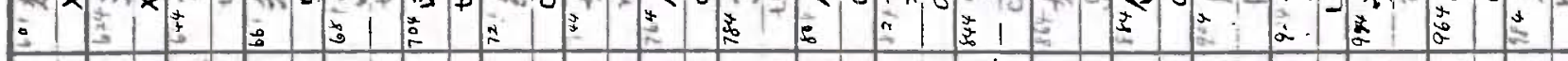

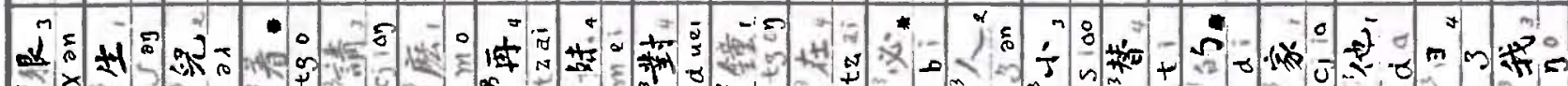

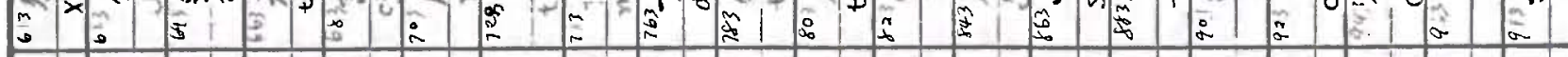

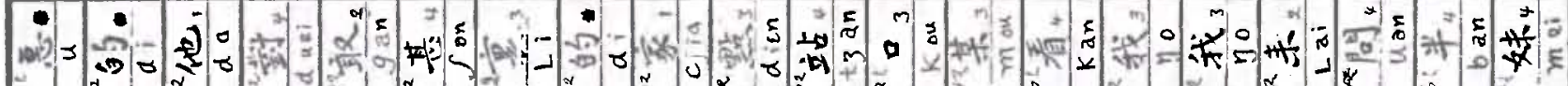

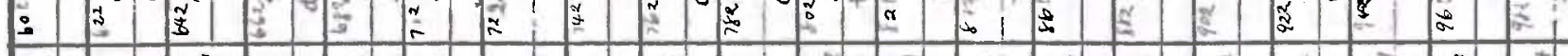
a

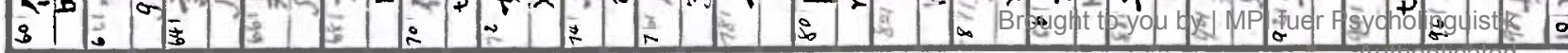




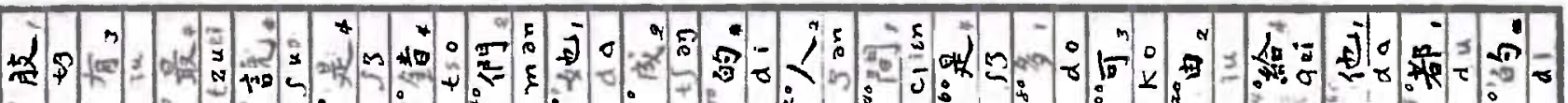

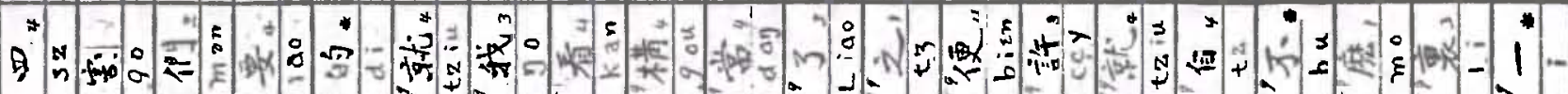

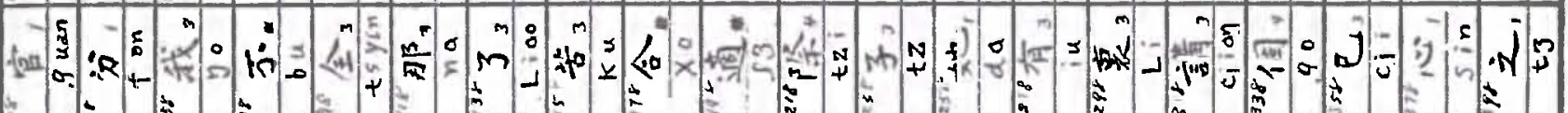

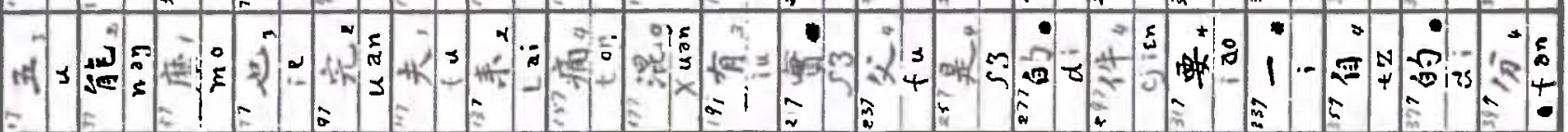

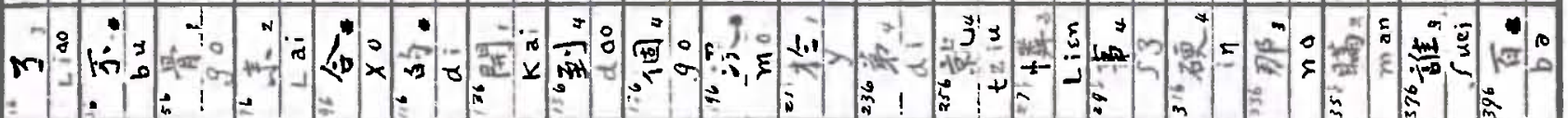

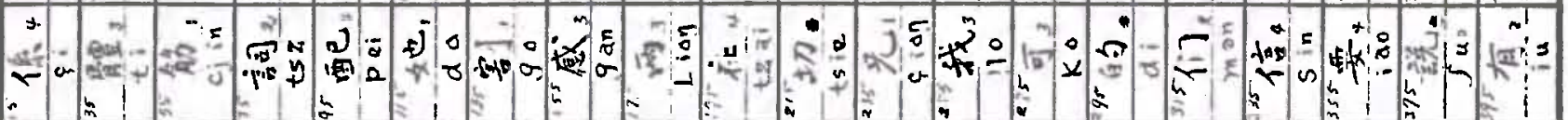

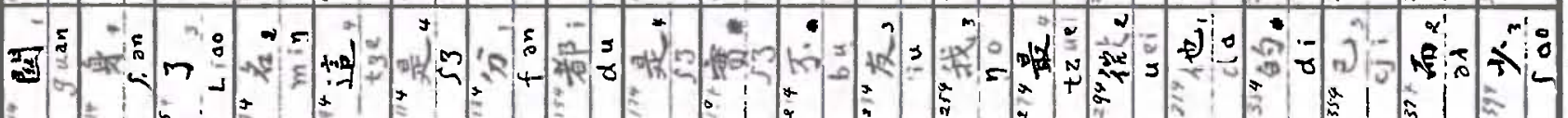

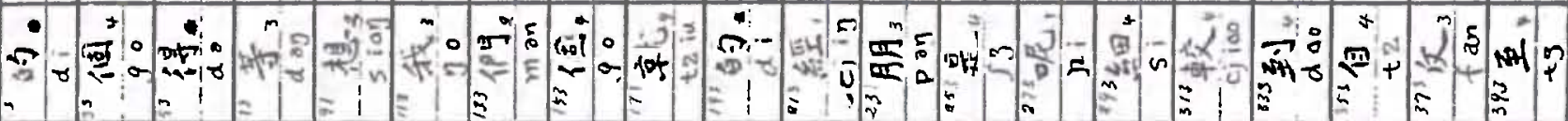

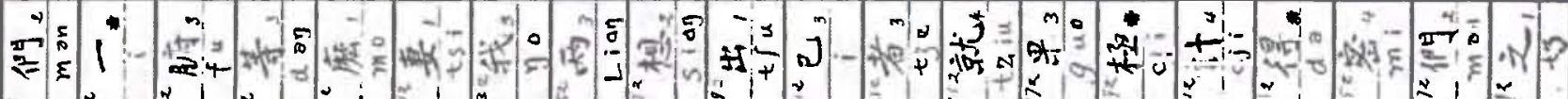

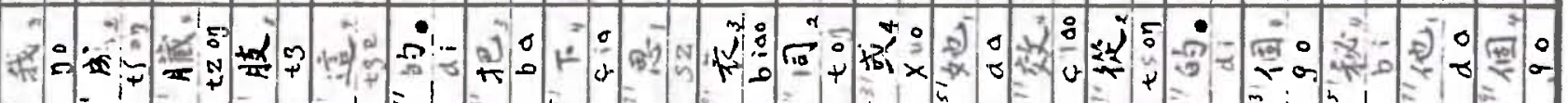

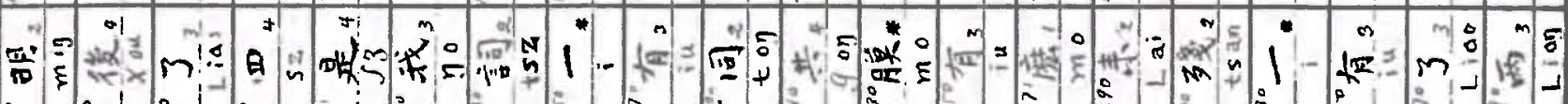

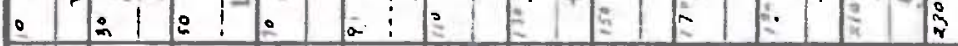

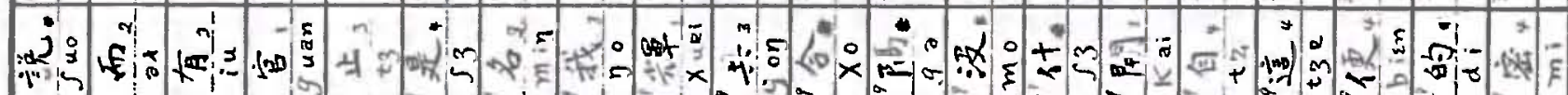

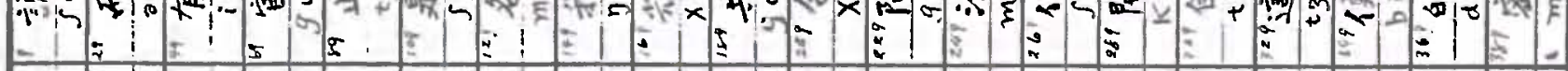

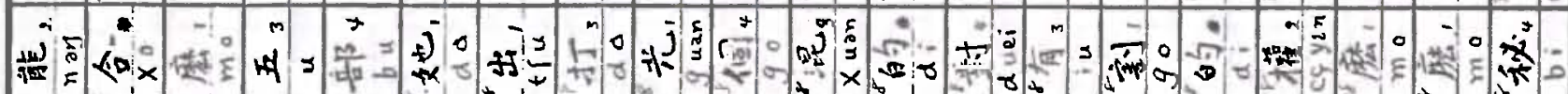

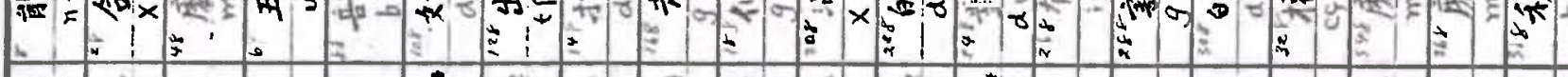

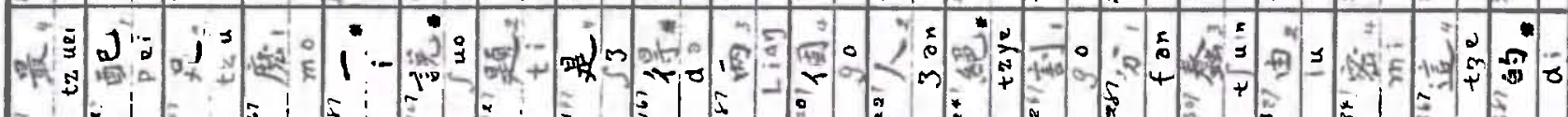

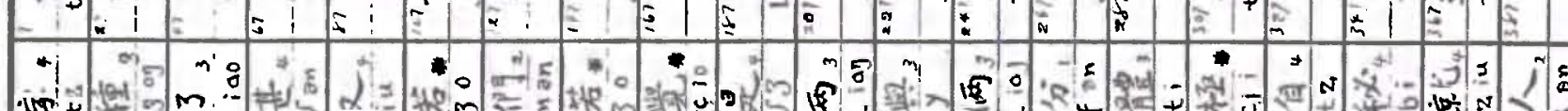

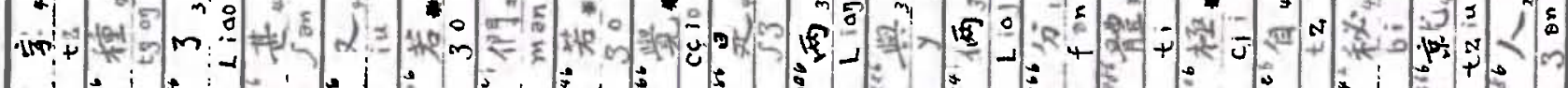

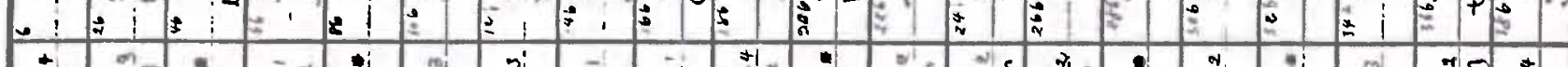

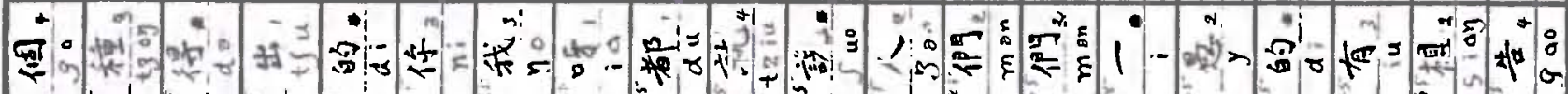
m!

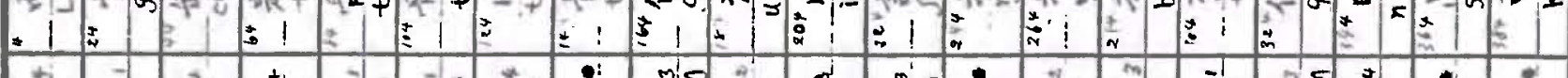
(n)

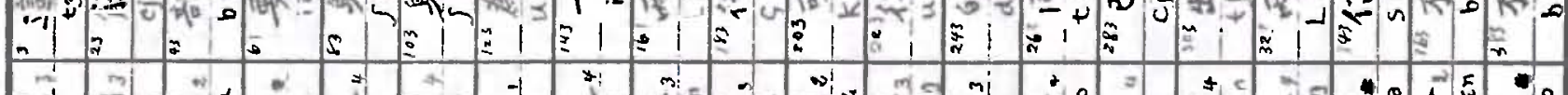

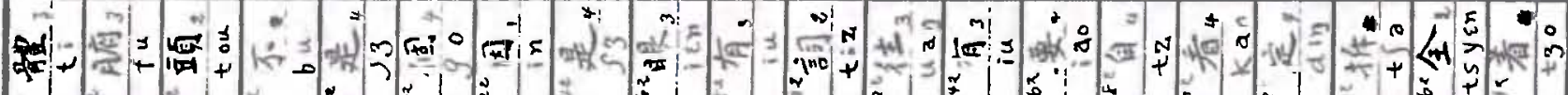
1 1.

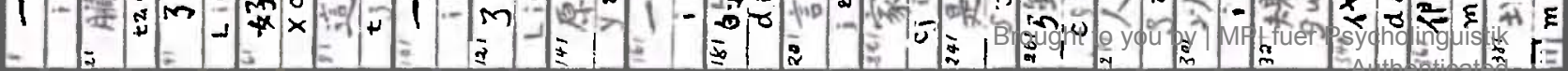


Q tot

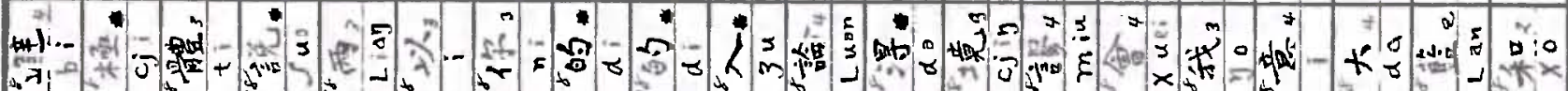

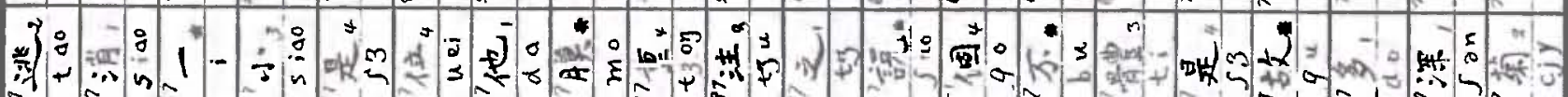

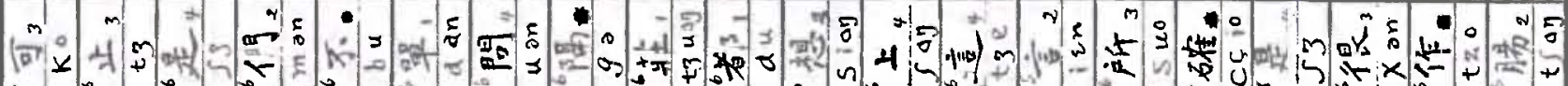

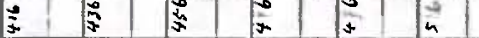

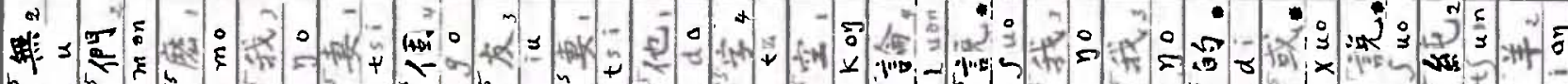
F.

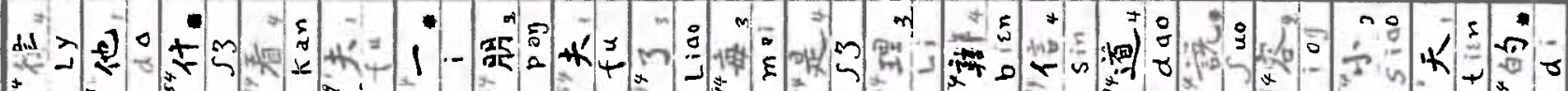

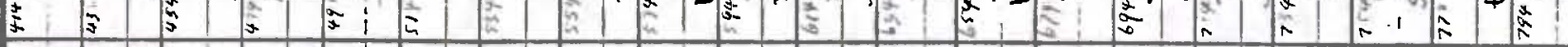

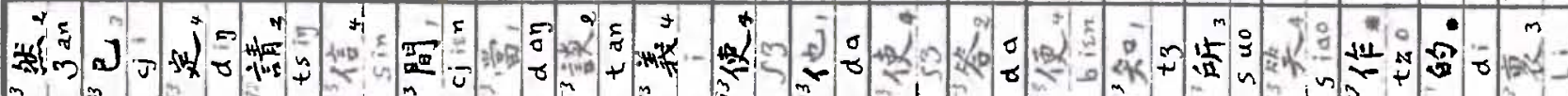

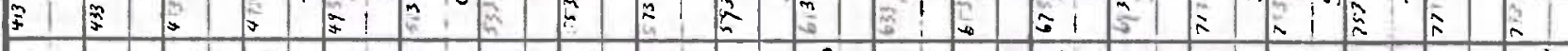

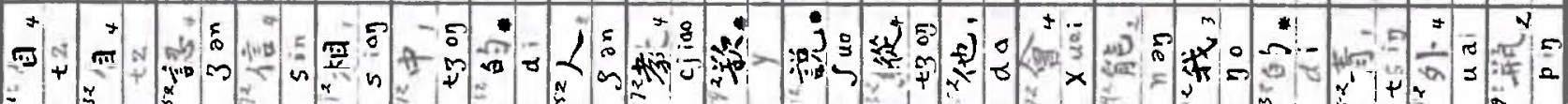

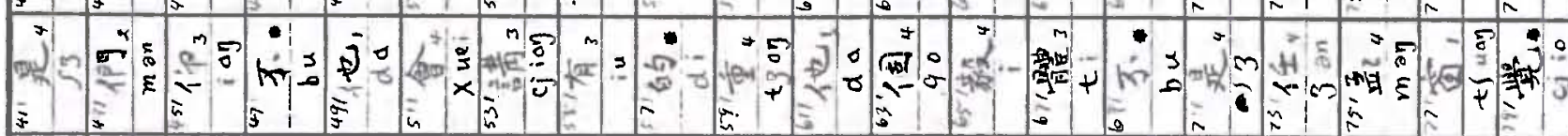

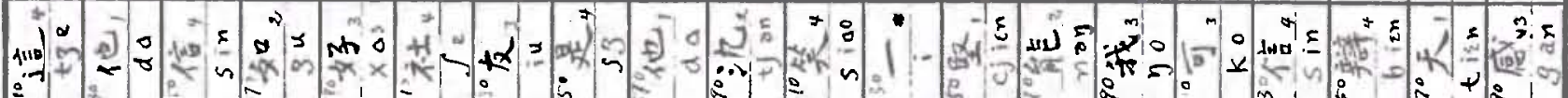

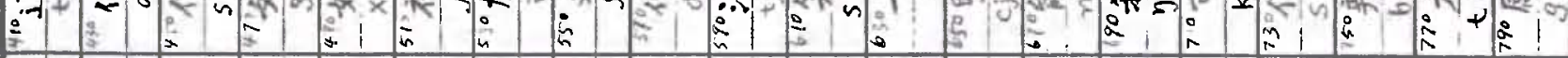

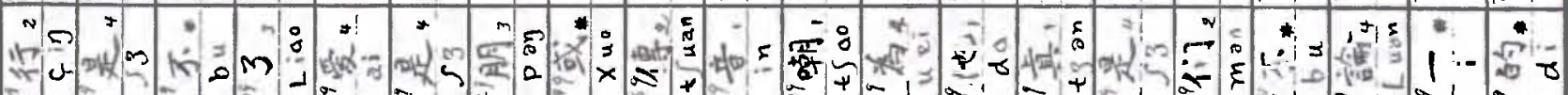

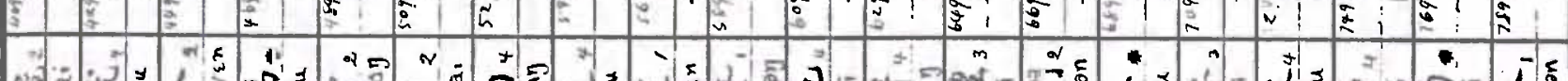

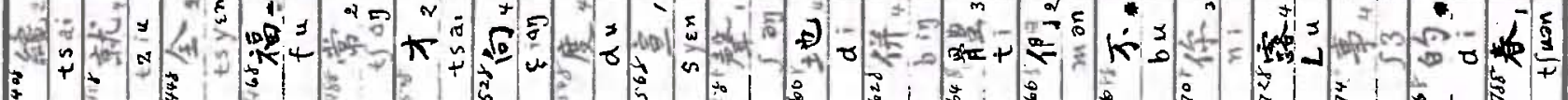

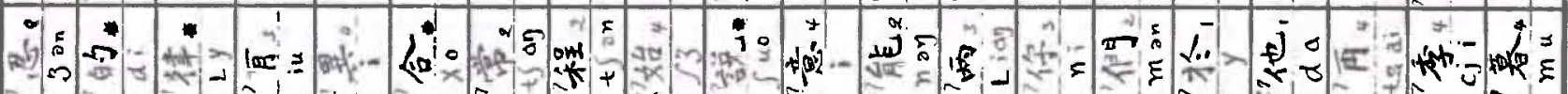
$j$
0

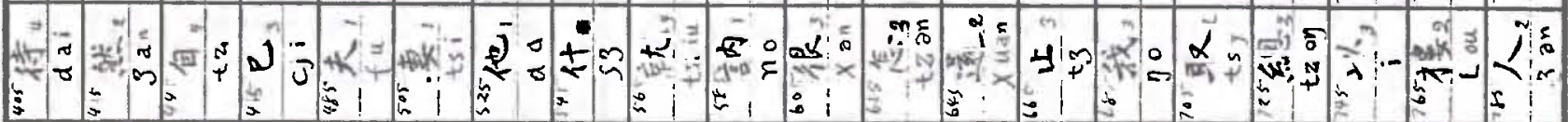

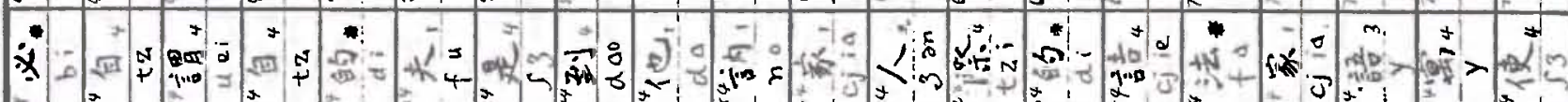

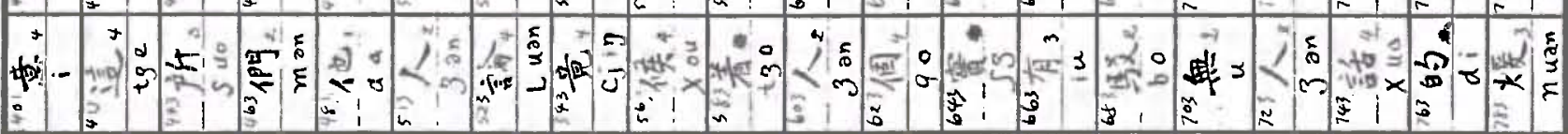
a

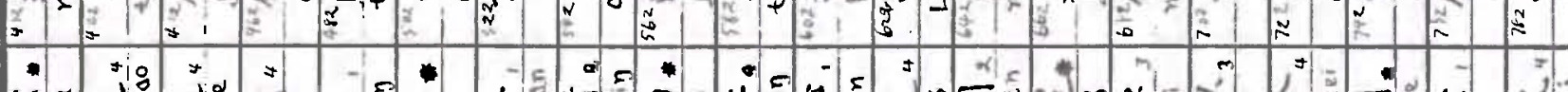

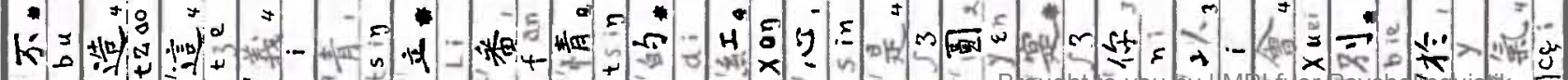

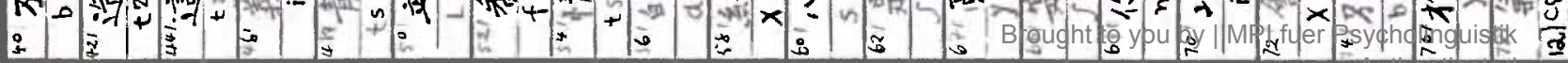




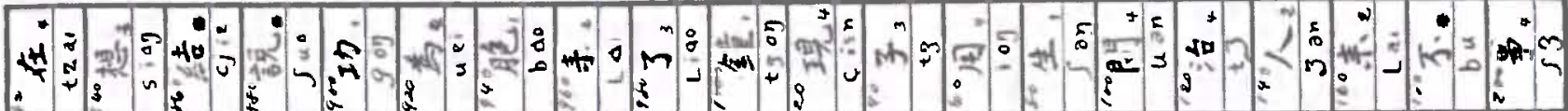

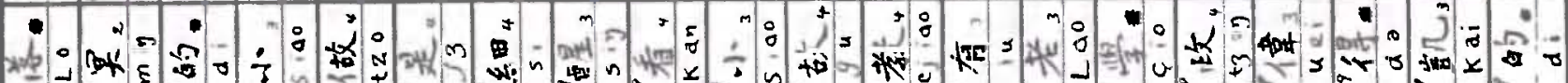

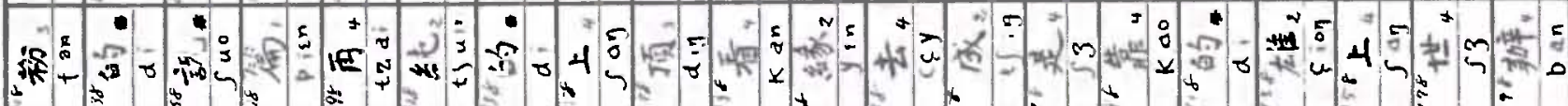

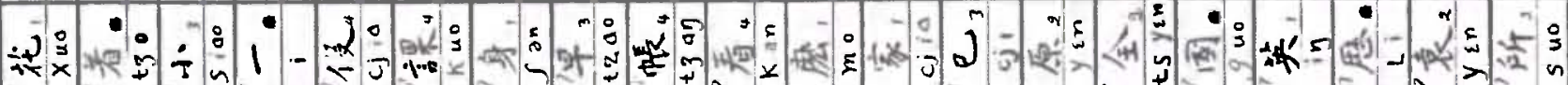

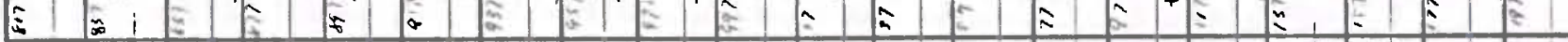

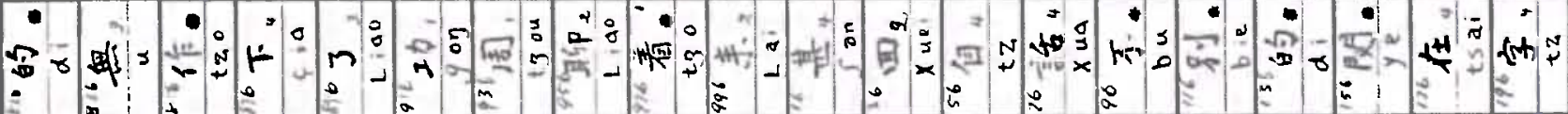

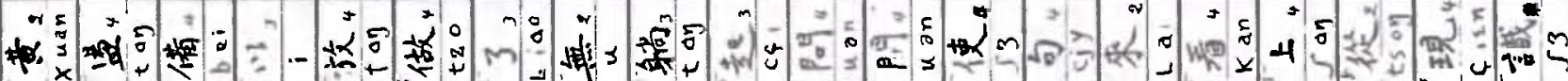
E

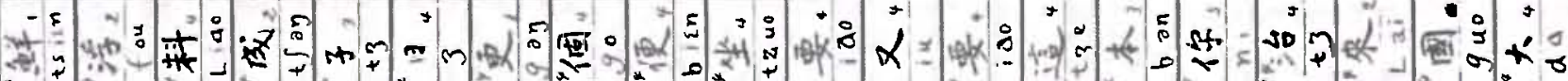

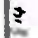

$\therefore$ (1)

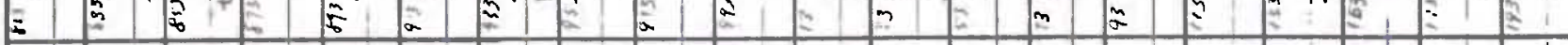

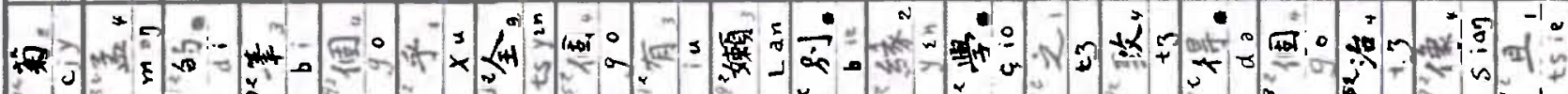

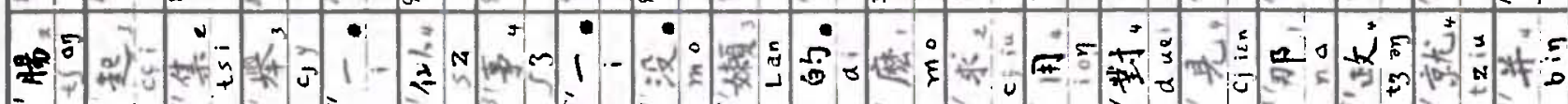

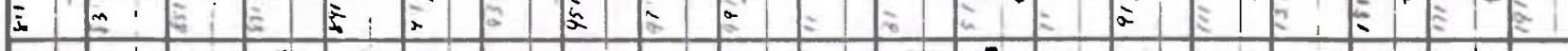

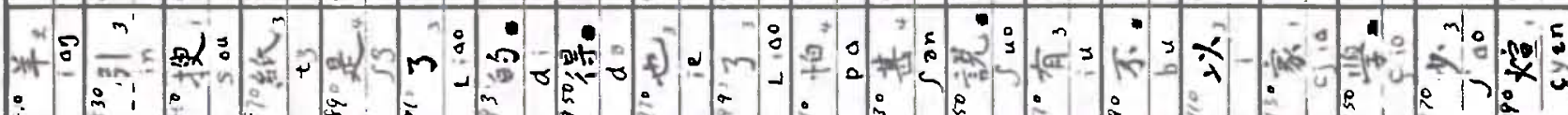

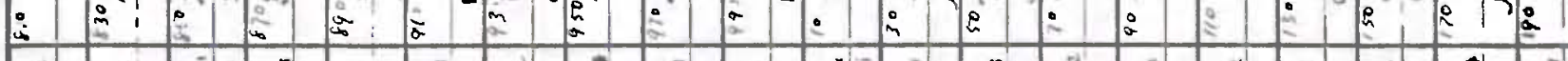

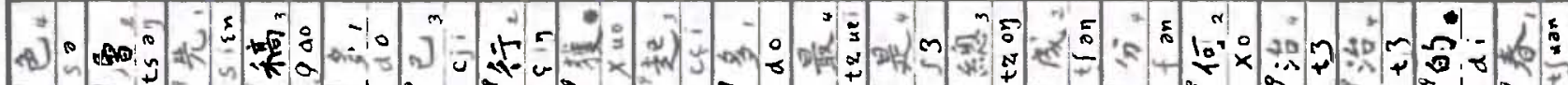

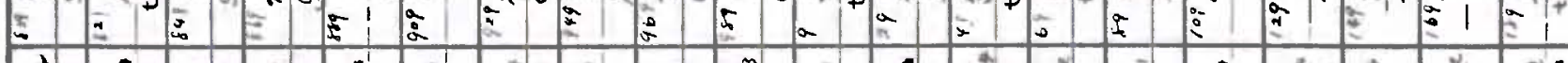

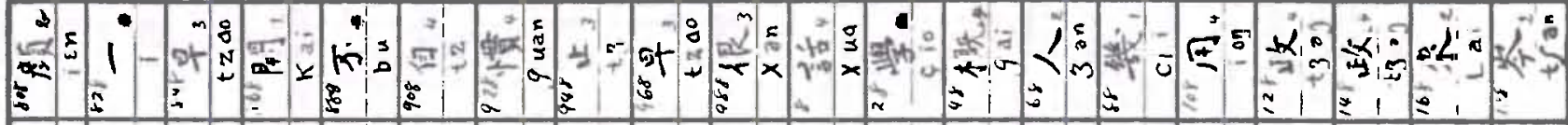

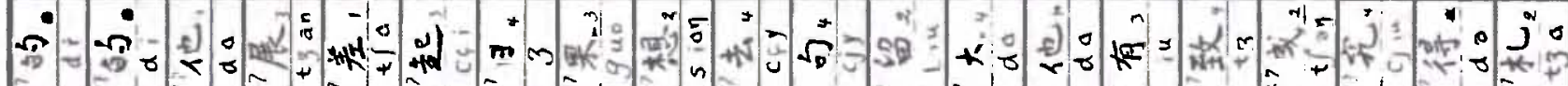

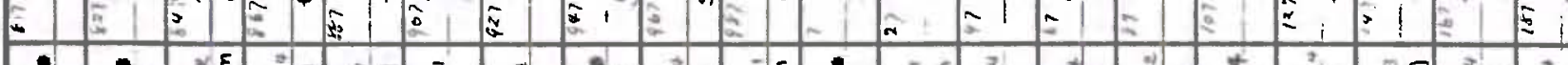

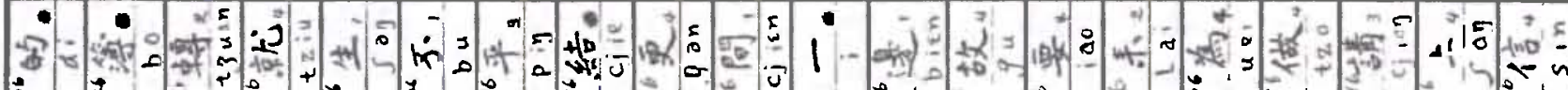

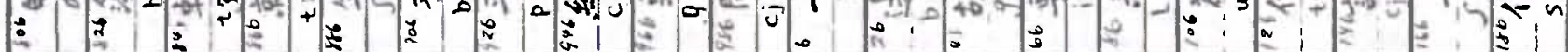
悔

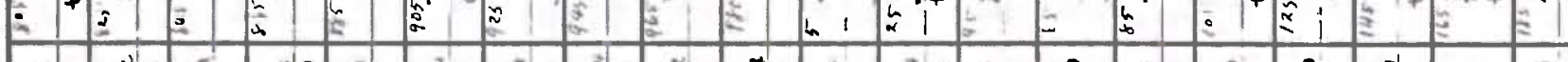

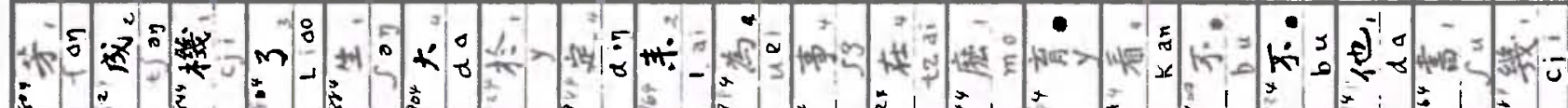

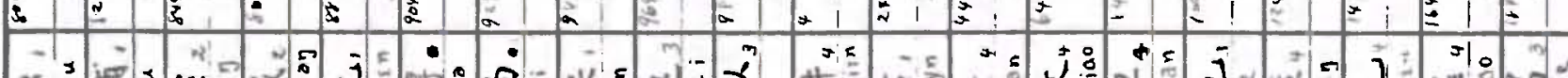
की

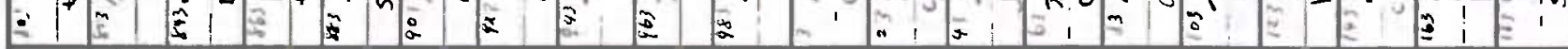

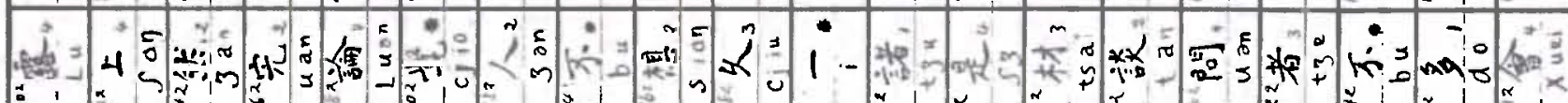

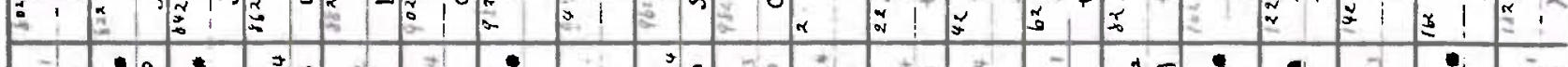

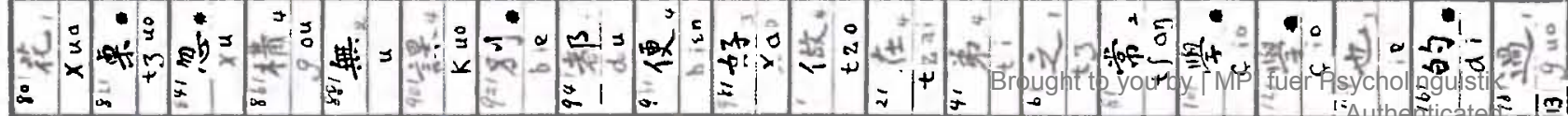




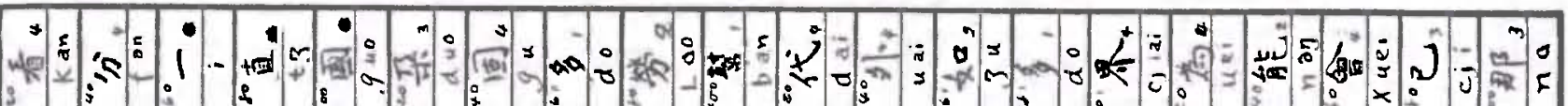

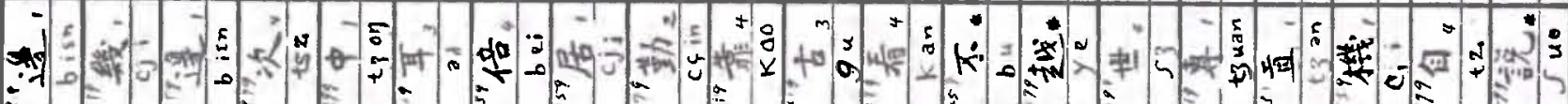

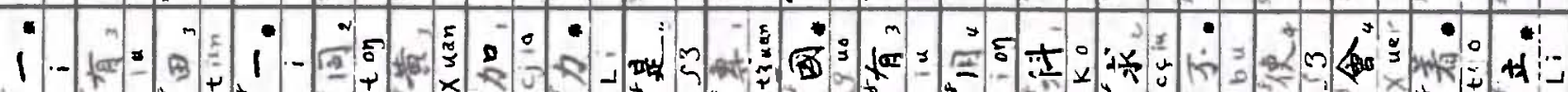
$\because$

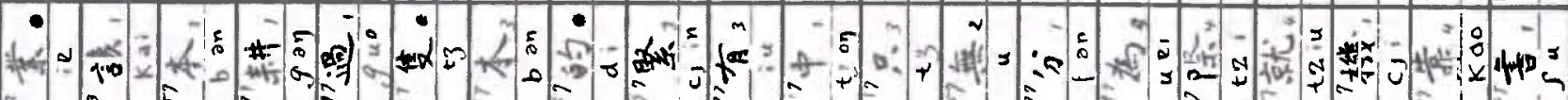
L a

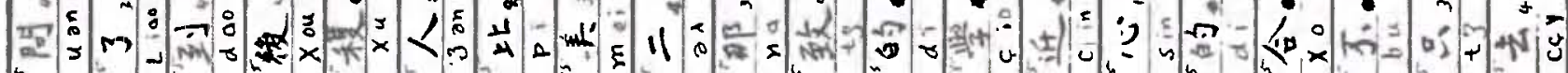

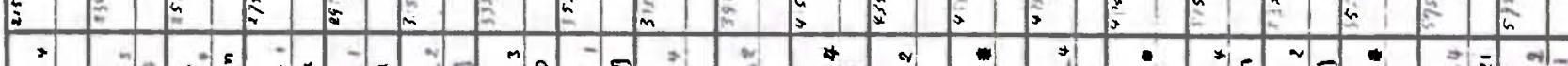

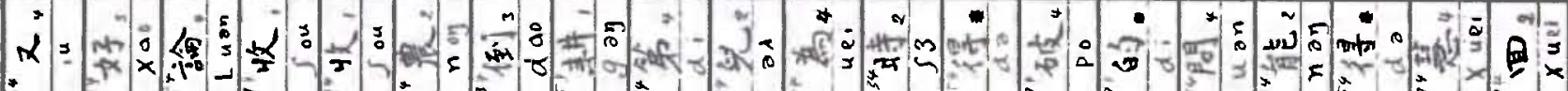

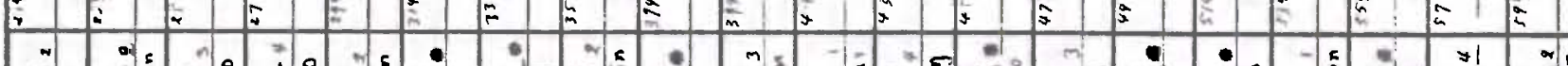

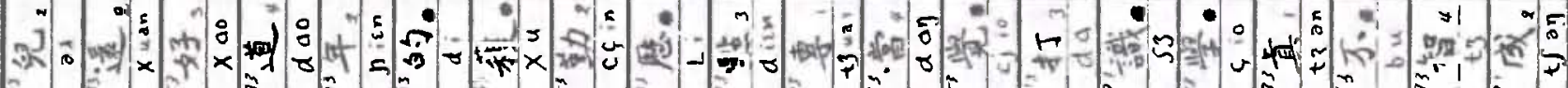

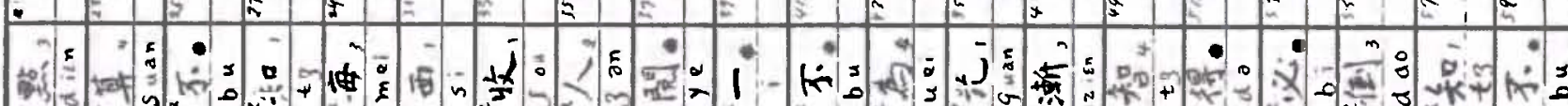

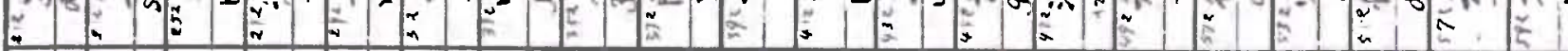

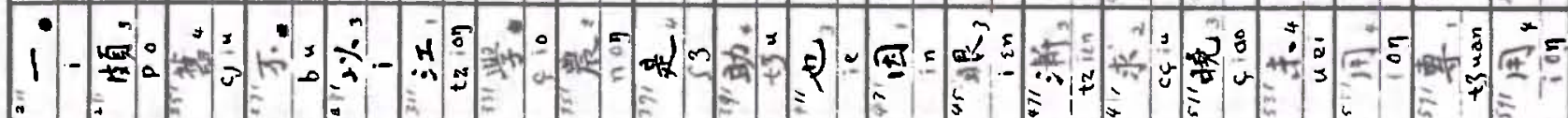

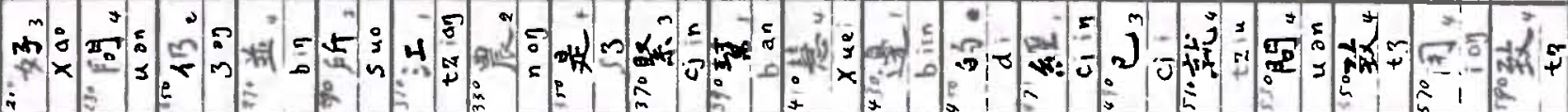

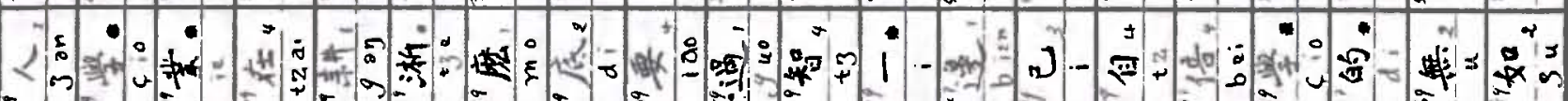
z

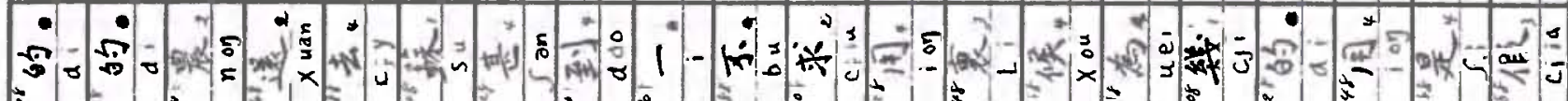

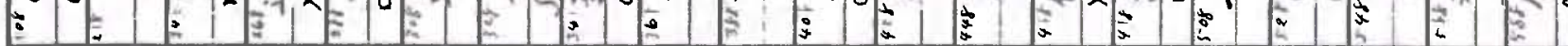

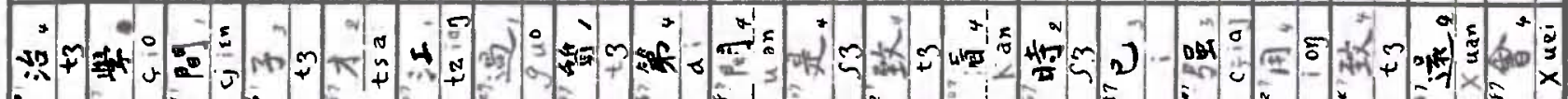

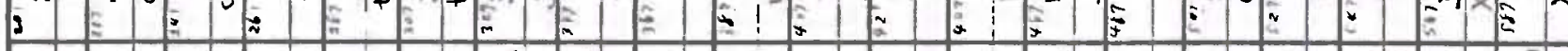

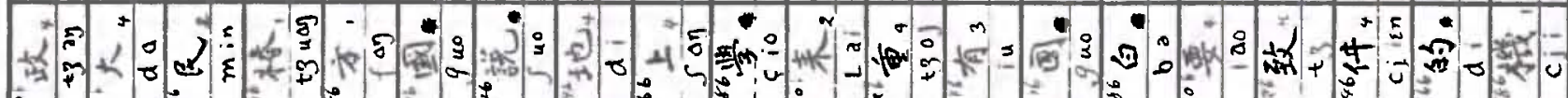

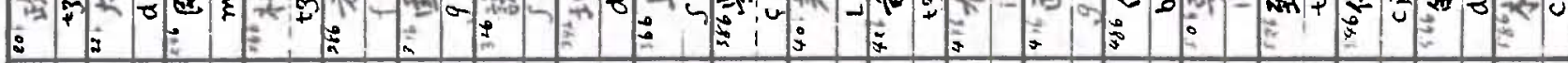

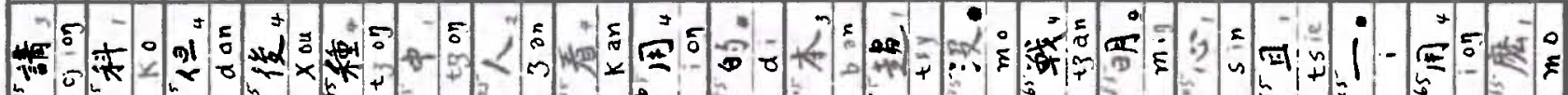

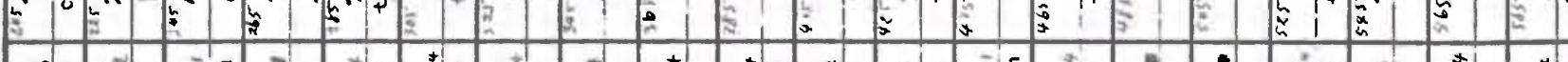

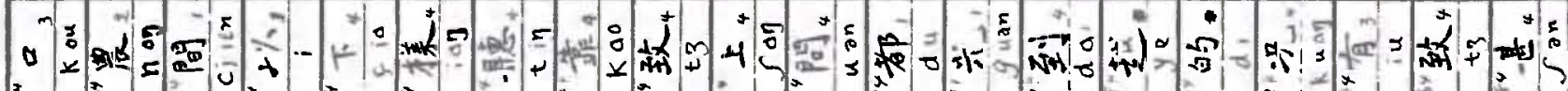

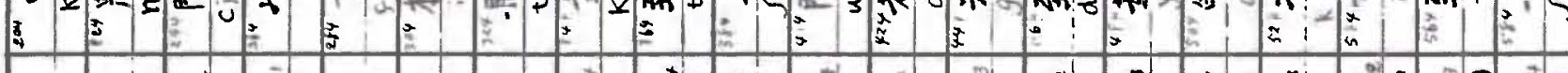

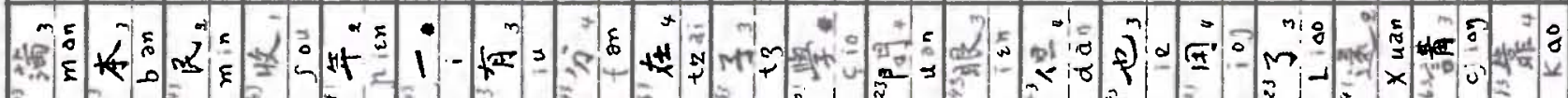

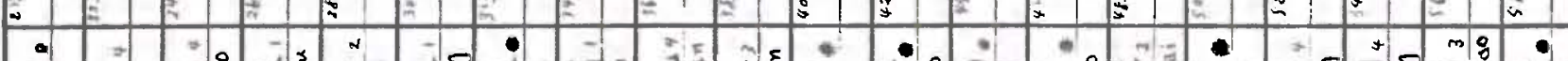

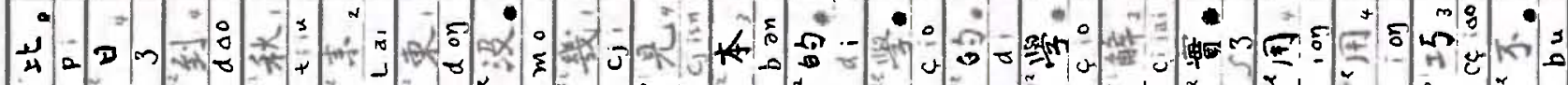

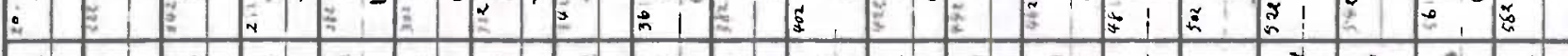

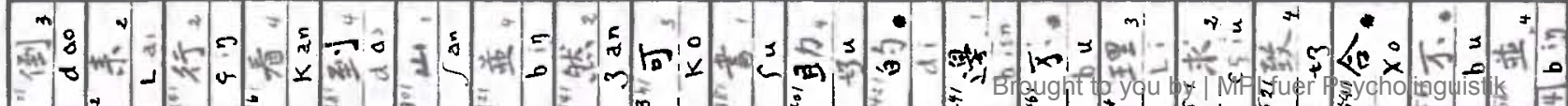




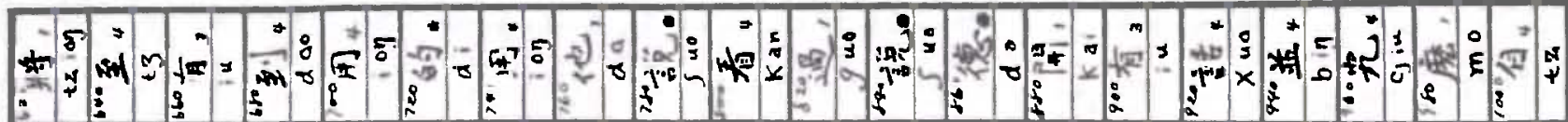
F

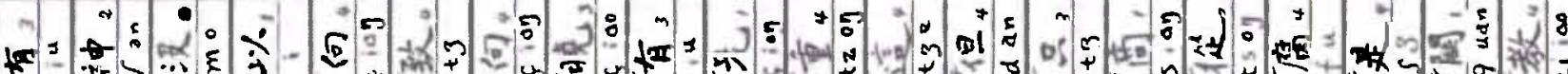

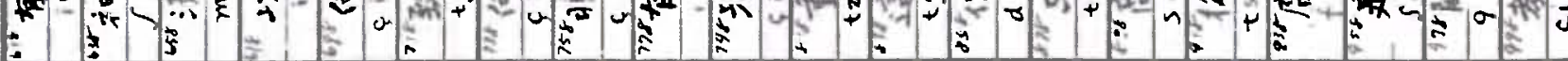

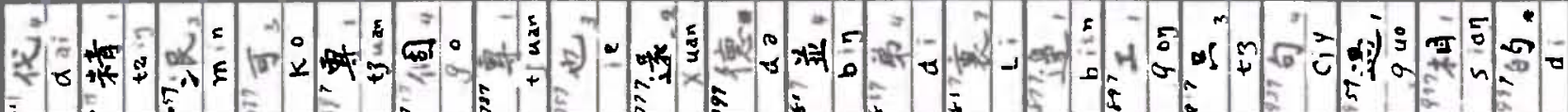

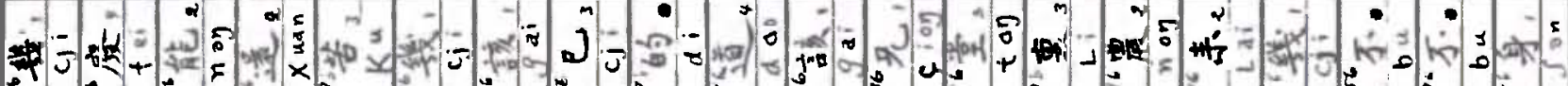

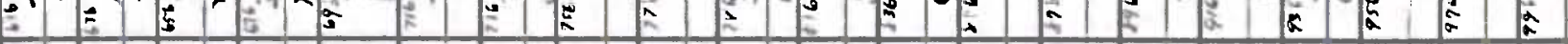
t:

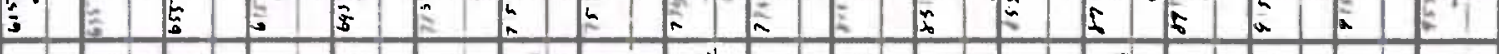

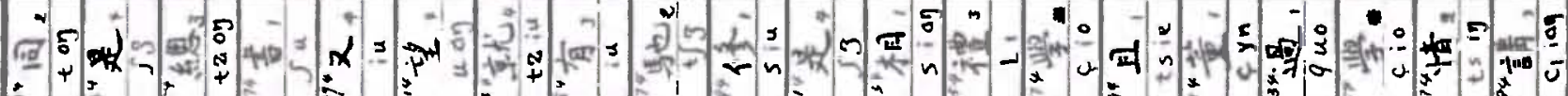

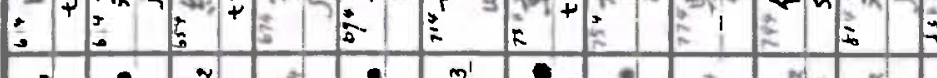

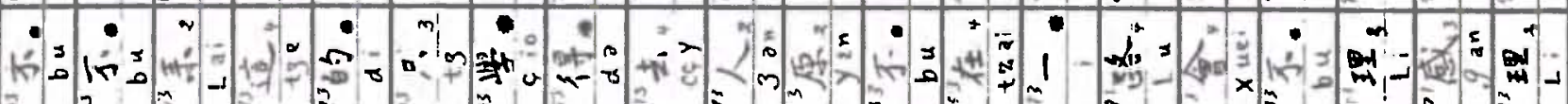

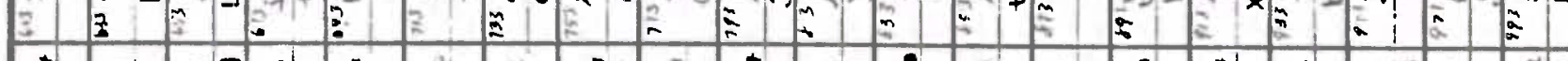

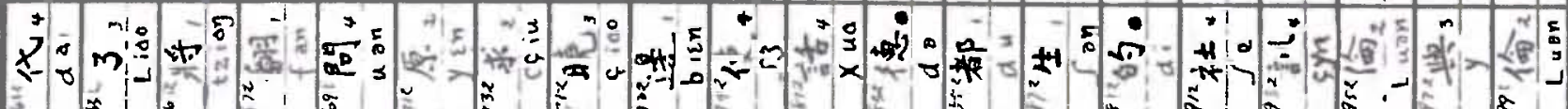
do.

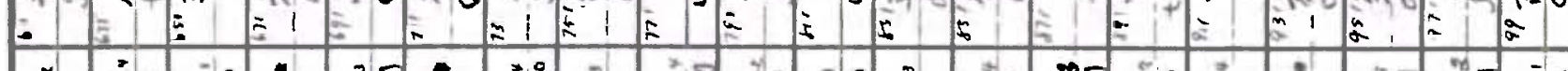

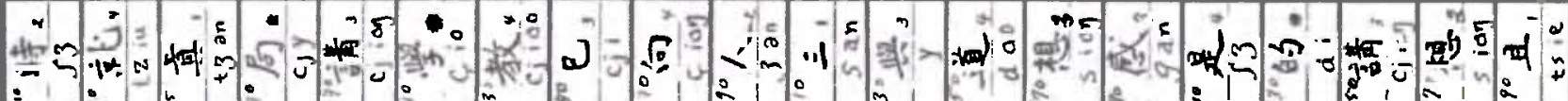

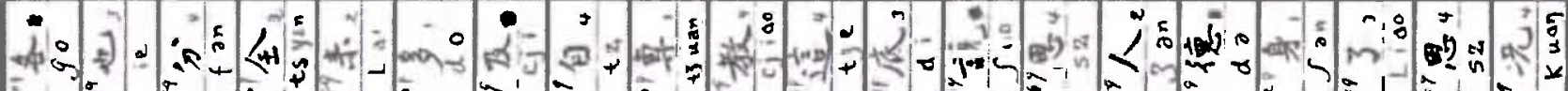

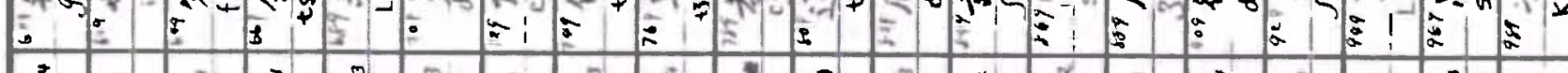

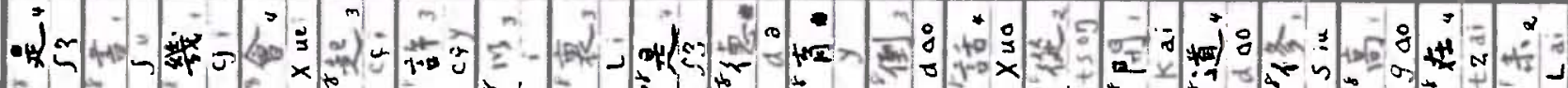

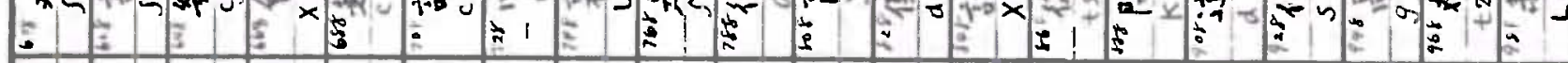

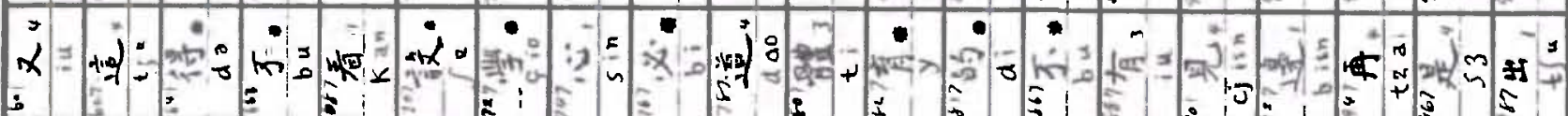

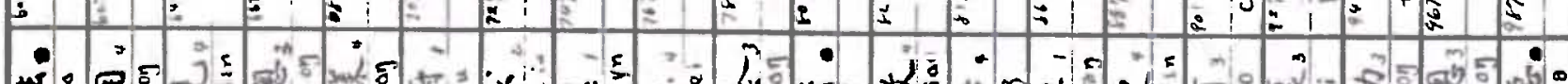

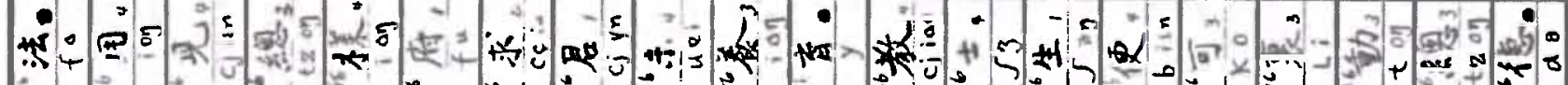

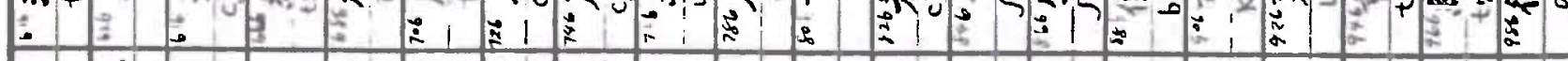

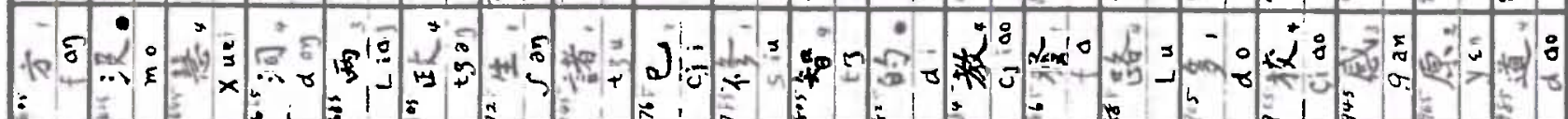

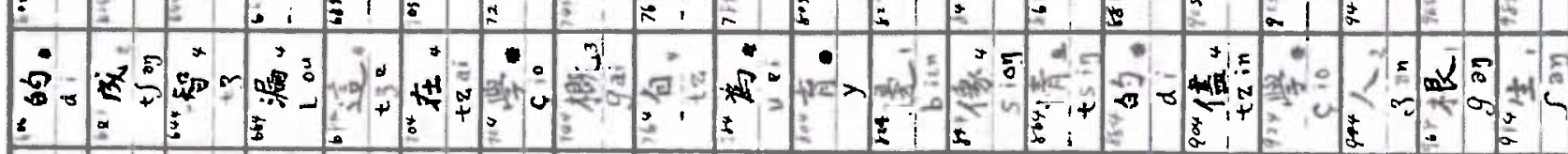
至定

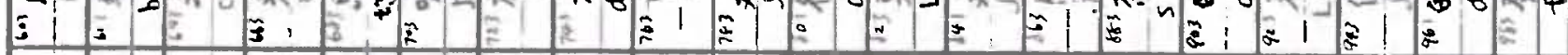

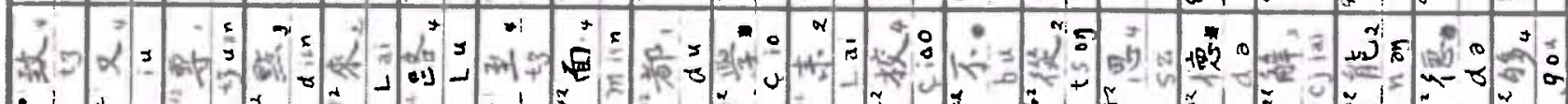

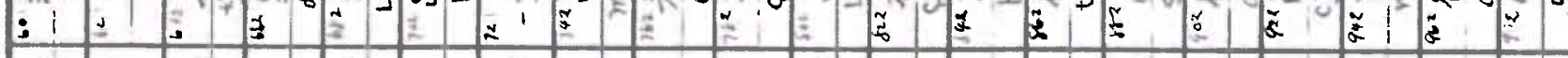
\#

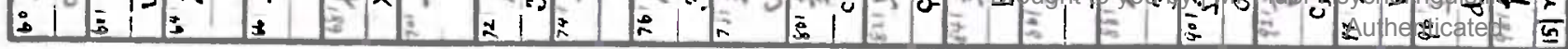




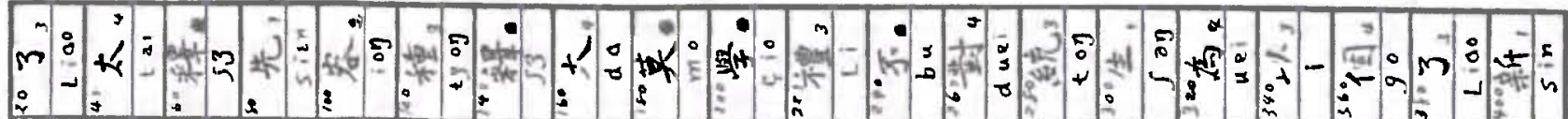

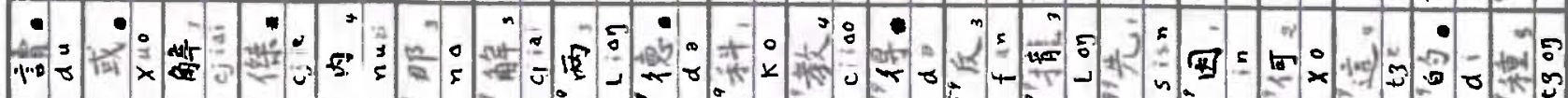

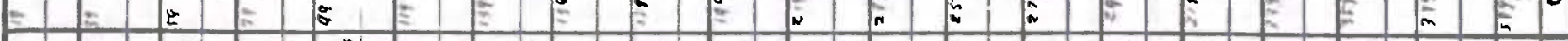

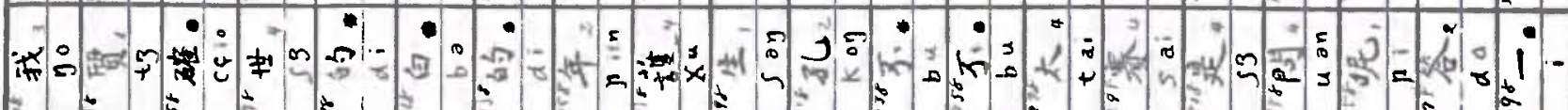

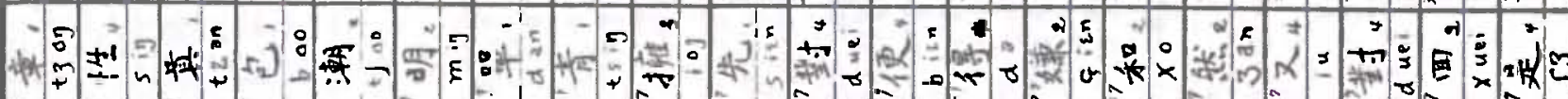

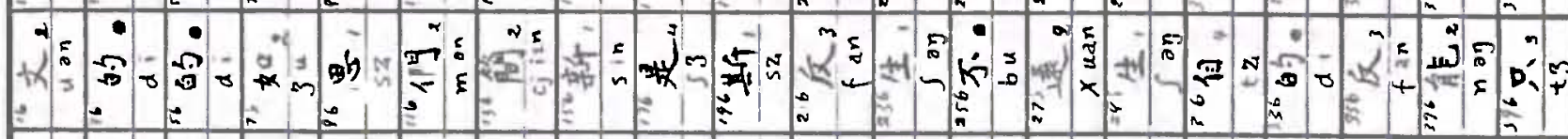

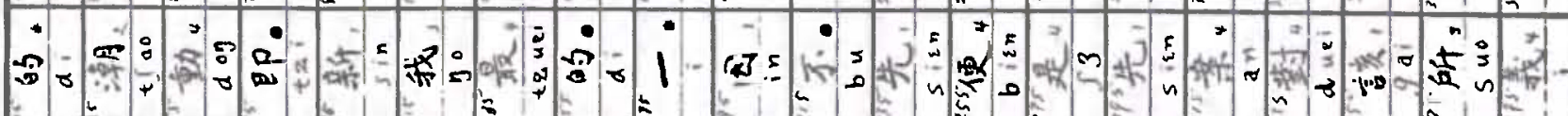

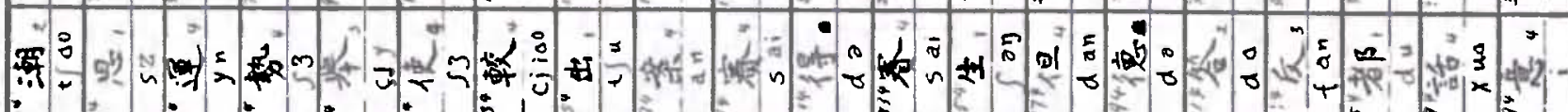

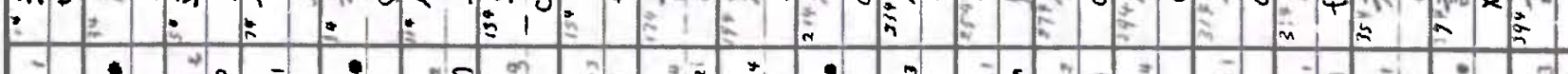

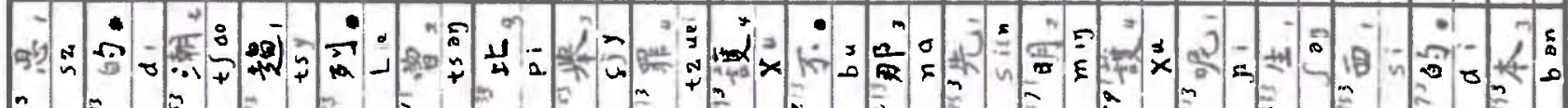

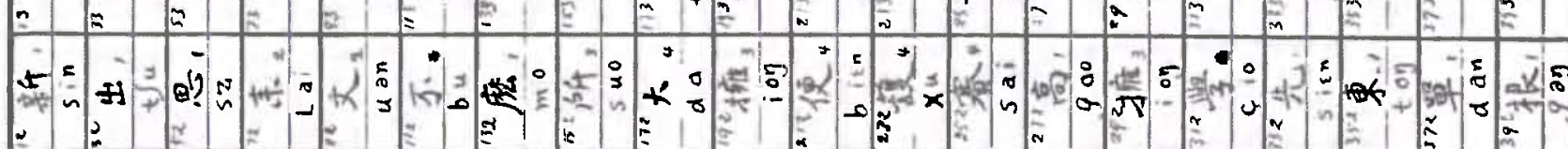

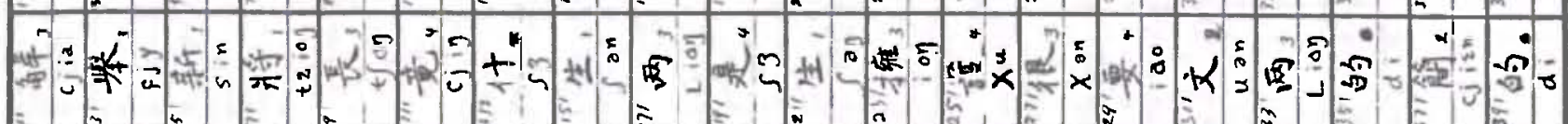

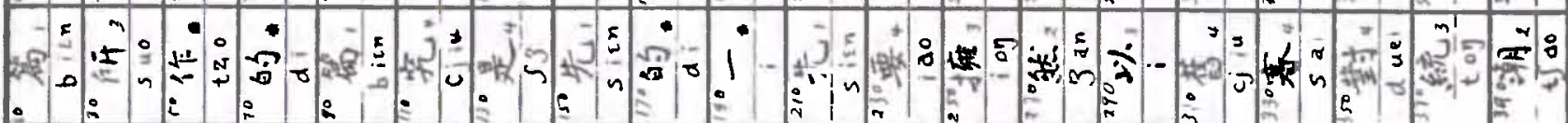

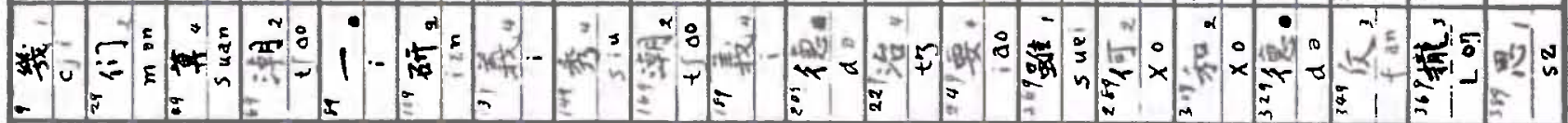

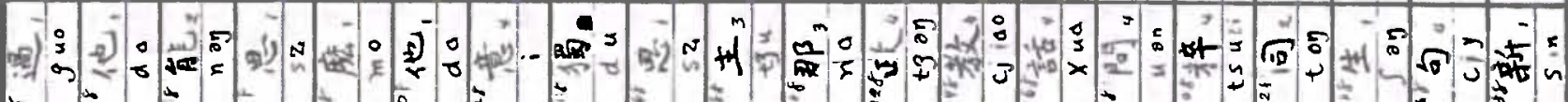

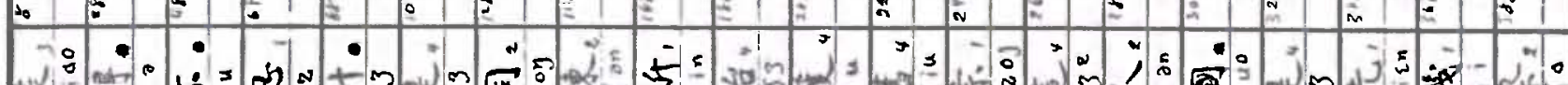
Hin

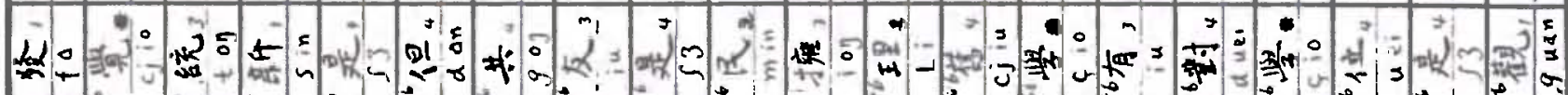

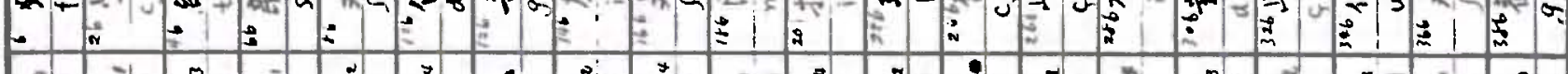

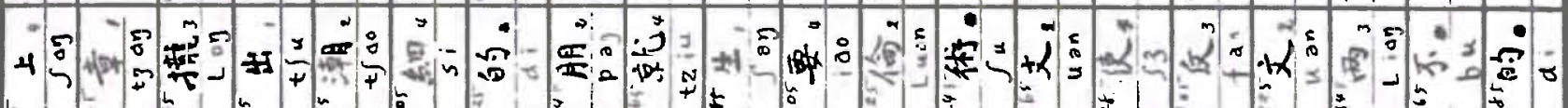

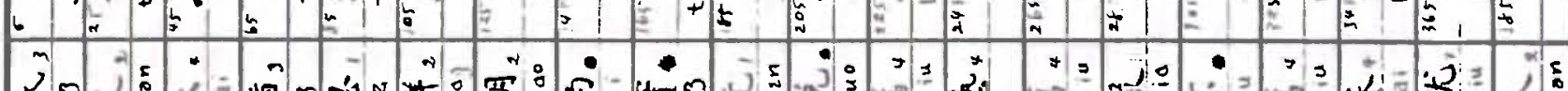

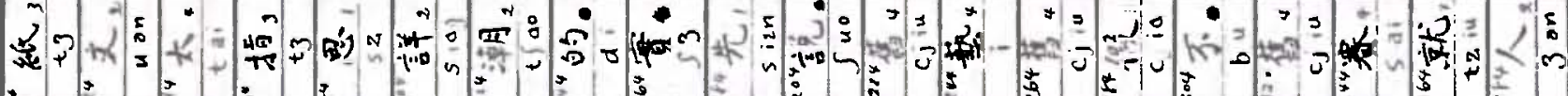

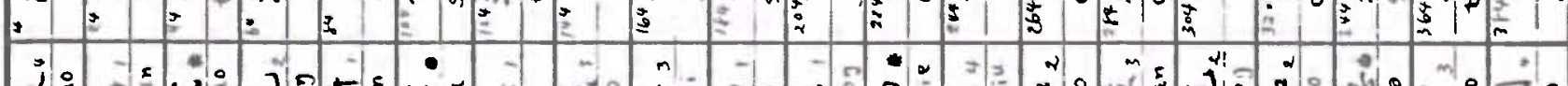

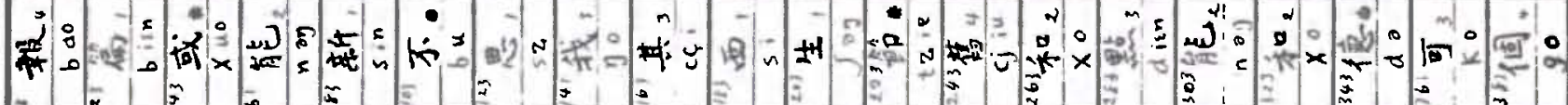

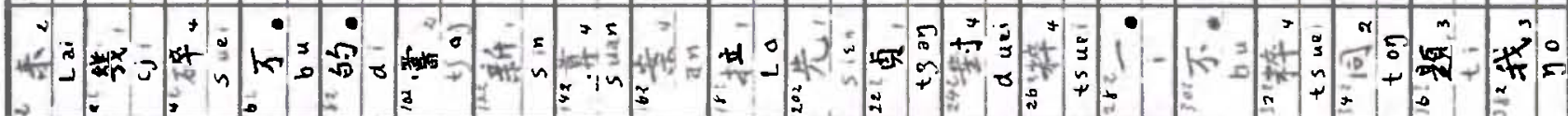

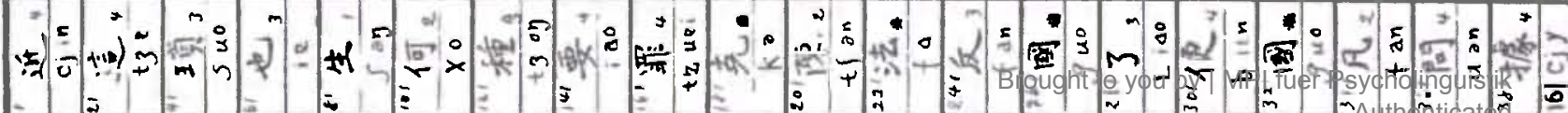




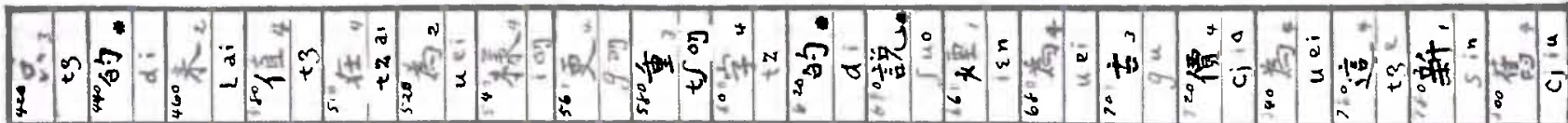

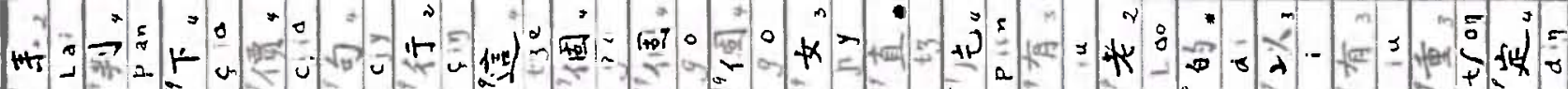

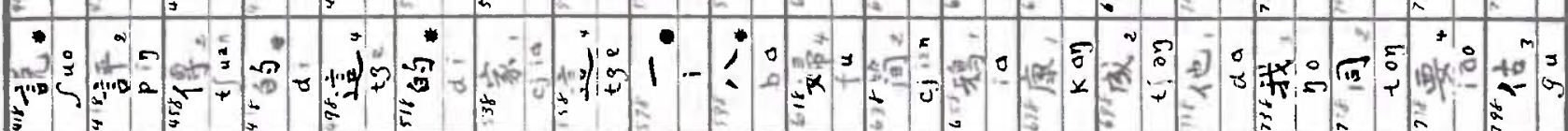

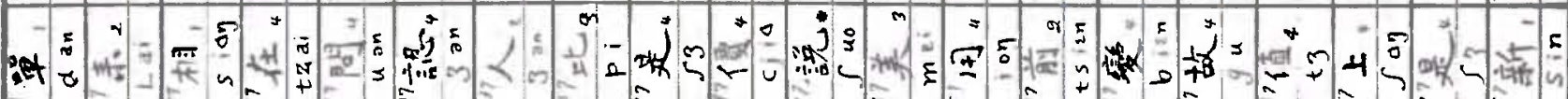

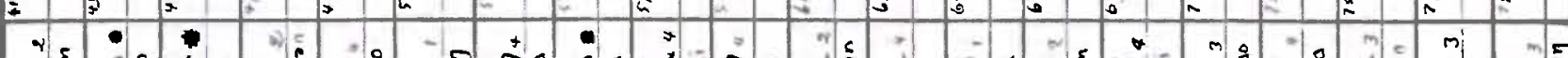

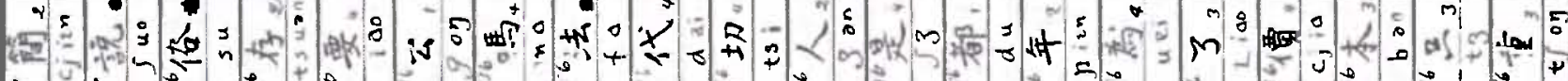

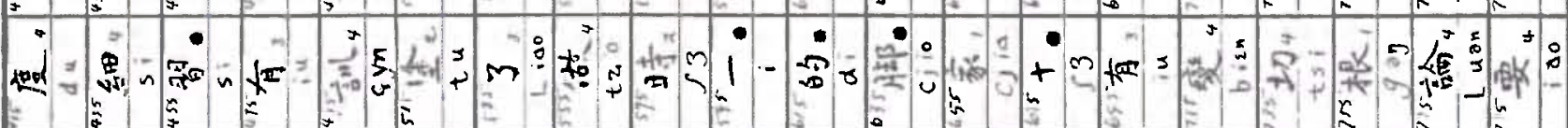

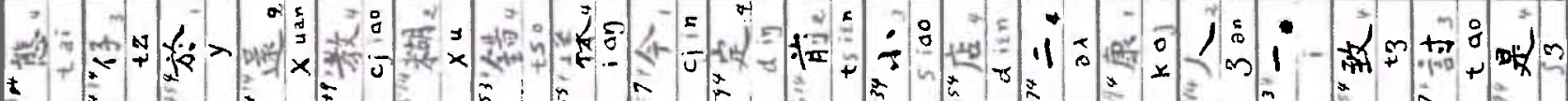

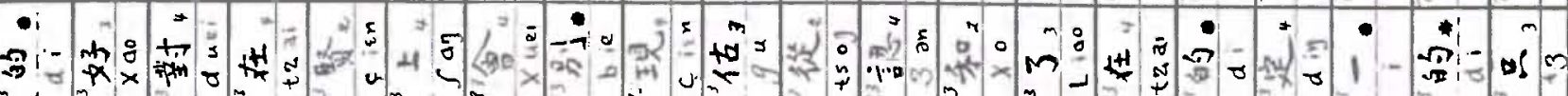

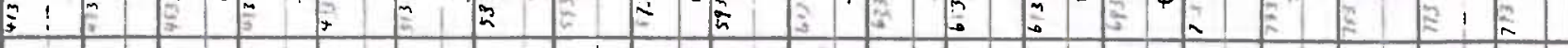

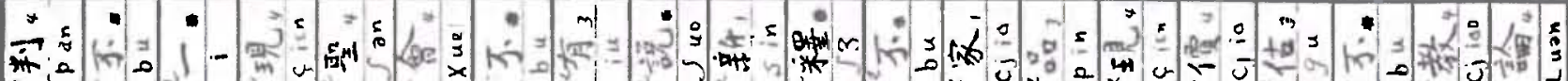

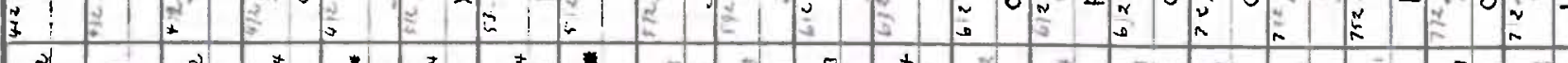

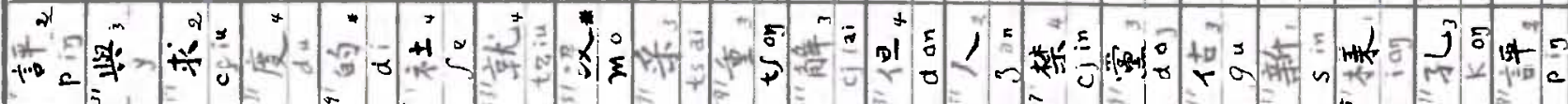

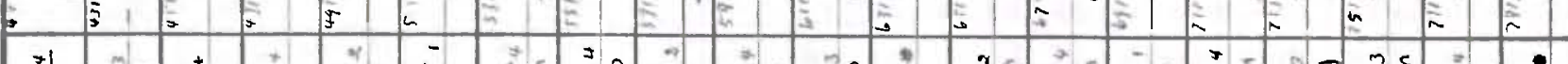

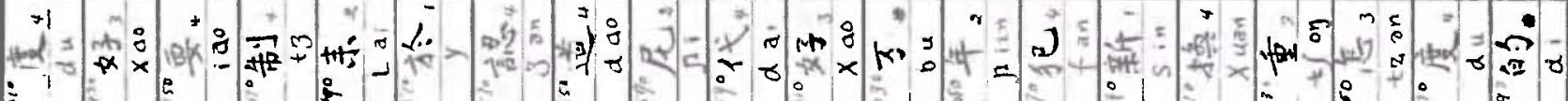

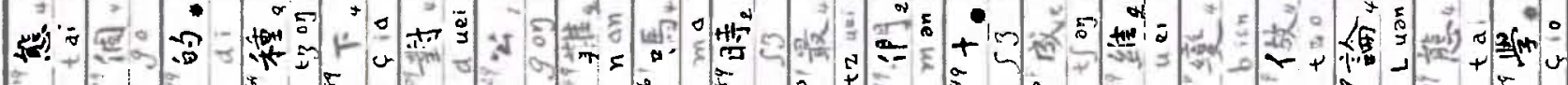

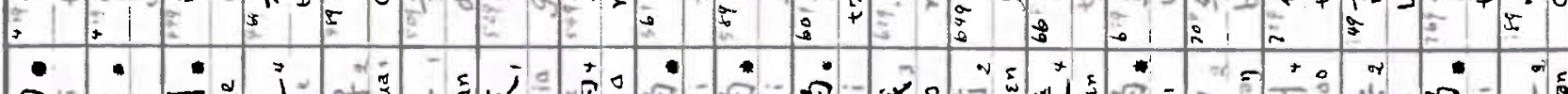

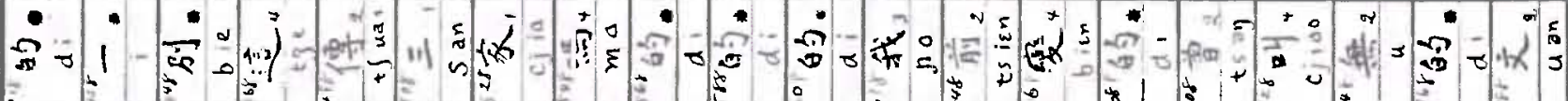
F.

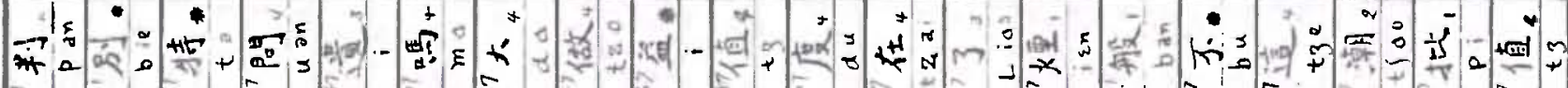

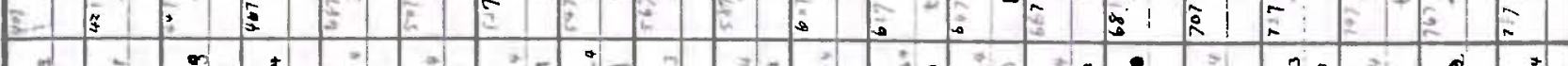

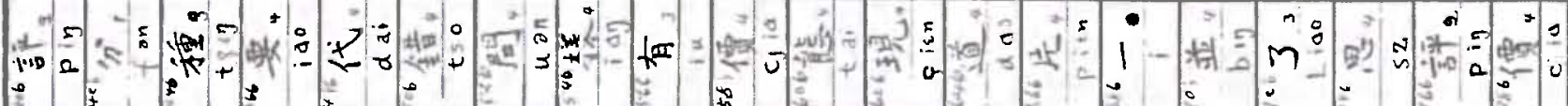

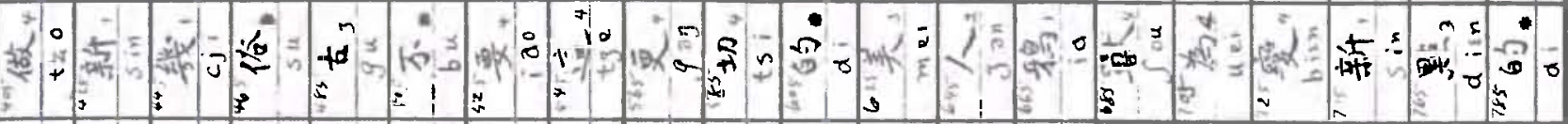

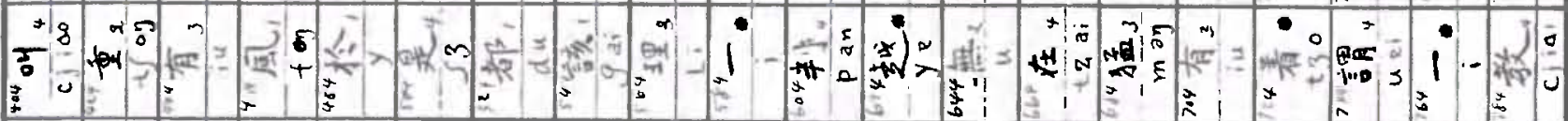

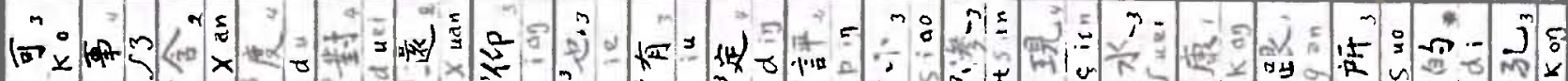

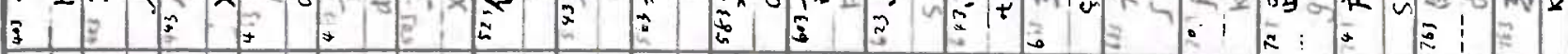

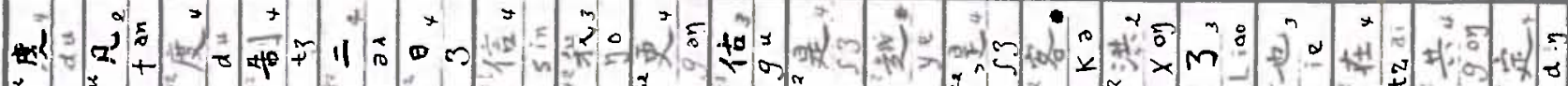

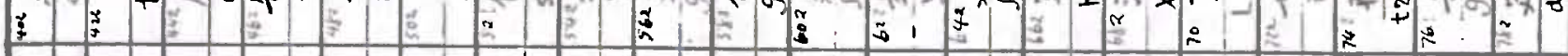

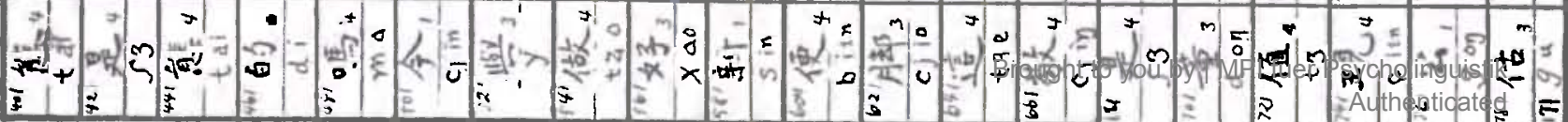




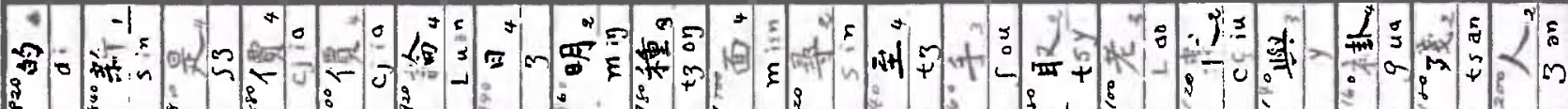

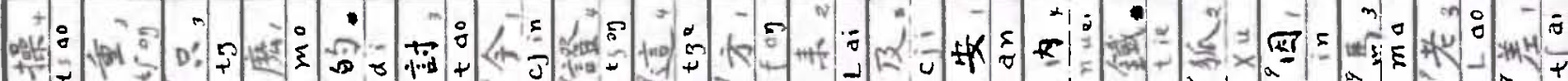

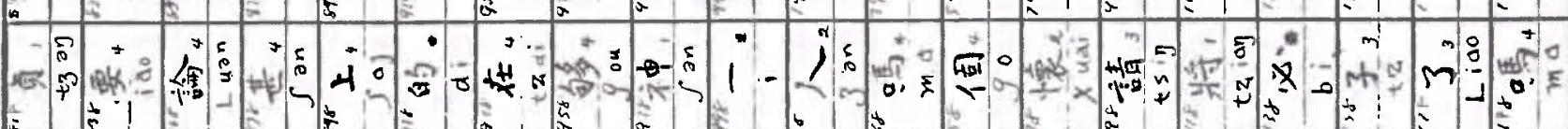

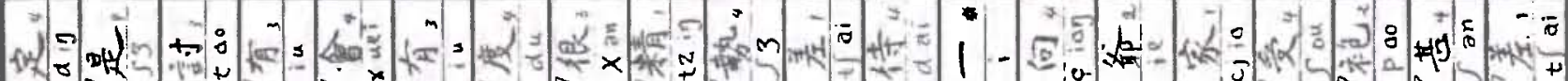
(a)

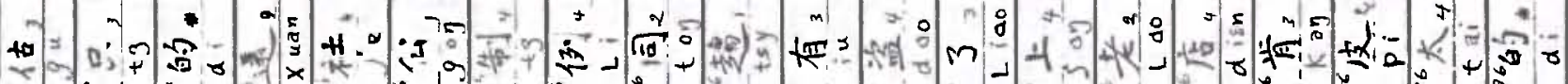

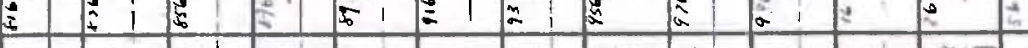

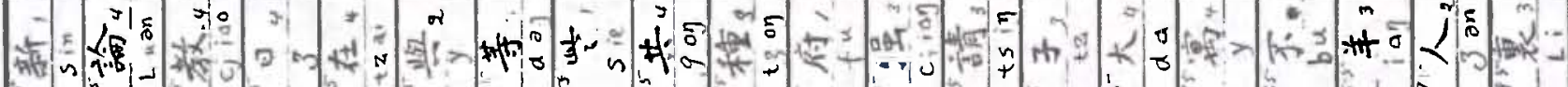

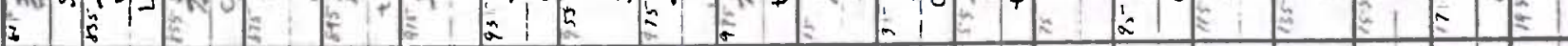

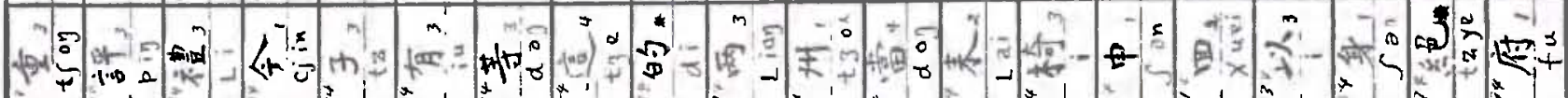

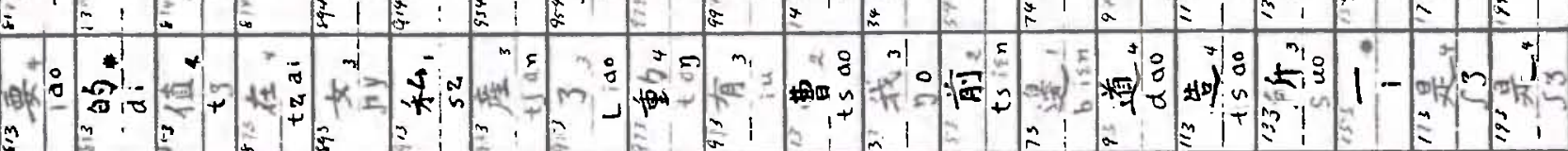

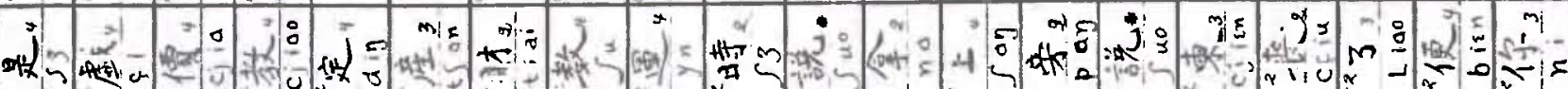

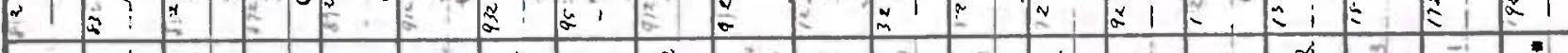

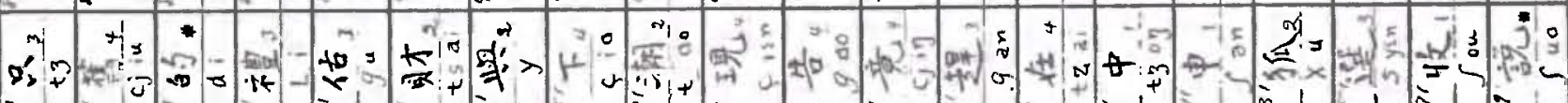

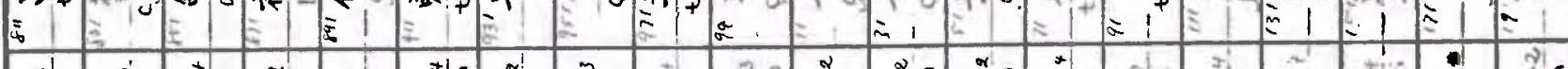

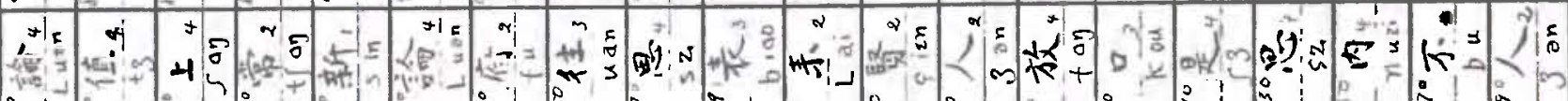

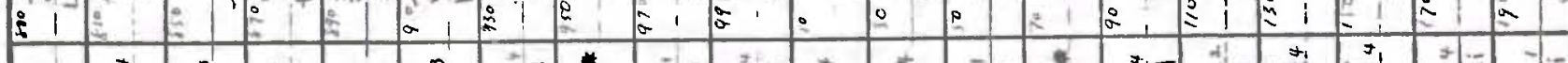

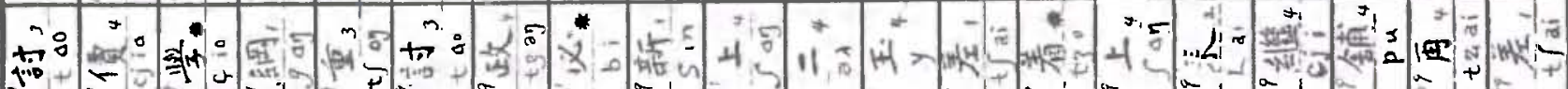

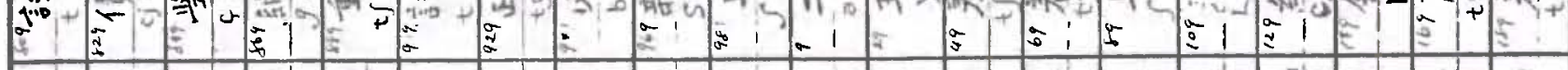

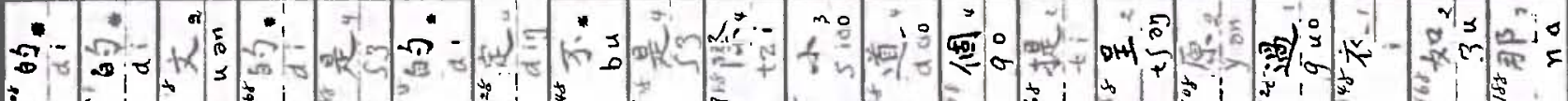

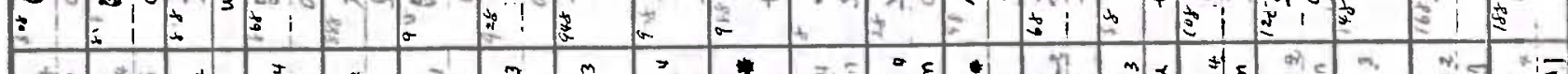

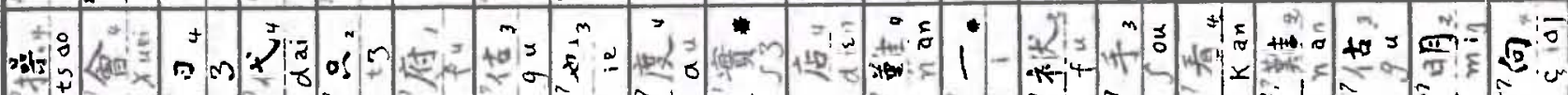

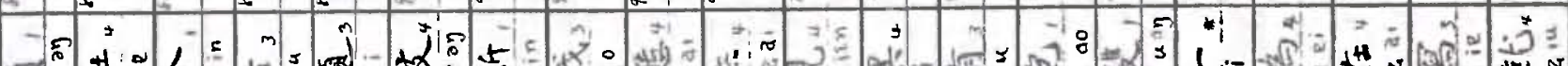

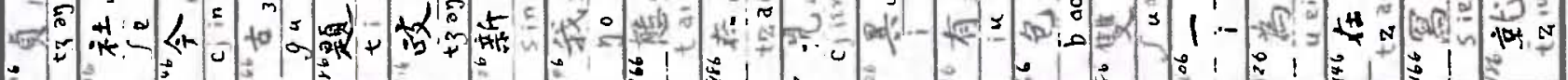

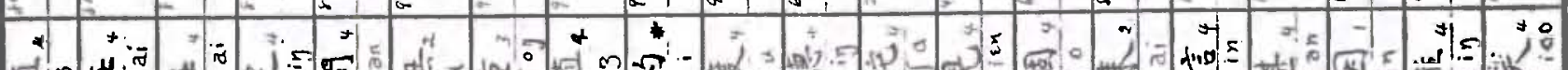

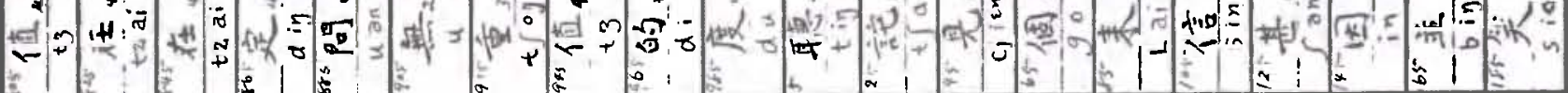

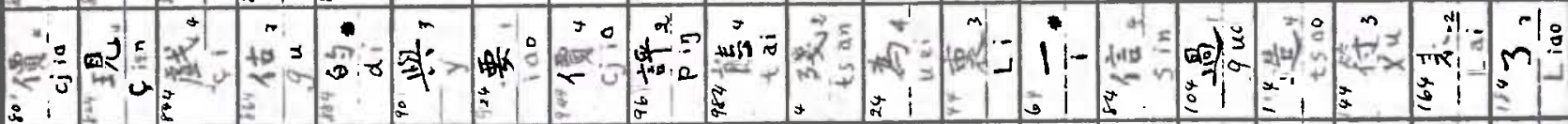

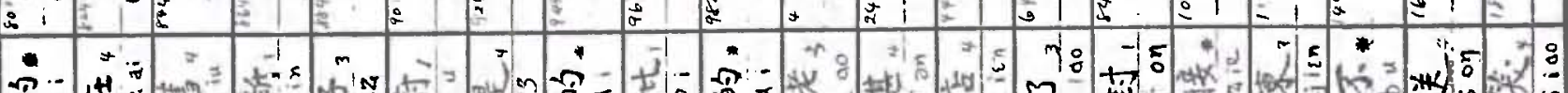
客-

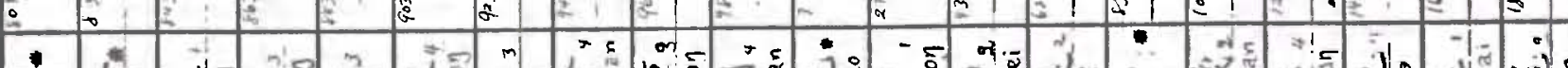

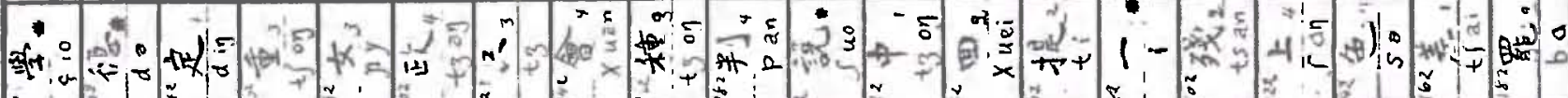

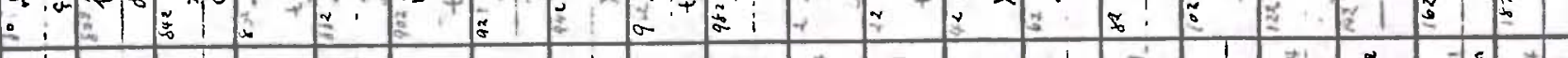
F|

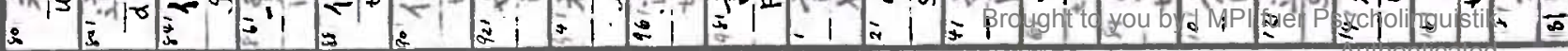




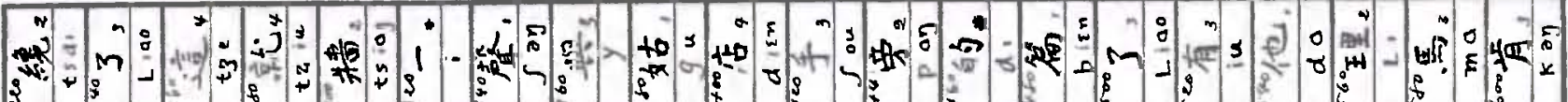

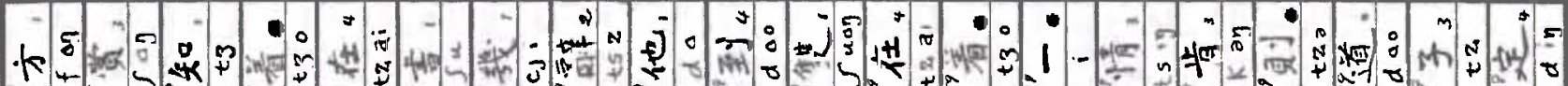
*

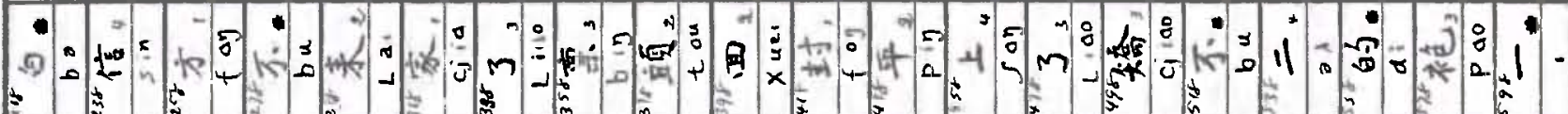

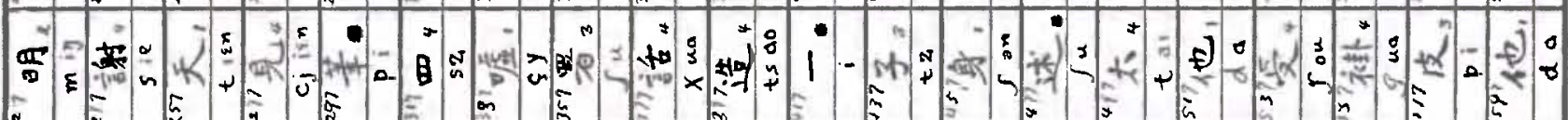

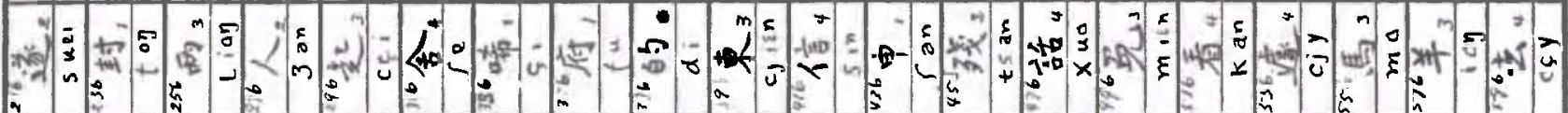
皮 皮 $+$

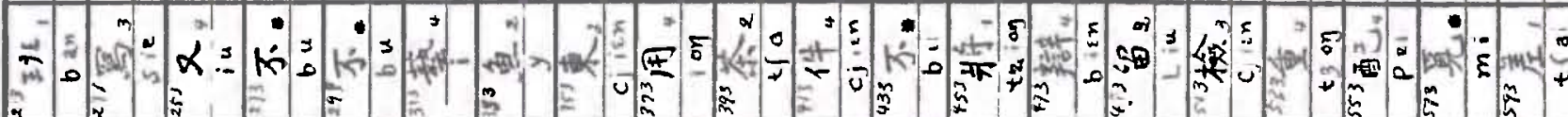

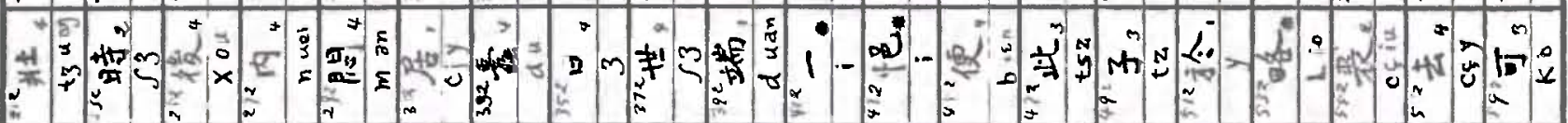

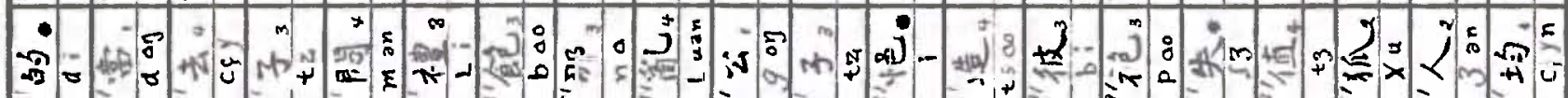

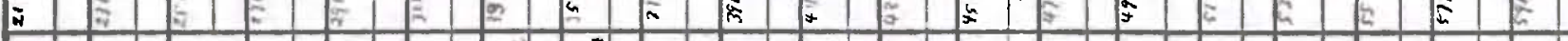

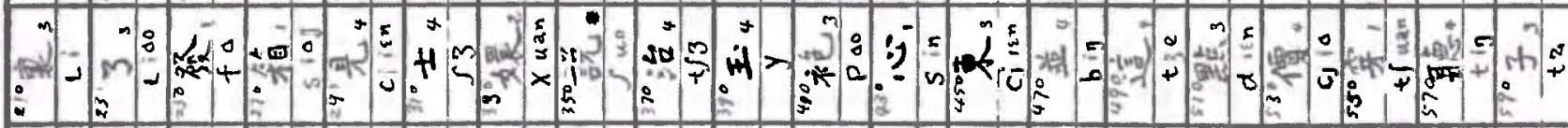

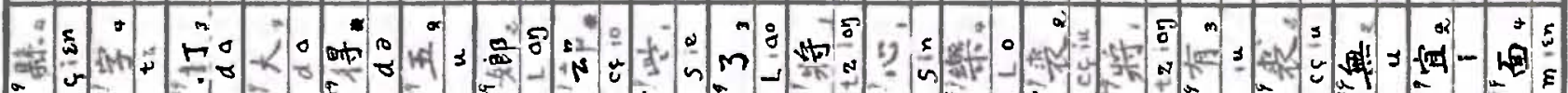

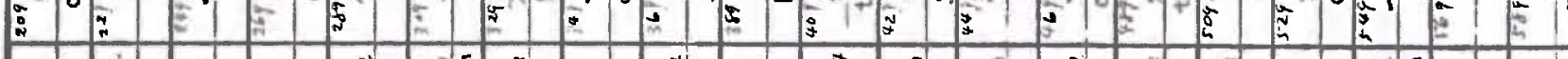

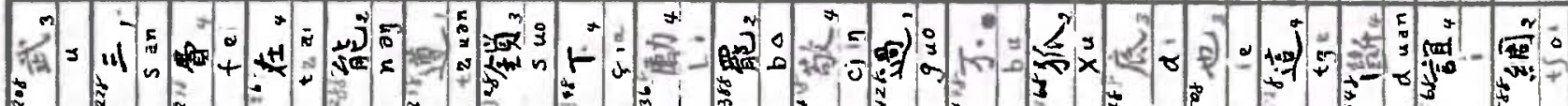

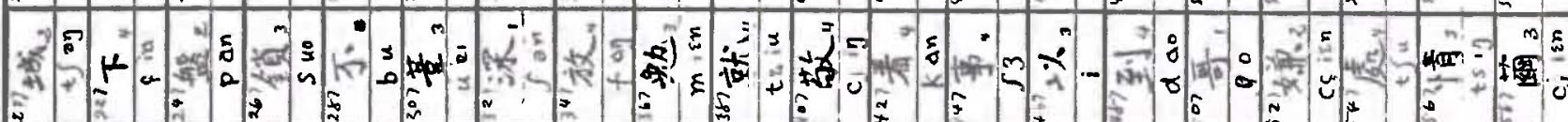

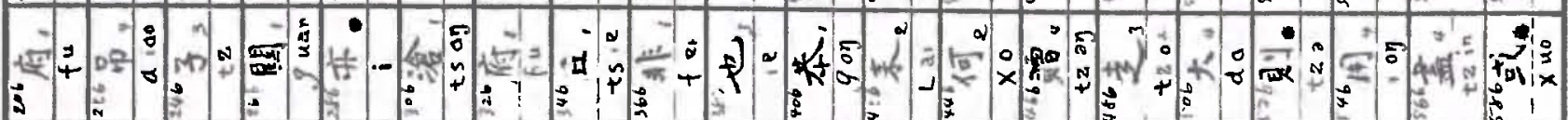

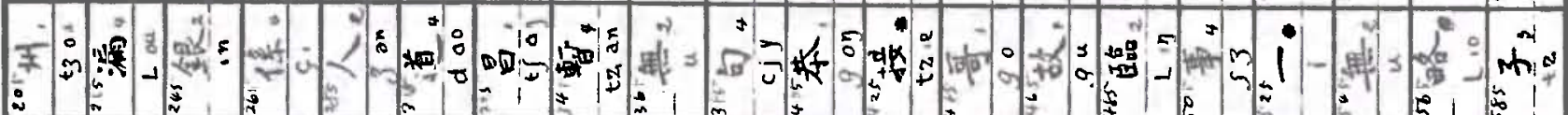

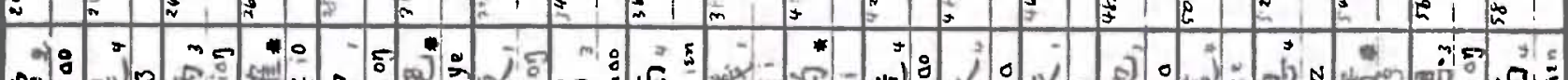
F)

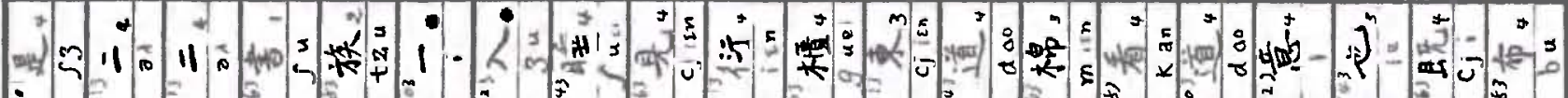

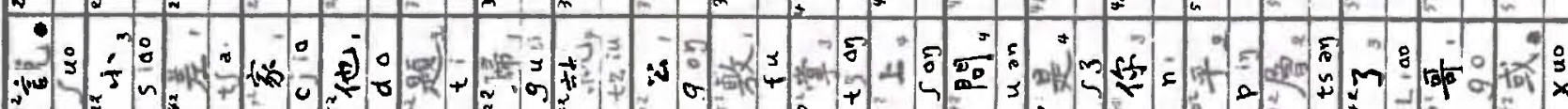

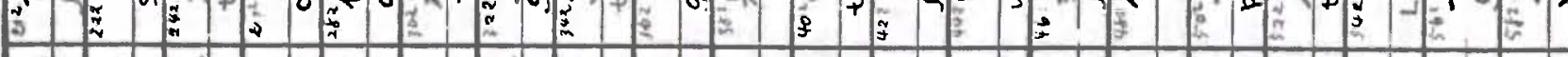

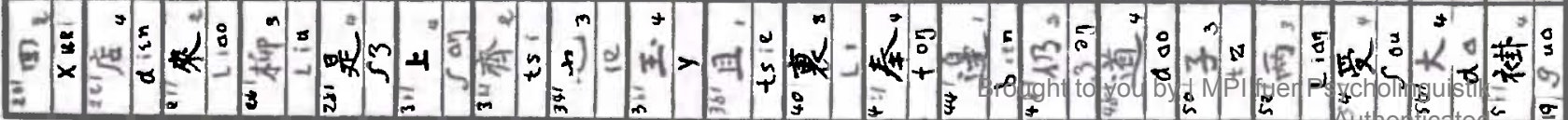




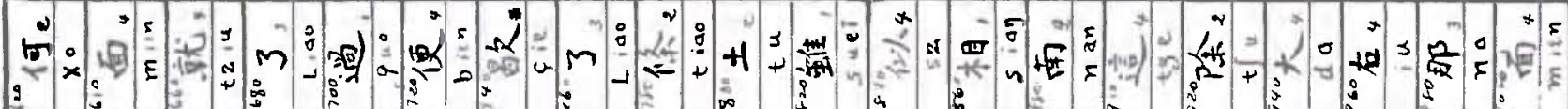

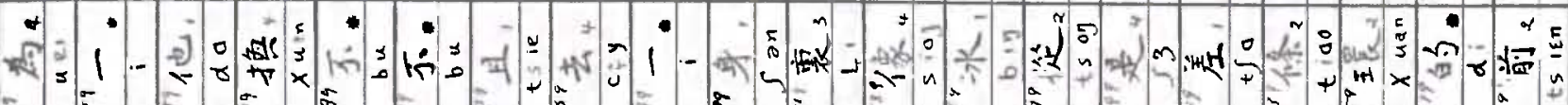

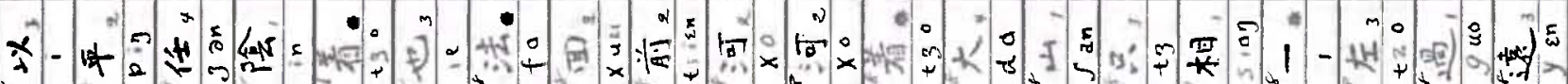

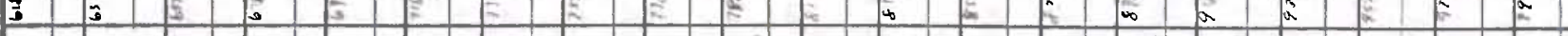

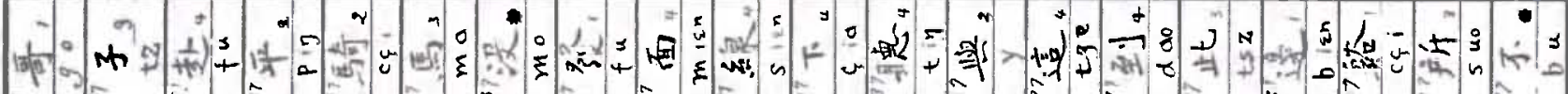

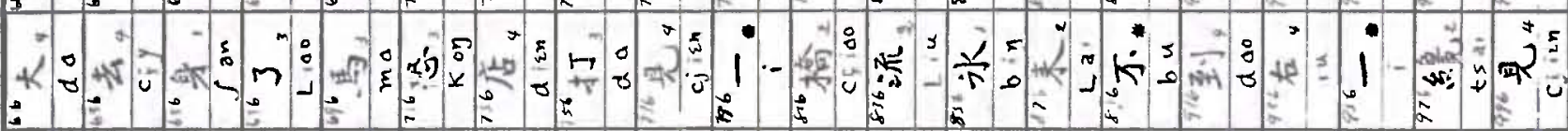

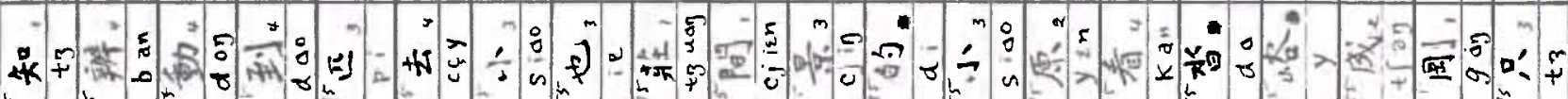

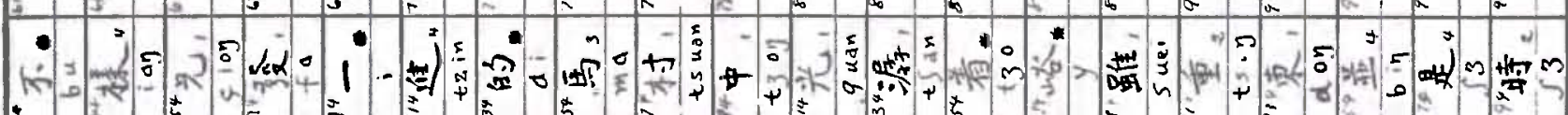

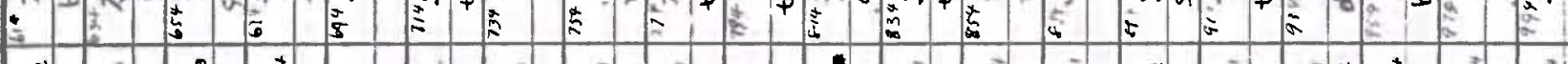

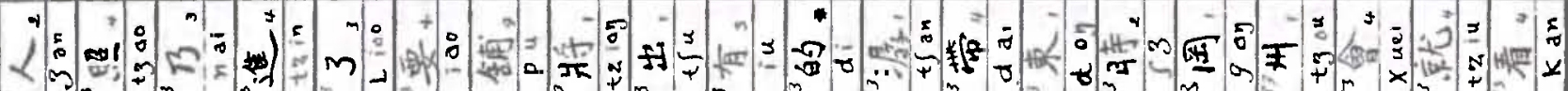

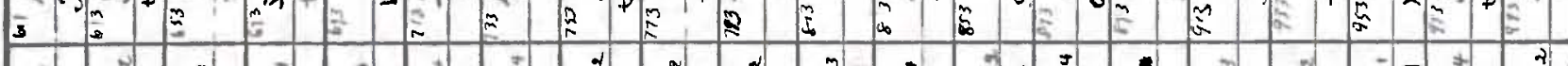

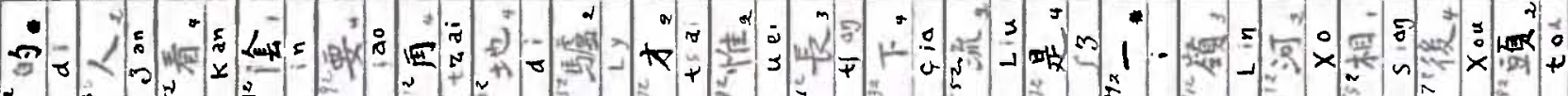

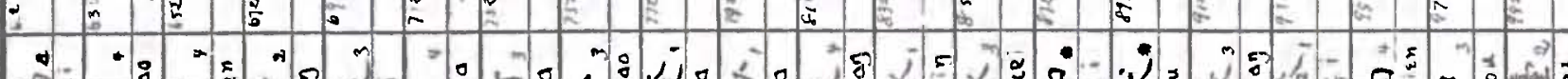

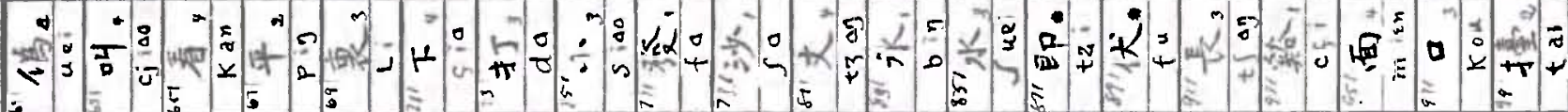

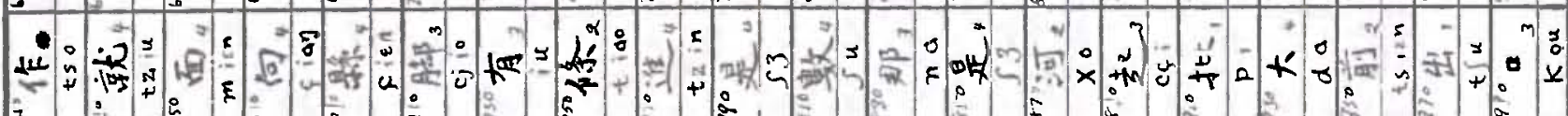

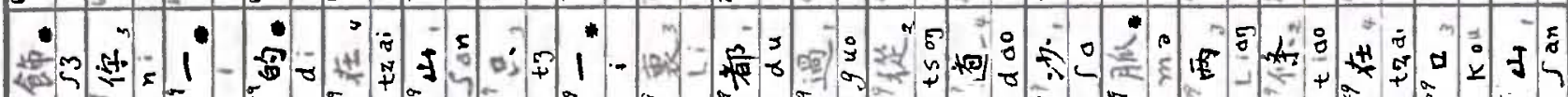

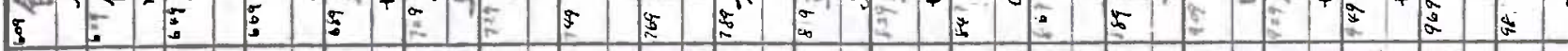

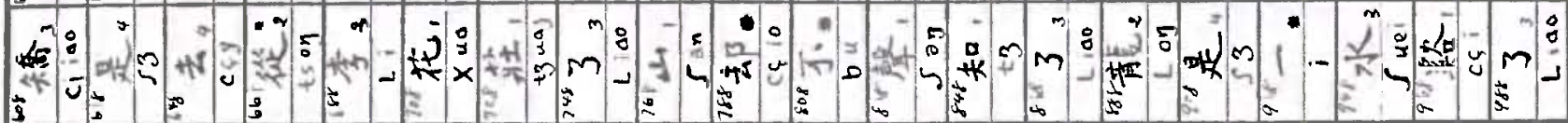

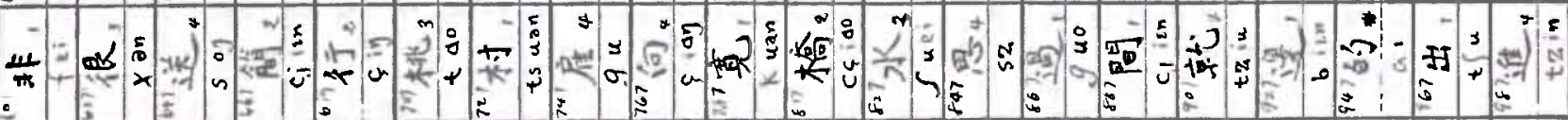

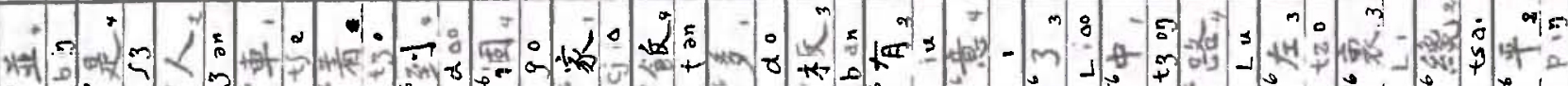

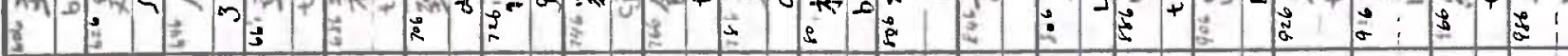

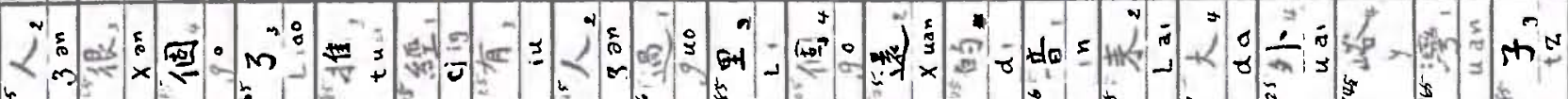

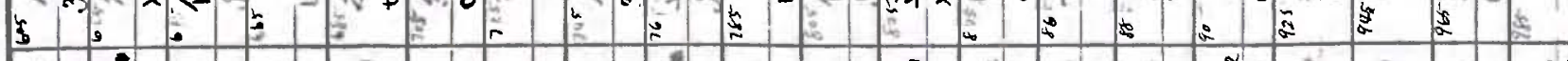

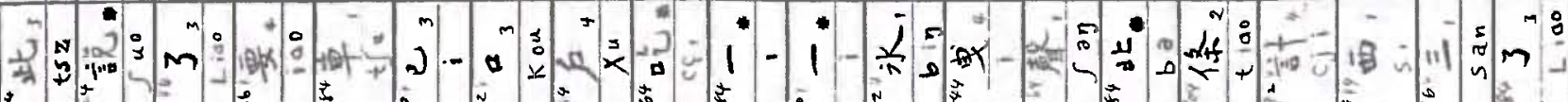

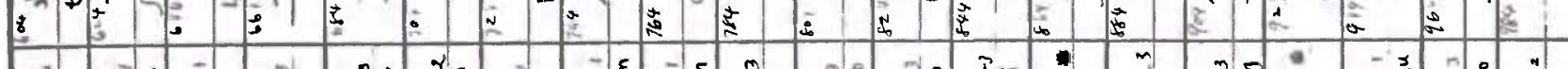

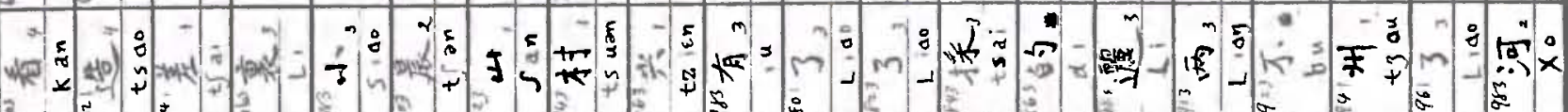
m० 350 j

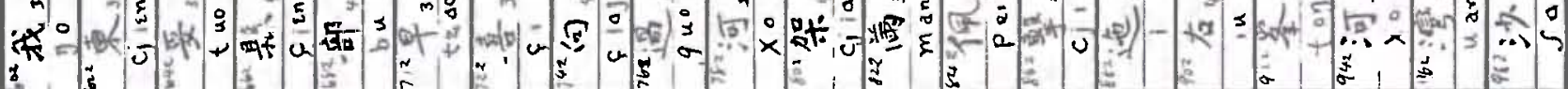

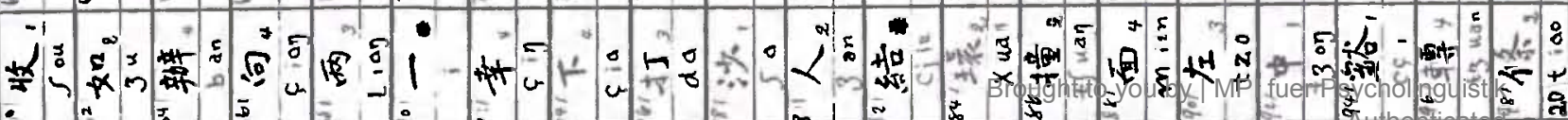


14a.

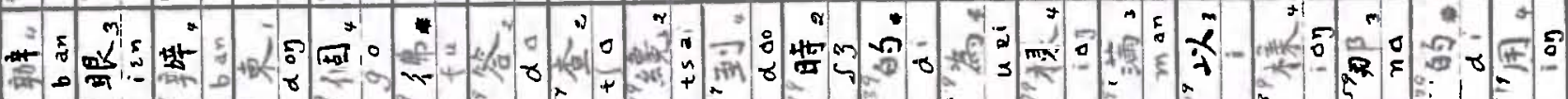

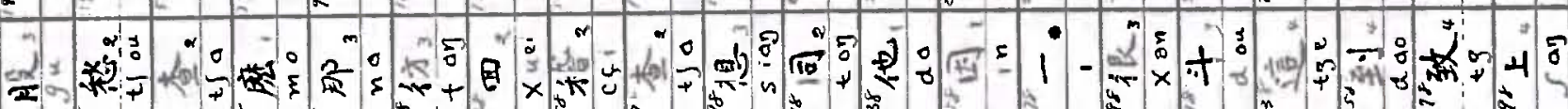

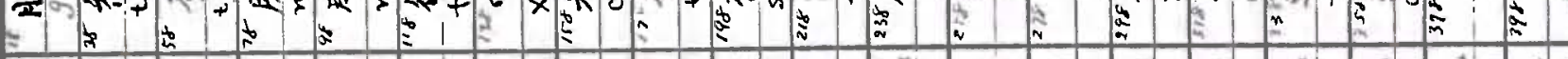

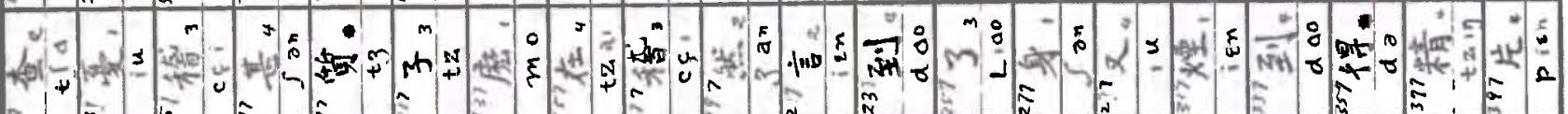
do

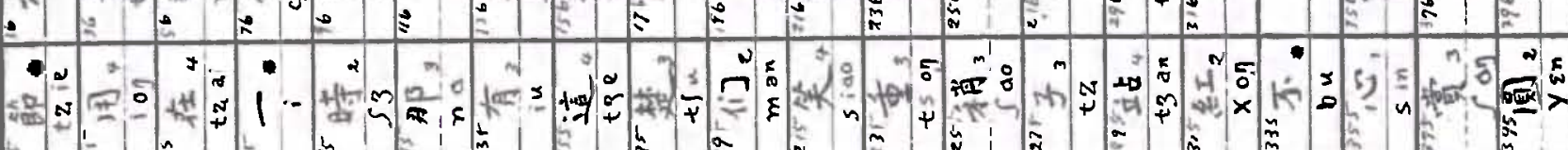

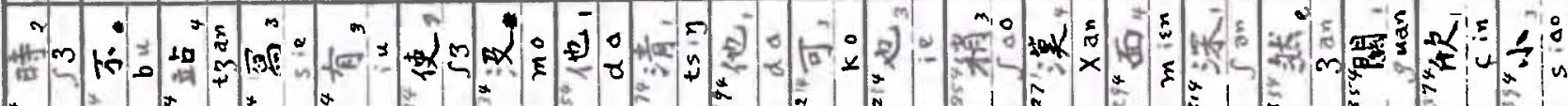
dor

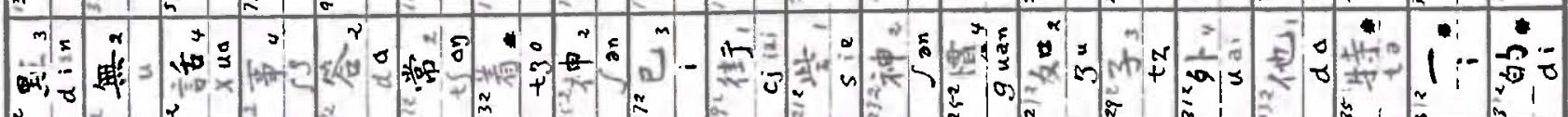

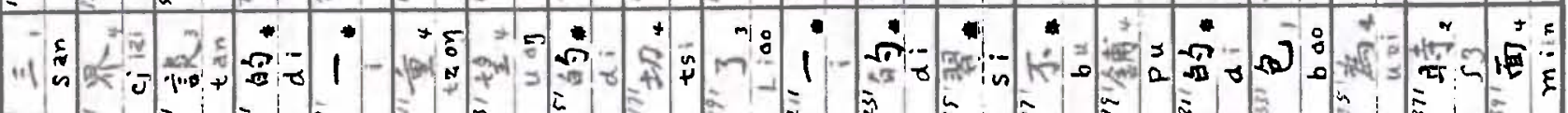

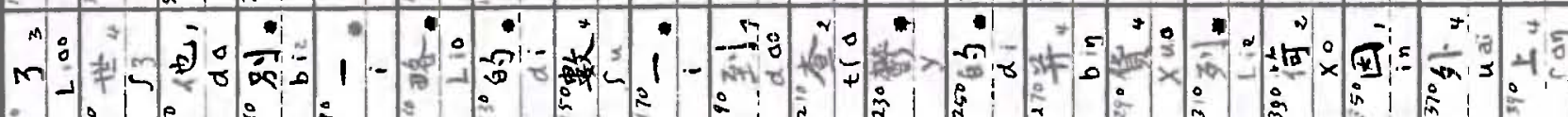

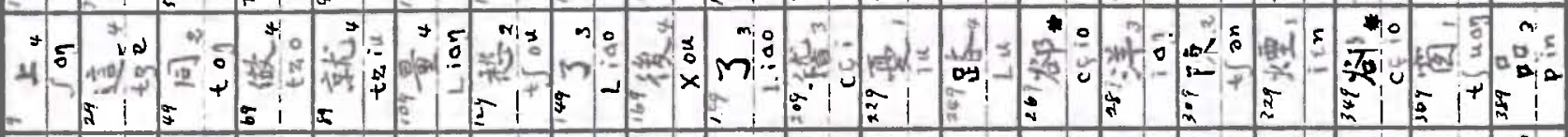

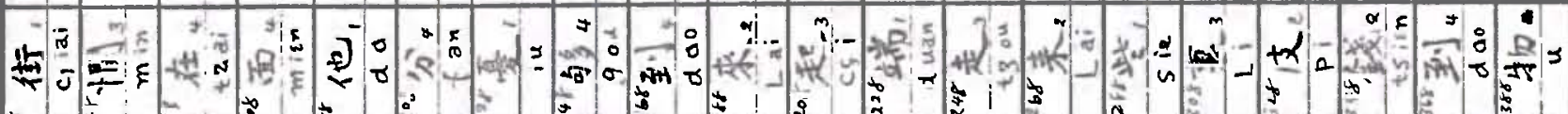

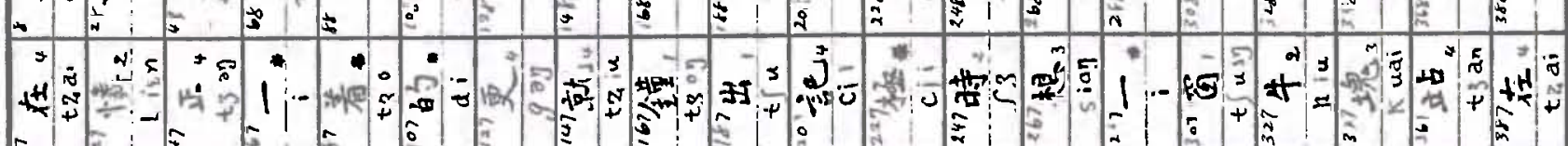

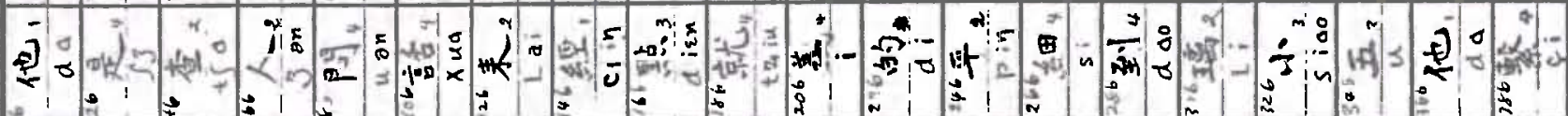

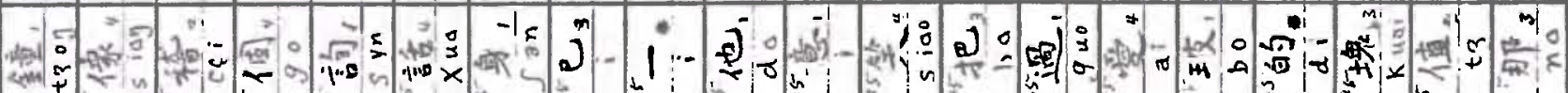

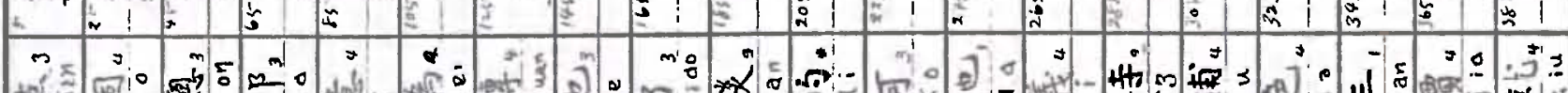
Lo:

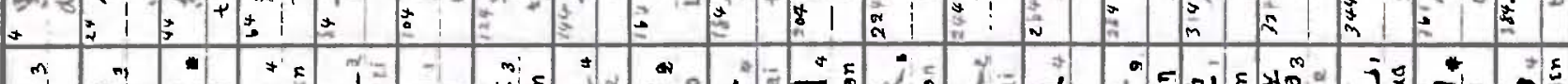

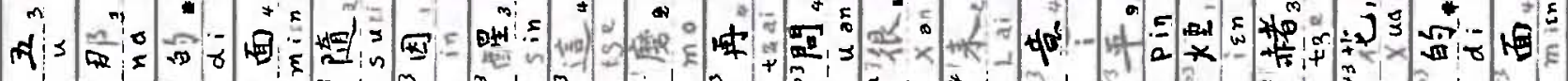

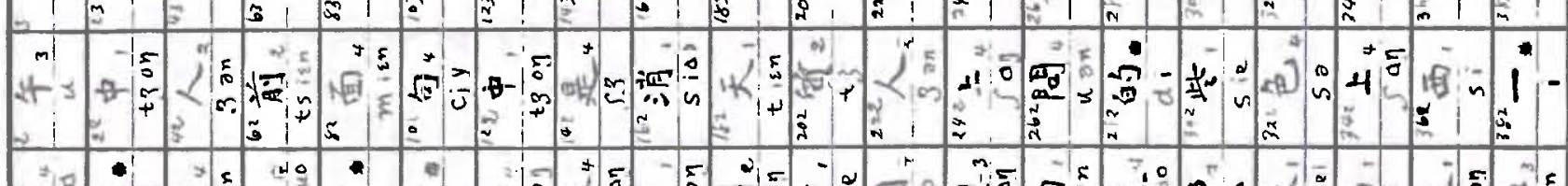

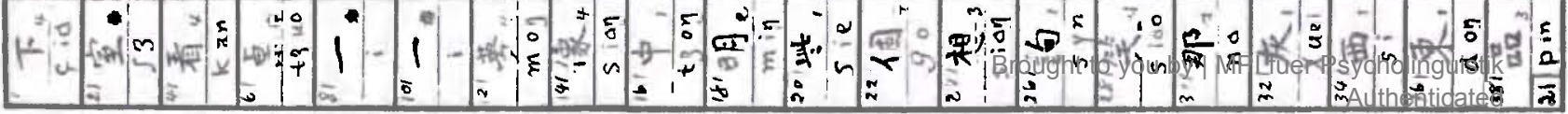


The

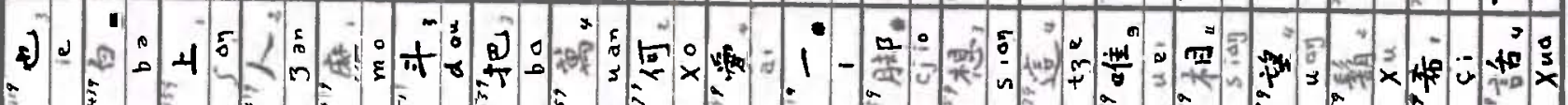

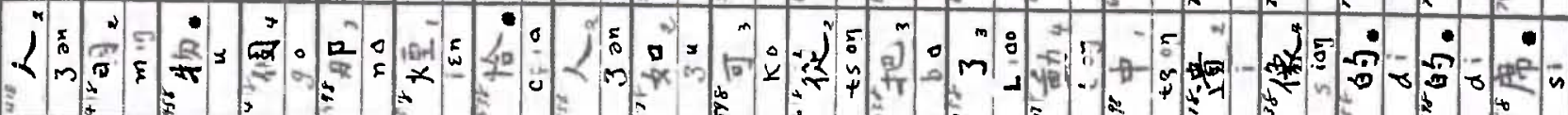
1 - -

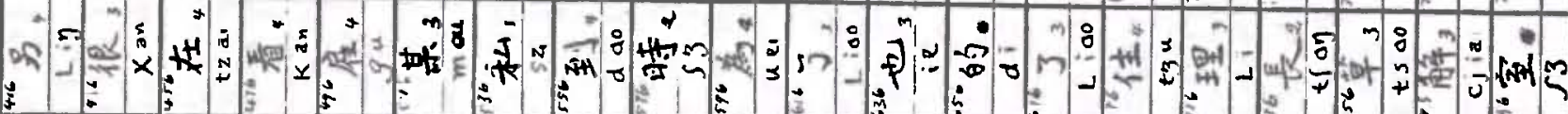

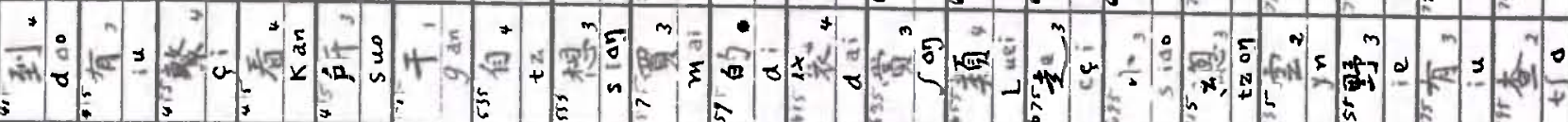
और

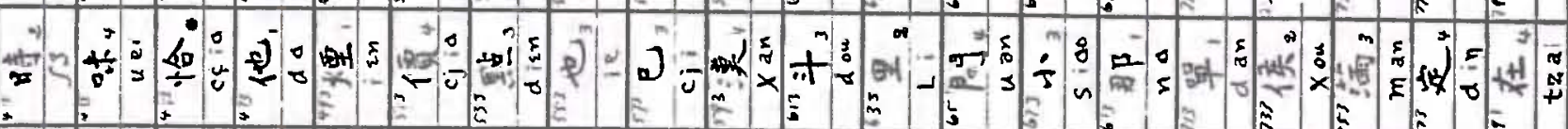

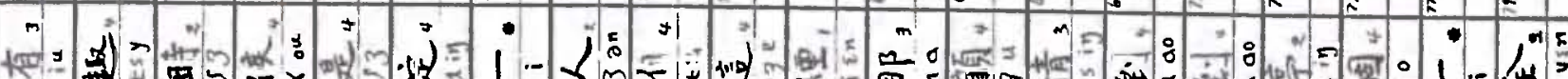

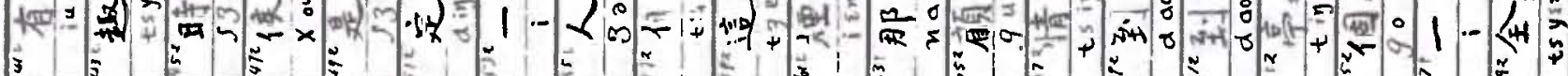

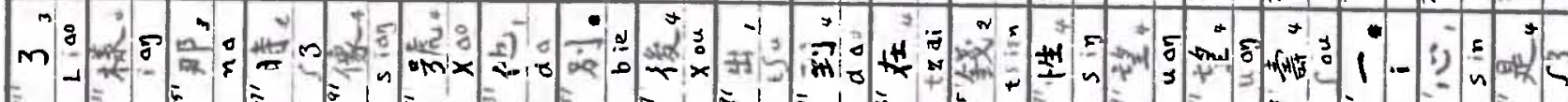

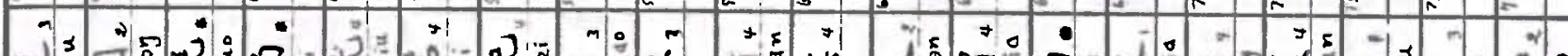

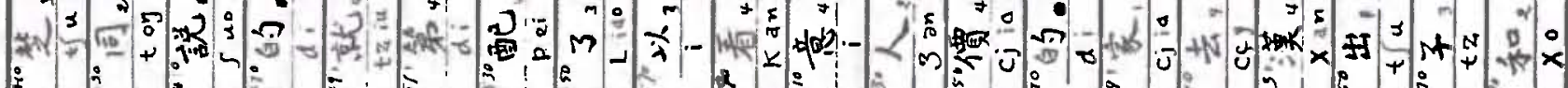

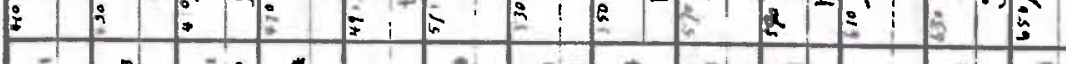

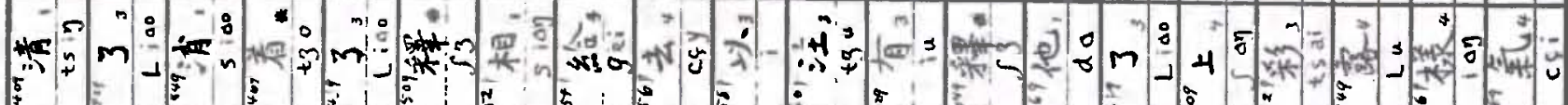

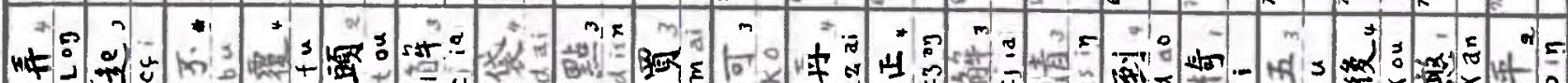

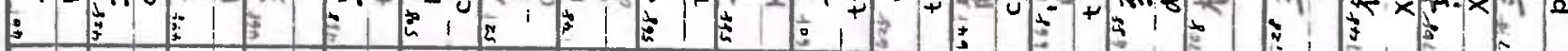

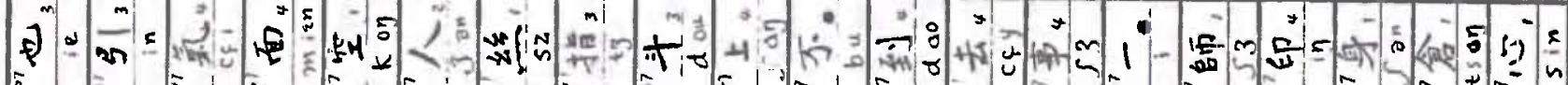

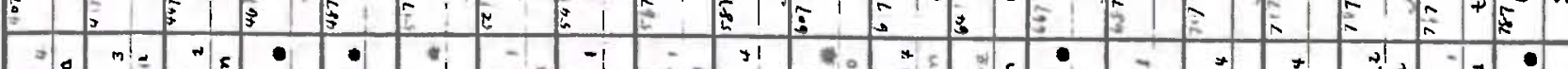

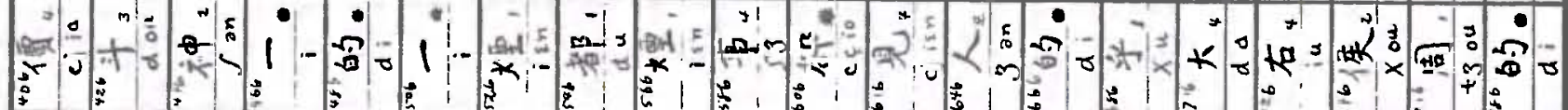

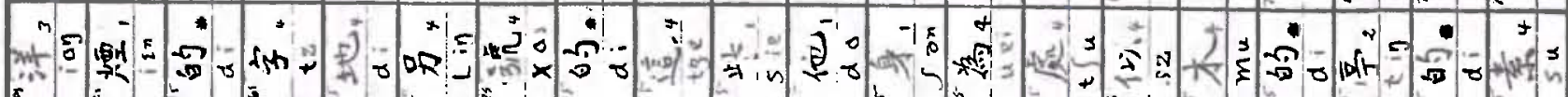
-

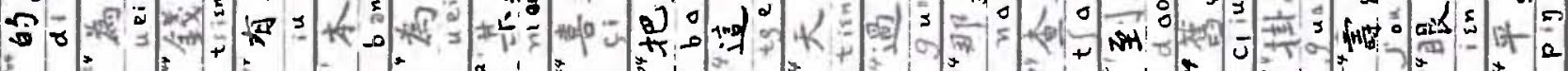

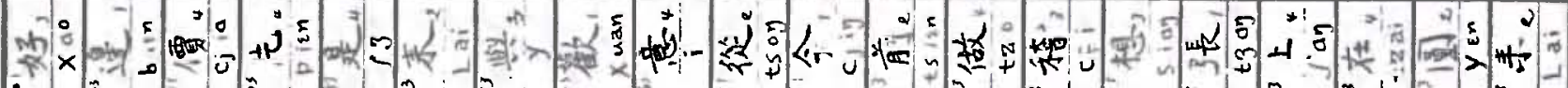

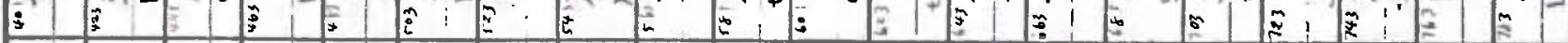

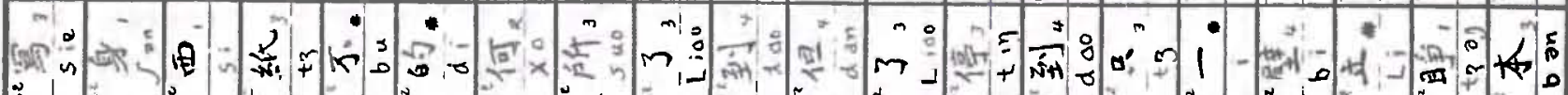

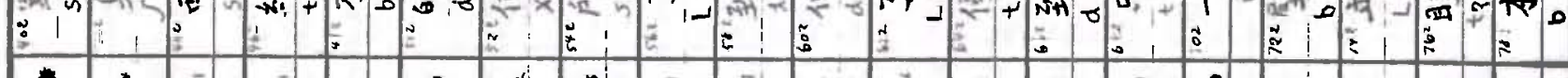

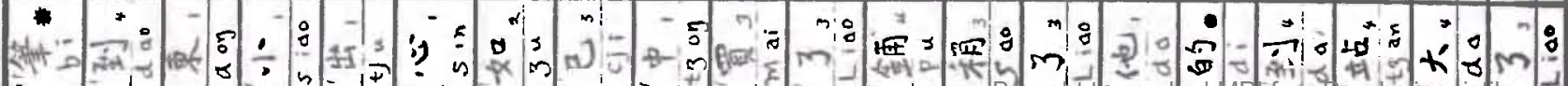

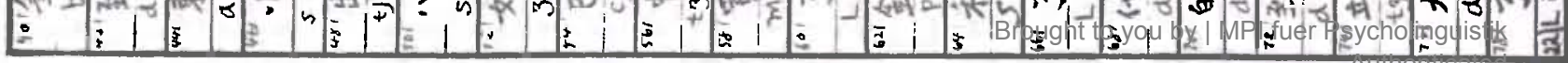




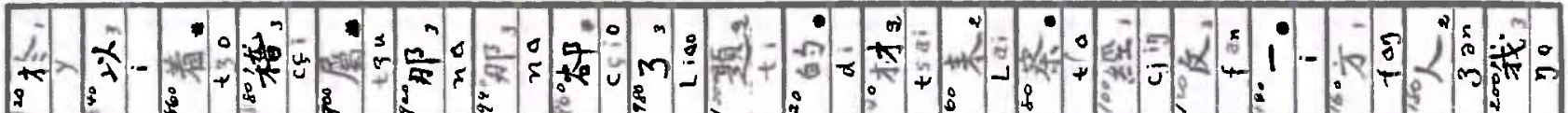

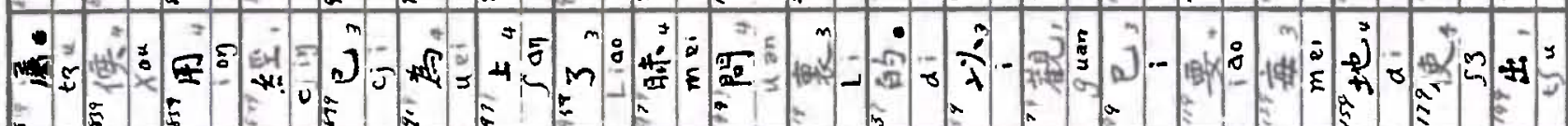

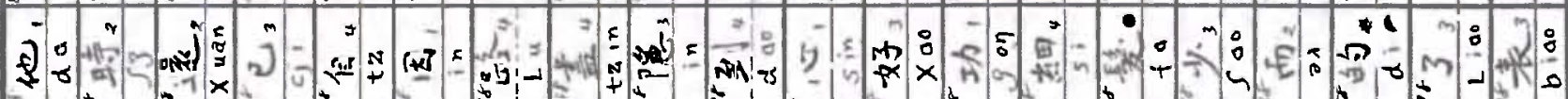

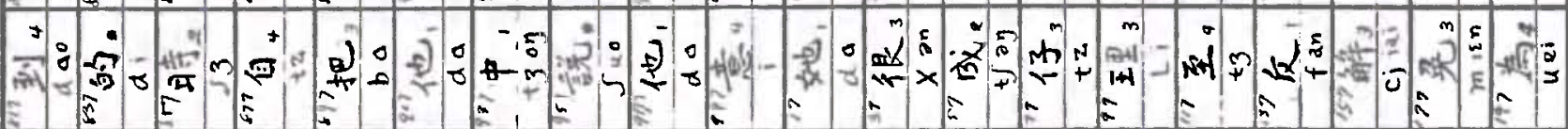

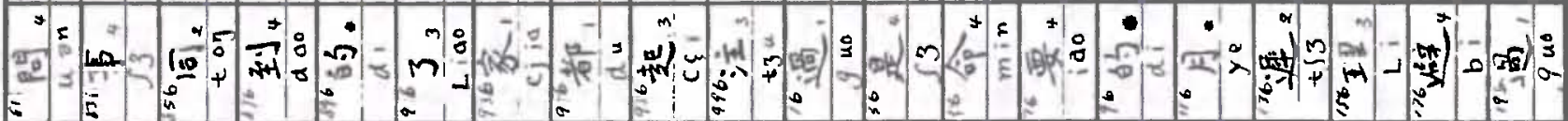

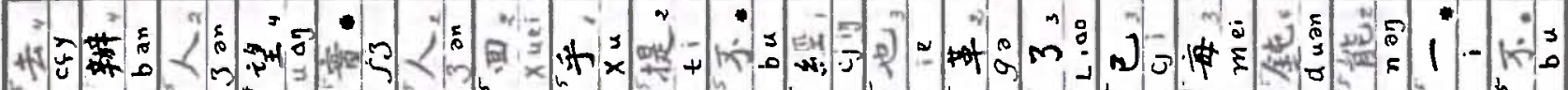

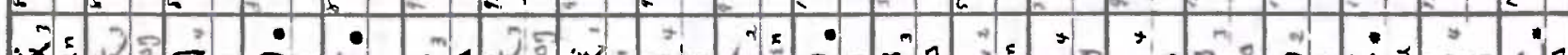

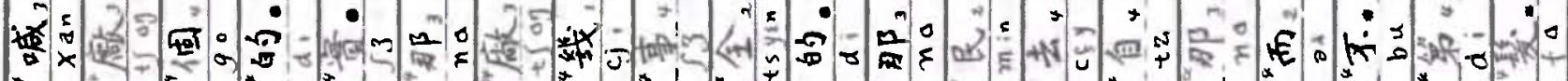

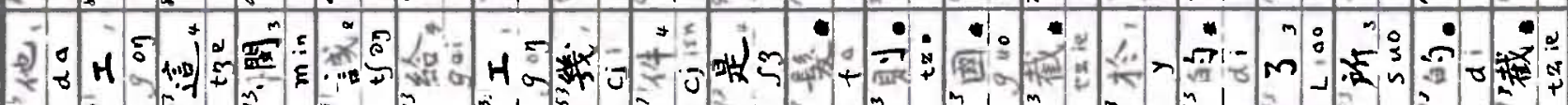

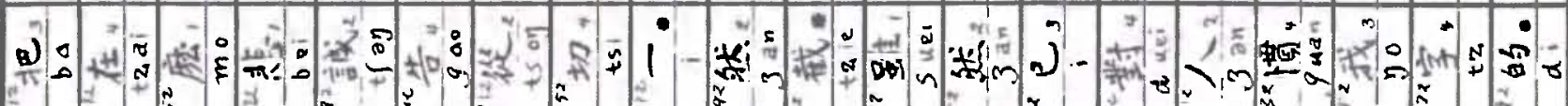

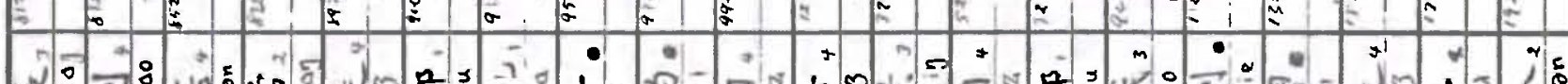

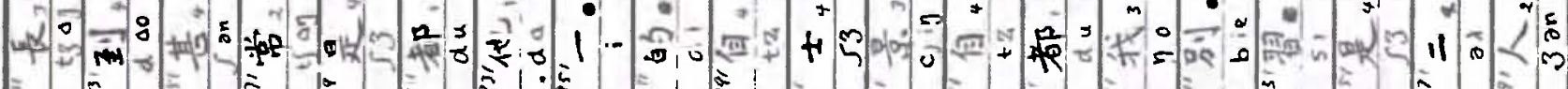
+4

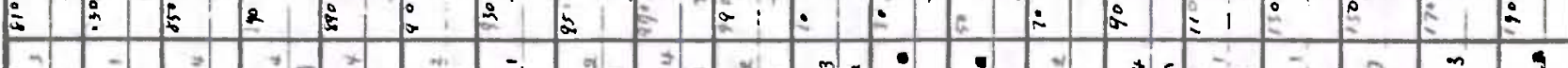

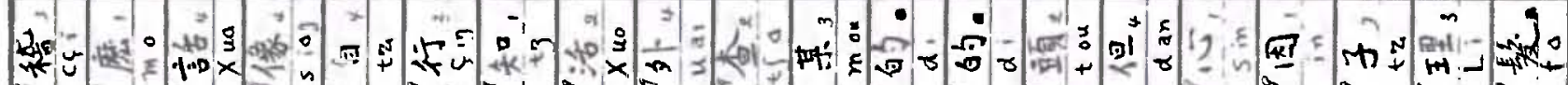

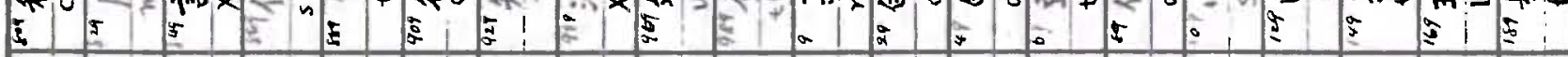

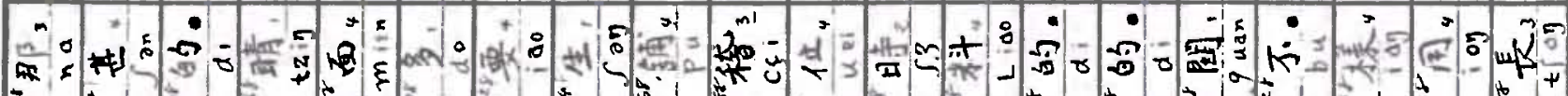

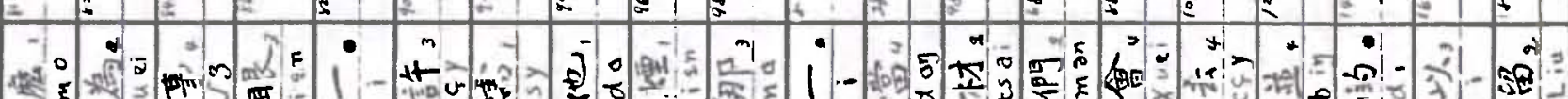

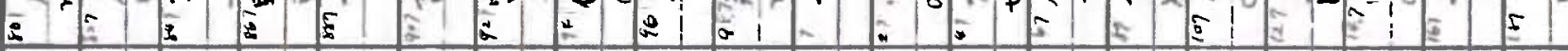

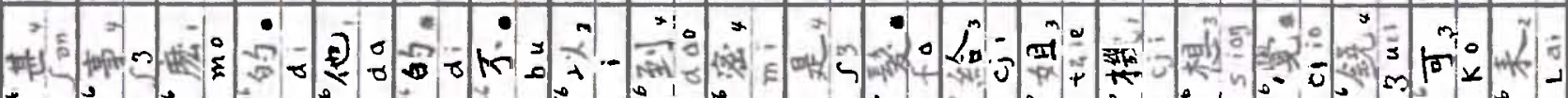

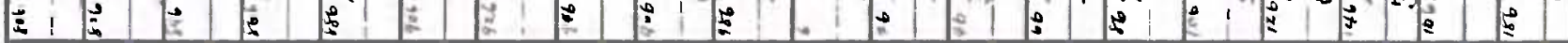

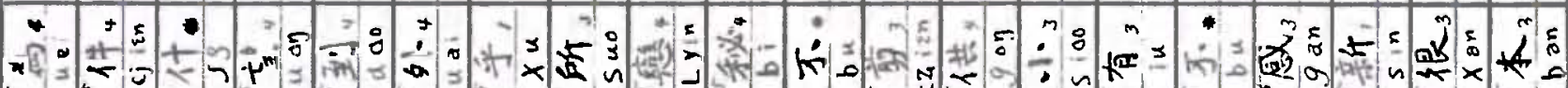
$E$ E (6)

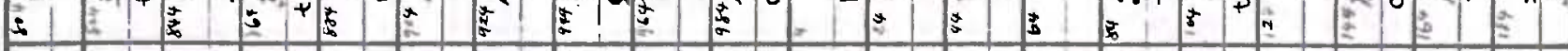

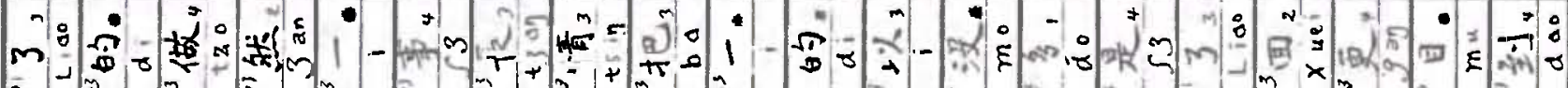

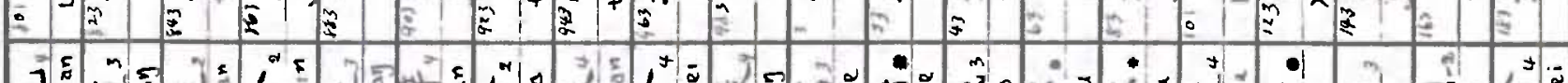

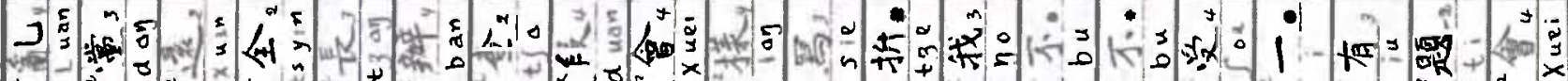

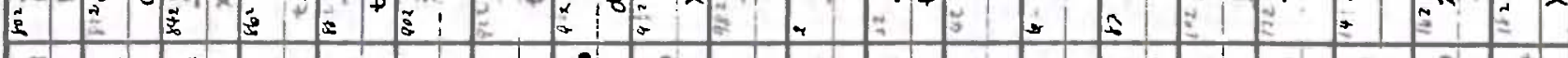

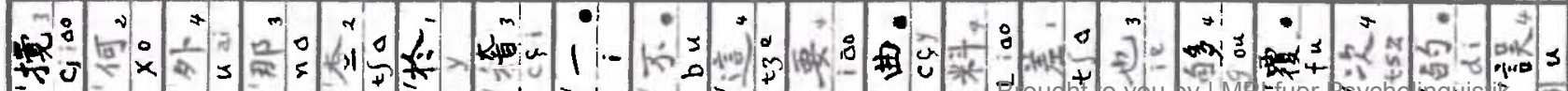
点

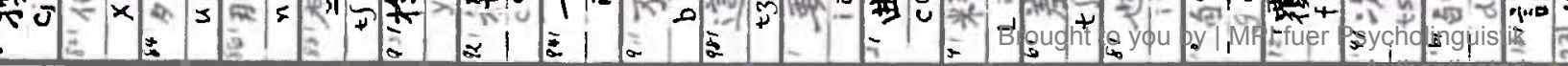




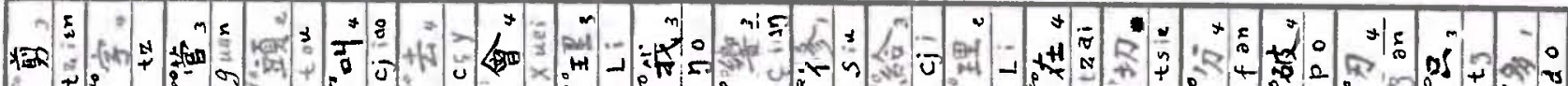

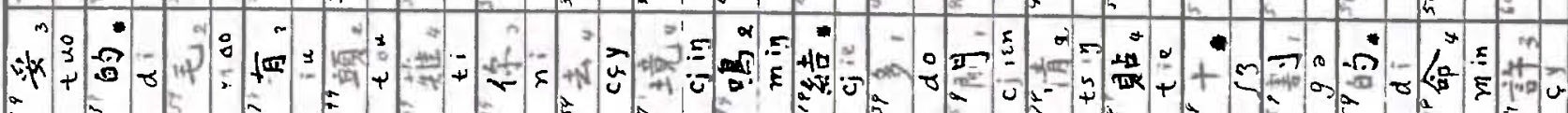

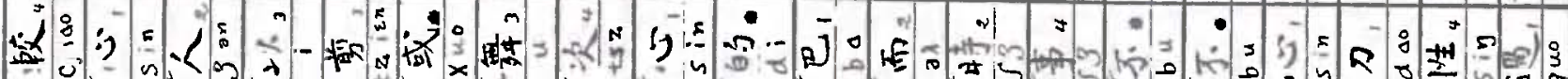

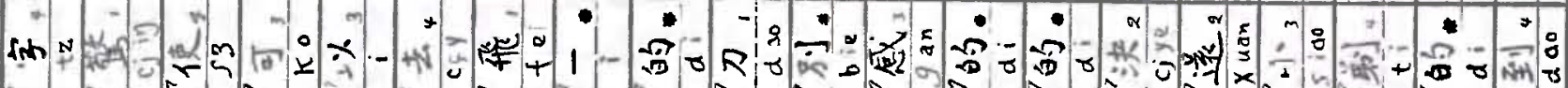

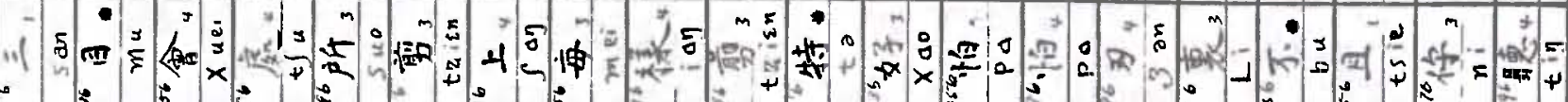

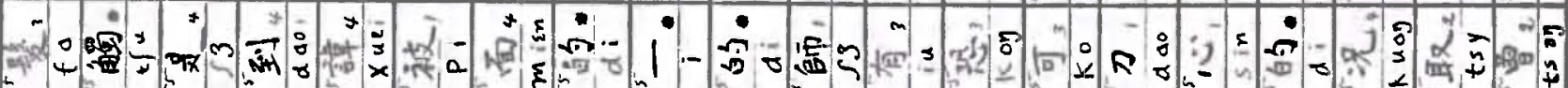

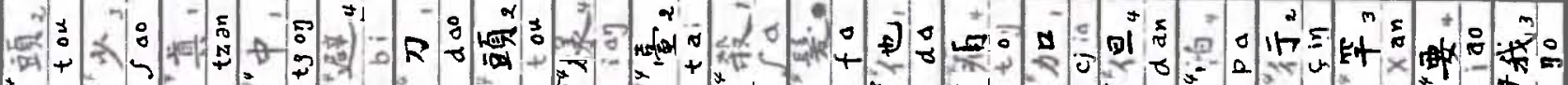

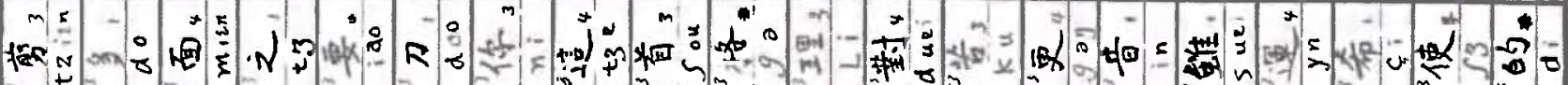
E.

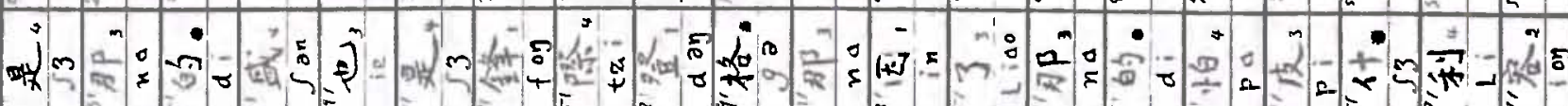

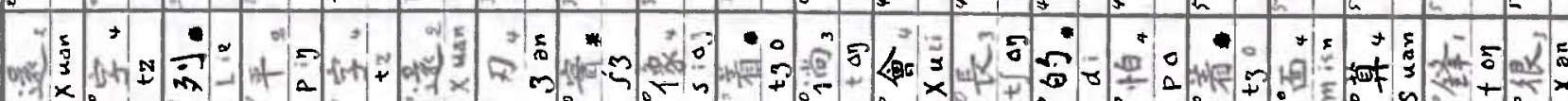

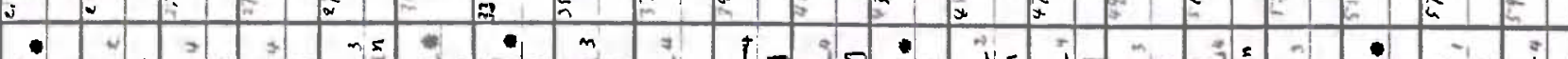

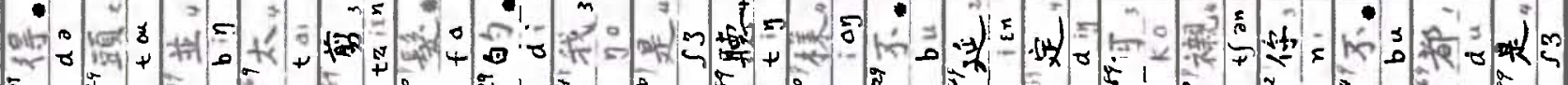
I.

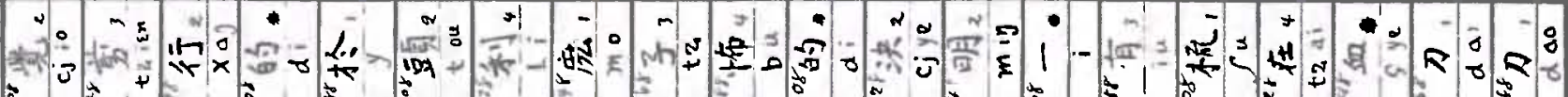

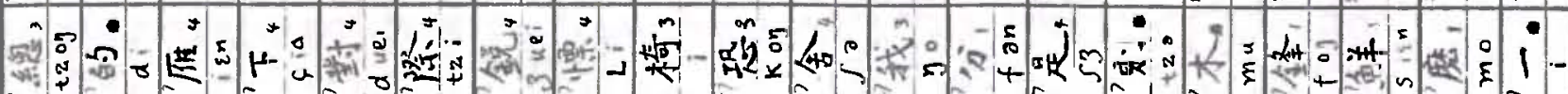
सी

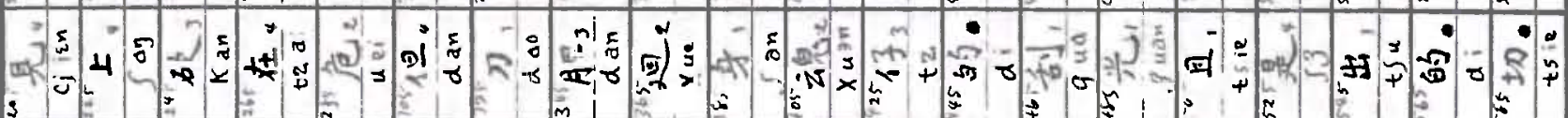

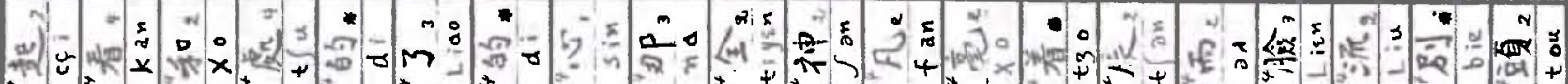

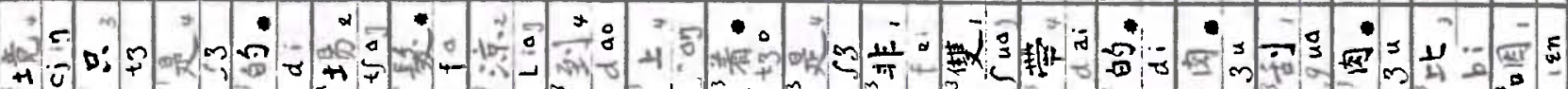

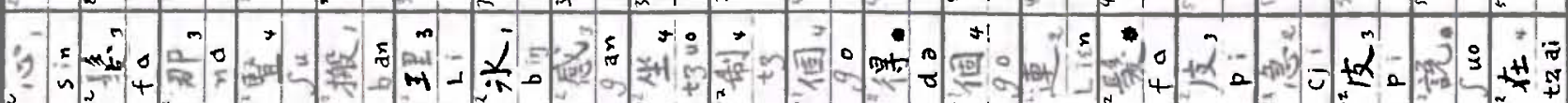

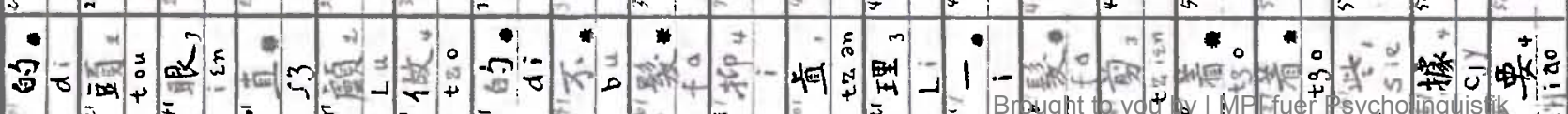




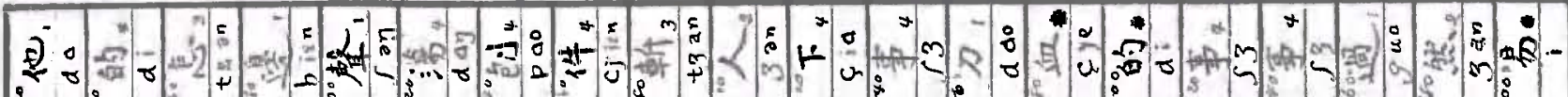
ง $\div$ :

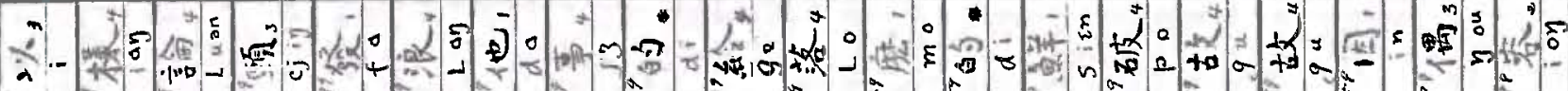

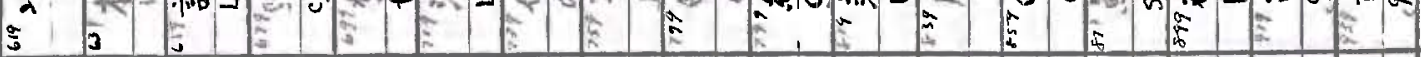

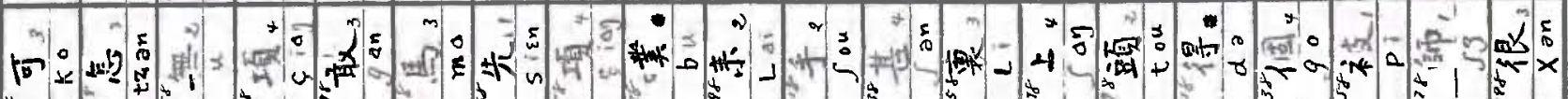

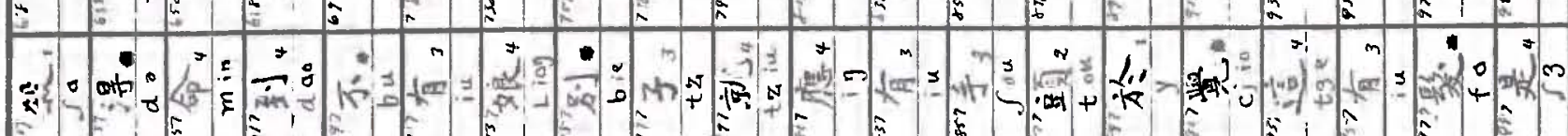

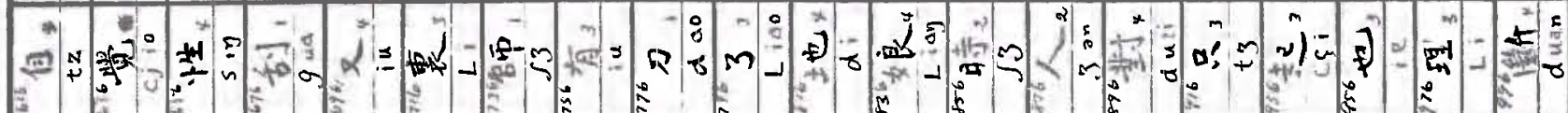

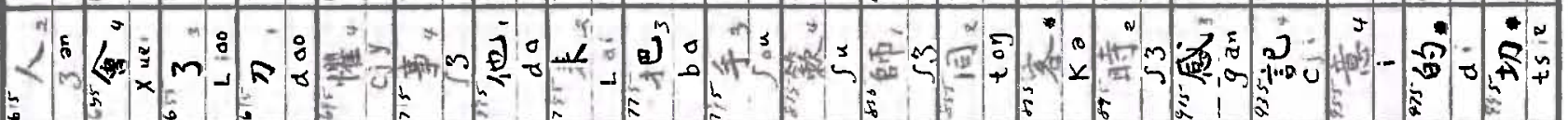

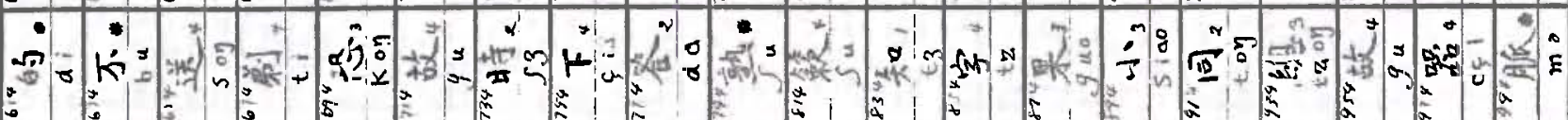

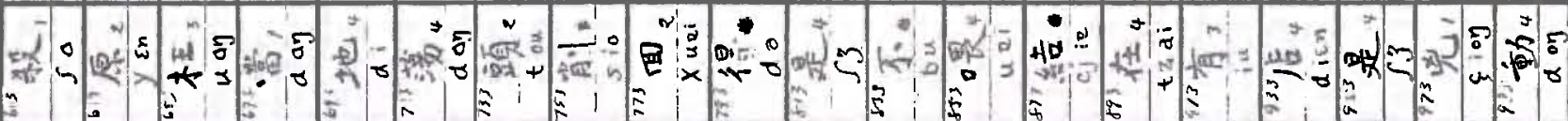

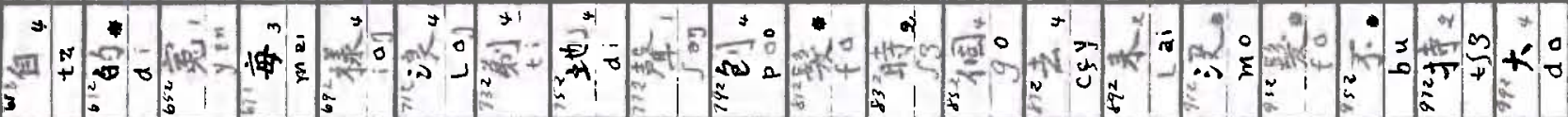

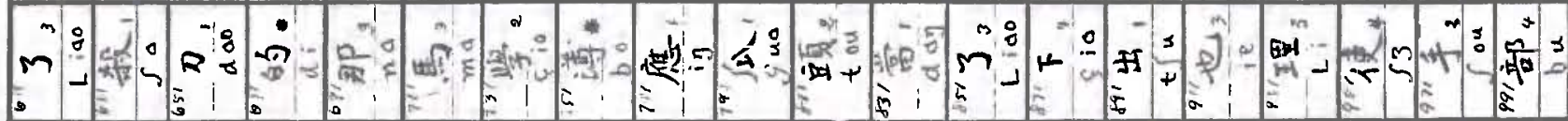
每

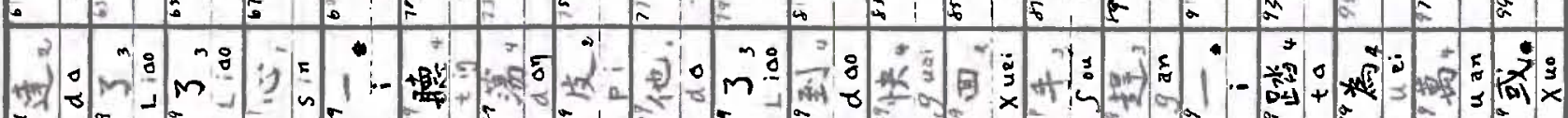
2.

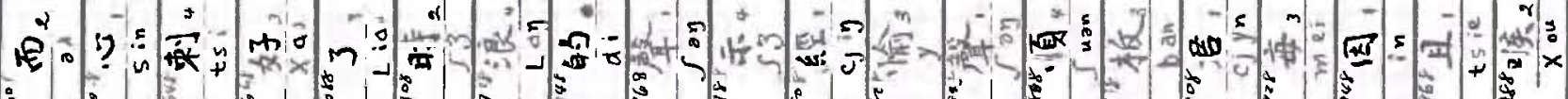

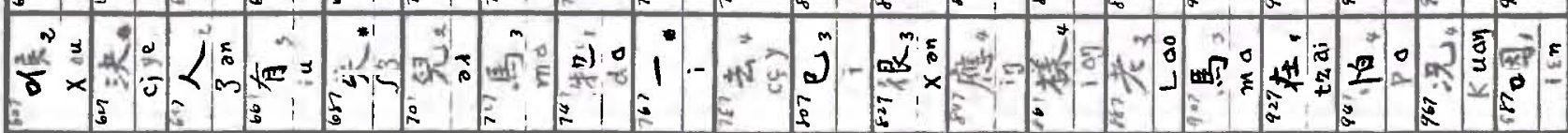

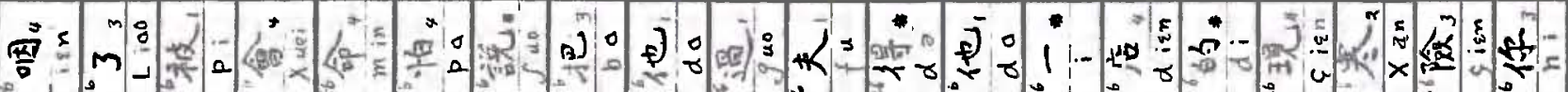

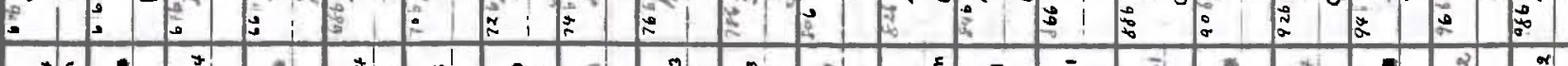

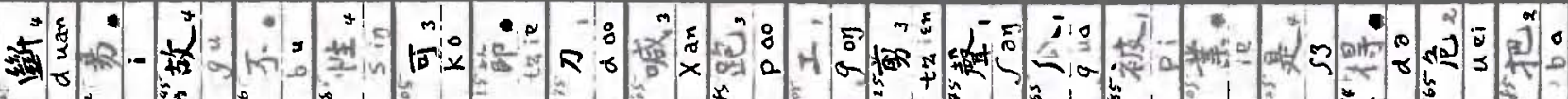

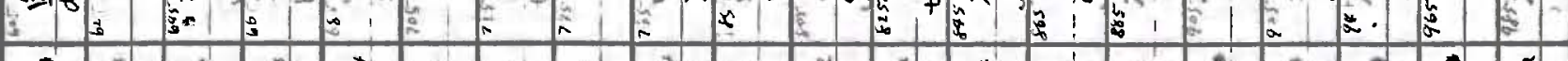

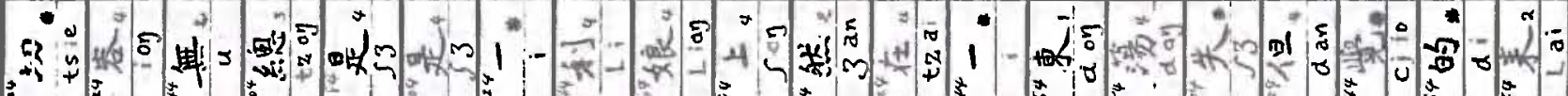

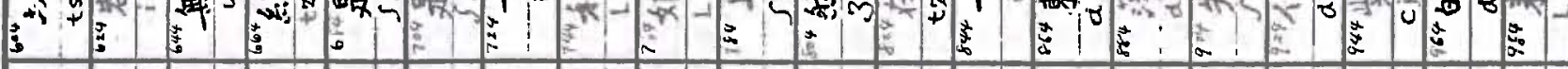

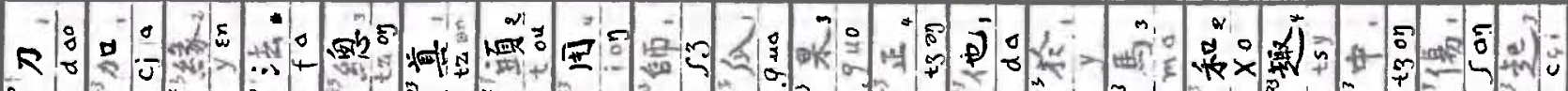
उ.

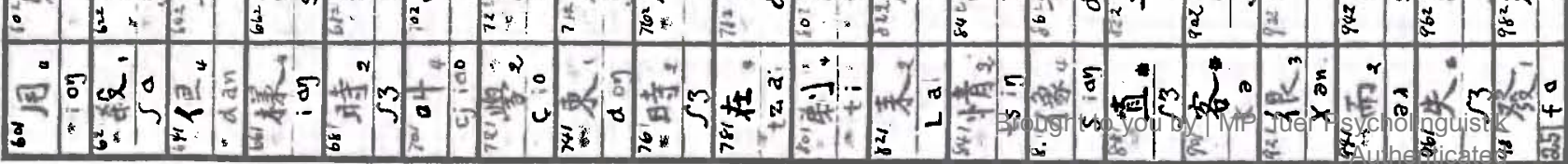




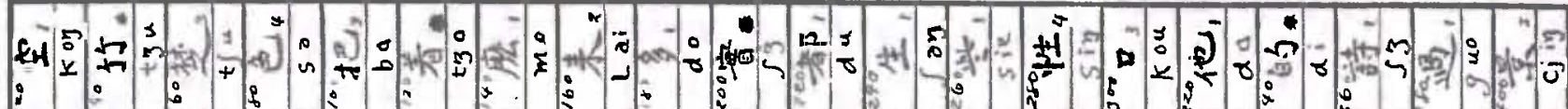

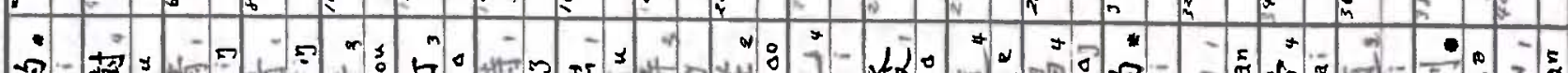

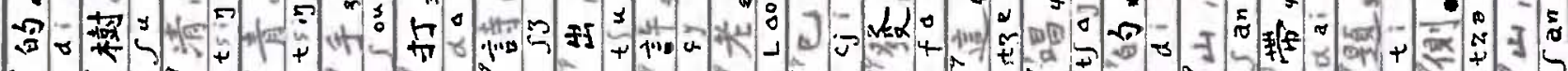

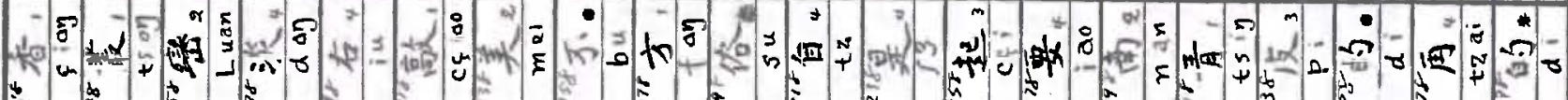

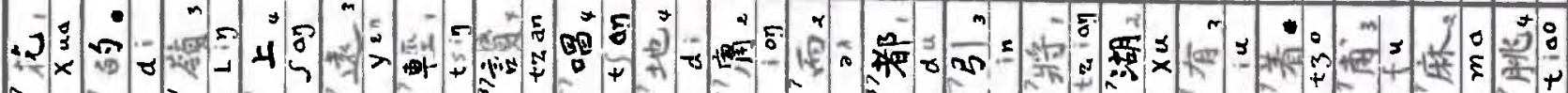
m : (j) II

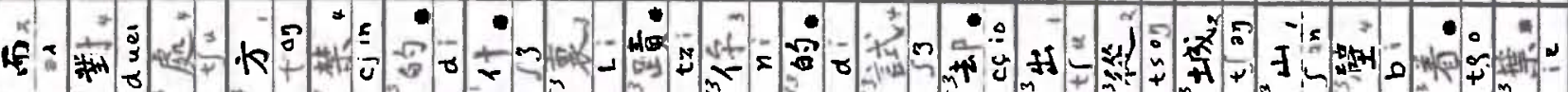

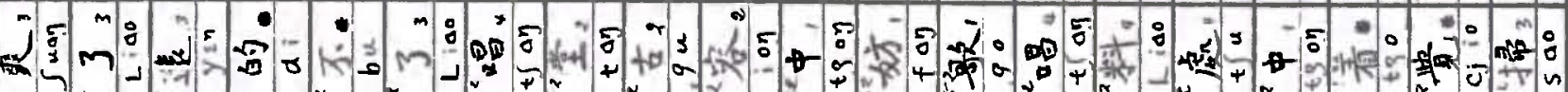
骨 0 过

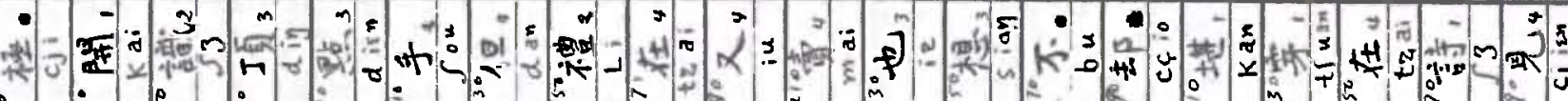

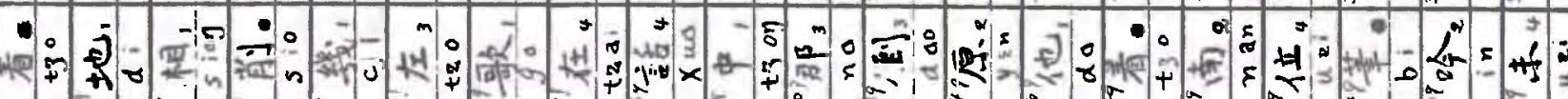

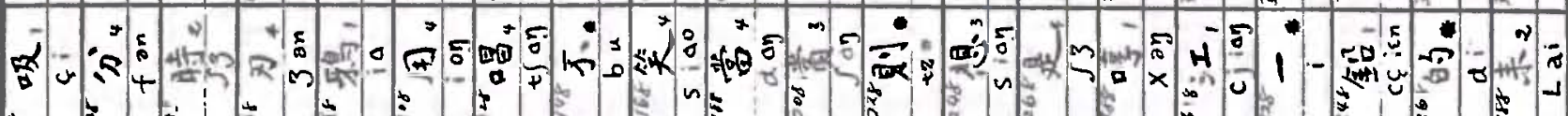

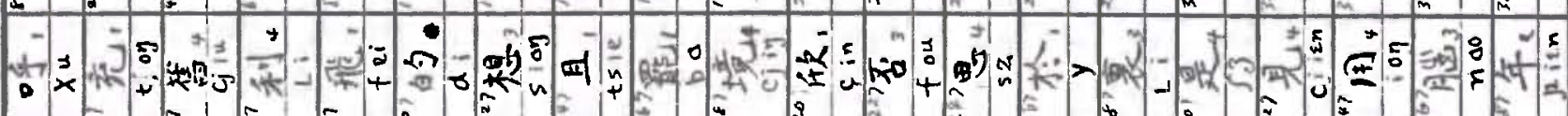

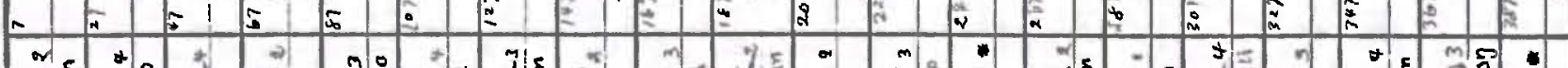

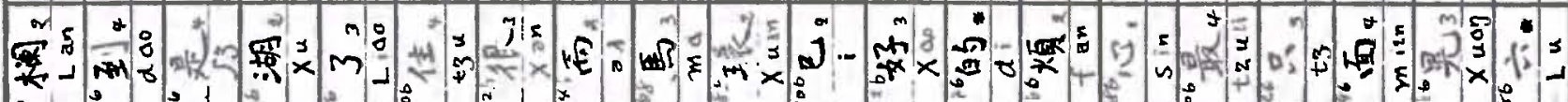

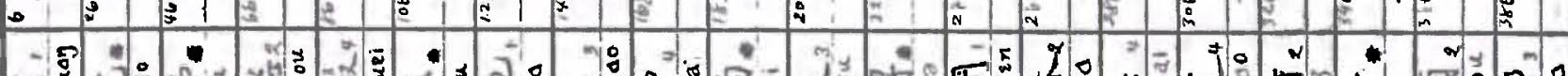

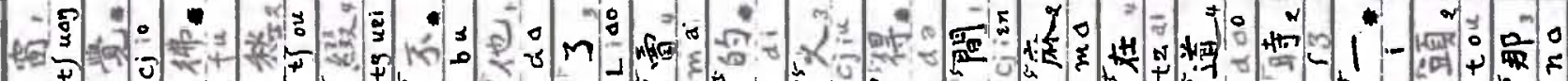

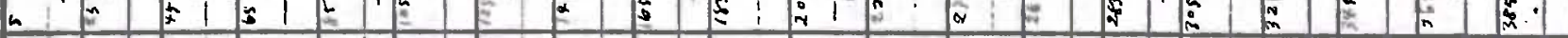

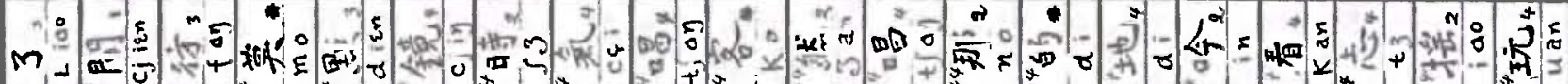

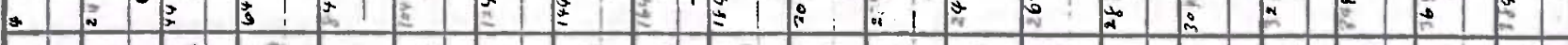

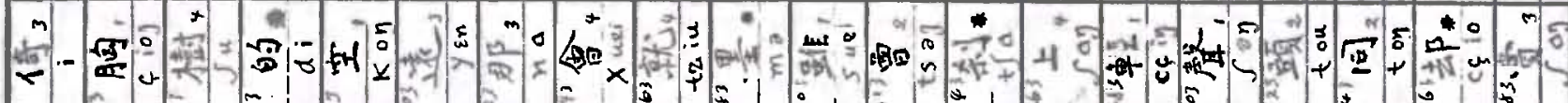

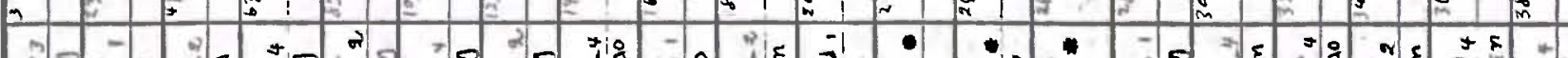

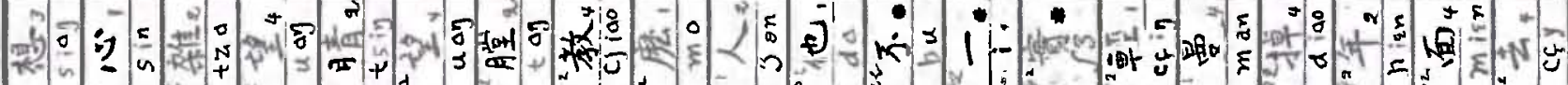

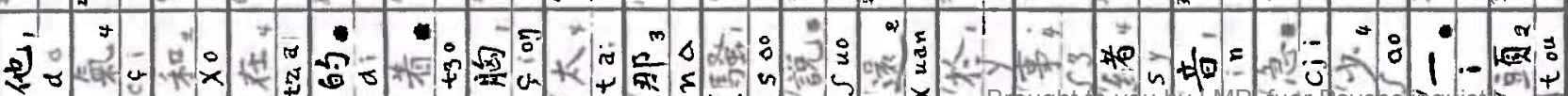

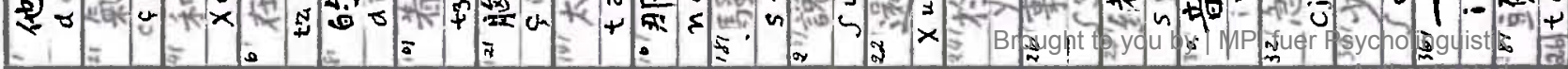




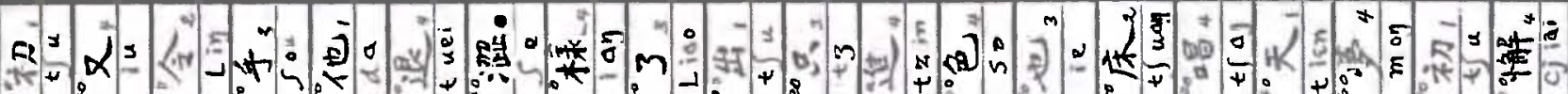
N阝

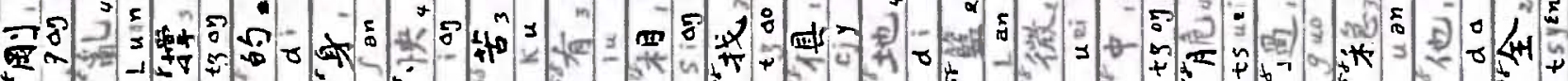

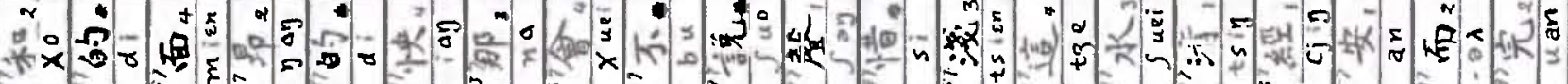

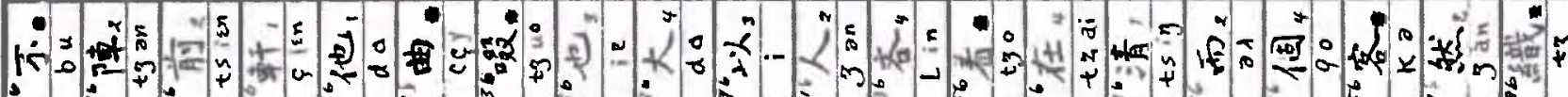
* F

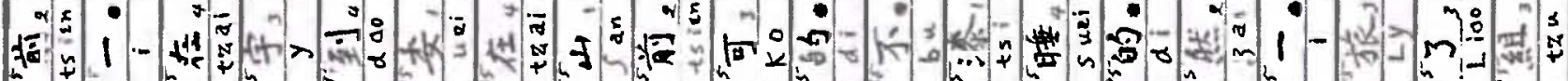

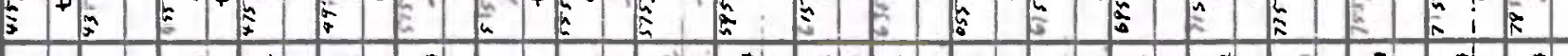

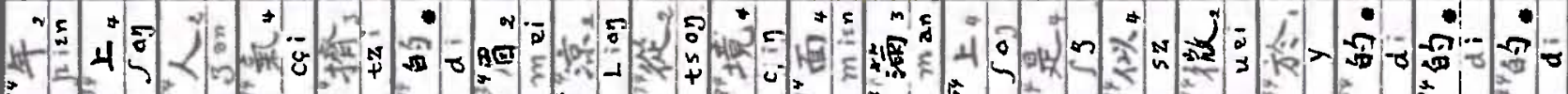

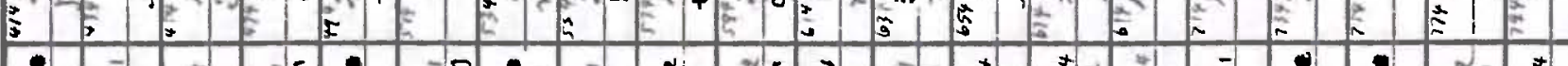

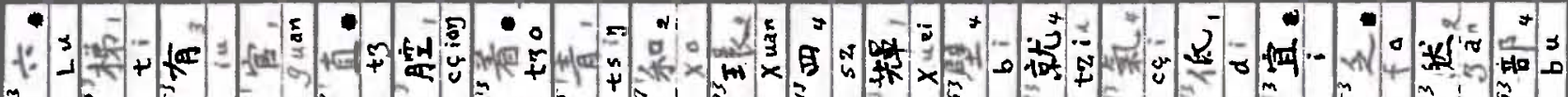

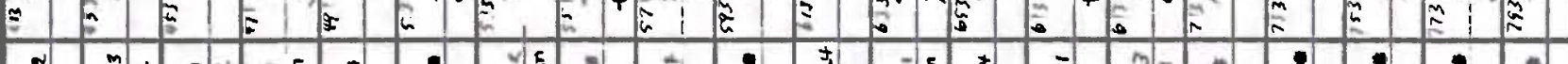

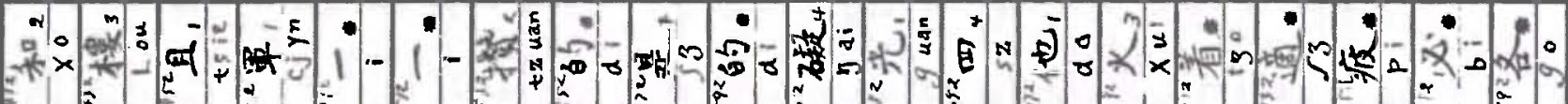

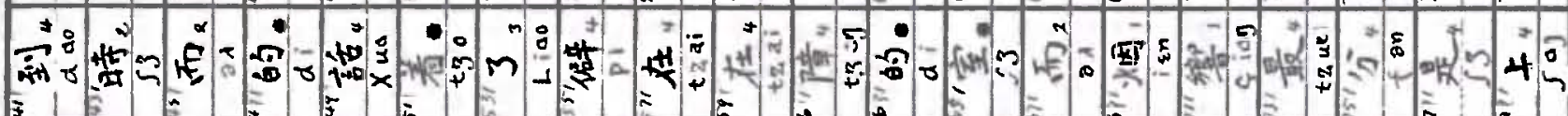

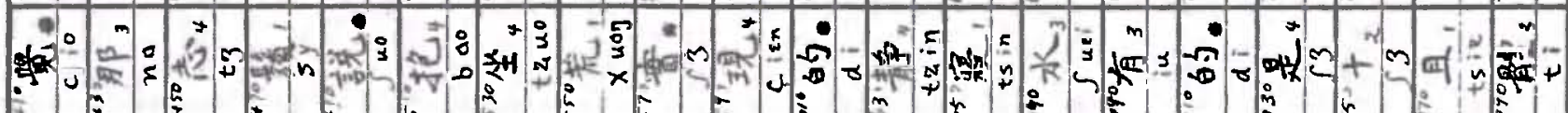

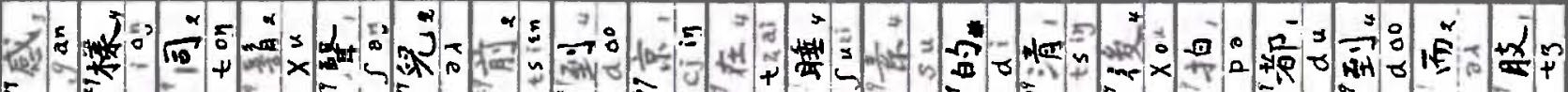

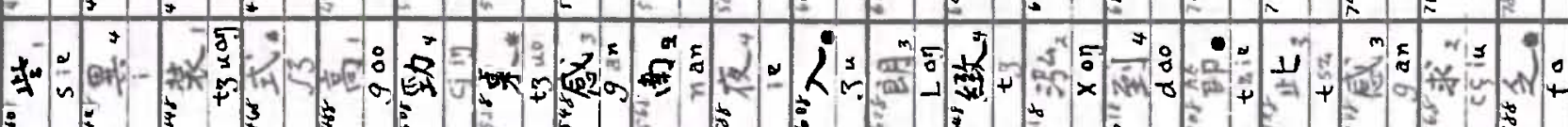

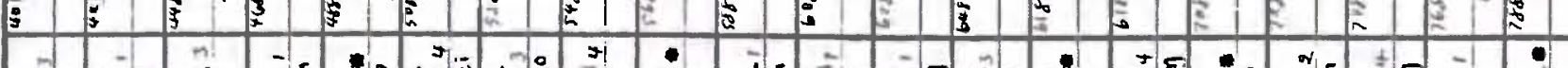

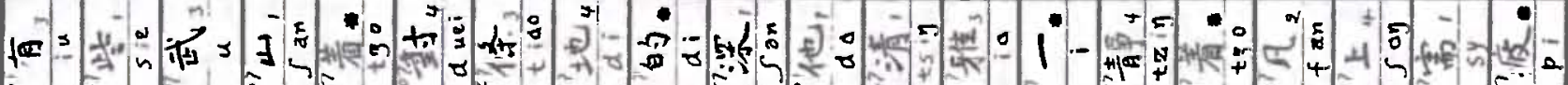

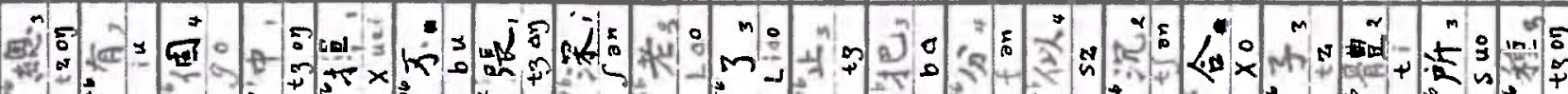

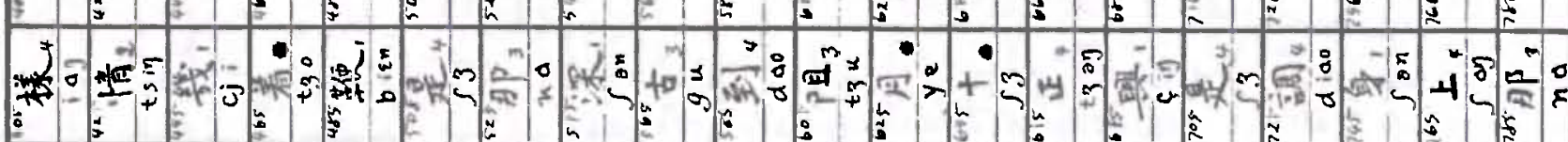

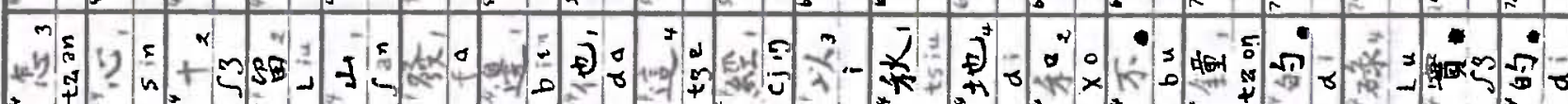

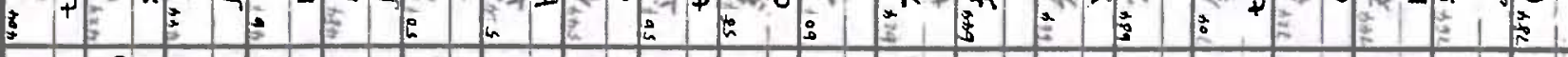

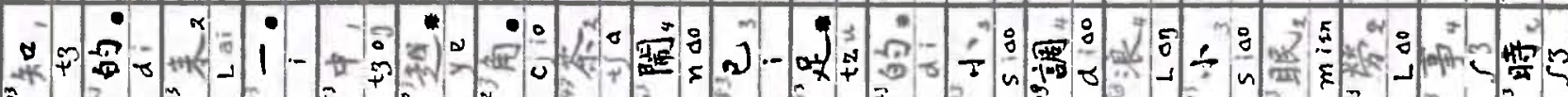

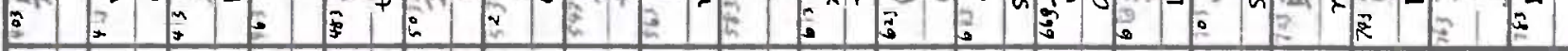

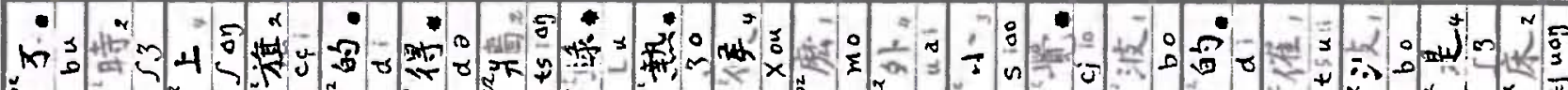
J

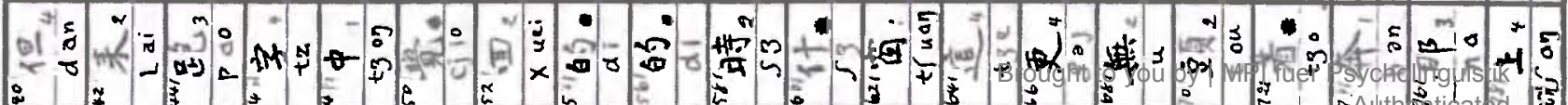




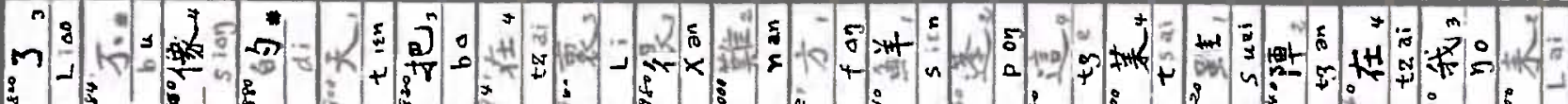

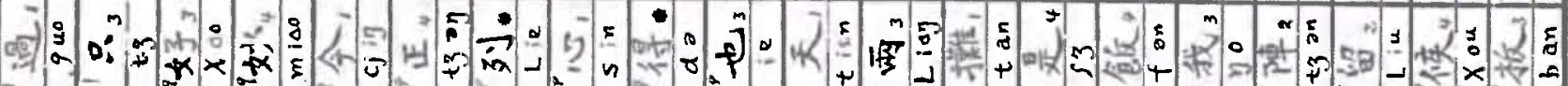
हل

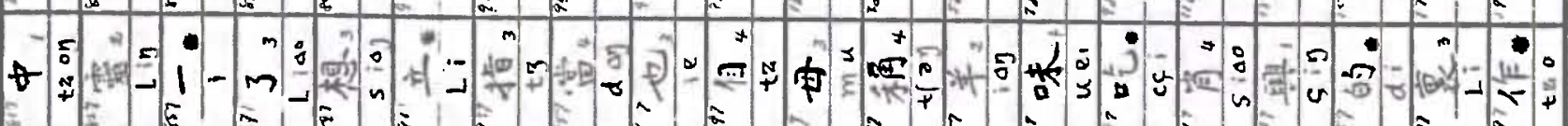

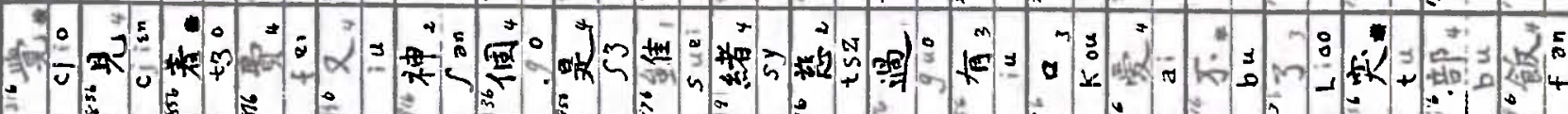

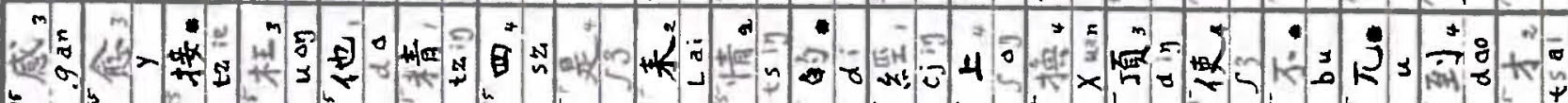

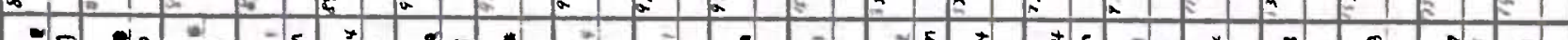
-

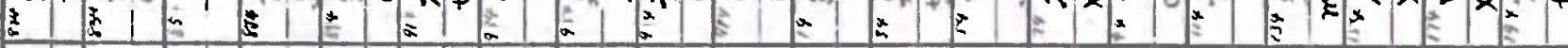

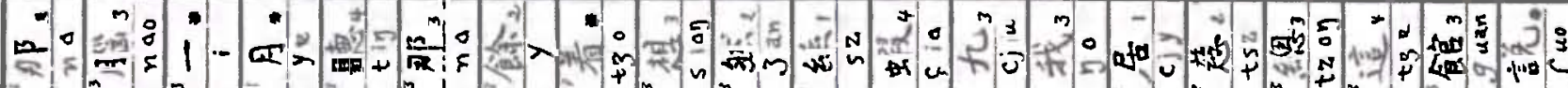
El E E E E E E

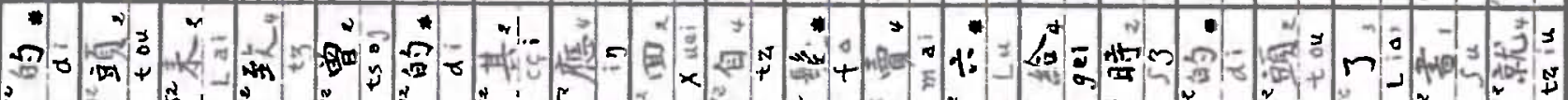

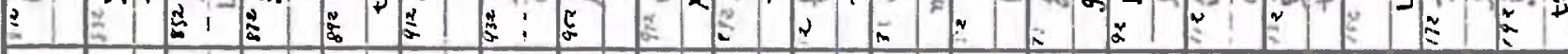

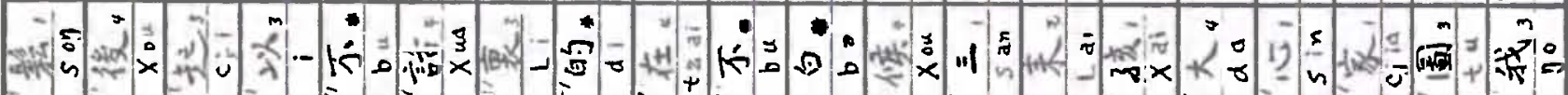

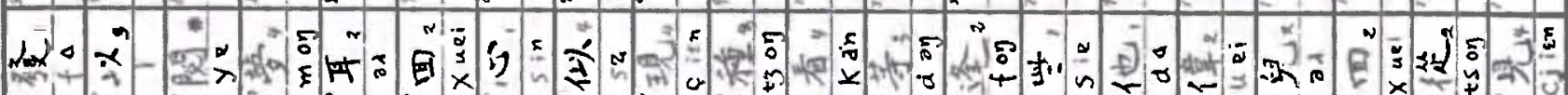

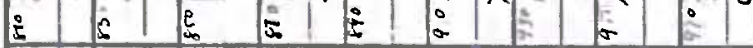

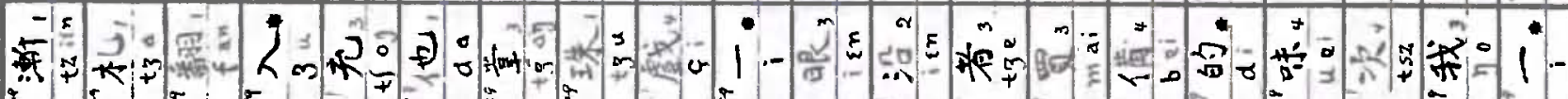

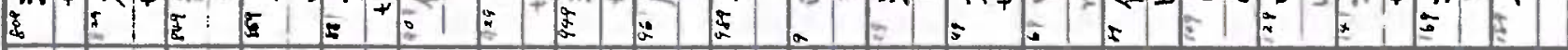

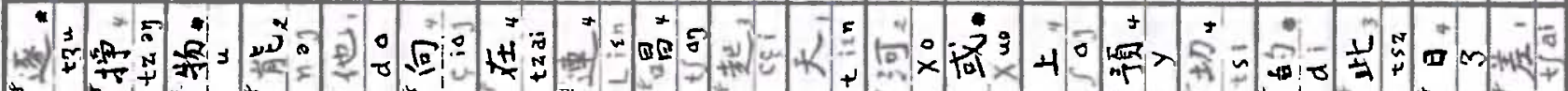
年

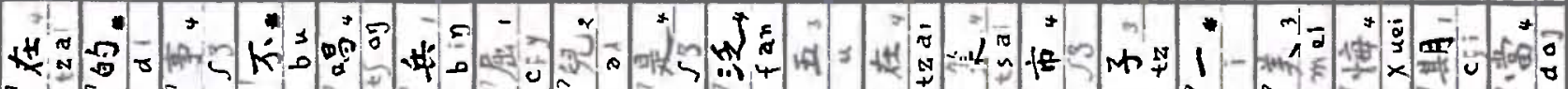

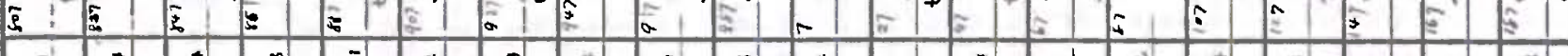

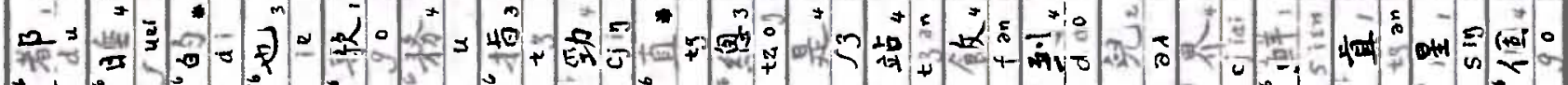
ह

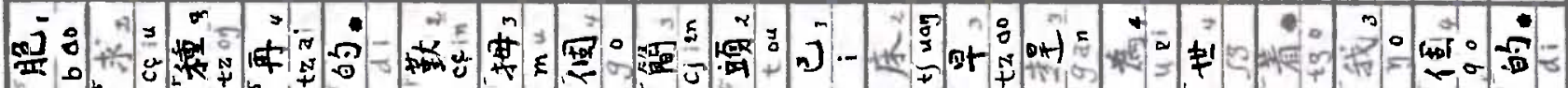

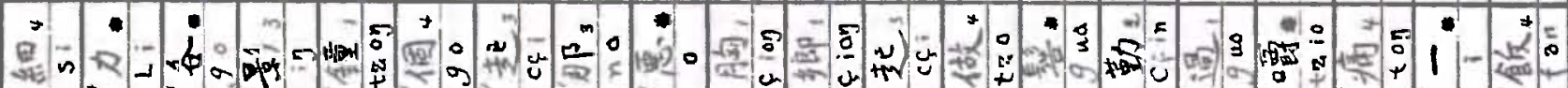

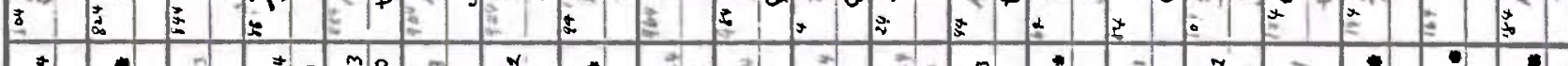

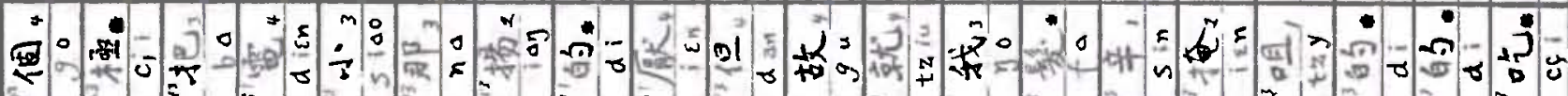
他宁 g.

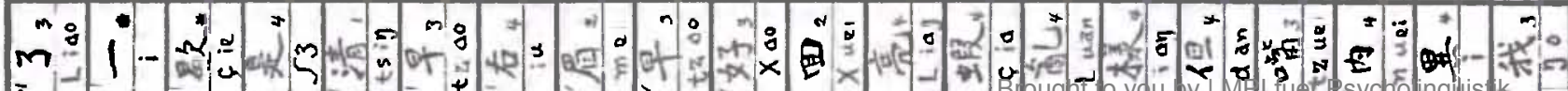

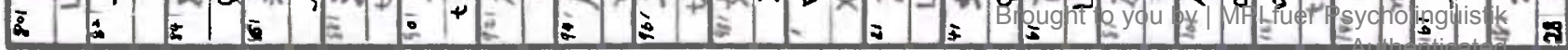




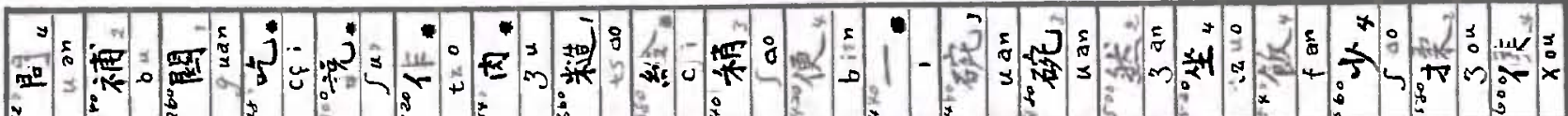

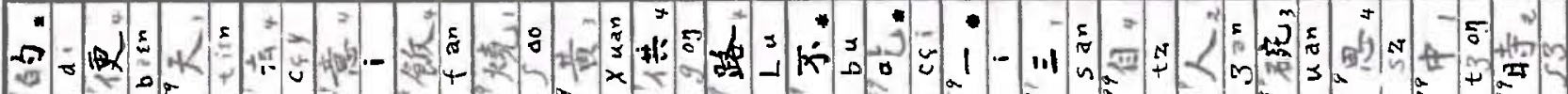

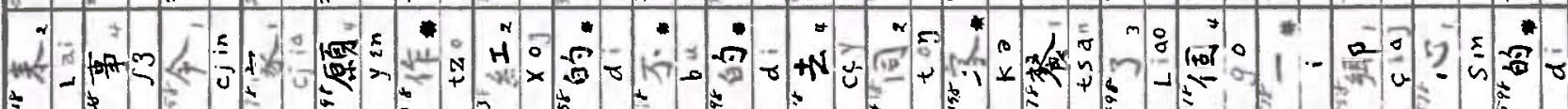

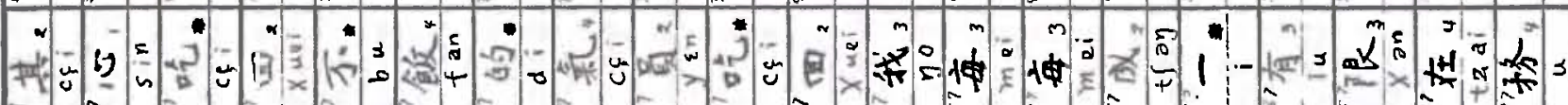

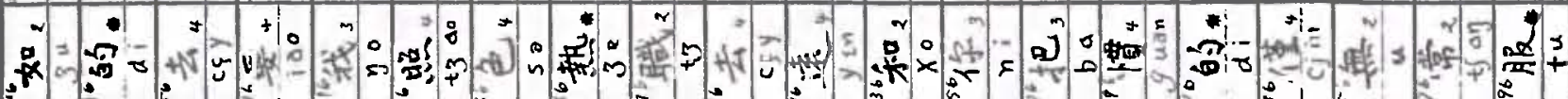

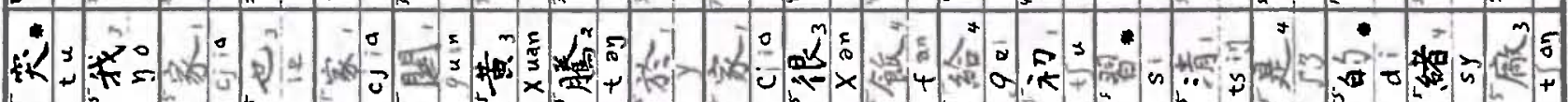

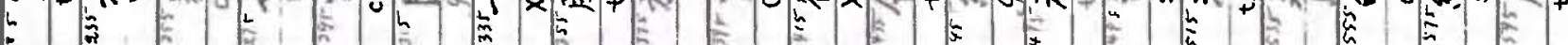

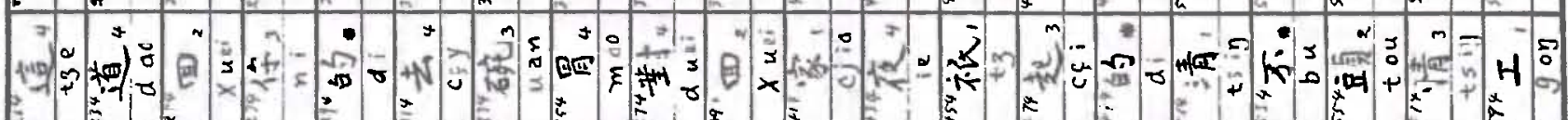
S

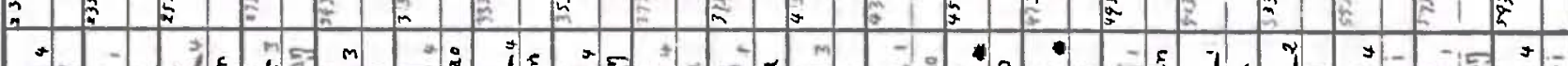

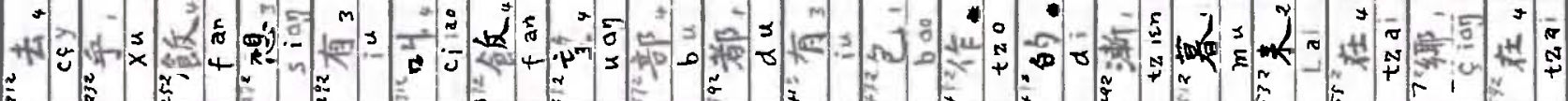

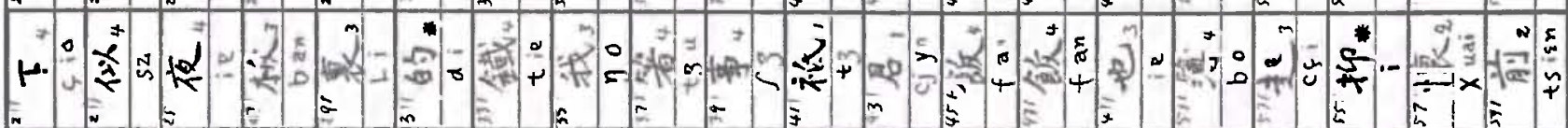

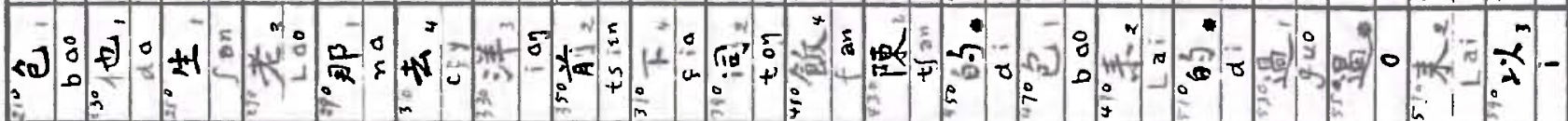

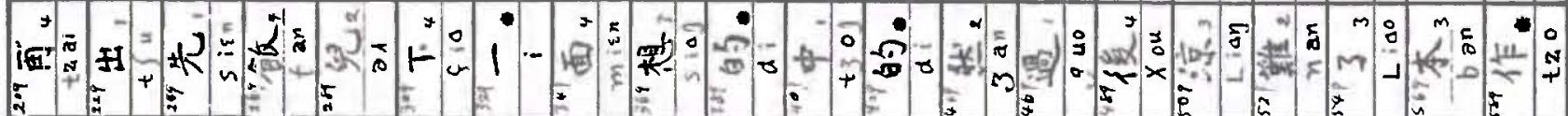

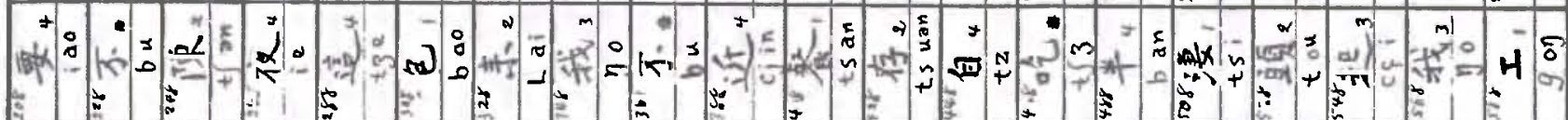

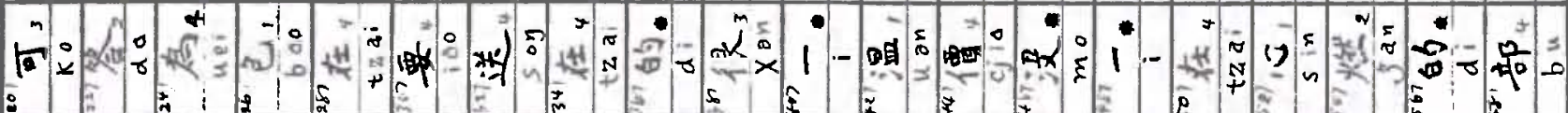

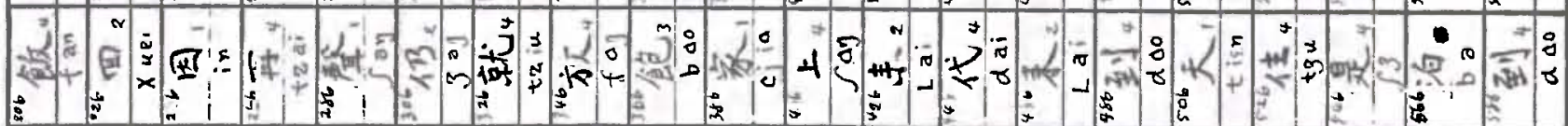

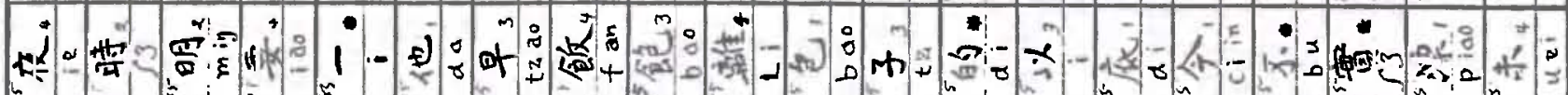

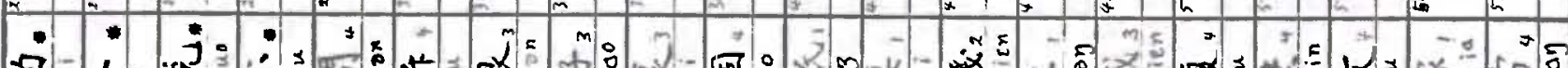

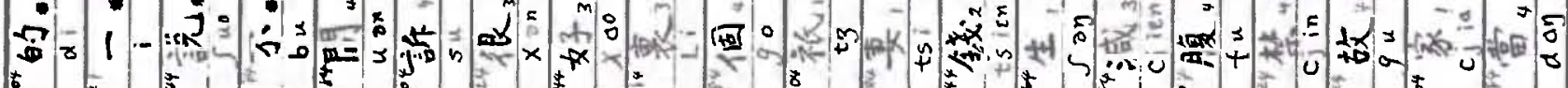

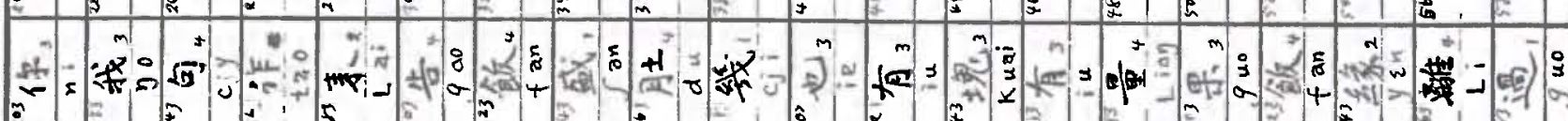

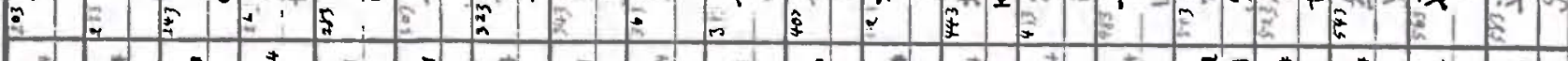

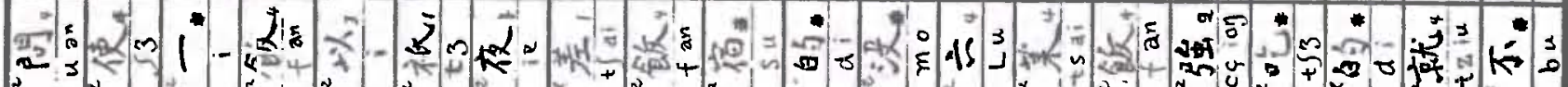

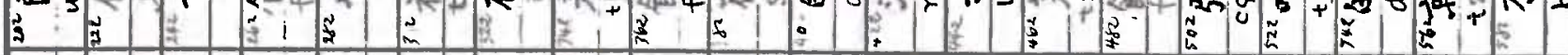

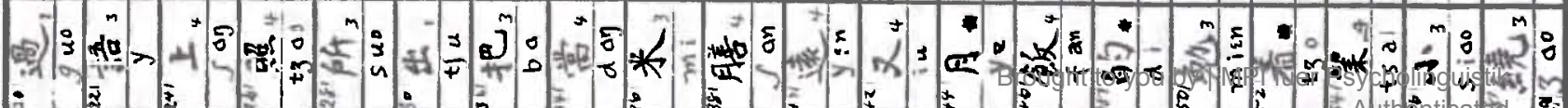

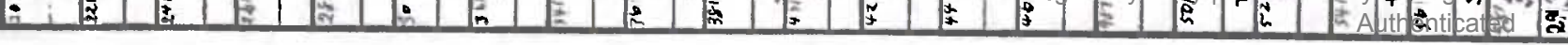




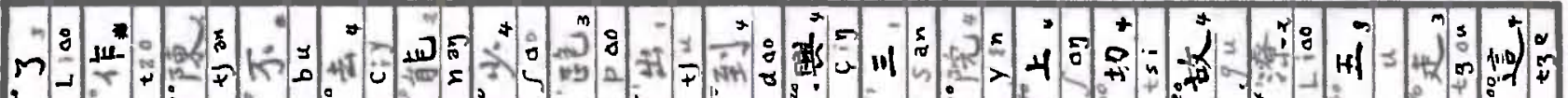

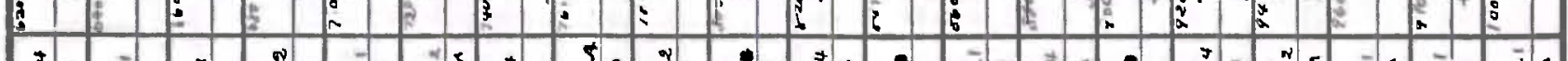

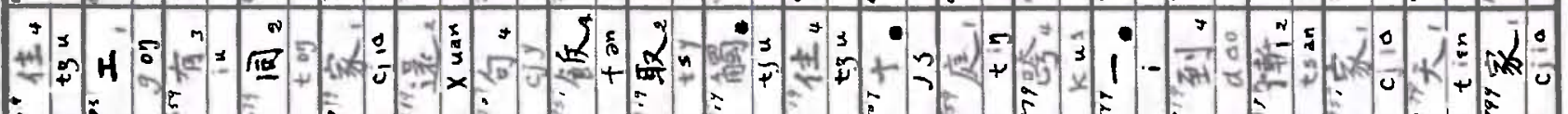

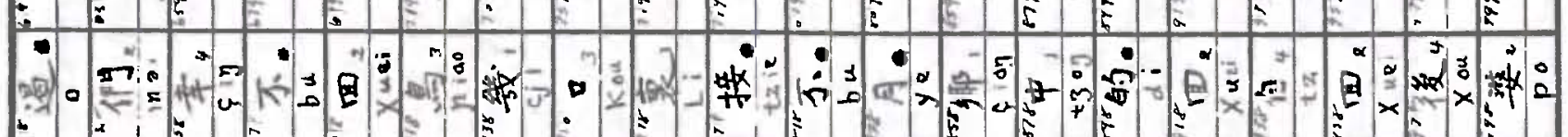

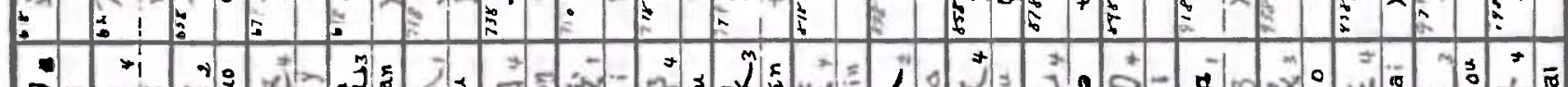

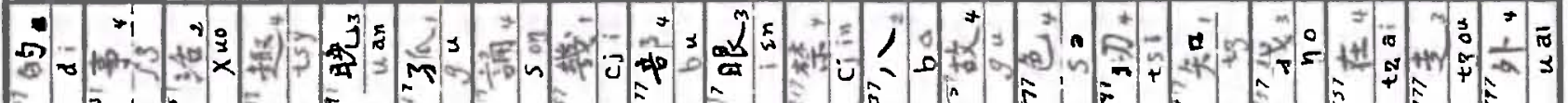

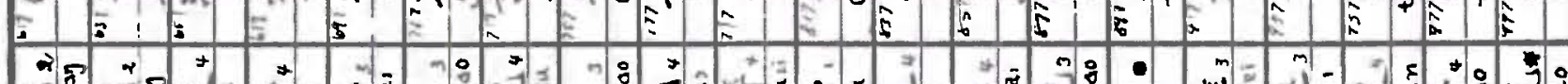

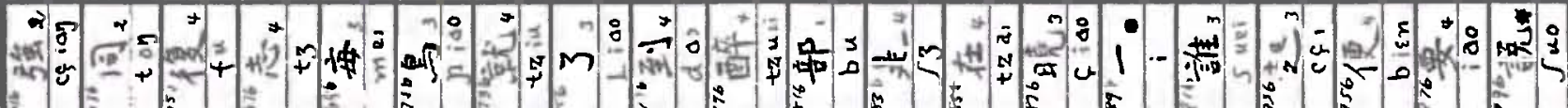

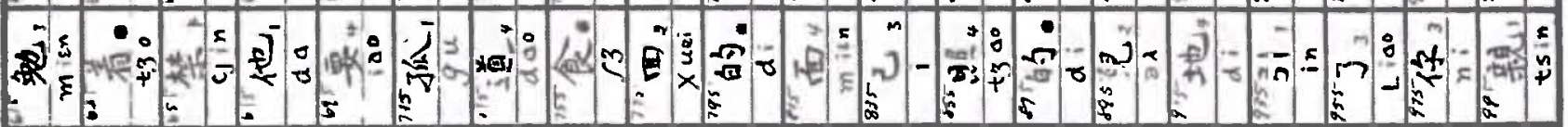

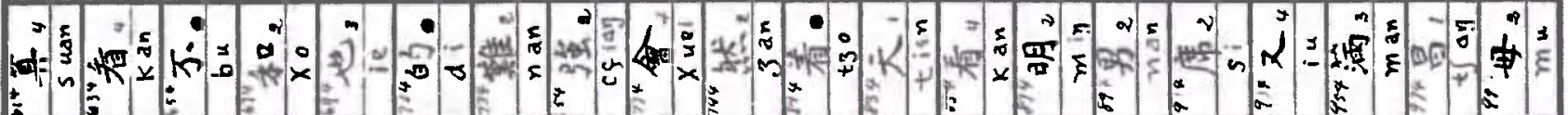

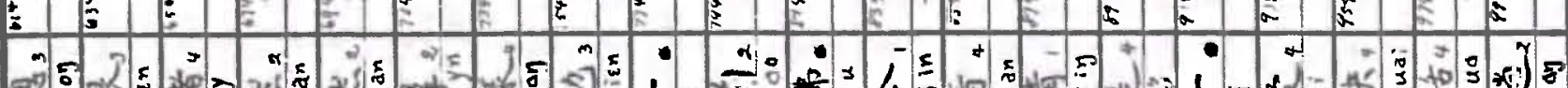

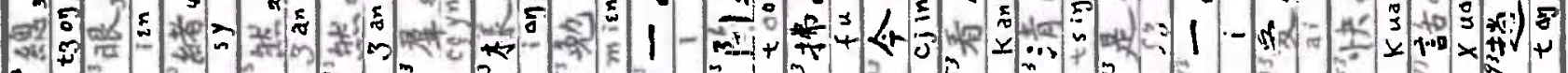

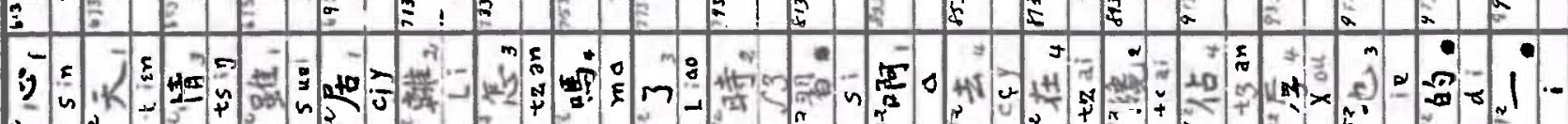

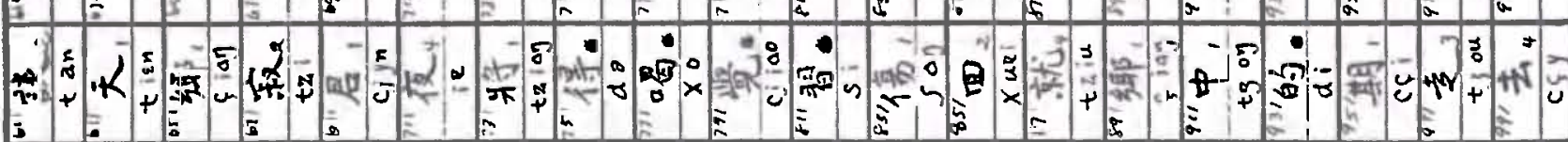

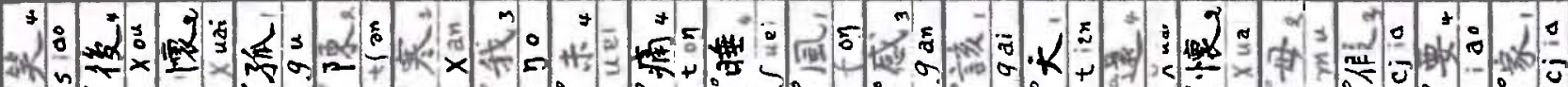

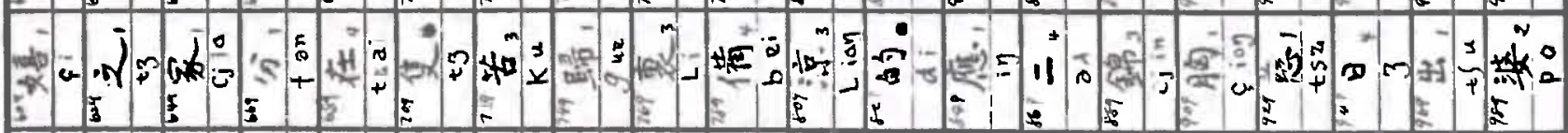

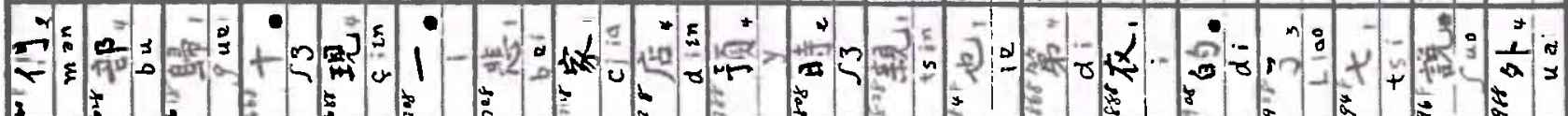
(D)

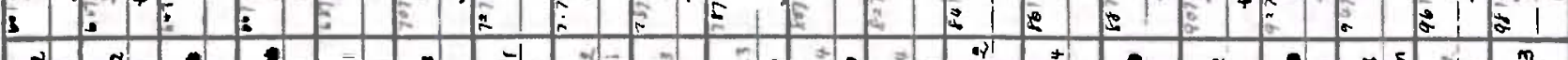

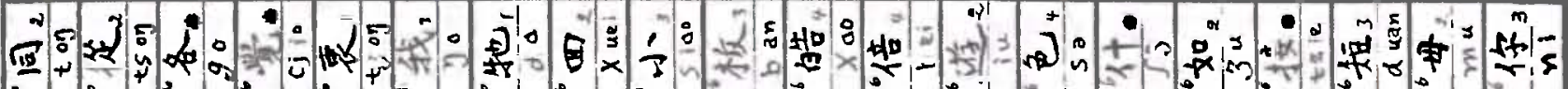

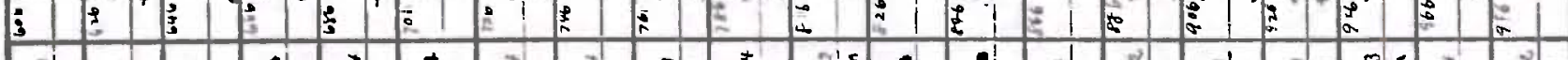

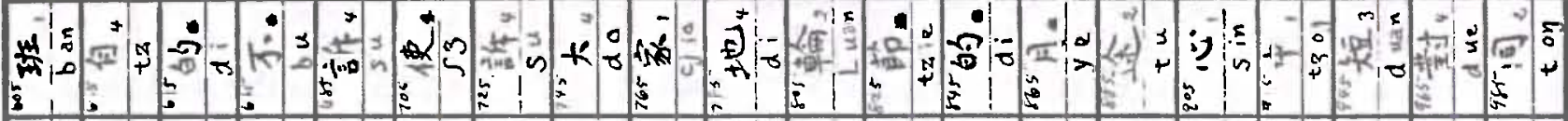

1 1. 5

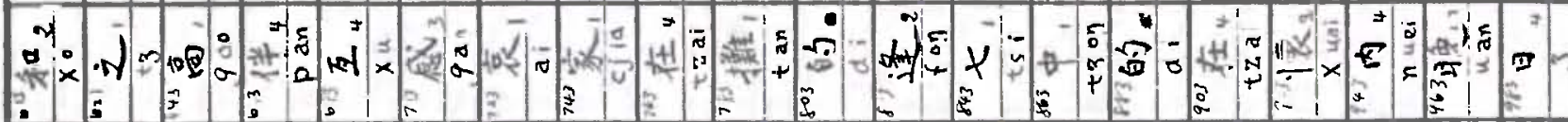

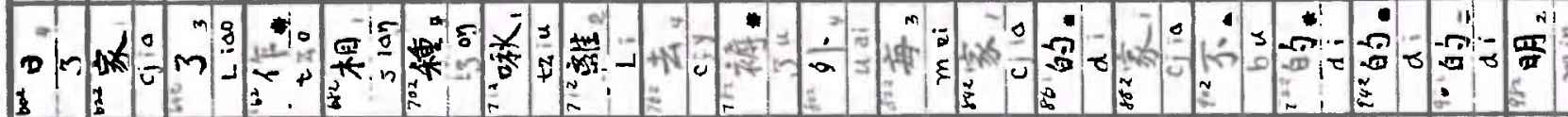

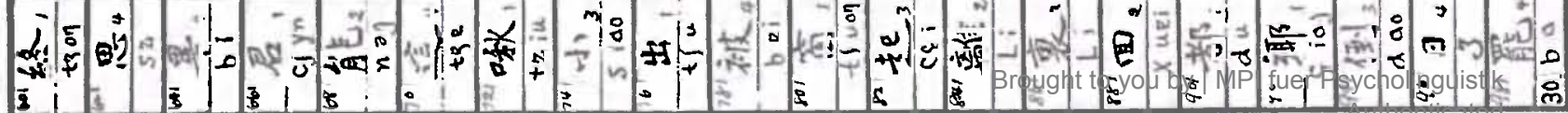




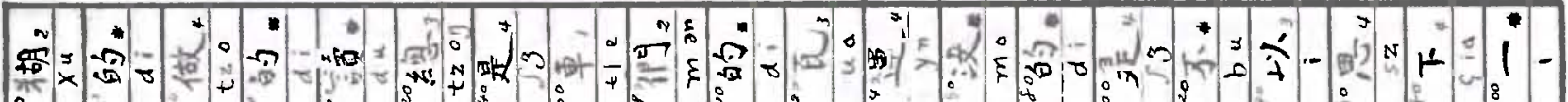

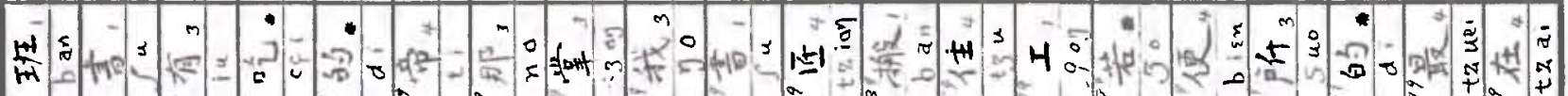

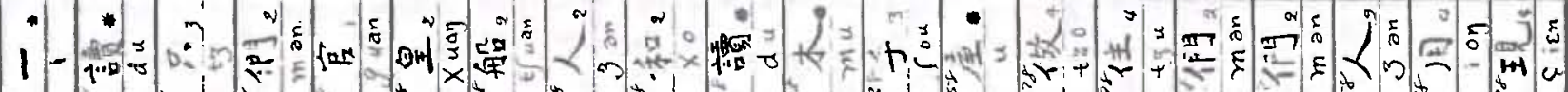

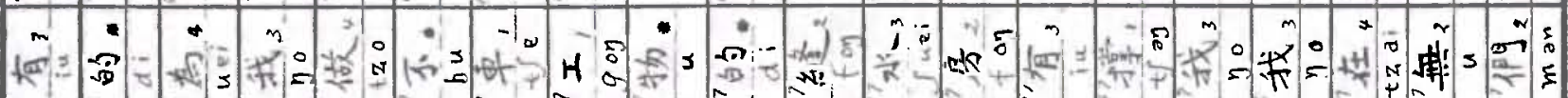
-

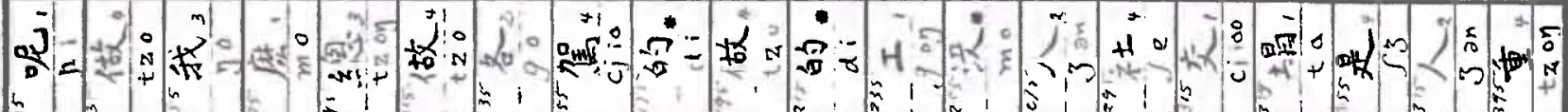

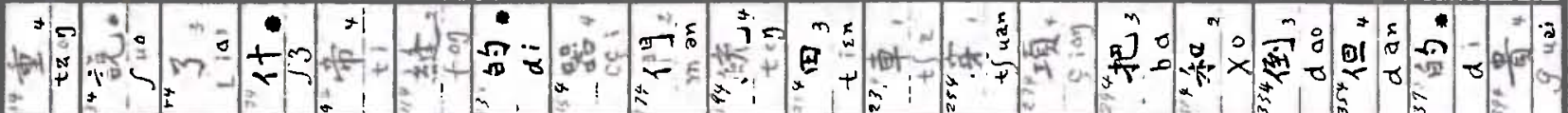
Na

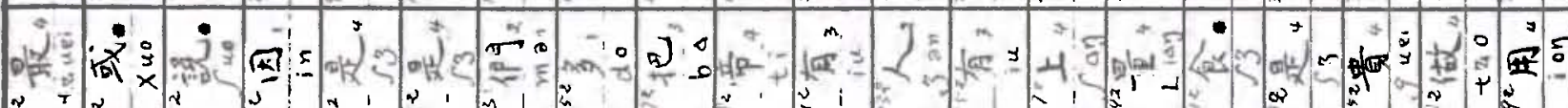

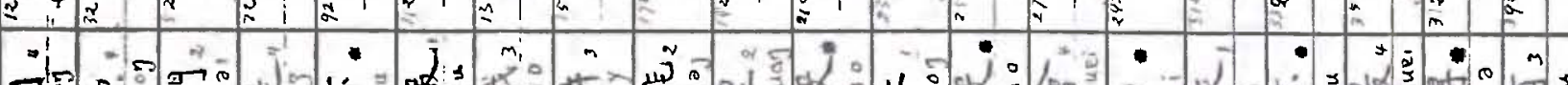
里国

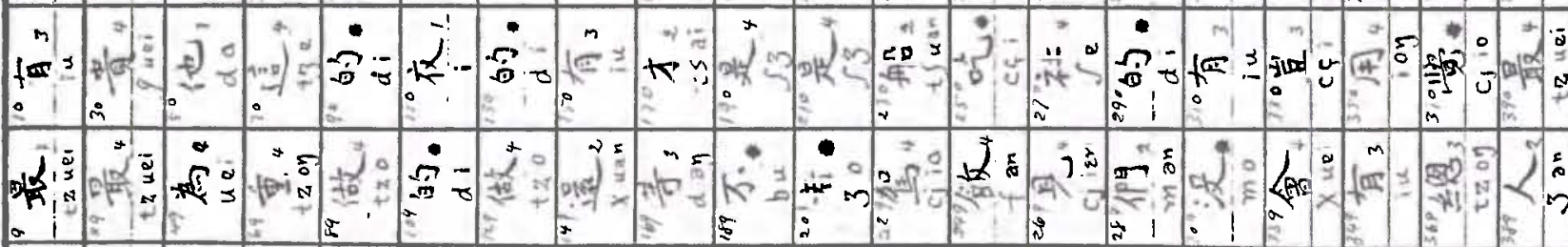

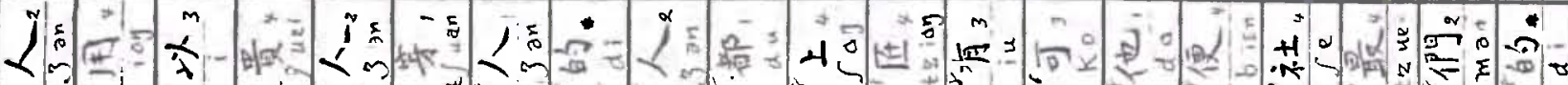

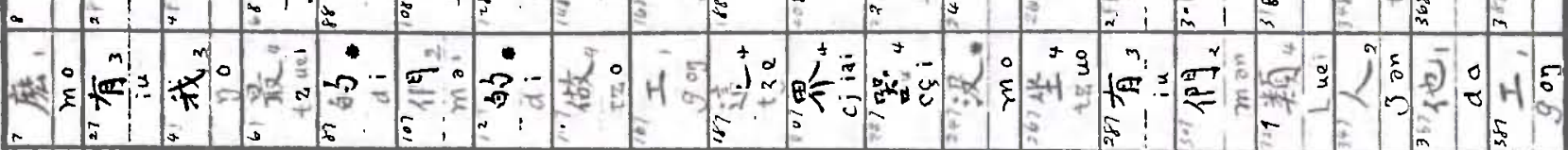

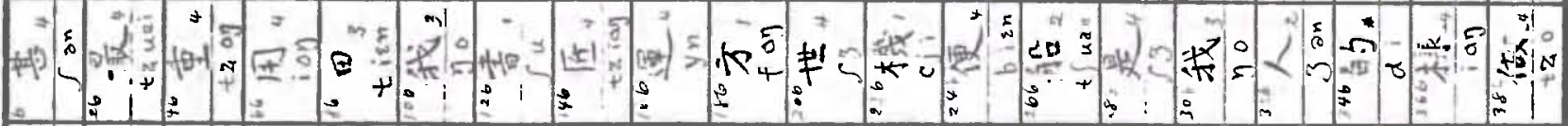

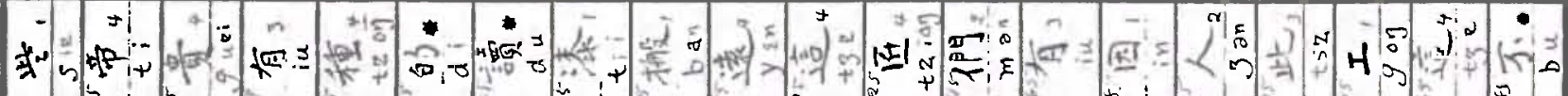

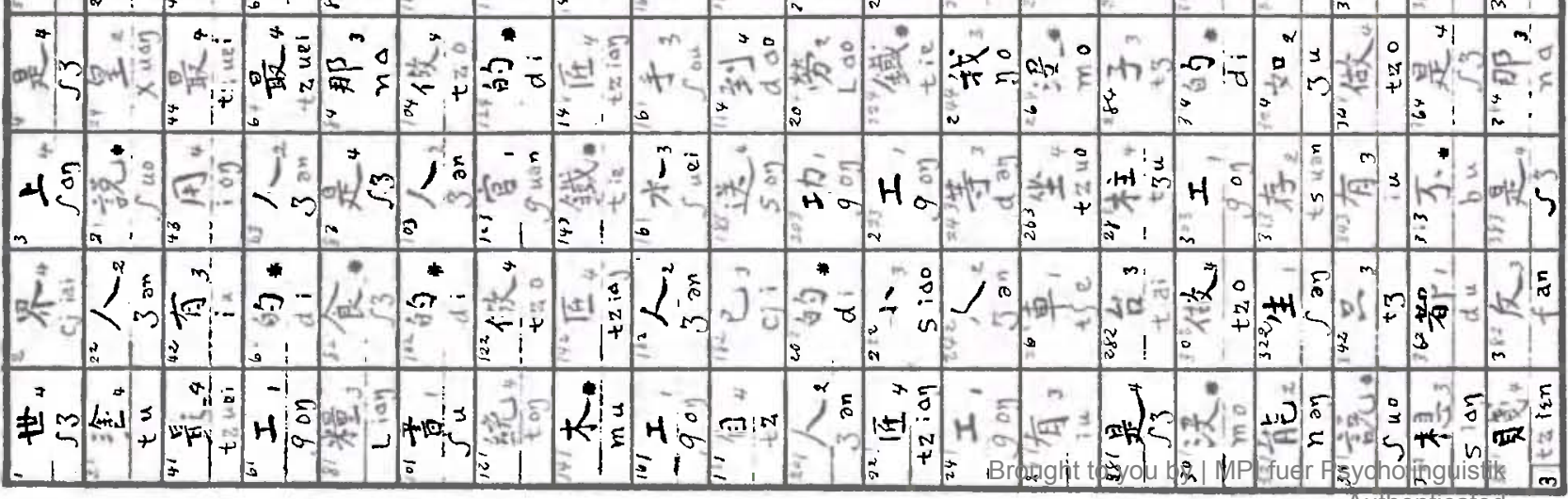




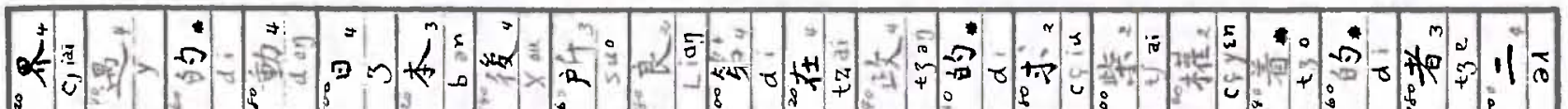

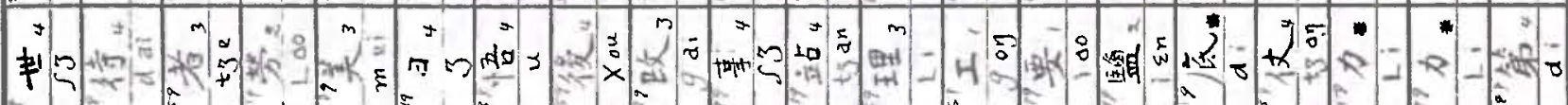

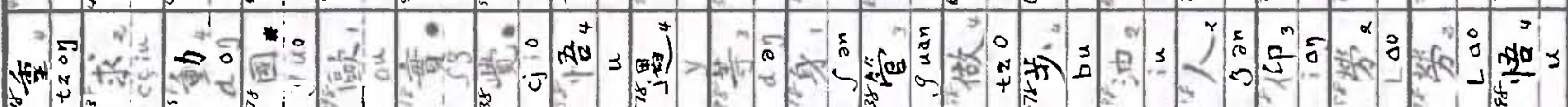

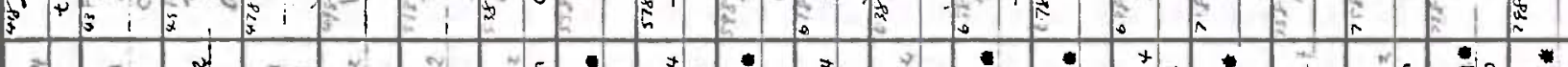

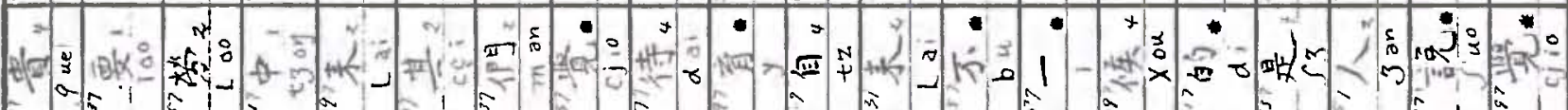

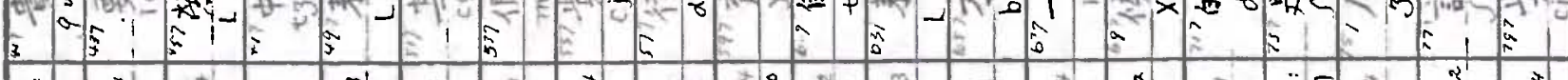
⿷匚⿳丨コ丨卜

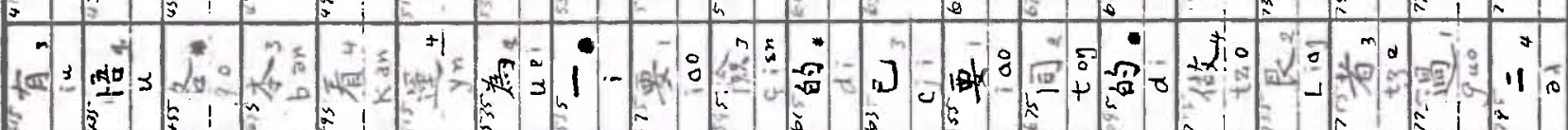

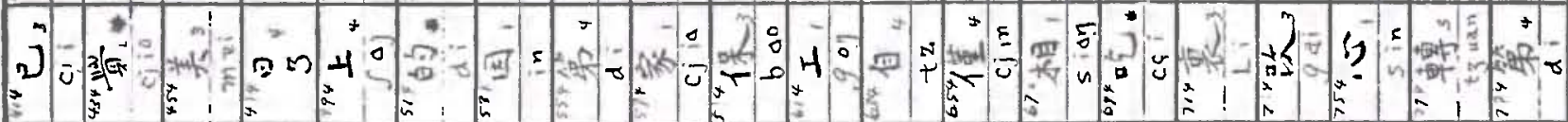
(19)

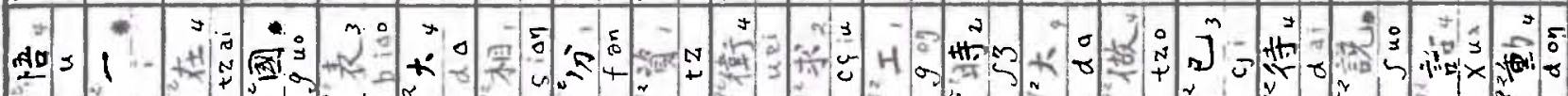

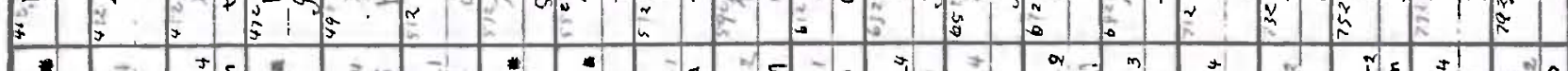
A

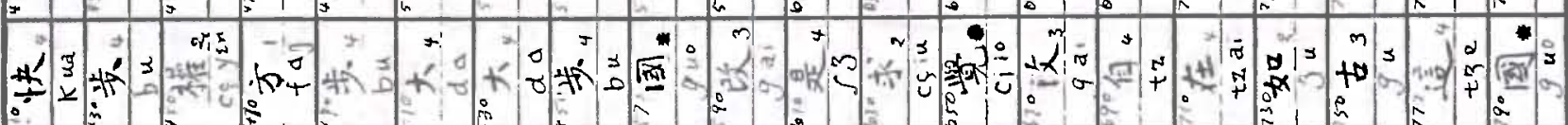

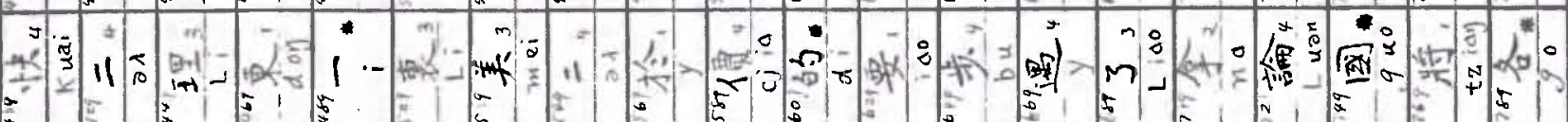

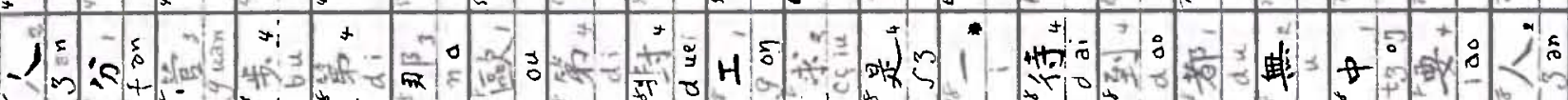

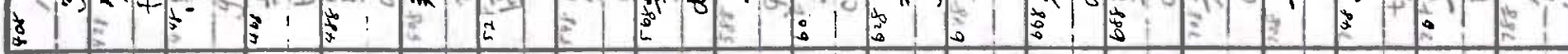

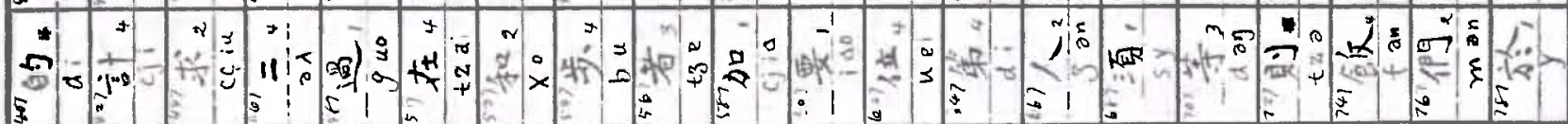

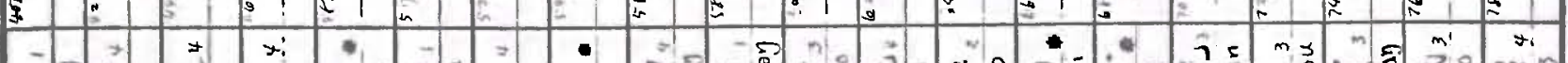

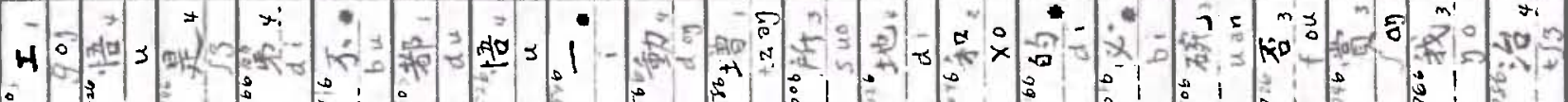

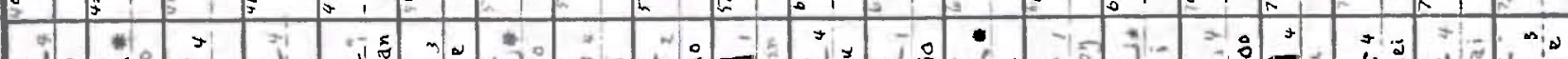
स.

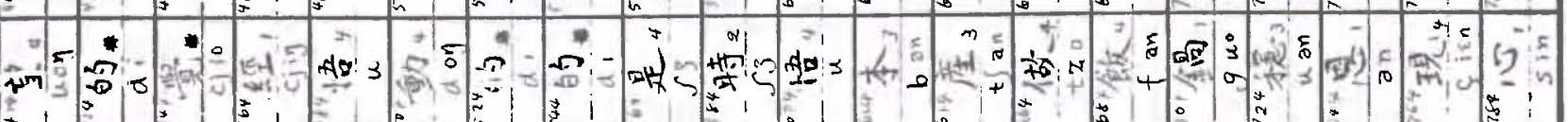

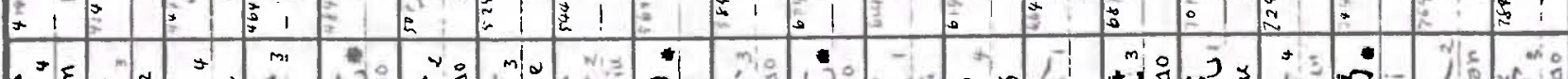

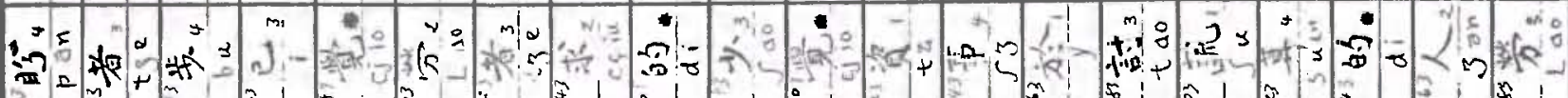

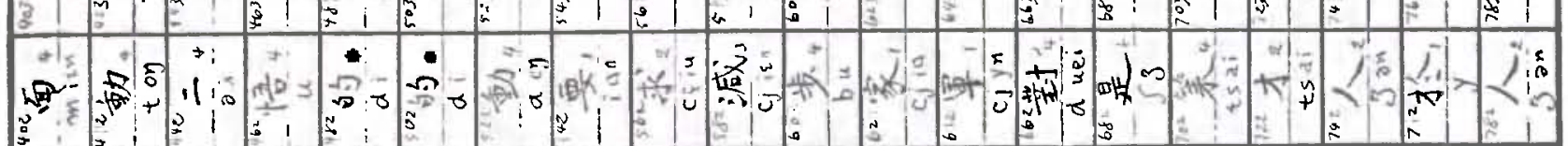

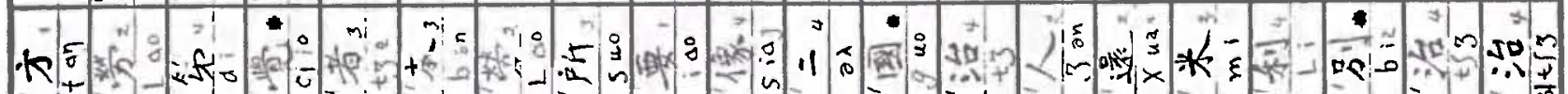

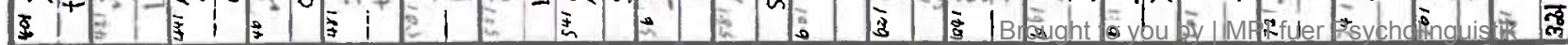




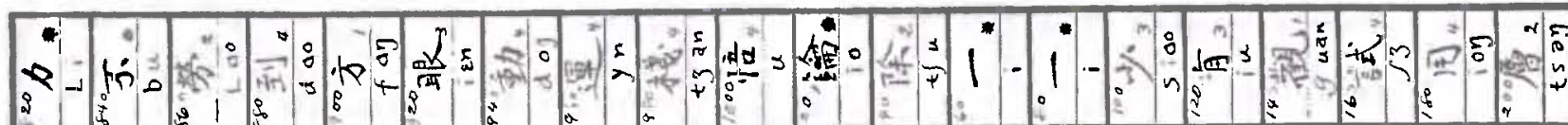

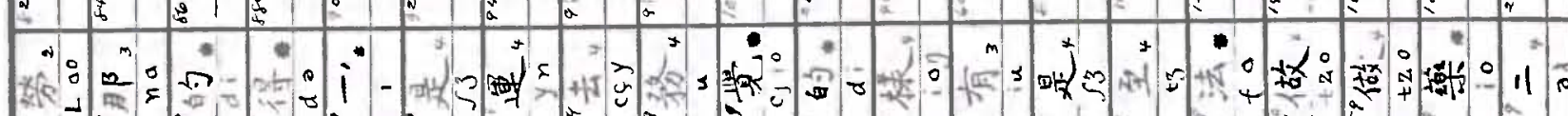

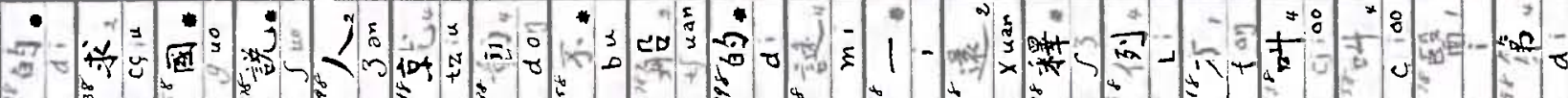

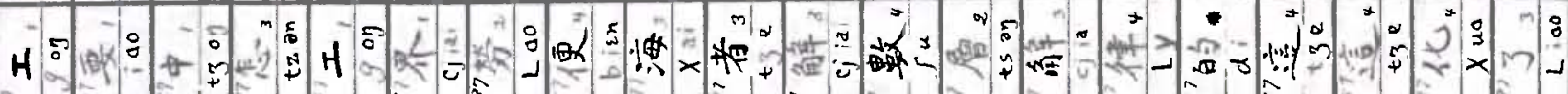

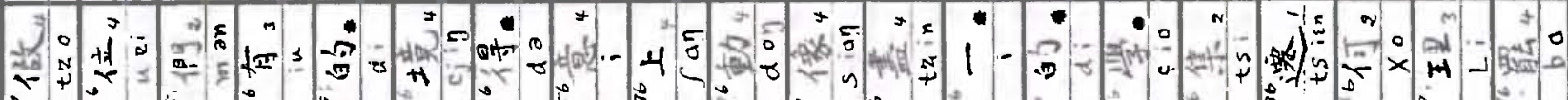

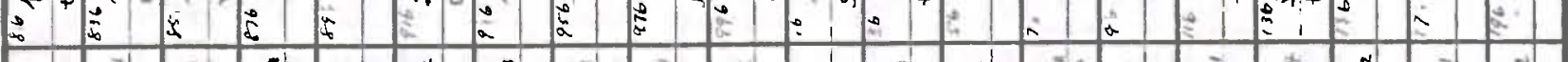

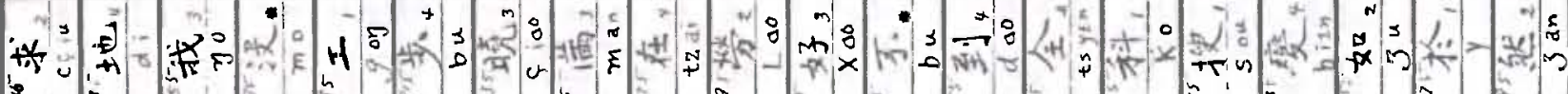
s

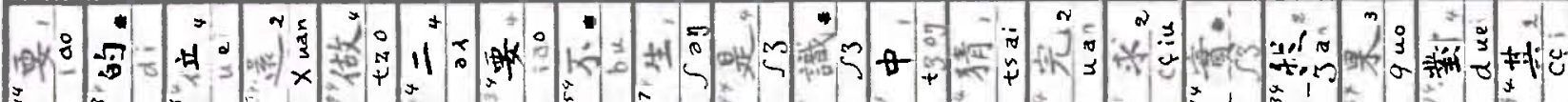

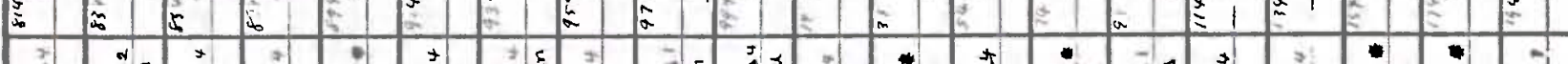

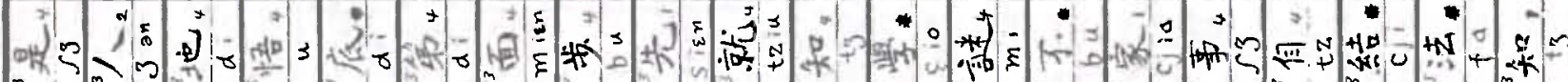

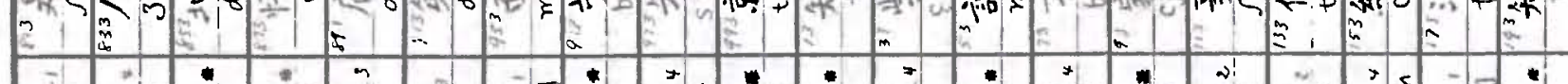

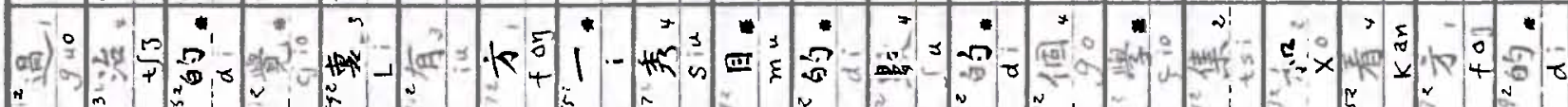

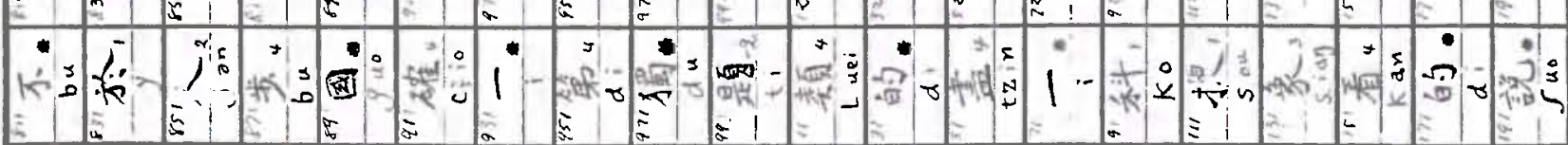

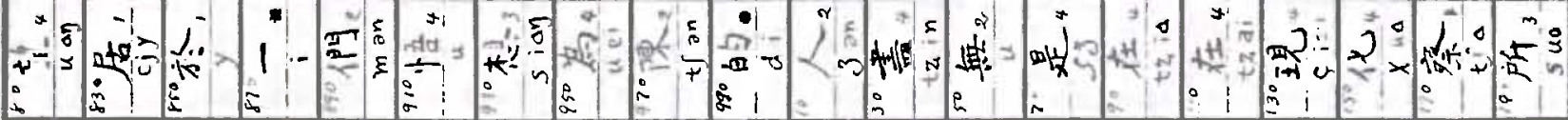

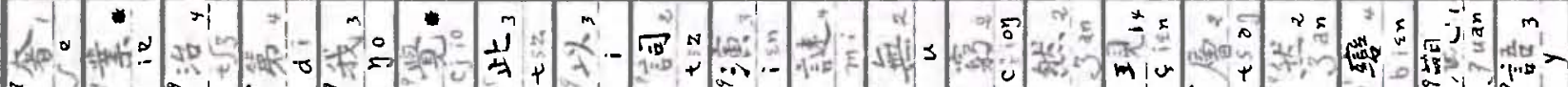

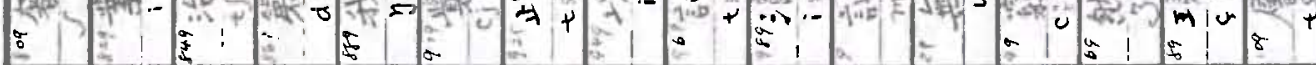

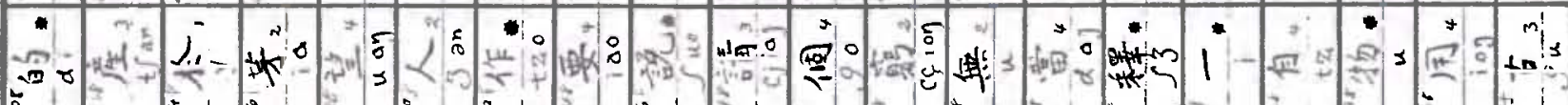

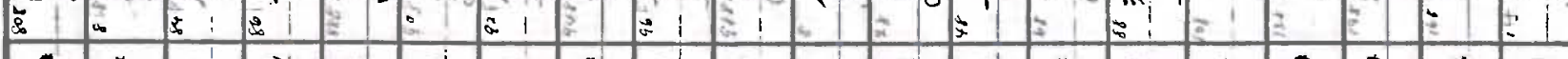

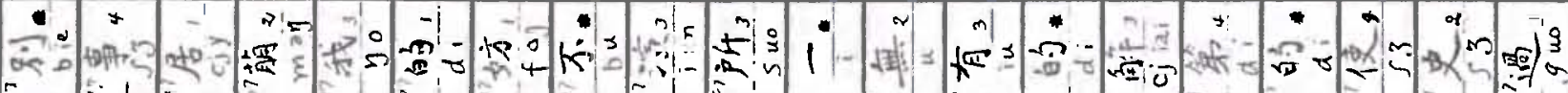

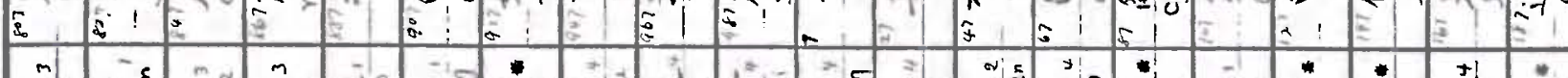

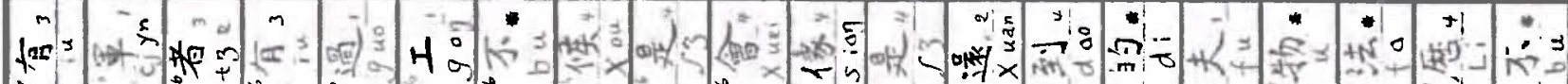

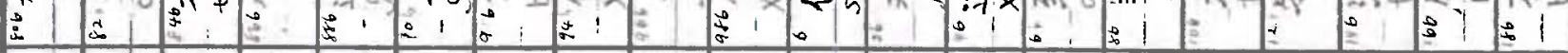

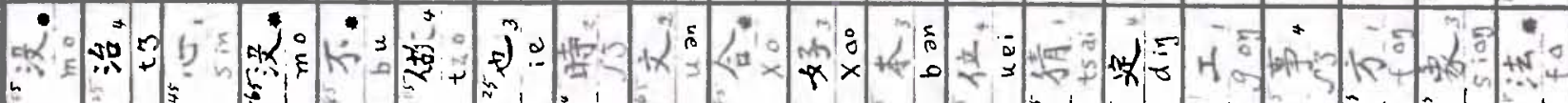

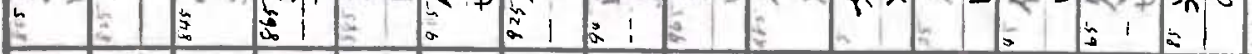

और * +

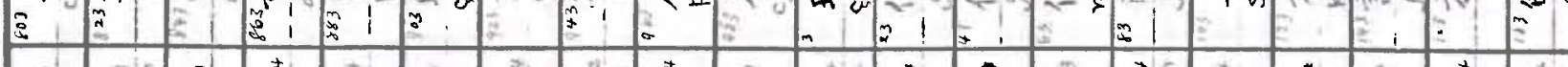

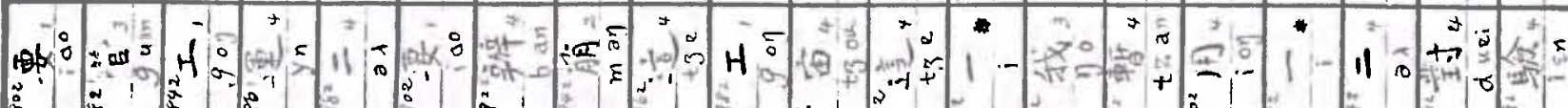

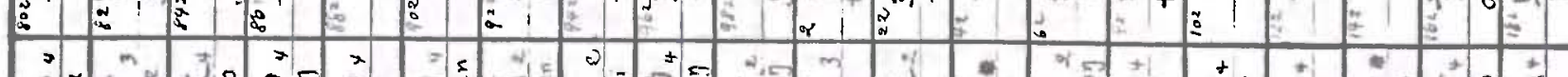

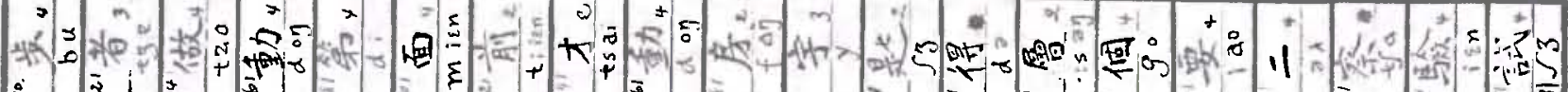
2. 


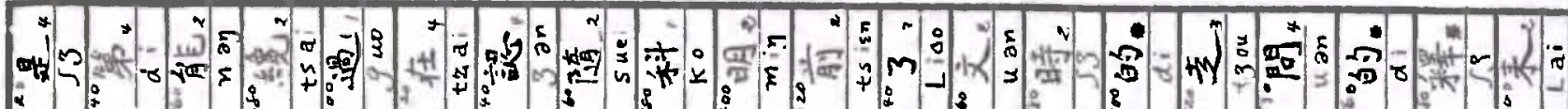

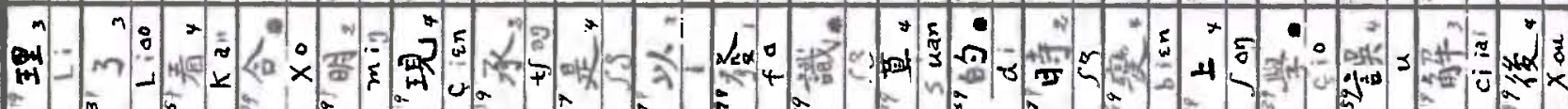
1.-

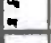

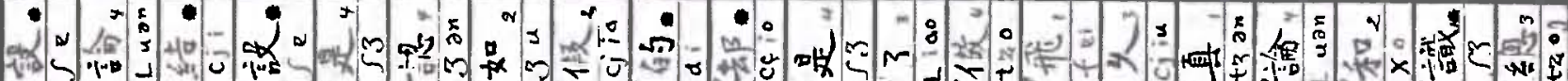

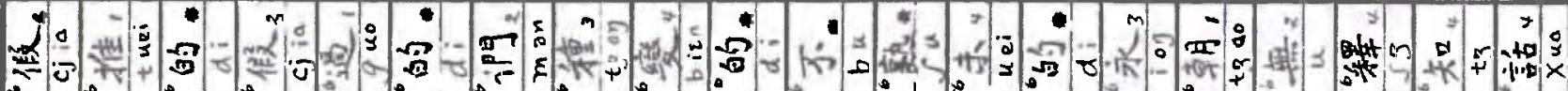

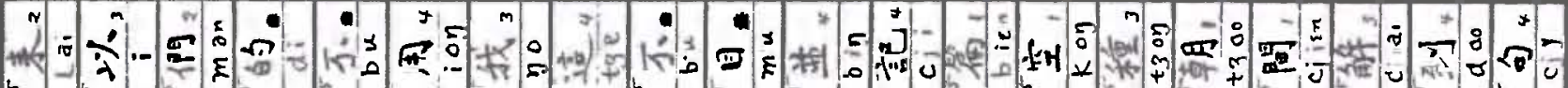

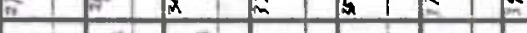

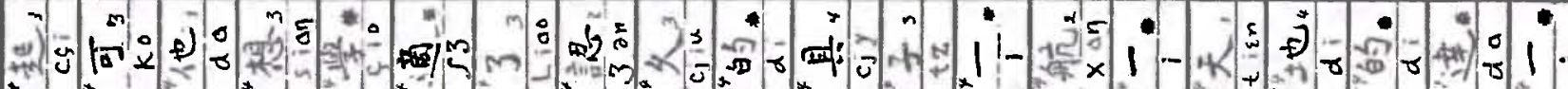

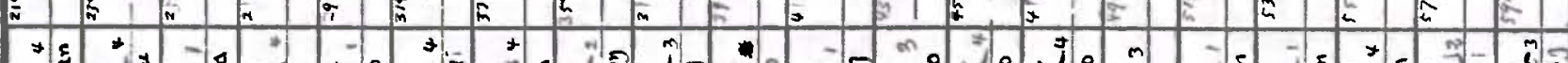
qu作

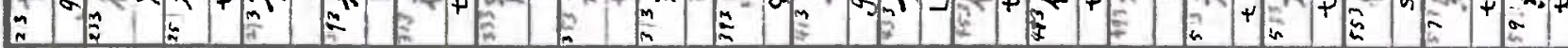

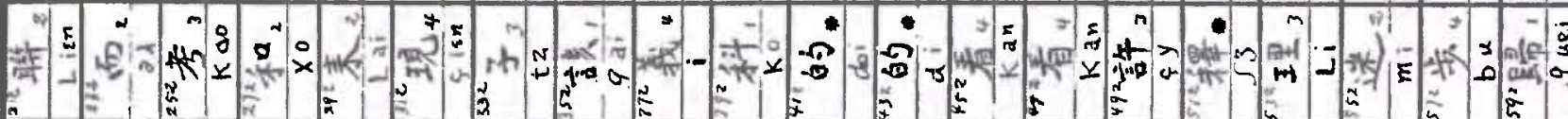

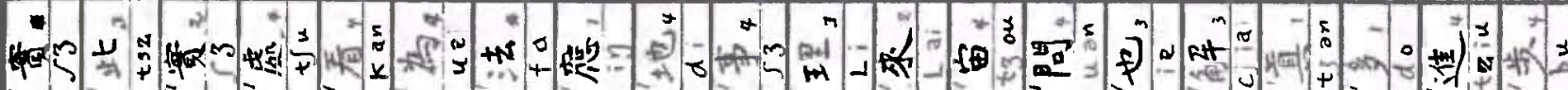

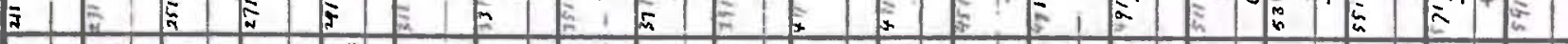

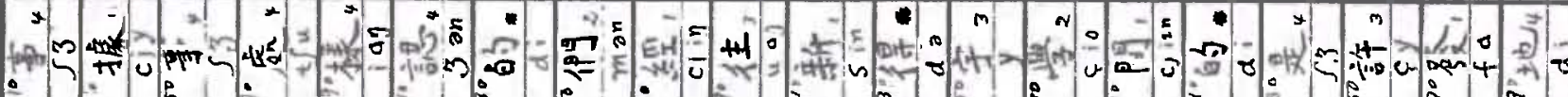

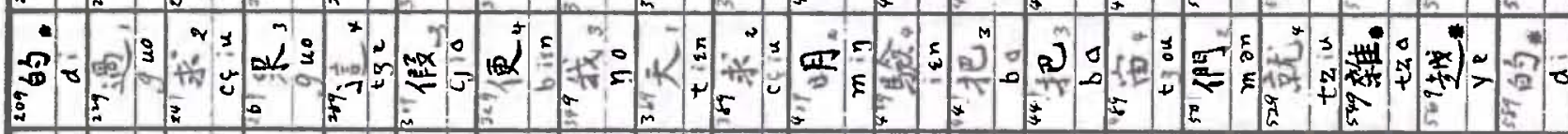

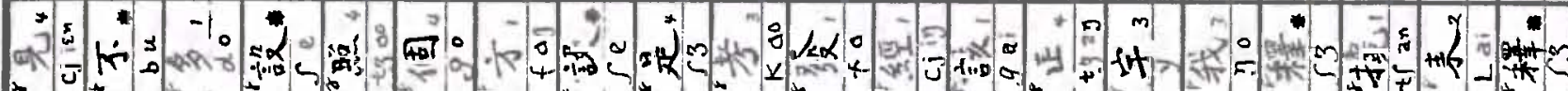

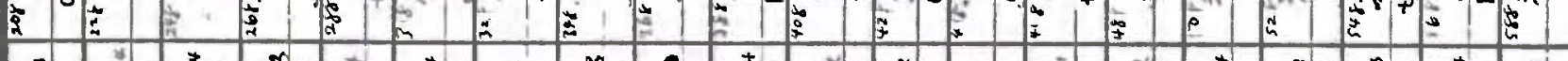

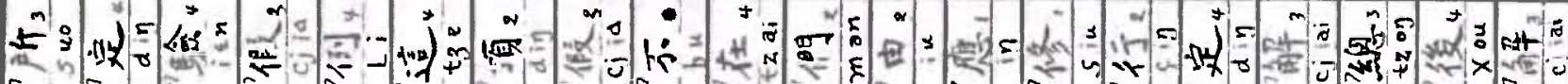

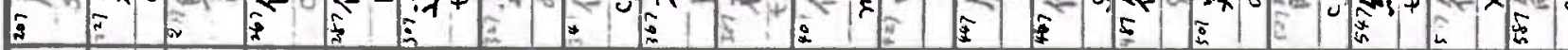

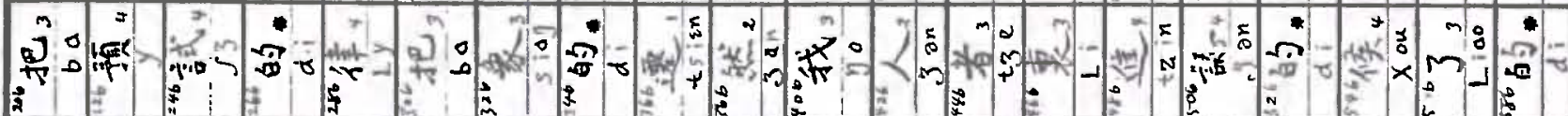

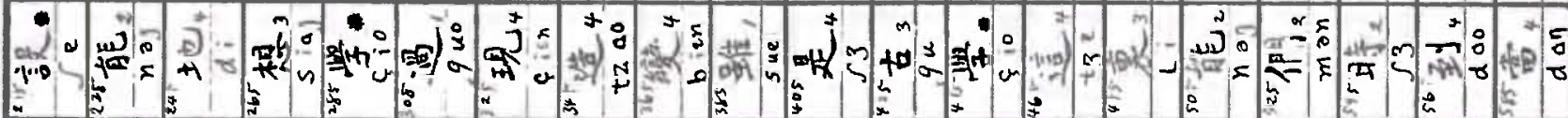
造 E 1 t a

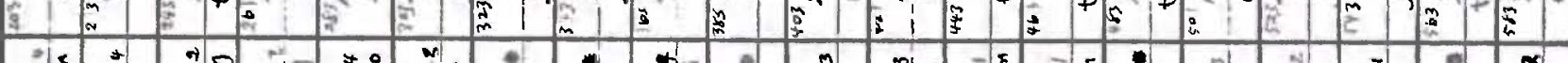

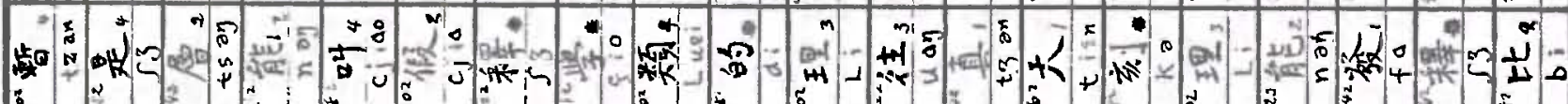

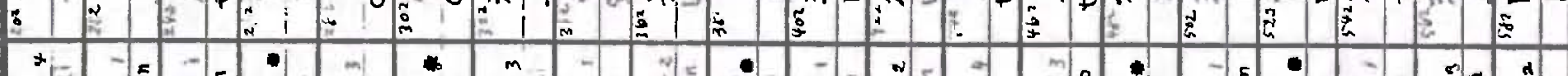

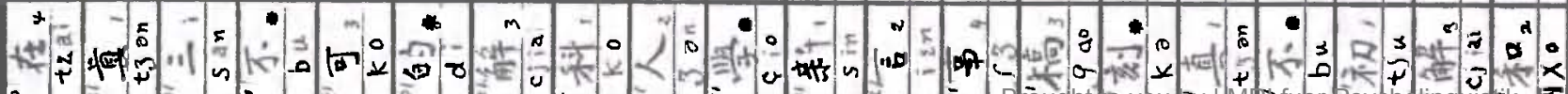

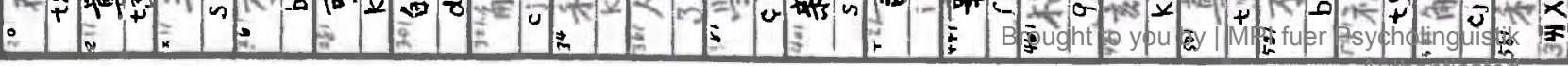




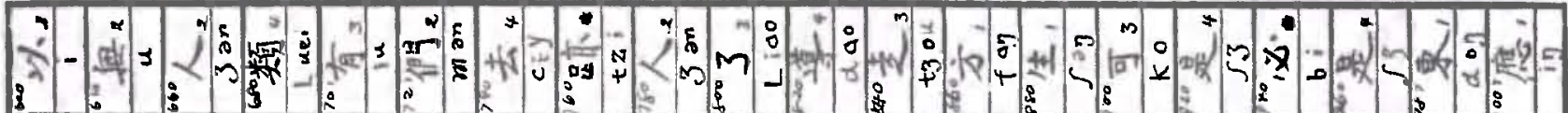
-

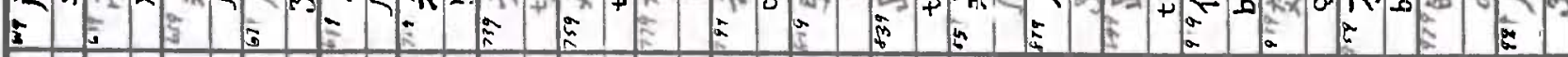

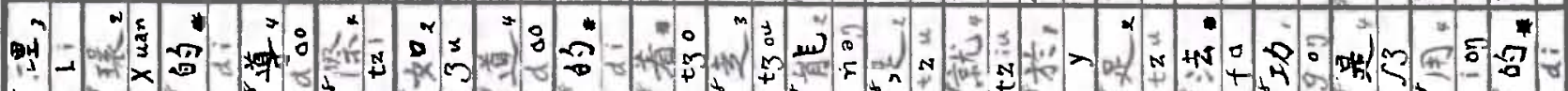
(5)

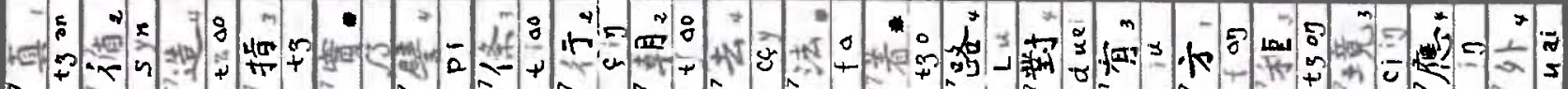

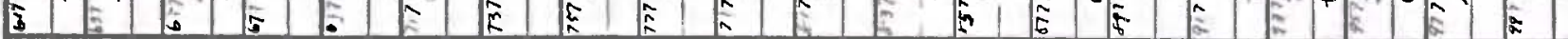

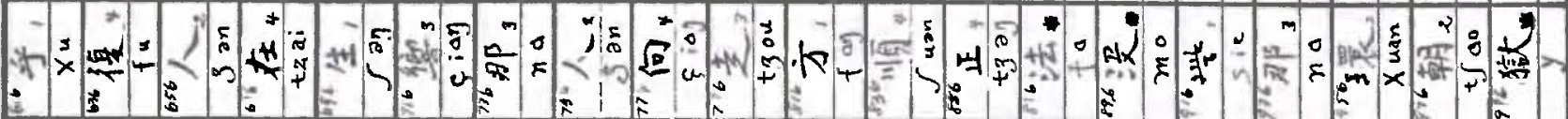

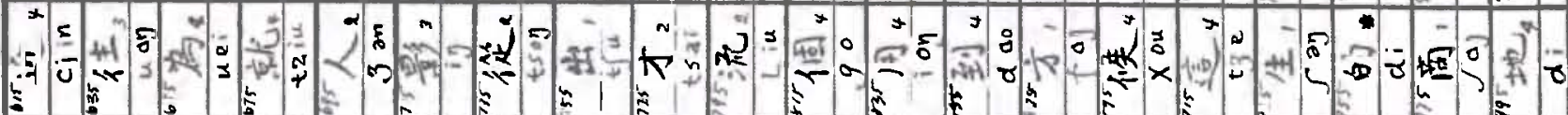

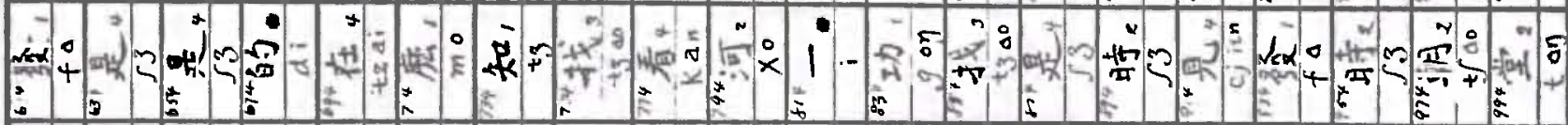

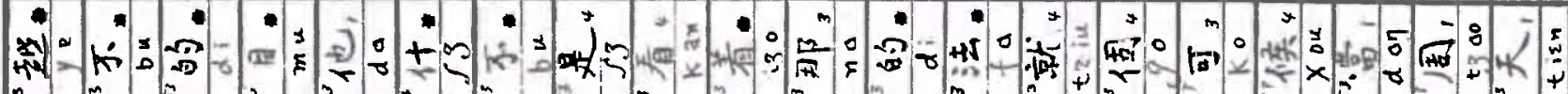

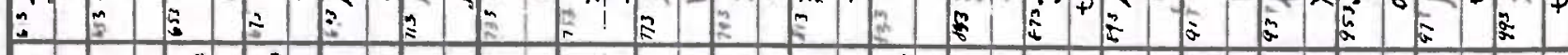

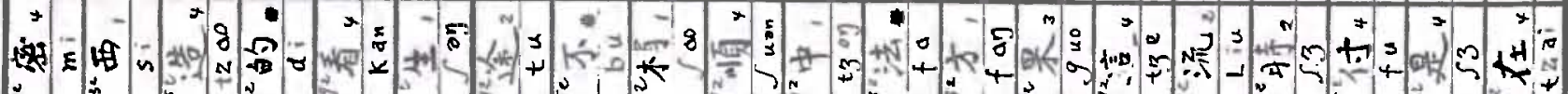

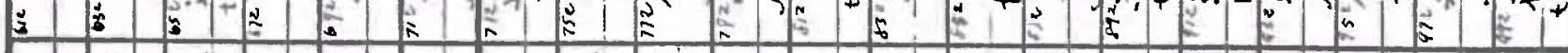

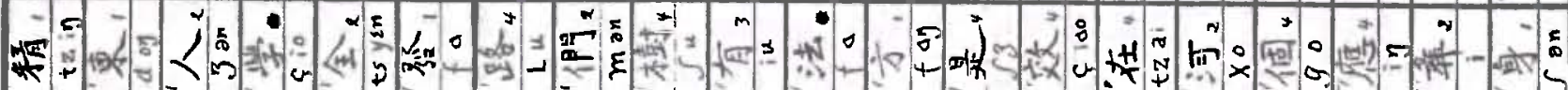

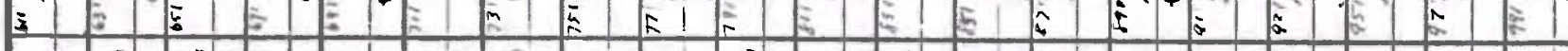

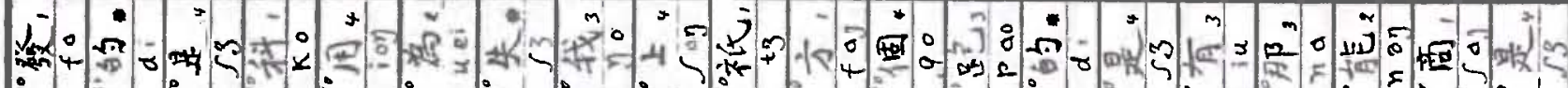

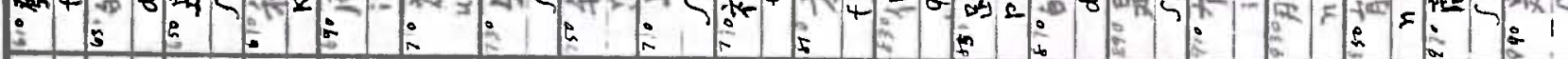

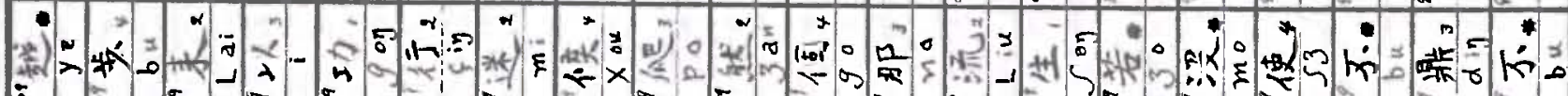

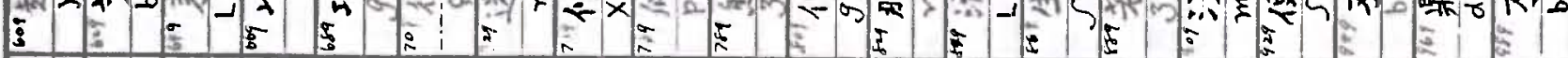

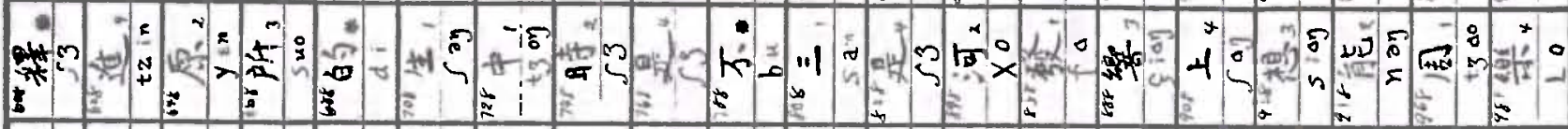

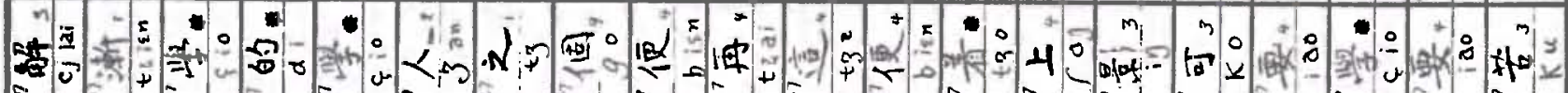

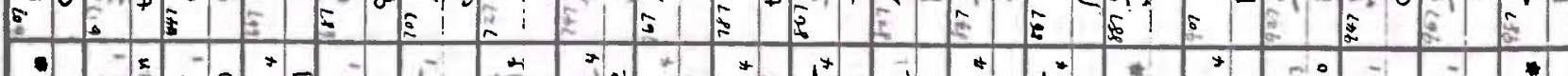

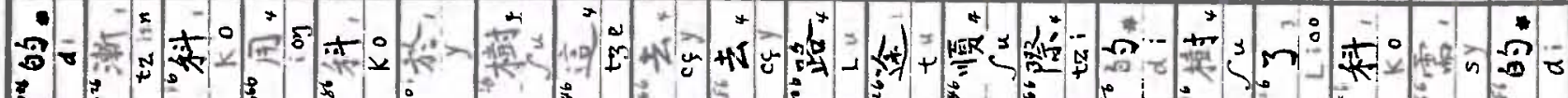

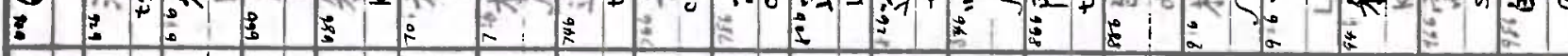

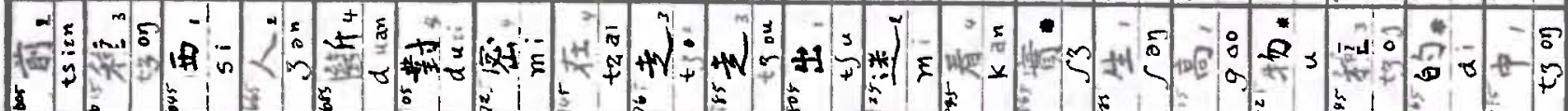

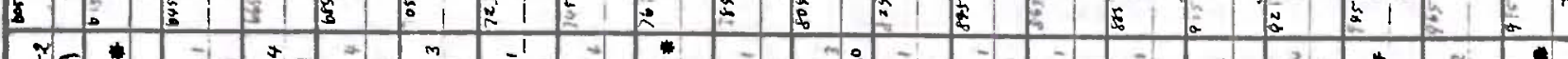

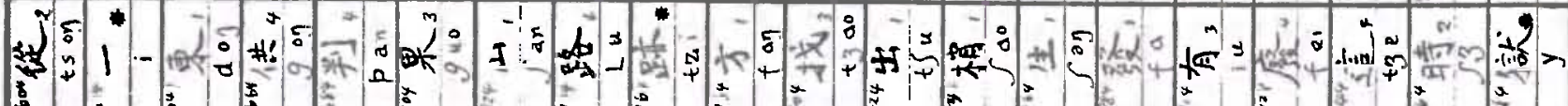
J. मे

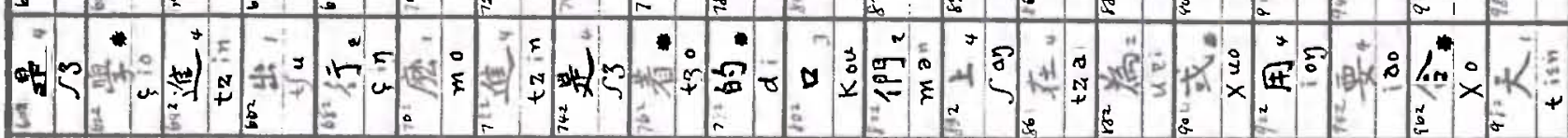

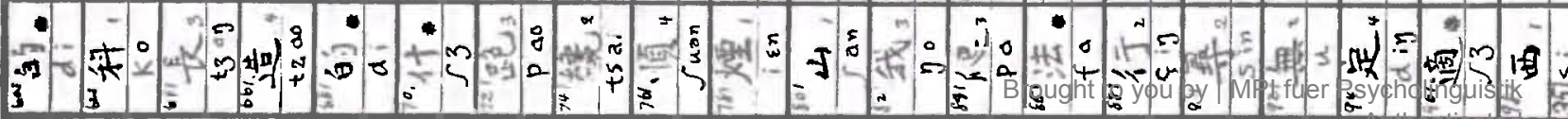




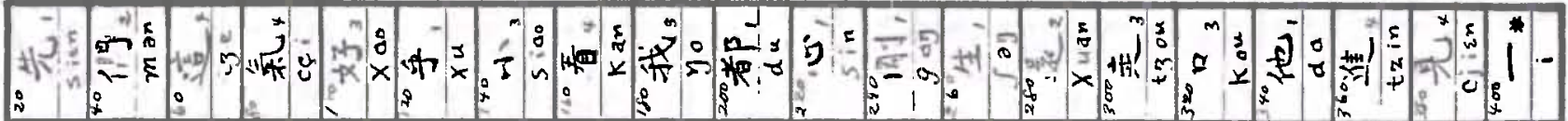

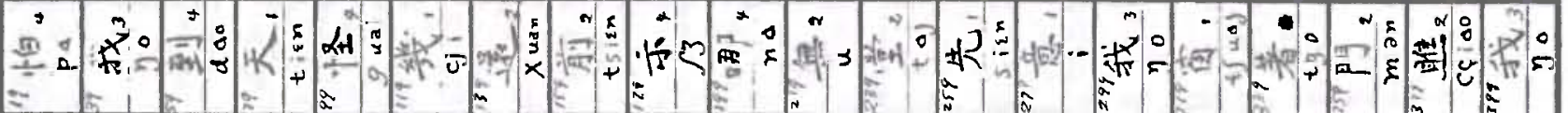
全 - 10 a 廿 j.

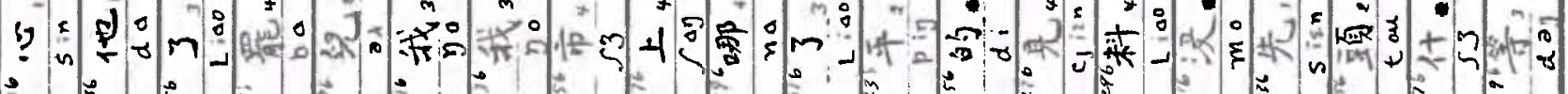

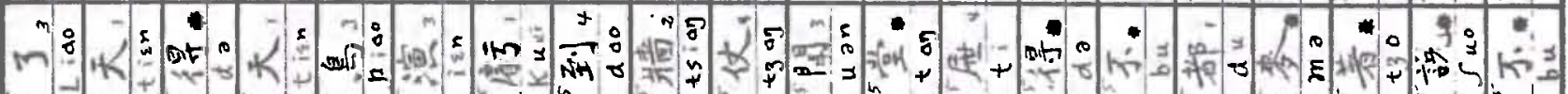

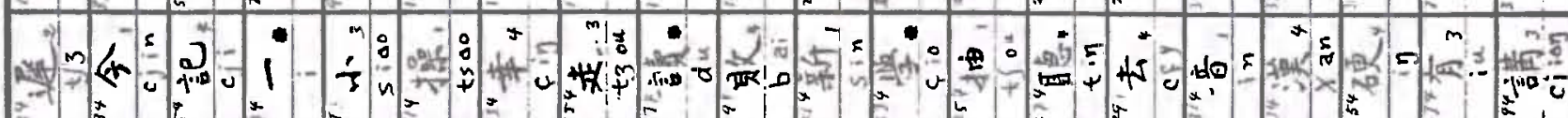

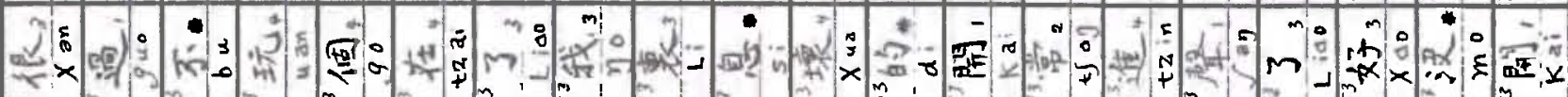

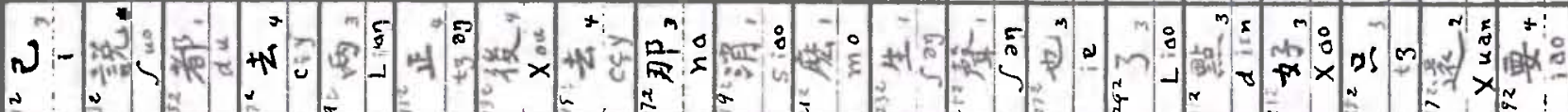

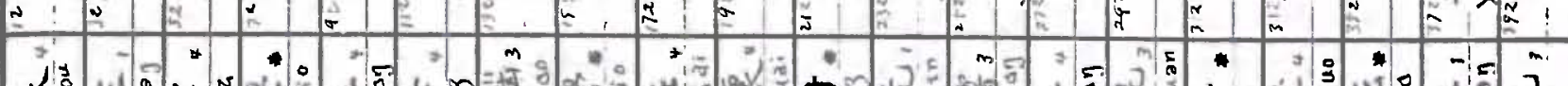

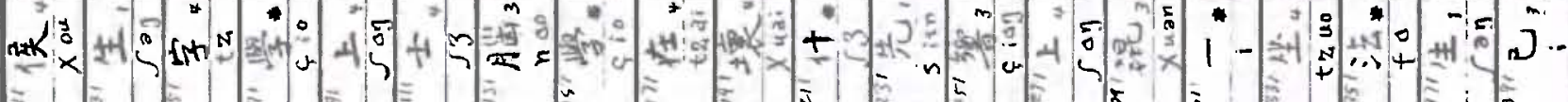

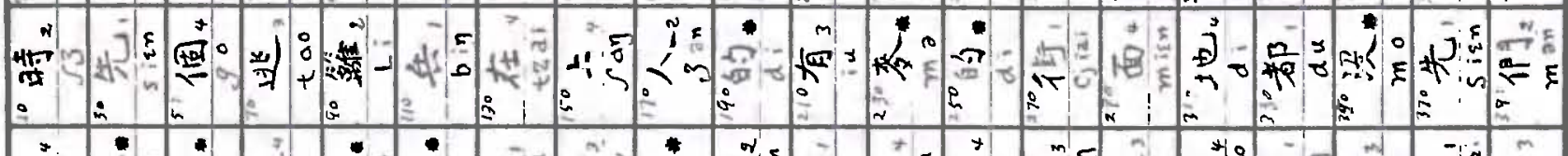

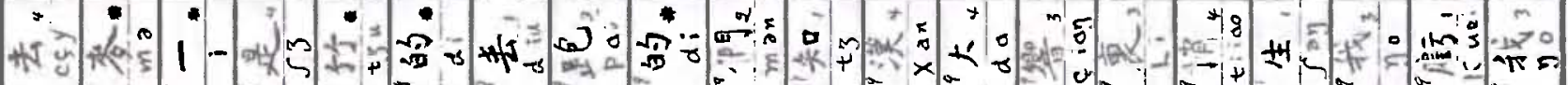

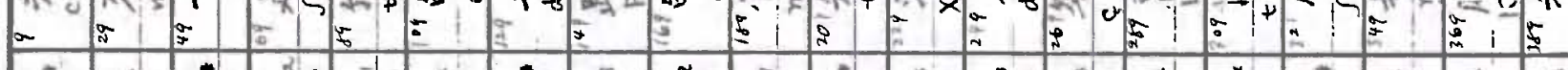

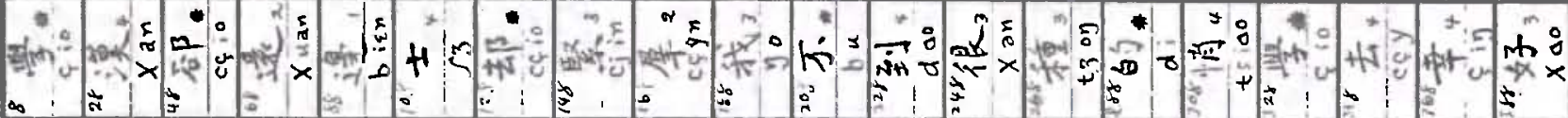

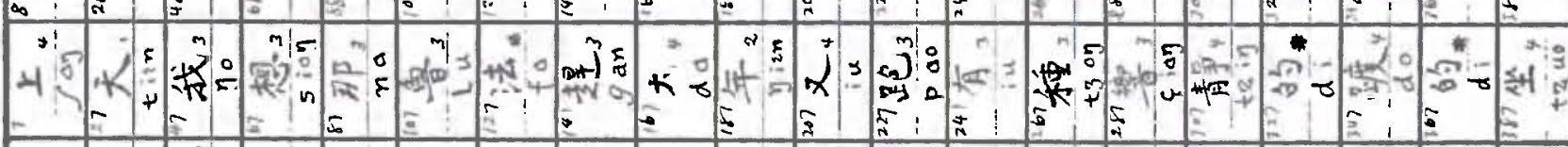

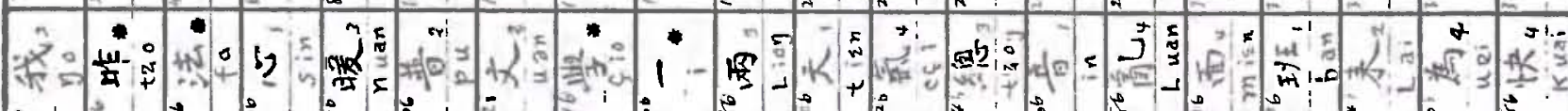

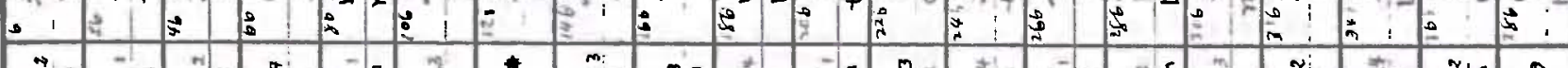

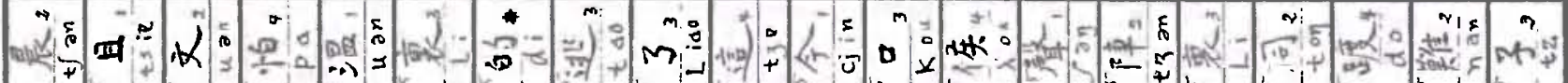

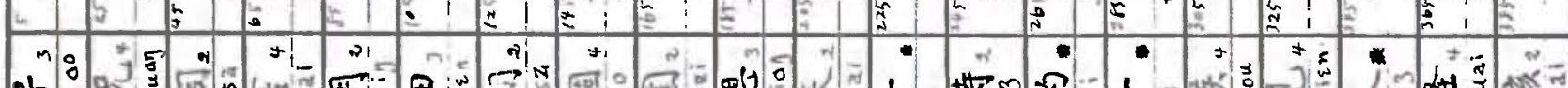

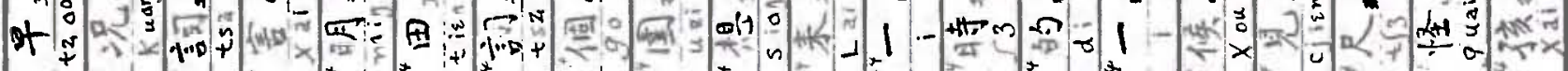

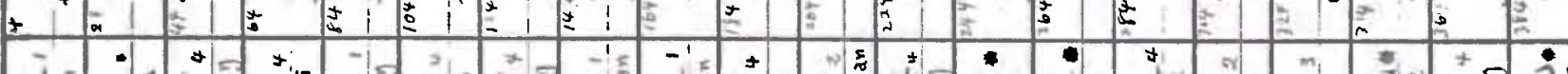
K

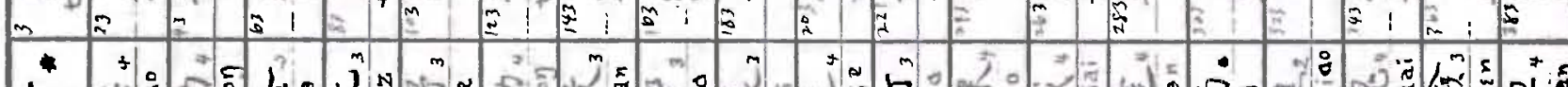

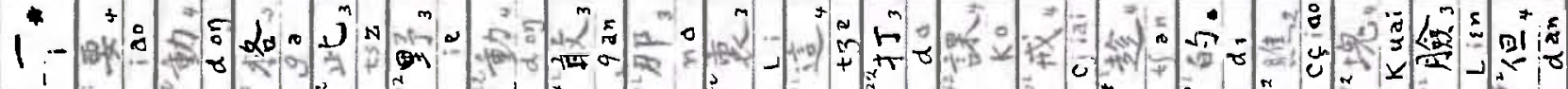
1. 
1.

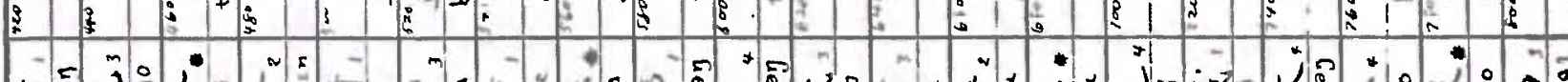

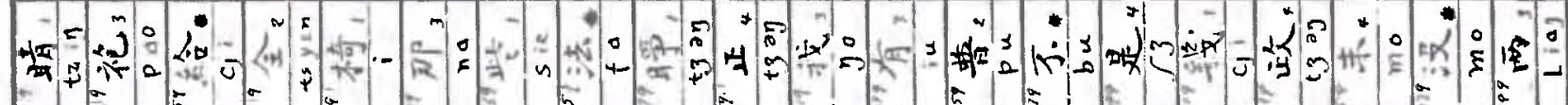
.

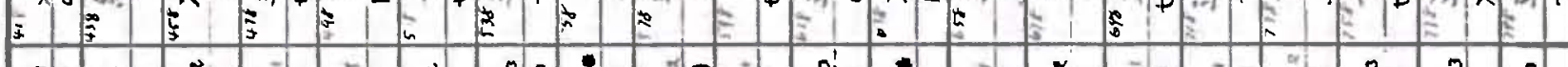

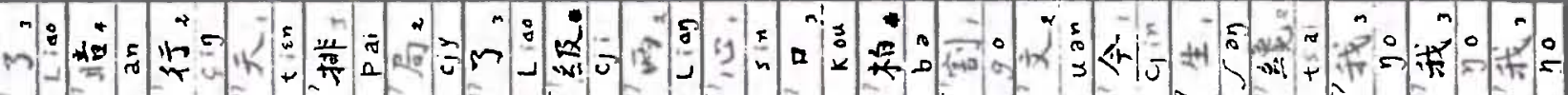
(4) $0=0$

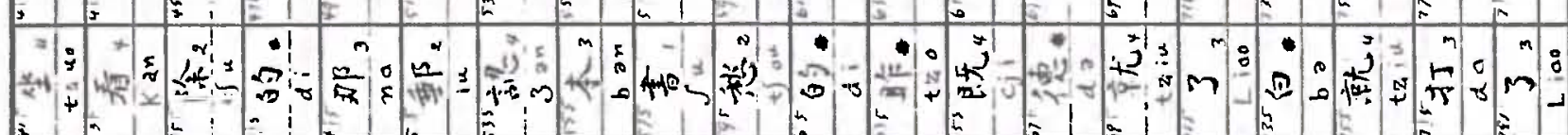

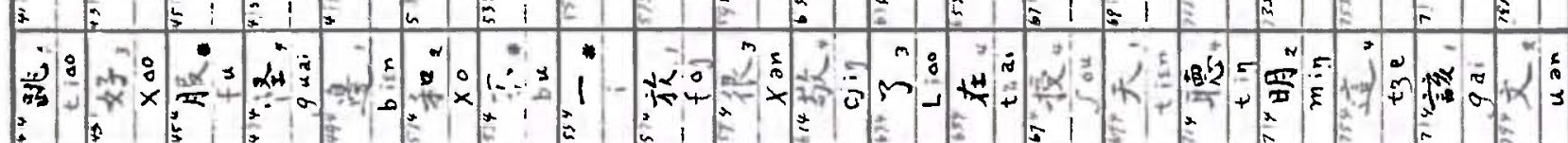

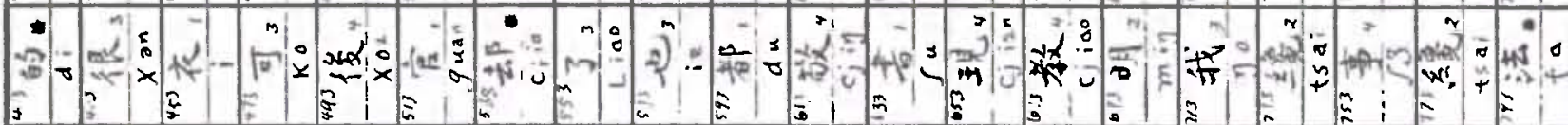

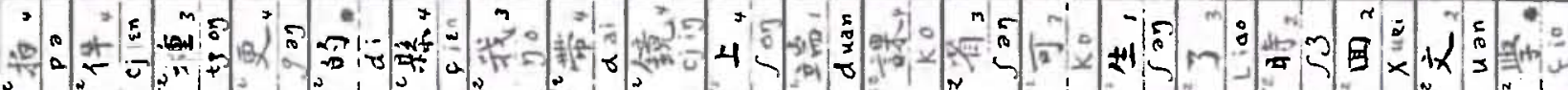

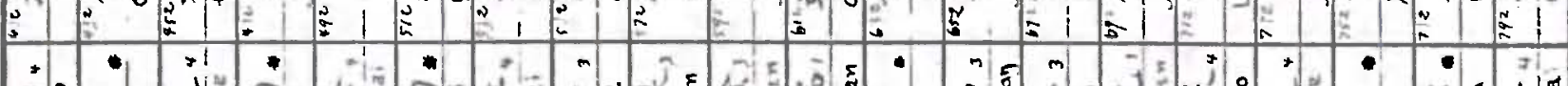

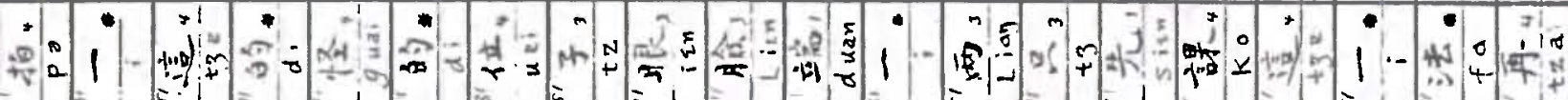

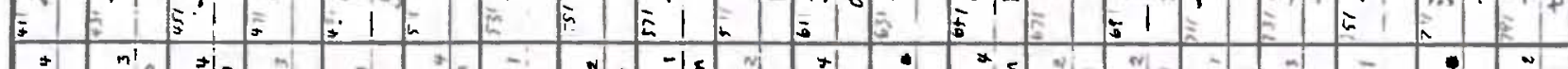

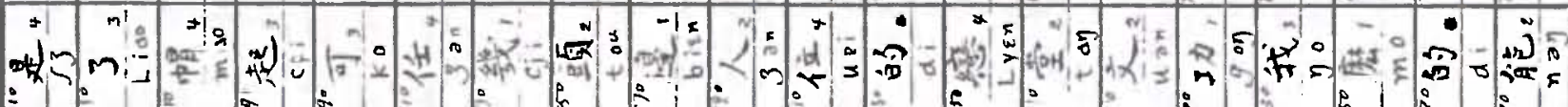

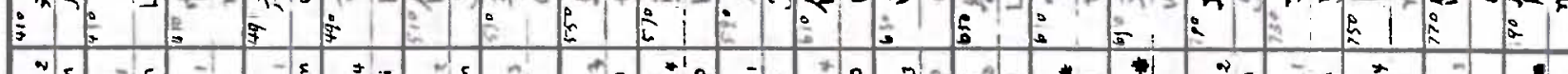

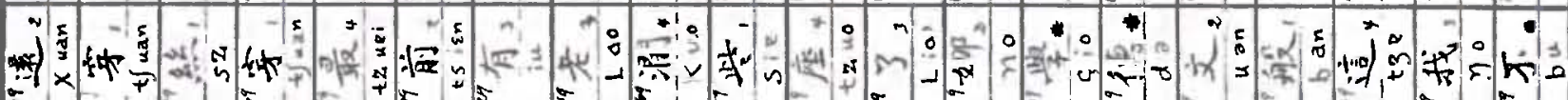
年

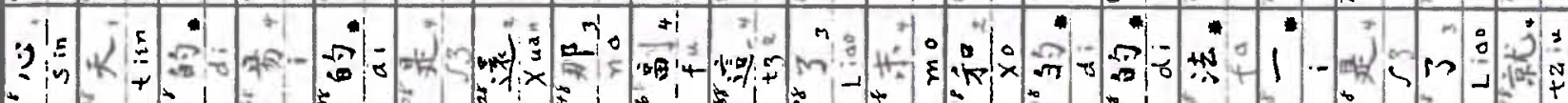

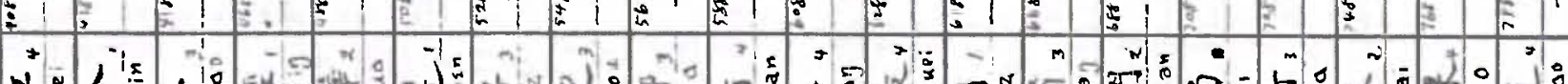
L

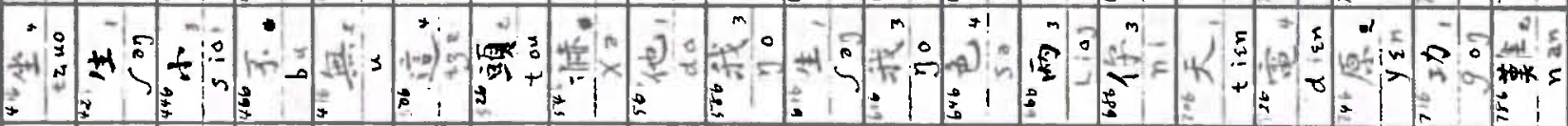

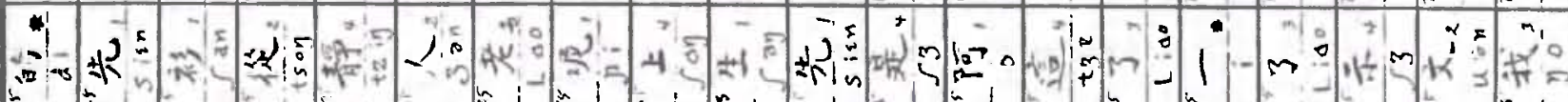

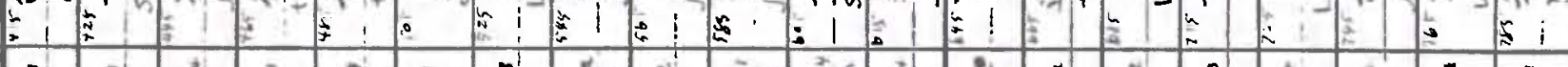

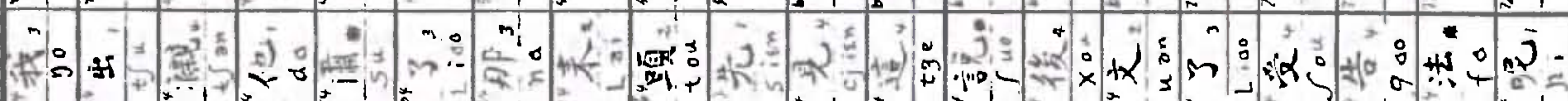

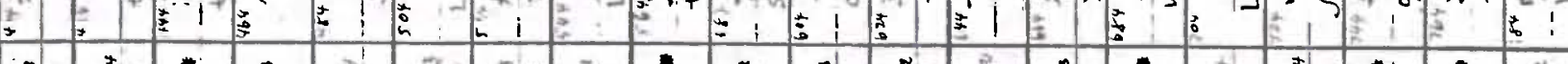

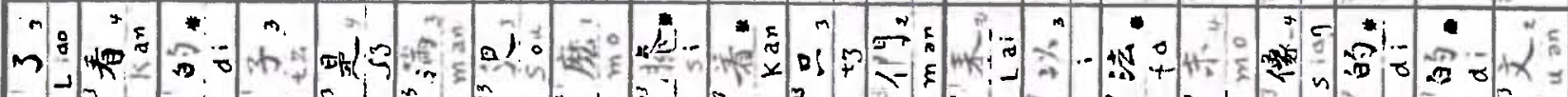

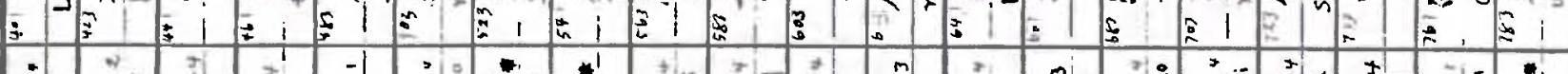

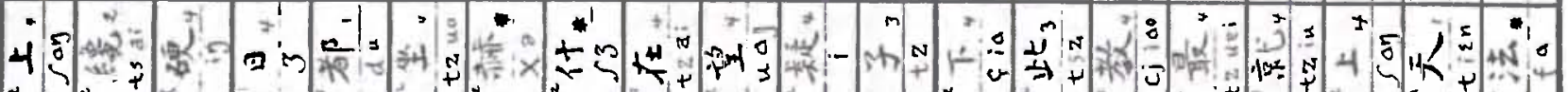

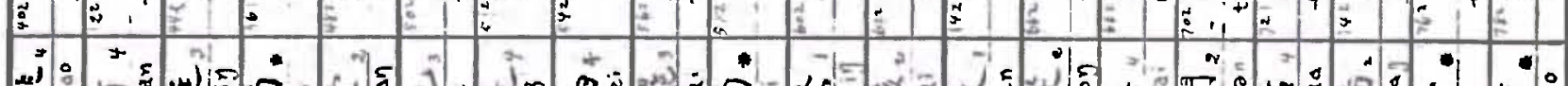
势

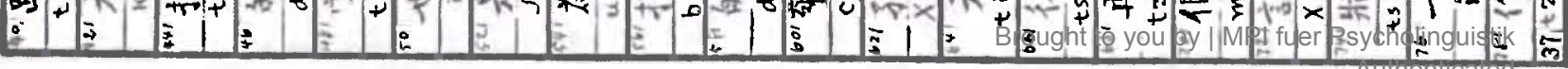




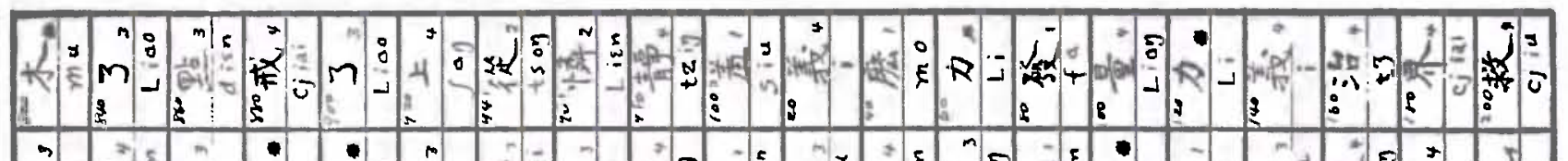
T) M)

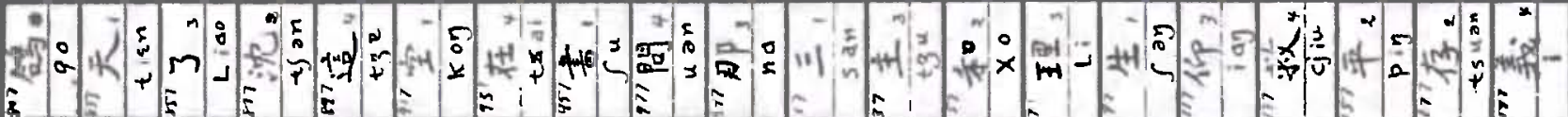

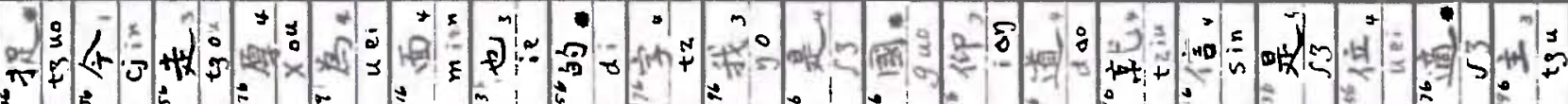

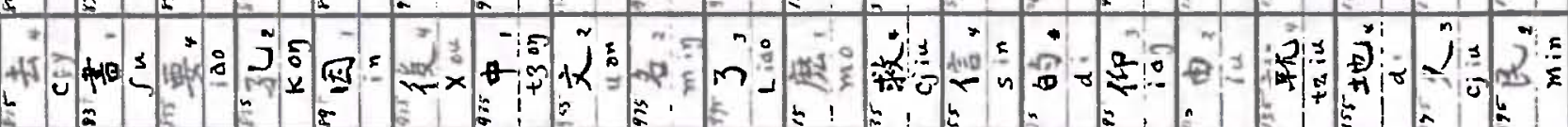

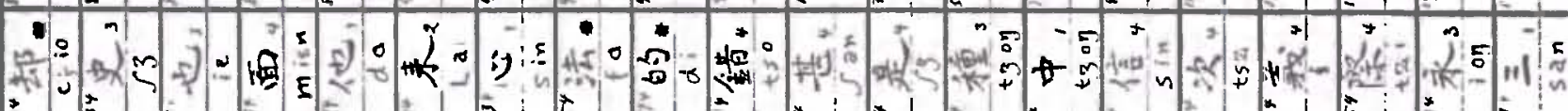

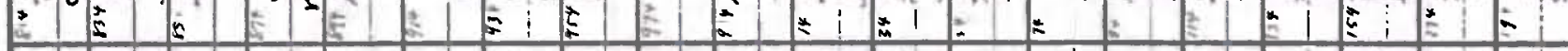

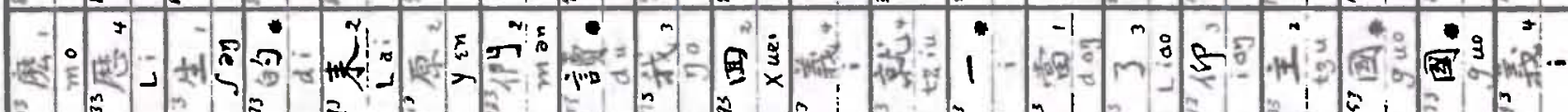

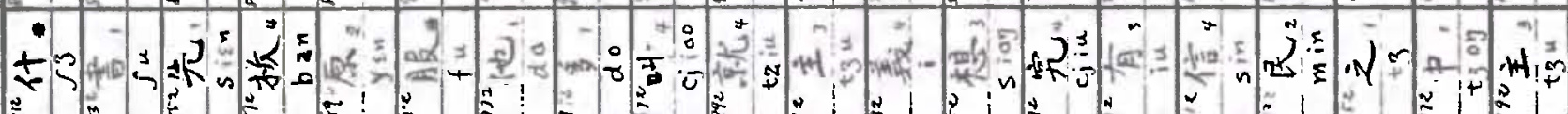

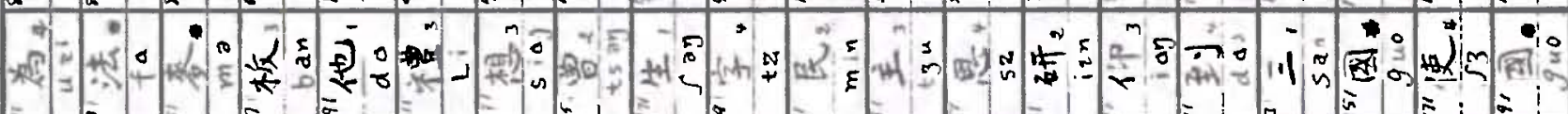

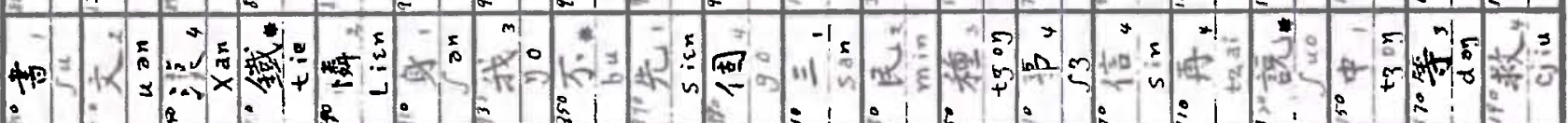

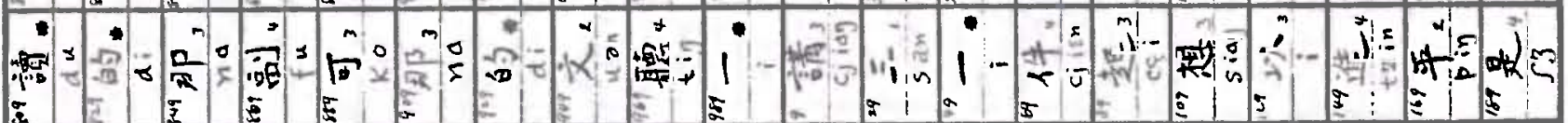

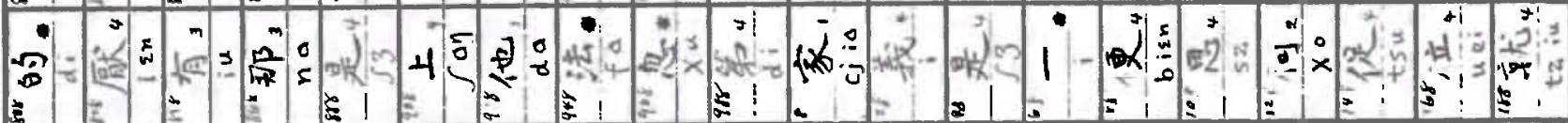

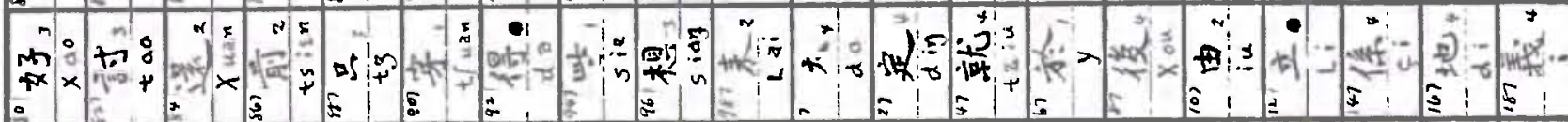

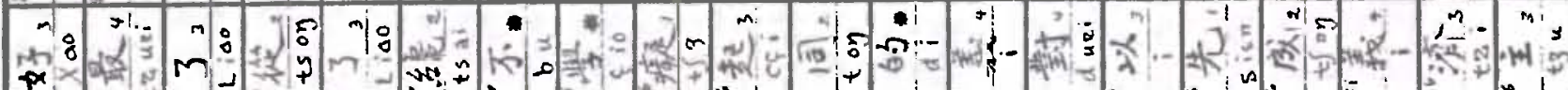
点

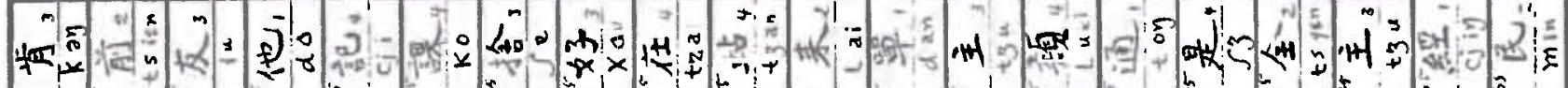
EIE

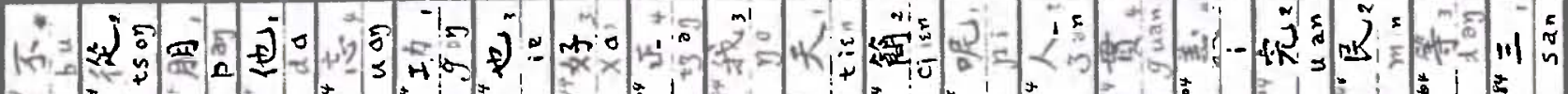

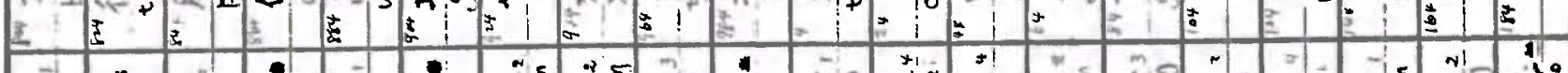

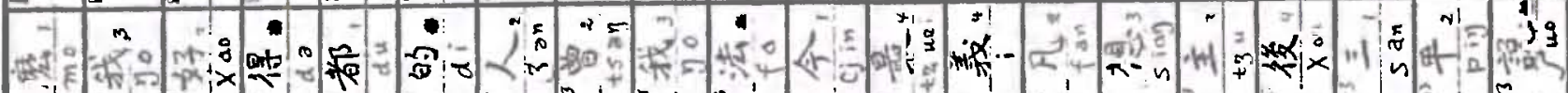

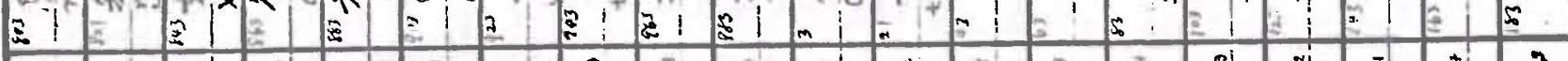

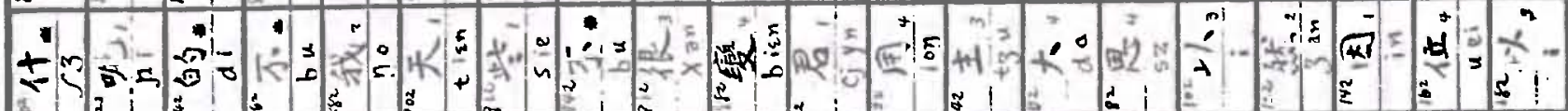

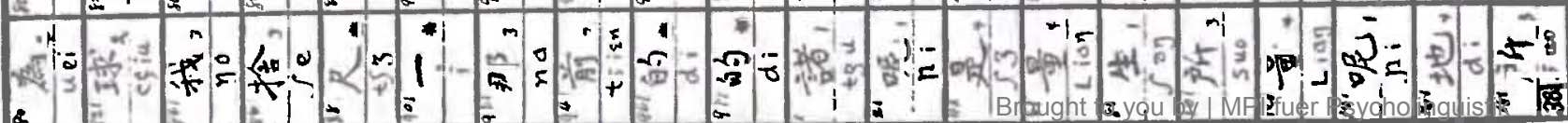




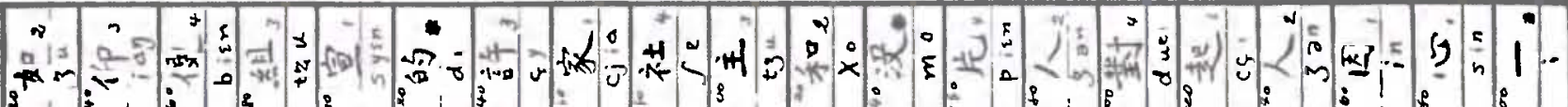
年

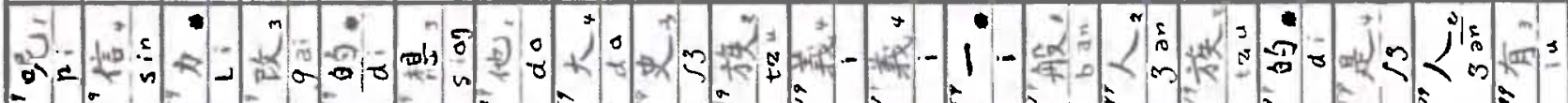

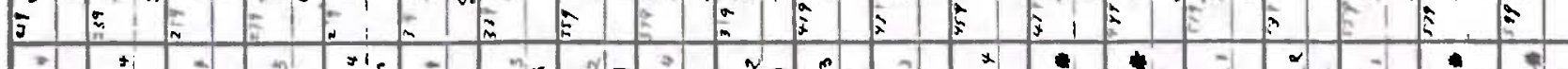

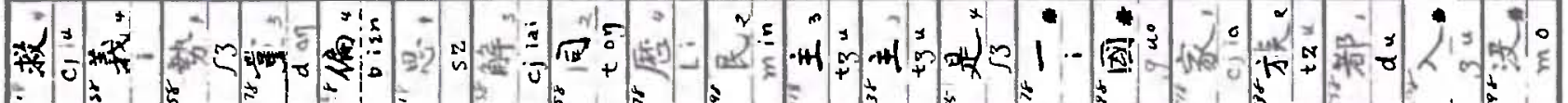
(n)

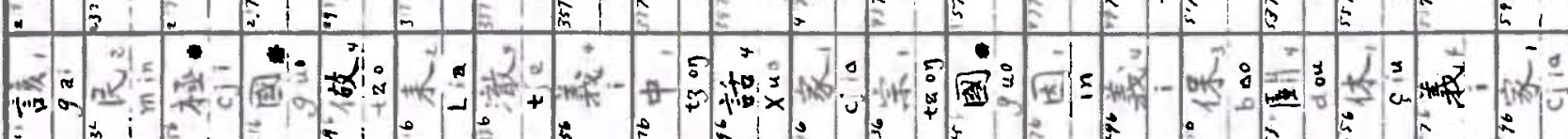

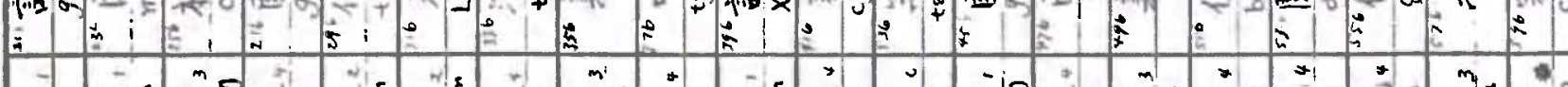

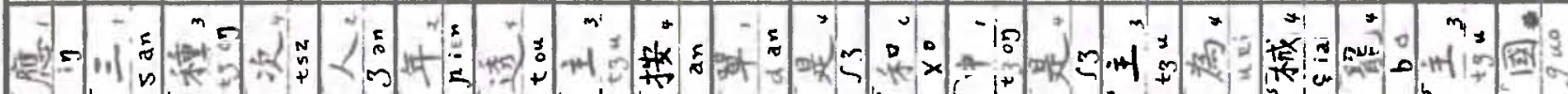

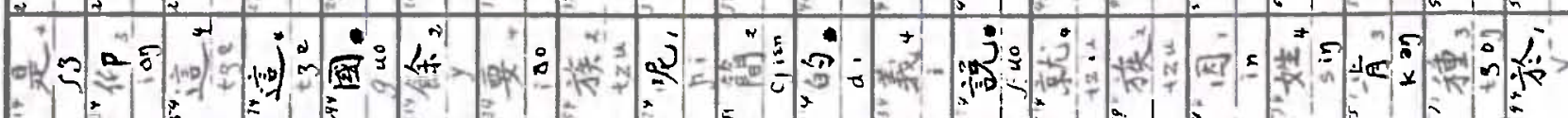

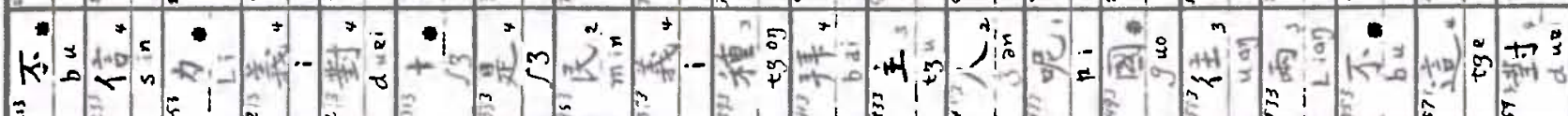

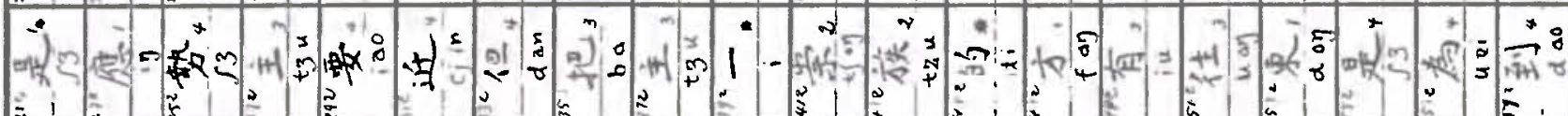

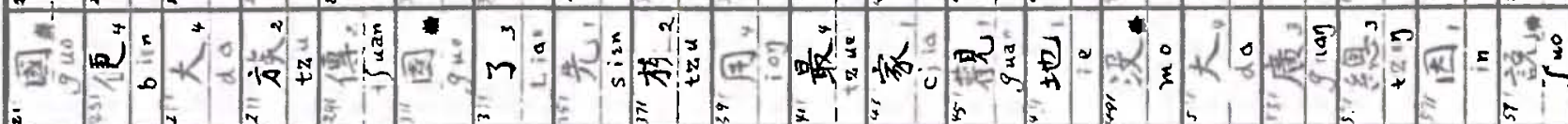

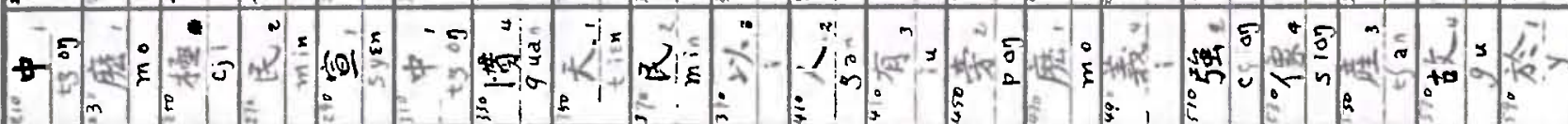

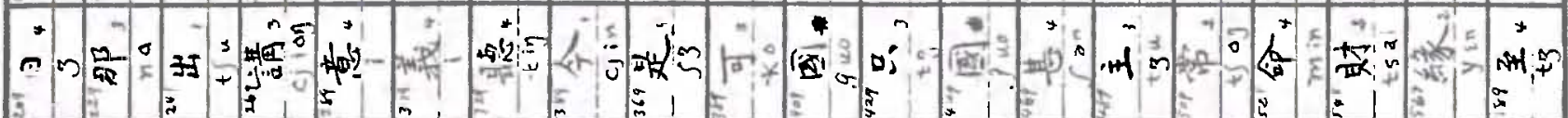

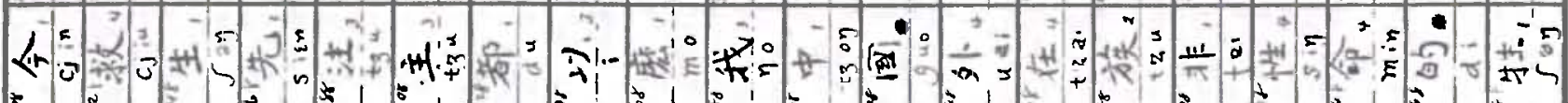

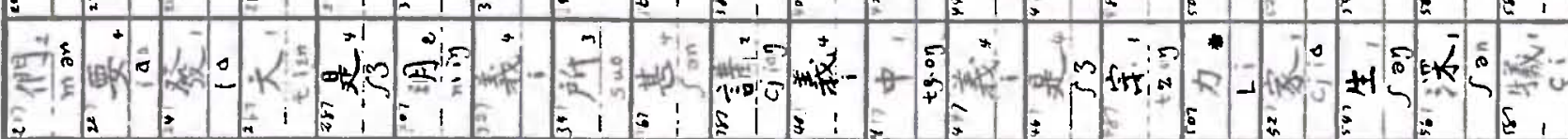

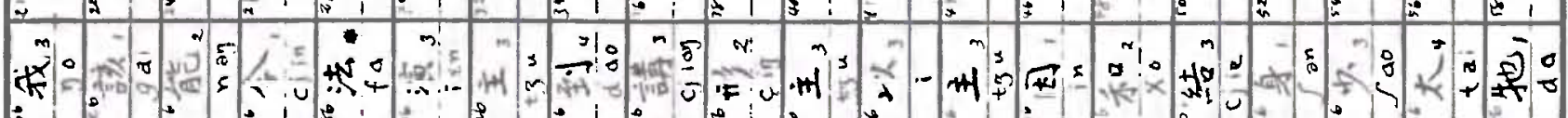

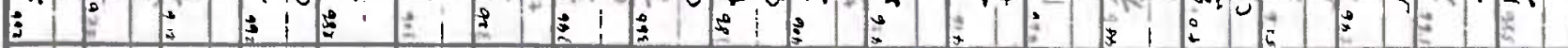

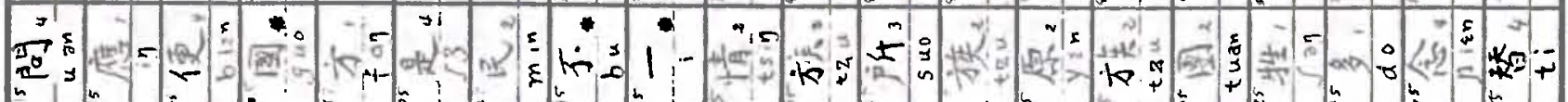

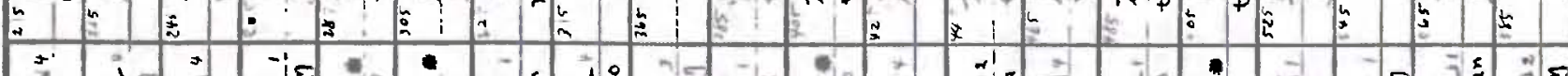

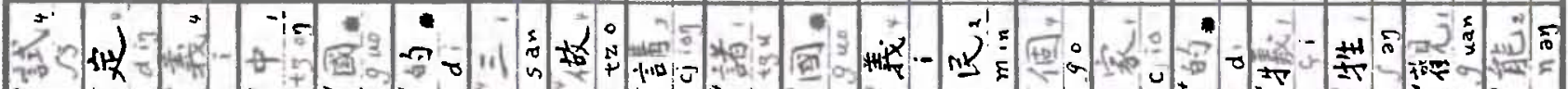

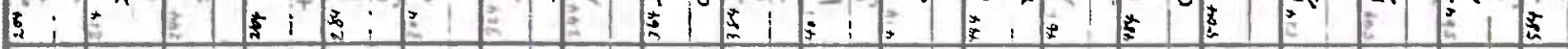

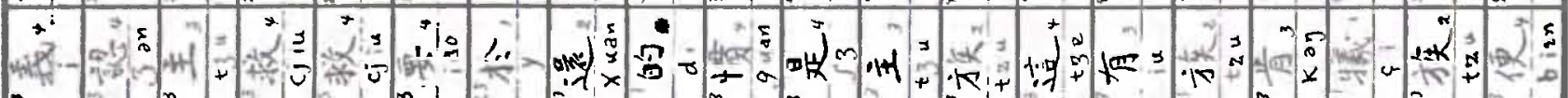

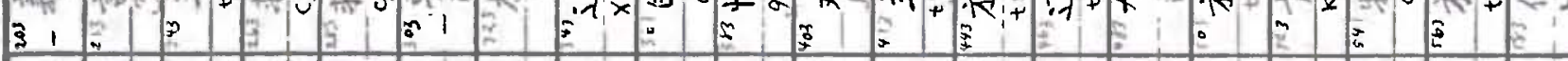
.

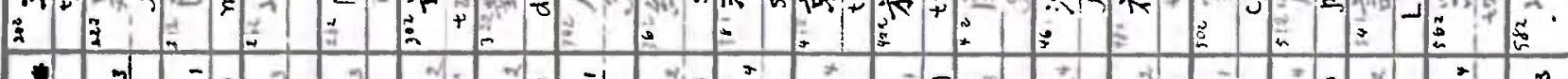

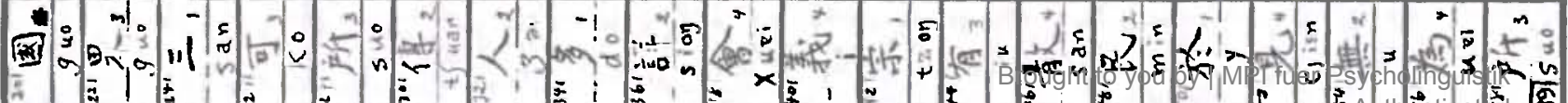




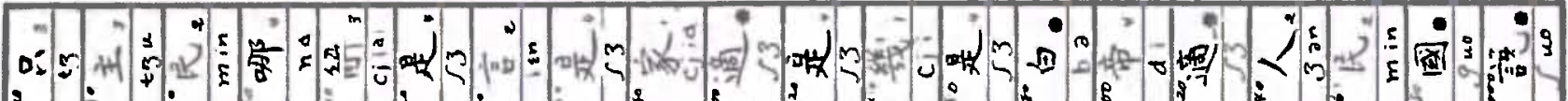

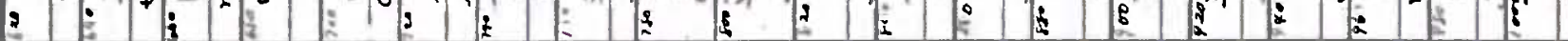

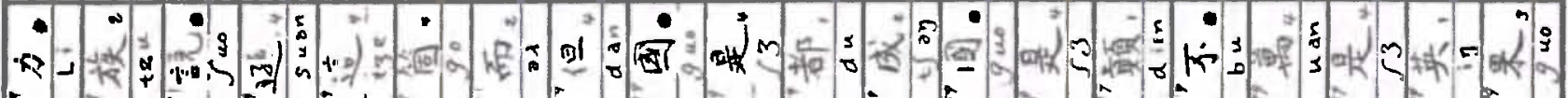

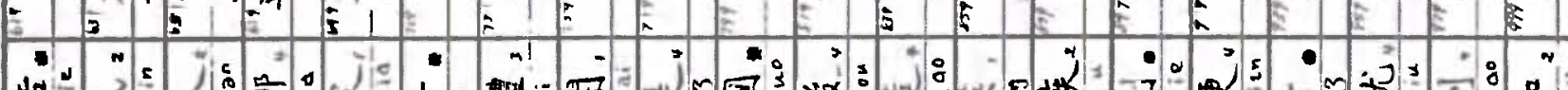

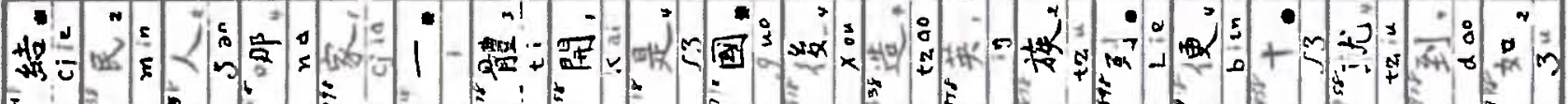

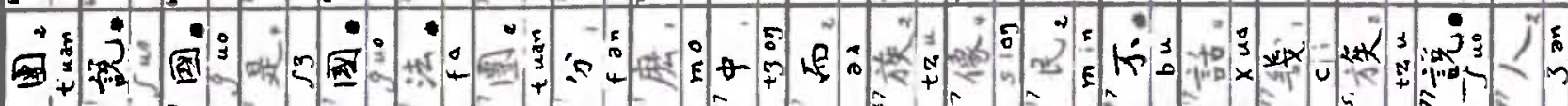

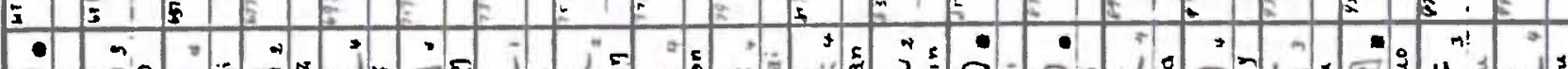
की

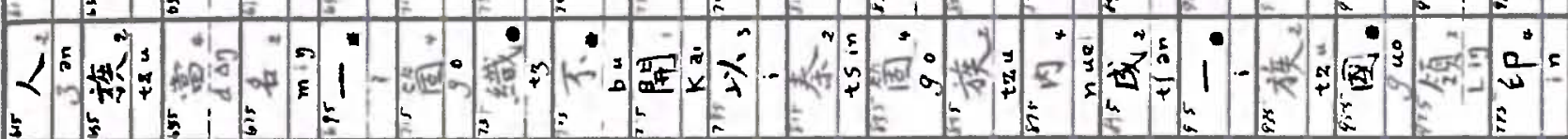

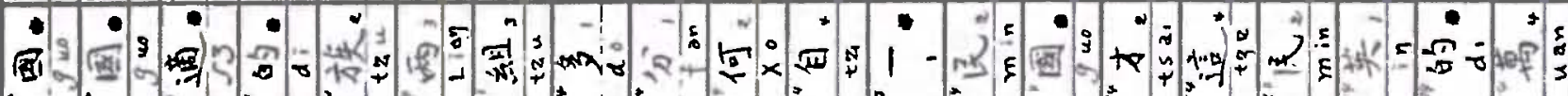

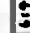

-

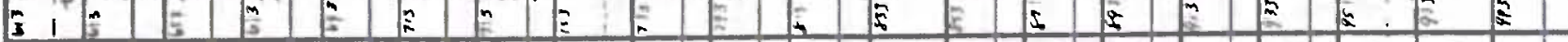

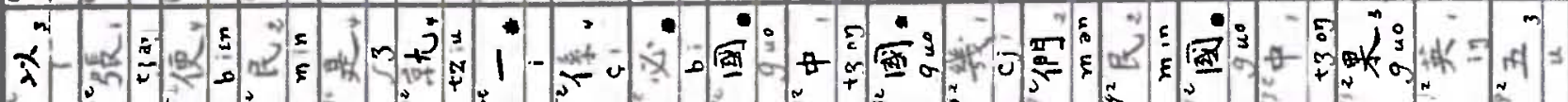

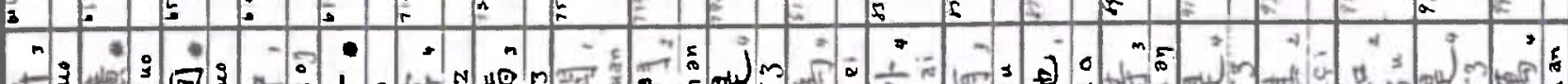

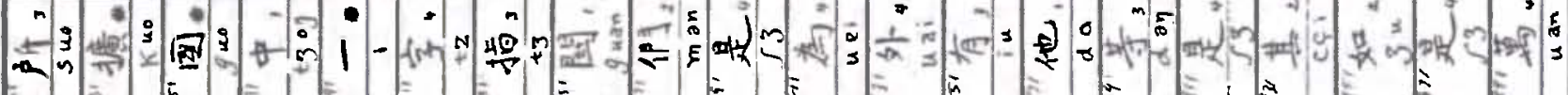

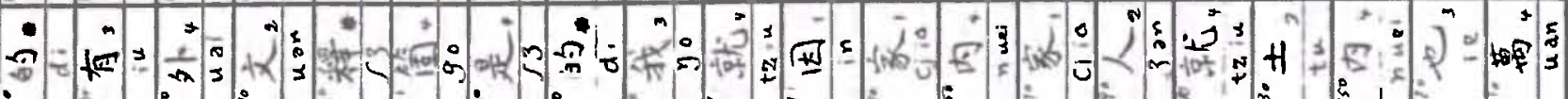

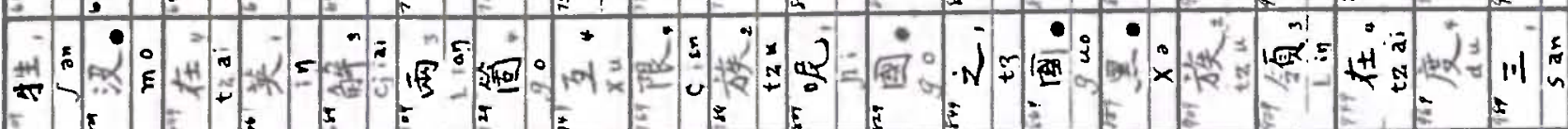

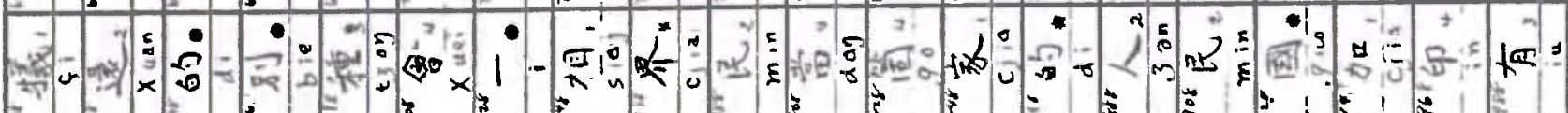

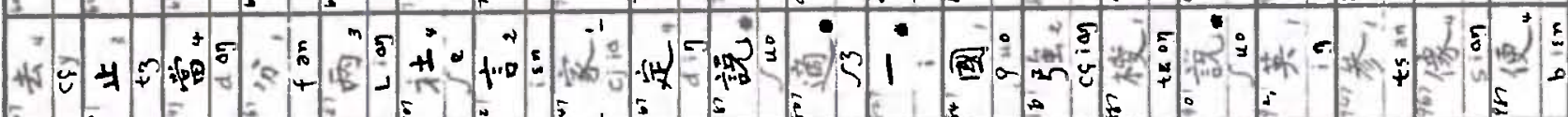

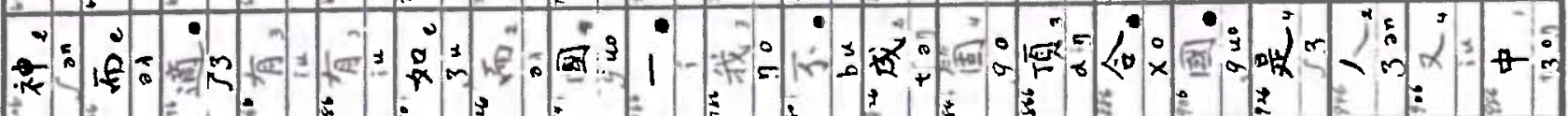

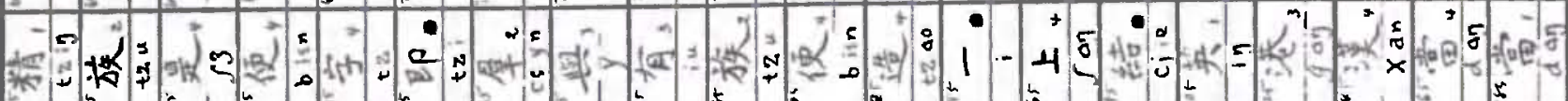

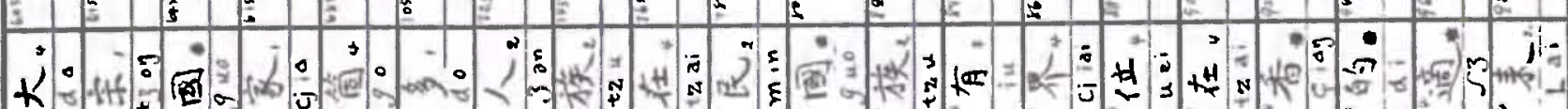

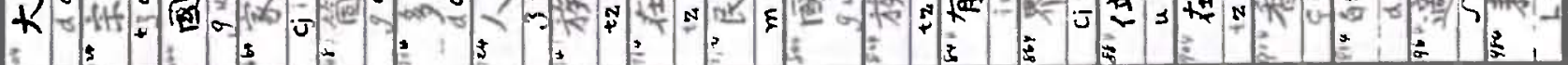

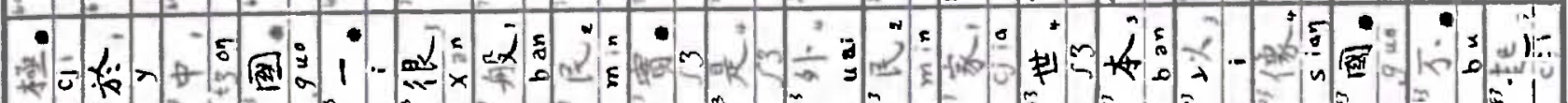

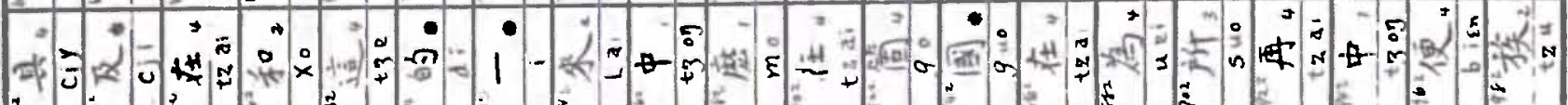

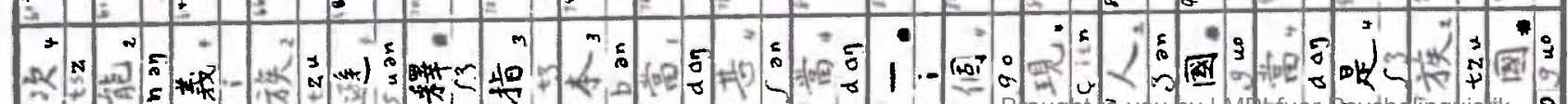

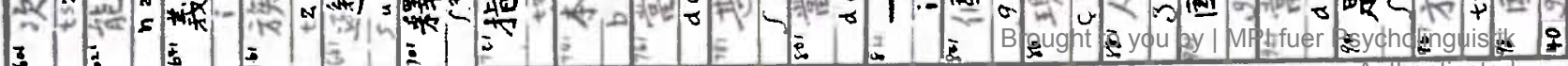




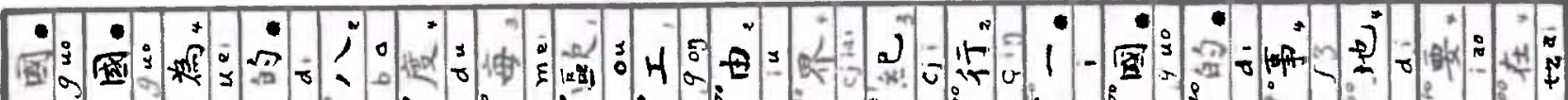
\&

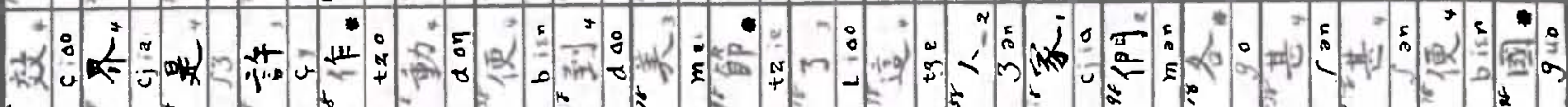

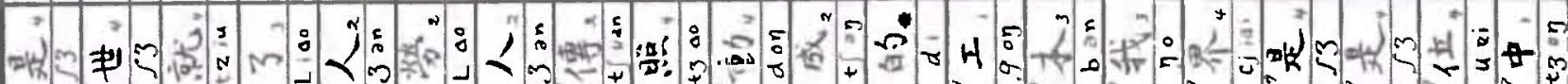

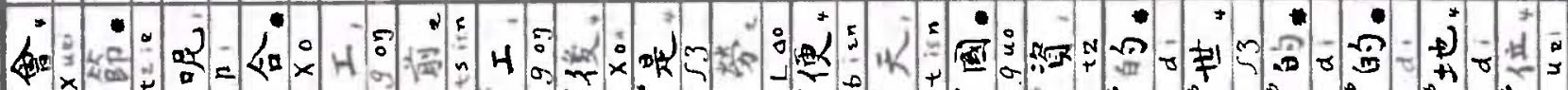

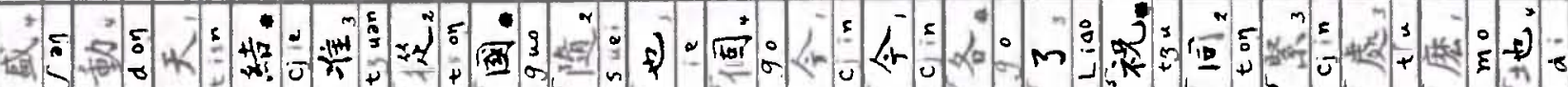

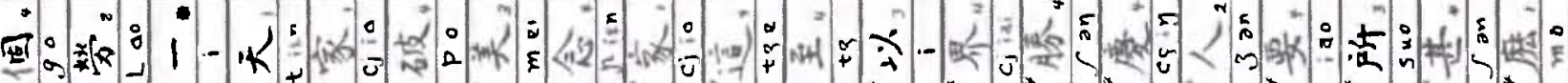

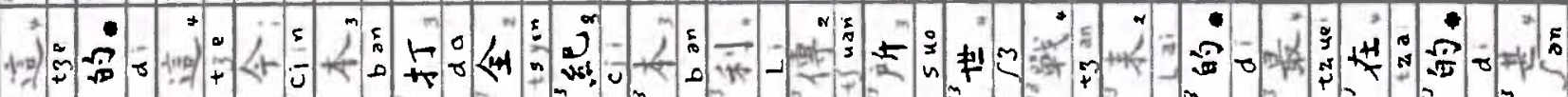

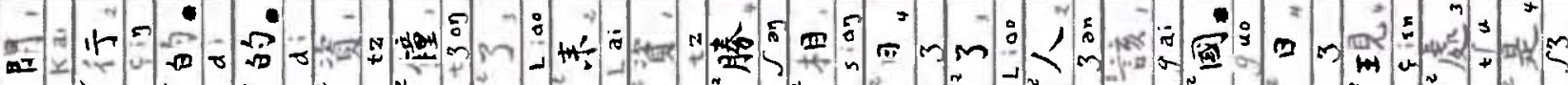

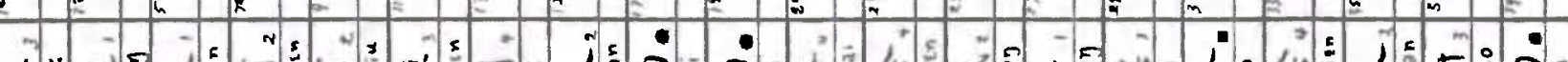

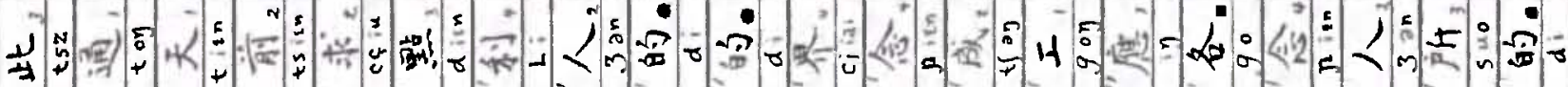
+1

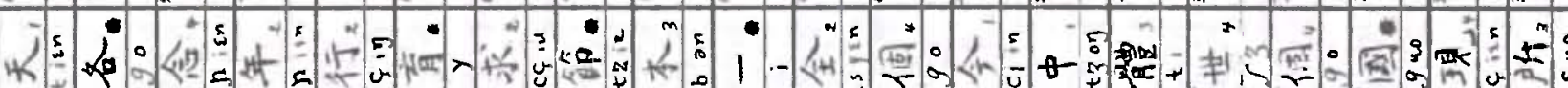

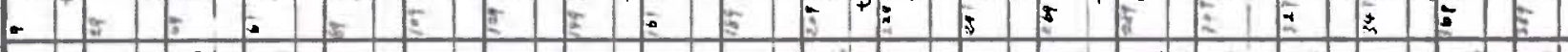

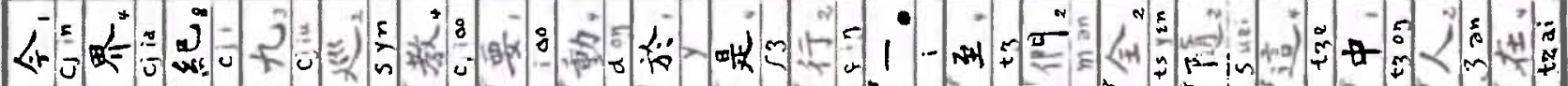

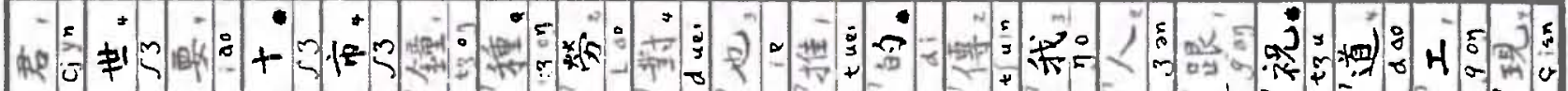

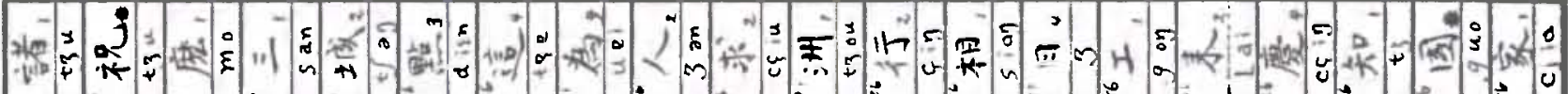
+h

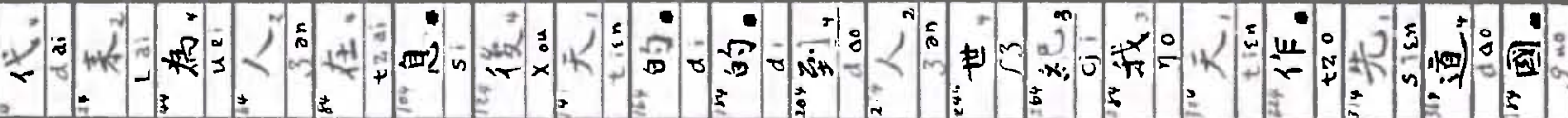

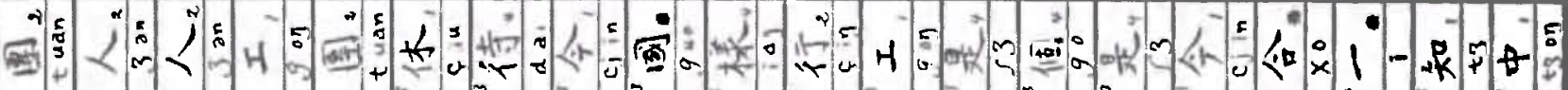

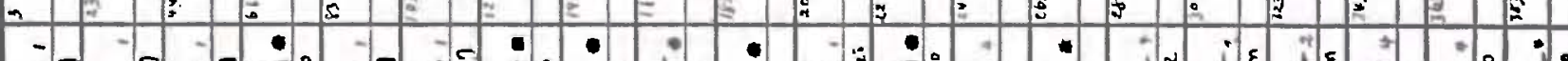
H

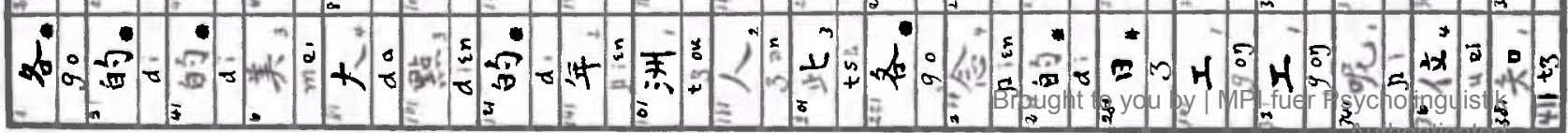




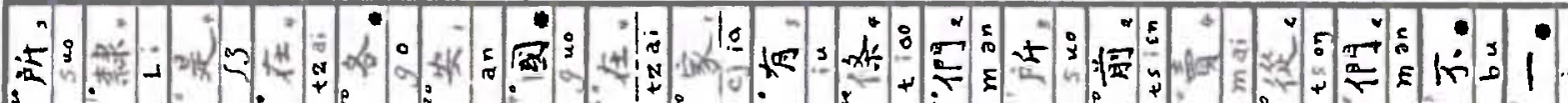
$\$$

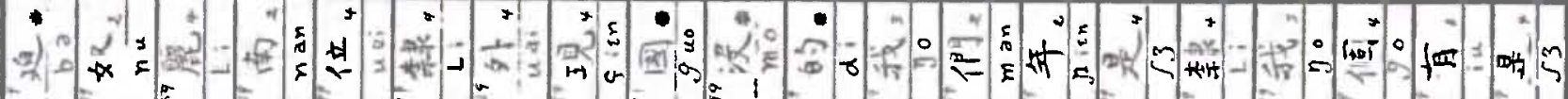

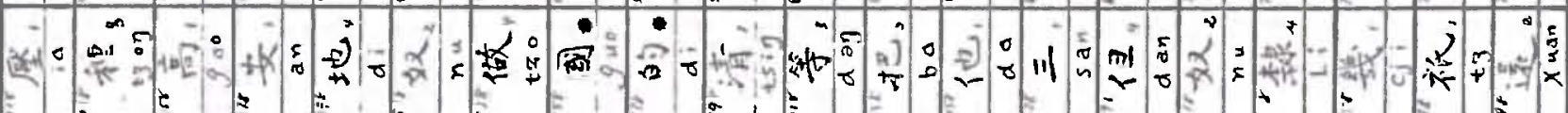
-

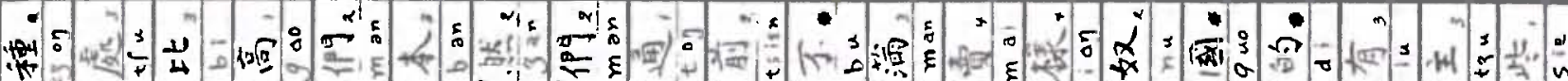

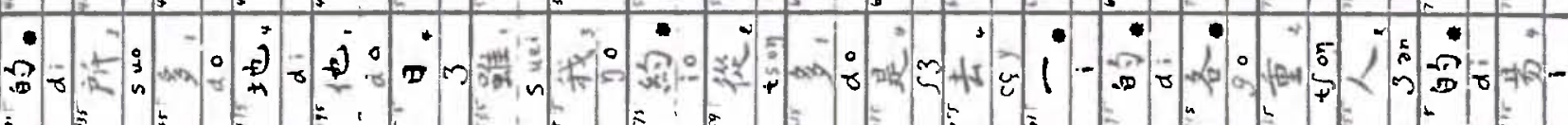

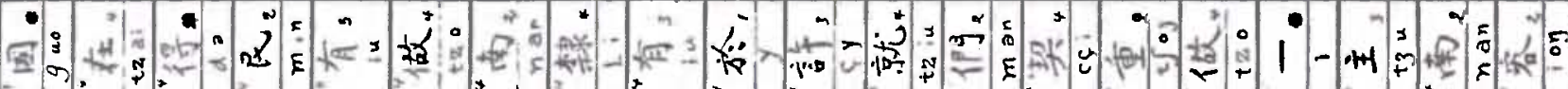

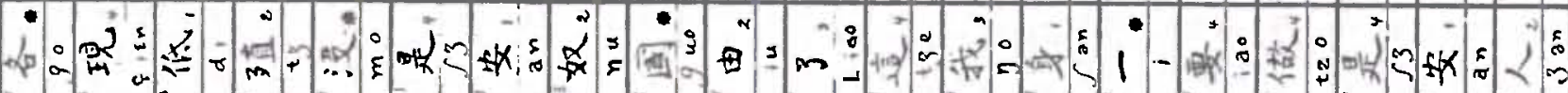

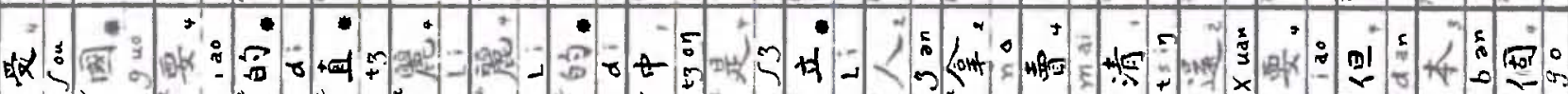
弓冫

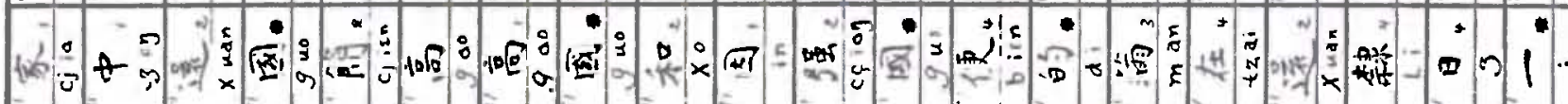

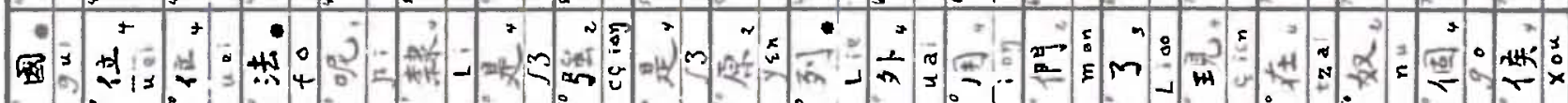
$\because \quad$

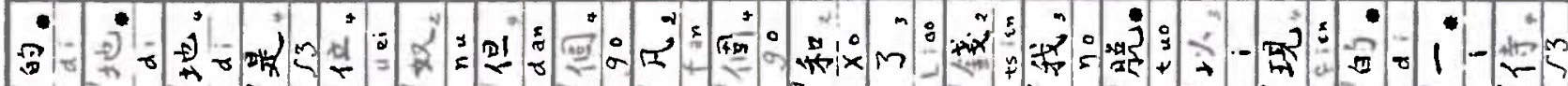

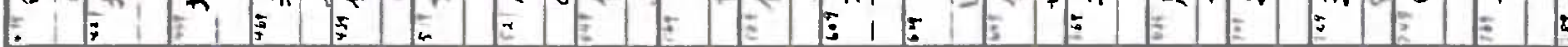

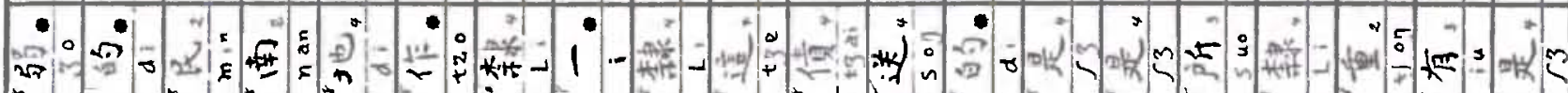
: 5 :

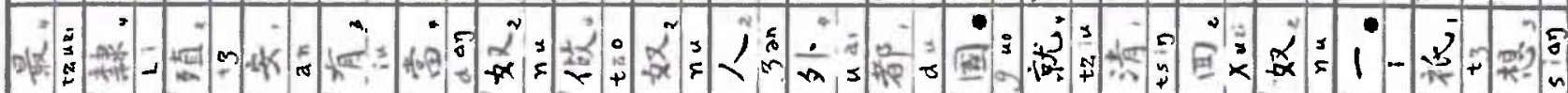

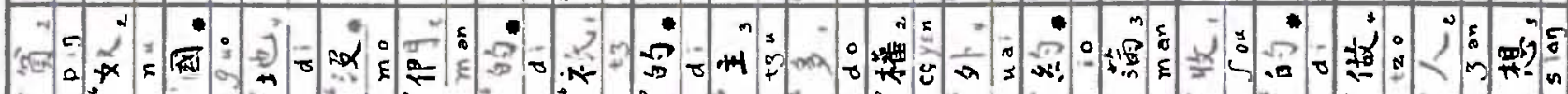

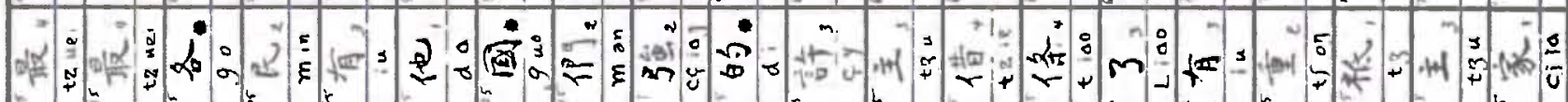

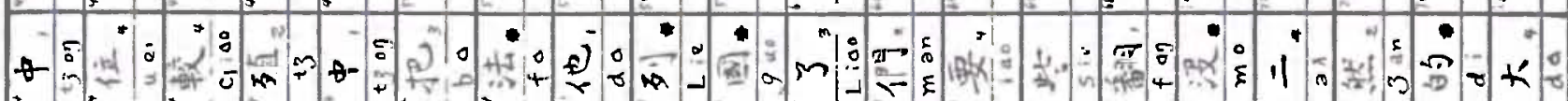

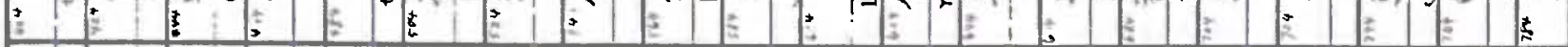

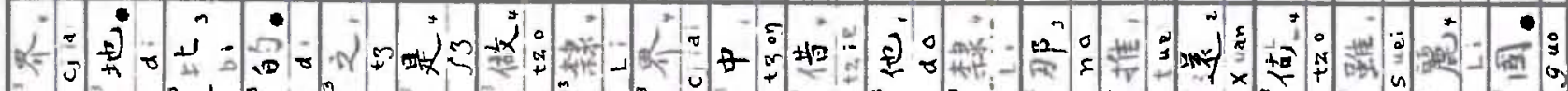

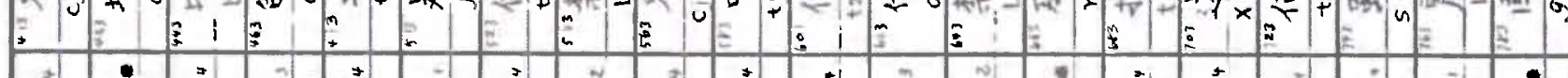

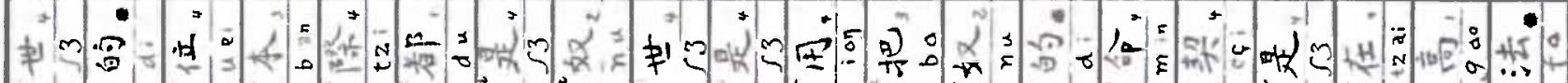

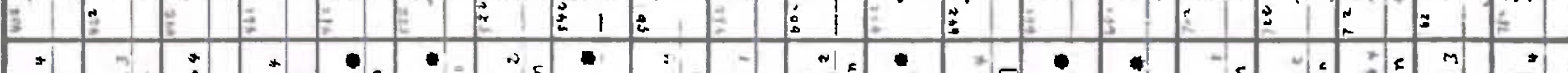

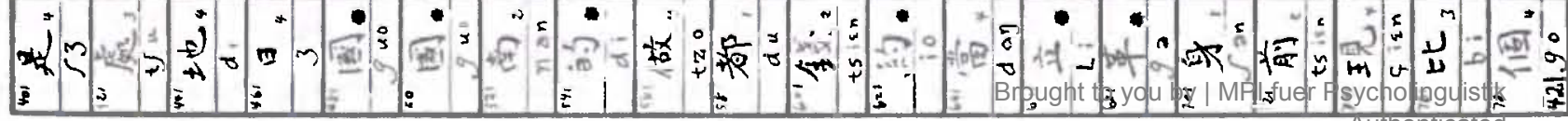




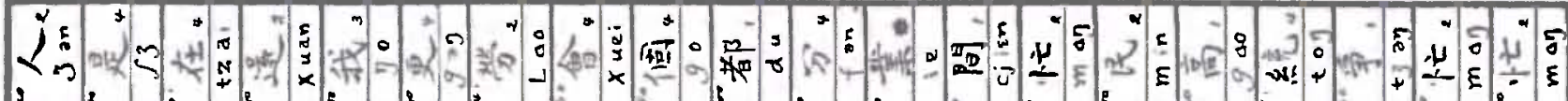

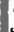

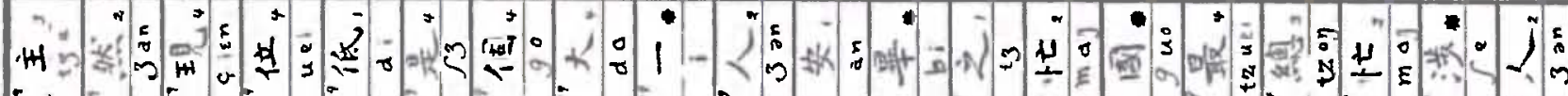
EIE E E E a E a E E

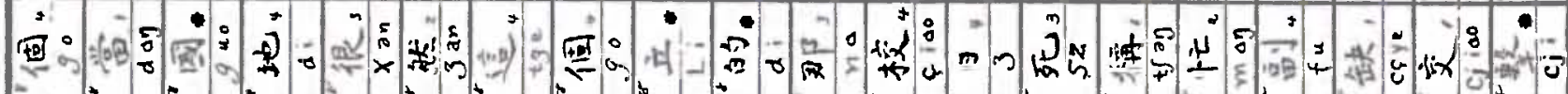

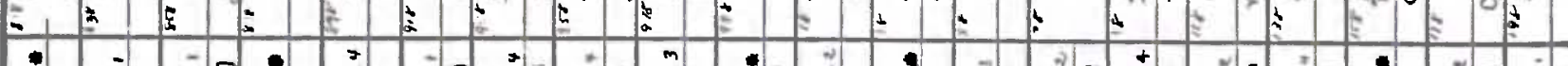

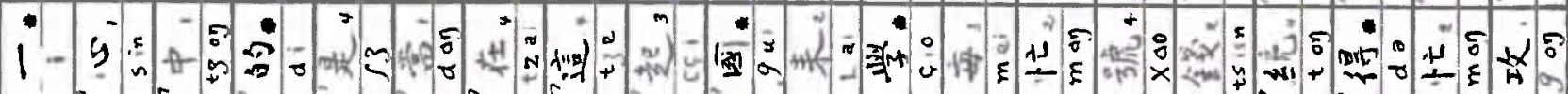

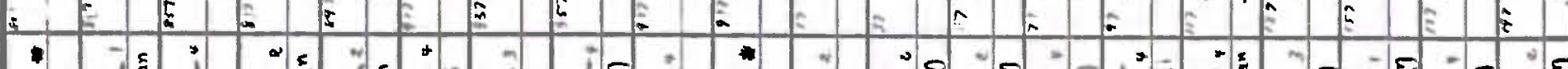

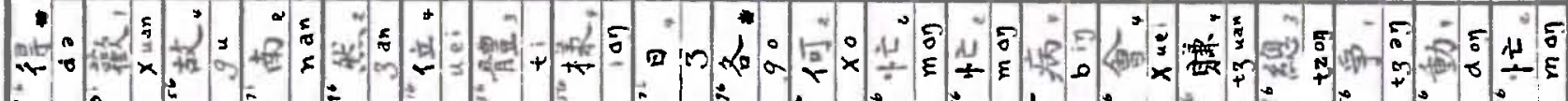

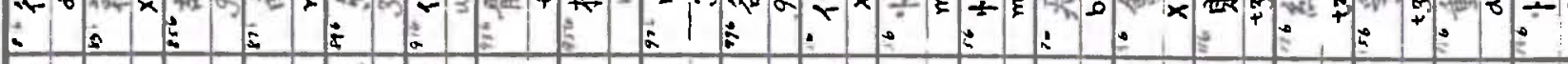

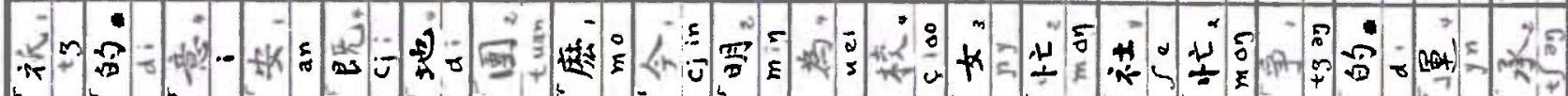

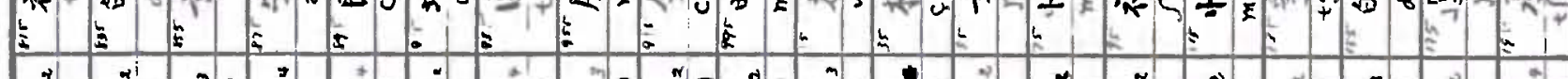

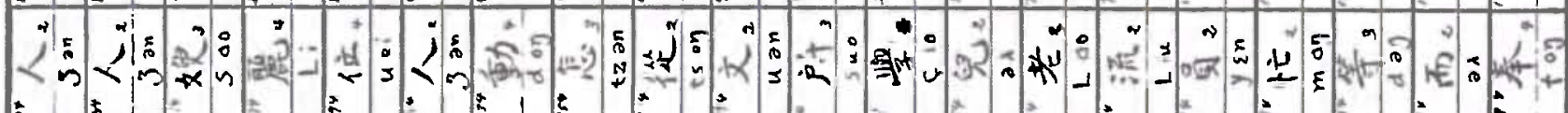

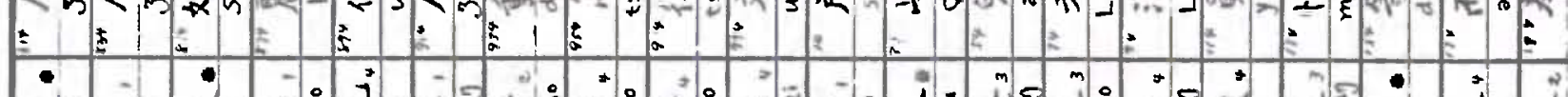

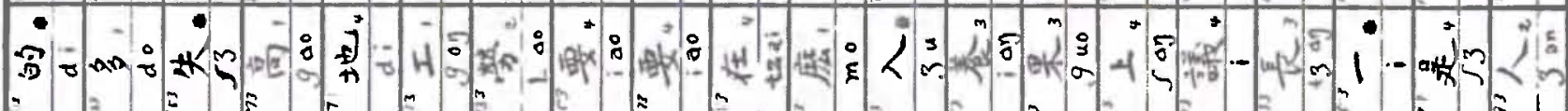

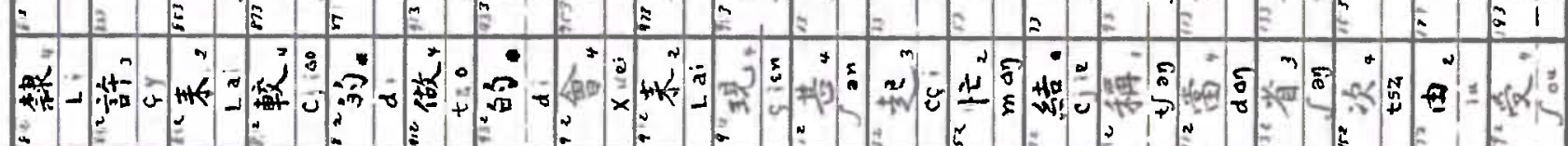

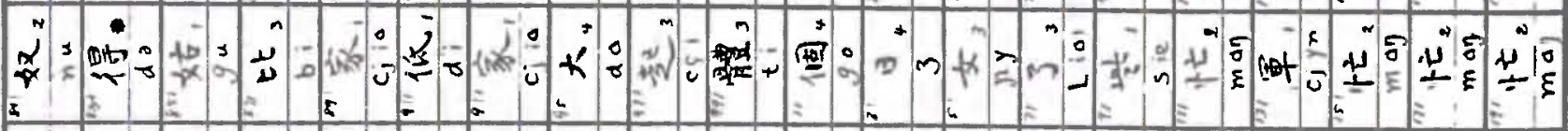

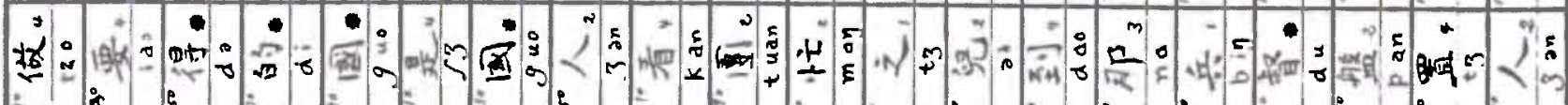

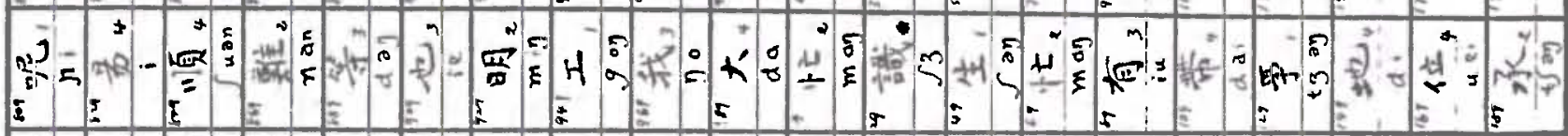

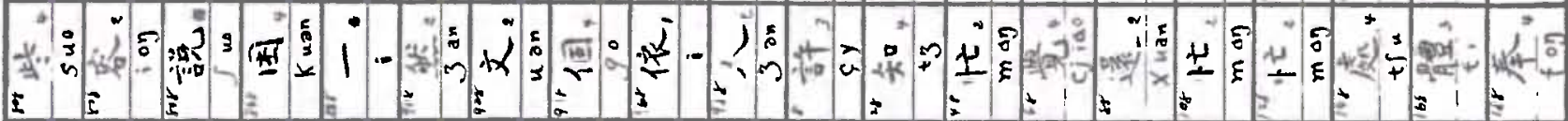

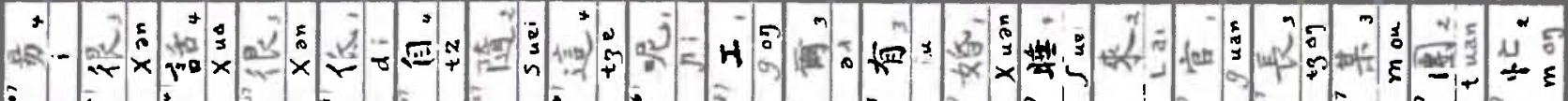

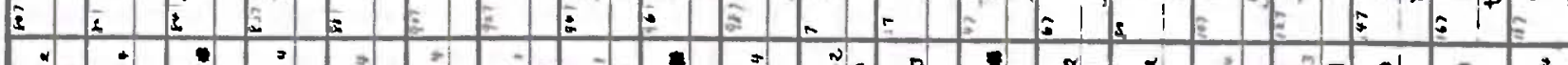

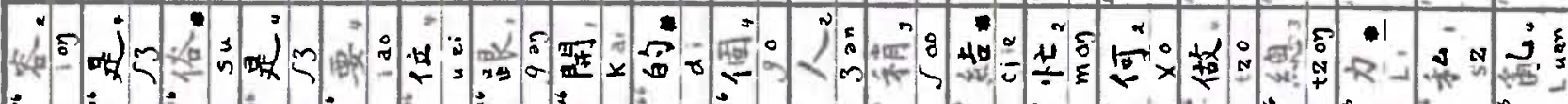

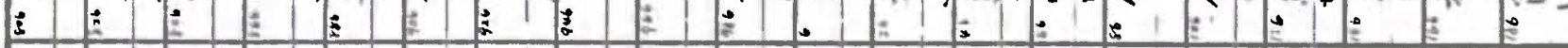
र $\begin{aligned} & 5 \\ & 0 \\ & 0\end{aligned}$

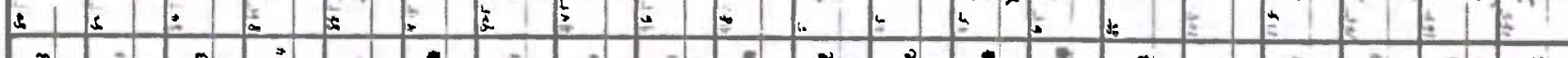

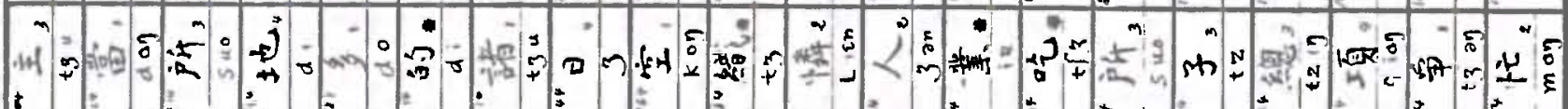

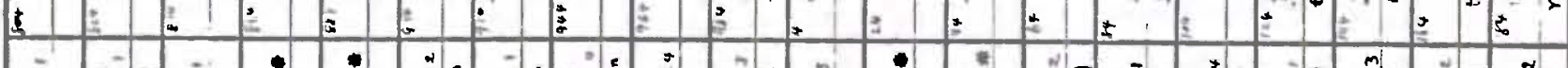

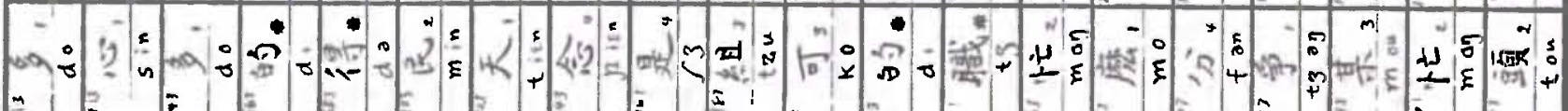

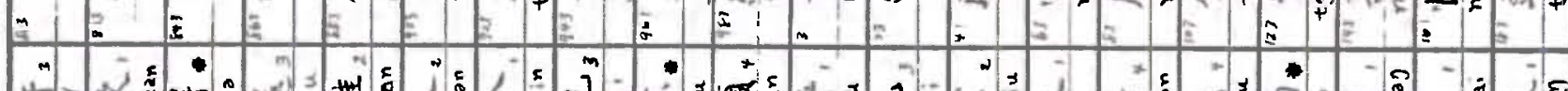

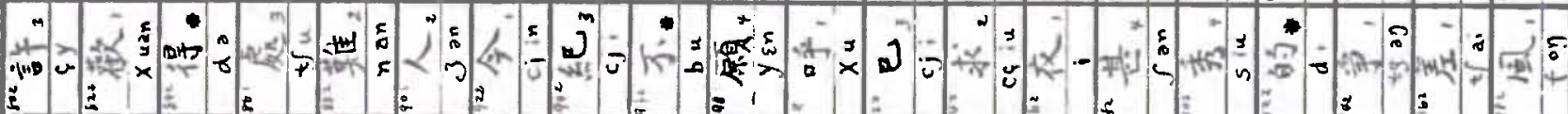
- 


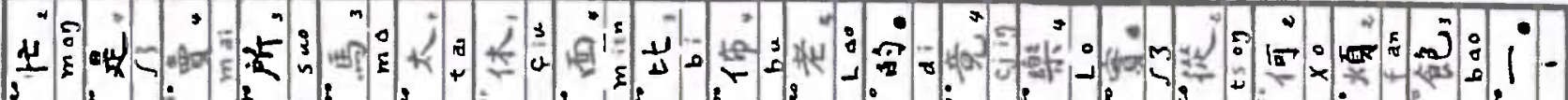

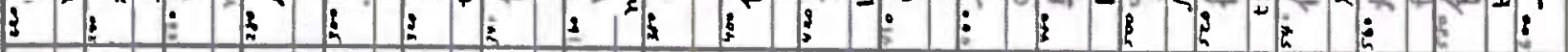

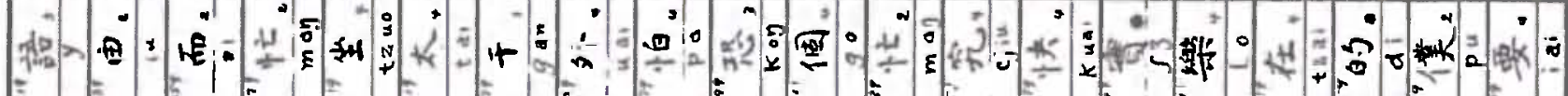
W

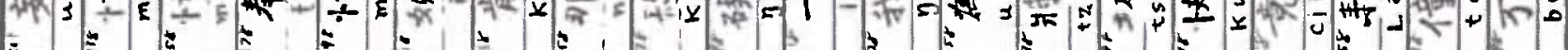

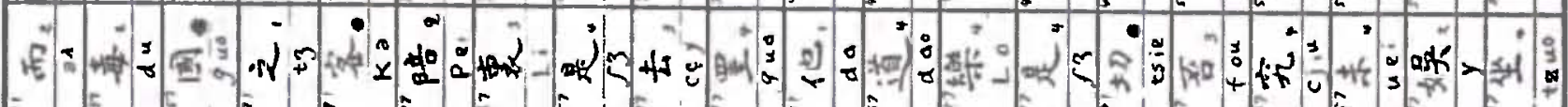

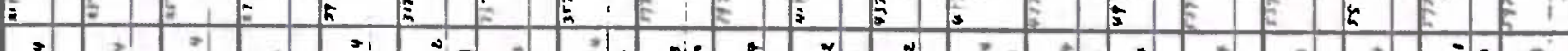

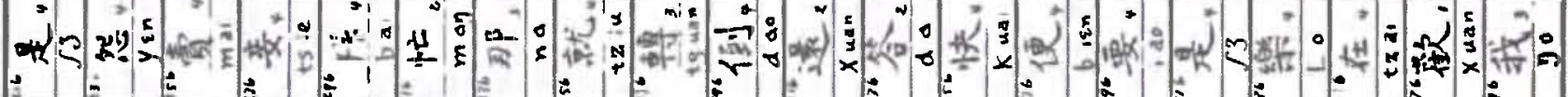
E) 3 L

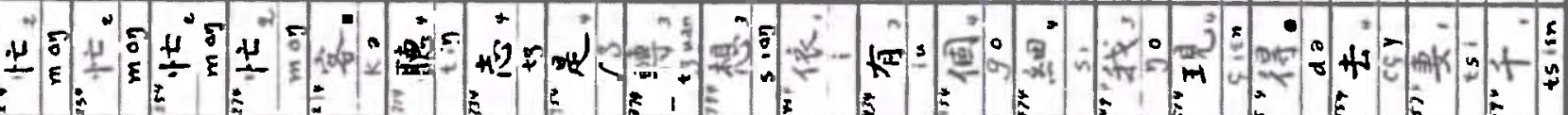

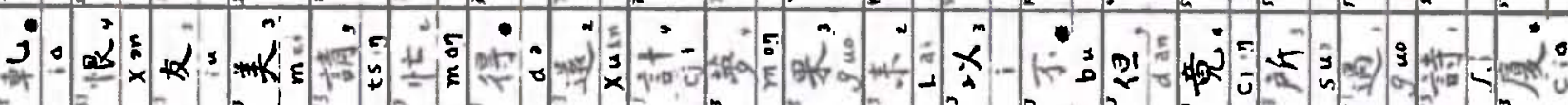

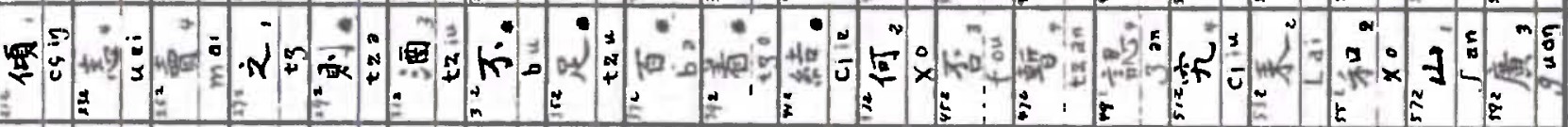

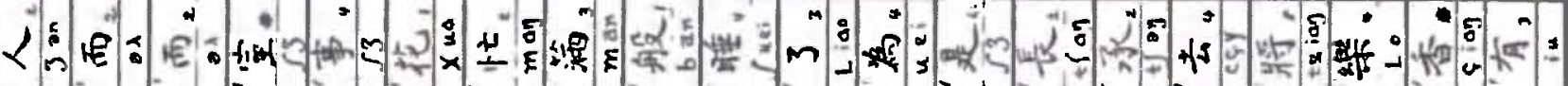

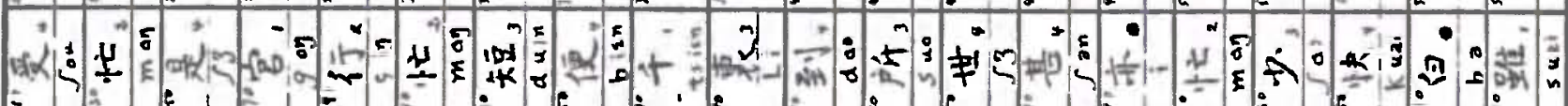

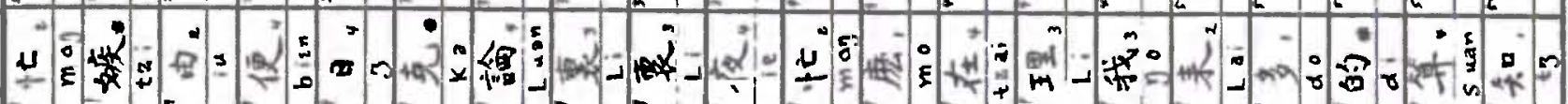

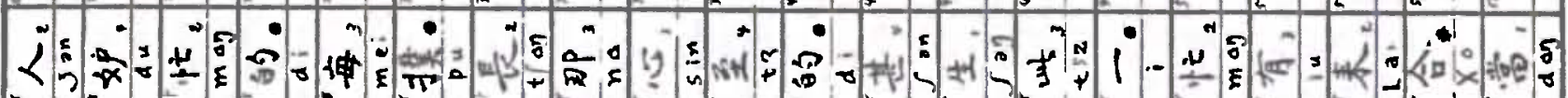

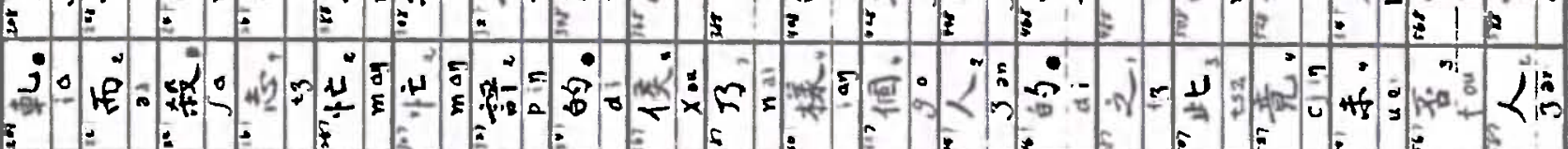

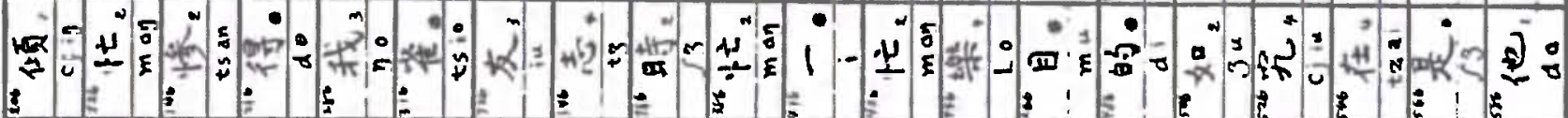

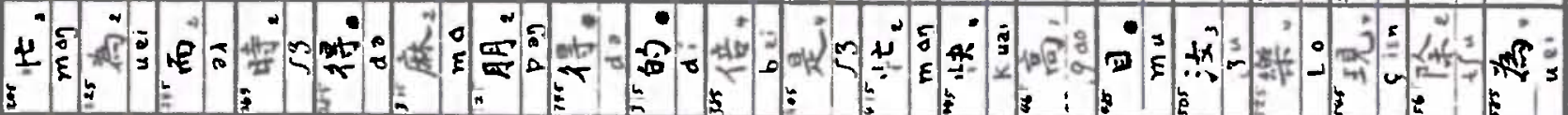

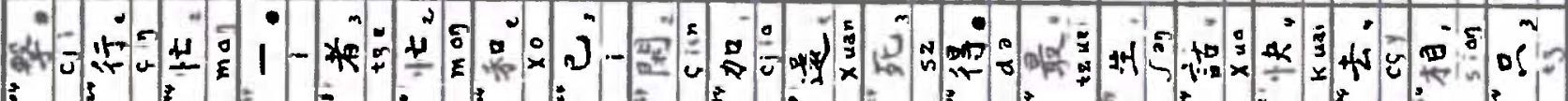

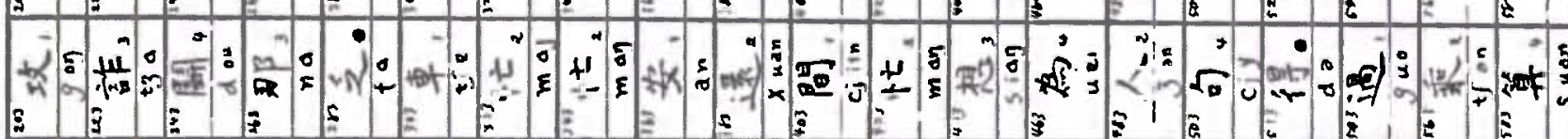

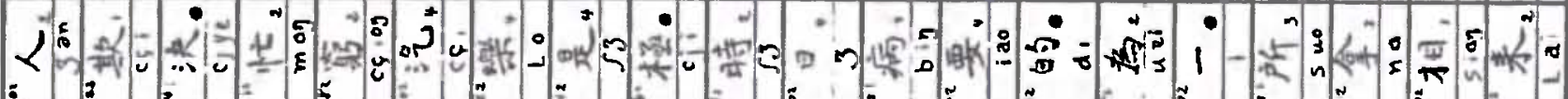

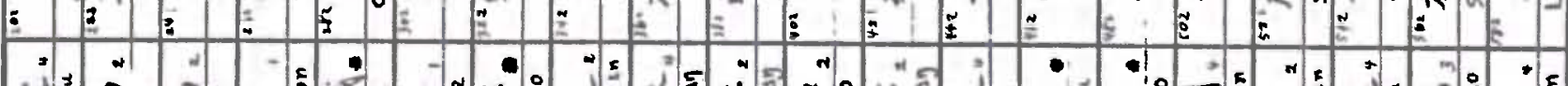

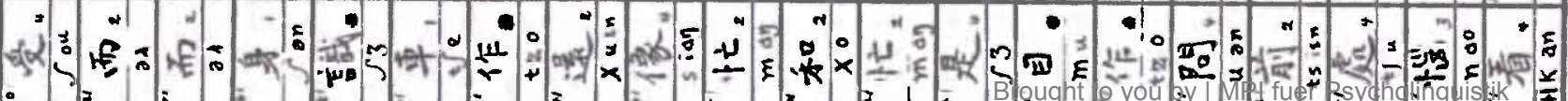




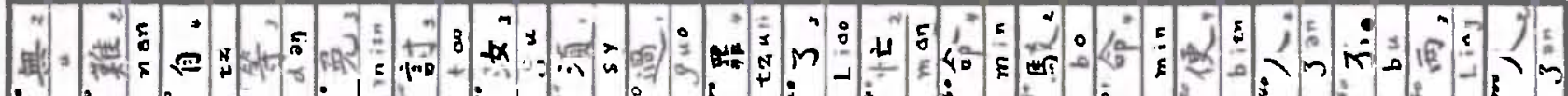

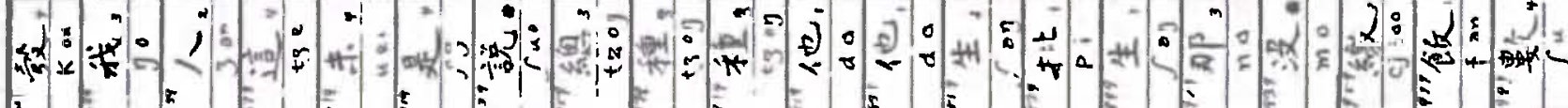

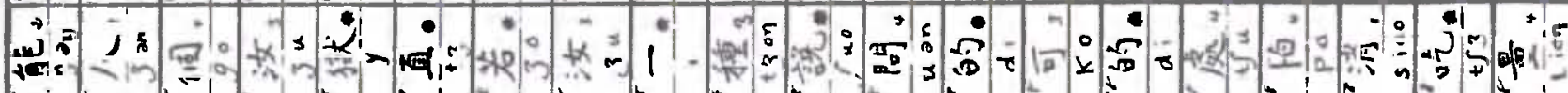

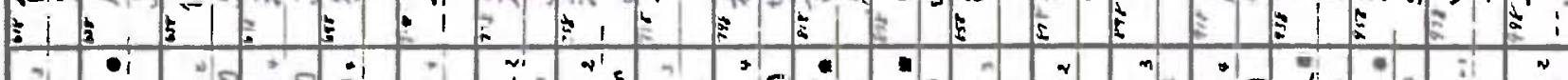

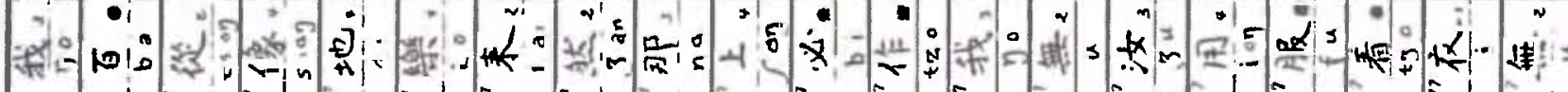

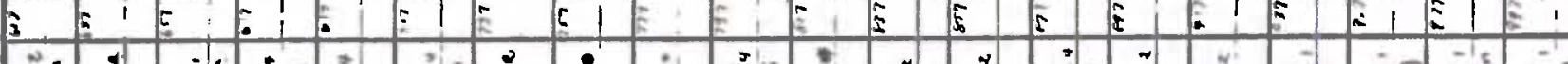

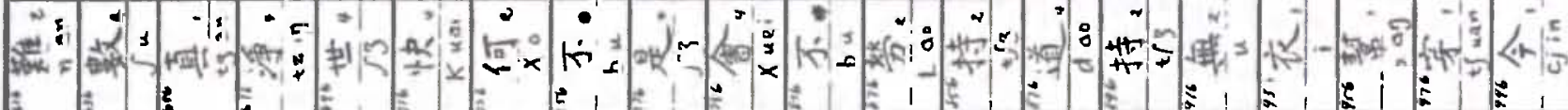

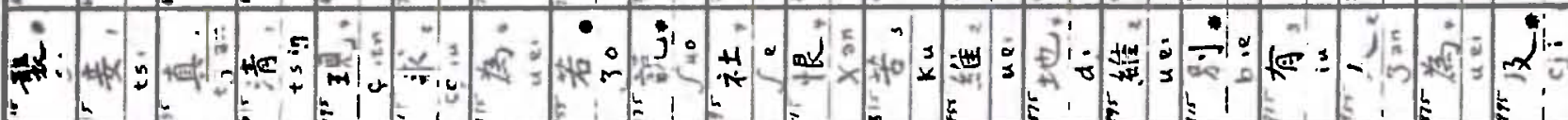
+

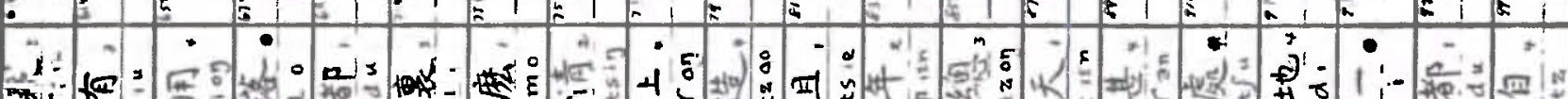

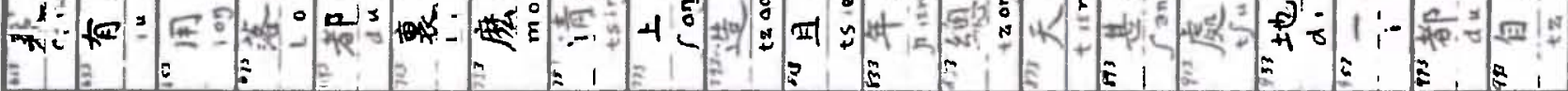
Nov

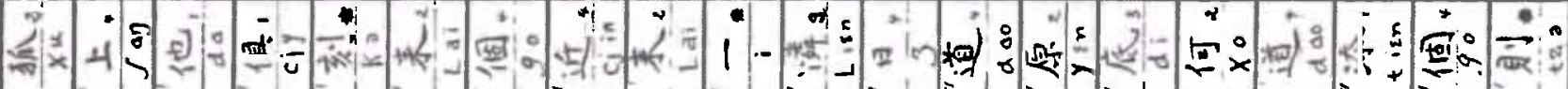

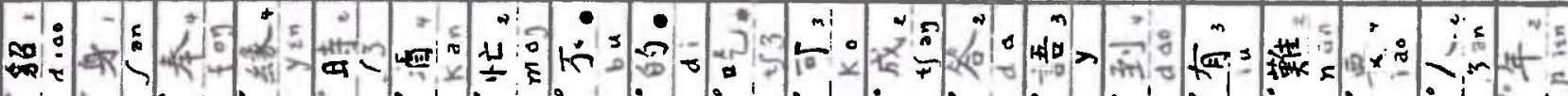

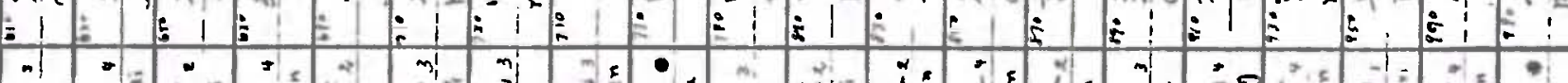

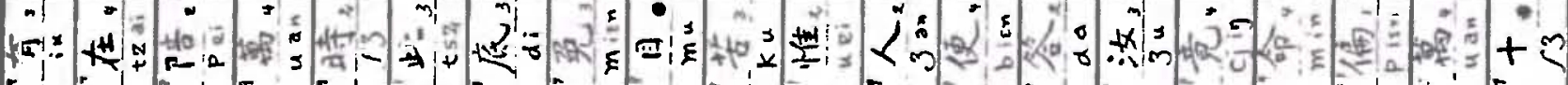

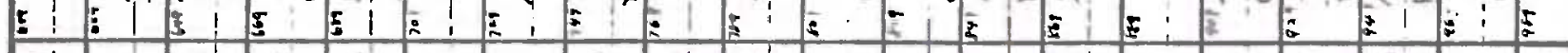

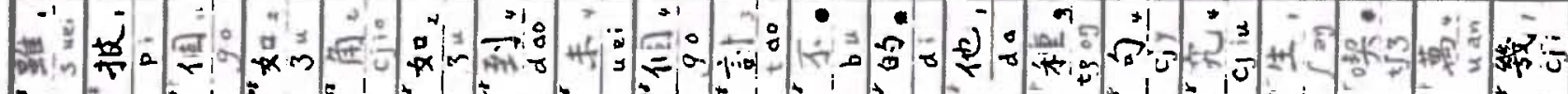

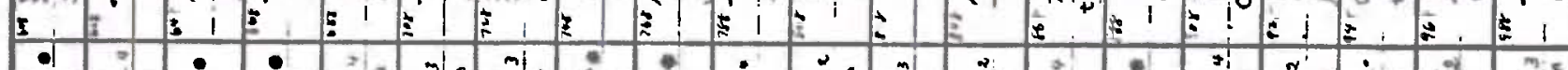

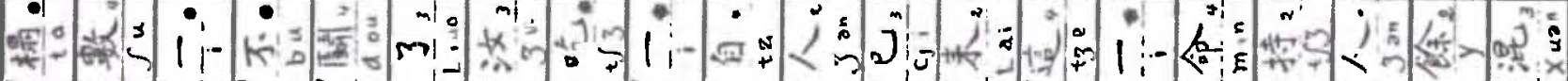

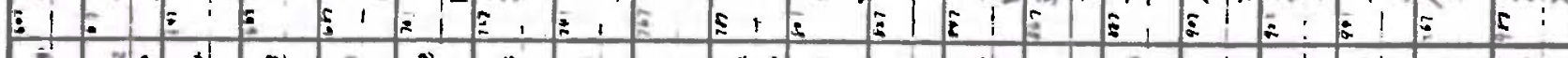

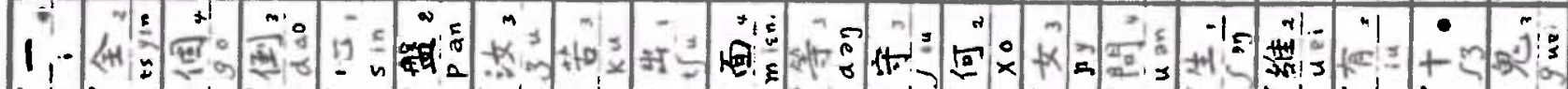

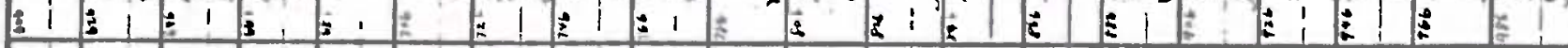
(4)

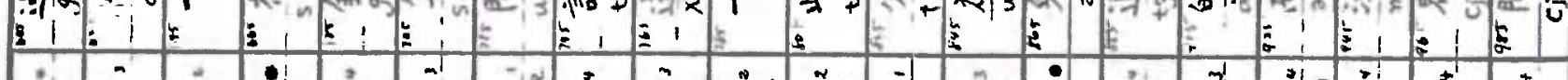

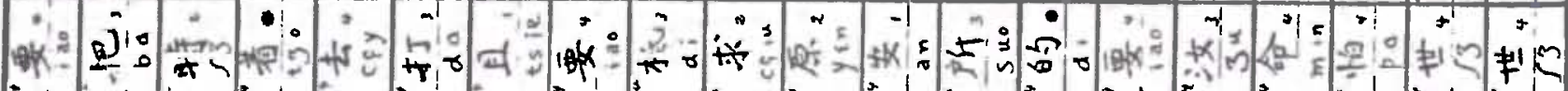

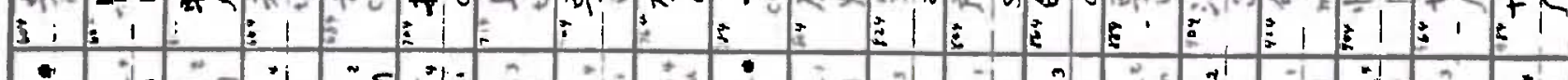

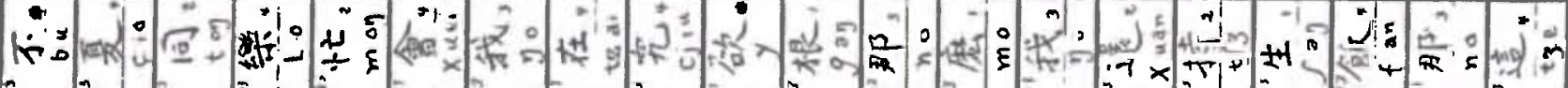

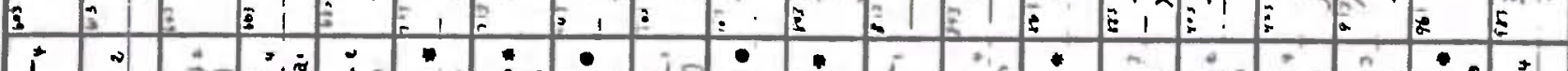

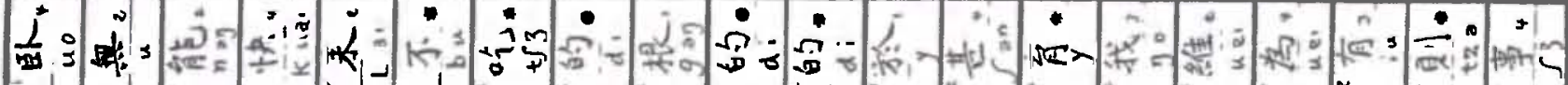

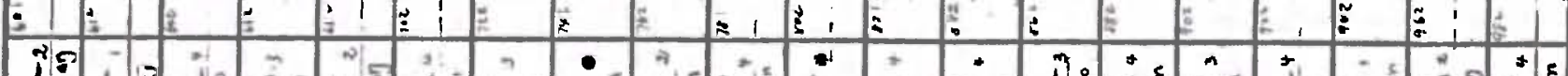

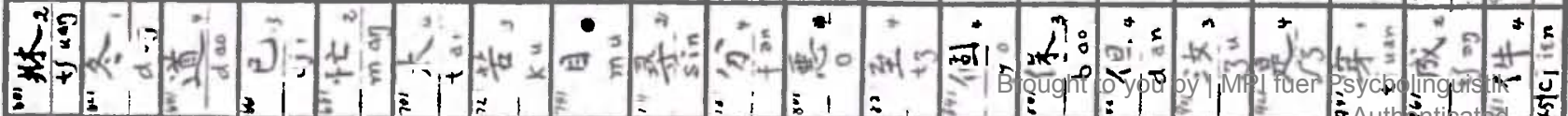




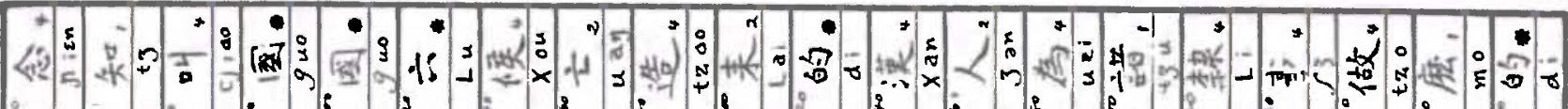

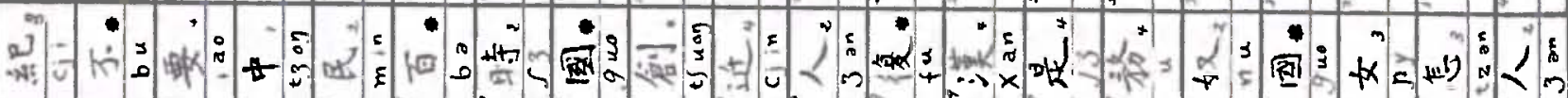

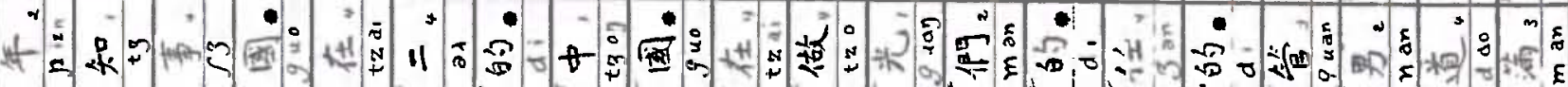

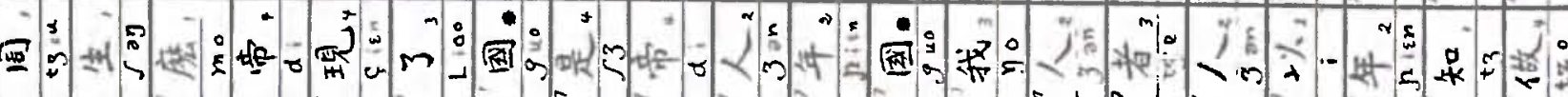
X)

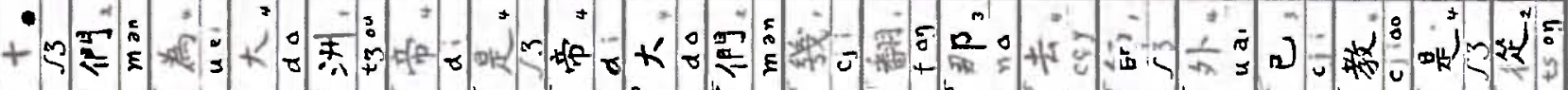

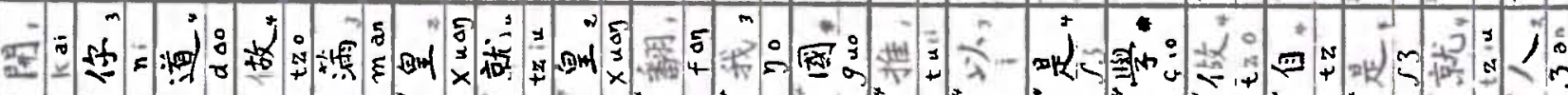

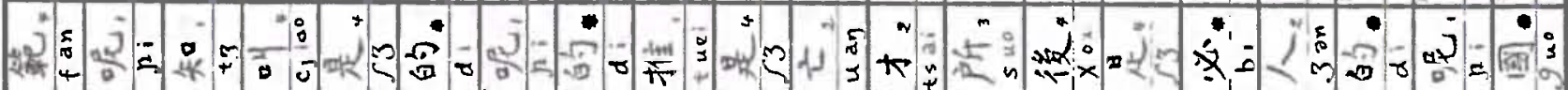

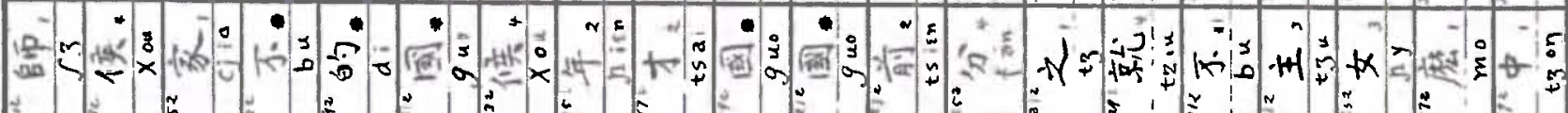

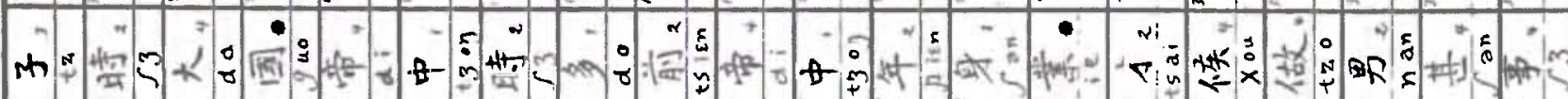

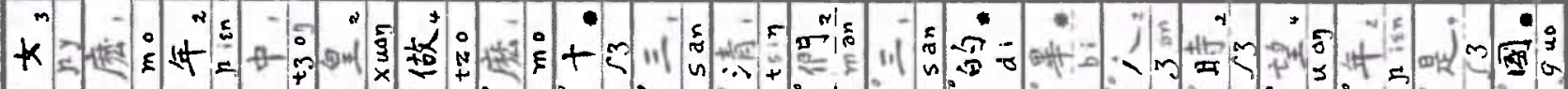

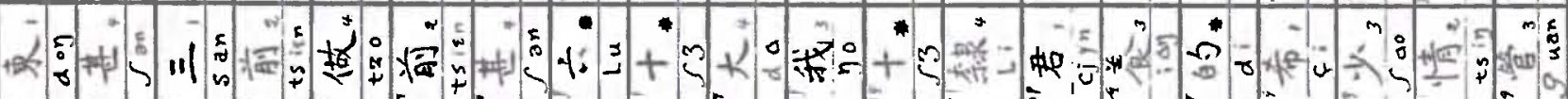

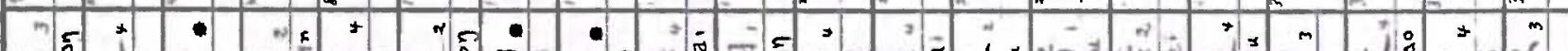

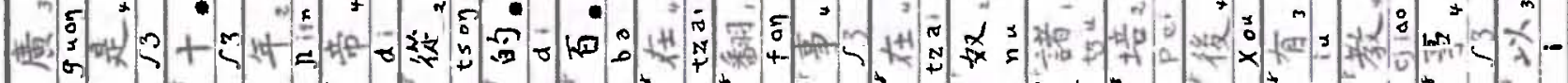

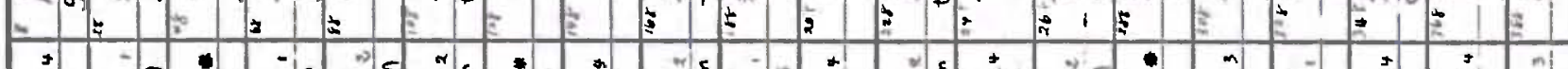

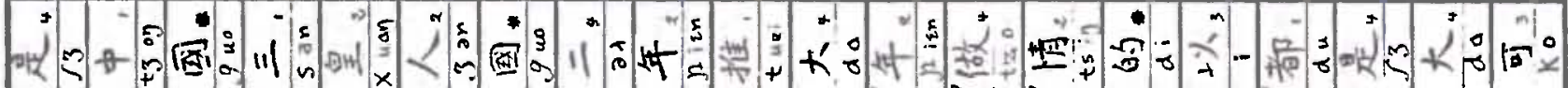

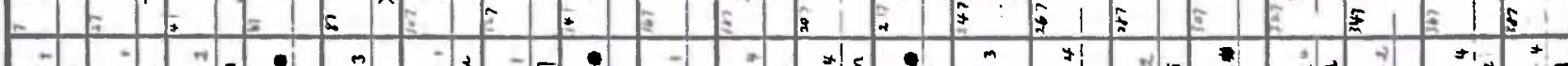
K

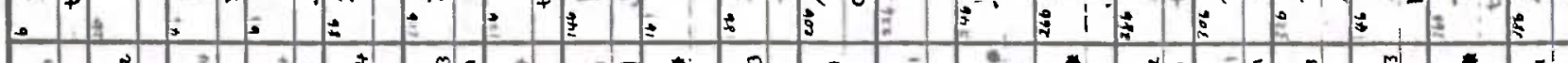

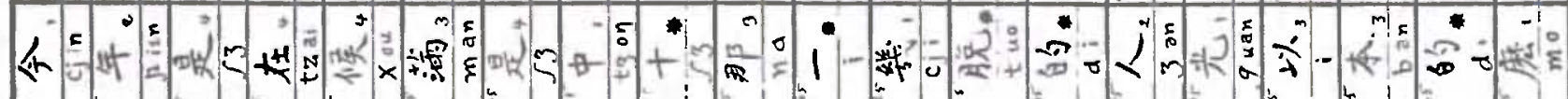

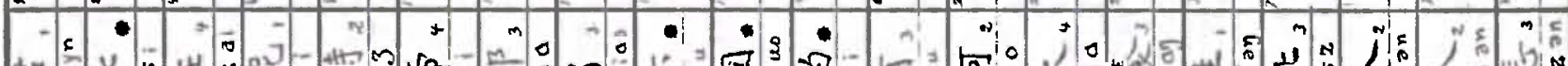

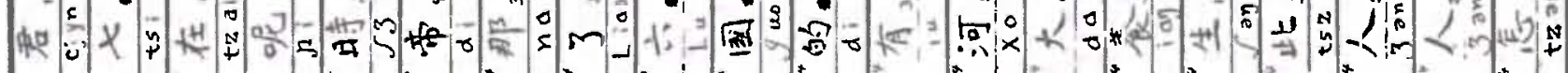

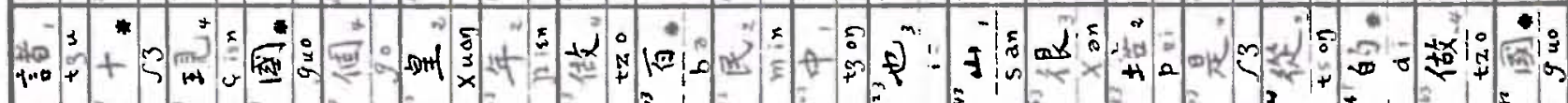

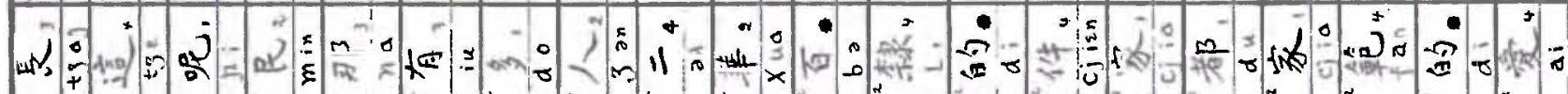
隹 
+

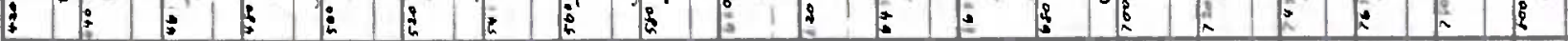

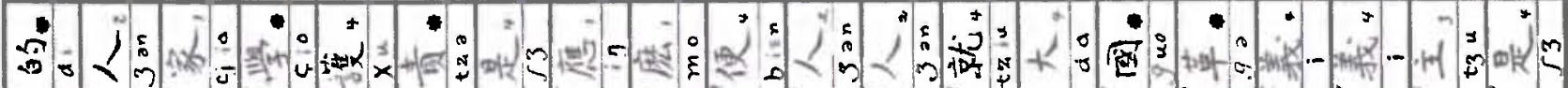

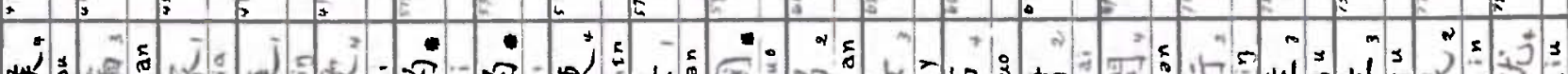

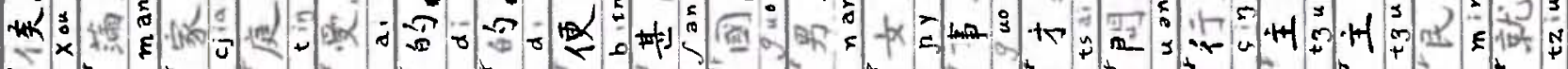

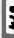

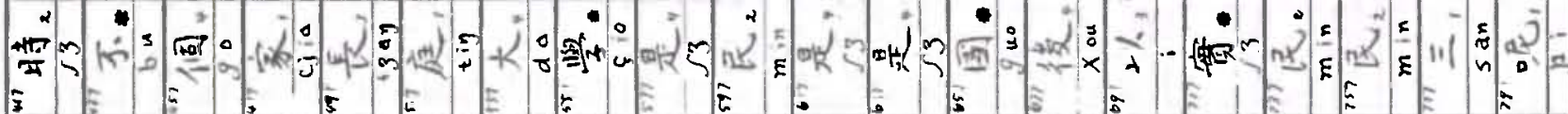

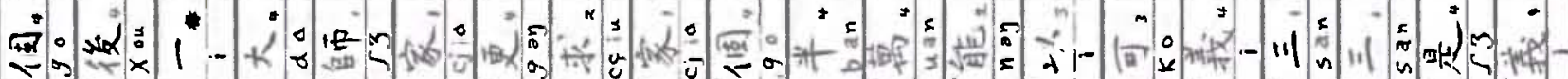

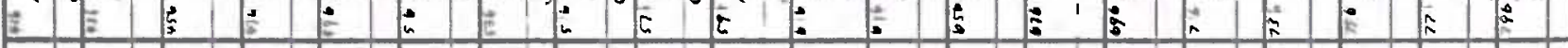

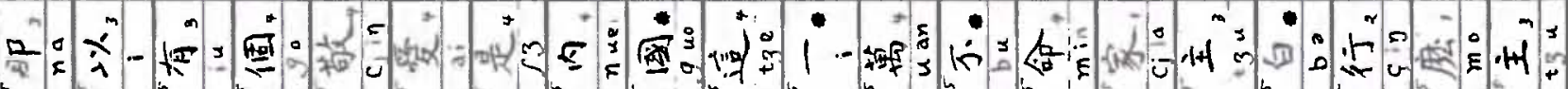

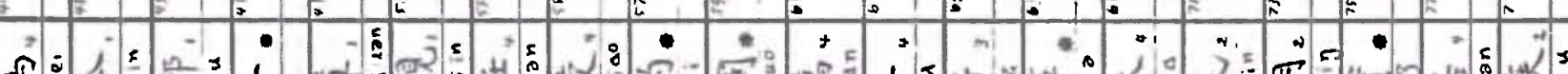

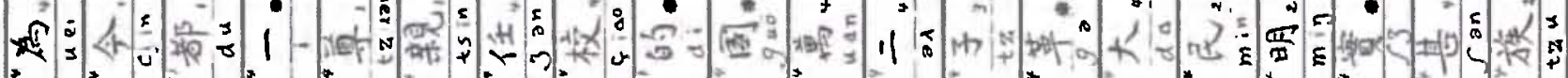

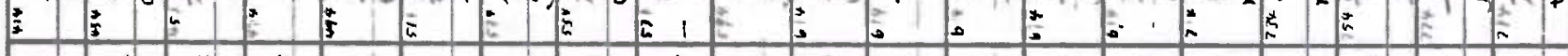

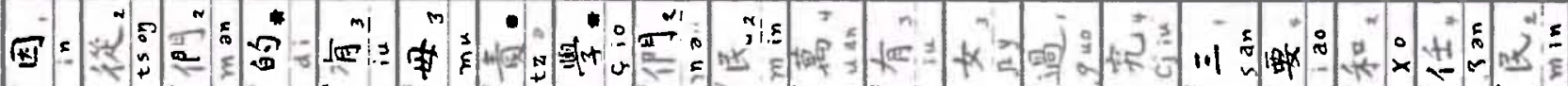

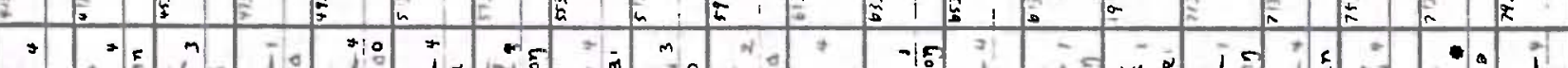

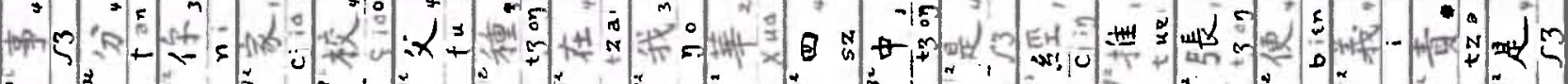

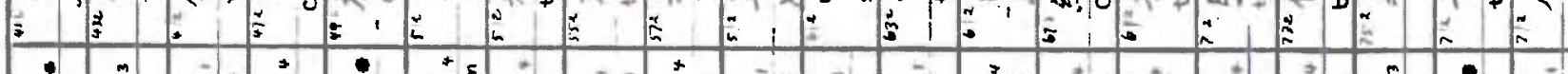

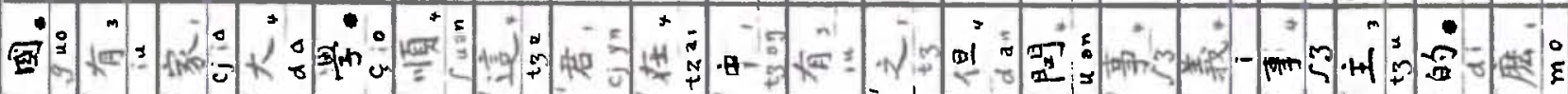

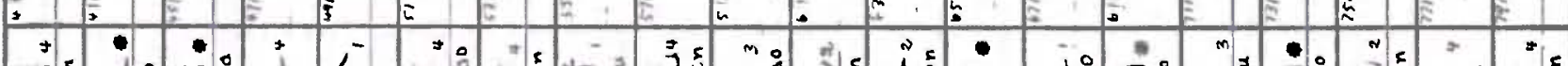

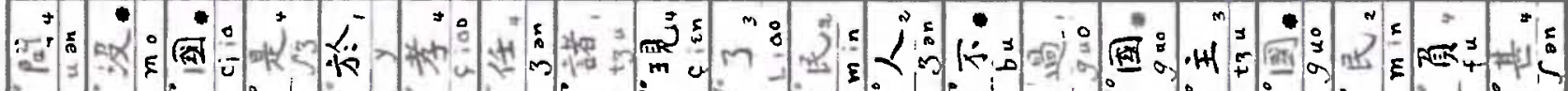

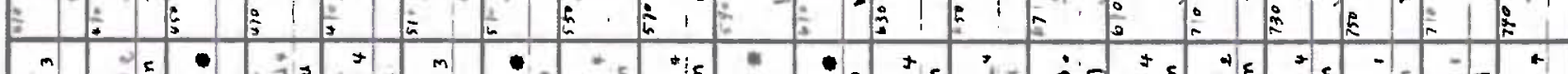

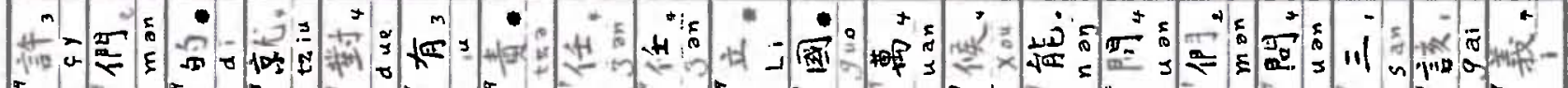

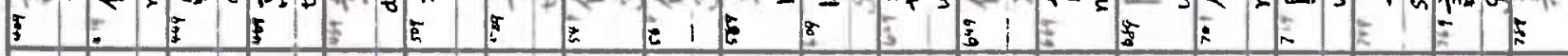
K

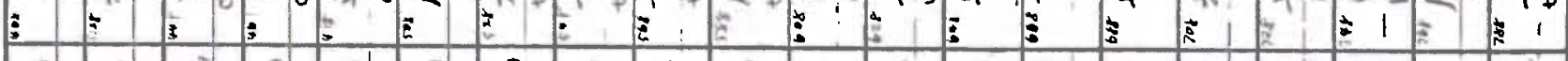

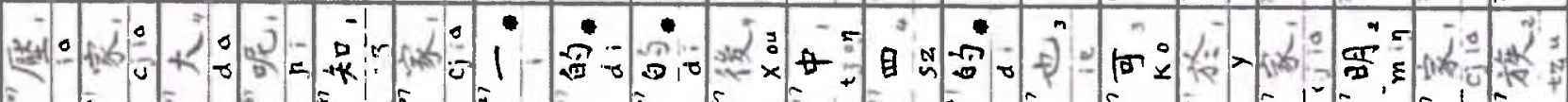

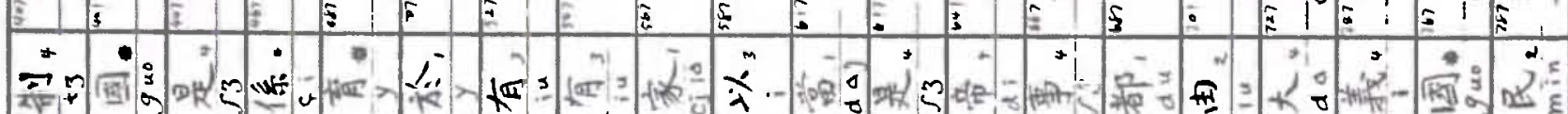

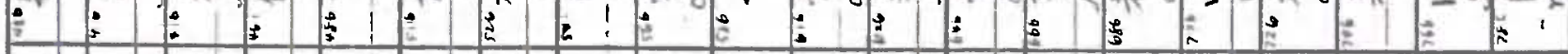

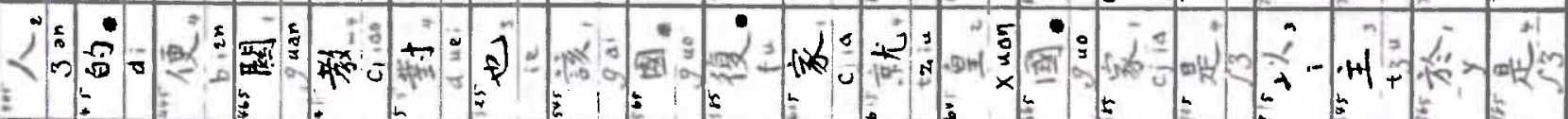

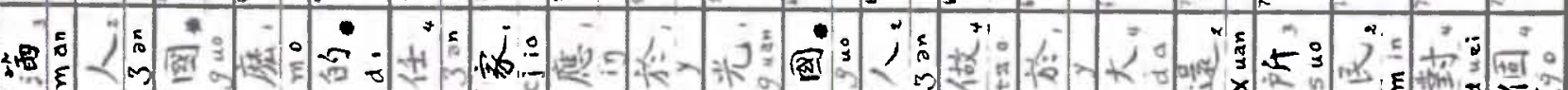
E E

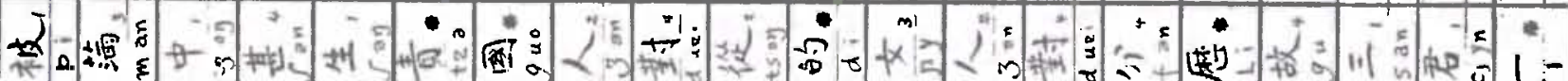

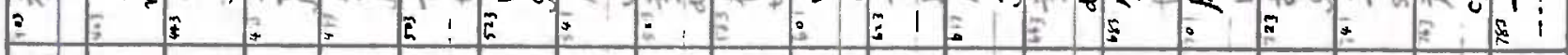

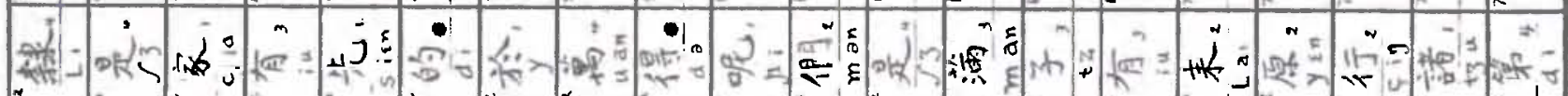

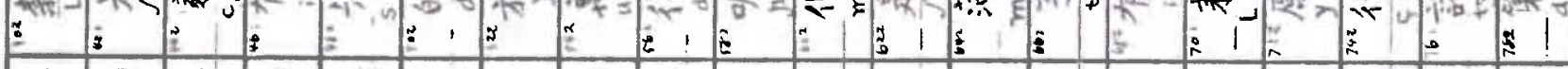

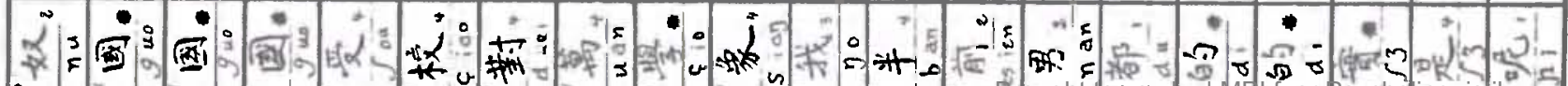

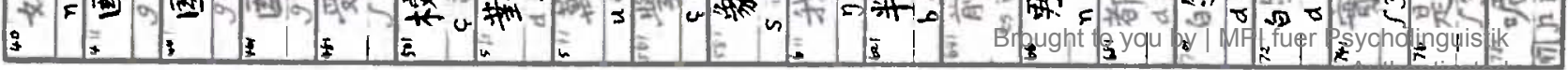




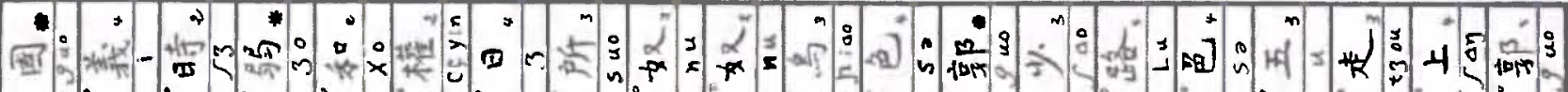

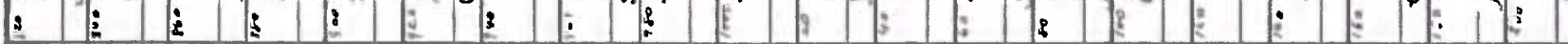
些党 $=5 \mathrm{~s}=\mathrm{E}$

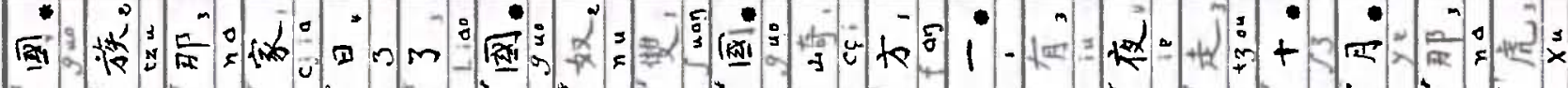

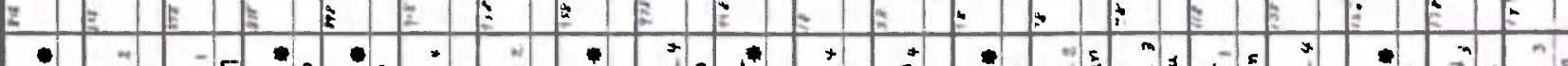

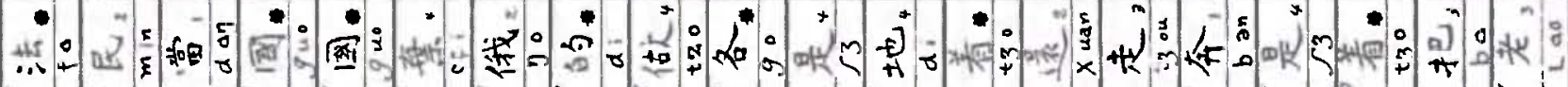

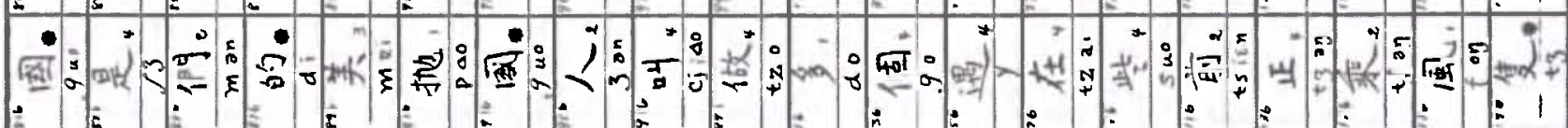

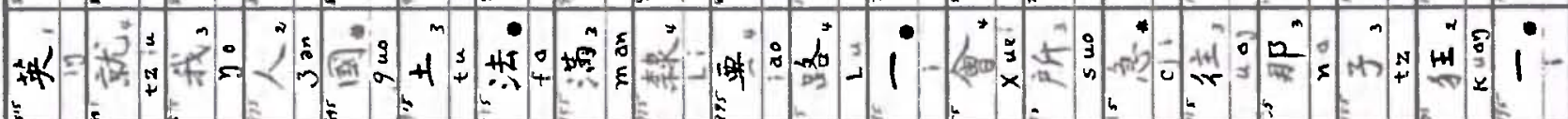

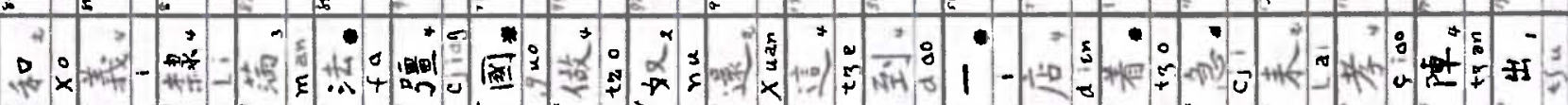
*

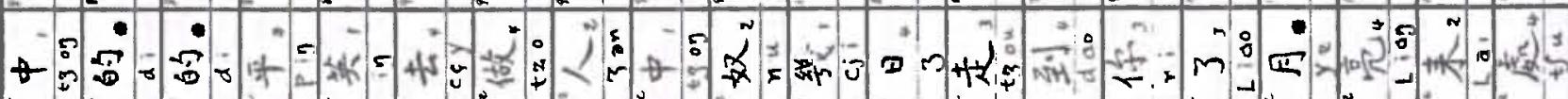

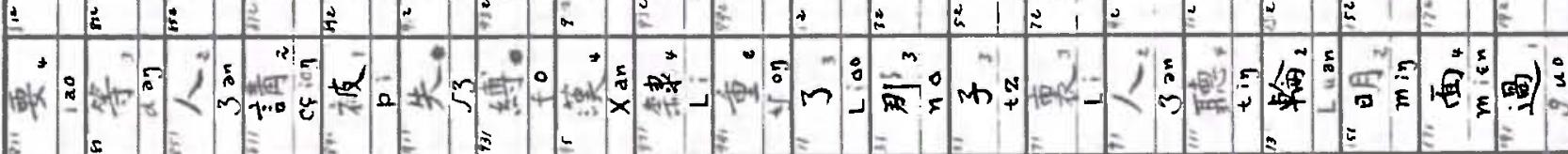

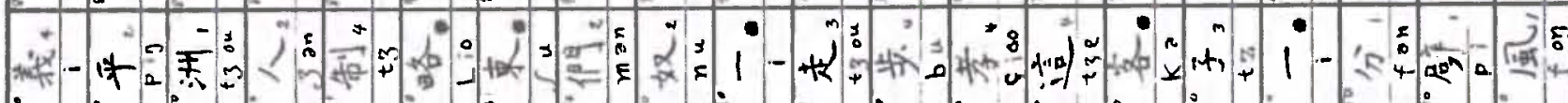

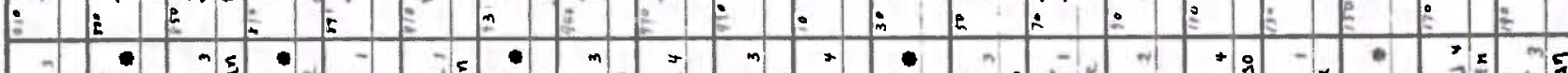
H.

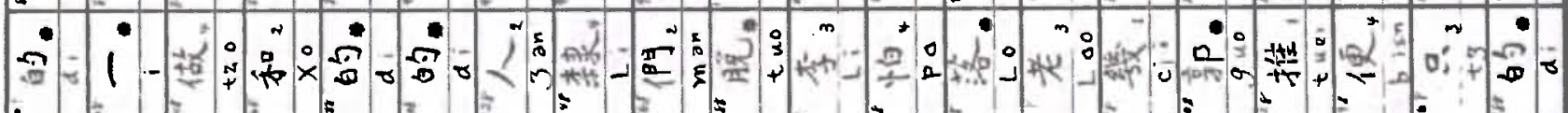

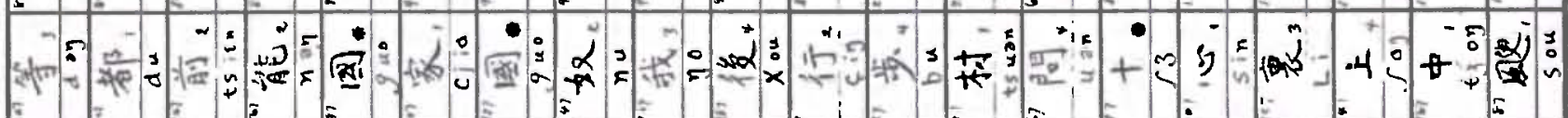

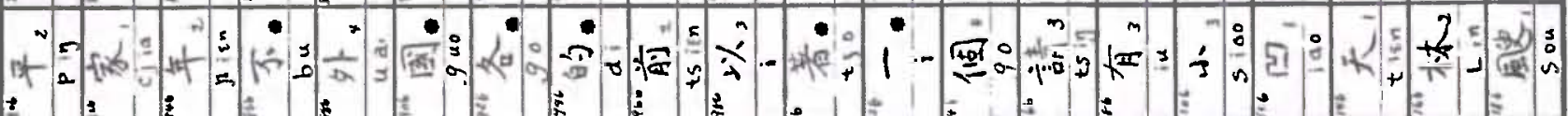

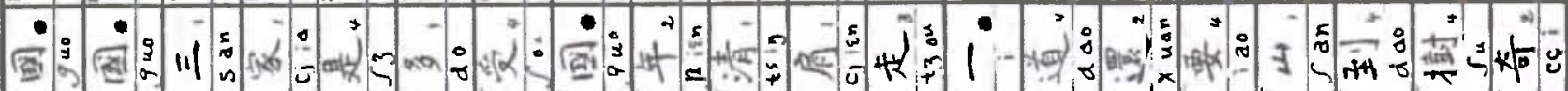

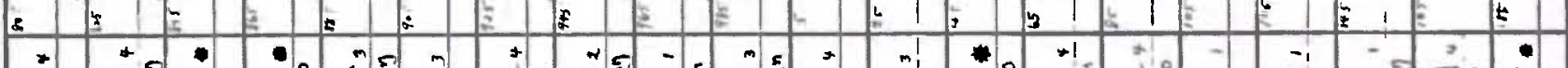

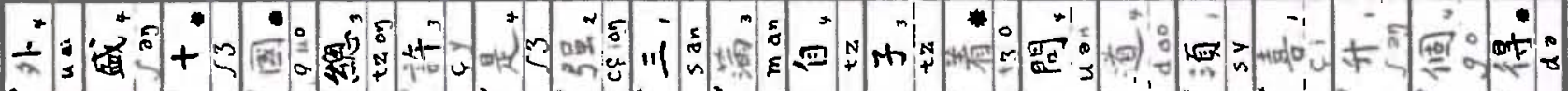

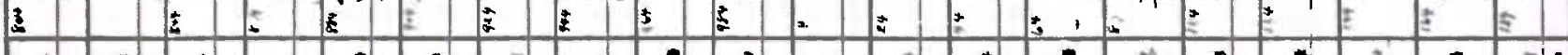

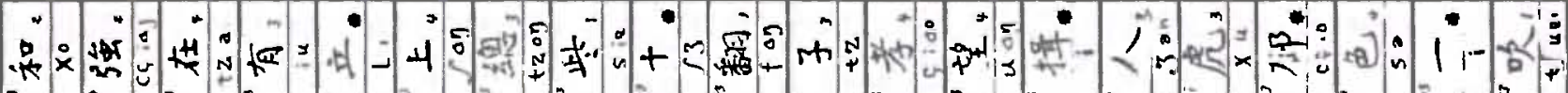

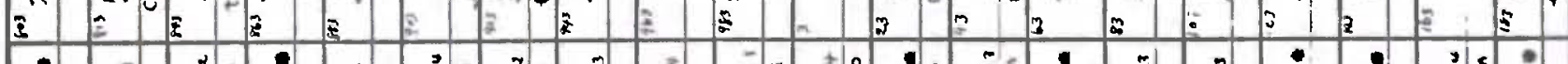

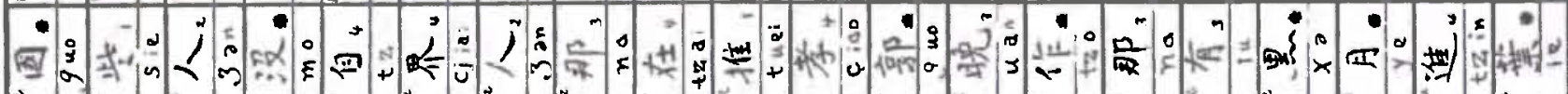

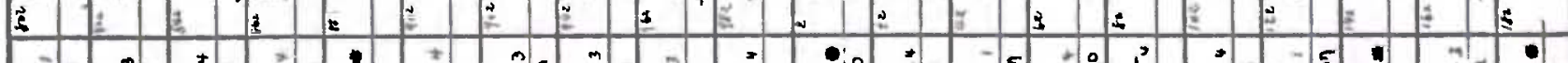

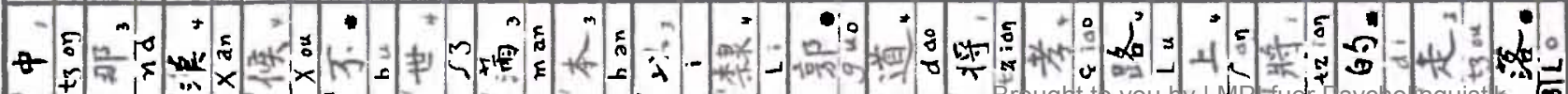

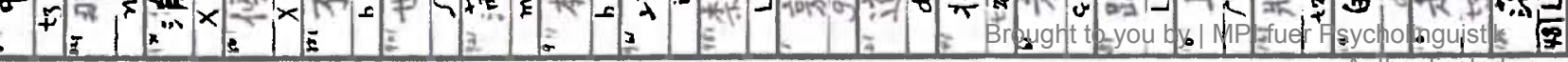




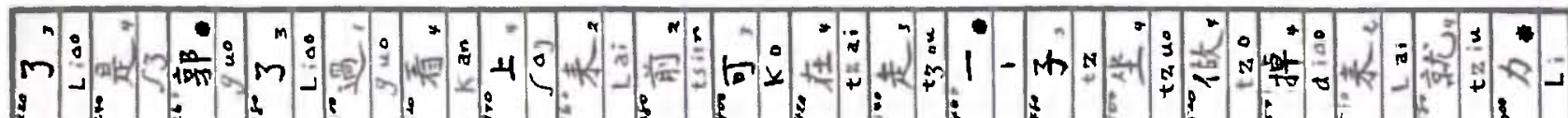

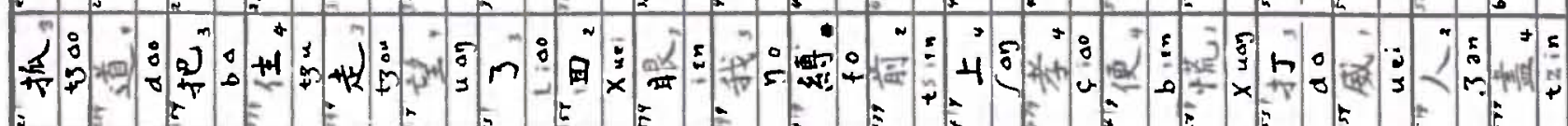

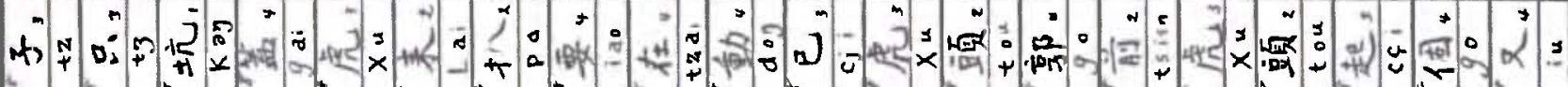

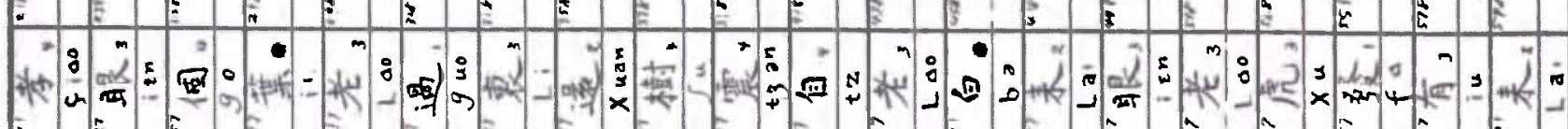

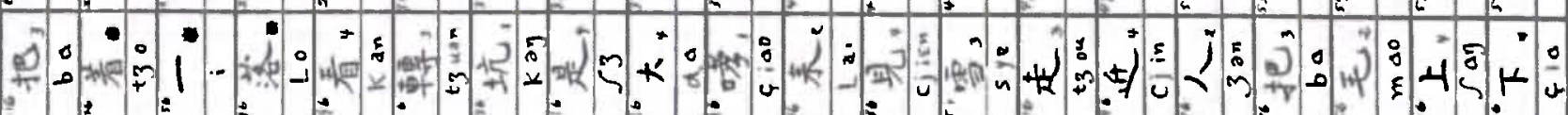

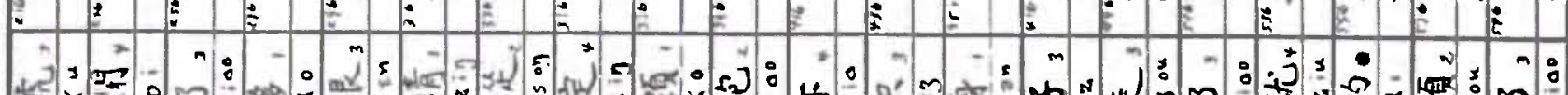

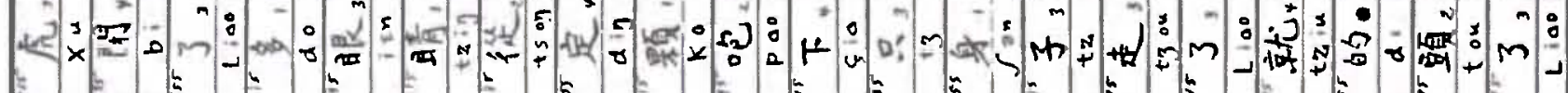

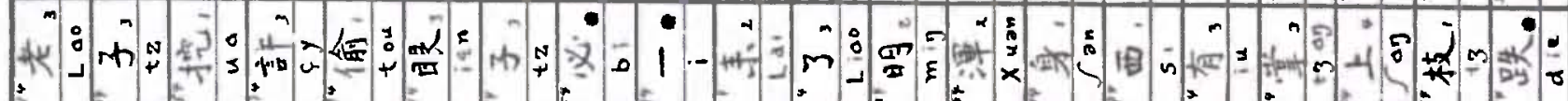

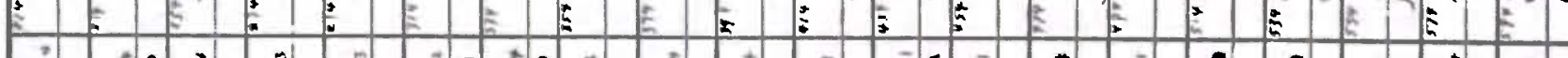

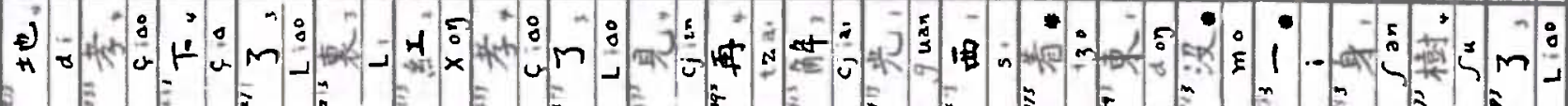

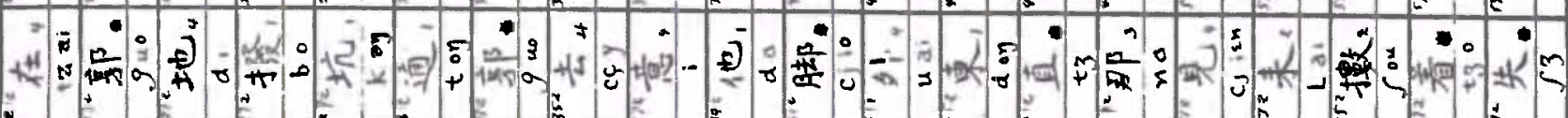

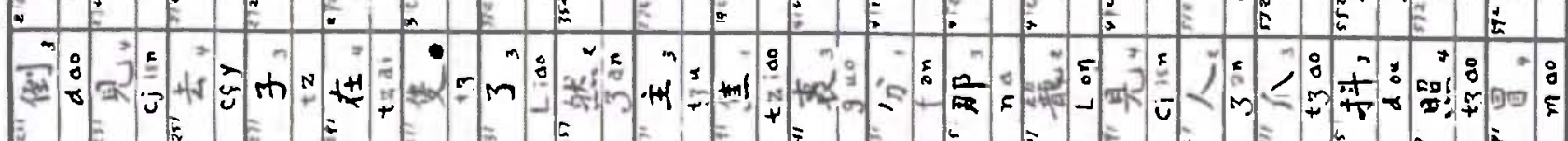

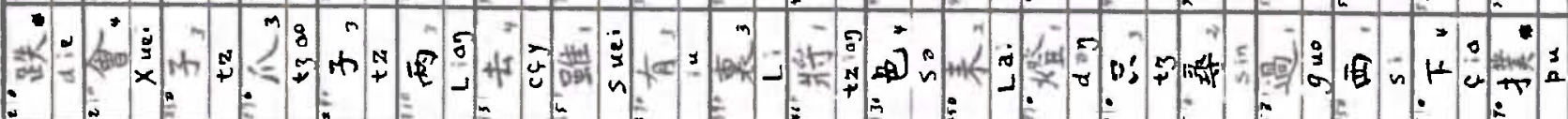

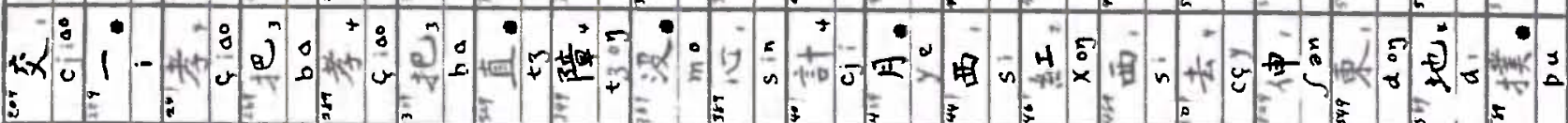

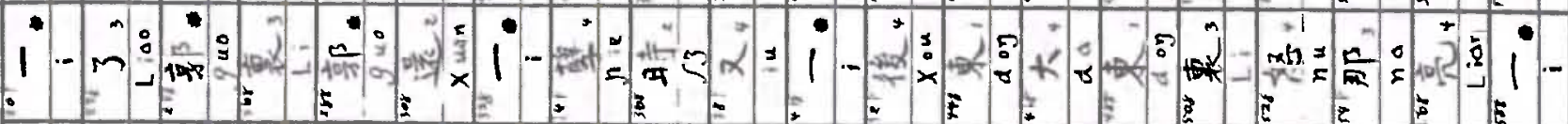

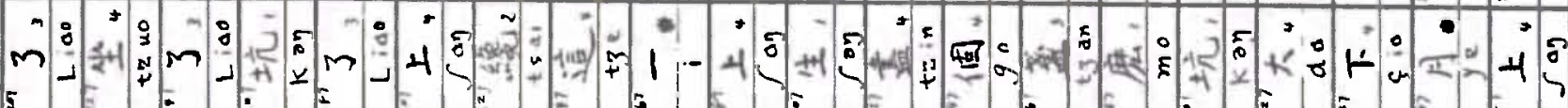

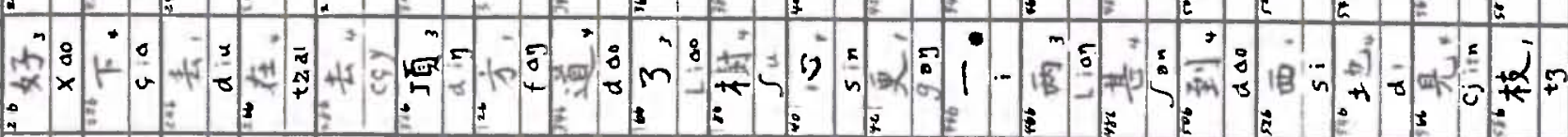

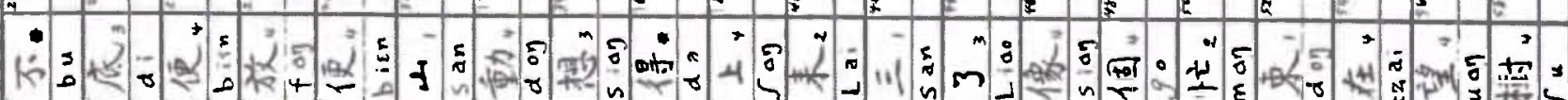

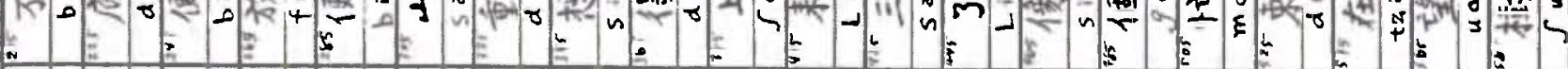

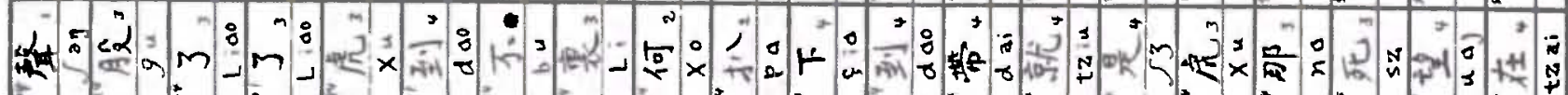

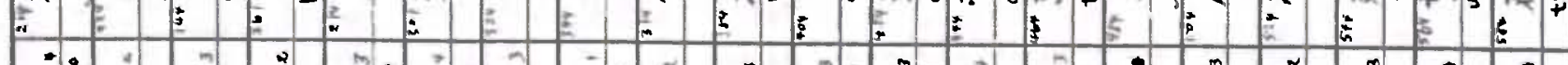
8

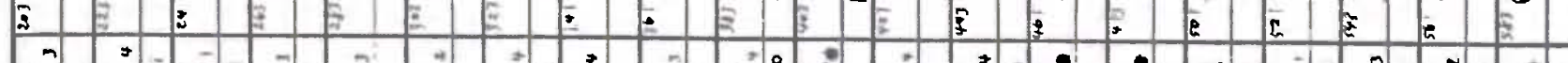
Th

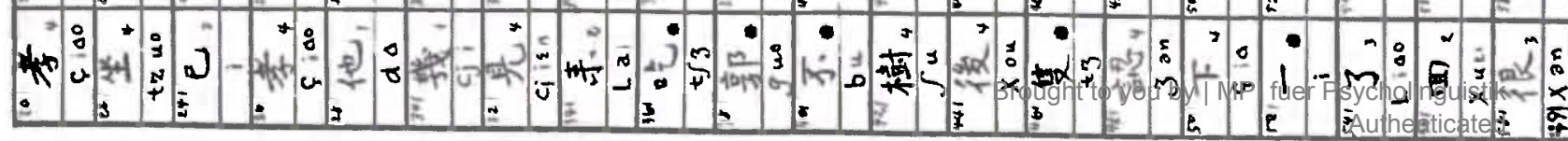




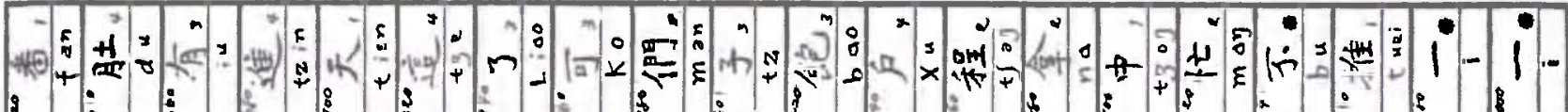

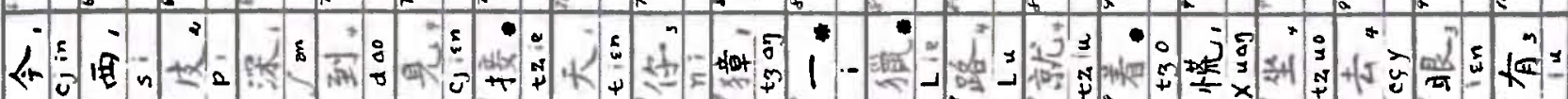

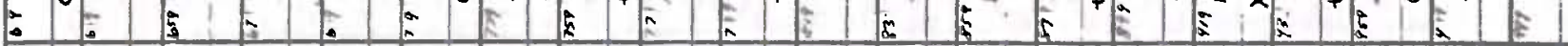

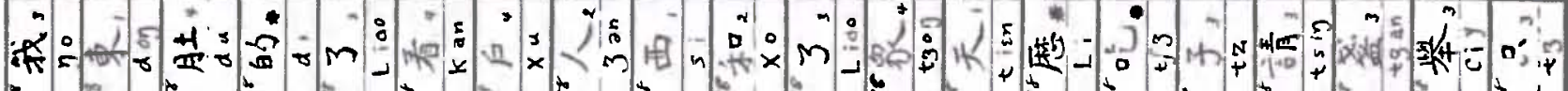
3

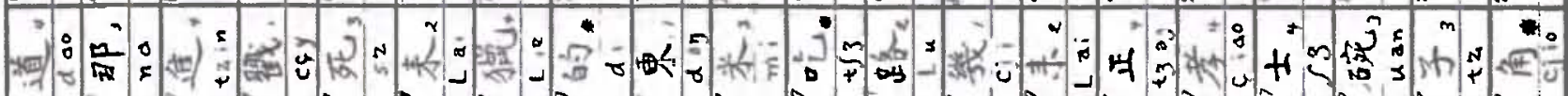

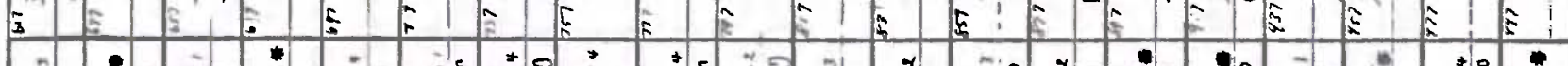

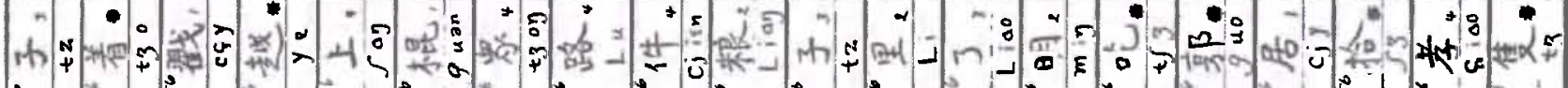

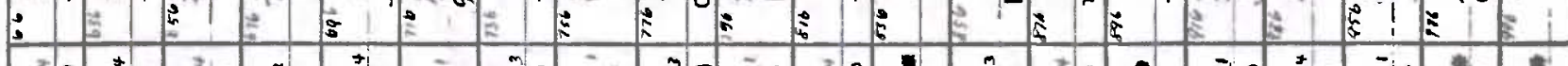
Hen

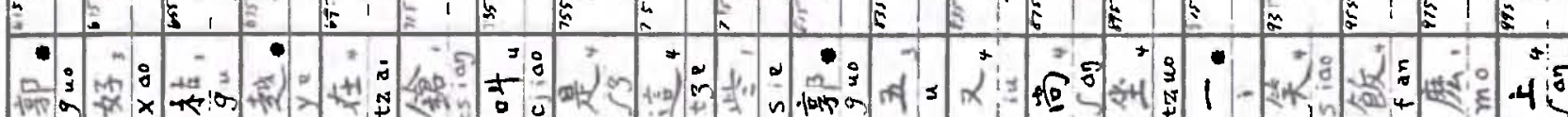

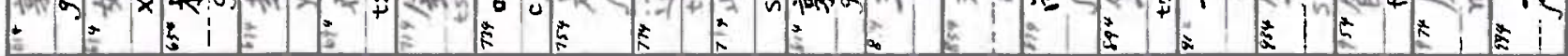
(5)

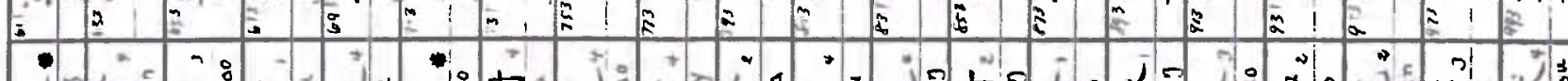

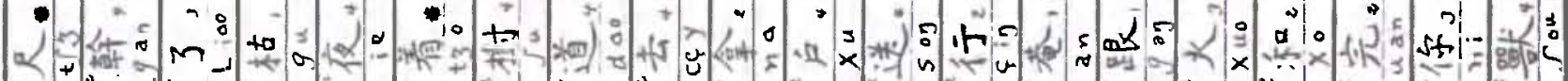

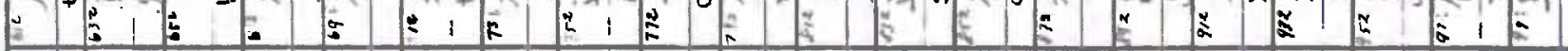

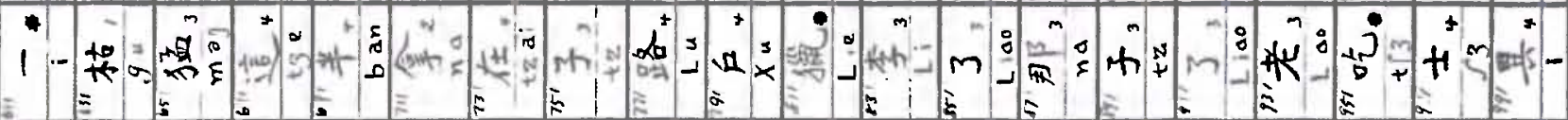

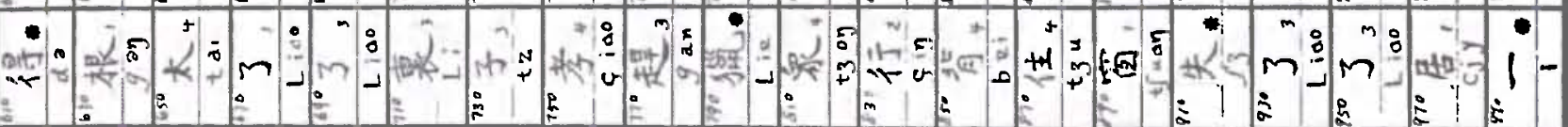

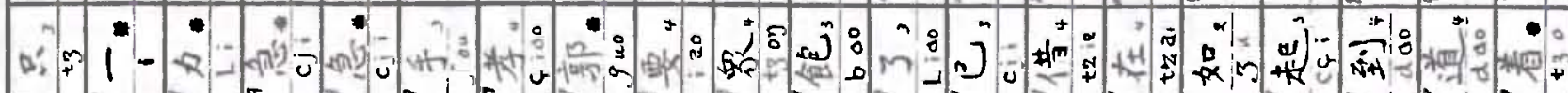

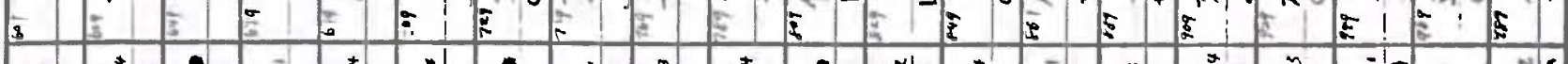
ht

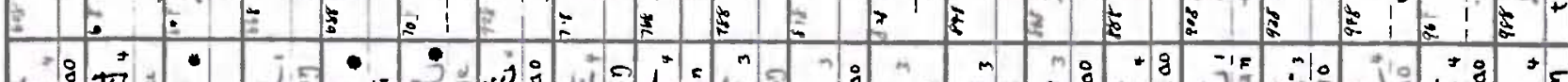

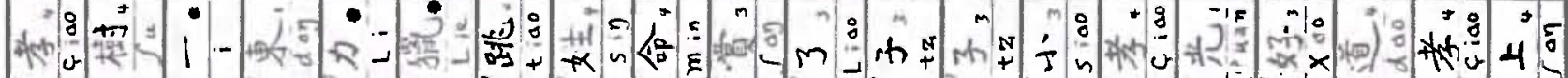

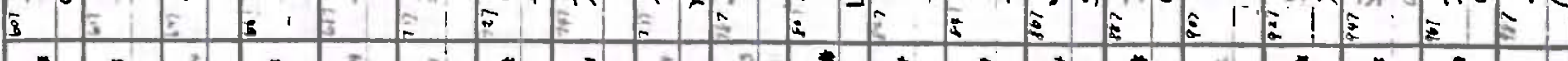

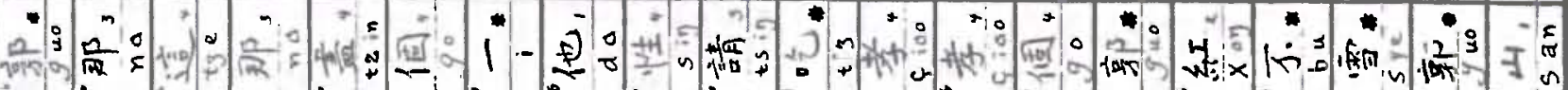

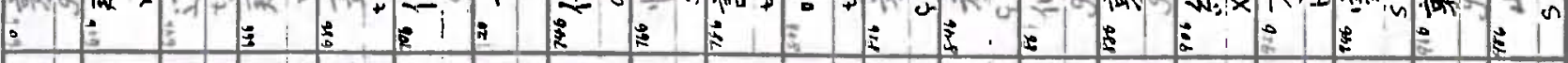

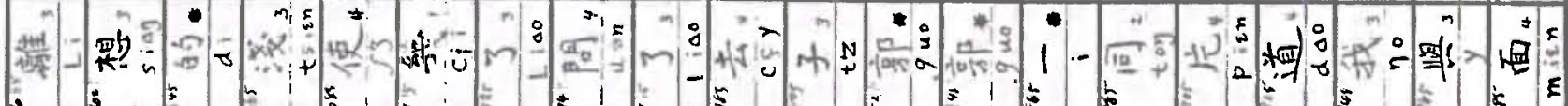

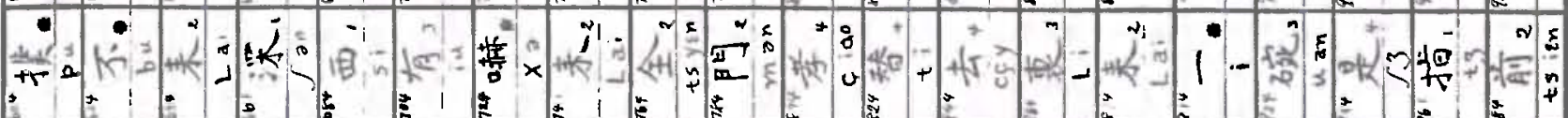

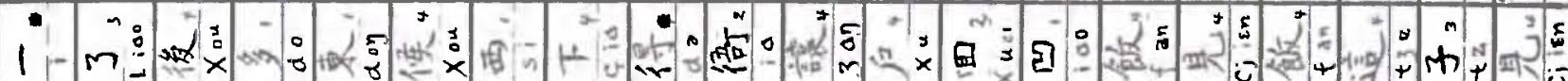

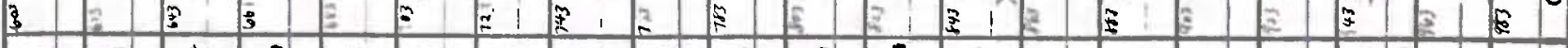

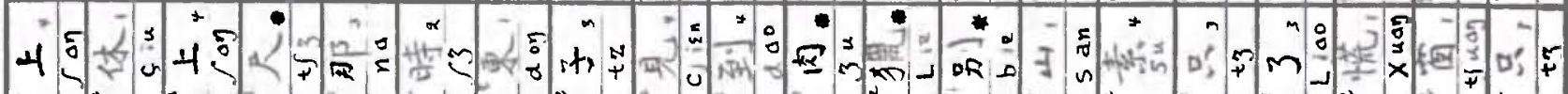

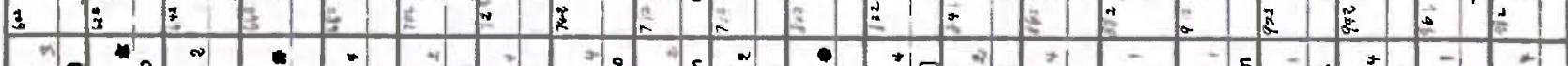

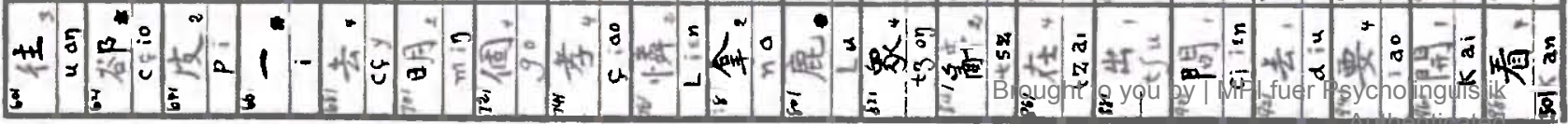




\section{APPENDIX C}


Brought to you by | MPI fuer Psycholinguistik Authenticated Download Date | 7/4/17 11:30 AM 


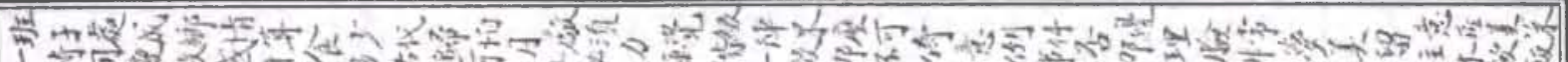
1 से

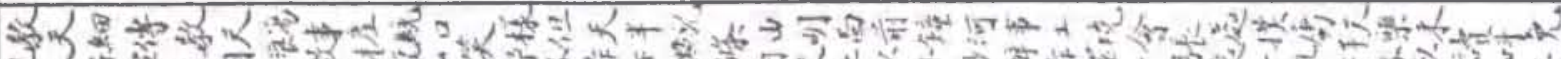

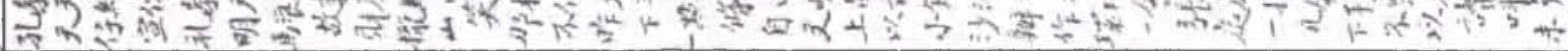

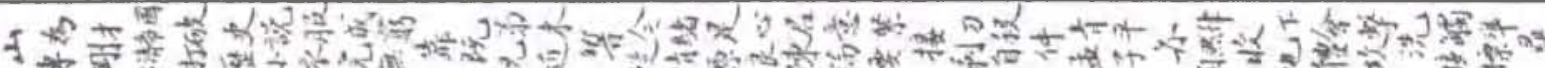

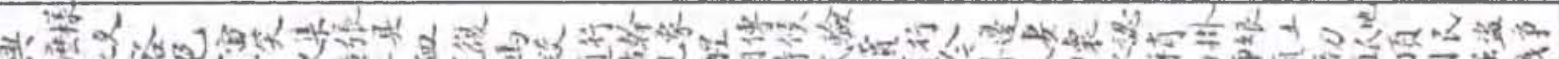
स:

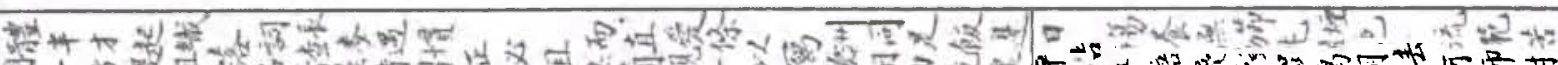

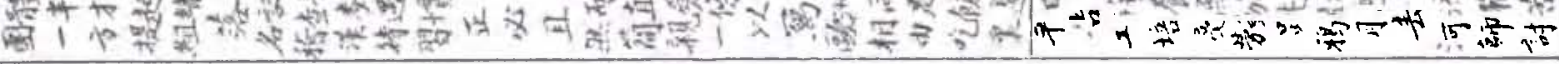

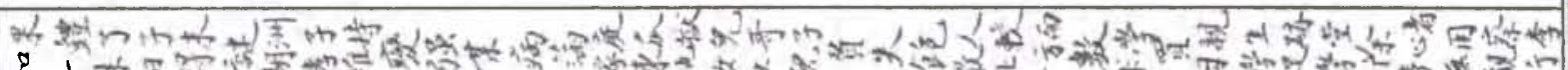

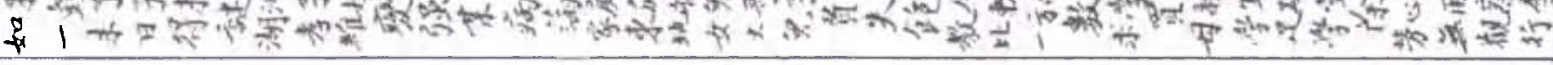

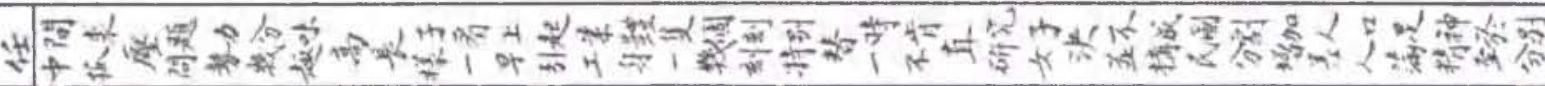
$\downarrow$

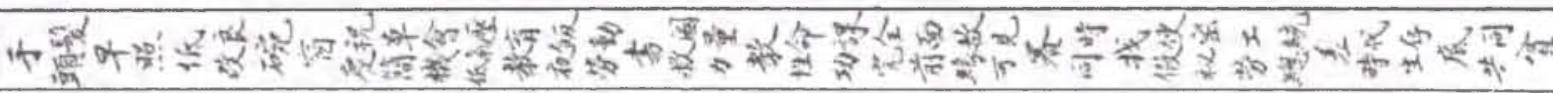

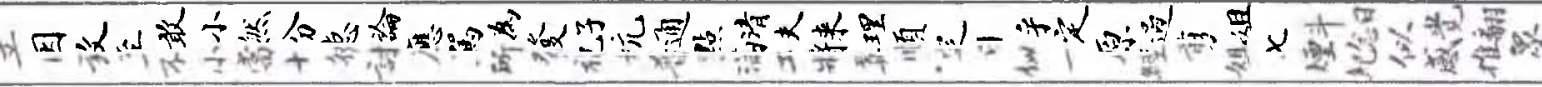

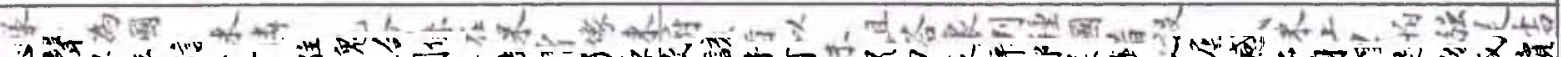

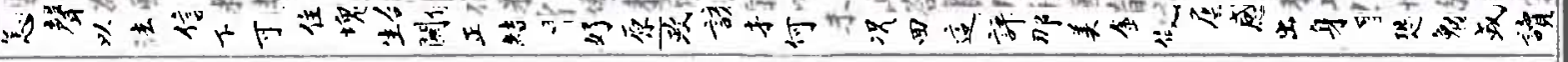
至 $\frac{3}{2}$ का w

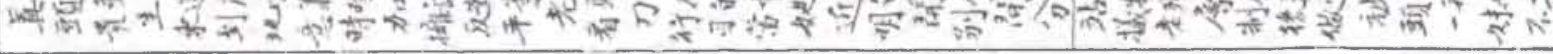

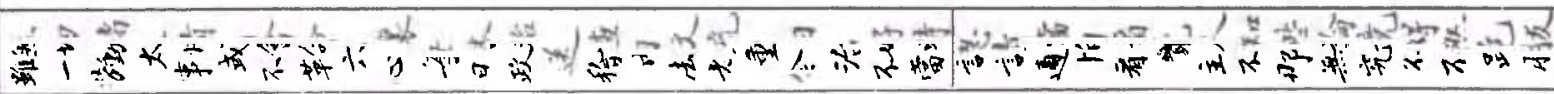

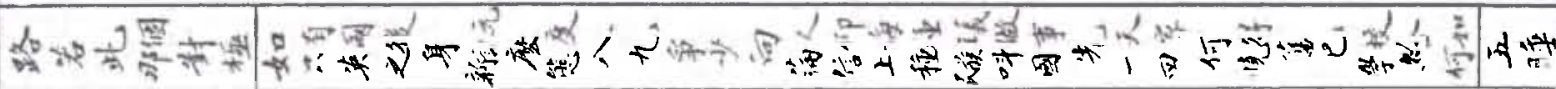

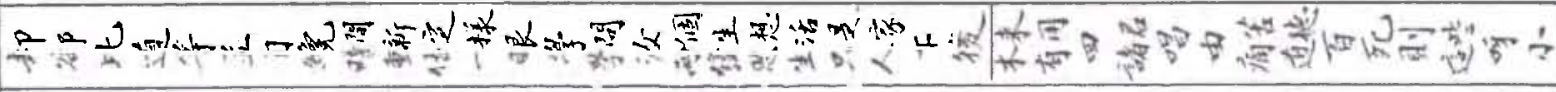

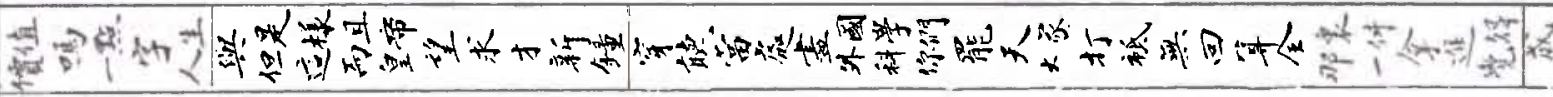

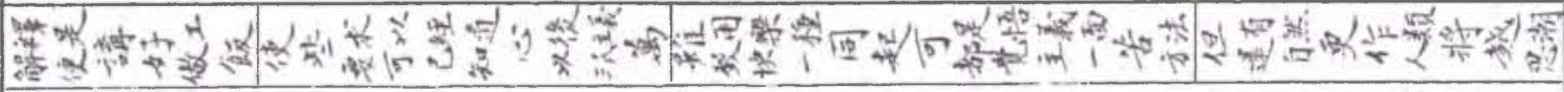

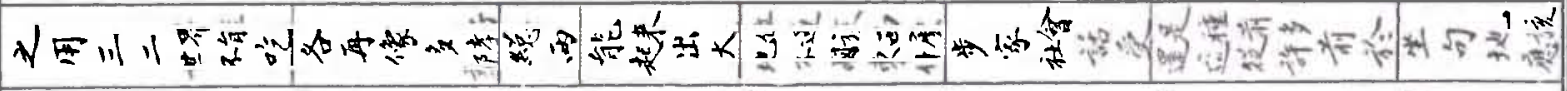

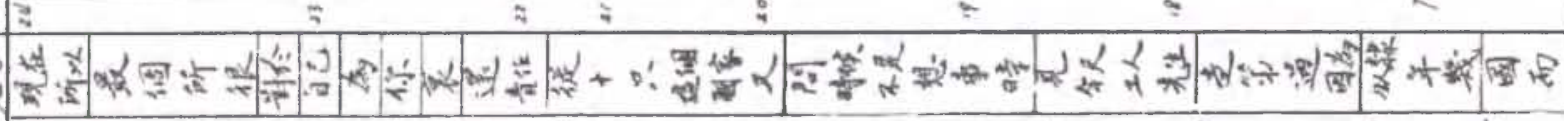

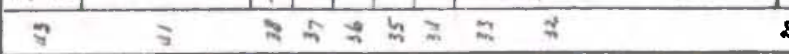

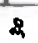

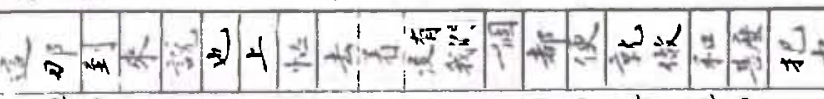




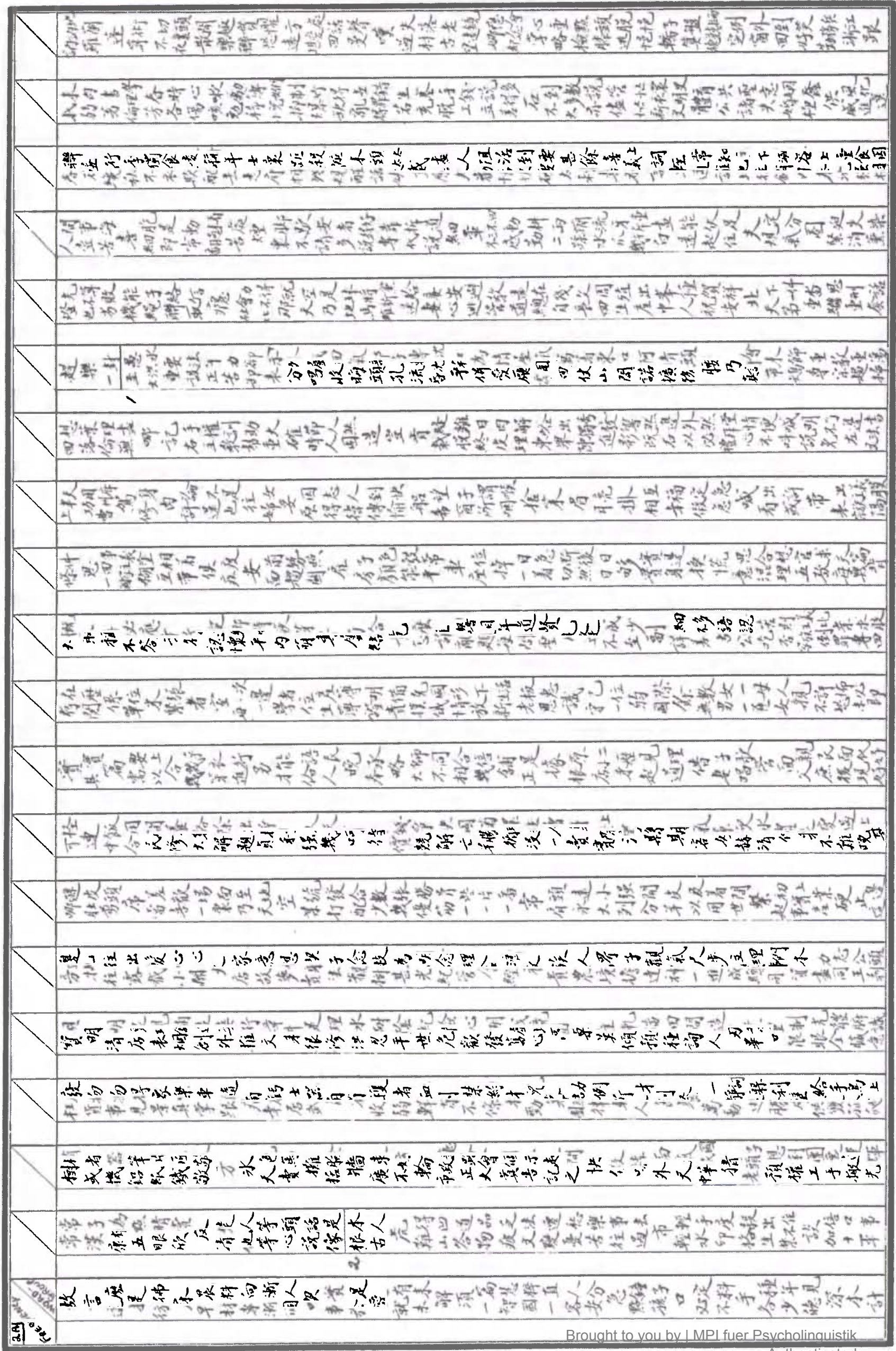




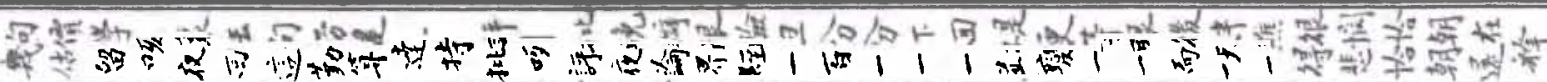

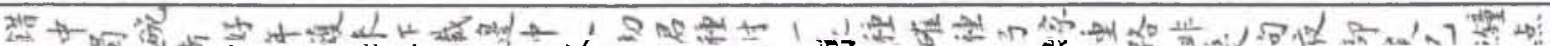

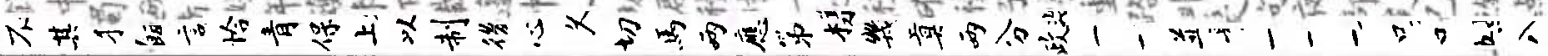

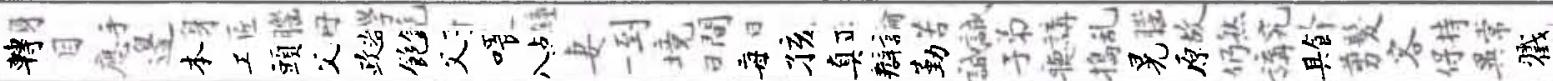

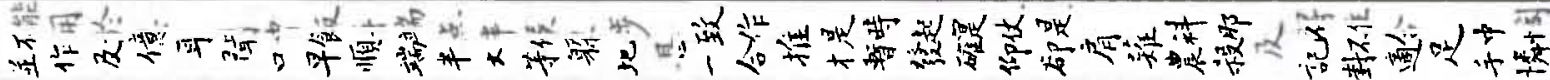

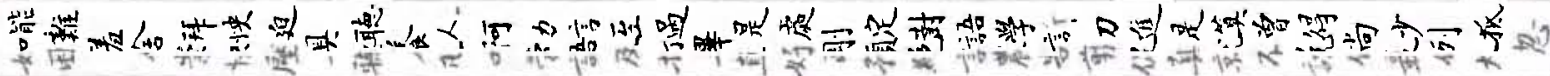

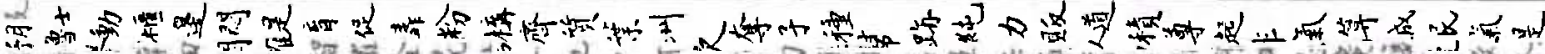

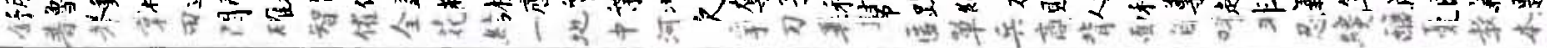
s.

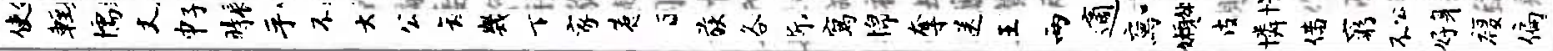

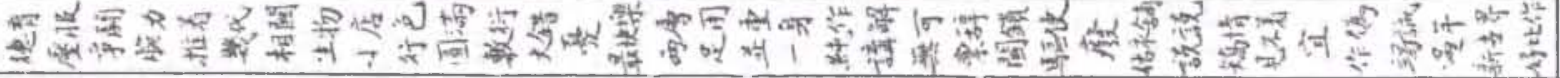

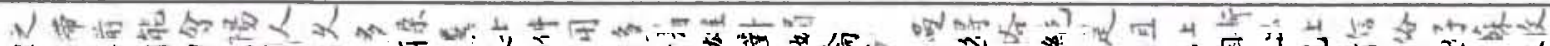

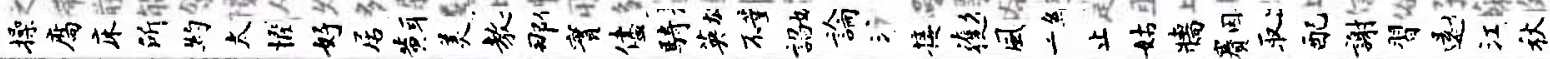

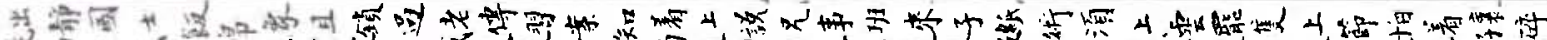

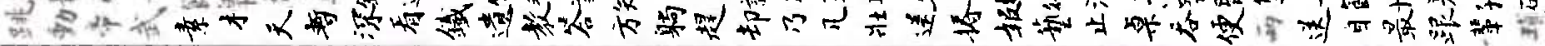

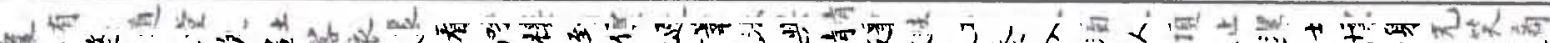

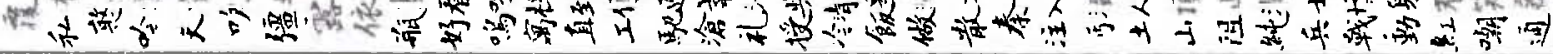

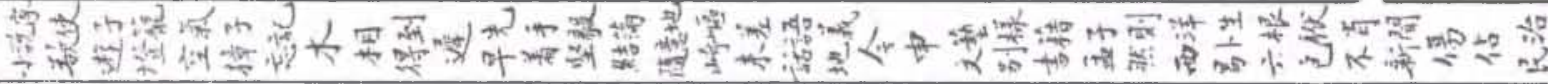

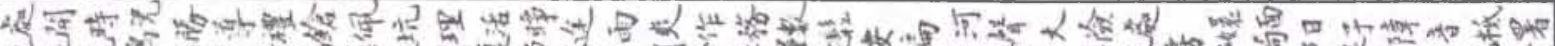

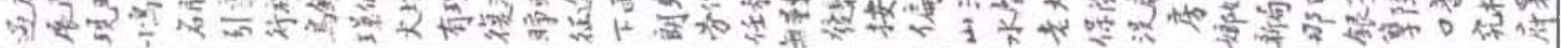
中心

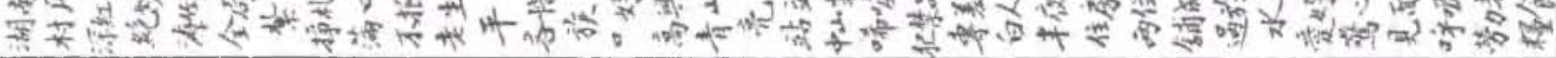
出

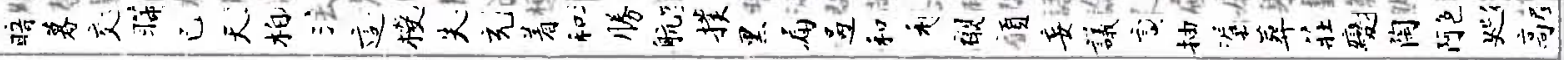

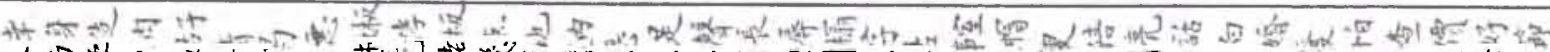

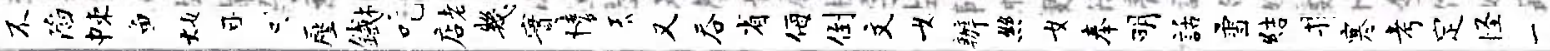

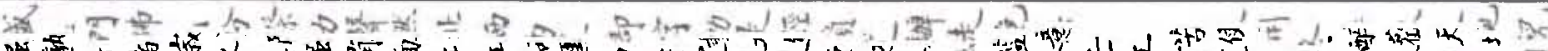

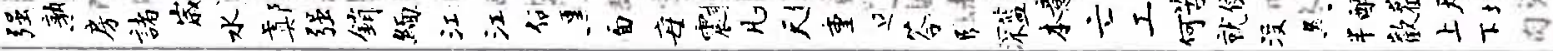

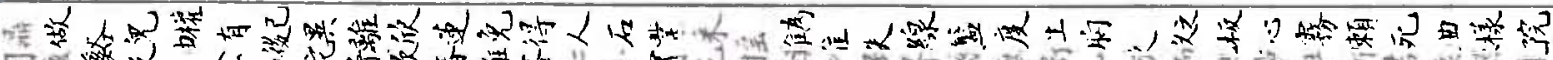

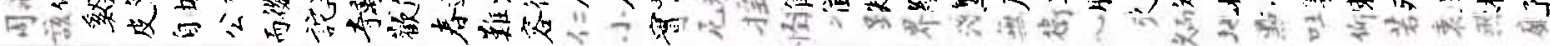

2.

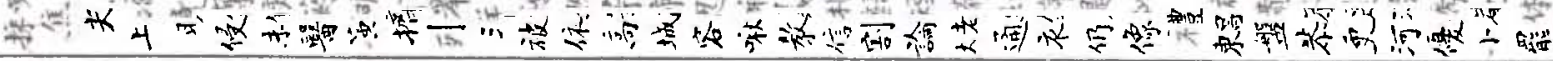

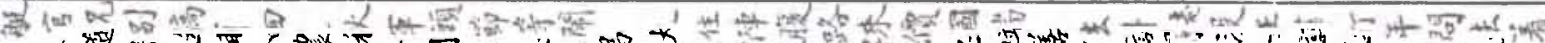

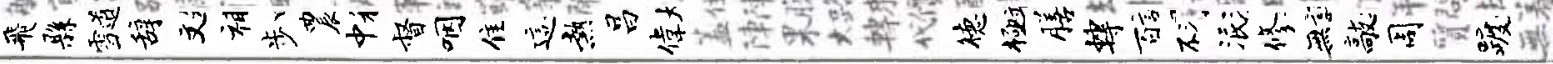




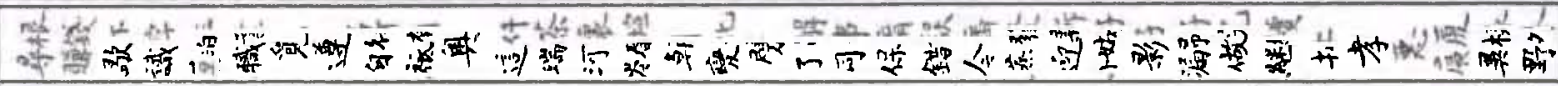

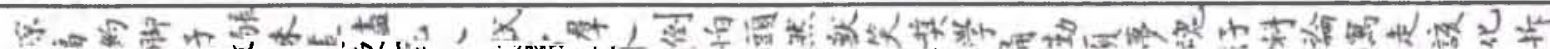

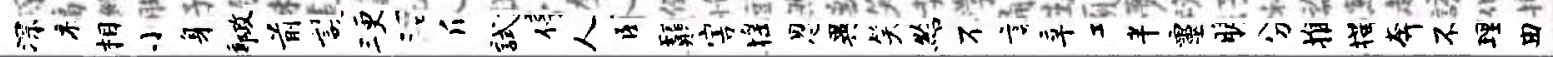

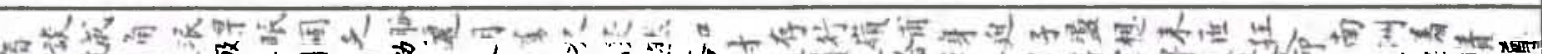

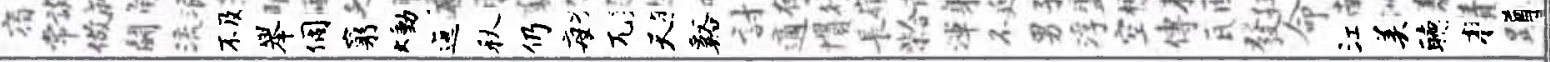

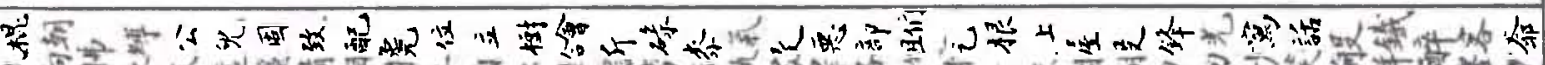

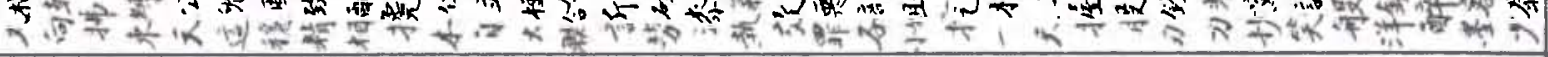

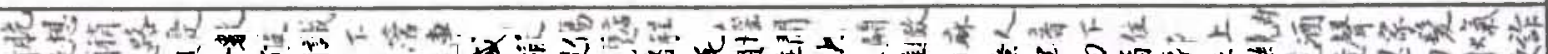

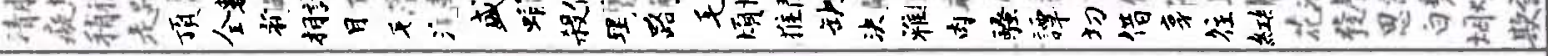

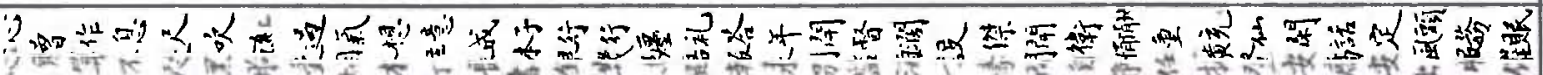

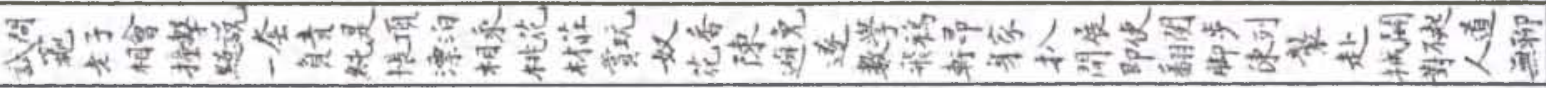

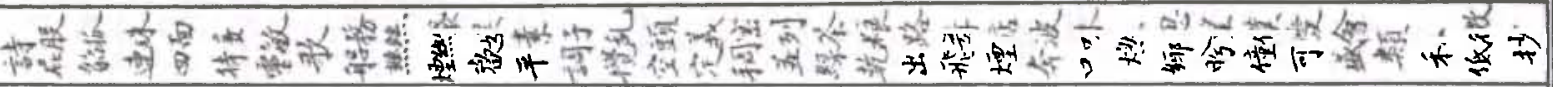

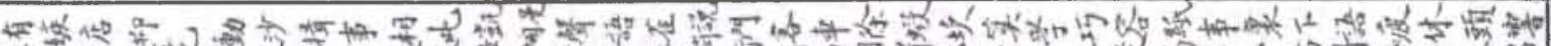

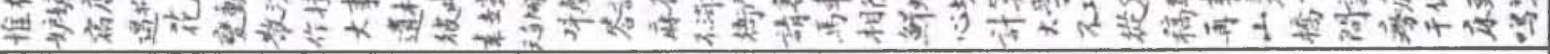

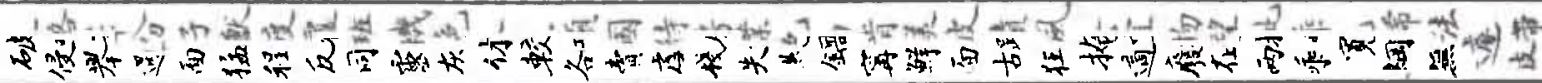

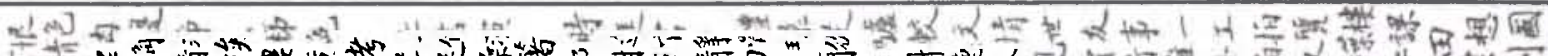

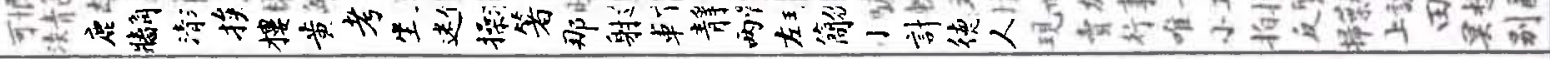

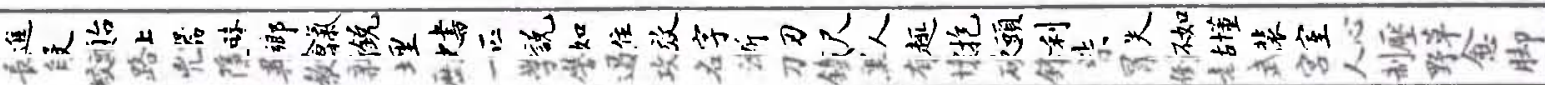

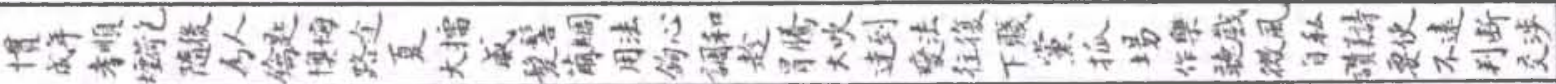

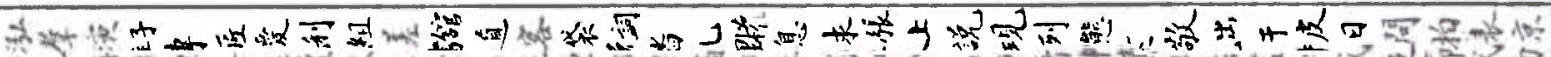

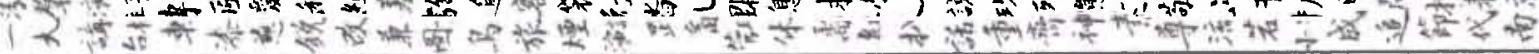

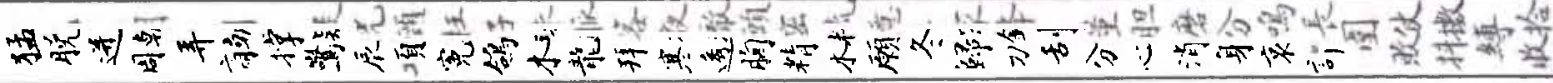
中

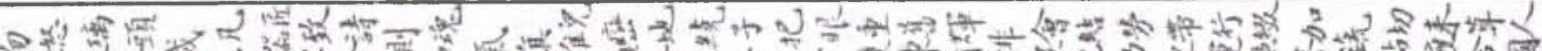

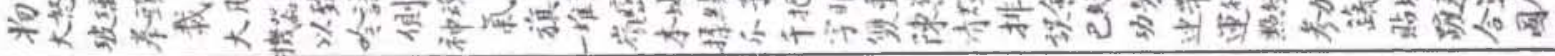

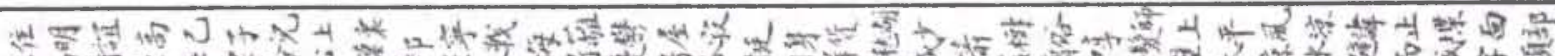

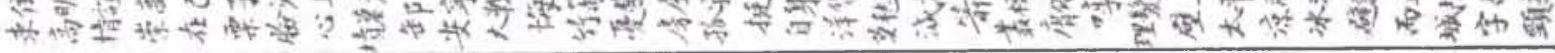

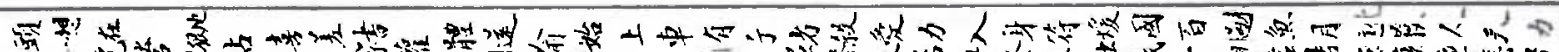

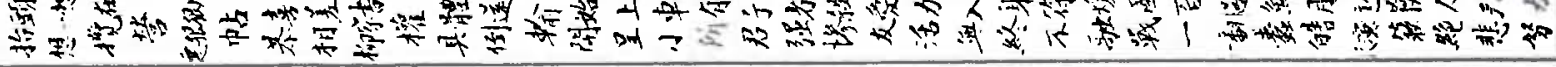

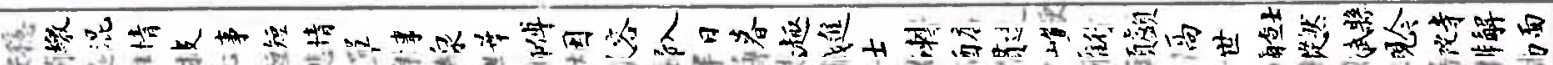




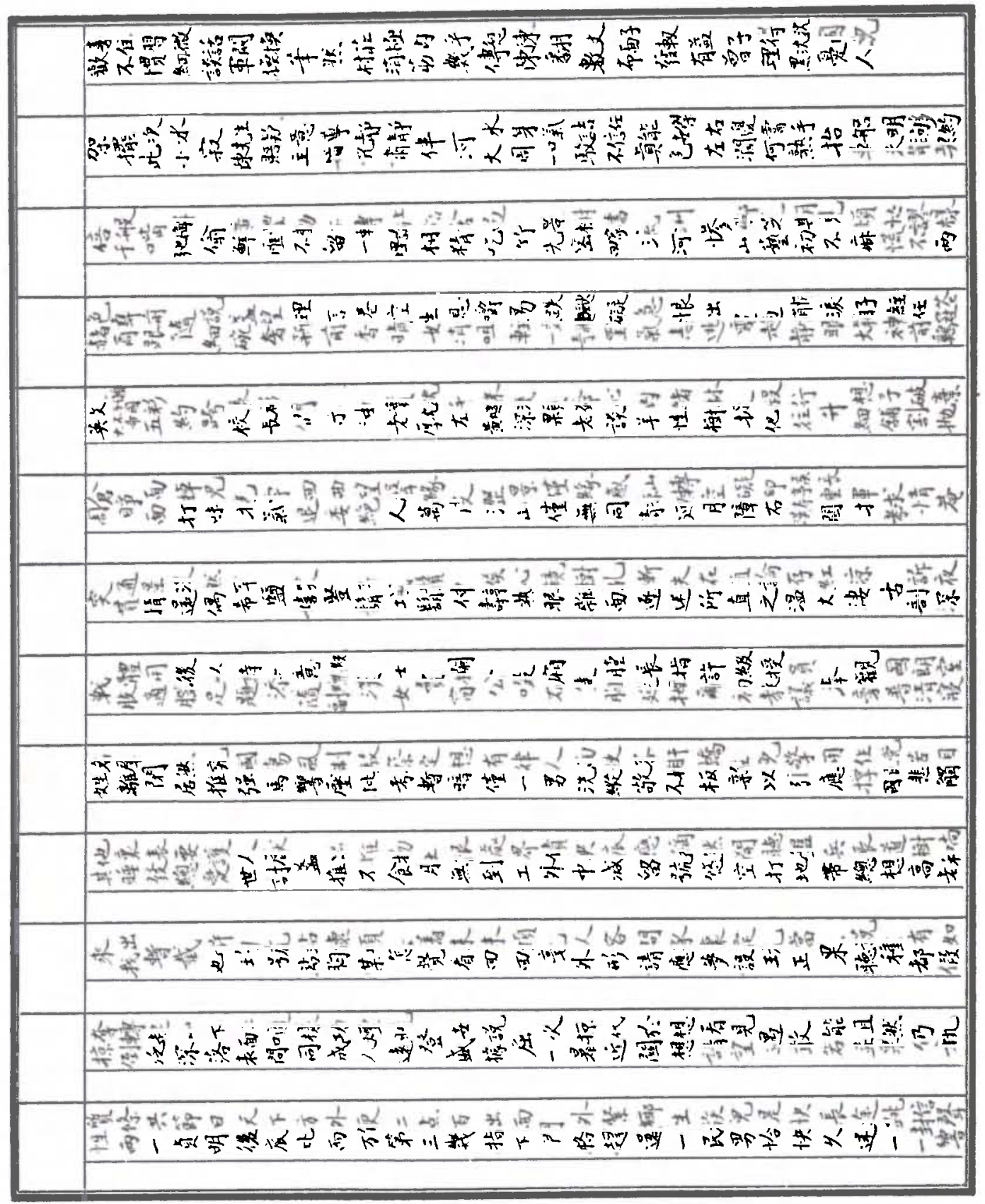


Brought to you by | MPI fuer Psycholinguistik Authenticated Download Date | 7/4/17 11:30 AM 


\section{APPENDIX D}


Brought to you by | MPI fuer Psycholinguistik Authenticated Download Date | 7/4/17 11:30 AM 


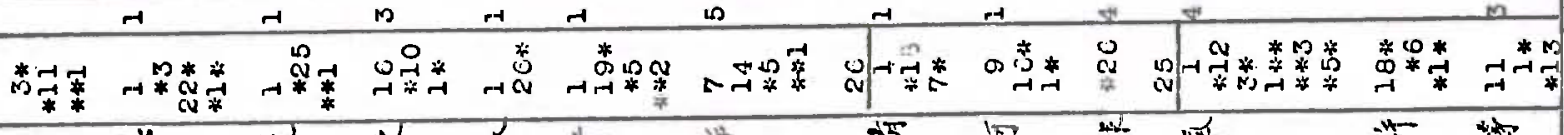
का

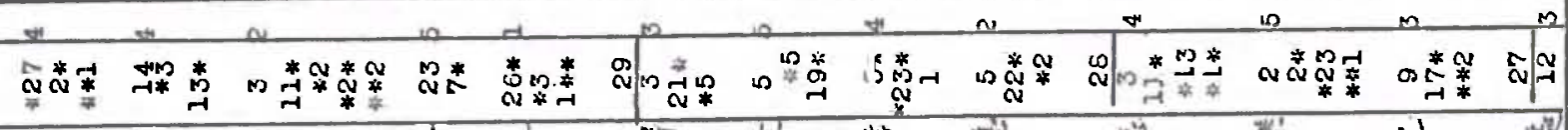

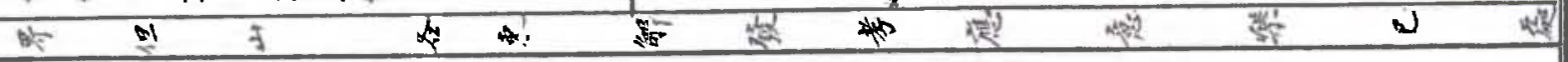

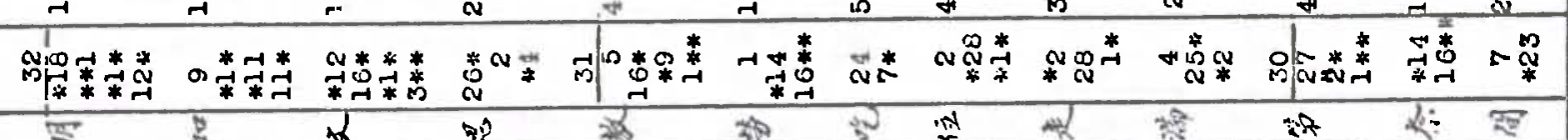

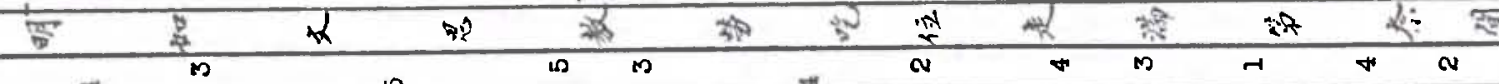

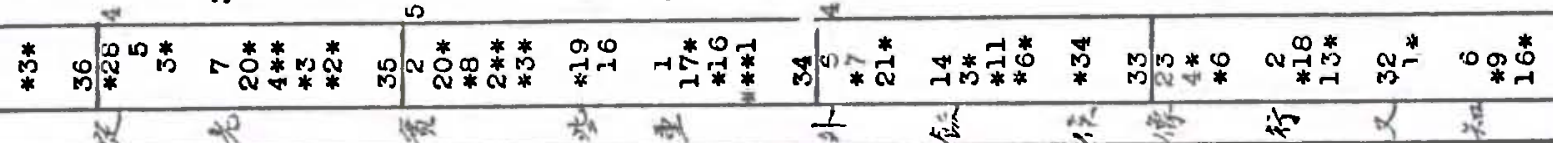

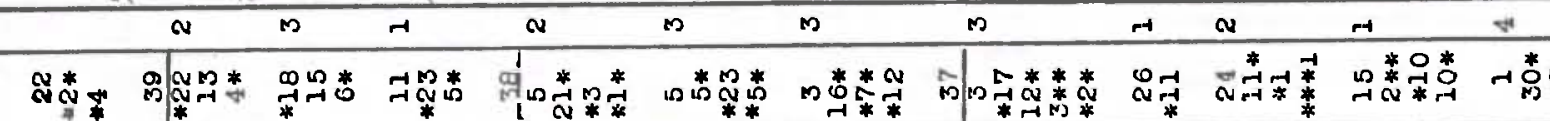

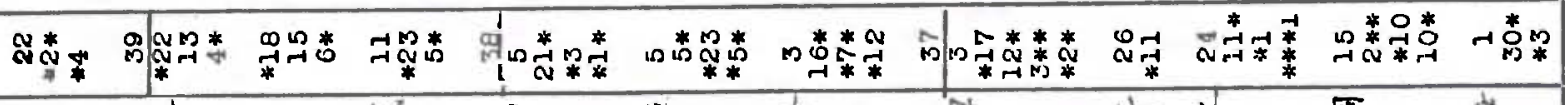

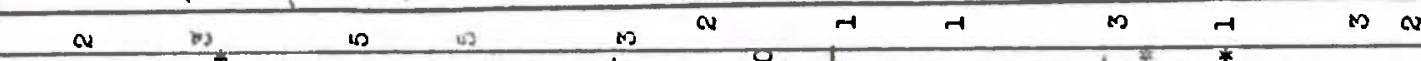

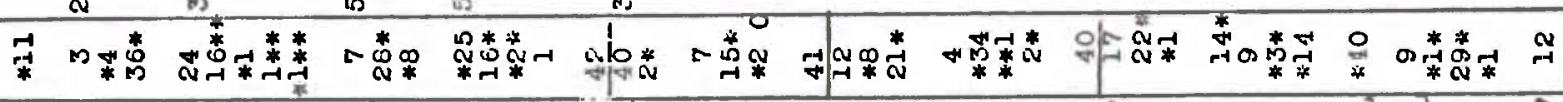

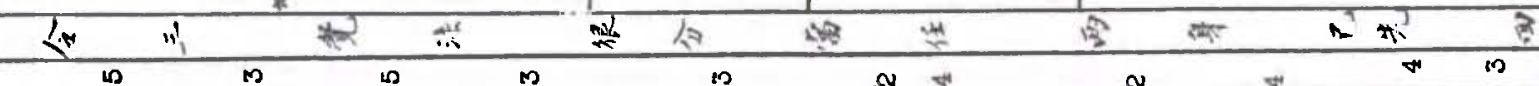
* 荤

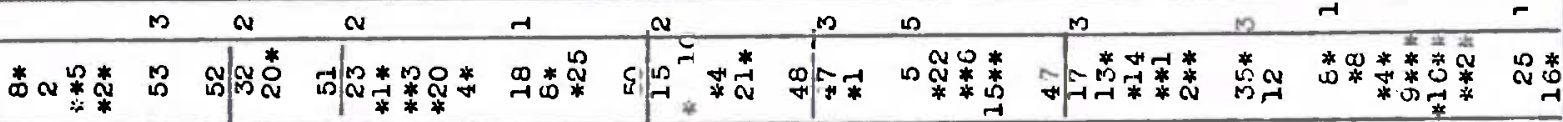

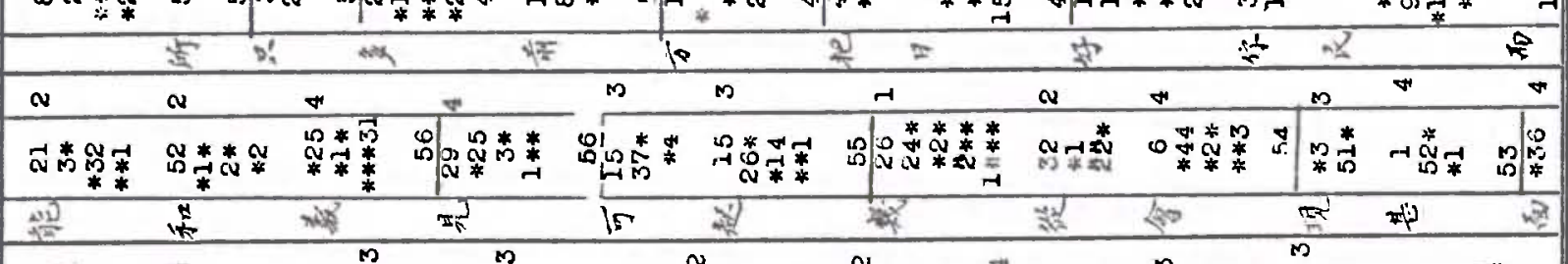

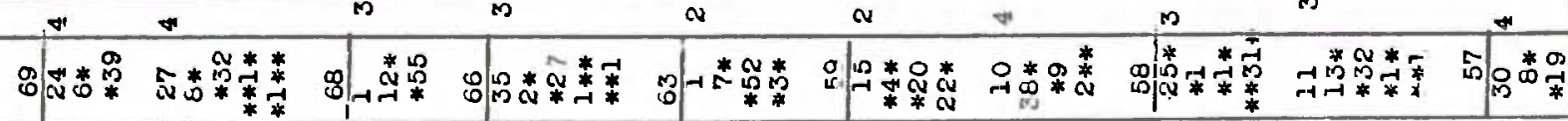
F का

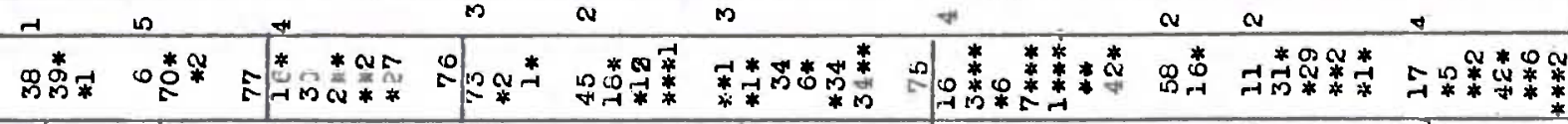
तो

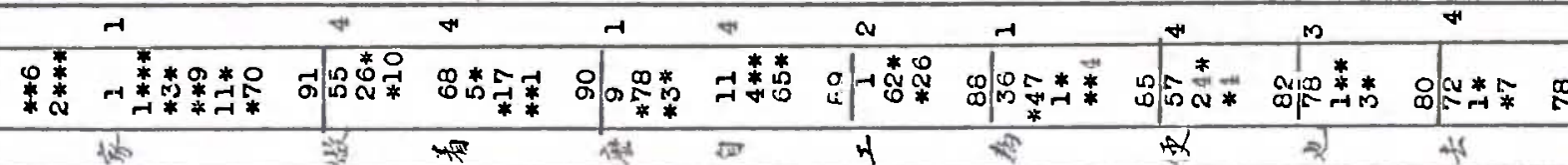

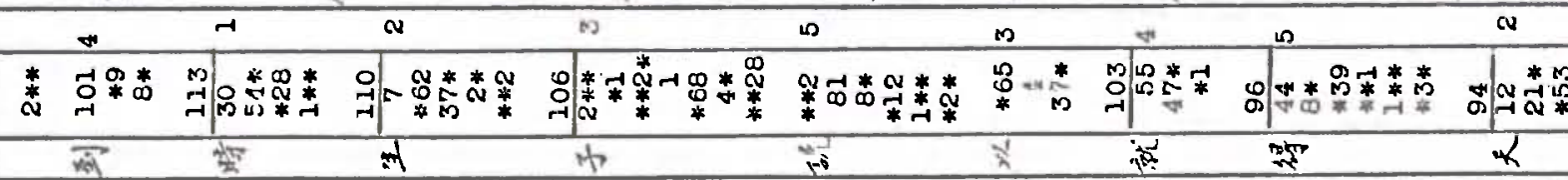

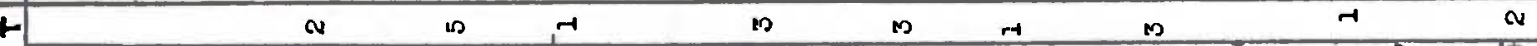
2 2.

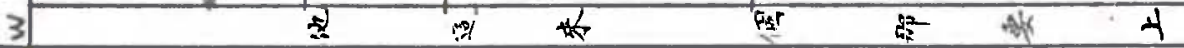

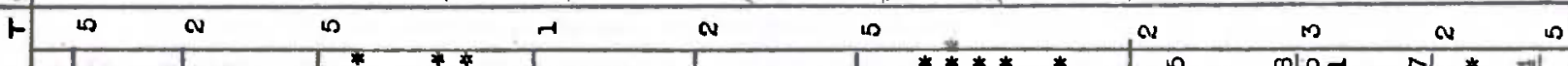

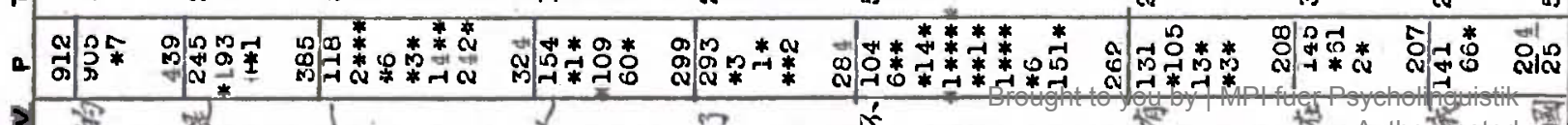
国 3 का 


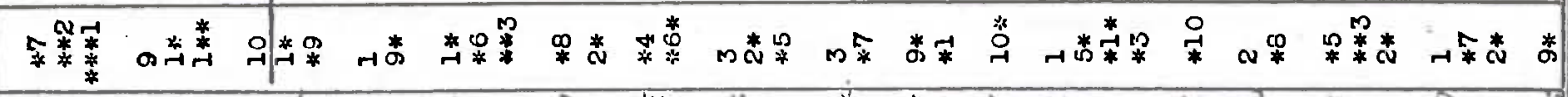

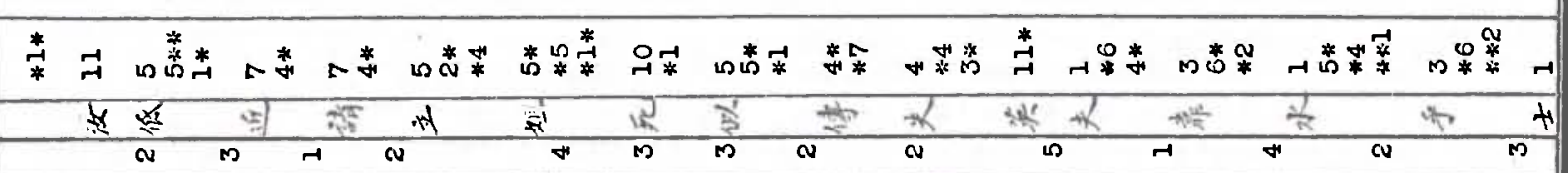

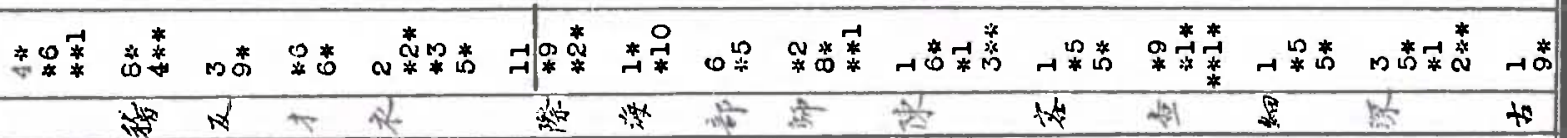

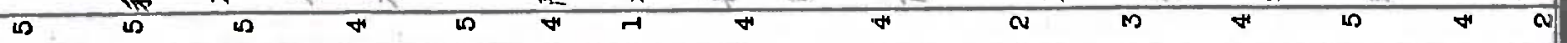

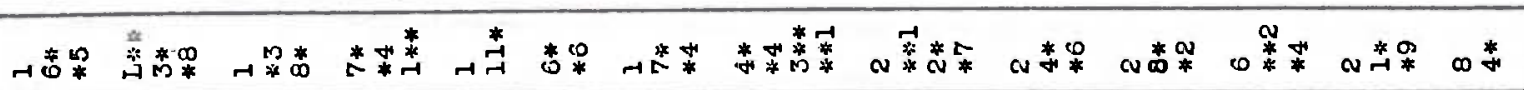

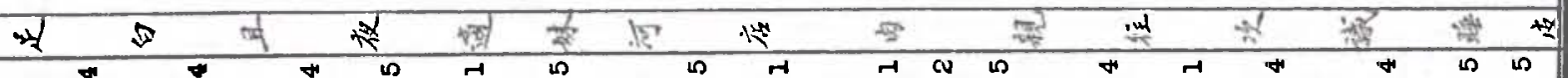

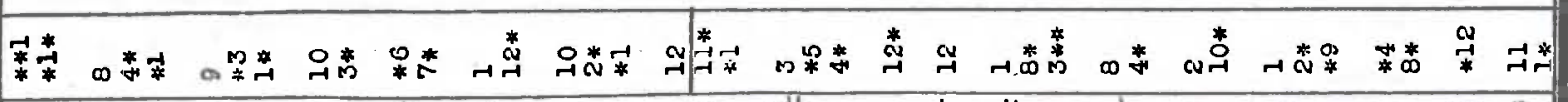

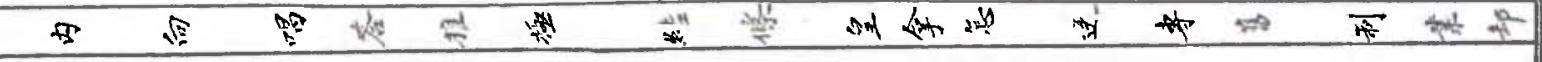

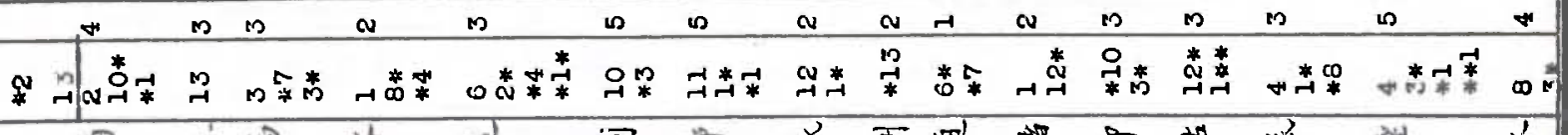

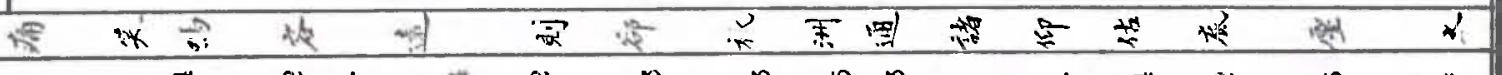

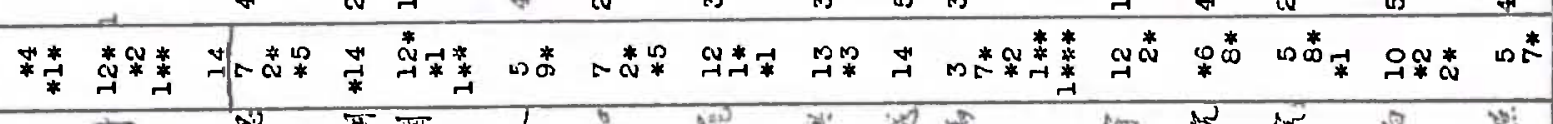

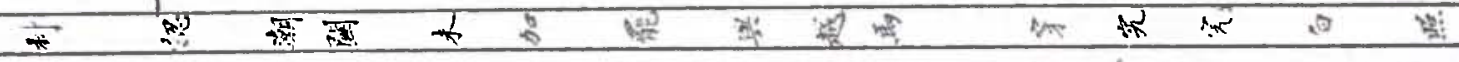
a $m$ \#

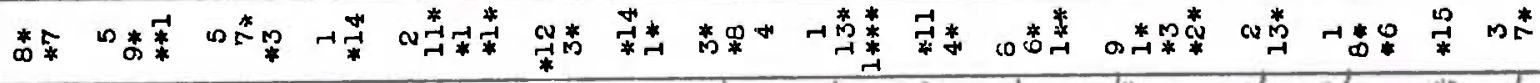

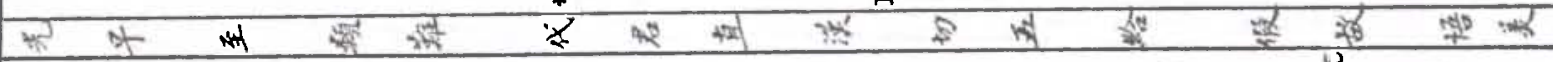

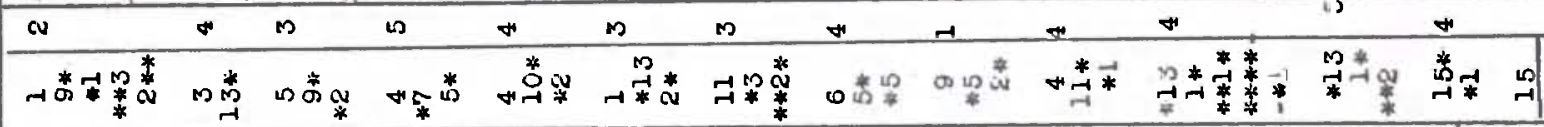
क

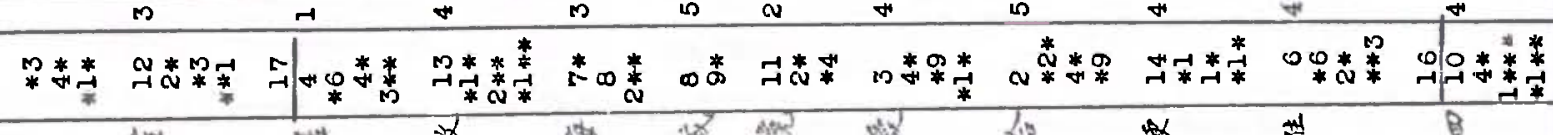

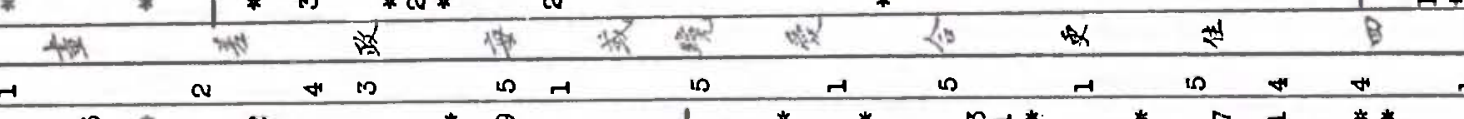

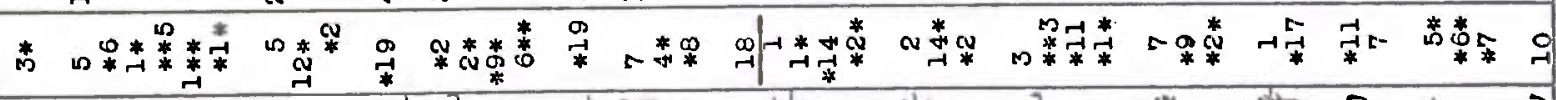

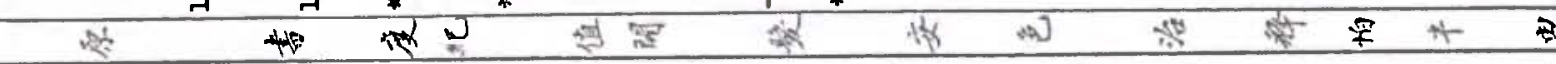

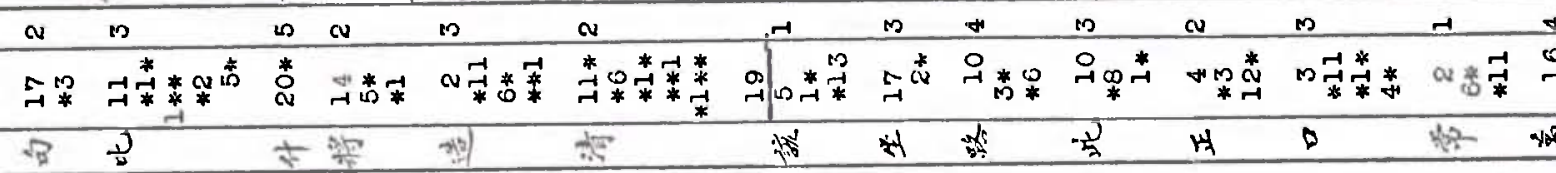

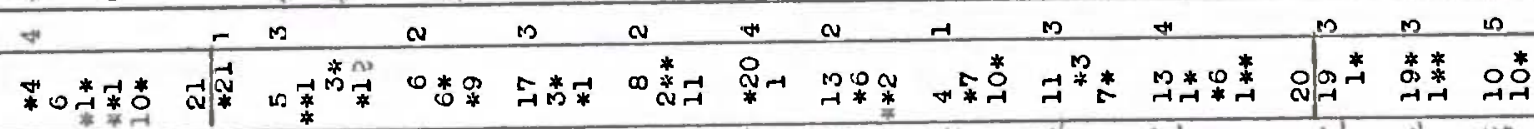

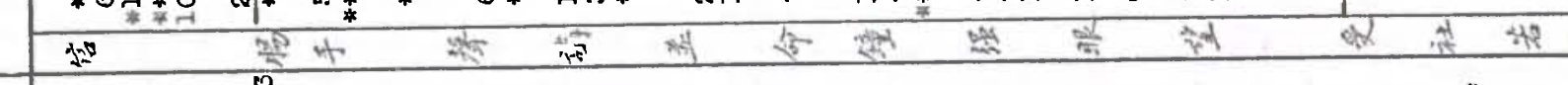

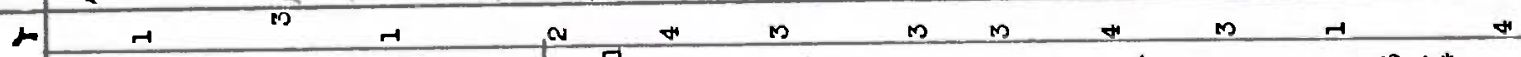

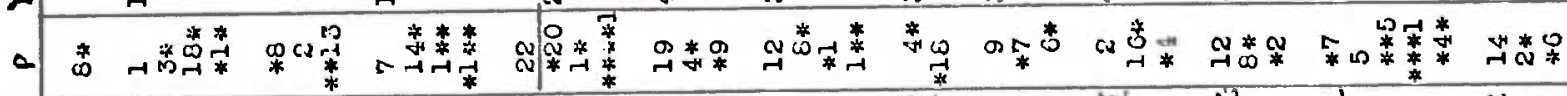
3 拿 情

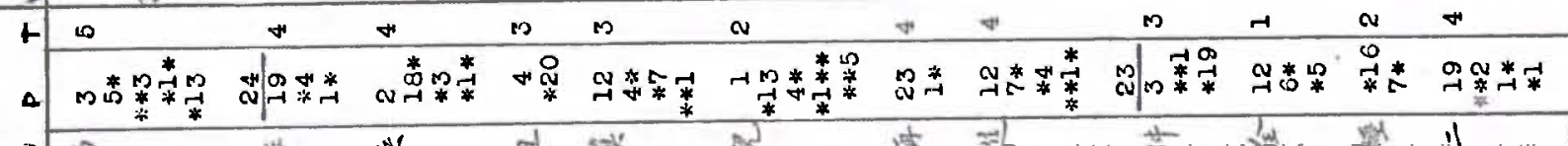
की 3 


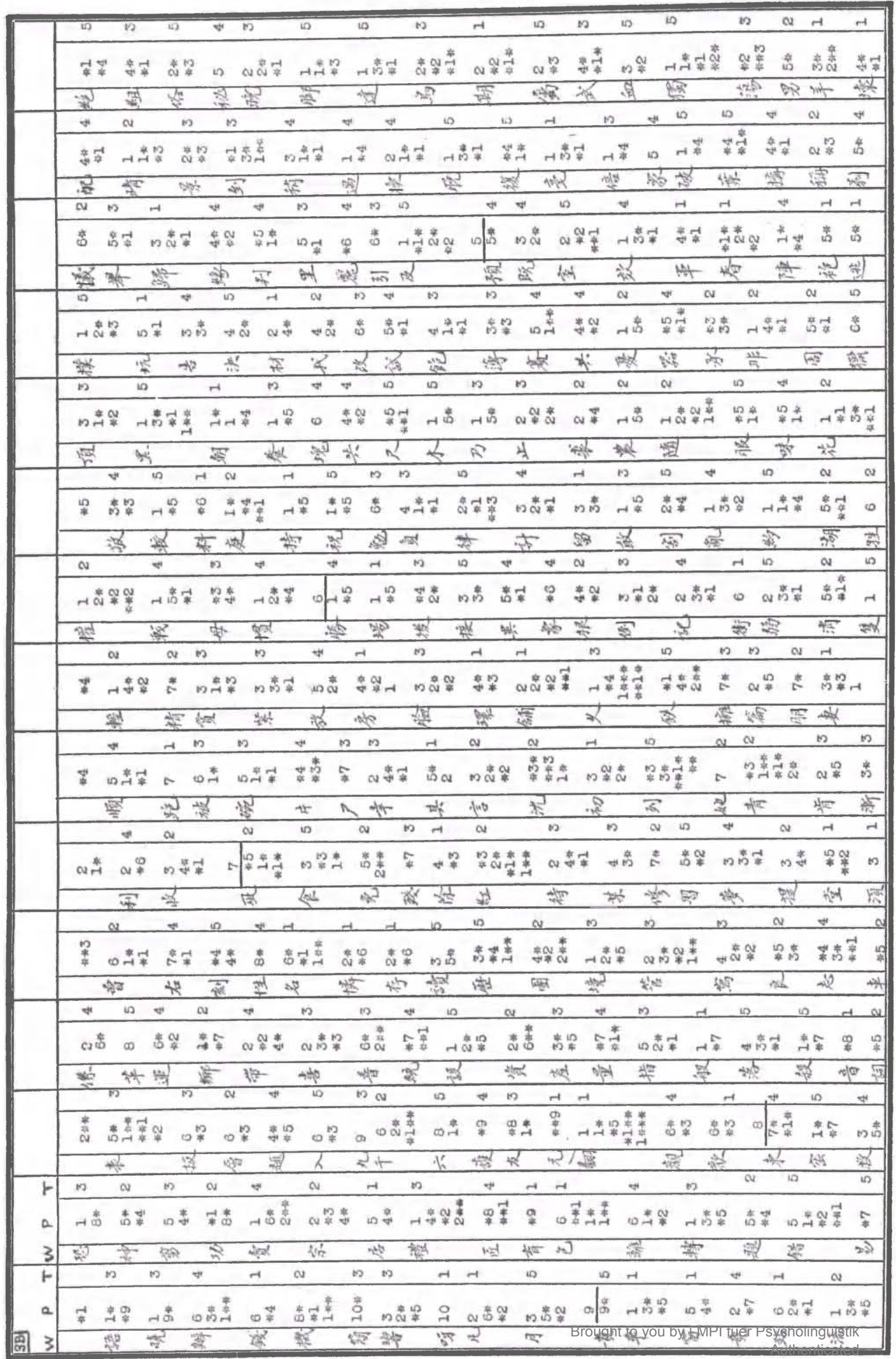




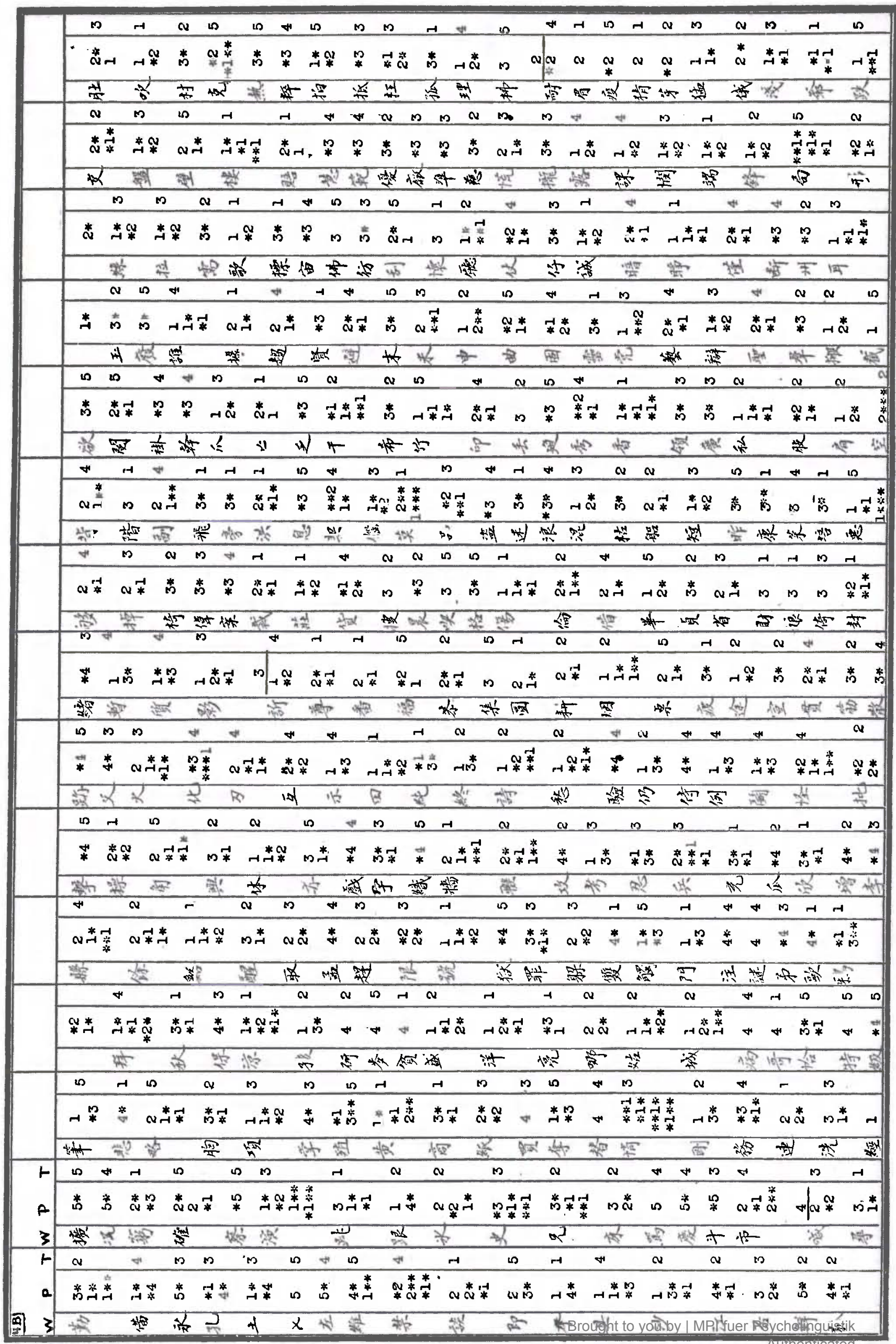




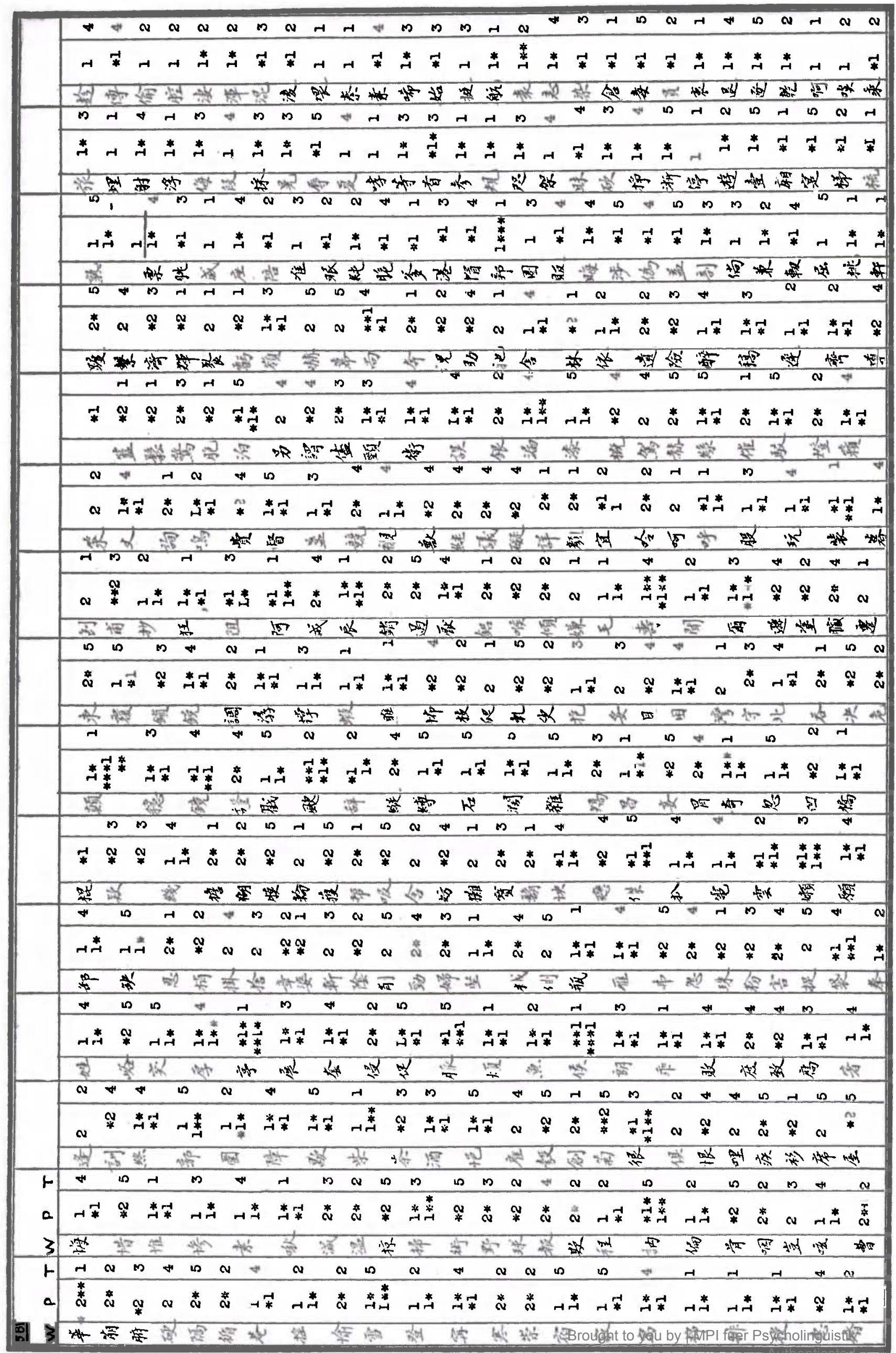




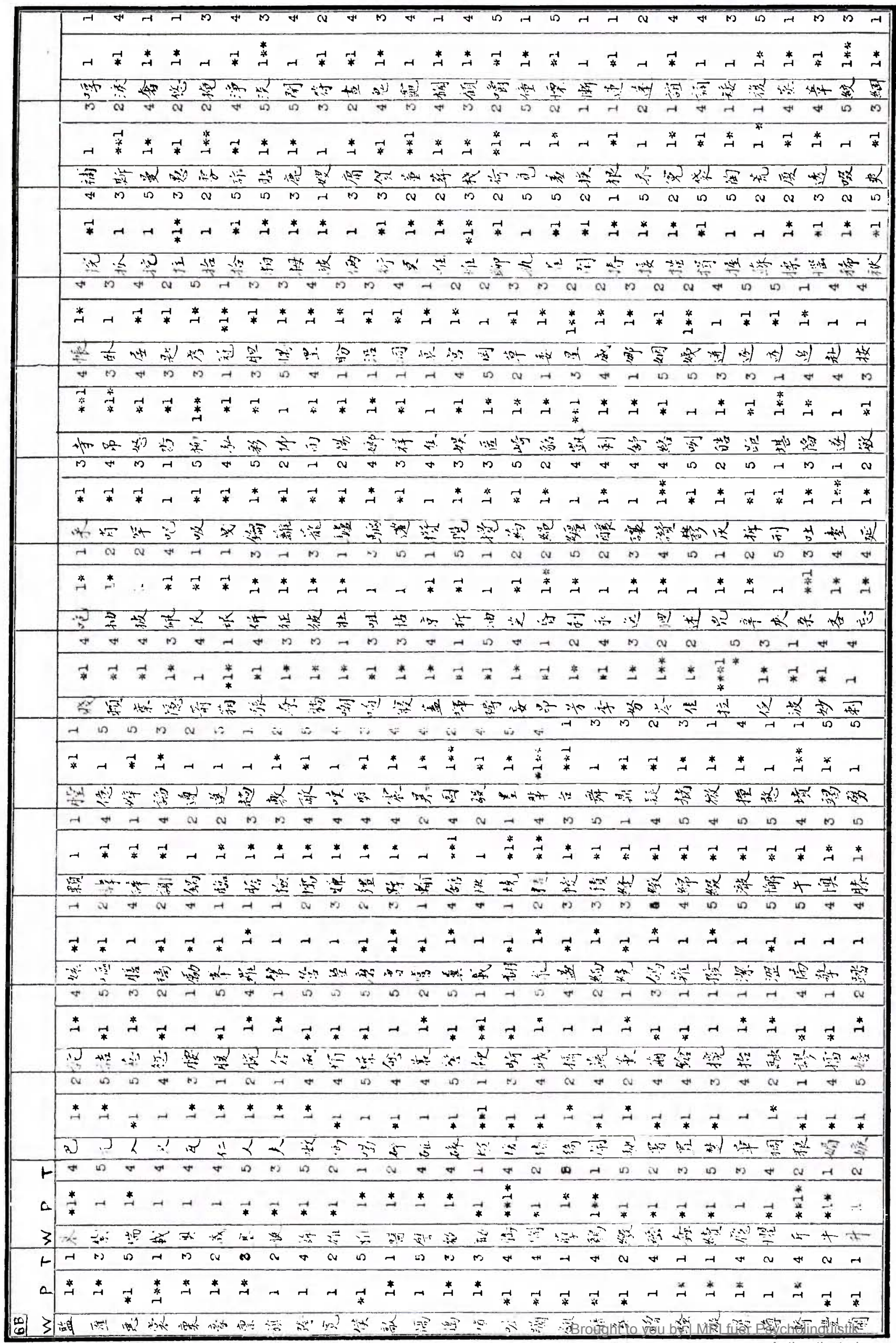




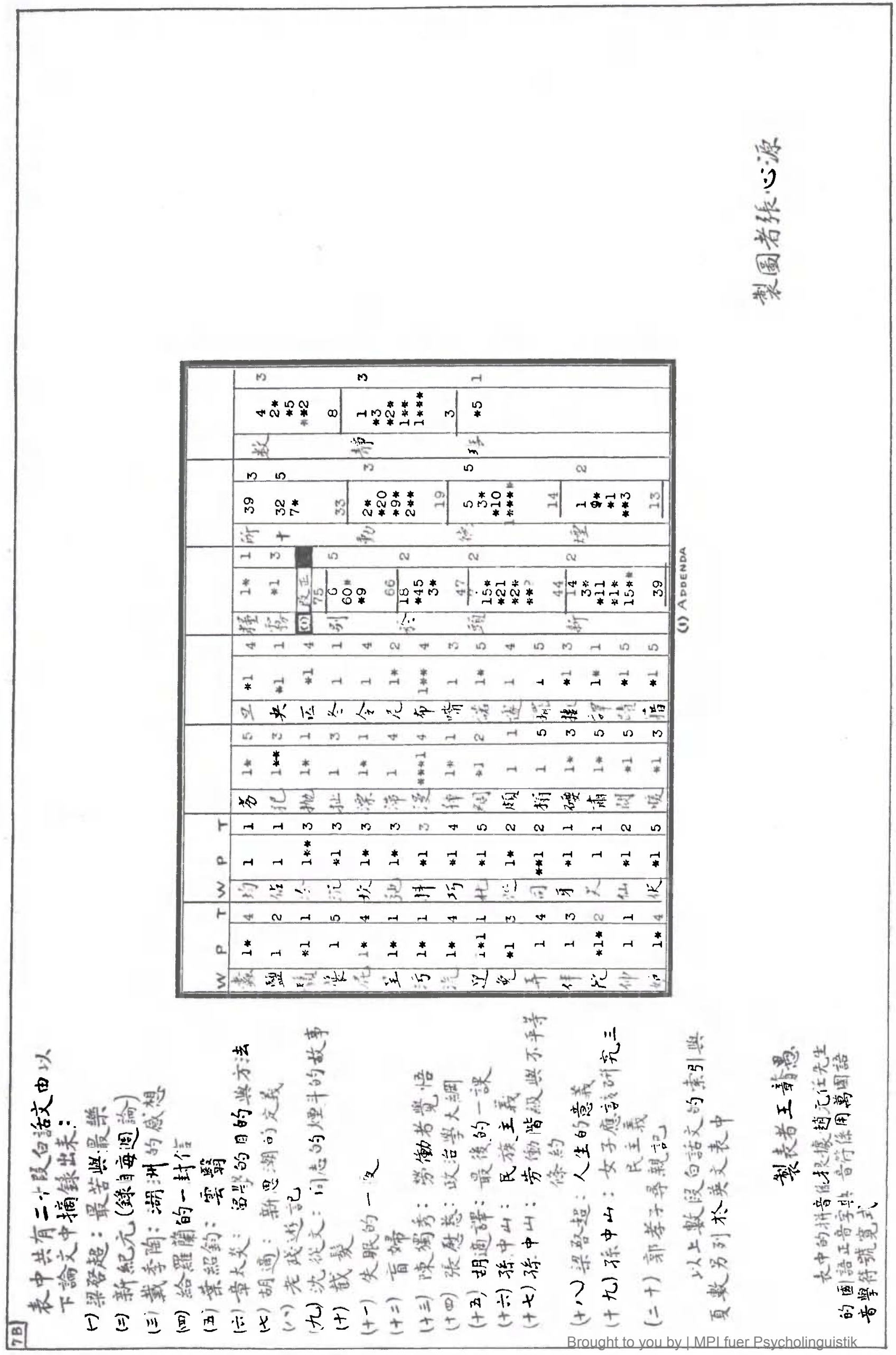

Brought to you by $\mid \mathrm{MPI}$ fuer Psycholinguistik 
Brought to you by | MPI fuer Psycholinguistik Authenticated Download Date | 7/4/17 11:30 AM 